\title{
Quarterly Report of RCRA Groundwater Monitoring Data for . Period October 1 through December 31, 1994
}

\author{
Earth and Environmental \\ Technical Services \\ Westirghouse Hanford Compary
}

Date Published

April 1995

\section{DISCLAIMER}

Thts report was prepured as an account of work sponsored by an ajency of the Uniked Sintes Gowernment. Nether the Uniled Stales Governemeat ner eny egency thersol, nor any of their employees, makes any wartanty, exptess or implied, or assumes any legal lisbility oc responsi-

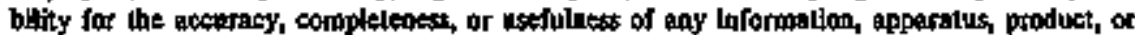
process disclowed, of repressepts that its ust would not inftinge privalely owated tights. Refor-

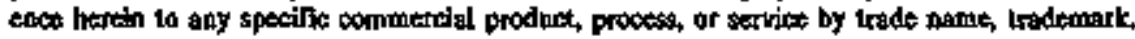

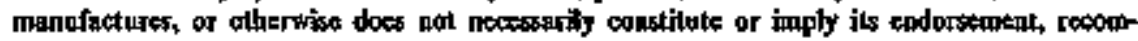

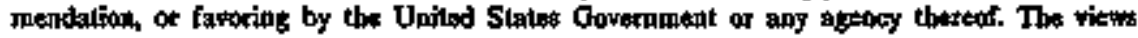
and options of auluors expressed berein do not nectsarily state er teflect those of the Uaitod States Gorenament or any asency thereof.

\section{United States}

Department of Energy

P.O. 80x 530

Richiand, Washington 99352

$$
\text { - }
$$




\section{DISCLAIMER}

Portions of this document may be illegible in electronic image products. Images are produced from the best available original document. 


\section{LIST OF TERHS}

\begin{tabular}{|c|c|}
\hline 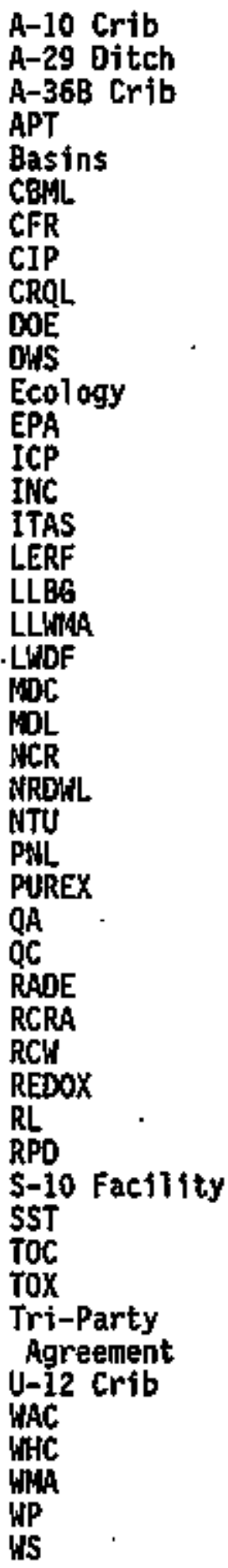 & 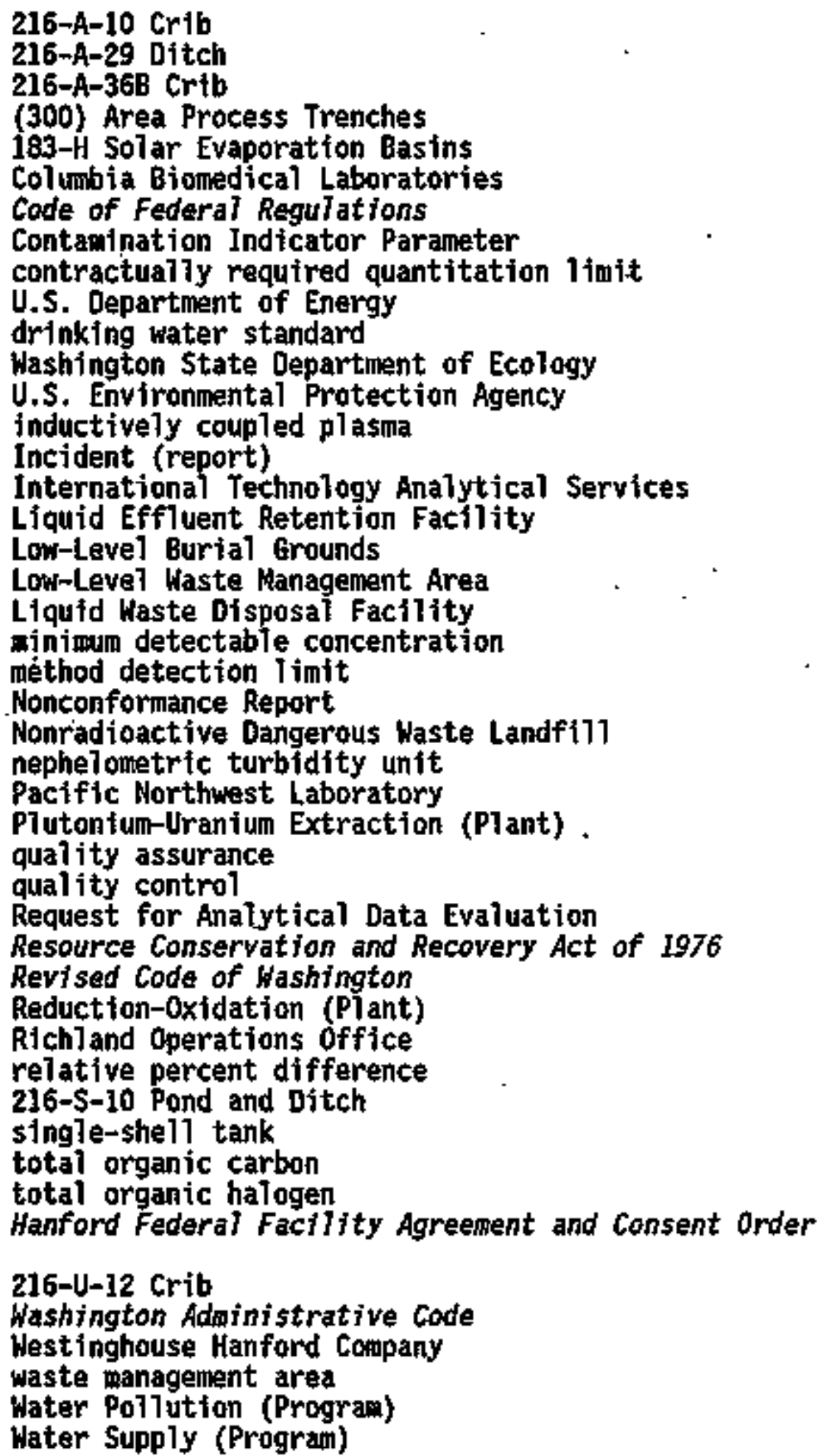 \\
\hline
\end{tabular}


DOE/RL-94-36-4

This page intentionally left blank. 
DOE/RL-94-36-4

CONTENTS

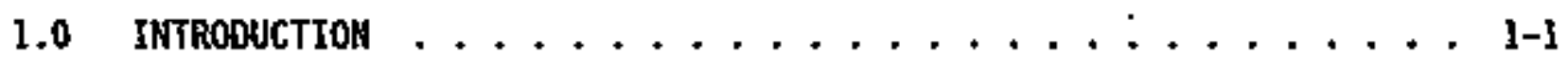

$2.0100-$ B POHDS . . . . . . . . . . . . . . 2-1

$3.0 \quad 183-H$ SOLAR EVAPORATIOH gasins $\ldots \ldots \ldots \ldots$

$4.0 \quad 100-H$ RCRA SITES ......................... 4-1

$5.0 \quad 216-8-3$ POHD SYSTEM $\ldots \ldots \ldots \ldots \ldots \ldots$. . . . . . . . . . .

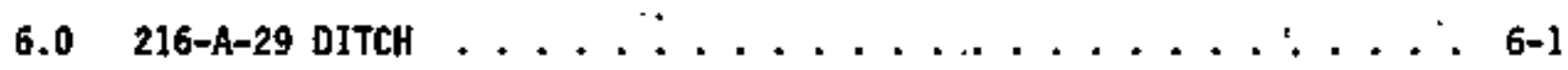

$7.0 \quad 216-A-36$ B CRIB $\ldots \ldots \ldots \ldots \ldots \ldots . \ldots \ldots$. . . . . . . . .

$8.0 \quad 216-A-10$ CRIB . . . . . . . . . . . . . . . . 8-1

9.0 216-8-63 TRENCH ..................... 9-1

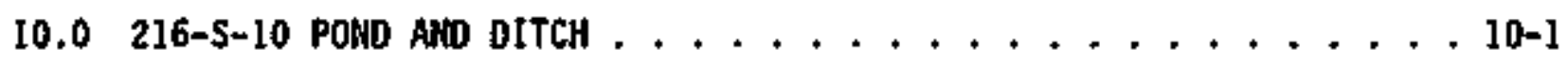

11.0 216-10-12 СR1B . . . . . . . . . . . . . . . . . 11-1

12.0 Liquid EfFluent RETEhtion Facility . . . . . . . . . . . 12-1

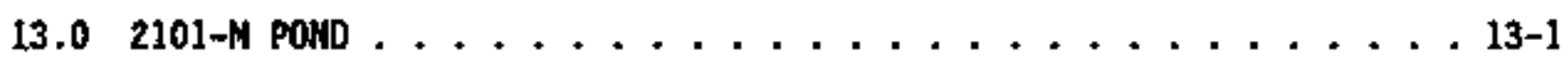

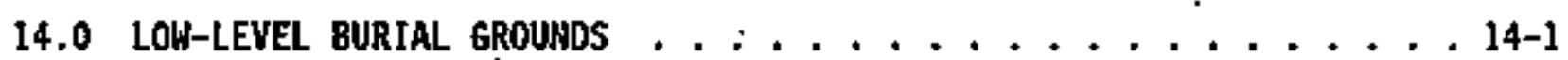

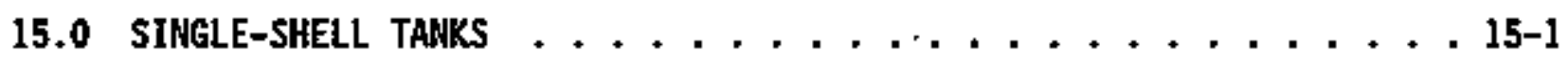

16.0300 AREA PROCESS TREACHES . . . . . . . . . . . . 16-1

17.0 nonradoactive dangerous waste Landfill . . . . . . . . . 17-1

18.0 RefEREnCES .................................. 


\section{DOE/RL-94-36-4}

This page intentionally left blank. 


\section{COATEHS}

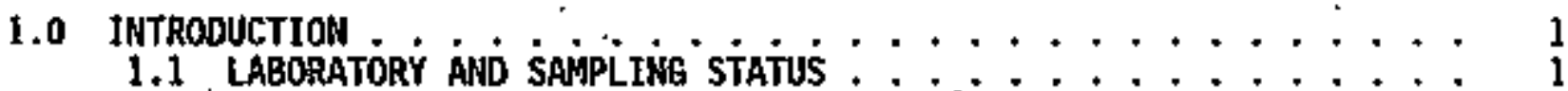

1.2 QUALITY CONTROL PROARAM $\ldots \ldots \ldots \ldots \ldots$

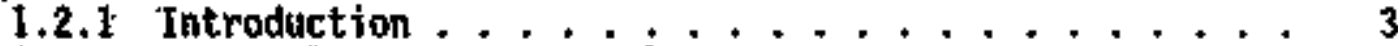

1.2.2 External Quality Control Program ........... 6

1.2.3 Interna] Qual tty Control Program ........... 9

1.2.4 Monconformance/Incident Reports .......... 11

1.2 .5 Qual ity Assurance Occurrences ........... 12

1.3 QUALITY CONTROL DEFINITIONS $\ldots \ldots \ldots \ldots \ldots$

1.4 DATA TABLES ...................... 18

1.4.1 Water Level Measurement Tabie ............ 18

1.4.2 Constituent Lifst and Summary of Results Table .... 18

1.4.3 Const 1 tuents with at Least One Detected Value Table ; 19

1.4.4 Contamination Indicator Parameters Table ...... 20

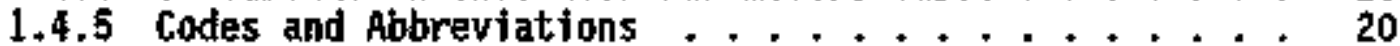

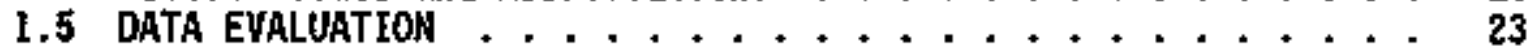




\section{LIST OF FIGURES}

1-1 Locatjons of the RCRA Groundwater Monitoring Projects and Landmarks on the Hanford site................. 2

\section{LIST OF TABLES}

1-1 Completeness ........................ 4

1-2 Field Duplicates and Fieid Blanks Evaluation Results, Fourth Quarter $1994 \ldots \ldots 6$

1-3 Breakdown of Field Dupificates Exceeding quality Control Limits : . 7

1-4 Breakdown of Fjeld 81anks Exceeding Quality Control Limits .... 8

1-5 Frequency of Quality control Samples. ............ B

1-6 Radiochemistry Quarterly Report Summary .............. 10

1-7 Sumbary of Perfornance Evaluation Samples ............. 11

1-8 RCRA Samples Flagged for Honconformance Report ......... 13

1-9 Analysis Hethod Code Definitions ............... 21

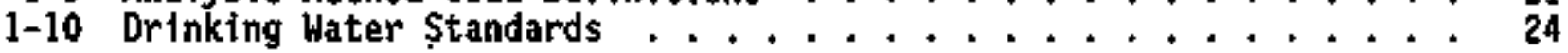


DOE/RL-94-36-4

QUARTERLY REPORT OF RCRA GROUNDWATER MONITORING DATA FOR PERTOD OCTOBER 1, 1994 THROUGH DECEMBER 31, 1994

\subsection{IHTRODUCTION}

Hanford Site interim-status groundwater monitoring projects are conducted as either background, indicator parameter evaluation, or groundwater quality assessment monitoring programs as defined in the Resource Conservation and Recovery Act of 1976 (RCRA); and "Interim Status Standards for Owners and Operators of Hazardous Waste Treatment, Starage, and Disposal Facilities" (Title 40 Code of Federal Regulations [CFR] Part 2655), as amended. Compliance with the 40 CFR 265 regulations is required by the Washington Administrative Cade (WAC) 173-303. This report contatns data from Hanford S1te groundwater monitoring projects. The location of each facility is shown in Figure 1-1.

Westinghouse Hanford Company (WHC) manages the RCRA groundwater monitoring projects for federal facilities on the Hanford site. Performing project management, preparing grotundrater monitoring p1ans, we 11 network design and installation, specifying groundwater data needs, performing quality control (QC) oversight, data management, and preparing project sampiing schedules are all parts of this responstbility. Pacific Northwest Laboratory (PHL) administers the contract for analyttical services and provides groundwater sampling services to WHC for the RCRA groundwater monjtoring progran.

This quarterly report contains data received between october and December 1994, winich are the cutoff dates for this reporting pertod. This report may contain not only data from the October through. Decentuer quarter, but a] so data from earl ter sampling events that were not prevlously reported.

\subsection{LABORATORY AND SAHPLING STATUS}

DataChem Laboratorles of Salt Lake City, Utah, performs the hazardous chemicals analyses for the Hanford $\$$ ite. Analyses for coliform bacteria are performed by Columbia Biomedtcal Laboratories (CBML) and analyses for dloxin are performed by TMS Analytical Services, Inc.; both are under subcontract to Datachen Laboratories. International Technology Analytical Services (ITAS) Richland, Washington, performs the radiochemical analyses. 


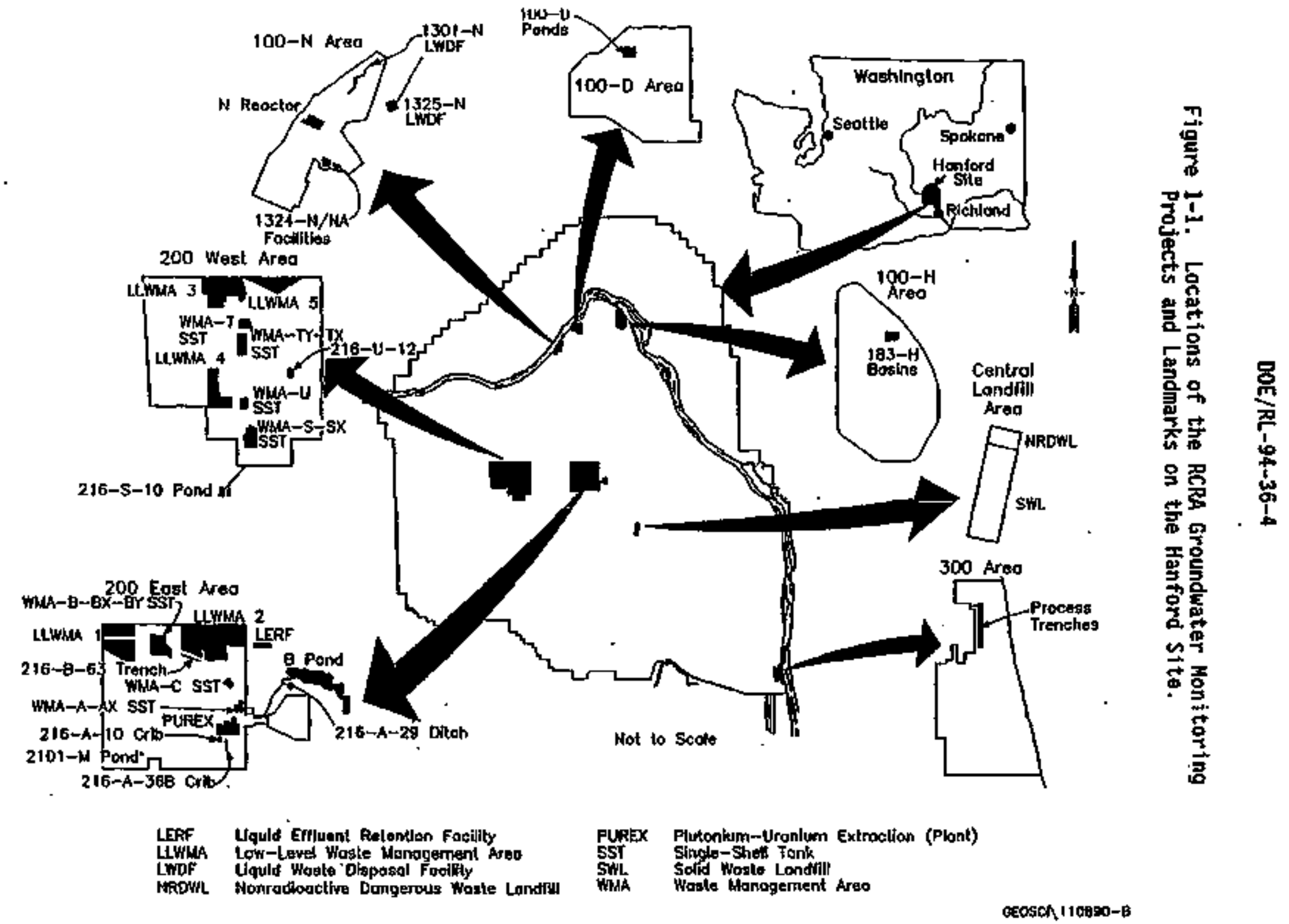




\subsection{QUALITY CONTROL PROGRAM}

H. H. Edrington/J. C. Johnston

Westinghouse lanford Company

\subsubsection{Introduction}

The QC Program is based on guidance from the U.S. Environmental Protection Agency (EPA), the RCRA Groundwater Honitoring Technfca7 Enforcenent Guidance Docurent (EPA 1986a), and Chapter 1.0, Quality Contro1, from Test Hethods for Evaluating Solfd Haste (EPA 19866).

1.2.1.1 Data quality Heasures. The QC Program uses the five measures of data qual jty: precision, accuracy, representativeness, completeness, and comparability, along with applicable program-specific quality parameters to evaluate the qua]ity of the data and the amalytical laboratories analyzing the samples. Target values for precision and accuracy are specified in the Quality Assurance Profect PJan for RCRA Groundwater Honitaring Activities (WHC 1992b).

1. Prectsion is evaluated using data results from laboratory dupl fcates, matrix spike duplicates (see Section-1.2.3), field . duplicates, and blind saaples (see Section 1.2.2).

2. Accuracy is evaluated using data results from Taboratory matrix sp1kes; laboratory control samples; EPA Water Pollution (WP), Hater Supply (WS), and Interlaboratory Performance Evaluation Programs (see Section 1.2.3); and by blind samples (see Section 1.2.2).

3. Representativeness expresses the dagree to which RCRA faciltty groundwater monitaring data represent the real camposition of the groundwater in the aquifer. Goals for data representativeness for groundwater monttoring programs are addressed qualitatively by the specification of well construction, saupling locations,., saapling intervals, and samp1ing and analyses techniques in the groundwater monitoring plan for each RCRA factlity.

4. Completeness is defined as the percentage of measurements that are judged to be valtd. "Completeness is determined by the number of data unflagged durfing validation, divided by the total number of data evaluated, and multiplied by 100 . The calculated percentages used in reporting compteteness are conservative figures and are based on the data $f$ lags ' $P,{ }^{\prime} ' F,{ }^{\prime}$ ' $Q$,' and ' $H$.' Table 1-l 111 ustrates project completeness for the data presented in this report.

5. Comparabtlity is used to ensure that samples analyzed by different 1aboratories or by the same laboratory over different time periods are comparable. For the October through December 1994 quarter, only the primary hazardous chealistry and radiochemjstry Taboratories were requested to analyze samples and submit data. Samples were analyzed in accordance with Test Methods for Evaluating Solfd Waste (EPA 1986b) and other applicable approved 
methods. Comparability of field measurements is determined by following approved sampling procedures that ensure consistency among sampling events.

Table 1-1. Completeness. (2 sheets)

\begin{tabular}{|l|c|c|c|}
\hline \multicolumn{1}{|c|}{ Project } & $\begin{array}{c}\text { Tota\} number } \\
\text { of evaluated } \\
\text { data }\end{array}$ & $\begin{array}{c}\text { Mumber of } \\
\text { unflagged } \\
\text { resuits }\end{array}$ & $\begin{array}{c}\text { Percent } \\
\text { complete } \\
\text { (unflagged) }\end{array}$ \\
\hline $100-D$ & 38 & 36 & 95 \\
\hline $100-N$ & 835 & 777 & 93 \\
\hline 183-H & 1112 & 990 & 89 \\
\hline $2101-M$ & 368 & 368 & 100 \\
\hline $216-A-10$ & 593 & 538 & 91 \\
\hline $216-A-29$ & 1869 & 1752 & 94 \\
\hline $216-A-36 B$ & 358 & 327 & 91 \\
\hline $216-B-3$ & 1246 & 1206 & 97 \\
\hline $216-B-63$ & 1299 & 1249 & 96 \\
\hline $216-S-10$ & 568 & 492 & 97 \\
\hline $216-U-12$ & 714 & 660 & 92 \\
\hline 300 APT & 973 & 872 & 90 \\
\hline LERF & 0 & 0 & N/A \\
\hline LLBG WMA-1 & 1810 & 1661 & 92 \\
\hline LLBG WMA-2 & 1793 & 1721 & 96 \\
\hline LLB6 WMA-3 & 339 & 299 & 88 \\
\hline LLBG LMA-4 & 1926 & 1729 & 90 \\
\hline LLBG WMA-5 & 178 & 166 & 93 \\
\hline NRDHL & 0 & 0 & N/A \\
\hline SST A/AX & 73 & 71 & 97 \\
\hline SST B/BX/BY & 246 & 232 & 94 \\
\hline SST C & 60 & 60 & 100 \\
\hline SST S/SX & 124 & 109 & 88 \\
\hline SST T & 746 & 683 & 92 \\
\hline
\end{tabular}


Table 1-1. Conpleteness. (2 shests)

\begin{tabular}{|l|c|c|c|}
\hline \multicolumn{1}{|c|}{ Project } & $\begin{array}{c}\text { Total number } \\
\text { of evaluated } \\
\text { data }\end{array}$ & $\begin{array}{c}\text { Number of } \\
\text { unflagged } \\
\text { results }\end{array}$ & $\begin{array}{c}\text { Percent } \\
\text { complete } \\
\text { (unflagged) }\end{array}$ \\
\hline SST TX/TY & 396 & 368 & 93 \\
\hline SST U & 73 & 72 & 99 \\
\hline
\end{tabular}

APT - Area Process Trench.

LERF = Liquid Effiuent Retention Facjlity.

LLBG = Low-Leve I Burial Grounds.

NRDWL = Monradioactive Dangerous Waste Landfitl.

SWL = Solid Waste Landfi门1.

SST = stngle-she? t tank.

WMA = waste management area.

1.2.1.2 Program-Specific Validation Flags. Program-\$pecific validation flags are assigned during the process of evaluating RCRA groundwater data. These flags include (1) $\mathrm{H}$ flags for samples exceeding holding times; (2) Q flags for fjefd blank and dupljcate data exceeding quality control limits; (3) F fiags for data under the Request for Analytical Data Evaluation (RADE) process; and (4) P flags for data identified as a "potential problem."

1. Holding tipe exceedances have been flagged in the quarterly reports with an $\mathrm{H}$ flag. This flag means that the samples were andyzed after the method-required holding times stated in the contract. The H-flagged data may be used qualitatively, but no regulatory decistons should. be ade based on a single-fiagged data point.

2. Data associated with field blanks that indicate contamination and data associated with field duplicate samples that show greater than $25 \%$ relative percent difference (RPD) are identified in the quarterly project data tables with a flag. This flag means that the field OC data associated with the sample data were outside limits established in the Environmental Engineering and Geotechnology function Procedures (WHC-CH-7-8). The 0 -f lagged data can be used qual itatively, but no regulatory decisions should be made based on a single-flagged data point.

3. The RADE systien is used to track and evaluate data that appear to be inconsistent with the current pattern or with historical trends. An ' $F$ ' flag indicates that the data point is submitted for data review through the RADE system.

4. Honconformance Reports (NCR) and Incident (INC) Reports are recelved from the laboratory and are used to document occurrences that may affect data qua]tty. Data points affected by these reports are flagged with ' $Y$,' or 'R'.

5. Data points related to isolated occurrences determined by the specific project scientist are flagged with a 'P,". Indicating a 
potential problem with the data. Explanations for use of the flag will be discussed in the specific project chapter.

\subsubsection{External Quality Control Program}

The externa] QC program uses three kinds of QC samples to evaluate the quality of data generated in the field and In the laboratory. These are field duplicates, field blanks, and blind samples. (See Section $\mathbf{1 . 3}$ for definitions of the oc sarpies.)

The analytical results of QC samples are judged to be acceptable if the following evaluation criteria are net.

- Fjeld duplicates--Results of field duplicate pairs must have precision as measurad by RPD within $\pm 25 \%$.

- Blanks--Four kinds of blanks are used to check for contamination resulting from fjeld activities and/or bottle preparation. These are full trip, daily trip, field transfer, and bottle blanks.

Except for common laboratory contaninants, results above the threshold of two times the method detection 1 init (HDL) are identified as suspected contamination. For common laboratory contaminants, such as acetone, methylene chloride, 2-butanone, toluene, and phthalate esters, sample results less than five times the MDL are qualified as nondetects.

- $\quad$ olind samples--Results must fail within 2.0 standard deviatjons from the mean recovery of the known concentration of samples submitted to the laboratory.

Table 1-2 summarizes the analytical results of QC samples available for the October through December 1994 quarter.

Samples associated with duplicate or blank analytes that exceeded the $Q C$ limits are discussed in this section and are flagged with a ' $Q$ ' in the data tables.

Table 1-2. Fiejd Duplicates and Field Blanks Evaluation Results, Fourth Quarter 1994.

\begin{tabular}{|l|c|c|c|}
\hline $\begin{array}{c}\text { Quality control } \\
\text { sample }\end{array}$ & $\begin{array}{c}\text { Number of } \\
\text { analyses }\end{array}$ & $\begin{array}{c}\text { Aumber of resulis } \\
\text { outsjde of criteria }\end{array}$ & $\begin{array}{c}\text { Acceptable } \\
\text { (\%) }\end{array}$ \\
\hline Ouplicates & 879 & 52 & 94.1 \\
\hline Blanks & 2794 & 137 & 95.1 \\
\hline
\end{tabular}

Precision based on field duplicate analys is was deternined to be acceptable. See Table 1-3 for a complete breakdown of field duplicates that. did not meet QC acceptance criteria. The criterla for determining the acceptabifity of dupficate data are currently belng reevaluated. Precisjon values for some field duplicate constituents (including both radiochemistry 
and hazardous chemistry constituents) have been consistently outsfde the acceptable 1 imit of 25\% RPD for several quarters. However, the 1aboratorios have not experienced similar problems with precision, based on their own limits. This led the QC team to be]ieve that the 25\% blanket RPD limit may need to be reevaluated to account more for instrument- and method-specific precision limits.

- of the 2,784 blanks analyzed during the October through Decenter quarter, 137 exceeded the QC 1 inet for $95.1 \%$ acceptabi71ty. Table 1-4 shows a breakdown of the constituents detected in the blanks. Al] the methylene chloride samples were qualified by the laboratory with a ' $B$, ' which indicates contamination occurred at the laboratory in the laboratory blanks.

Table 1-5 shows the required and actual frequencies of the field QC samples for the October through Decewber 1994 quarter. Sampl ing frequencies were slightly high this quarter for field dupi icates and full trip blanks. Field transfer biank frequency came in below the required $5 \%$ at $4.1 \%$. The frequency for daily trip blanks did not meet the requijement of one per day. In November, one dajly trip blank was not taken into the field.

Table 1-3. Brakdown of Field Buplicates Exceeding . Quality Control Limits.

\begin{tabular}{|c|l|c|c|c|}
\hline $\begin{array}{c}\text { Mathod } \\
\text { analysis } \\
\text { code }\end{array}$ & \multicolumn{1}{|c|}{ Method description } & $\begin{array}{c}\text { Nugber of } \\
\text { flagged } \\
\text { results }\end{array}$ & $\begin{array}{c}\text { Total } \\
\text { number of } \\
\text { results }\end{array}$ & $\begin{array}{c}\text { Percent of } \\
\text { total out } \\
\text { of imits }\end{array}$ \\
\hline 25 & $\begin{array}{l}\text { Volatile organics (gas } \\
\text { chromatography) }\end{array}$ & 3 & 85 & 3.5 \\
\hline 34 & $\begin{array}{l}\text { Inductive1y coupled } \\
\text { plasma metals }\end{array}$ & 25 & 380 & 6.6 \\
\hline 67 & Total organtc halogen & 4 & 15 & 26.7 \\
\hline 122 & Total organic carbon & 6 & 11 & 54.5 \\
\hline 124 & Anions & 3 & 84 & 3.6 \\
\hline 129. & Anmonium lon & 1 & 2 & 50.0 \\
\hline 135 & Gross alpha & 1 & 7 & 14.3 \\
\hline 136 & Gross beta & 1 & 6 & 50 \\
\hline 137 & Radium & 1 & 1 & 100 \\
\hline 139 & Iodine-129, Low 7evel & 3 & 28 & 10.7 \\
\hline 140 & $\begin{array}{l}\text { ITAS Gamma scan (8e-7, } \\
\text { K-40, Rul-106) }\end{array}$ & 1 & 3 & 33.3 \\
\hline 145 & Urantum & & & 33.3 \\
\hline
\end{tabular}


Table 1-4. Breakdown of Field BTanks Exceeding Quality Control Limits.

\begin{tabular}{|c|l|c|c|c|}
\hline $\begin{array}{c}\text { Method } \\
\text { analysis } \\
\text { code }\end{array}$ & \multicolumn{1}{|c|}{ Method description } & $\begin{array}{c}\text { Nenaber of } \\
\text { flagged } \\
\text { results }\end{array}$ & $\begin{array}{c}\text { Total } \\
\text { number of } \\
\text { results }\end{array}$ & $\begin{array}{c}\text { Percent of } \\
\text { total out } \\
\text { of limits }\end{array}$ \\
\hline 16 & Volattle organtcs (GC/MS B240) & 15 & 330 & 4.5 \\
\hline 25 & Volatile organics (GC) & 3 & 85 & 3.5 \\
\hline 34 & $\begin{array}{l}\text { Inductively coupled plasma } \\
\text { metals }\end{array}$ & 20 & 551 & 3.6 \\
\hline 40 & $\begin{array}{l}\text { Lead by graphite furnace } \\
\text { atomic absorption }\end{array}$ & 3 & 10 & 30 \\
\hline 41 & $\begin{array}{l}\text { Mercury by cold vapor atomic } \\
\text { absorption }\end{array}$ & 1 & 9 & 11.1 \\
\hline 67 & Total organtc halogen & 12 & 142 & 8.45 \\
\hline 122 & Total organic carbon & 4 & 48 & 8.3 \\
\hline 124 & Anions & 20 & 126 & 15.9 \\
\hline 126 & Turbildity & 15 & 15 & 100 \\
\hline 127 & Total carbon & 1 & 1 & 100 \\
\hline 140 & ITAS Gamia scan (Be-7, K-40) & 4 & 64 & 6.3 \\
\hline 182 & Volatile organics (GC/MS 8260) & 38 & 792 & 4.8 \\
\hline 357 & Alkalinity & 1 & 9 & 11.1 \\
\hline
\end{tabular}

Table 1-5. Frequency of Quality Control Samples.

\begin{tabular}{|l|c|c|c|c|}
\hline $\begin{array}{c}\text { Qual ity control } \\
\text { samples }\end{array}$ & $\begin{array}{c}\text { Required } \\
\text { frequenctes }\end{array}$ & $\begin{array}{c}\text { Number of } \\
\text { wells sampled }\end{array}$ & $\begin{array}{c}\text { Number of } \\
\text { samples } \\
\text { taken }\end{array}$ & $\begin{array}{c}\text { Actual } \\
\text { frequency } \\
\text { (\%) }\end{array}$ \\
\hline Duplicates & $5 \%$ & 219 & 12 & 5.5 \\
\hline Dajly trip blank & $\begin{array}{c}\text { One each } \\
\text { sampling day } \\
(45)\end{array}$ & $\begin{array}{c}44 \\
(45 \text { required) }\end{array}$ & 44 & 97.8 \\
\hline Full trip blank & $5 \%$ & 219 & 12 & 5.5 \\
\hline Field transfer blank & $5 \%$ & 219 & 9 & 4.1 \\
\hline Bottle blanks & $\begin{array}{c}\text { Per lot of } \\
\text { bottles }\end{array}$ & N/A & 2 & N/A \\
\hline
\end{tabular}

N/A - not applicabie. 


\subsubsection{Internal quality Control Progran}

The internal $Q C$ progran uses four types of QC data to estab]ish and monitor perfornance in the laboratory. These data are laboratory blanks, matrix spikes, matrix duplicates, and EPA studies (WP and WS).

Every quarter each contracted laboratory supplies its own QC report, which includes data quality information on matrix spikes, matrix duplicates, and blanks in the form of precision and accuracy. Each contracted laboratory also supplies to WHC a report of its results of EPA's WP, WS, and radiochemical intercomparison studies on a quarterly basis. The results of EPA studies independently verify the continuation of iaboratory performance and are expressed as the percentage of EPA-actepted results. In addition, every 6 months each contracted 7 aboratory suppiies an $\mathrm{ML} / \mathrm{min}$ imum detectable concentration (MDC) report. The MOLS and BDCS are requited to be below the contractually required quantitation $13 \mathrm{~m}^{i} \mathrm{t}$ (CRQL). The CRQL is not assocfated with a quantitation limit as the name suggests; it is intended to be the lowest analyte concentration in a given matrix that a laboratory can be expected to detect consistentiy. The CRQL is agreed on under the contractual statenent of work. A laboratory NCR is issued when the MDL is greater than the CRQL. WHC reviews each of these reports; the results are summarized in Tabie 1-6.

Radiochemistry precision and accuracy figures are gleaned from the radiochenistry laboratory's quarterly report, with percent acceptability calculated from duplicates and spikes, respectively. These results, along wt th radjochenistry blank data, are summartzed in Table 1-6.

Precision and accuracy results from the radiochemistry laboratory, summarized in Table 1-6, indicate the performance of a.17 custamers submitting water matrix samples. WHC samples represent only a part of the performance summary. These figures represent samples analyzed during the period of October through Decenter 1994.

For the October through Decemoer 1994 quarter, the radiochemistry laboratory analyzed 163 duplicates, of which 160 were within control injts $( \pm 3 \sigma)$, for an acceptability of $98.2 \%$. The radiochemistry laboratory analyzed 265 spikes, of which 261 felT within control 1 imits, for an acceptability of $98.5 \%$.

The $95 \%$ confidence interval on the mean is used to establish acceptance 7 imits for performance evaluation sample resul ts (see WHC-CM-7-8, Section 2.4, "Evaluation of RCRA Groundwater Performance Evaluation Sample Data"). The hazardous chealistry laboratory's mean results for 15 of the 15 perfarmance evaluation sample values fell within the limits of the $95 \%$ confidence interval and are acceptable.

Tox results could not be evaluated at this time because raw data were nat available. ToX results shall be evaluated when these data arrive. Fourteen results from phenol and ICP tetal analyses could not be evaluated per WHC-CM-7-8 because of a 1 ack of results and differing analytical methods used by the jaboratories. Three ICP metal results (iron, potassium, and sodium) could not be evaluated and were rejected because the percent bias and/or the relative standard deviation exceeds $100 \%$, per $\mathrm{WHC}-\mathrm{CM}-7-8$. Based on this 
information, HC w111 resend ICP metal and phenol performance evaluation samples the next time standards are sent.

Tabie 1-6. Radiochemistry Quarterly Report Summary.

\begin{tabular}{|c|c|c|c|}
\hline $\begin{array}{c}\text { Fourth } \\
\text { quarter 1994 }\end{array}$ & $\begin{array}{c}\text { OupT icates } \\
\text { (precision, } \\
\text { \%cceptable) }\end{array}$ & $\begin{array}{c}\text { Spikes } \\
\text { (accuracy, } \\
\text { acceptable) }\end{array}$ & $\begin{array}{c}\text { Blanks } \\
\text { (\% acceptabie) }\end{array}$ \\
\hline $\begin{array}{c}\text { October } \\
\text { through } \\
\text { December }\end{array}$ & 98.2 & 98.5 & 97.1 \\
\hline
\end{tabular}

EPA intercomparison samples were analyzed by the radiochemistry laboratory during October and November 1994. Seven samples were ana7yzed for gross alpha, gross beta, cobalt-60, cestum-134 and -137 , barium-133, and zinc-65. Ai] sampie results were within the $\pm 25 \%$ bias acceptability criterlon. This translates to seven out of seven acceptabie results, or $100 \%$ acceptability.

Environmental Sciences sent out performance evaluation (PE) samples to four laboratories during November 1994. The PE samples were sent out in duplleate to each Jaboratory and contained known amounts TOC, TOX, nitrate, and carbon tetrachloride. The laboratories that participated in the analysis of these samples are the primary hazardous themistry ']aboratory (Lab 1), two comparison laboratories (Labs 2 and 3 ), and the prjuary laboratory for ToX analysis (Lab 4). The laboratories recalved all four sample sets, with the exception of $\mathrm{Lab} 4_{t}$ which only recejved TOX.

The results for these analyses are shown in Table 1-7. Lab l's results were within the linits of the $95 \%$ confidence interval for all four analytes. A 95\% confjdence interval is used to establish acceptance limits for the PE samples. This is in accordance with WHC-CH-7-8, Section 2.4 .

Lab $4^{\prime} \mathrm{s}$ results were outsfide the acceptance limits for the rox samples. Lab 4 has been analyzing RCRA groundwater samples for Environmental \$ciences since Hovenber 1994. Lab 4 received TOX PE samples in May 1994 and had results within acceptable 7inits at that tipe. The PE procedure, MHC-CM-7-8, Section 2.4, requires that another set of $P E$ samples be sent as soon as possible to a laboratory that does not get results in acceptable 1 imits. 
Table 1-7. Summary of Performance Evaluation Samples.

\begin{tabular}{|c|c|c|c|c|c|c|}
\hline inalyte & 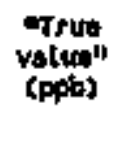 & $\begin{array}{c}\text { Lab } 1 \text { (prinitry } \\
\text { hazerdous } \\
\text { chetifstry lab) } \\
\text { moan rosult (a) }\end{array}$ & $\begin{array}{c}\text { Lab } 2 \\
\text { (comparison } \\
\text { lob) metr } \\
\text { reatitc (ppb) }\end{array}$ & $\begin{array}{c}\text { Lob } 3 \\
\text { (comparison } \\
\text { (als) neen } \\
\text { rexult (pot) }\end{array}$ & $\begin{array}{l}\text { Lab } 4 \\
\text { (prignory Tox } \\
\text { lab) mean } \\
\text { result tppo) }\end{array}$ & $\begin{array}{c}95 x \\
\text { conf tdence } \\
\text { interval } \\
(p p b)\end{array}$ \\
\hline TOC & 978 & 1,000 & 1,300 & 2,370 & $*$ & $901-2,213$ \\
\hline Tox & 17.97 & 17 & 17 & 14.75 & 22.75 & $35.39-20.36$ \\
\hline Mitrute & 205 & 350 & 327 & 69 & $\cdot$ & $112-386$ \\
\hline $\begin{array}{l}\text { Carber- } \\
\text { tetra- } \\
\text { chlorido }\end{array}$ & 10.3 & 7.7 & 6.0 & 8.0 & - & $6.0+8.5$ \\
\hline
\end{tabular}

\subsubsection{Nonconformance/Incjdent Reports}

\section{T. X. Washington}

Mestinghouse tlanford Company

NCR and INC Reports are methiods of doctmentation by which contract laboratories inform laboratory contractors and their customers of any problems encountered with the anajysis, data, and/or data deliverable. This nethod of documentation identifies occurrences, deficiencies, and/or issues that may potent1ally affect the data integrity. These may include, but are not limited to, the following:

- Lost sampte

- Broken bottle

- Instrument majfunction

- Calibration standard out of acceptable range

- Laboratory control standard out of acceptable range

- Matrfx spjke recovery out of acceptable range

- Procedural nonconpliance

- Chajn-of-custody dtscrepancies

- Shipping temperature out of acceptable range

- Misreported data. 
During the October through Decenber quarter, 16 NCR/IAC Reports were transeitted, affecting 35 data points. Fourteen HCR/INC Reports were transmitted by the hazardous chentstry laboratory and two were transmitted by the radiochemistry laboratory. These reports describe incidents that potentially affect the integrity of either groundwater samples or external QC samples (e.g., trip blanks, bottle blanks).

The Incident reparts transintted by the hazardous chenistry laboratory affect 32 data points, 18 of which were rejected. The rojected data were attributed to sample containers received broken at the laboratories. In addition, eight analyticai results-were evaluated as being suspect. These data should be used for trending purposes only. Ninety percent of the suspect data are attributed to ToX samples recefved broken at the laboratory. The sample shipping departient will be informed of the problea and instructed to package samples conscientiously.

The incident reports transuitted by the radiochenistry laboratory affect. three data points. The incidents documented were determined to have had no adverse effect on the data. The radiochenistry laboratory had incorrectiy calculated a result using sample weight rather than sample volume, the sample was recalculated and rereported. The two renaining data points were determined to have a high beta activity and, therefore, the sample sizes were reduced to a voiume less than that prescribed by the analytical procedure.

Table 1-8 presents the sample numbers adversely affected by the NCR and incjdent reports, along with the associated well numbers, analyt lca] methods, and flags assigned to these samples as a result of the HHC NCR and tncident report evaluations (see Section 1.4 .5 for definttions of flags found in Table $1-8)$.

\subsubsection{Qualfty Assurance Occurrences}

Quality assurance (QA) occurrences are situations that potentialiy affect the quality of the data. These situations are discussed through simaries of inspections of laboratory services and/or systen and performance audits/surveit tances.

Inspection of laboratory services and/or system and performance audits/surveillances for the RCRA groundwater monitoring program are performed throughout the year by the laboratory contractor, WHC, and/or the U.S. Department of Energy (BOE) on various aspects of this program. QA requirements and data quality objectives are defined in the Quality Assurance Project PJan for RCRA Groundwater Nonftoring Activities (WHC 1992b). Results of these oversight activities are documented with the andit/ surveillance and/or inspecting organjzation(s). 
Table 1-8. RCRA Saroples Flagged for Nonconformance Report. (2 sheets)

\begin{tabular}{|c|c|c|c|c|c|}
\hline F1ag Name & Flag & Hethod Name & Code & Well Name & Number \\
\hline Rejected & R & $\begin{array}{l}\text { TOC } \\
\text { TOX } \\
\text { TRITIUK }\end{array}$ & $\begin{array}{l}122 \\
67\end{array}$ & $\begin{array}{l}199-N 1-74 \\
299-W_{11}-27 \\
299-W 11-28 \\
299-W 22-22 \\
299-W 6-3 \\
199-K-27\end{array}$ & $\begin{array}{l}\text { BOCgH4 } \\
\text { BOC107 } \\
\text { BOC122 } \\
\text { BOC124 } \\
\text { BOCZK4 } \\
\text { BOCgY4 } \\
\text { BoCYf2 }\end{array}$ \\
\hline Suspect & $Y$ & $\begin{array}{l}\text { ALPHA } \\
\text { ANIOHS } \\
\text { HERBICIOES } \\
\text { TOX }\end{array}$ & $\begin{array}{l}49 \\
67\end{array}$ & $\begin{array}{l}199-K-109 A \\
199-N-16 \\
199-N-17 \\
199-N-21 \\
199-N-25 \\
299-W 7-11 \\
299-N 7-12 \\
299-W 7-9 \\
299-N 8-1 \\
699-41-35 \\
299-E 25-46 \\
. \\
299 \text { E333-36 } \\
299-E 33-42 \\
299-E 33-43\end{array}$ & 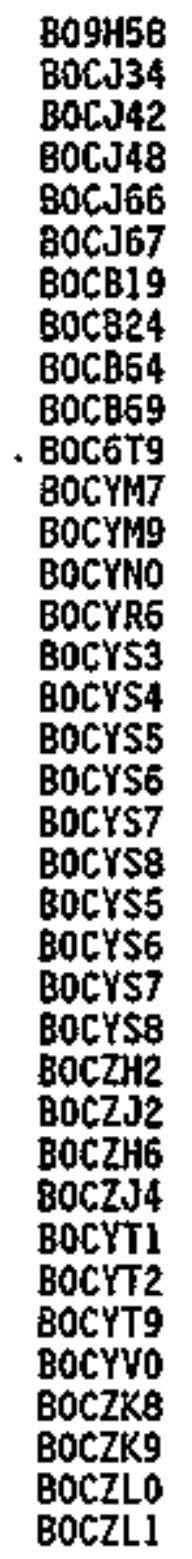 \\
\hline
\end{tabular}


Table 1-8. RCRA Samples Flagged for Nionconformance Report. (2 sheets)

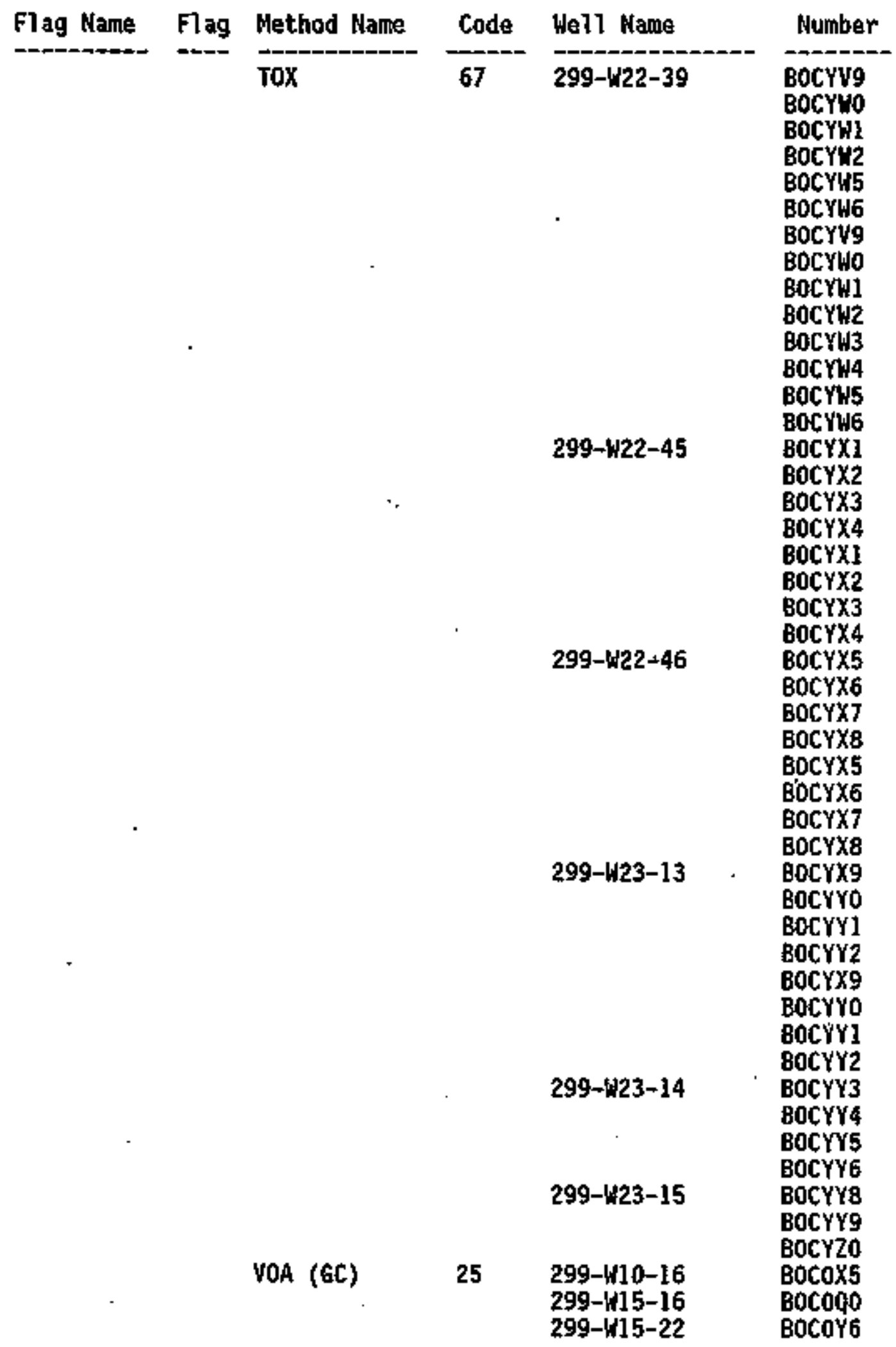




\section{Findings:}

- The contractor fajled to track and verify finplementatton of corrective actions, as required by the contract.

- Honcompliances were found vith the laboratories procedures governing document control. Huttiple instances were found of effective dates for procedures proceeding approval dates.

- Honcompliances were found wth the implementation of the contractuat requirenent for annua] review of procedures.

- Deficlencies exist In the traceabtlity to specific procedures and standards used for the analysis of samples.

- Noncompliances were found to the laboratories procedura] and contractual requirenents for training.

- Deficiencies and inconsjstencies exist in the control charting and tracking for both analysis and reporting purposes.

\section{Observations:}

- The contract laboratory was unable to clearly explain the responsibilities of its QA organizatton as applicable to WHC samples.

- It was unclear whether the laboratory has an effective program in p]ace to control contamination.

The corrective action for the findings and observations is scheduled to be provided after the printing of this report.

The second inspection was perforwed at the radiochemtstry laboratory on Juty 6-8, 1994. The offictal report has not been released at this time and a sumbary with be provided during the next reporting period.

A third inspection of services was performed at CBML on August 8-10, 1994. The purpose of the inspection was to determine compltance by CBML With the PNL contract regarding $Q A$ and $Q C$ for coliform and biological oxygen demand analyses.

The inspection tean found evidence supporting - two findings:

Finding \#1: ' CBMl had not followed the requirements delineated in their QA manua?.

Finding \#2: CBOML had not met all PNL contract and CBML QA manual requirements for planning and implementing surve13] ances and audits.

The corrective action for the findings. is scheduled to be provided after the printing of this report. 


\subsection{QUALITY CONTROL DEFIHITIOHS}

Accuracy--The closeness of agreement between an observed value and a true value. Accuracy is assessed by means of reference samples and percent recoveries.

Blind sample--A sample that contains a concentration of analyte that is known to the supp]ier but unknown to the anafyzing laboratory. The ana]yzing laboratory is informed that the sample is a $Q C$ sanple and not a field sample. The biind, double blind, and matrix-matched double bifnd samples are used to assess accuracy and monitor the pertormance of the analytical laboratory(jes) with prepared or purchased materlals from EPA QC samples/concentrates or primary materials.

Bottle blank--A sampie that contains oniy Type II roagent water. The bottle blank contajns one sample for each bottle size, with at least enough bottles to include all constituents analyzed by a specific project, except radionuclides. Bottle blanks shall be submitted to the priary laboratory per lot of bottles. Bottle blanks are filled in the analytical laboratory under the sample preparatjon procedures. Bottle blanks do not go into the fteld.

- Contractually required quantitation limit--A value intended to be the lowest analyte concentration in a given matrix that the laboratory can be expected to achieve consistently; agreed upon under the contract statement of work.

Daily trip blank--A sampie that contains oniy Type If reagent water. The daily trip b]ank is used to check for sample contanination by volatile organic compounds arising from conditions encotntered during the collection of samples. The datly trtp blank is not opened in the fteld. One daily trip biank is collected for each day that sampling occurs.

Double bilind samplo--A sample that contains a concentration of analyte that ts known to the suppljer but is unknown to the analyzing laboratory. The analyzing laboratory is not informed that the sample is a QC sample. Ail attempts are made to make this sample appear like a fteld sample. For example, the double blind sampie should be subnitted to the laboratory within the same tione period and with a sample identification number similar to that. of the field samples. The double blind sample does not include matrix matching.

External quality control samplo--Any Qc sample prepared without the knowledge of the analytical laboratory.

Fjeid duplicate sample--A sample used to deternine repeatability of an analytical meastrement on identical samples collected as close as possible to the same time at the sare location. These samples are stored in separate containers and are analyzed independently by the same laboratory.

Field transfor blank--A sampie that contains only Type 11 reagent water. The fleld transfer blank is used to check for sample contagination by volatile organic compounds arising from conditions oncountered during the collection of samples. The fleld transfer blank is taken during the collection of samples. The fleid transfer biank is filied at the sampling site by pouring Type II 
reagent water from a cleaned contajner into a volatile organic analysis vial. At least 1 field transfer blank is collected for each 20 samples, or 1 per sampling battch.

Full trip blank--A sample that contains only Type II reagent water and preservative, as required. A full trip blank is used to check for contamination in sariple bottles and sample preparation. The full trip blank is analyzed for all constituents of jnterest on all types of sample bottles used during that saming period. The freqtency of collection for a full trip blank is 1 per 20 samples, or I per salpling batch. A full trip blank is ftlled in the analytical laboratory under the sample preparation procedures. The full trip blank is not opened in the fiejd.

Internal guality control sample--Any QC sample prepared by the analytfcal laboratory and used to establish and monitor the quality of the analytical 1aboratory.

Limft of detection--The lowest concentration level that is statistically different from a blank. This level is calculated by the average blank signal plus three standard deviations of the blank analyses.

Matrix-matched double bi ind sample-A matrix-matched double blind sample contains a concentration of ana]yte that ts known to the supplier but unknown to the analyzing laboratory. The sample matrix has been altered to closely match that of the field samples.

Nethod detection Timit--The minfmum concentration of a substance that can be measured and reported with $99 \%$ confidence that the analyte concentration is greater than zero and is deternined from analys is of a sample in a given matrix type containing the analyte.

Mininum detectable concentration--Required Tevel of analytical detection for radiochemical samples.

Precision--The agreement among a set of individual measurements of the same property, usually under prescribed similar conditions. Precision is calculated by using the RPD of the duplicate/replicate analyses. These samples should contain concentrations of analyte above the $\mathrm{HDL}$ and may involve the use of matrix spikes.

Reliable detection level--A detection limit set at two times the concentration of the MDL, so the risk of both false positives and false. negatives falls below $1 \%$.

Type II reagent water--Distilled or deionized water that is free of contaminants that may interfere with the analytical test in question. 


\subsection{DATA TABLES}

\section{J. A. Serkouski \\ Westinghouse Hanford Company}

Four different formats are used to display water leve] measurements and groundwater sample analytical results. This section descrtbes the meaning of each table column heading, defines the abbreviations used, explajns the data qua] 1 fters and flags, and $\mathrm{l}$ ists analysis method codes and relevant regulatory standards.

\subsubsection{Hater Level Measurement Table.}

The water level measurement table has four columens as follows:

- Hell--Well in which measurement was made.

- Date--Date of measurement.

- Depth to water--Depth, in feet, from well casing reference point to top of water in well.

- Hater level--Elevation, in feet above mean sea leve1, of water level computed by subtracting depth to water from casing reference elevation.

Wells are grouped according to the zone that they monitor, beginning with the top of the unconfined aquifer and continuing downward to the confined aquifer.

Only measurements made during the reporting quarter are included in the tables. Measurements made during sampling are reported with an asterisk (*). Measurements are also routinely performed at other t1mes to permit collection of data for an entire network within a 1- or 2-day time period.

Data that are judiged to be suspect by the project scientist are fiagged with $a{ }^{\prime}+$ ' in the table. Before data are designated as suspect, checks are conducted to determine if a transcription error occurred between the field sheet and the electronic database.

\subsubsection{Constituent List and Summary of Results Table}

The Constituent List and Summary of Results table (Summary table) is the first of three tables that present the results of groundwater sample analyses. The Sunmary table displays statistics based on the complete data set for the reportíng pertod. The complete data set conststs of all analysis results requested by the project associated with the samples collected before the end of the current reporting quarter, which arrived at WHC before the cutoff date for the current quarterly report (see Section 1.1 for the current cutoff date), and have not been reported in a previous quarterly report. Therefore, data collected during previous quarters may appear in this document if the laboratory did not report the data until the current reporting period. 
The fields in the Summary table are as follows:

- Constituent name--pame of the analyzed constituent being summarized; the Short name is an abbreviation used in the Constituents with at Least One Detected Value table (Section 1.4.3); the (Method), When present, is a code (5ection 1.4.5) used to dtstinguish between different analytical methouss for the same constituent.

- Units--Unjts in wich the MDL and drinking water standards (DHS) are reported in this table.

- Laboratory MDL--The MOL computed by the laboratory for a constituent. This field is blank for radionuclides where the MDL is defined as the value of the total error reported by the laboratory for each result.

- OUS limit/agency--The DWS used for comparison to the reported resuits; see Section $\mathbf{1 . 4 . 5}$ for Aqency definitions and a complete ifsting of standards used.

- Number of samples--Iotal 15 the number of sample results from all wells in the project contained in the compiete data set for that constituent; $\geq \mathrm{HDL}$ is the number of results that exceed the MOL; $\geq D \mathrm{DWS}$ is the number of results that exceed the listed DWS.

Suntariarized constituents are grouped as Contamination Indicator Parameters (CIP), Orinking Water Parameters, Groundwater Qua] tty Parameters, and SiteSpecific and other Constituents. Within groups, rows are ordered a]phabetically by the full constituent name.

If a constftuent has an MDL that exceeds the OWS, exceedance of the DWS cannot be determined, so an asterisk $\left(^{*}\right)$ is placed in the $\geq D W S$ field. Some radtonuclides do not have an explicit MoL; the asterisk is used if the DWS exceeds the total error associated with at least one of the results.

\subsubsection{Constftuents with at Least one Detected Value Table}

The Constituents with at Least One Detected Value table (Betected table) is a subset of the complete data set. Only constituents that were detected above the MOL in at least one sample collected for the project during the current reportfing perfod are reported in the Detected table.

The first three column headings are as follows:

- Hell name--Well from which sample was collected.

- Collection date--Date on which the sample was collected.

- Sample number-unique nuaber asstgned to a well sample. 
The remaining colums contain analytical results and qualifiers for the constituent. The structure of the colurn header is as follows.

Constituent short name

Method code/reporting units

MOL/DWS (suffix).

The Summary table (Section 1.4.2) shous the re\}ationship between the full constituent name and the short name. The analytical method code is defined in Table 1-9. Abbreviations used for reporting units are 1isted in Section 1.4.5. On the third line, a period appears in place of the MDL or DNS if a value does not exist. The one-letter suffix that may be added to the DWS identifies the regulation associated with the standard and is described in Section 1.4 .5 .

Analysis results are displayed with two digits to the right of the dectmal point. This is a consequence of the generating software and does not reflect the precision of the resuits. The laboratories normally report the result with three signiftcant digits. Radionuclide values may be negative because the laboratory subtracts a background reading from the measured result. The letter(s) that may foliow the result are data qualifiars and flags. A complete explanation of what each flag means is providad in Section 1.4 .5

\subsubsection{Contamination Indicator Paraneters Table}

The CIP table has a format similar to the Detected table (seo Section 1.4.3) but includes only data for $\mathrm{pH}$, specific conductance, TOC, and TOX. All results for these parameters, whether detected or not, are listed in the CIP tabie. The analysis method code is not part of the column header in this report.

\subsubsection{Codes and Abbreviations}

Abbreviations--The abbreviations used in the data tables are as follows:

COL colifora colonies per 100 millititers

DWS drinking water standard

ft foet

MDL method detection 1 imit

msl mean sea Tevel

NTU nephelometric turbidity unit

$\mathrm{pCi} / \mathrm{L} \quad$ picocuries per liter

ppb parts per bilition

$\mathrm{ppm} \quad$ parts per nillion

$\mu \mathrm{mho} / \mathrm{cri}$ micromhos per centimeter. 
Table 1-9. Ana]ysis Method Code Definitions. (2 sheets)

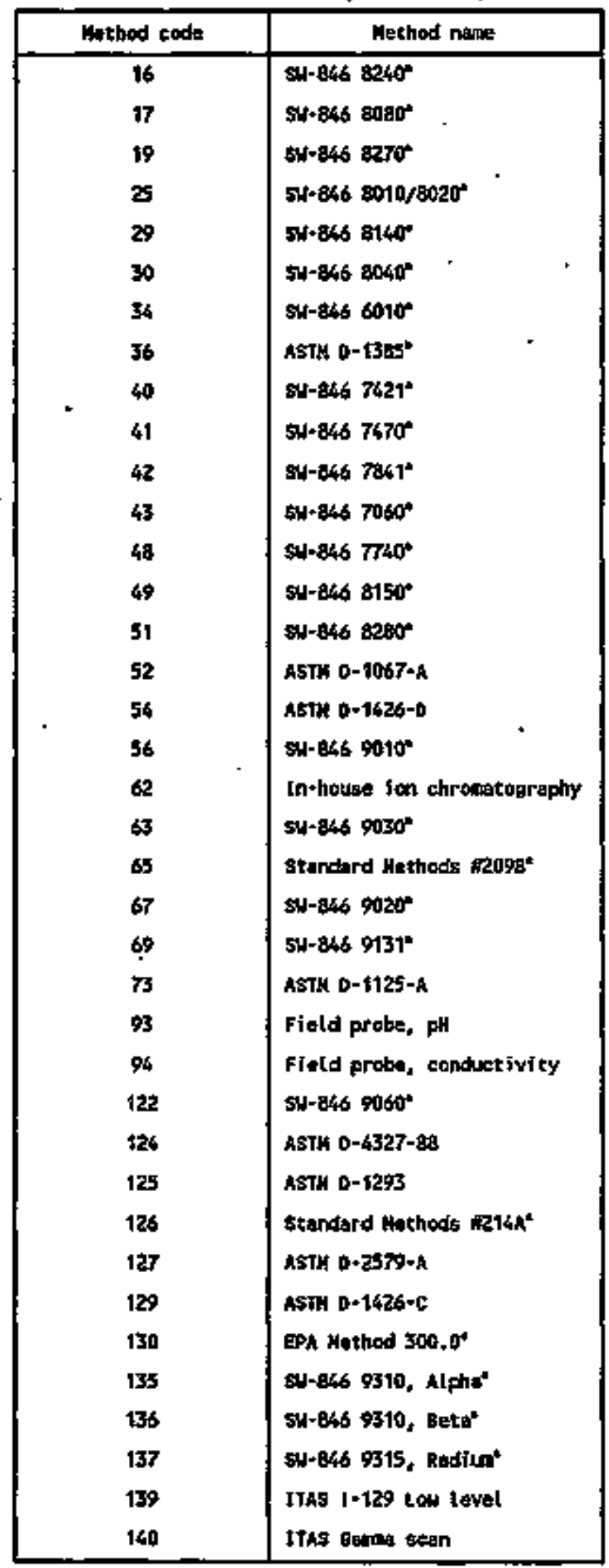


Table 1-9. Analysis Method Code

Definjtions. (2 sheets)

\begin{tabular}{|c|c|c|}
\hline & Nethod codls & Whethod name \\
\hline \multirow{11}{*}{ • } & 141 & ITA8 $S r=100$ \\
\hline & 142 & 1IA: $\|-3$ \\
\hline & 143 & ITAS TC- 99 \\
\hline & 144 & sth-846 s132 \\
\hline & 145 & ITAS Grogs U \\
\hline & 146 & JJAS Jgotople PU \\
\hline & 147 & ITAS At.24t \\
\hline & 148 & JTAS Jsotoplic U \\
\hline & 168 & ฟSEPA HACH $\cos ^{a}$ \\
\hline & 357 & EPh 600, 310.2 \\
\hline & 358 & $51+8469012$ \\
\hline
\end{tabular}

(CEA t986).

"(A5TH 1991).

'(EPA I97\%).

(APPHA 1989).

Agencies and suffixes--Agency codes and DWS suffixes jdentify the regulatory origin of the standard of the DWS. Agency codes are used in the Summary tables, and DWS suffixes are used in the Detected and CIP tables. The codes are defined as follows:

$\begin{array}{lll}\text { Agency Suffix } & \text { Reguthatary basts } \\ \text { EPA } & \text { Hone } & \text { Paximum contasinant levels in } 40 \text { CFR 141, "National } \\ & & \text { Primary Drinking Hater Regulations." }\end{array}$

EPAS S Secondary maximum contaninant levels in 40 CFR 143, "Wational Secondary Drinking Water Regulations."

EPAI $\quad \mathrm{E}$ EPA interim primary DHSs in 40 CFR 265, Appendix III.

Data qualifiers and flags--Bata qualifiers and fiags used in the Detected and CIP tables are asstgned by the laboratory and WHC personnel, respectively. Qualifiers reflect conditions occurring in the laboratory relating to the analytical procedure. Flags serve a wider function of alerting the data user to the 1 imitations of the reported value. Qualifiers and flags can be appended to each other to form a string of ietters when several factors apply to a result. The qualffiers and flags used are as follows:

\section{Laboratory qualifiers:}

B - B]ank associated with analyte is contaninated

D - Analyzed sample is diluted

E - Concentration is out of instrunent calibration range

$\checkmark$ - Concentration is estimated 
L - Concentration is below the contractually required quantitation iimit but above the HOL-

$U$ - Concentration is below the indtcated value.

Data flags:

F - Suspect data currently under revien

H - Laboratory holding time exceeded

$G$ - Reviewed data that are considered valld

P - Potential problen; see text associated with table

Q - Result associated with suspect QC data

$R$ - Reviewed data that have been rejected

$Y$ - Reviewed data that continue to be suspect

+ - Suspect water level data

* - MDL is greater than DHS, so exceedance of DHS is undetermined.

More conplete descriptions of some of these qualifiers and fiags are presented in Section 1.2, Quality Control Program.

Analysis method codes-Anatysis method codes are used as an abireviation for the laboratory method used to perfora an analysis. A complete listing of the analysis method codes used in the Sumlary and Detected tables is shown in Tab7e 1-10.

DWS5--DWSs are used in all of the chemistry tables to provide a standard with whtch to compare sample results. The DWSs are based on standards found in 40 CFR 265, 40 CFR 141, and 40 CFR 143. Where there is overlap in constituents addressed by these regulations, 40 CFR 265 takes precedence over 40 CFR 141, which, in turn, takes precedence over 40 CFR 143. A complete listing of the DWSs used in the Summary, Detected, and CIP tables is shown in Table 1-10.

\subsection{DATA EVALUATIOH}

\section{A. V. Gray}

Hestinghouse Hanford Company

Data evaluation is a process through which suspect data are identified and/or investigated. At present, the data evaluation process consists of the evaluation of data called out in RADEs, subritited by data users, and the required statistical evaluation of CIP data. The statistical evaluations of CIP data are presented utthin the chapters for individual sites.

Eighteen RADEs were submitted during the third quarter of 1994 . Of the submitted RADEs, two are for radionuclides, two are for field parameters ( $\mathrm{PH}$ ), five are for ICP metals, two are for TOC, five are for TOX, one is for turbidity, and one for ammonium ion. 
Tabie 1-10. Drinking Water Standards. (2 sheets)

\begin{tabular}{|c|c|c|c|c|}
\hline Const I tuent neme & shoct nowe & 945 & Unite & Aatency \\
\hline $1,1,1-t$ s ieblorowthens & $1,1,1-7$ & 200 & ppb & EPA \\
\hline 1,1-0lehlorothane & DICETHY & 7 & Fpb & EPA \\
\hline 2,z-bibrong-z-chloropropent & DJBRerit. & 0.2 & $p \neq b$ & EPA \\
\hline 1, 2 -0 ibromethen & DIARETH & 0,05 & pat & EPA \\
\hline 3,2-0tichl arobenzene & 12-dben & 600 & طקp & EPA \\
\hline 3,2-0tchlaroethants & $1,2-0 I C$ & 5 & apb & PA \\
\hline 1, Z-Dich Loropropane & o1cphtise & 5 & ppb & EPh \\
\hline 1,4-Dtchlarobanzere & 14-diben & 75 & ppb & $\operatorname{EPA}$ \\
\hline $2,4,5-\mathrm{TP}$ & $2,4,5 \mathrm{TP}$ & 50 & pb & EPA \\
\hline $2,4-0$ & $2,6-\mathrm{B}$ & 100 & ppb & EPAI \\
\hline ursenic & NRSEMtC & 50 & ppb & EA \\
\hline Aariun & BARIUH & 1,000 & ppb & EPA] \\
\hline Benzen: & aEHZEASE & 5 & ppb & $\operatorname{gPk}$ \\
\hline colditus & crantur & 10 & $\mathrm{apb}$ & Eend \\
\hline Carbon tetrechlorids & TETRAHE & 5 & ppb & EPA \\
\hline C45ility-137 & $\operatorname{cs}-137$ & 200 & $\mathrm{PCH} / \mathrm{L}$ & EPA \\
\hline chlordans & chandis: & 2 & . $P$ P & EPA \\
\hline chloride & CHACRID & 250,000 & ato & EPAs \\
\hline Chlorobentene & Cherogs & 100 & bop & EPA \\
\hline Chromiun & CARONIM & 50 & ppb & EPAI \\
\hline cis-1,z-৩tichlorgettoylene & CIs120E & 70 & ppb & Erh \\
\hline Cobalt -60 & $60-60$ & 100 & $\mathrm{pC} i / \mathrm{L}$ & EPA \\
\hline coll fortis & Cotseran & 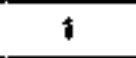 & COL & EPAI \\
\hline Copper & COPpER & 1,000 & ppo & EPAS \\
\hline Endtin & EMDRIE & 0.2 & طקp & EPA \\
\hline Ethytbenzene & etheraz & 700 & pか内 & EPA \\
\hline Fluoride & FLUORID & 1,400 & pob & EPA1 \\
\hline germa-ahc (Lintane) & g-ahis & 6 & ppos & EPA1 \\
\hline Grogs alpha & ALPHA & 95 & $\mathrm{PC}: / \mathrm{c}$ & EPA \\
\hline Heptachlor & HEPTLOR & 0.4 & Pp & tek \\
\hline Heptuchlor apoxtde & GEPTIDE & 0.2 & pph & EPA \\
\hline todino-129 & $1=129$ & 1 & $\mathrm{pCi} / \mathrm{L}$ & EDh \\
\hline tron & IROA & 300 & płt & EPAS \\
\hline lood & LEND & 50 & ppb & EPA1 \\
\hline Hengunose" & Mureste & 50 & $p \mathbf{p b}$ & EPAS \\
\hline Narcury & MERCLIFY & 2 & ppb & EPA \\
\hline Hathoxyator & METHLOR & 100 & $\mathrm{ppb}$ & EPAI \\
\hline
\end{tabular}


Table 1-10. Drinking Water Standards. (2 sheets)

\begin{tabular}{|c|c|c|c|c|}
\hline Constituent mane & short nape & ots & Units & Agency \\
\hline Mitrate & Mt TRAJE & 45,000 & ppb & EPA \\
\hline Hjtritce & AETAJTE & 1,000 & $p p$ & EPA \\
\hline Pentocht orophenol & PEUTC:AP & 1 & ppb & EFA \\
\hline PH & PH & $6.5-8.5$ & PH & EPAS \\
\hline Rediun & RADThy & $\mathbf{5}$ & pestl & EPAt \\
\hline Ruthenium 106 & $R+106$ & 30 & $\mathrm{pci} / \mathrm{L}$ & EPA \\
\hline Solentum & SELEAIXI & 10 & $\mathbf{p p b}$ & EPAI \\
\hline stiver & SILIER & so & Ppb & EPAI \\
\hline Strontiun- 90 & sh- 00 & $\mathbf{B}$ & $p c t / 1$ & EPA \\
\hline styrant & STYREHE & 100 & ppb & EPR \\
\hline sultate & SULFATE & 250,000 & pps & EPAS \\
\hline Technetfurry & TC+5g & 900 & pCt/L & EPA \\
\hline Tetrachlorcethene & PERCELE & $\mathbf{s}$ & Ppob & EPA \\
\hline Toluene & TOLUENE & 1,000 & bop & EPA \\
\hline Total dissolved solldy & Tos & 500 & eq & EPAs \\
\hline Toxaghene & TOKAEE⿰亻弋 & 5 & $\mathbf{p p b}$ & EPRI \\
\hline trans-1,2-bichlorothylano & TRALPCE & 100 & , pob & EPA \\
\hline Trichioraethen: & TRICENE & $\mathbf{5}$ & ppb & $\operatorname{sen}$ \\
\hline Trittun & TRITIUS: & 20,000 & $\mathrm{pCI} / \mathrm{t}$ & apd \\
\hline vimy chlorids & vinrtoe & 2 & pob & EPA \\
\hline Xylenes \{total\} & XrLene & 10,000 & ppb & EPA \\
\hline Zinc: & ZIt:Pe & 5,000 & Ppb & ePAs \\
\hline
\end{tabular}

Gov Staction 1.4.5 for egency listing.

con * coll form colontes par 100 mitilititars.

peill a picocuries per titer.

ppb = ports per-billion.

An F flag is used to indicate that a RADE has been submitted. Suspect analytical data shall be evaluated for the following, as applicable: htstorica) trends, site geohydrology, contaminant distribution (e.g., plumes), and tnternal consistency to determine if the data are acceptable. After evaluation, the $F$ flag will be changed to ' $G$,' indicating acceptable data; ' $Y$, ' indicating suspect data; or ' $R$,' indicating unusable data. A stummary of the results will be presented in the anntal report. 


\section{DOE/RL-94-36-4}

This page intentionally Teft blank. 


\section{DOE/RL-94-36-4}

\section{CONTERTS}

$2.0 \quad 100-D$ PONDS

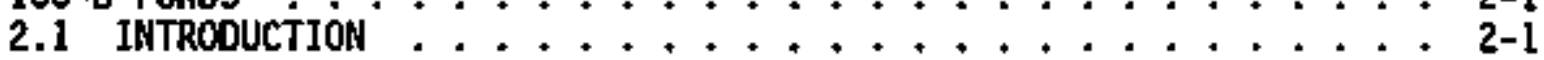

2.2 WATER LEVEL MEASUREMENTS $\ldots \ldots \ldots \ldots \ldots \ldots$

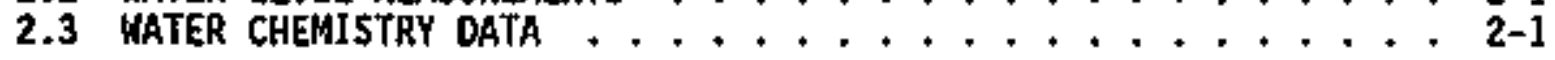


DOE/RL-94-36-4

\section{LIST OF FIG̈URES}

2-1 Monitoring Well Location Map for the 100-0 Ponds ........ 2-2

\section{LIST OF TABLES}

2-1 Monitoring Mell Purpose and Sampling Schedule in the 100-D Ponds Network ............ 2-3

2-3 Constituent L1st and Summary of Results for the 100-0 Pond Data for Reporting Period October 1 through Oecember 31, 1994 . . . 2-7

2-4 Constituents with at Least One Detected value for the 100-D Pond Data for Reporting Period October 1 through December 31, 1994 . . . 2-8

2-5 Contamination Indicator Paraneters for the 100-D Pond Data for Reporting Period October 1 through December 31, $1994 \ldots$ 2-9 


\title{
2.0 100-D POHDS
}

\author{
M. J. Hartman \\ Nestinghouse Hanford Company
}

\subsection{INTRODUCTIOH}

The 100-0 Ponds system is an interim-status RCRA dtsposal unit located in the 100-0 Area of the Hanford Site (Figure 2-1). Four wells make up the 100-D Ponds sampling network (Table 2-I). The 100-D Ponds are monitored under an indicator evaluation program (40 CFR 265).

The 100-D Ponds unit was constructed in 1977 for disposal of nonradioactive effluents derived fron 100-D Area operating facilities. The 100-D Ponds are located in the former 188-D ash disposal basin and consist of a settiling pond and a percolation pond separated by a dike.

Effluent to the 100-D Ponds originated from two sources: a filter plant and engineering testing laboratories. Some past discharges any have included corrosive waste or mercury (Hartman 1991). All discharges to the 100-D Ponds ceased in May 1994.

\subsection{WATER LEVEL MEASUREAENTS}

Water levels are measured monthly in wells in and near the 100-D Area to track changes caused by river-stage fluctuations and the cessation of effluent dtscharge. Water level data are Tisted in Table 2-2.

\subsection{WATER CHEMISTRY DATA}

Upgradient well 05-13 was sampled for field conductivity, field pH, and Tox; no other samples were scheduled during the reporting period. Bata are reported in Tables 2-3 through 2-5. Field $\mathrm{pH}$, conductivity, and temperature data from August 26, 1994, are also 3 ísted in the tabTes because they were not included in the 1ast quarterly report. Field parameters and laboratory $\mathrm{pH}$ were measured in we11 05-13 on that date to verify a $\mathrm{pH}$ exceedance. The August 26, 1994, data did not exceed the critical range for $\mathrm{pH}$, indicating that the previous value was in error. Data flags are discussed in Sections 1.2 and 1.4. Ho OuSs were exceeded. 


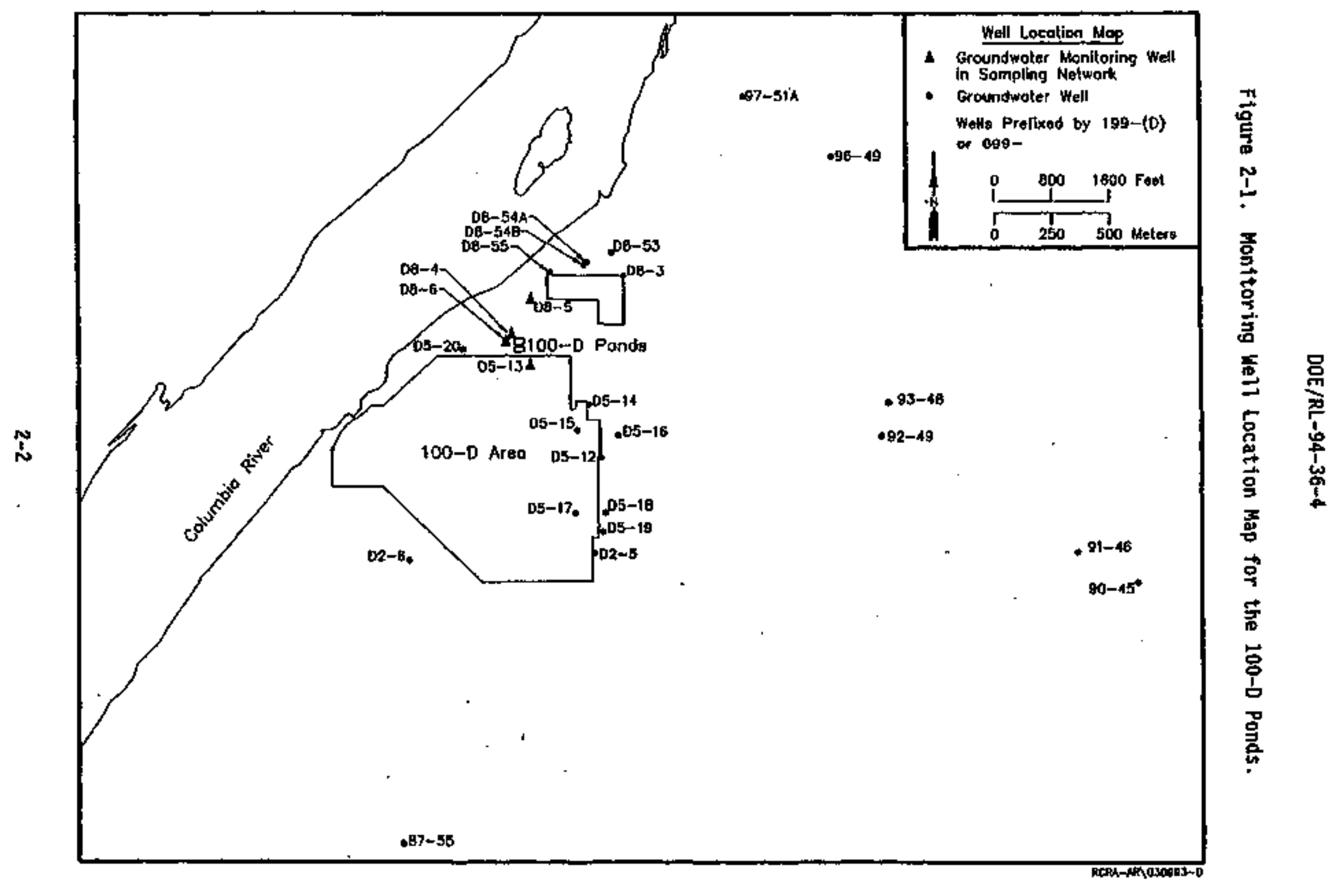


Table 2-1. Monitoring Nell Purpose and Sampling Schedule in the 100-D Ponds Metwork.

\begin{tabular}{|c|c|c|c|}
\hline $\begin{array}{c}\text { Wel1 no- } \\
(199-)\end{array}$ & $\begin{array}{c}\text { Relat1ve } \\
\text { position }\end{array}$ & $\begin{array}{c}\text { Sample } \\
\text { frequency }\end{array}$ & $\begin{array}{c}\text { Sample date, } \\
\text { 4th Qtr 1994 }\end{array}$ \\
\hline D5-13 & Upgradient & Quarterly & Il/3/94 \\
\hline D8-4 & Downgradient & Semiannually & Hot scheduled \\
\hline D8-5 & Downgradient & Semiannually & Hot scheduled \\
\hline D8-6 & Downgradient & Semianinually & Hot scheduled \\
\hline
\end{tabular}

Note: The hydrogeologic unit monitored is the upperwost portion of the unconfined aquifer, which conprises unconsolidated sand and gravels of the Ringold Formation. 
Table 2-2 * RCRA Water Level Measurement Report
100-D Ponds - Fourth Quarter 1994.

(sheet 1 of 3 )

\begin{tabular}{|c|c|c|c|c|}
\hline He11 & gate & $\begin{array}{l}\text { Depth to } \\
\text { water (ft) }\end{array}$ & $\begin{array}{l}\text { Wate } \\
\text { elevatto } \\
\text { (ft) }\end{array}$ & $\begin{array}{l}\text { ve msl } \\
\text { (m) }\end{array}$ \\
\hline \multicolumn{5}{|c|}{ Wells Honttoring the Top of the Unconfined Aquifer } \\
\hline 199-02-5 & $\begin{array}{l}10 / 17 / 94 \\
11 / 07 / 94 \\
12 / 06 / 94\end{array}$ & $\begin{array}{l}75.56 \\
75.71 \\
75.79\end{array}$ & $\begin{array}{l}384.74 \\
384.59 \\
384.51\end{array}$ & $\begin{array}{l}117.27 \\
117.22 \\
117.20\end{array}$ \\
\hline $\begin{array}{c}199-02-6 \\
.\end{array}$ & $\begin{array}{l}10 / 17 / 94 \\
11 / 07 / 94 \\
12 / 06 / 94\end{array}$ & $\begin{array}{l}85.94 \\
86.04 \\
85.95\end{array}$ & $\begin{array}{l}383.34 \\
383.24 \\
383.33\end{array}$ & $\begin{array}{l}116.84 \\
116.81 \\
116.84\end{array}$ \\
\hline $199-05-12$ & $\begin{array}{l}10 / 17 / 94 \\
11 / 07 / 94 \\
12 / 06 / 94\end{array}$ & $\begin{array}{l}85.89 \\
85.03 \\
85.05\end{array}$ & $\begin{array}{l}383.72 \\
383.58 \\
383.56\end{array}$ & $\begin{array}{l}116.96 \\
116.92 \\
116.91\end{array}$ \\
\hline $199-05-13$ & $\begin{array}{r}10 / 17 / 94 \\
11 / 07 / 94 \\
12 / 06 / 94\end{array}$ & $\begin{array}{l}89.22 \\
89.21 \\
89.16\end{array}$ & $\begin{array}{l}382.27 \\
382.28 \\
382.33\end{array}$ & $\begin{array}{l}116.52 \\
116.52 \\
116.53\end{array}$ \\
\hline $199-05-17$ & $\begin{array}{l}10 / 17 / 94 \\
11 / 07 / 94 \\
12 / 06 / 94\end{array}$ & $\begin{array}{l}85.15 \\
85.29 \\
85.37\end{array}$ & $\begin{array}{l}384.34 \\
384.20 \\
384.12\end{array}$ & $\begin{array}{l}117.15 \\
117.10 \\
117.08\end{array}$ \\
\hline $199-05-20$ & $\begin{array}{l}10 / 17 / 94 \\
11 / 07 / 94 \\
12 / 06 / 94\end{array}$ & $\begin{array}{l}86.71 \\
85.43 \\
85.86\end{array}$ & $\begin{array}{l}381.39 \\
381.67 \\
382.24\end{array}$ & $\begin{array}{l}116.25 \\
116.33 \\
116.51\end{array}$ \\
\hline $199-08-3$ & $\begin{array}{l}10 / 17 / 94 \\
11 / 07 / 94\end{array}$ & $\begin{array}{l}69.11 \\
68.62\end{array}$ & $\begin{array}{l}379.85 \\
380.34\end{array}$ & $\begin{array}{l}115.78 \\
115.93\end{array}$ \\
\hline $199-08-4$ & $\begin{array}{l}10 / 17 / 94 \\
11 / 07 / 94 \\
12 / 06 / 94\end{array}$ & $\begin{array}{l}87.13 \\
87.04 \\
86.79\end{array}$ & $\begin{array}{l}381.60 \\
381.69 \\
381.94\end{array}$ & $\begin{array}{l}116.31 \\
116.34 \\
116.42\end{array}$ \\
\hline $199-D 8-5$ & $\begin{array}{l}10 / 17 / 94 \\
11 / 07 / 94 \\
12 / 06 / 94\end{array}$ & $\begin{array}{l}71.97 \\
71.52 \\
71.03\end{array}$ & $\begin{array}{l}380.52 \\
380.97 \\
381.46\end{array}$ & $\begin{array}{l}115.98 \\
116.12 \\
116.27\end{array}$ \\
\hline 199-08-53 & $\begin{array}{l}10 / 17 / 94 \\
11 / 07 / 94 \\
12 / 06 / 94\end{array}$ & $\begin{array}{l}56.42 \\
55.77 \\
54.91\end{array}$ & $\begin{array}{l}379.61 \\
380.26 \\
381.12\end{array}$ & $\begin{array}{l}115.71 \\
115.90 \\
116.17\end{array}$ \\
\hline $199-08-54 A$ & $10 / 17 / 94$ & 63.09 & 379.69 & 115.73 \\
\hline
\end{tabular}


Table 2-2. RCRA Water Level Measurement Report 100-D Ponds - Fourth Quarter 1994.

(sheet 2 of 3 )

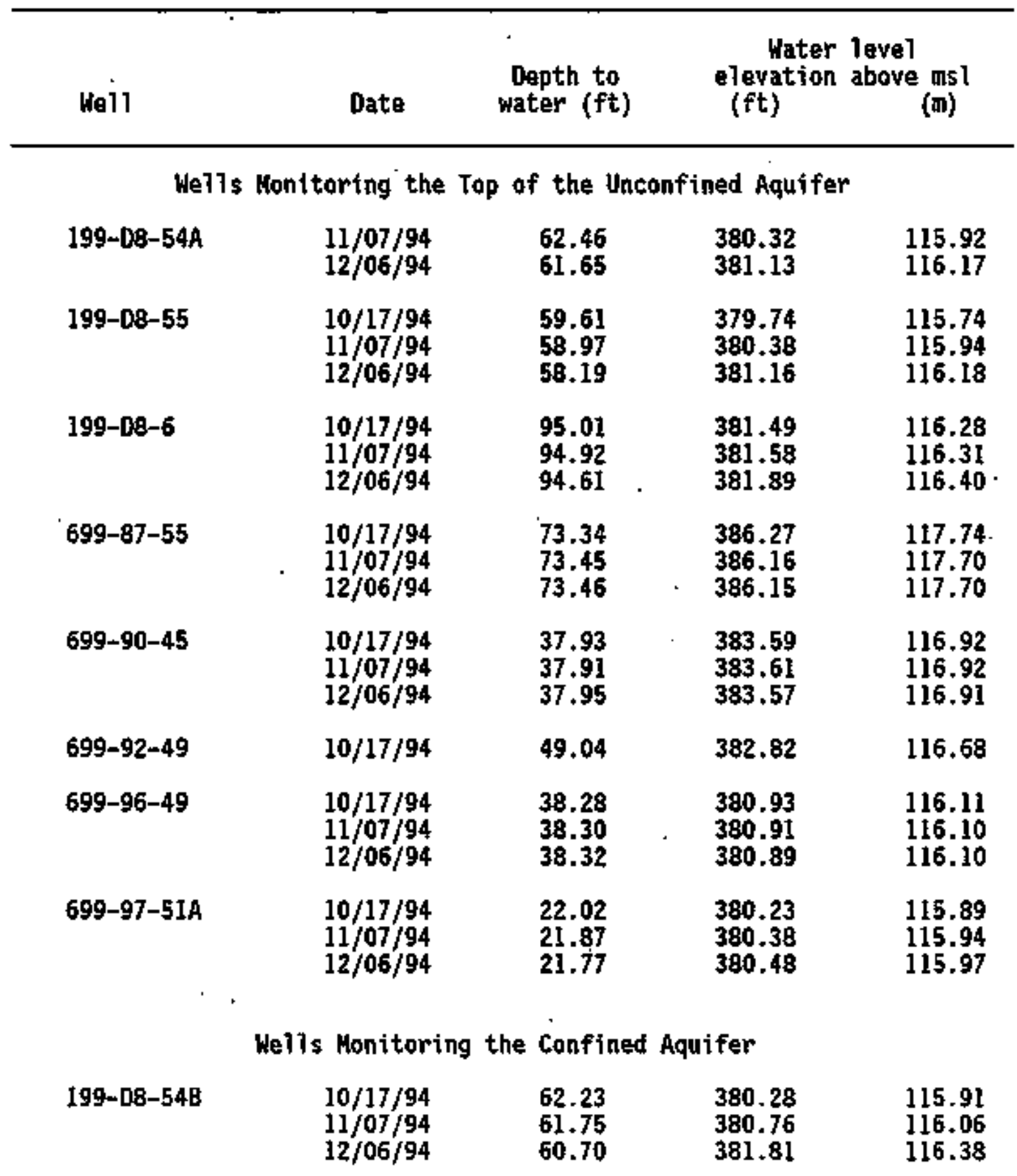


Table 2-2. RCRA Water Leve] Measurement Report 100-0 Ponds - Fourth Quarter 1994. (sheet 3 of 3 )

MOTES: 1. Water level elevations are calculated by subtracting the measured depth-to-water from the surveyed elevation for the we]l.

2. Depth-to-water values are transcribed frow fleld records.

3. Elevations marked with an ' $*$ ' were measured at the time of sampling.

4. Elevations marked with $a^{\prime}+{ }^{\prime}$ are outside of the expected range, and are suspected of error. 
Table 2-3. Constituent List and Summary of Results for the 100-0 Pond Data for Reporting Period October 1 through December 31, 1994.

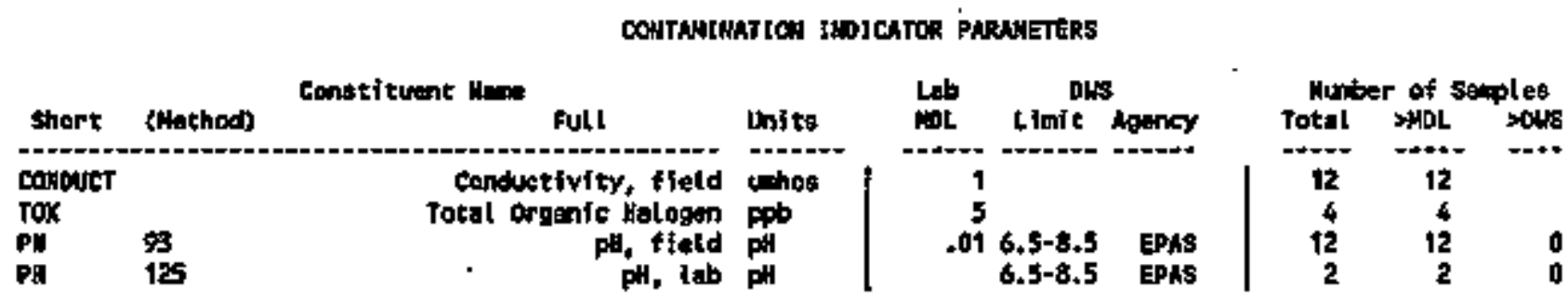

SITE SFECIFIC No OTHER COHSTI TUEhtS

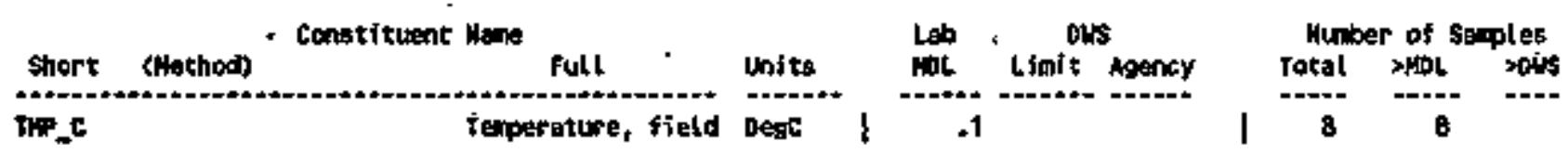

For exphanetion of this table, set section 1.4 of report. 
Table 2-4. Constituents with at Least One Detected Value for the 100-D Pond Data for Reporting Perfod Octaber 1 through December 31, 1994.

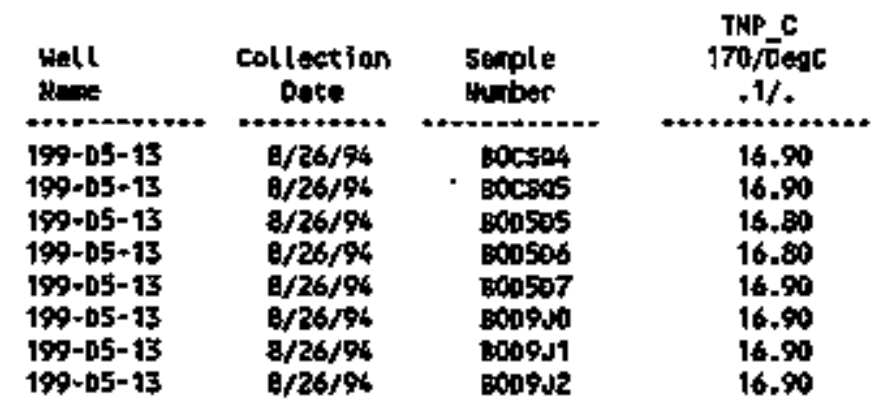

For explenotion of this table, sas Section 1.4 of report. 
Table 2-5. Contanination Indicator Parameters for the 100-0 Pond Data for Reporting Perilod October I through December 31, I994.

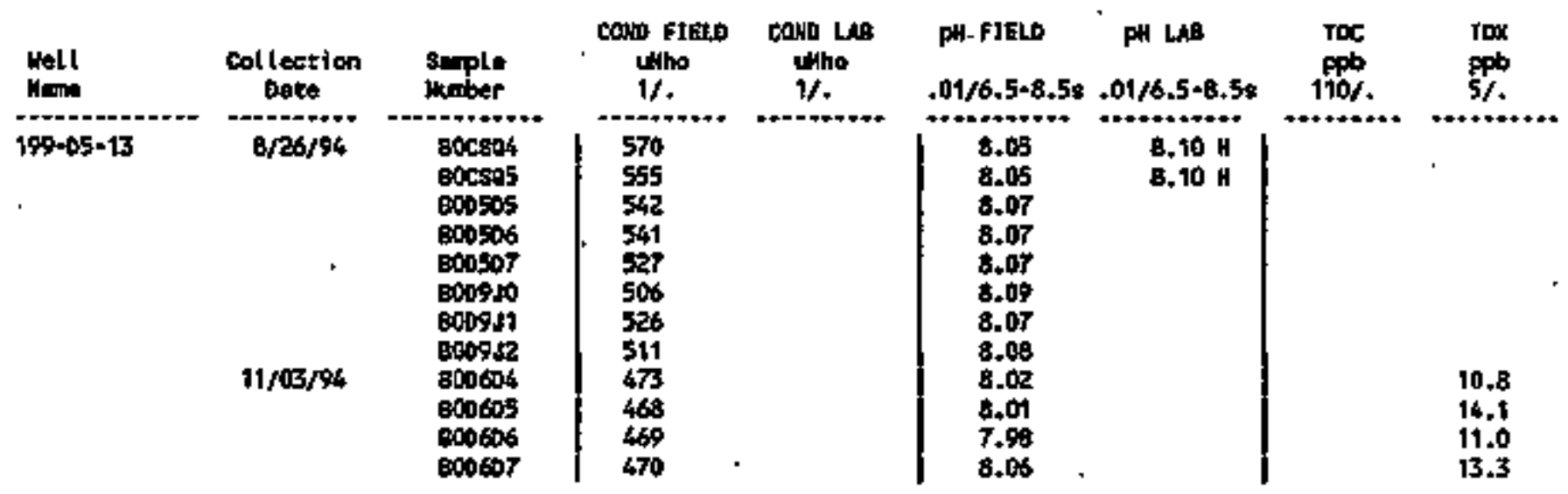

For explenation of this tofile, san section 1.4 of report. 
D0E/RL-94-36-4

This page intentionally left blank. 
DOE/RL-94-36-4 .

\section{contants}

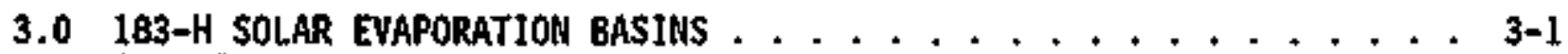

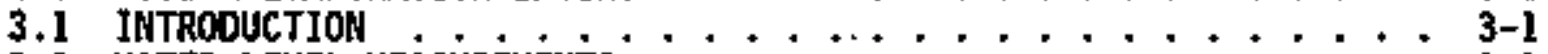

3.2 WATER LEVEL MEASUREHENTS $\ldots \ldots \ldots \ldots \ldots$

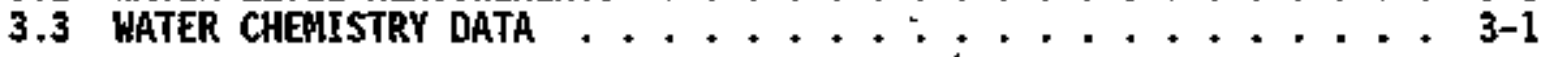


DOE/RL-94-36-4

\section{LIST OF FIGURES}

3-1 Nell Location Map for the 183-H Solar Evaporation Basins Metwork ................ 3-2

\section{LIST OF TABLES}

3-1 Menitoring Well Position and Sampling Schedule for the 183-H Solar Evaporation Basins Metwork . . . . . . . 3-3

3-2 RCRA Nater Level Measurement Report 183-H Solar Evaporation Basins, Fourth Quarter 1994 ... . . . . . 3-4

3-3 Constituent List and Summary of Results for 183-H Solar Evaporation Bastns Data for Reporting Perfod October 1 through December $31,1994 \ldots . . . . . . . . .3$

3-4 Constituents with at Least One Detected Value for the 183-H Solar Evaporation Basins Data for Reporting Perjod October 1 through December 31, $1994 \ldots . . . . . . . . . . . .3-10$

3-5 Contamination Indicator Parameters for the 183-H 5olar Evaporation Basins Data for Reporting Period October 1 through

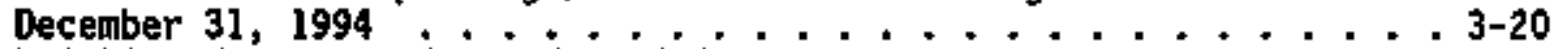

3-6 Orinking Water Standards Exceeded for the 183-H Basins . . . . 3-21

3-7 Requests for Analytical Data Evaluation Submitted for the 183-H Solar Evaporation Basins, Fourth Quarter $1994 \ldots$. . . 3-22 
DOE/RL-94-36-4

\title{
3.0 183-H SOLAR EUAPORATION BASIMS
}

\author{
M. J. Hartman
}

Nestinghouse Hanford Company

\subsection{IHTRODUCTION}

The 183-H Solar Evaporation Basins (Basins) are a RCRA-regutatid facility located near the Columbia River in the 100-H Area of the Hanford Site. Originaliy the Basins were part of the water treatment facility that prepared coolant for the 105-H Reactor. Subsequent to that mission, between 1973 and 1985, the Basins were used for storage and volume reduction (by solar evaporation) of 1 iquid waste from nuclear fuel processing activities conducted in the 300 Area. The predonitnant waste consisted of nitric acid solutions that had been neutralized with sodium hydroxide before discharge into the Basins. The solutions contained various metals and radioactive constituents (e.g., chromium, uranlum, and technetfum). Hazardous waste has been removed froin the facility and the basin walls have been partialiy decontaminated.

Groundwater quality has been affected by leakage fron the Basins. Groundwater monitoring has been conducted as an assessment-level program under 40 CFR 265 interin-status standards, as mandated by a consent agreement and complfance order (Ecology and EPA 1986). The 193-H Basins are included in the new Hanford Facility RCRA Perait and, in the future, will be monitored under final-status requirements (Ecology 1994). A final-status groundwater nonitoring pian is being prepared.

The purpose of each RCRA we11, its sampling frequency, and the dates sampled during the quarter, are summarized in Table 3-1. Well Iocations are shown in Figure 3-I.

\subsection{WATER LEVEL MEASURĖNEKTS}

Water levels were weasured monthly during the past quarter to track changes caused by river stage fluctuations. Water levels also were recorded at the time of sample collection for water quality anajyses. Water levels are presented in Table 3-2.

\subsection{NATER CHEHTSTRY DATA}

Quarterly sampling was completed as scheduled in 183-H network wells. Three wel.ls were sampled monthly to provide enhanced nonitoring of the contaminant plume. Results are presented in Tables 3-3 through 3-5. Data fiags are discussed in Sections 1.2 and 1.4. DuSs exceeded are listed in Tabje 3-6. Several results appear anomalous, and RADEs have been submitted. Table 3-7 1 ists RADEs subnitted for the past quarter and the reason for the request. 
Figure 3-1. Well Location Map for the 193-H Solar Evaporation Basins Hetwork.

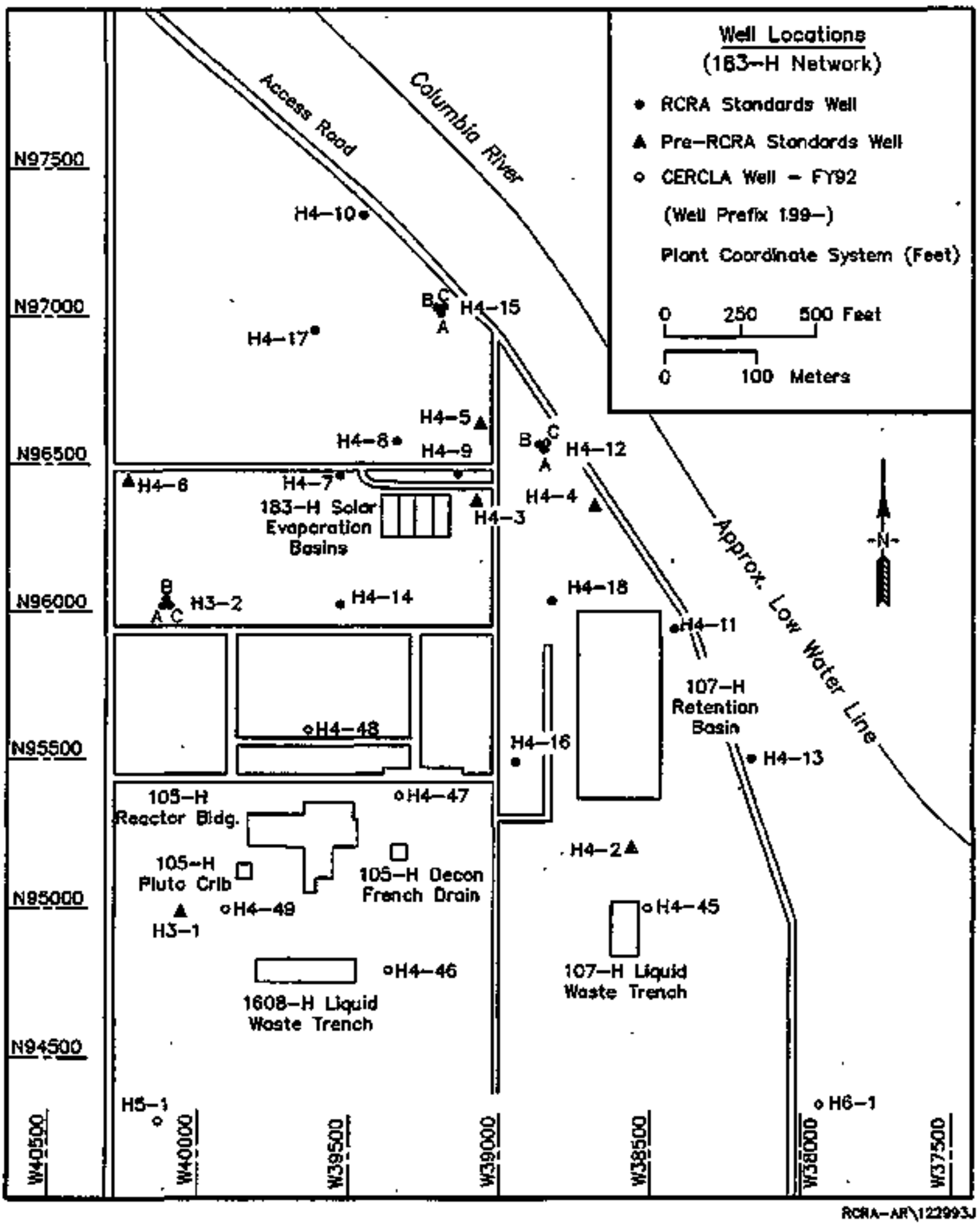


Table 3-1. Monitoring Mell Position and Sampling Schedule for the 183-H Solar Evaporation Basins Hetwork.

\begin{tabular}{|c|c|c|c|c|}
\hline $\begin{array}{c}\text { Wel1 no. } \\
\text { (199-) }\end{array}$ & $\begin{array}{l}\text { Relative } \\
\text { position }\end{array}$ & Hydrogeologic unit" & $\begin{array}{l}\text { Sample } \\
\text { frequency }\end{array}$ & $\begin{array}{l}\text { Sample date, } \\
\text { 4th Qtr } 1994\end{array}$ \\
\hline $\mathrm{HB}-2 \mathrm{~A}$ & Upgradient & Hanford: Water Table & Quarteriy & $12 / 2 / 94$ \\
\hline $\mathrm{H3}-2 \mathrm{C}$ & Upgradient & Ringo7d: st7ty Sand & Quarterly & $12 / 2 / 94$ \\
\hline $\mathrm{H} 4-11$ & Downgradtent & Hanford: Water Table & Quarterjy & $12 / 5 / 94$ \\
\hline H4-12A & Downgradient & Hanford: Water Table & Quarter]y & $12 / 14 / 94$ \\
\hline H4-12C & Downgradient & Ringold: \$ilty Sand & Quarter7y & $12 / 5 / 94$ \\
\hline H4-14 & Upgradient & Hanford: Water Table & Quarter7y & $12 / 5 / 94$ \\
\hline H4-18 & Downgradient & Hanford: Water Table & Quarteriy & $12 / 5 / 94$ \\
\hline H4-3 & Downgradient & Hanford: Water Table & Monthly & $\begin{array}{l}10 / 03 / 94 \\
11 / 22 / 94 \\
12 / 05 / 94\end{array}$ \\
\hline $\mathrm{H} 4-4$ & Downgradient & Hanford: Hater Table & Month]y & $\begin{array}{l}10 / 03 / 94 \\
11 / 16 / 94 \\
12 / 05 / 94\end{array}$ \\
\hline $\mathrm{H} 4-5$ & Downgradient & Hanford: Water Table & Quarteriy & $12 / 05 / 94$ \\
\hline H4-6 & Upgradient & Hanford: Water Table & Quarterly & $12 / 2 / 94$ \\
\hline H4-7 & Upgradient & Hanford: Water Table & Quarterly & $12 / 5 / 94$ \\
\hline H4-8 & Upgradient & Hanford: Water Table & Quarterly & $12 / 2 / 94$ \\
\hline H4-9 & Downgradient & Hanford: Water Table & Monthly & $\begin{array}{c}10 / 15 / 94 \\
N / S \\
12 / 12 / 94\end{array}$ \\
\hline
\end{tabular}

Hydrogeologic units include the sandy gravels of the Hanford formation and silty sands of the Ringold Formation. Water levels are measured monthly in all wells.

Wot saxpled; pump above water. Pump was lowered before 12/94 sampling. 
Table 3-2. RCRA Water Level Measurement Report. 183-H Solar Evaporation Basins, Fourth Quarter 1994. (sheet 1 of 4 )

\begin{tabular}{|c|c|c|c|c|}
\hline We11 & Date & $\begin{array}{l}\text { Depth to } \\
\text { water (ft) }\end{array}$ & $\begin{array}{l}\text { Water } \\
\text { elevation } \\
\text { (ft) }\end{array}$ & $\begin{array}{l}\text { leveî } \\
\text { above ms } \\
\text { (m) }\end{array}$ \\
\hline \multicolumn{5}{|c|}{ Wells Monjtoring the } \\
\hline $199-\mathrm{H} 3-\mathrm{I}$ & $\begin{array}{l}10 / 17 / 94 \\
11 / 07 / 94 \\
12 / 06 / 94\end{array}$ & $\begin{array}{l}46.15 \\
46.39 \\
46.52\end{array}$ & $\begin{array}{l}375.33 \\
375.09 \\
374.96\end{array}$ & $\begin{array}{l}114.40 \\
114.33 \\
114.29\end{array}$ \\
\hline $199-H 3-2 A$ & $\begin{array}{l}10 / 17 / 94 \\
11 / 07 / 94 \\
12 / 02 / 94 \\
12 / 06 / 94\end{array}$ & $\begin{array}{l}42.99 \\
43.20 \\
43.26 \\
43.26\end{array}$ & $\begin{array}{l}375.33 \\
375.12 \\
375.06 * \\
375.06\end{array}$ & $\begin{array}{l}114.40 \\
114.34 \\
114.32 \\
114.32\end{array}$ \\
\hline $199-\mathrm{H3}-2 \mathrm{~B}$ & $\begin{array}{l}10 / 17 / 94 \\
11 / 07 / 94 \\
12 / 06 / 94\end{array}$ & $\begin{array}{l}43.59 \\
43.78 \\
43.85\end{array}$ & $\begin{array}{l}374.83 \\
374.64 \\
374.57\end{array}$ & $\begin{array}{l}114.25 \\
114.19 \\
114.17\end{array}$ \\
\hline $199-\mathrm{H} 4-10$ & $\begin{array}{l}10 / 17 / 94 \\
11 / 07 / 94 \\
12 / 06 / 94\end{array}$ & $\begin{array}{l}31.96 \\
31.45 \\
30.95\end{array}$ & $\begin{array}{l}372.48 \\
372.99 \\
373.49\end{array}$ & $\begin{array}{l}113.53 \\
113.69 \\
113.84\end{array}$ \\
\hline 199-H4-11 & $\begin{array}{l}10 / 17 / 94 \\
11 / 07 / 94 \\
12 / 06 / 94\end{array}$ & $\begin{array}{l}45.29 \\
44.54 \\
43.75\end{array}$ & $\begin{array}{l}371.55 \\
372.30 \\
373.09\end{array}$ & $\begin{array}{l}113.25 \\
113.48 \\
113.72\end{array}$ \\
\hline $199-H 4-12 A$ & $\begin{array}{l}11 / 07 / 94 \\
12 / 06 / 94\end{array}$ & $\begin{array}{l}40.69 \\
40.09\end{array}$ & $\begin{array}{l}372.81 \\
373.41\end{array}$ & $\begin{array}{l}113.63 \\
113.82\end{array}$ \\
\hline 199-H4-12B & $\begin{array}{l}10 / 17 / 94 \\
11 / 07 / 94 \\
12 / 06 / 94\end{array}$ & $\begin{array}{l}41.29 \\
40.69 \\
40.09\end{array}$ & $\begin{array}{r}372.23 \\
372.83 \\
373.43\end{array}$ & $\begin{array}{l}113.46 \\
113.64 \\
113.82\end{array}$ \\
\hline $199-\mathrm{H} 4-13$ & $\begin{array}{l}10 / 17 / 94 \\
11 / 07 / 94 \\
12 / 06 / 94\end{array}$ & $\begin{array}{l}47.10 \\
46.20 \\
45.27\end{array}$ & $\begin{array}{l}371.10 \\
372.00 \\
372.93\end{array}$ & $\begin{array}{l}113.11 \\
113.39 \\
113.67\end{array}$ \\
\hline $199-\mathrm{H} 4-14$ & $\begin{array}{l}10 / 17 / 94 \\
11 / 07 / 94 \\
12 / 05 / 94 \\
12 / 06 / 94\end{array}$ & $\begin{array}{l}46.14 \\
46.26 \\
45.15 \\
46.30\end{array}$ & $\begin{array}{l}374.94 \\
374.82 \\
375.93^{*}+ \\
374.78\end{array}$ & $\begin{array}{l}114.28 \\
114.25 \\
114.58 \\
114.23\end{array}$ \\
\hline 199-H4-15A & $\begin{array}{l}10 / 17 / 94 \\
11 / 07 / 94 \\
12 / 06 / 94\end{array}$ & $\begin{array}{l}34.78 \\
34.22 \\
33.74\end{array}$ & $\begin{array}{l}372.93 \\
373.49 \\
373.97\end{array}$ & $\begin{array}{l}113.67 \\
113.84 \\
113.99\end{array}$ \\
\hline
\end{tabular}


Table 3-2. RCRA Water Level Measurement Report 183-H Solar Evaporation Basins - Fourth Quarter 1994.

(sheet 2 of 4)

Well Date $\quad \begin{gathered}\text { Depth to } \\
\text { water (ft) }\end{gathered} \quad$\begin{tabular}{c}
$\begin{array}{c}\text { Water leve? } \\
\text { elevation above ms } 1 \\
(\mathrm{ft})\end{array}$ \\
\hline
\end{tabular}

Wells Monitoring the Jop of the Unconfined Aquifer (Hanford Foration)

\begin{tabular}{|c|c|c|c|c|c|}
\hline 199-H4-15B & $\begin{array}{l}10 / 17 / 94 \\
11 / 07 / 94 \\
12 / 06 / 94\end{array}$ & $\begin{array}{l}34.51 \\
33.94 \\
33.44\end{array}$ & & $\begin{array}{l}372.41 \\
372.98 \\
373.48\end{array}$ & $\begin{array}{l}113.51 \\
113.68 \\
113.84\end{array}$ \\
\hline 199-H4-16 & $\begin{array}{l}10 / 17 / 94 \\
11 / 07 / 94 \\
12 / 06 / 94\end{array}$ & $\begin{array}{l}50.43 \\
50.49 \\
50.44\end{array}$ & & $\begin{array}{l}373.80 \\
373.74 \\
373.79\end{array}$ & $\begin{array}{l}113.93 \\
113.92 \\
113.93\end{array}$ \\
\hline $199-\mathrm{H} 4-17$ & $\begin{array}{l}10 / 17 / 94 \\
11 / 07 / 94 \\
12 / 06 / 94\end{array}$ & $\begin{array}{l}45.95 \\
45.68 \\
45.70\end{array}$ & . & $\begin{array}{l}373.64 \\
373.91 \\
373.89\end{array}$ & $\begin{array}{l}113.89 \\
113.97 \\
113.96\end{array}$ \\
\hline $\begin{array}{c}199-H 4-18 \\
.\end{array}$ & $\begin{array}{l}10 / 17 / 94 \\
11 / 07 / 94 \\
12 / 05 / 94 \\
12 / 06 / 94\end{array}$ & $\begin{array}{l}48.73 \\
48.54 \\
48.47 \\
48.36\end{array}$ & & $\begin{array}{l}373.59 \\
373.78 \\
373.85 * \\
373.96\end{array}$ & $\begin{array}{l}113.87 \\
113.93 \\
113.95 \\
113.98\end{array}$ \\
\hline 199-H4-3 & $\begin{array}{l}10 / 03 / 94 \\
10 / 17 / 94 \\
11 / 07 / 94 \\
11 / 22 / 94 \\
12 / 05 / 94 \\
12 / 06 / 94\end{array}$ & $\begin{array}{l}47.85 \\
47.40 \\
47.16 \\
47.00 \\
48.45 \\
46.96\end{array}$ & . & $\begin{array}{l}372.44^{*} \\
372.89 \\
373.13 \\
373.29^{*} \\
371.84^{*}+ \\
373.33\end{array}$ & $\begin{array}{l}113.52 \\
113.66 \\
113.73 \\
113.78 \\
113.34 \\
113.79\end{array}$ \\
\hline $199-\mathrm{H} 4-4$ & $\begin{array}{l}10 / 03 / 94 \\
10 / 17 / 94 \\
11 / 07 / 94 \\
11 / 16 / 94 \\
12 / 05 / 94 \\
12 / 06 / 94\end{array}$ & $\begin{array}{l}42.72 \\
41.77 \\
41.06 \\
41.02 \\
40.92 \\
40.38\end{array}$ & . & $\begin{array}{l}371.52^{\star} \\
372.47 \\
373.18 \\
373.22^{\star} \\
373.32^{\star} \\
373.86\end{array}$ & $\begin{array}{l}113.24 \\
113.53 \\
113.75 \\
113.76 \\
113.79 \\
113.95\end{array}$ \\
\hline 199-H4-5 & $\begin{array}{l}10 / 17 / 94 \\
11 / 07 / 94 \\
12 / 05 / 94 \\
12 / 06 / 94\end{array}$ & $\begin{array}{l}43.87 \\
43.37 \\
42.25 \\
43.01\end{array}$ & & $\begin{array}{l}372.34 \\
372.84 \\
373.96{ }^{\star}+ \\
373.20\end{array}$ & $\begin{array}{l}113.49 \\
113.64 \\
113.98 \\
113.75\end{array}$ \\
\hline 199-H4-6 & $\begin{array}{l}10 / 17 / 94 \\
11 / 07 / 94 \\
12 / 02 / 94\end{array}$ & $\begin{array}{l}44.75 \\
44.95 \\
45.02\end{array}$ & & $\begin{array}{l}374.83 \\
374.63 \\
374.56\end{array}$ & $\begin{array}{l}114.25 \\
114.19 \\
114.17\end{array}$ \\
\hline
\end{tabular}


Table 3-2. RCRA Water Level Measurement Report. 183-H Solar Evaporation Basins, Fourth Quarter 1994.

(sheet 3 of 4 )

We13 Date $\quad \begin{gathered}\text { Depth to } \\
\text { water (ft) }\end{gathered} \quad \begin{gathered}\text { Water Tevel } \\
\text { elevation above ms1 } \\
(\mathrm{ft})\end{gathered} \quad$\begin{tabular}{l}
$(\mathrm{m})$ \\
\hline
\end{tabular}

Wells Monitoring the Top of the Unconfined Aquifer (Hanford Formation)

$199-\mathrm{H} 4-6 \quad 12 / 06 / 94.45 .00 \quad 374.58: 114.17$

199-H4-7

$10 / 17 / 94$

$11 / 07 / 94$

46.77

46.75

46.75

$12 / 05 / 94$

46.69

373.82

373.84

373. $84 *$

373.90

113.94

$12 / 06 / 94$

46. 78

373.22

373.38

46.62

46.55

46.45

373. 45 *

373.55

113.95

113.95

113.96

199-H4-9

$$
\begin{aligned}
& 10 / 17 / 94 \\
& 11 / 07 / 94 \\
& 11 / 15 / 94 \\
& 12 / 06 / 94
\end{aligned}
$$

45. 36

44.97

46.81

44.78

373.22

373.61

$371.77^{*}+$

373.80

113.76

113.81

113.83

113.86

113.76

113.88

113.32

113.93

Wetls Mon1toring Upper Leveis in the Ringold Formation

$\begin{array}{lllll}199-H 3-2 C & 10 / 17 / 94 & 43.62 & 374.60 & 114.18 \\ & 11 / 07 / 94 & 43.73 & 374.49 & 114.14 \\ & 12 / 06 / 94 & 43.70 & 374.52 & 114.15 \\ 199-H 4-12 \mathrm{C} & & & & \\ & 10 / 17 / 94 & 41.63 & 371.89 & 113.35 \\ & 11 / 07 / 94 & 40.84 & 372.68 & 113.59 \\ & 12 / 05 / 94 & 40.55 & 372.97 * & 113.68 \\ & 12 / 06 / 94 & 40.21 & 373.31 & 113.78 \\ 199-H 4-15 C S & 10 / 17 / 94 & 35.39 & 372.05 & 113.40 \\ & 11 / 07 / 94 & 34.49 & 372.95 & 113.68 \\ & 12 / 06 / 94 & 34.73 & 372.71 & 113.60\end{array}$

Well Monitoring Mid-Level in the Ringold Formation

199-H4-15CR

$$
\begin{aligned}
& 10 / 17 / 94 \\
& 11 / 07 / 94 \\
& 12 / 06 / 94
\end{aligned}
$$

33.40

33.46

34.23

373.97

373.91

373.14

113.99

I13.97

113.73 
Table 3-2. RCRA Water Level Heasurement Report 183-H Solar Evaporation Basins, Fourth Quarter 1994. (sheet 4 of 4 )

NOTES: 1. Nater level elevations are calculated by subtracting the measured depth-to-water fron the surveyed elevation for the well.

2. Depth-to-water values are transcribed fron field records.

3. Elevations marked with an $" *$ " were measured at the time of sampling.

4. Elevations marked with a ' + ' are outside of the expected range, and are suspected of error. 
Table 3-3. Constituent List and Stmmary of Results for 183-H Solar Evaporation Basins Data for Reporting Period

October 1 through Decerber 31, 1994.

(sheet 1 of 2 )

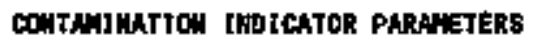

\begin{tabular}{|c|c|c|c|c|c|c|c|c|}
\hline & & Constituent Hune & & Lab & & Huat & of $s$ & Sample \\
\hline stion & (Hethod) & Eval! & Unito & $\mathrm{NOL}$ & Agency & Total & Fot & 3015 \\
\hline $\begin{array}{l}\text { conputet } \\
\text { conpuret } \\
\text { iot } \\
\operatorname{tox} \\
\text { PH }\end{array}$ & $\begin{array}{l}94 \\
73\end{array}$ & $\begin{array}{l}\text { Conductivfty, fiold } \\
\text { Conductivity, th } \\
\text { Totel Orgenic Corbon } \\
\text { Total organic Haiogen } \\
\text { pH, field }\end{array}$ & $\begin{array}{l}\text { whos } \\
\text { wihos } \\
\text { pho } \\
\text { ph }\end{array}$ & $\begin{array}{l}1 \\
110 \\
5 \\
.01 \\
6.5-8.5\end{array}$ & EPAS & $\begin{array}{l}19 \\
15 \\
15 \\
15 \\
19\end{array}$ & $\begin{array}{l}19 \\
15 \\
15 \\
13 \\
19\end{array}$ & 1 \\
\hline
\end{tabular}

DRIAKLIG WTER PARANETERS

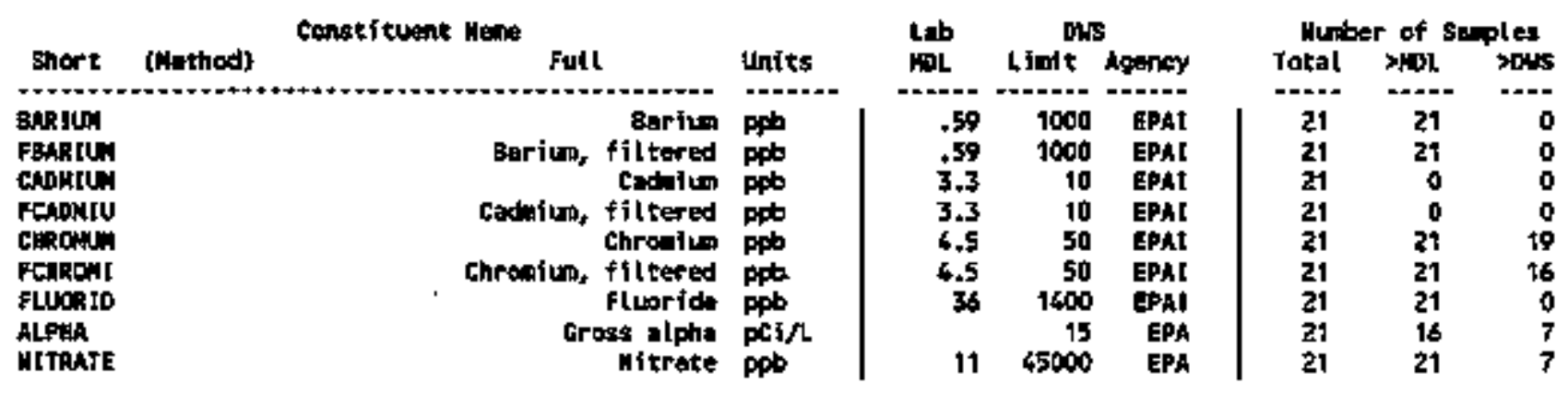

GOJUDUATER CUALJTY PARNETERS

\begin{tabular}{|c|c|c|c|c|c|c|c|c|c|}
\hline Short & (Hethod) & constituent Míline Full & Inits & Lab & Limt to & Sigency & $\begin{array}{c}\text { Hunb } \\
\text { Tatal }\end{array}$ & ir of & $\underset{>0245}{\operatorname{maplos}}$ \\
\hline 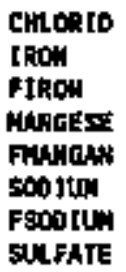 & & 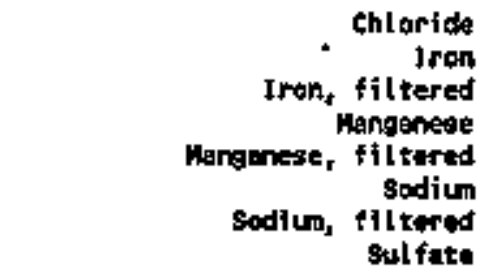 & 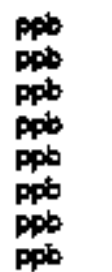 & $\begin{array}{r}14 \\
5.24 \\
3.24 \\
.72 \\
.72 \\
61 \\
61 \\
37\end{array}$ & $\begin{array}{r}250000 \\
300 \\
300 \\
50 \\
50\end{array}$ & $\begin{array}{l}\text { EFAS } \\
\text { EPAS } \\
\text { EPAS } \\
\text { EFAS } \\
\text { EPAS } \\
\text { EPAS }\end{array}$ & $\begin{array}{l}21 \\
21 \\
21 \\
21 \\
21 \\
21 \\
21 \\
21\end{array}$ & $\begin{array}{l}21 \\
21 \\
19 \\
19 \\
14 \\
21 \\
21 \\
21\end{array}$ & $\begin{array}{r}0 \\
11 \\
0 \\
3 \\
0\end{array}$ \\
\hline
\end{tabular}


Table 3-3. Constituent List and Summary of Results for 183-H 507ar Evaporation Basins Data for Reporting Period

October I through December 31, 1994.

(sheet 2 of 2)

SITE SPECIFJC ND OTHER CONSTITUETS

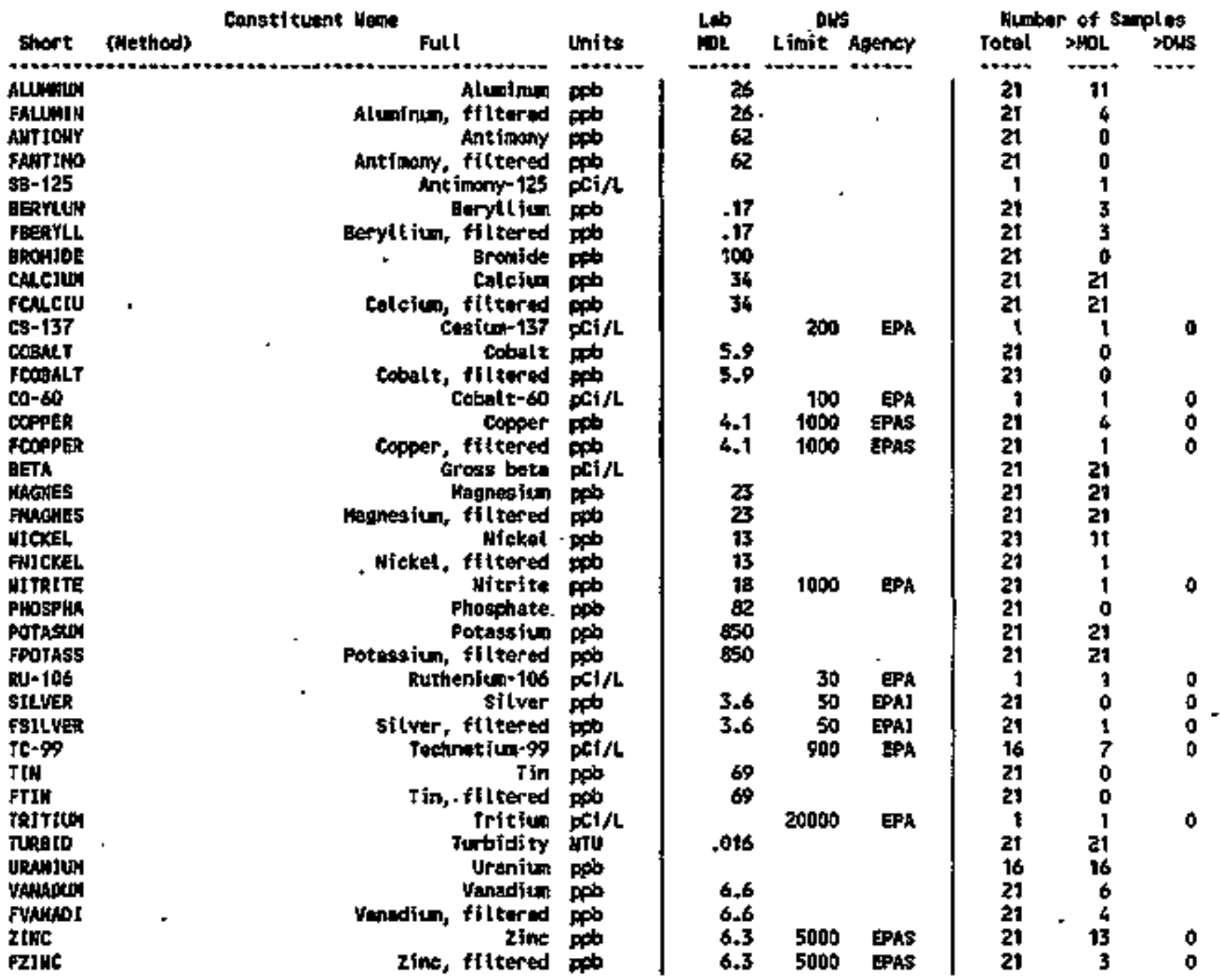

Far axplanation af this table, see section" 1,4 of repart. 
Table 3-4. Constituents with at Least One Detected Value for the 183-H Solar Evaporation Basins Data for Reporting Period October 1 through December 31, 1994.

(sheet 1 of 10)

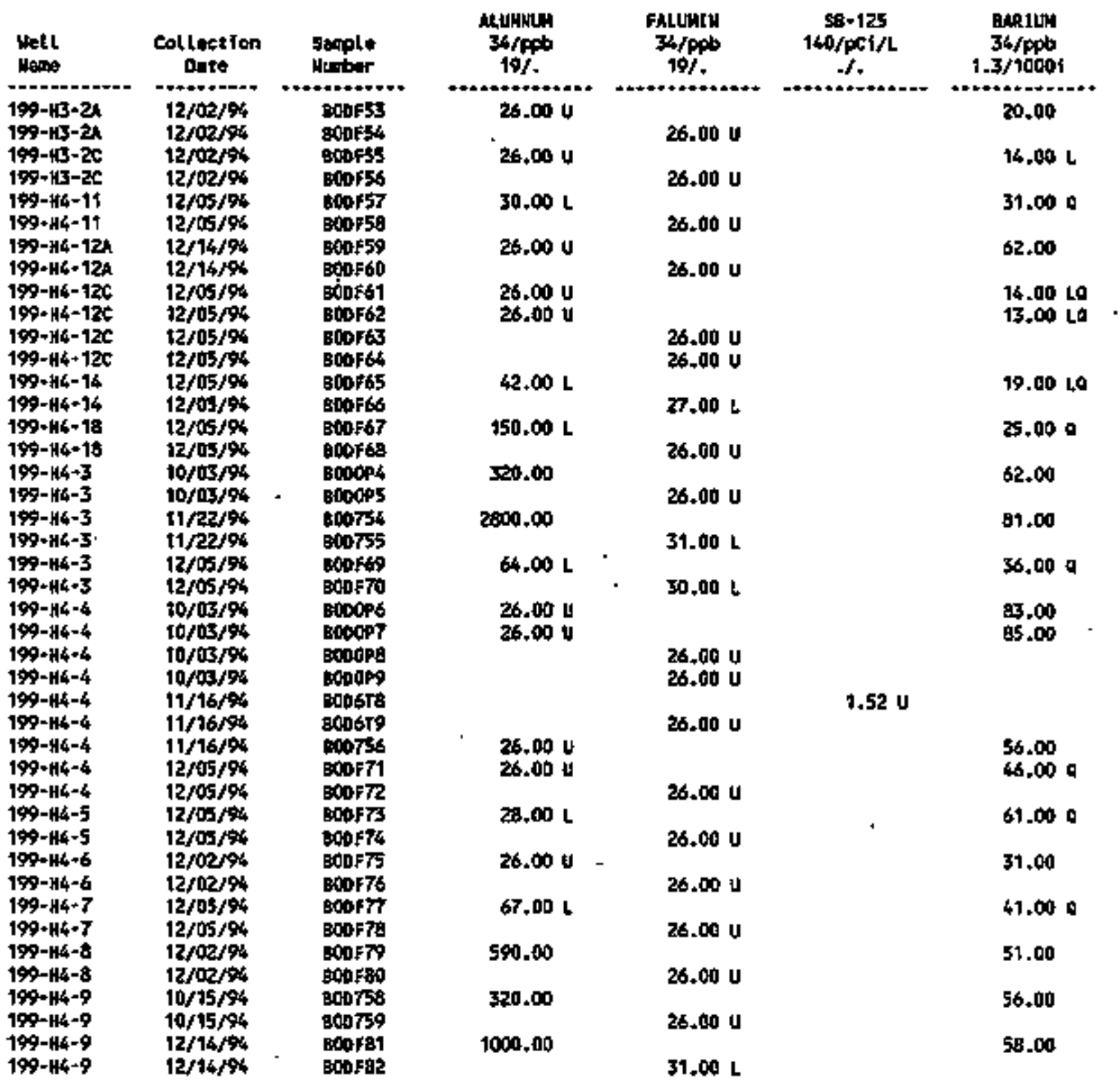


Table 3-4. Constituents with at Least One Detected Value for the 183-H Solar Evaporation Basins Data for Reporting Period October 1 through Decenaber 31, 1994.

(sheet 2 of 10 )

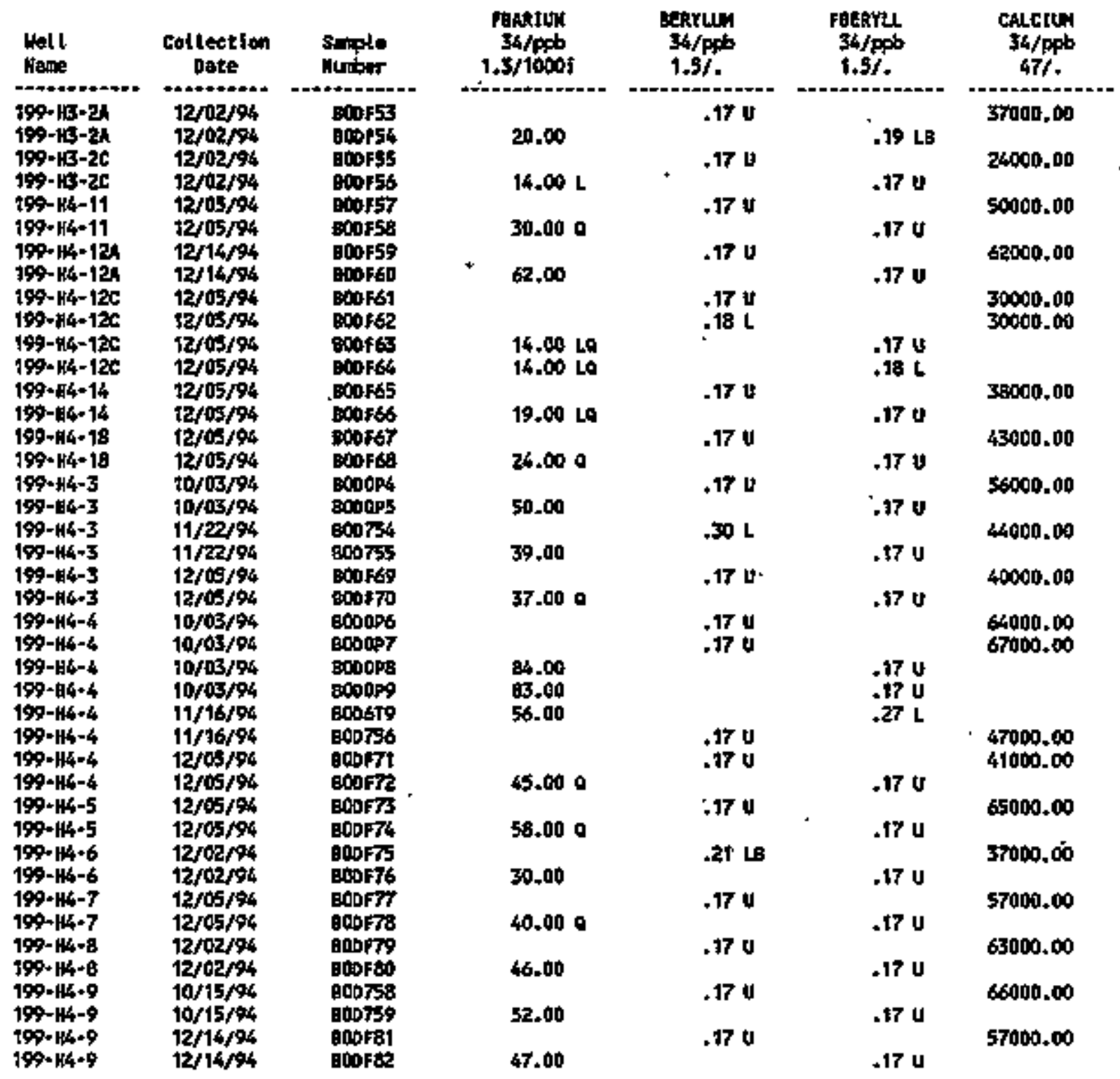


Table 3-4. Constituents with at Least One Detected Value for the 183-H Solar Evaporation Basins Data for Reporting Period

Dctober 1 through December 31, 1994.

(sheet 3 of 10)

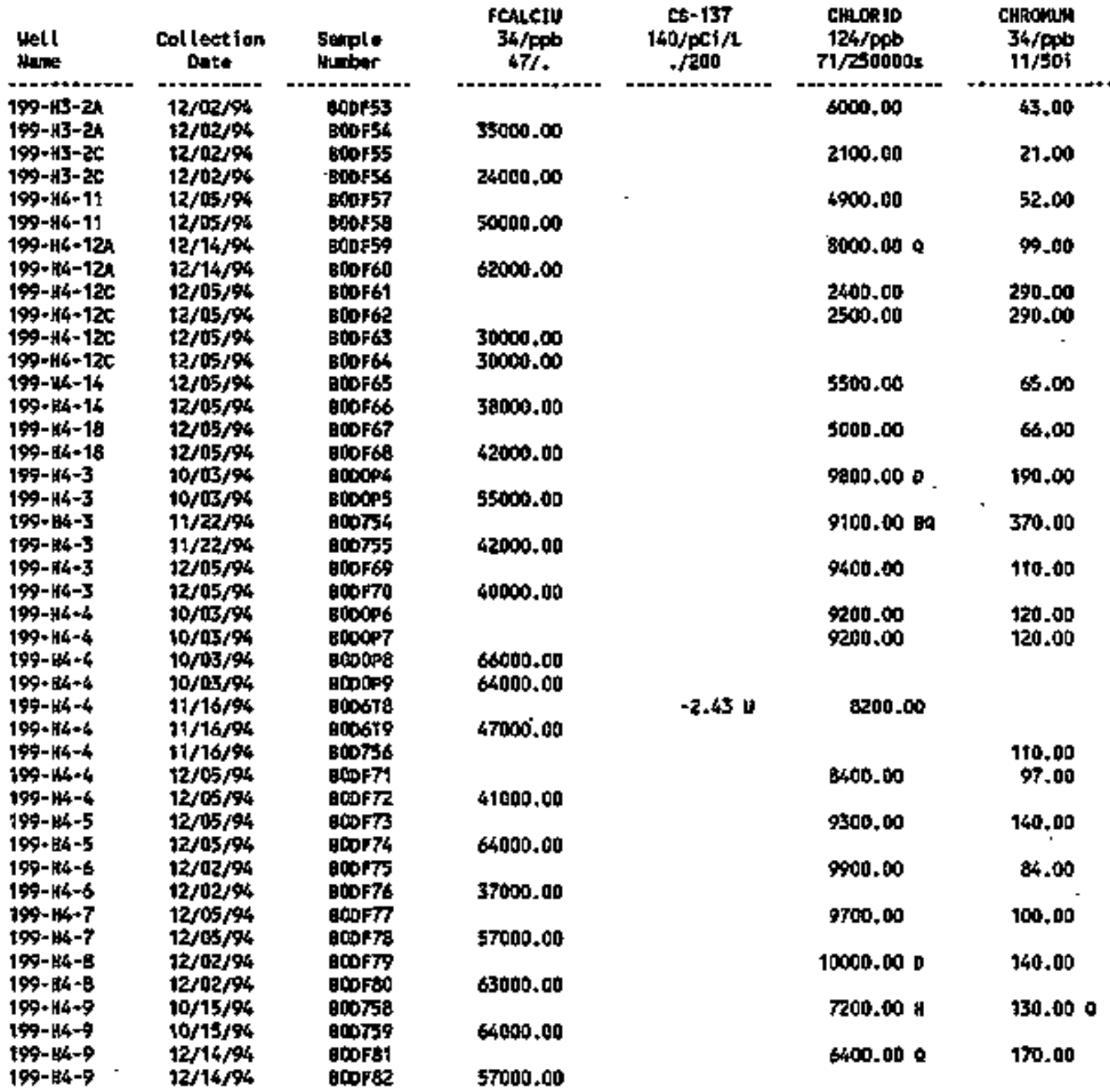


Tabie 3-4. Constituents with at Least One Detected Vajue for the 183-H Solar Evaporation Basins Data for Reporting Period

October 1 through December 31, 1994. (sheet 4 of 10)

\begin{tabular}{|c|c|c|c|c|c|c|}
\hline Well & $\begin{array}{l}\text { Collection } \\
\text { Oute }\end{array}$ & $\begin{array}{l}\text { sumple } \\
\text { Munber }\end{array}$ & $\begin{array}{l}\text { FCHRonl } \\
34 / \mathrm{kpb} \\
11 / 501\end{array}$ & $\begin{array}{c}c 0-60 \\
140 / \mathrm{pcl} / \mathrm{C} \\
=/ 100\end{array}$ & $\begin{array}{c}\text { COPPER } \\
34 / 0 \% b \\
2, \Delta / 10008\end{array}$ & $\begin{array}{c}\text { FCOPPER } \\
34 / \mathrm{POO} \\
2,6 / 1000 \mathrm{~S}\end{array}$ \\
\hline 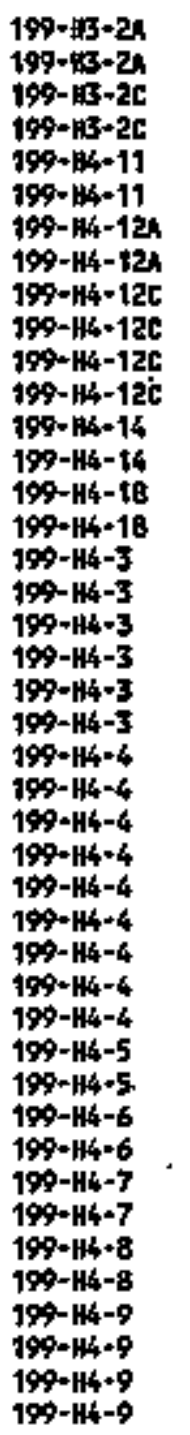 & $\begin{array}{l}12 / 02 / 94 \\
12 / 02 / 94 \\
12 / 02 / 94 \\
12 / 02 / 44 \\
12 / 05 / 94 \\
12 / 05 / 94 \\
12 / 14 / 94 \\
12 / 14 / 94 \\
12 / 05 / 94 \\
12 / 05 / 94 \\
12 / 05 / 94 \\
12 / 05 / 94 \\
12 / 05 / 94 \\
12 / 05 / 94 \\
12 / 05 / 94 \\
12 / 05 / 94 \\
10 / 03 / 94 \\
10 / 03 / 94 \\
11 / 22 / 94 \\
11 / 22 / 94 \\
12 / 05 / 94 \\
12 / 05 / 94 \\
10 / 03 / 94 \\
10 / 03 / 94 \\
10 / 03 / 94 \\
10 / 03 / 94 \\
11 / 15 / 94 \\
11 / 16 / 94 \\
11 / 16 / 94 \\
12 / 05 / 94 \\
12 / 05 / 94 \\
12 / 05 / 94 \\
12 / 05 / 94 \\
12 / 02 / 94 \\
12 / 02 / 94 \\
12 / 05 / 94 \\
12 / 05 / 94 \\
12 / 02 / 94 \\
12 / 02 / 94 \\
10 / 15 / 94 \\
10 / 15 / 94 \\
12 / 14 / 94 \\
12 / 16 / 94\end{array}$ & 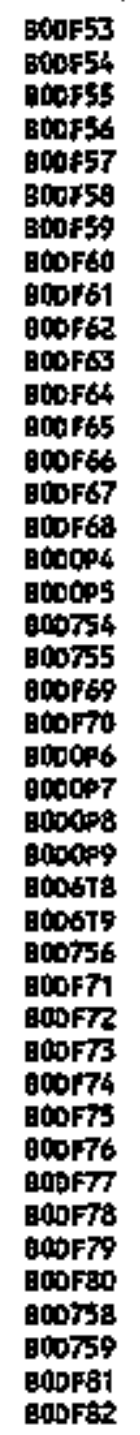 & $\begin{array}{l}28.00 \\
13.00 \mathrm{~L} \\
40.00 \\
96.00 \\
280.00 \\
280.00 \\
61.00 \\
36.00 \\
82.00 \\
77.00 \\
86.00 \\
110.00 \\
19.00 \\
96.00 \\
88.00 \\
83.00 \\
42.00 \\
90.00 \\
100.00 \\
56.00 \mathrm{a} \\
53.00\end{array}$ & U & $\begin{array}{l}4.10 \mathrm{U} \\
4.10 \mathrm{U} \\
4.10 \mathrm{U} \\
4.10 \mathrm{U} \\
4.90 \mathrm{U} \\
4.10 \mathrm{U} \\
6.90 \mathrm{~L} \\
5.80 \mathrm{~L}\end{array}$ & $\begin{array}{l}4.10 \mathrm{U} \\
4.10 \mathrm{U} \\
4.10 \mathrm{U} \\
4.10 \mathrm{U} \\
4.10 \mathrm{U} \\
4.10 \mathrm{U} \\
4.10 \mathrm{U} \\
4.10 \mathrm{U} \\
4.10 \mathrm{U} \\
4.30 \mathrm{U} \\
4.10 \mathrm{U} \\
4.10 \mathrm{U} \\
4.10 \mathrm{U} \\
4.10 \mathrm{U} \\
4.10 \mathrm{U} \\
4.10 \mathrm{U} \\
4.10 \mathrm{U} \\
4.10 \mathrm{U} \\
4.10 \mathrm{U} \\
4.10 \mathrm{U} \\
4.10 \mathrm{U}\end{array}$ \\
\hline
\end{tabular}


Table 3-4. Constituents wth at Least One Detected Value for the 183-H Solar Evaporation Basins Data for Reporting Period

October 1 through December 31, 1994.

(sheet 5 of 10 )

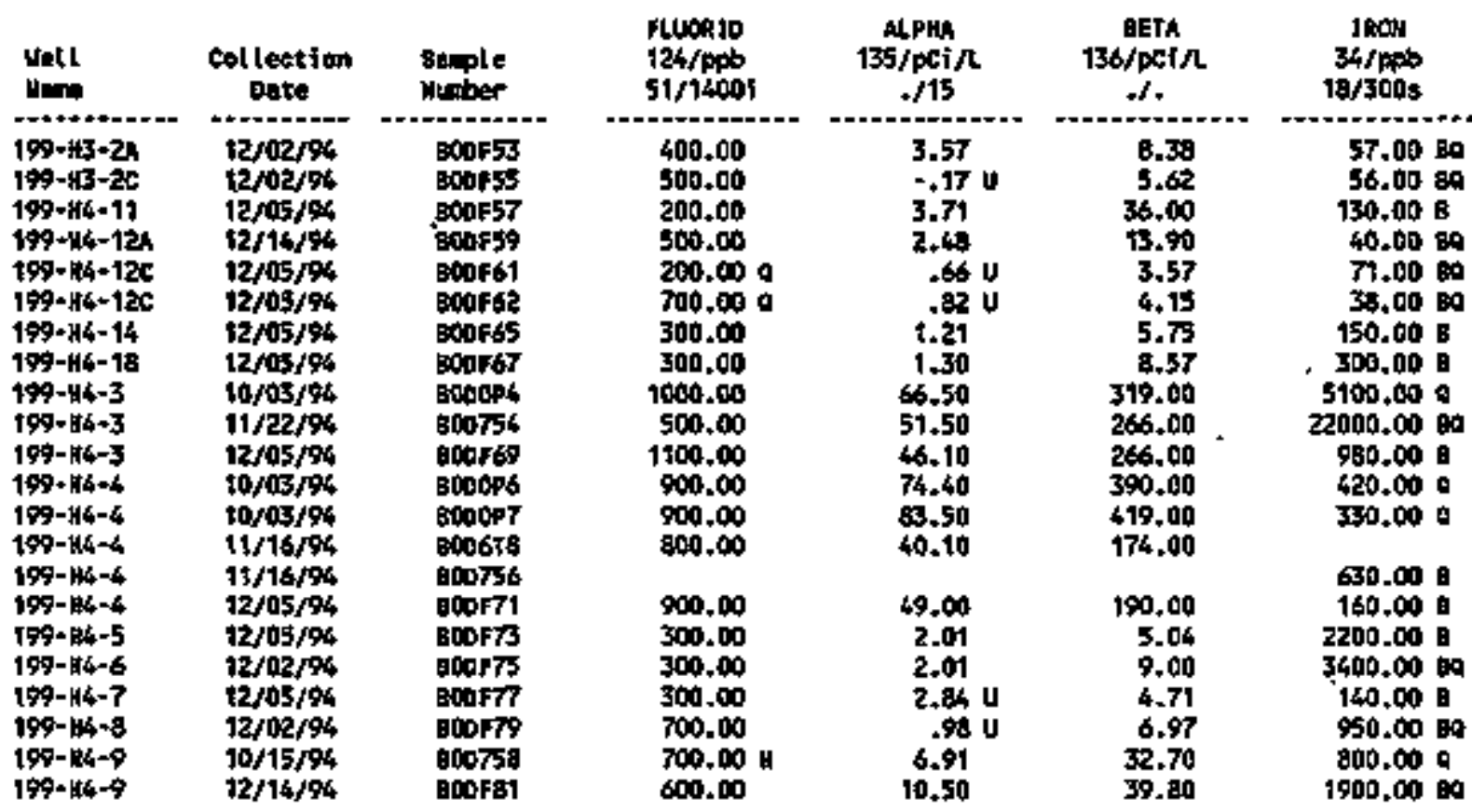

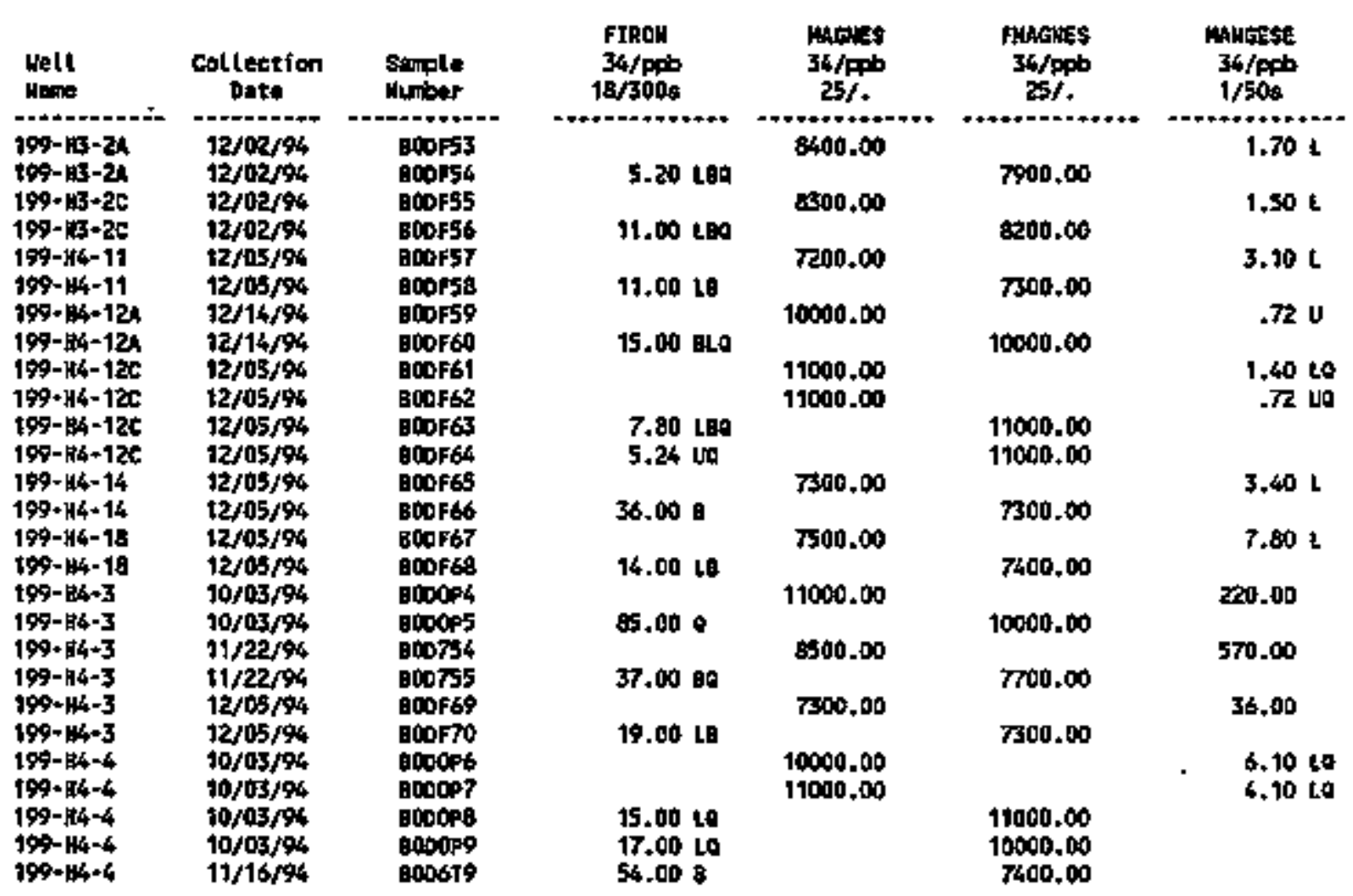


Table 3-4. Constituents w1th at Least One Detected Value for the 183-H Sotar Evaporation Basins Data for Reporting Period October 1 through Decenther 31, 1994.

(sheet 6 of 10)

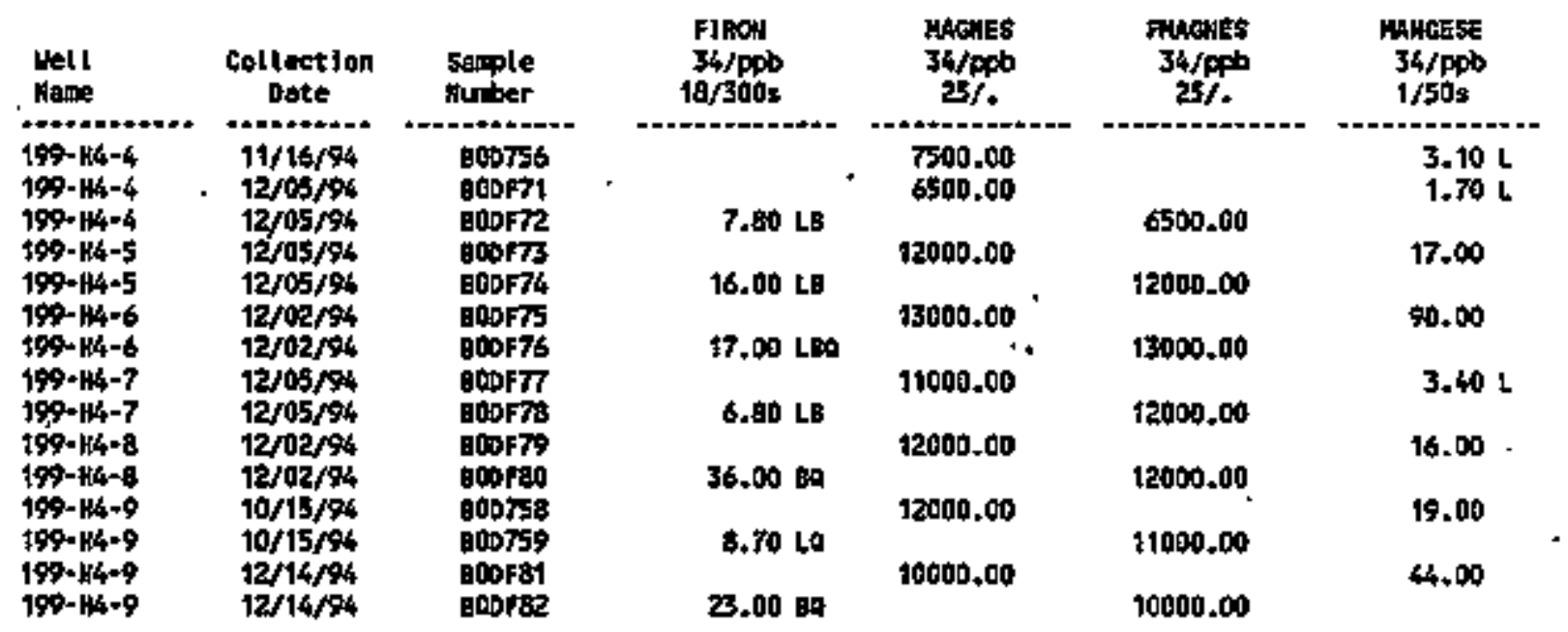

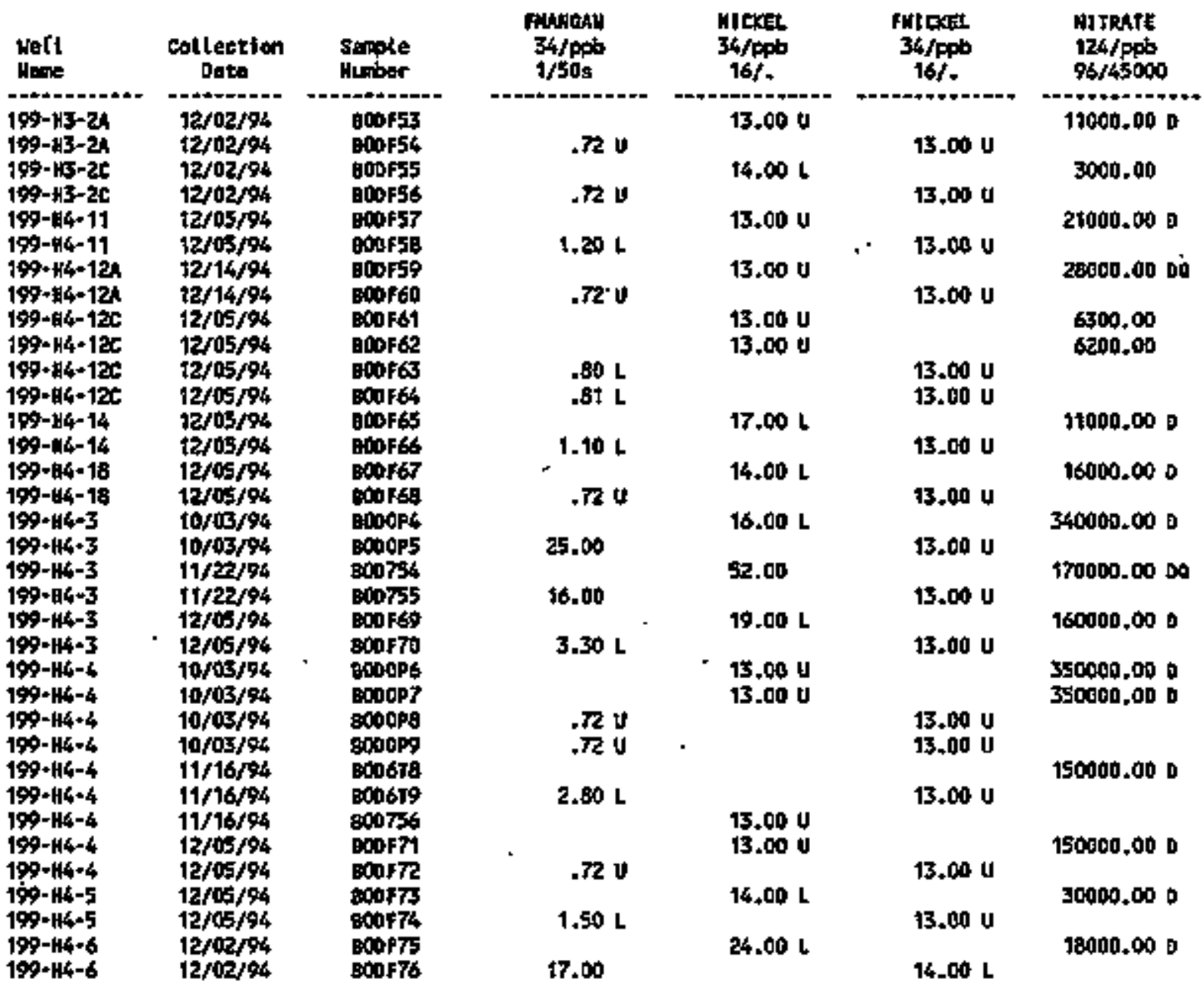


Table 3-4. tonstituents with at Least One Detected Value for the 183-H Solar Evaporation Basins Data for Reporting Period October 1 through December 31, 1994.

(sheet 7 of 10)

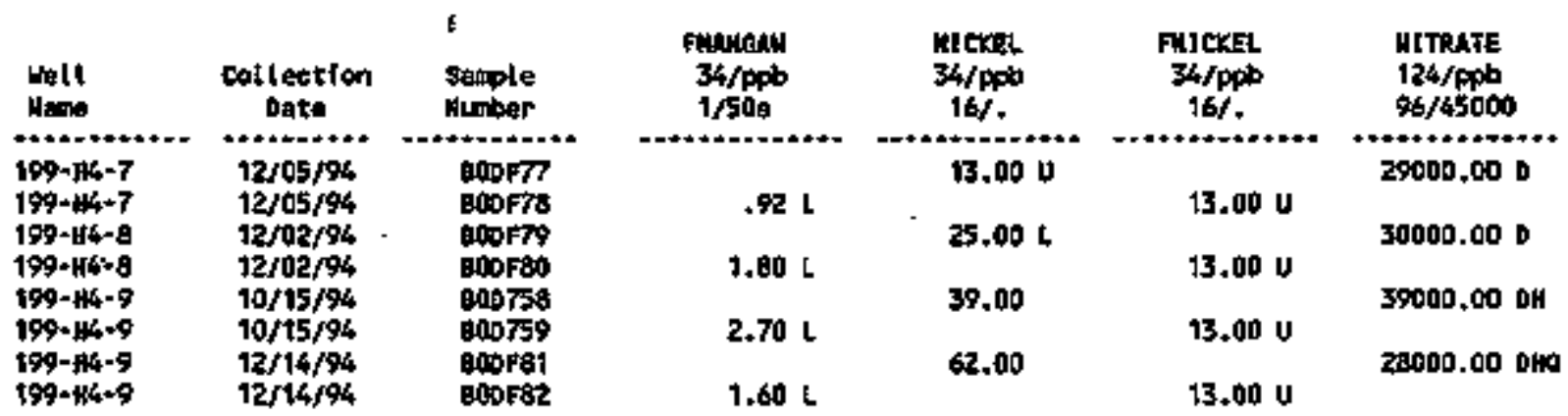

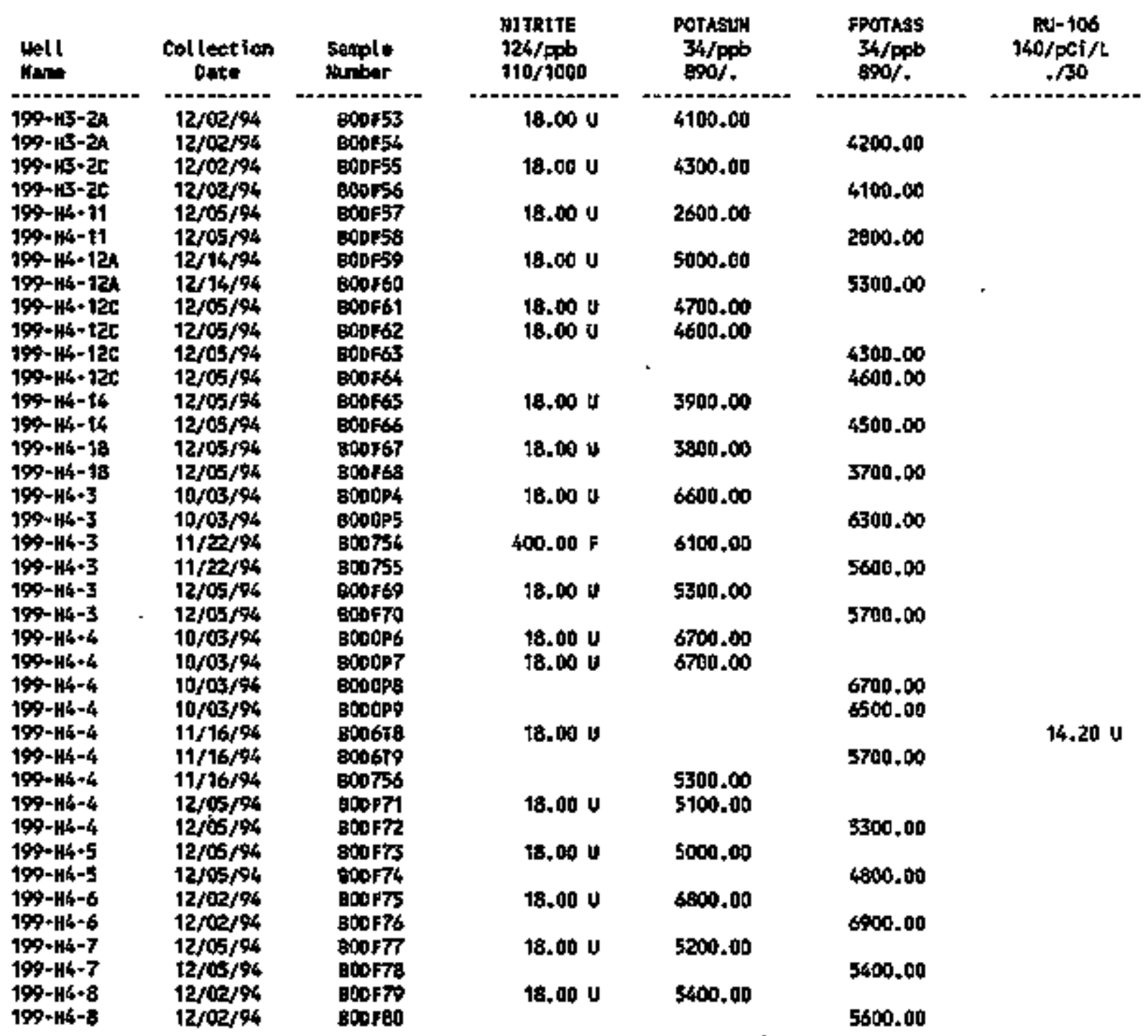


Table 3-4. Constituents with at Least One Detected Value for the 183-1 Solar Evaporation Basins Data for Reporting Period

October 1 through December 31, 1994.

(sheet 8 of 10 )

\begin{tabular}{|c|c|c|c|c|c|c|}
\hline Well & $\begin{array}{c}\text { Collection } \\
\text { Dnte }\end{array}$ & $\begin{array}{l}\text { Semple } \\
\text { Numbar }\end{array}$ & $\begin{array}{l}\text { MLTRIiE } \\
124 / \mathrm{ppb} \\
110 / 100 \mathrm{D}\end{array}$ & $\begin{array}{l}\text { POTASUA. } \\
34 / \mathrm{pph} \\
8 \% 0 .\end{array}$ & $\begin{array}{l}\text { FPOTASS } \\
34 / p p b \\
\text { B90/. }\end{array}$ & $\begin{array}{c}\mathrm{RU}+10 \mathrm{DS} \\
140 / \mathrm{pCD} / \mathrm{L} \\
-/ 30\end{array}$ \\
\hline $\begin{array}{l}199-144-9 \\
199-44-9 \\
199-144-9 \\
199-14-9\end{array}$ & $\begin{array}{l}10 / 15 / 94 \\
10 / 15 / 94 \\
12 / 14 / 94 \\
12 / 14 / 94\end{array}$ & $\begin{array}{l}\text { 800758 } \\
\text { 800759 } \\
000 f 81 \\
\text { 80078d }\end{array}$ & 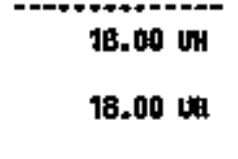 & 5200.00 & $\begin{array}{r}5100.00 \\
5200.00\end{array}$ & . \\
\hline
\end{tabular}

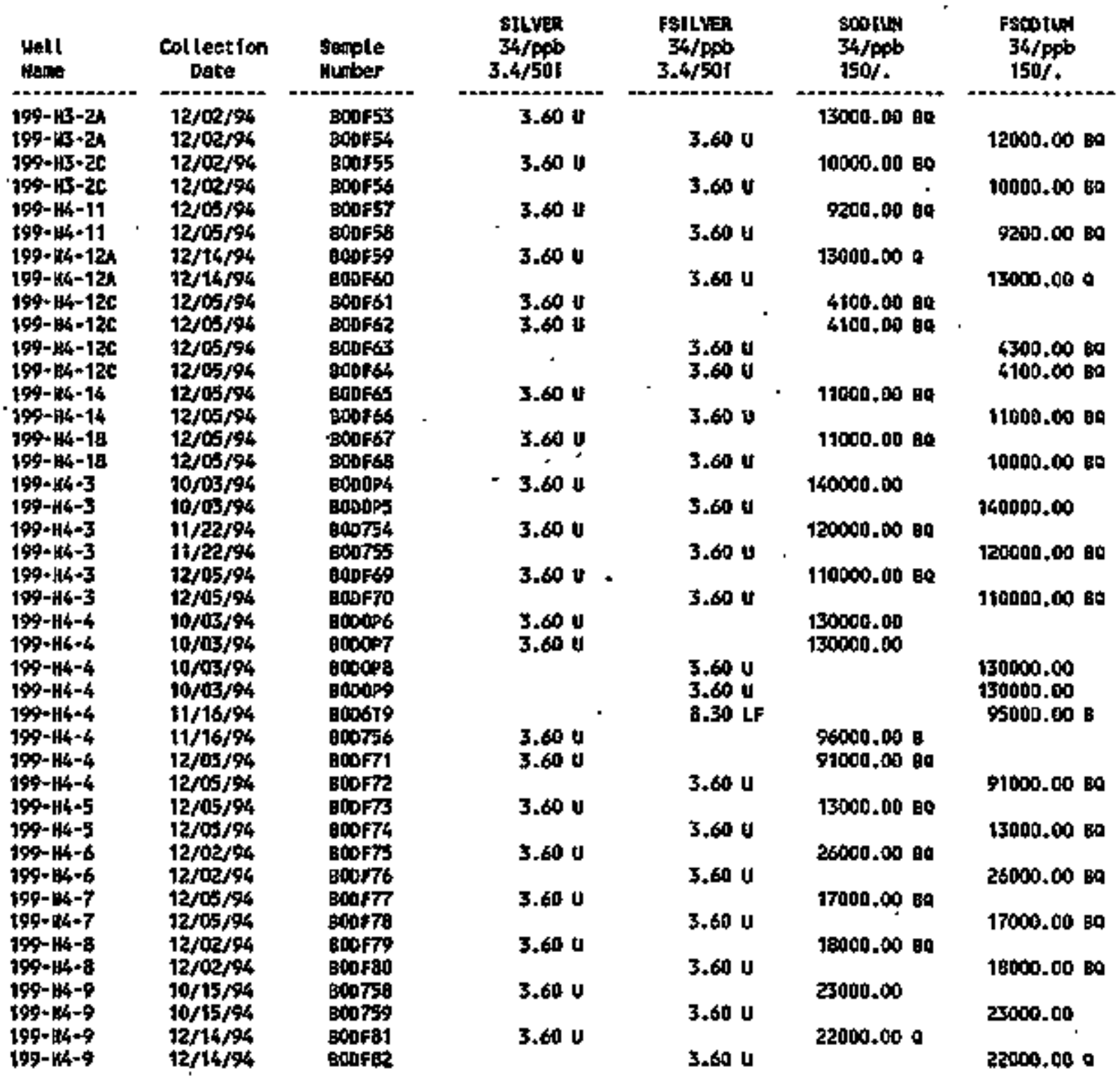


Table 3-4. Constituents with at Least One Detected Value for the 183-H Solar Evaporation Basins Data for Reporting Perlod October 1 through December 31, 1994.

(sheet 9 of 10 )

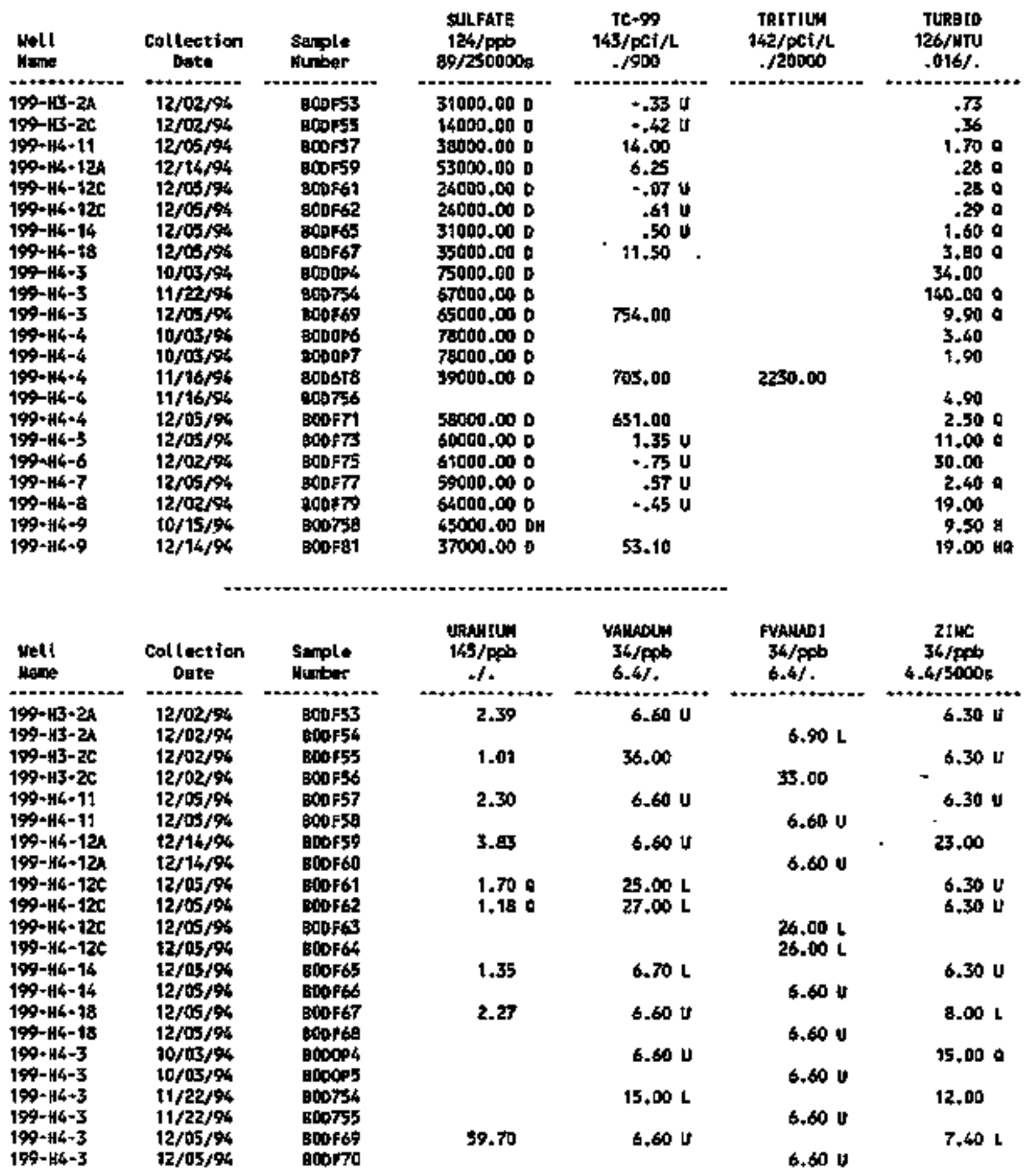


Tabie 3-4. Constituents with at Least One Detected Value for the 183-H Solar Evaporation Basins Data for Reporting Period

October I through Decerther 31, 1994. (sheet 10 of 10 )

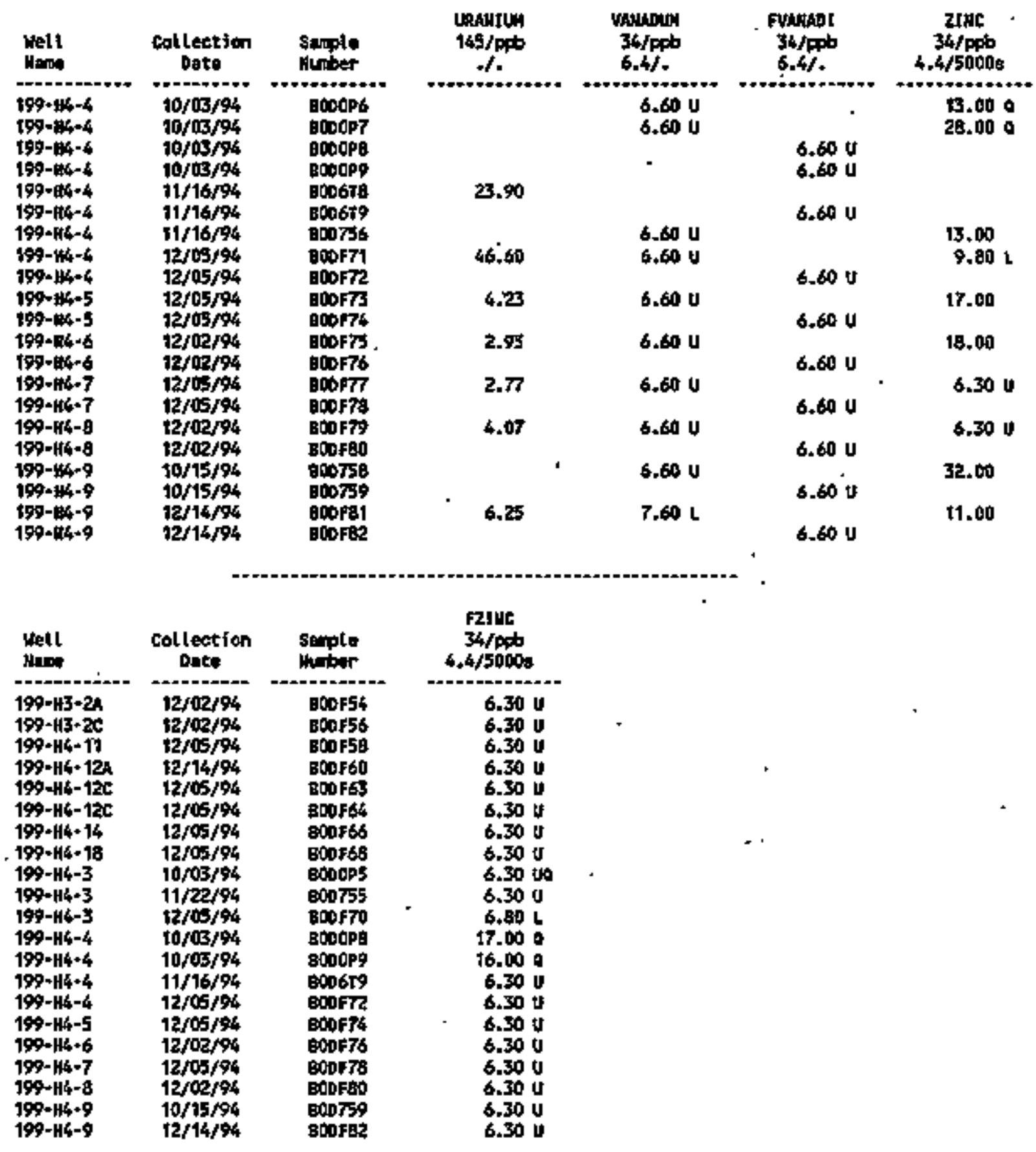

For explandion of thio toble, see Section 1.4 of report. 
Tabie 3-5. Contamination Indicator Parameters for the 183-H Solar Evaporation Basins Data for Reporting Period Octaber 1 through December 31, 1994.

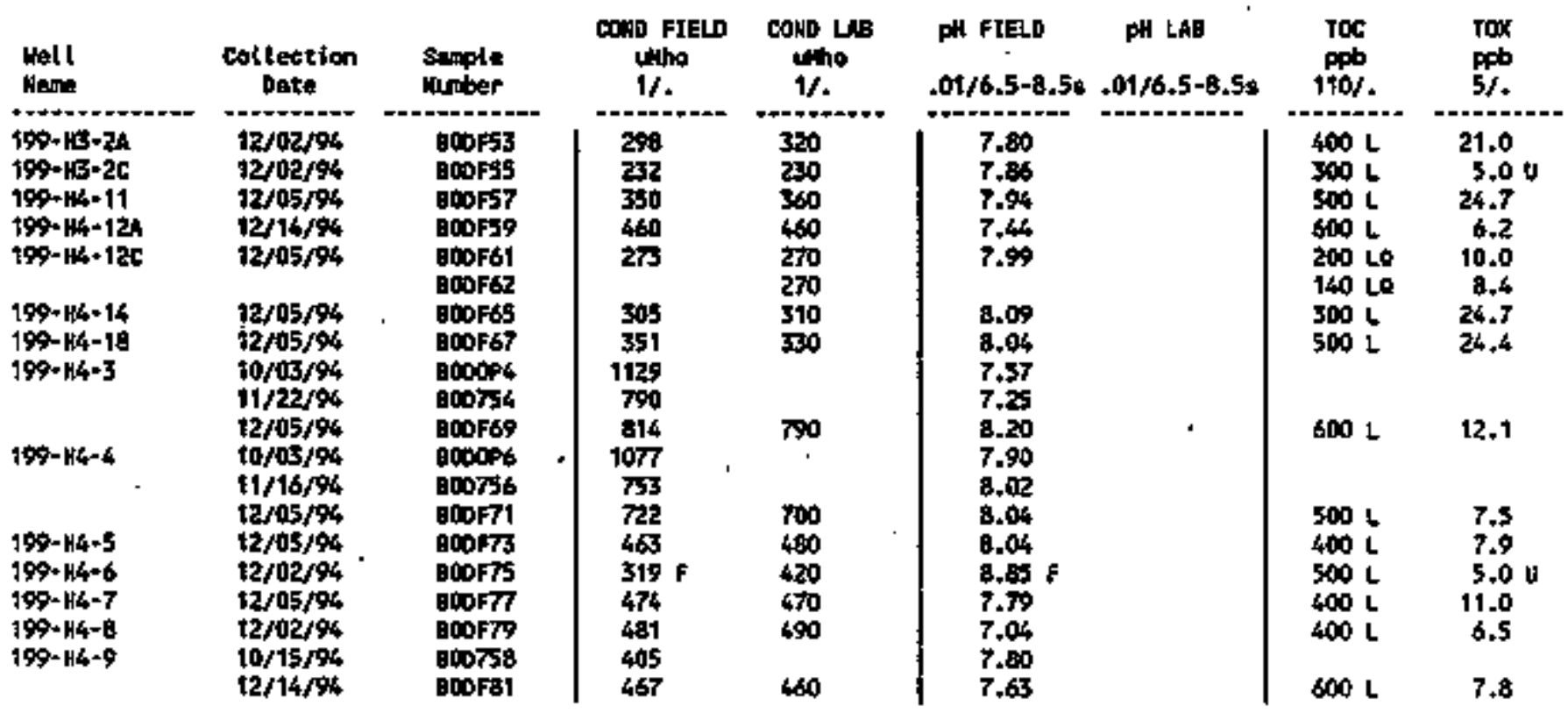


Table 3-6. Orinking Water Standards Exceeded for the 183-H lasins, Fourth Quarter 1994.

\begin{tabular}{|c|c|}
\hline Constituent (OWS) & $\begin{array}{l}\text { Wells exceeding Dus } \\
\text { (number of samples) }\end{array}$ \\
\hline Field conductivity $(700 \mu \mathrm{mho} / \mathrm{cm})$ & $\mathrm{H} 4-3(3) ; \mathrm{H} 4-4$ (3) \\
\hline Lab conductivity $(700 \mu \mathrm{mho} / \mathrm{cmt})$ & $\mathrm{H} 4-3(1)$ \\
\hline Fietd pH $(6.5-8.5)$ & $44-6^{\circ}$ \\
\hline Chromium, unfiltered samples (50 ppb) & A11 wells except $\mathrm{H} 3-2 \mathrm{~A}$ and $\mathrm{H} 3-2 \mathrm{C}$ \\
\hline Chromium, filtered samples (50 ppb) & $\begin{array}{l}\text { A11 wells except } \mathrm{H3}-2 \mathrm{~A}, \mathrm{H3}-2 \mathrm{C}, \mathrm{H} 4- \\
11 \text {, } \mathrm{H4}-18 \text {, and } \mathrm{H} 4-6 \text {. }\end{array}$ \\
\hline Gross alpha (15 pC1/L) & $\mathrm{H} 4-3(3), \mathrm{H} 4-4(3)$ \\
\hline Iron, unfiltered samples (300 $\mathrm{ppb})$ & $\begin{array}{l}\mathrm{H} 4-3 \text { (3), } \mathrm{H} 4-4(3), \mathrm{H} 4-5, \mathrm{H} 4-6, \\
\mathrm{H} 4-8, \mathrm{H}-\mathrm{H}-9 \text { (2) }\end{array}$ \\
\hline Iron, filtered sample (300 ppb) & none. \\
\hline Manganese, unfiltered samples ( $50 \mathrm{ppb}$ ) & $H 4-3(2), H 4-6$ \\
\hline Manganese, filtered sample (50 ppb) & none. \\
\hline Nitrate $(45,000 \mathrm{pDb})$ & $\mathrm{H} 4-3$ (3), H4-4 (3) \\
\hline
\end{tabular}

Oata are in question. Requests for Analytical Data Evaluation were submitted.

OWS - drinking water standard. 
Table 3-7. Requests for Analytical Data Evaluation Submitted for the 183-H Solar Evaporation Basins, Fourth Quarter 1994.

\begin{tabular}{|c|c|l|l|}
\hline Wel1 no. & $\begin{array}{c}\text { Sample } \\
\text { date }\end{array}$ & \multicolumn{1}{|c|}{ Constituent } & \multicolumn{1}{|c|}{ Reason } \\
\hline H4-3 & $11 / 22 / 94$ & Nitrite & Higher than historical trend. \\
\hline H4-6 & $12 / 2 / 94$ & Conductivity (field) & Lower than historical trend. \\
\hline H4-6 & $12 / 2 / 94$ & pH (field) & Higher than historical trend. \\
\hline H4-4 & $11 / 16 / 94$ & Silver (filtered) & $\begin{array}{l}\text { First detection in this well; } \\
\text { higher than unfiltered result. }\end{array}$ \\
\hline
\end{tabular}




\section{CONTENTS}

$4.0 \quad 100-H$ RCRA SITES .................... 4 4-1

4.1 IMTRODUCTIOH $\ldots \ldots \ldots . \ldots . \ldots . \ldots . \ldots . \ldots$

4.2 MATER LEYEL MEASUREMENTS $\ldots \ldots \ldots$. . . . . . .

4.3 WATER CHEHISTRY DATA $\ldots \ldots \ldots \ldots \ldots \ldots$ 


\section{DOE/RL-94-36-4.}

\section{LIST OF FIGURES}

4-1 Well Location Map for the 1301-N, 1324-N/MA, and $1325-N$ Facilities ................. $4-2$

\section{LIST OF TABLES}

4-1 Monftaring Well Purpose and 5ampling Schedule for the 100-N Area . 4-3

4-2 RCRA Water Level Meknontentwa Refmutth Quarter 1994 ... . . . . 4-4

4-3 Constituent List and Summary of Results for 100-N RCRA Sites

Data for Reporting Perlod October 1 through December 31, 1994 . . 4-9

4-4 Constituents with at Least One Detected Value

for the 100-N RCRA Sites Data for Reporting Period

October 1 through December $31,1994 \ldots$ 4-12

4-5 Contamination Indicator Parameters for the 100- $\mathrm{A}$ RCRA Sites

Data for Reporting Period October 1 through December 31, 1994 . . 4-21

4-6 Drinking Water Standards Exceeded for 100-N Area RCRA Sites ... 4-22

4-7 Requests for Analytical Data Evaluation

for the 100-N RCRA Sites, Fourth Quarter $1994 \ldots \ldots$. . . . 4-23 
DOE/RL-94-36-4

\title{
4.0 IOO-H RCRA SITES
}

\author{
H. J. Hartman \\ Hestinghouse. Hanford Company
}

\subsection{IMTROOUCTIOH}

Four interim-5tatus RCRA disposal units are located in the 100-N Area of the Hanford Site: (1) the 1301-N Liquid Waste Disposal Facility (LWDF), (2) the 1324-N Surface Impoundment, (3) the 1324-NA Percolation Pond, and (4) the 1325-N LWDF (F tgure 4-1). The 1324-N and 1324-NA units are mont tored as a single stte. RCRA monitoring began in Tate 1987. The 1324-N/NA site is monitored under a grounduater quality assessment program because of elevated levels of TOX (40 CFR 265.93). The 1325-N LWOF is monitored under an indicator parameter eva]uation program (40 CFR 265.92). Background concentrations are bejng reestab] ished for the 1301-N LWDF.

The 1301-N and 1325-N LWDFs were used for disposal of Tiquid effluent from the $N$ Reactor and associated facilities. Primary waste constituents were radionuclides, including strontium-90, tritfum, and coba7t-60. Minor amounts of hazardous waste aiso were present in the waste streams (e.g., hydrazine). The 1324-N/NA units recelved corrosive waste. The 100-N RCRA units are currently, not in use.

HeIl locations in the 100-N Area are ilfustrated in Figure 4-1. Many of these wells are monitored monthly for water levels. Fewer wells are used for. groundrater sampling. Sampling networks are listed in Table 4-1.

\subsection{MATER LEVEL. MEASUREMEKTS}

Water levels are measured monthly to most 100-N Area wel]s to track changes caused by river-stage fluctuations. Oata are listed in Table 4-2.

\subsection{WATER CHEHISTRY DATA}

All the wells in the 1324-N/NA network were scheduled for sampling this quarter, but well $\mathrm{B}-59$ had insufficient water. Only the upgradient wel1s for the 1301-N LWOF were scheduled for sampling. Hel1 M-34 was sampled, but well H-57 had a broken pump. Well N-74, upgradient of the 1325-N LWOF, was sampled only for field conductivity, field $\mathrm{pH}$, and $\mathrm{TOX}$, as planned. Mell $\mathrm{N}-43$, downgradient of the 1325-N LWDF, was sampled on october 31,1994 , because it had been rescheduled the previous quarter.

Groundwater chemistry data from samples collected during the fourth quarter of 1994 are listed in Tables 4-3 through 4-5. These tables also include data from earlier quarters that were not reported previously. Data qualifiers and data flags are discussed in Sections 1.2 and 1.4, respectlvely. ONSs were exceeded for the constituents 7isted in Table 4-6. Several analytical resuits appear anomalous and RABEs were submitted (Table 4-7). 


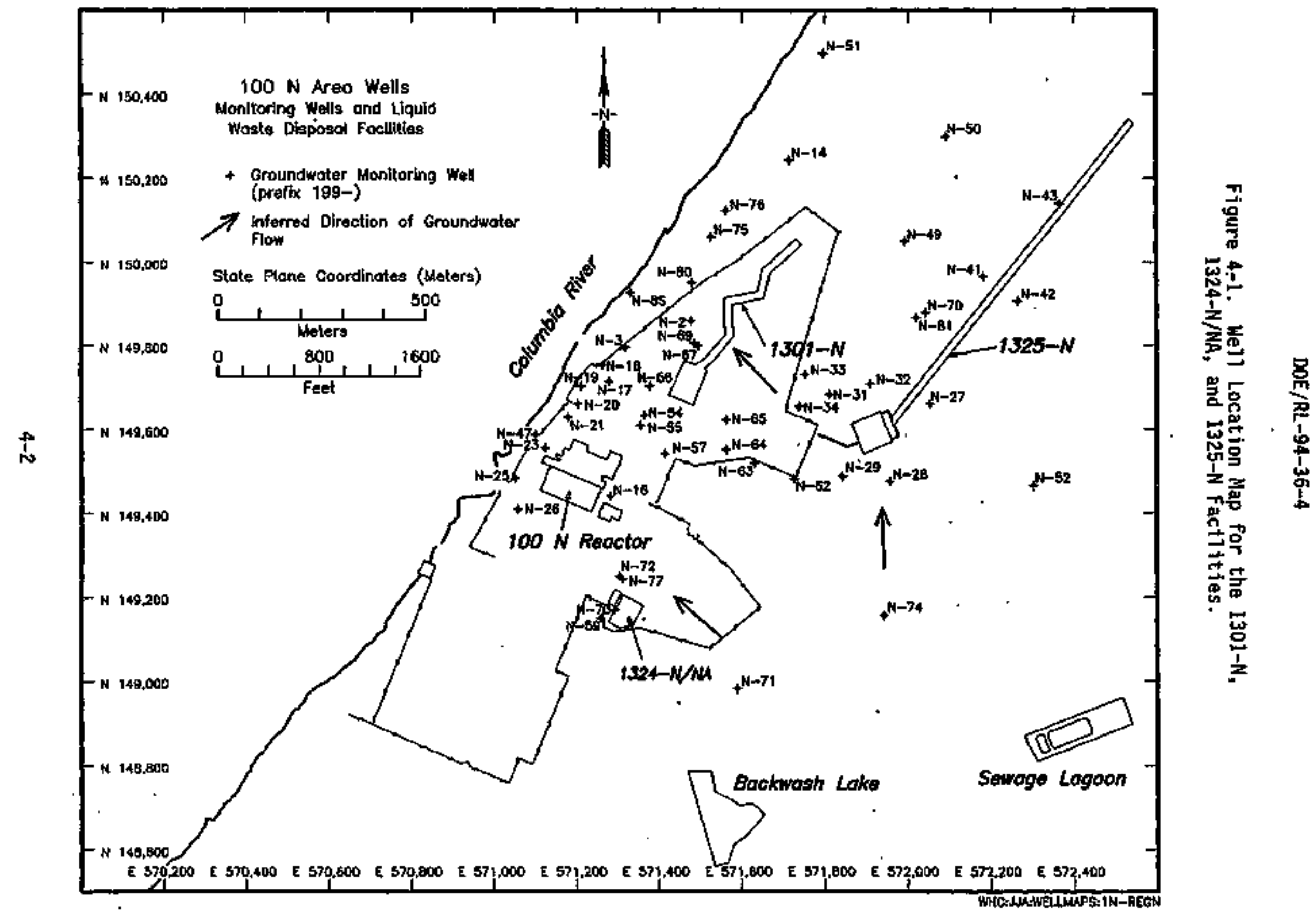


Table 4-1. Monitoring We]l Purpose and

Sampling Schedule for the 100-N Area.

\begin{tabular}{|c|c|c|c|c|}
\hline $\begin{array}{l}\text { Well no. } \\
\text { (199-) }\end{array}$ & RCRA site & Relative position & $\begin{array}{c}\text { Sample } \\
\text { frequency }\end{array}$ & $\begin{array}{l}\text { Sample date, } \\
\text { 4th Qtr } 1994\end{array}$ \\
\hline $\mathrm{N}-2$ & $1301-N$ & Downgradient & Quarteriy & Not scheduled \\
\hline $\mathrm{N}-3$ & $1301-N$ & Downgradient & Quarter'yy & Not scheduled \\
\hline H-14 & $1301-\mathrm{N}$ & Downgradient & Quarterly & Not scheduled \\
\hline$H-27$ & $1325-\mathrm{N}$ & Downgradient & Serliainnua Jly & Not scheduled \\
\hline $\mathrm{N}-29$ & $1325-N$ & Downgradient & Semi annually & Not scheduled \\
\hline $\mathrm{H}-32$ & $1325-N$ & Downgradient & Seriannually & Not scheduled \\
\hline$N-34$ & $1301-N$ & Upgṛadient & Quarterly & $11 / 4 / 94$ \\
\hline$N-41$ & $1325-\mathrm{N}$ & Downgradient & Seniannually & Not scheduled \\
\hline$N-43$ & $1325-N$ & Downgradient & Semfannua $7 y$ & $10 / 31 / 94^{a}$ \\
\hline $\mathrm{N}-57$ & $1301-N$ & Upgradient & Quarter]y & Not sampled ${ }^{b}$ \\
\hline$N-59$ & $1324-N / N A$ & Downgradient & Quarter7y & Not sampled \\
\hline$N-67$ & $1301-\mathrm{N}$ & Downgradient & Quarteriy & Not scheduled \\
\hline $\mathrm{H}-70$ & $1325-N$ & $\begin{array}{l}\text { Downgradient; } \\
\text { bottom of aquifer }\end{array}$ & Quarterly & Not scheduled \\
\hline$N-71$ & $1324-N / N A$ & Upgradient & quarterly & $11 / 4 / 94$ \\
\hline$N-72$ & 1324-N/NA & Downgradient & Quarterly & $11 / 7 / 94$ \\
\hline $\mathrm{N}-73$ & 1324-N/NA & Bowngradient & Quarterly & $11 / 7 / 94$ \\
\hline $\mathrm{N}-74$ & $1325-\mathrm{N}$ & Upgradient & Quarter\}y & $11 / 4 / 94$ \\
\hline $\mathrm{N}-75$ & $1301-\mathrm{N}$ & Downgradjent & Quarterly & Not scheduled \\
\hline $\mathrm{N}-76$ & $1301-\mathrm{N}$ & Downgradjent & Quarterly & Not scheduled \\
\hline $\mathrm{N}-77$ & $1324-N / N A$ & $\begin{array}{l}\text { Downgradient; } \\
\text { bottom of aqujfer }\end{array}$ & Quarterly & $11 / 7 / 94$ \\
\hline $\mathrm{N}-81$ & $1325-N$ & Downgradient & Semi annuall $7 y$ & Not scheduled \\
\hline
\end{tabular}

Note: The hydrogeologic unit monitored is the unconfined aquifer, which comprises sands and gravels of the Ringold Formation.

"RescheduTed because of logistical problems during 3rd quarter.

Pump broken.

'Insufficient water in well.

RCRA = Resource Conservation and Recovery Act of 1976. 
Table 4-2. RCRA Water Level Measurement Report 100-N Area, Fourth Quarter 1994.

(sheet 1 of 5 )

\begin{tabular}{|c|c|c|c|c|}
\hline Wel1 & Gate & $\begin{array}{l}\text { Depth to } \\
\text { water (ft) }\end{array}$ & $\begin{array}{l}\text { Water } \\
\text { eTevation } \\
\text { (ft) }\end{array}$ & $\begin{array}{l}\text { level } \\
\text { above ms1 } \\
\text { (II) }\end{array}$ \\
\hline \multicolumn{5}{|c|}{ Wells Monitoring the Top of the Unconfined Aquifer } \\
\hline $199-N-14$ & $\begin{array}{l}11 / 09 / 94 \\
12 / 07 / 94\end{array}$ & $\begin{array}{l}70.16 \\
69.50\end{array}$ & $\begin{array}{l}383.50 \\
384.16\end{array}$ & $\begin{array}{l}116.89 \\
117.09\end{array}$ \\
\hline $199-N-16$ & $\begin{array}{l}10 / 17 / 94 \\
11 / 08 / 94 \\
12 / 07 / 94\end{array}$ & $\begin{array}{l}72.48 \\
72.31 \\
71.99\end{array}$ & $\begin{array}{l}384.74 \\
384.91 \\
385.23\end{array}$ & $\begin{array}{l}117.27 \\
117.32 \\
117.42\end{array}$ \\
\hline $199-N-19$ & $\begin{array}{l}10 / 17 / 94 \\
11 / 08 / 94 \\
12 / 07 / 94\end{array}$ & $\begin{array}{l}71.87 \\
71.27 \\
69.91\end{array}$ & $\begin{array}{l}382.55 \\
383.15 \\
384.51\end{array}$ & $\begin{array}{l}116.60 \\
116.78 \\
117.20\end{array}$ \\
\hline $199-M-2$ & $\begin{array}{l}10 / 18 / 94 \\
11 / 09 / 94 \\
12 / 07 / 94\end{array}$ & $\begin{array}{l}76.16 \\
76.22 \\
75.56\end{array}$ & $\begin{array}{l}383.58 \\
383.52 \\
384.18\end{array}$ & $\begin{array}{l}116.92 \\
116.90 \\
117.10\end{array}$ \\
\hline $199-N-20$ & $\begin{array}{l}10 / 17 / 94 \\
11 / 08 / 94 \\
12 / 07 / 94\end{array}$ & $\begin{array}{l}73.21 \\
72.81 \\
71.69\end{array}$ & $\begin{array}{l}383.12 \\
383.52 \\
384.64\end{array}$ & $\begin{array}{l}116.77 \\
116.90 \\
117.24\end{array}$ \\
\hline$\quad 199-N-21$ & $\begin{array}{l}10 / 17 / 94 \\
11 / 08 / 94 \\
12 / 07 / 94\end{array}$ & $\begin{array}{l}74.42 \\
73.94 \\
72.94\end{array}$ & $\begin{array}{l}383.03 \\
383.51 \\
384.51\end{array}$ & $\begin{array}{l}116.75 \\
1.16 .89 \\
117.20\end{array}$ \\
\hline $199-N-23$ & $\begin{array}{l}10 / 17 / 94 \\
11 / 08 / 94 \\
12 / 07 / 94\end{array}$ & $\begin{array}{l}73.93 \\
73.39 \\
72.37\end{array}$ & $\begin{array}{l}382.84 \\
383.38 \\
384.40\end{array}$ & $\begin{array}{l}116.69 \\
116.85 \\
117.17\end{array}$ \\
\hline $199-N-25$ & $\begin{array}{l}10 / 17 / 94 \\
11 / 08 / 94 \\
12 / 07 / 94\end{array}$ & $\begin{array}{l}44.91 \\
43.98 \\
41.66\end{array}$ & $\begin{array}{l}381.32 \\
382.25 \\
384.57\end{array}$ & $\begin{array}{l}116.23 \\
116.51 \\
117.22\end{array}$ \\
\hline $199-N-26$ & $\begin{array}{l}10 / 17 / 94 \\
11 / 08 / 94\end{array}$ & $\begin{array}{l}74.20 \\
73.82\end{array}$ & $\begin{array}{l}382.04 \\
382.42\end{array}$ & $\begin{array}{l}116.45 \\
116.56\end{array}$ \\
\hline $199-N-26$ & $12 / 07 / 94$ & 71.76 & 384.48 & 117.19 \\
\hline $199-N-27$ & $\begin{array}{l}10 / 18 / 94 \\
11 / 08 / 94 \\
12 / 07 / 94\end{array}$ & $\begin{array}{l}62.53 \\
62.69 \\
62.80\end{array}$ & $\begin{array}{l}387.04 \\
386.88 \\
386.77\end{array}$ & $\begin{array}{l}117.97 \\
117.92 \\
117.89\end{array}$ \\
\hline
\end{tabular}


Table 4-2. RCRA Water Level Measurement Report 100-N Area, Fourth Quarter 1994.

(sheet 2 of 5)

\begin{tabular}{|c|c|c|c|c|}
\hline Well & Date & $\begin{array}{l}\text { Depth to } \\
\text { water (ft) }\end{array}$ & $\begin{array}{l}\text { Wator } \\
\text { elevation } \\
\text { (ft) }\end{array}$ & $\begin{array}{l}\text { level } \\
\text { above ms } \\
\text { (田) }\end{array}$ \\
\hline \multicolumn{5}{|c|}{ Wells Monttoring the Top of the Unconfined Aqutfer } \\
\hline $199-H-28$ & $\begin{array}{l}10 / 18 / 94 \\
11 / 08 / 94 \\
12 / 07 / 94\end{array}$ & $\begin{array}{l}77.29 \\
77.07 \\
77.00\end{array}$ & $\begin{array}{l}387.43 \\
387.65 \\
387.72\end{array}$ & $\begin{array}{l}118.09 \\
118.16 \\
118.18\end{array}$ \\
\hline $199-\mathrm{N}-29$ & $\begin{array}{l}10 / 18 / 94 \\
11 / 08 / 94 \\
12 / 07 / 94\end{array}$ & $\begin{array}{l}77.97 \\
78.12 \\
78.28\end{array}$ & $\begin{array}{l}387.76 \\
387.61 \\
387.45\end{array}$ & $\begin{array}{l}118.19 \\
118.14 \\
118.09\end{array}$ \\
\hline $199-N-3$ & $\begin{array}{l}10 / 18 / 94 \\
11 / 09 / 94 \\
12 / 07 / 94\end{array}$ & $\begin{array}{l}76.47 \\
75.90 \\
75.18\end{array}$ & $\begin{array}{l}382.90 \\
383.47 \\
384.19\end{array}$ & $\begin{array}{l}116.71 \\
116.88 \\
117.10\end{array}$ \\
\hline $199-N-31$ & $\begin{array}{l}10 / 18 / 94 \\
12 / 07 / 94\end{array}$ & $\begin{array}{l}76.78 \\
76.83\end{array}$ & $\begin{array}{l}386.33 \\
386.28\end{array}$ & $\begin{array}{l}117.75 \\
117.74\end{array}$ \\
\hline $199-[1-32$ & $\begin{array}{l}10 / 18 / 94 \\
11 / 08 / 94 \\
12 / 07 / 94\end{array}$ & $\begin{array}{l}76.04 \\
76.19 \\
76.23\end{array}$ & $\begin{array}{l}385.52 \\
386.37 \\
386.33\end{array}$ & $\begin{array}{l}117.81 \\
117.77 \\
117.75\end{array}$ \\
\hline $199+N-33$ & $10 / 18 / 94$ & 74.71 & 385.64 & 117.54 \\
\hline $199-[\mathrm{H}-34$ & $\begin{array}{l}10 / 18 / 94 \\
11 / 04 / 94 \\
11 / 08 / 94 \\
12 / 07 / 94\end{array}$ & $\begin{array}{l}73.45 \\
78.83 \\
73.55 \\
74.00\end{array}$ & $\begin{array}{l}386.68 \\
381.30^{\star}+ \\
386.58 \\
386.13\end{array}$ & $\begin{array}{l}117.86 \\
116.22 \\
117.83 \\
117.69\end{array}$ \\
\hline $199-N-41$ & $\begin{array}{l}10 / 18 / 94 \\
11 / 08 / 94 \\
12 / 07 / 94\end{array}$ & $\begin{array}{l}74.56 \\
74.25 \\
74.08\end{array}$ & $\begin{array}{l}383.53 \\
383.84 \\
384,01\end{array}$ & $\begin{array}{l}116.90 \\
116.99 \\
117.05\end{array}$ \\
\hline $199-\mathrm{H}-42$ & $\begin{array}{l}10 / 18 / 94 \\
11 / 08 / 94 \\
12 / 07 / 94\end{array}$ & $\begin{array}{l}71.57 \\
71.52 \\
71.23\end{array}$ & $\begin{array}{l}384.05 \\
384.10 \\
384.39\end{array}$ & $\begin{array}{l}117.06 \\
117.07 \\
117.16\end{array}$ \\
\hline $199-14-43$ & $\begin{array}{r}10 / 18 / 94 \\
10 / 31 / 94 \\
11 / 09 / 94 \\
12 / 07 / 94\end{array}$ & $\begin{array}{l}65.79 \\
61.89 \\
65.51 \\
65.08\end{array}$ & $\begin{array}{l}383.73 \\
387.63^{\star}+ \\
384.01 \\
384.44 .\end{array}$ & $\begin{array}{l}116.96 \\
118.15 \\
117.05 \\
117.18\end{array}$ \\
\hline
\end{tabular}


Table 4-2. RCRA Water Level Measurement Report 100-N Area, Fourth Quarter 1994.

(shert 3 of 5)

\begin{tabular}{|c|c|c|c|c|c|}
\hline Wel] & & Date & $\begin{array}{l}\text { Depth to } \\
\text { water (ft) }\end{array}$ & $\begin{array}{l}\text { Hater } \\
\text { elevation } \\
\text { (ft) }\end{array}$ & $\begin{array}{l}\text { Jevel } \\
\text { above msl } \\
\text { (m) }\end{array}$ \\
\hline \multicolumn{6}{|c|}{ Wells Monitoring the Top of the Unconfined Aquifer } \\
\hline $199-\mathrm{N}-50$ & & $\begin{array}{l}11 / 08 / 94 \\
12 / 07 / 94\end{array}$ & $\begin{array}{l}80.62 \\
80.20\end{array}$ & $\begin{array}{l}383.26 \\
383.68\end{array}$ & $\begin{array}{l}116.82 \\
116.95\end{array}$ \\
\hline $199-N-51$ & & $\begin{array}{l}10 / 18 / 94 \\
11 / 08 / 94 \\
12 / 07 / 94\end{array}$ & $\begin{array}{l}80.38 \\
79.79 \\
79.02\end{array}$ & $\begin{array}{l}382.31 \\
382.90 \\
383.67\end{array}$ & $\begin{array}{l}116.53 \\
116.71 \\
116.94\end{array}$ \\
\hline $199-N-52$ & - & $\begin{array}{l}10 / 18 / 94 \\
11 / 08 / 94 \\
12 / 07 / 94\end{array}$ & $\begin{array}{l}76.18 \\
76.25 \\
76.38\end{array}$ & $\begin{array}{l}388.00 \\
387.93 \\
387.80\end{array}$ & $\begin{array}{l}118.26 \\
118.24 \\
118.20\end{array}$ \\
\hline $199-N-54$ & $\cdot$ & $\begin{array}{l}10 / 17 / 94 \\
11 / 08 / 94 \\
12 / 07 / 94\end{array}$ & $\begin{array}{l}73.55 \\
73.42 \\
73.04\end{array}$ & $\begin{array}{l}384.43 \\
384.56 \\
384.94\end{array}$ & $\begin{array}{l}117.17 \\
117.21 \\
117.33\end{array}$ \\
\hline 199-N-55 & & $\begin{array}{l}10 / 17 / 94 \\
11 / 08 / 94 \\
12 / 07 / 94\end{array}$ & $\begin{array}{l}73.89 \\
73.78 \\
73.76\end{array}$ & $\begin{array}{l}384.44 \\
384.55 \\
384.57\end{array}$ & $\begin{array}{l}117.18 \\
117.21 \\
117.22\end{array}$ \\
\hline $199-N-56$ & $\ldots$ & $\begin{array}{l}10 / 17 / 94 \\
11 / 08 / 94 \\
12 / 07 / 94\end{array}$ & $\begin{array}{l}74.57 \\
74.42 \\
74.01\end{array}$ & $\begin{array}{l}384.00 \\
384.15 \\
384.56\end{array}$ & $\begin{array}{l}117.04 \\
117.09 \\
117.21\end{array}$ \\
\hline $199-N-57$ & & $\begin{array}{l}10 / 17 / 94 \\
11 / 08 / 94 \\
12 / 07 / 94\end{array}$ & $\begin{array}{l}72.20 \\
72.26 \\
72.14\end{array}$ & $\begin{array}{l}385.04 \\
385.98 \\
386.10\end{array}$ & $\begin{array}{l}117.66 \\
117.65 \\
117.68\end{array}$ \\
\hline $199-N-59$ & & $10 / 18 / 94$ & 74.79 & 385.26 & 117.43 \\
\hline $199-N-62$ & & $\begin{array}{l}10 / 18 / 94 \\
11 / 08 / 94 \\
12 / 07 / 94\end{array}$ & $\begin{array}{l}76.31 \\
76.48 \\
76.65\end{array}$ & $\begin{array}{l}387.78 \\
387.61 \\
387.44\end{array}$ & $\begin{array}{l}118.20 \\
118.14 \\
118.09\end{array}$ \\
\hline $199-N-63$ & & $\begin{array}{l}10 / 18 / 94 \\
11 / 08 / 94 \\
12 / 07 / 94\end{array}$ & $\begin{array}{l}79.86 \\
80.03 \\
79.94\end{array}$ & $\begin{array}{l}387.34 \\
387.17 \\
387.26\end{array}$ & $\begin{array}{l}118.06 \\
118.01 \\
118.04\end{array}$ \\
\hline $199-N-64$ & & $\begin{array}{l}10 / 18 / 94 \\
11 / 09 / 94 \\
12 / 07 / 94\end{array}$ & $\begin{array}{l}68.38 \\
6 B .48 \\
68.57\end{array}$ & $\begin{array}{l}386.75 \\
386.65 \\
386.56\end{array}$ & $\begin{array}{r}117.88 \\
117.85 \\
117.82\end{array}$ \\
\hline
\end{tabular}


Table 4-2. RCRA Water Leve1 Measurement Report 100-N Area, Fourth Quarter 1994.

(sheet 4 of 5)

\begin{tabular}{|c|c|c|c|c|}
\hline WeT7. & Date & $\begin{array}{l}\text { Depth to } \\
\text { water (ft) }\end{array}$ & $\begin{array}{l}\text { Water } \\
\text { elevation } \\
\text { (ft) }\end{array}$ & $\begin{array}{l}\text { level } \\
\text { above msl } \\
\text { (tr) }\end{array}$ \\
\hline \multicolumn{5}{|c|}{ Hells Monitoring the Top of the Unconfined Aqujfer } \\
\hline $\begin{array}{c}199-N-65 \\
.\end{array}$ & $\begin{array}{r}10 / 18 / 94 \\
11 / 09 / 94 \\
12 / 07 / 94\end{array}$ & $\begin{array}{l}70.73 \\
70.82 \\
70.81\end{array}$ & $\begin{array}{l}386.23 \\
386.14 \\
386.15\end{array}$ & $\begin{array}{l}117.72 \\
117.70 \\
117.70\end{array}$ \\
\hline $199-\mathrm{H}-67$ & $\begin{array}{l}10 / 18 / 94 \\
11 / 09 / 94 \\
12 / 07 / 94\end{array}$ & $\begin{array}{l}74.80 \\
74.70 \\
74.39\end{array}$ & $\begin{array}{l}384.15 \\
384.25 \\
384.56\end{array}$ & $\begin{array}{l}117.09 \\
117.12 \\
117.21\end{array}$ \\
\hline $199-14-71$ & $\begin{array}{l}10 / 18 / 94 \\
11 / 04 / 94 \\
11 / 08 / 94 \\
12 / 07 / 94\end{array}$ & $\begin{array}{l}73.97 \\
73.79 \\
74.17 \\
74.48\end{array}$ & $\begin{array}{l}389.02 \\
389.20^{*} \\
388.82 \\
388.51\end{array}$ & $\begin{array}{l}118.57 \\
118.63 \\
118.51 \\
118.42\end{array}$ \\
\hline $199-11-72$ & $\begin{array}{l}10 / 17 / 94 \\
11 / 07 / 94 \\
11 / 08 / 94 \\
12 / 07 / 94\end{array}$ & $\begin{array}{l}72.49 \\
72.55 \\
72.45 \\
72.43\end{array}$ & $\begin{array}{l}386.46 \\
386.40^{*} \\
386.49 \\
386.52\end{array}$ & $\begin{array}{l}117.79 \\
117.77 \\
117.80 \\
117.81\end{array}$ \\
\hline $199-14-73$ & $\begin{array}{r}10 / 18 / 94 \\
11 / 07 / 94 \\
11 / 08 / 94 \\
\cdot 12 / 07 / 94\end{array}$ & $\begin{array}{l}75.59 \\
75.60 \\
76.61 \\
76.67\end{array}$ & $\begin{array}{l}386.64 \\
387.63^{*}+ \\
386.62 \\
386.56\end{array}$ & $\begin{array}{l}117.85 \\
118.15 \\
117.84 \\
117.82\end{array}$ \\
\hline $199-14-74$ & $\begin{array}{l}10 / 18 / 94 \\
11 / 04 / 94 \\
11 / 08 / 94 \\
12 / 07 / 94\end{array}$ & $\begin{array}{l}68.65 \\
58.68 \\
68.77 \\
68.94\end{array}$ & $\begin{array}{l}388.97 \\
388.94^{\star} \\
388.85 \\
388.68\end{array}$ & $\begin{array}{l}118.56 \\
118.55 \\
118.52 \\
118.47\end{array}$ \\
\hline . $199-H-75$ & $\begin{array}{l}10 / 18 / 94 \\
11 / 09 / 94 \\
12 / 07 / 94\end{array}$ & $\begin{array}{l}74.37 \\
73.70 \\
72.93\end{array}$ & $\begin{array}{l}382.41 \\
383.08 \\
383.85\end{array}$ & $\begin{array}{l}116.56 \\
116.76 \\
117.00\end{array}$ \\
\hline $199-4-76$ & $\begin{array}{l}10 / 18 / 94 \\
11 / 08 / 94 \\
12 / 07 / 94\end{array}$ & $\begin{array}{l}69.74 \\
69.09 \\
68.33\end{array}$ & $\begin{array}{l}382.38 \\
383.03 \\
383.79\end{array}$ & $\begin{array}{l}116.55 \\
116.75 \\
116.98\end{array}$ \\
\hline $199-H-81$ & $\begin{array}{l}10 / 18 / 94 \\
11 / 08 / 94 \\
12 / 07 / 94\end{array}$ & $\begin{array}{l}77.30 \\
77.43 \\
76.54\end{array}$ & $\begin{array}{l}385.41 \\
385.28 \\
386.17\end{array}$ & $\begin{array}{l}117.47 \\
117.43 \\
117.70\end{array}$ \\
\hline
\end{tabular}


Table 4-2. RCRA Hater Level Measurement Report 100-N Area, Fourth Quarter 1994.

(sheet 5 of 5 )

We11 Date water (ft) $\begin{gathered}\text { Depth to } \\ \text { water leve1 } \\ \text { (ft) }\end{gathered}$

Nells Monitoring the Top of the Unconfined Aquifer

199-N-8S

$10 / 18 / 94$

$11 / 09 / 94$

24.10

23.32

$12 / 07 / 94$

21.17

381.00

381.78

383.93

116.13

116.37

127.02

$699-81-58$

$\begin{array}{llll}10 / 18 / 94 & 47.30 & 392.19 & 119.54 \\ 11 / 08 / 94 & 47.31 & 392.18 & 119.54 \\ 12 / 07 / 94 & 47.40 & 392.09 & 119.51\end{array}$

Mells Monitoring the Botton of the Unconfined Aquifer $199-N-69$

$10 / 18 / 94$
$11 / 09 / 94$
$12 / 07 / 94$

$199-\mathrm{N}-70$

199-N-77
$10 / 18 / 94$

$11 / 08 / 94$

$12 / 07 / 94$

$10 / 17 / 94$

$11 / 07 / 94$

$11 / 08 / 94$

$12 / 07 / 94$
75.35

75.18

74.85

69.44

69.55

69.50

72.83

72.79

72.80

72.80
383.98

384.15

384.48

385.24

385.13

385.18

386.61

$386.65^{*}$

386.64

386.64
117.04

117.09

117.19

117.42

117.39

117.40

117.84

117.85

117.85

117.85

NOTES: 1. Water levet elevations are calculated by subtracting the measured depth-to-water from the surveyed elevation for the well.

2. Depth-to-water values are transcribed from fieid records.

3. Elevations marked with an ' $*$ ' were measured at the time of sampling.

4. Elevations marked with a ' + ' are outside of the expected range, and are suspected of error. 
Table 4-3. Constituent List and Summary of Results for 100-N RCRA Sites Data for Reporting Period October 1 through December 31, 1994. (sheet 1 of 3 )

\section{CONTAMIWTION IADICATOR PARNETERS}

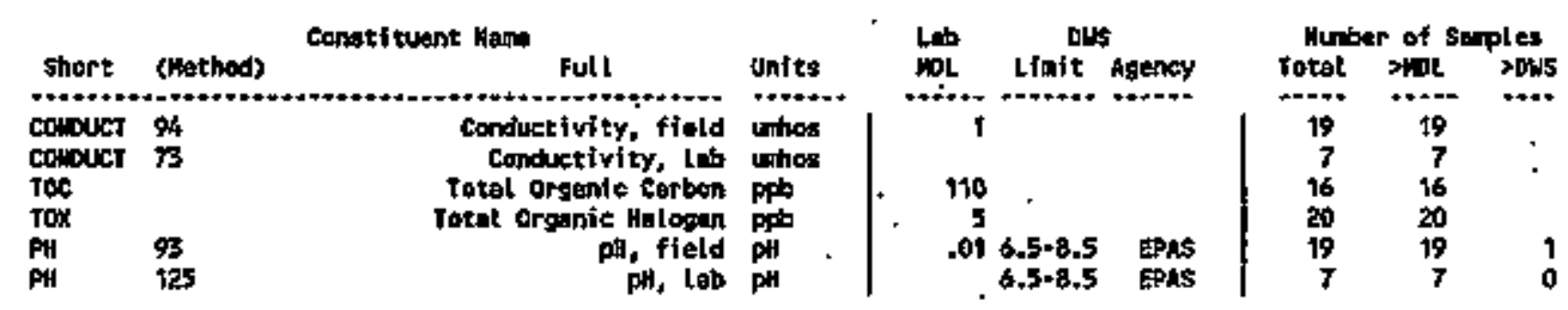

DRINKSWG WATER PARAHETERS

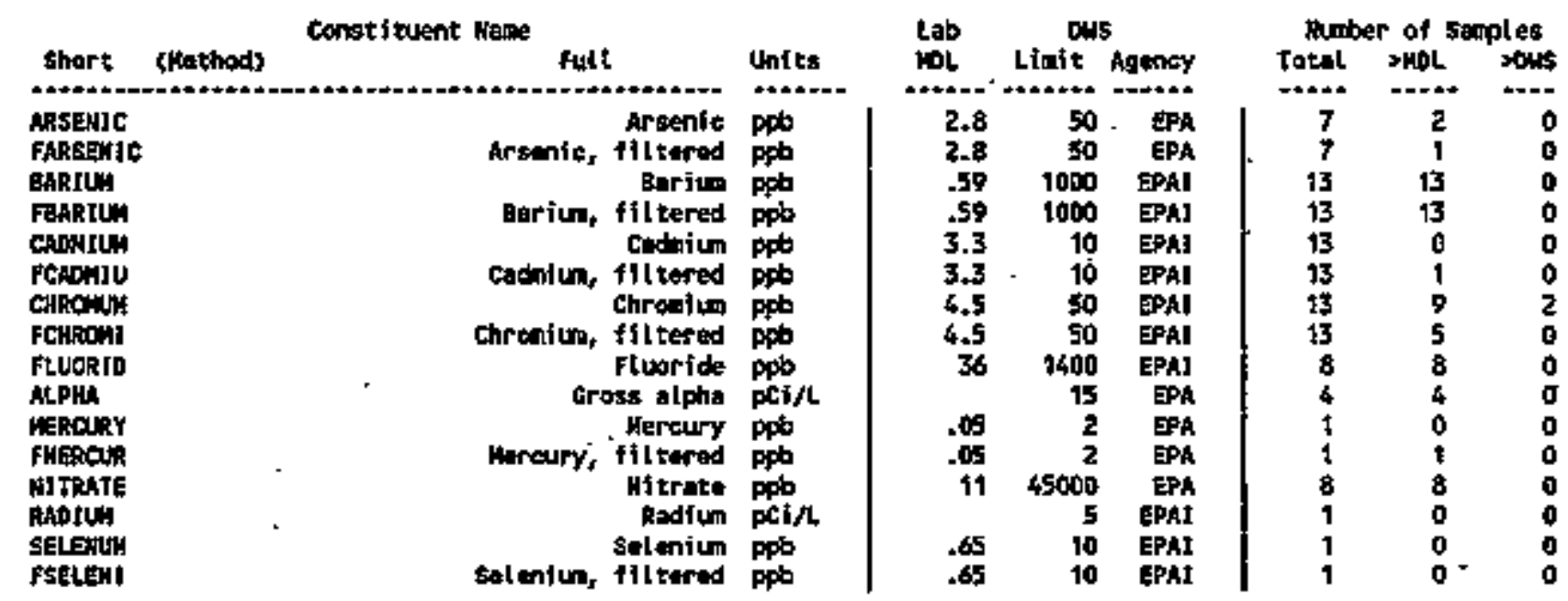

GROULOUKTER WALITY PARAHTERS

\begin{tabular}{|c|c|c|c|c|c|c|c|c|c|}
\hline & & Constituent Hams & & Lab & Mtris & & Hent & c of $\mathrm{s}$ & plas \\
\hline Shart & (Whethod) & Fult & Unties & MBL & Lfait & Asency & Total & 3WL & $>$ Dhts \\
\hline . & & m. & $++\infty+2$ & $+\cdots+\infty$ & $-2+++\cdots$ & $+\cdots$ & $+\infty-\infty$ & $+*+\infty$ & $\cdots$ \\
\hline CHLOR10 & & Chloride & ppb & 14 & 250000 & EPAS & & 8 & $\mathbf{0}$ \\
\hline 1ROA & & tron & ppb & 5.24 & 300 & EPAS & -13 & 13 & $\delta$ \\
\hline Fistor & & Iron, 11ltered & Pps & 5.24 & $\$ 00$ & EPA's & 13 & 7 & \\
\hline MAMGESE & & Minganese & ppb & .72 & 50 & EPAS & 13 & 12 & a \\
\hline FHAKBAH & & Menqunese, filtered & pab &. $\bar{\pi}$ & 50 & EPAS & 13 & 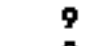 & 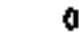 \\
\hline $\begin{array}{l}\text { LPHEAdOL } \\
\text { SODtux }\end{array}$ & $\cdot$ & Pbenol & ppos & .31 & & & 13 & 0 & \\
\hline fscoluk & & Sodium, filtered & ppob & 61 & & & 13 & 13 & \\
\hline SILLFATE & & sulfate & ppb & 37 & 250000 & EPAS & $\mathbf{B}$ & 8 & 2 \\
\hline
\end{tabular}


Table 4-3. Constituent List and Summary of Results for 100-N RCRA Sites Data for Reporting Period October 1 through December 31, 1994.

(sheet 2 of 3 )

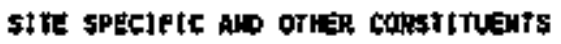

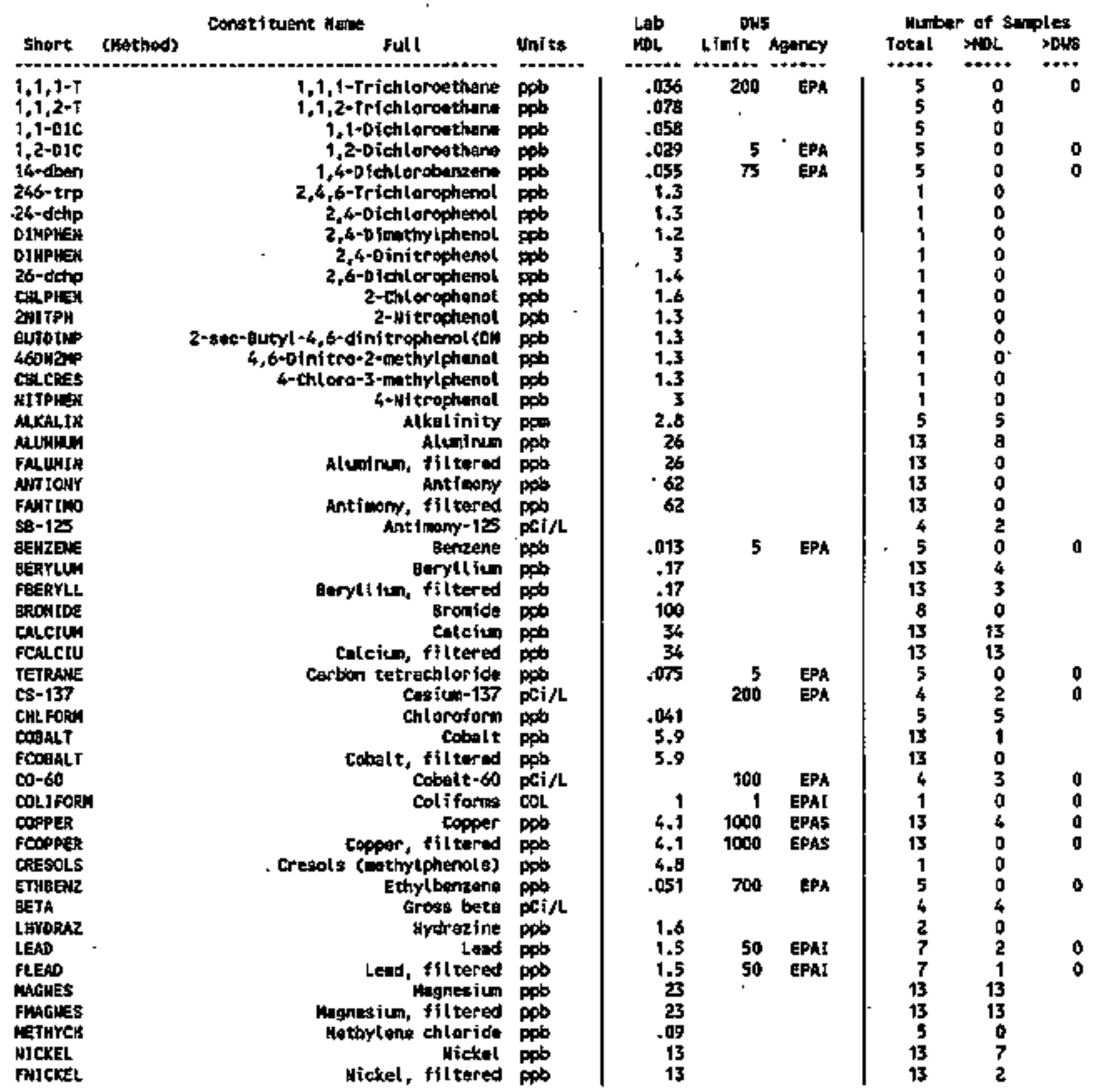


Table 4-3. Constituent List and Summary of Results for 100-N RCRA Sites Data for Reporting Period October 1 through Decenber 31, 1994. (sheet 3 of 3 )

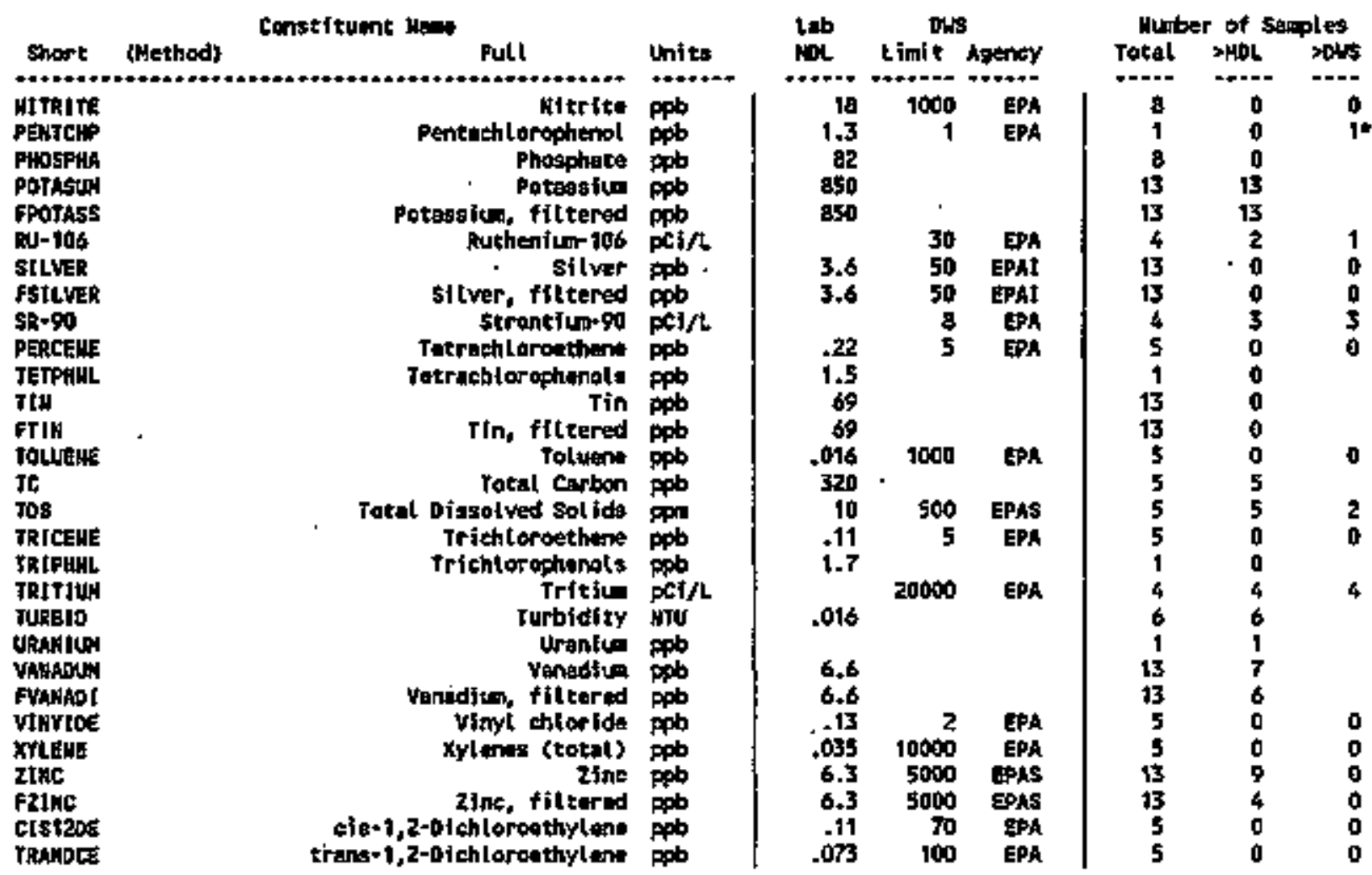

far explanation of this tabla, sose section 1.4 of raport. 
Table 4-4. Constituents with at Least One Detected Value for the 100-N RCRA Sites Data for Reporting Period October 1 through December 31, 1994.

(sheet 1 of 9)

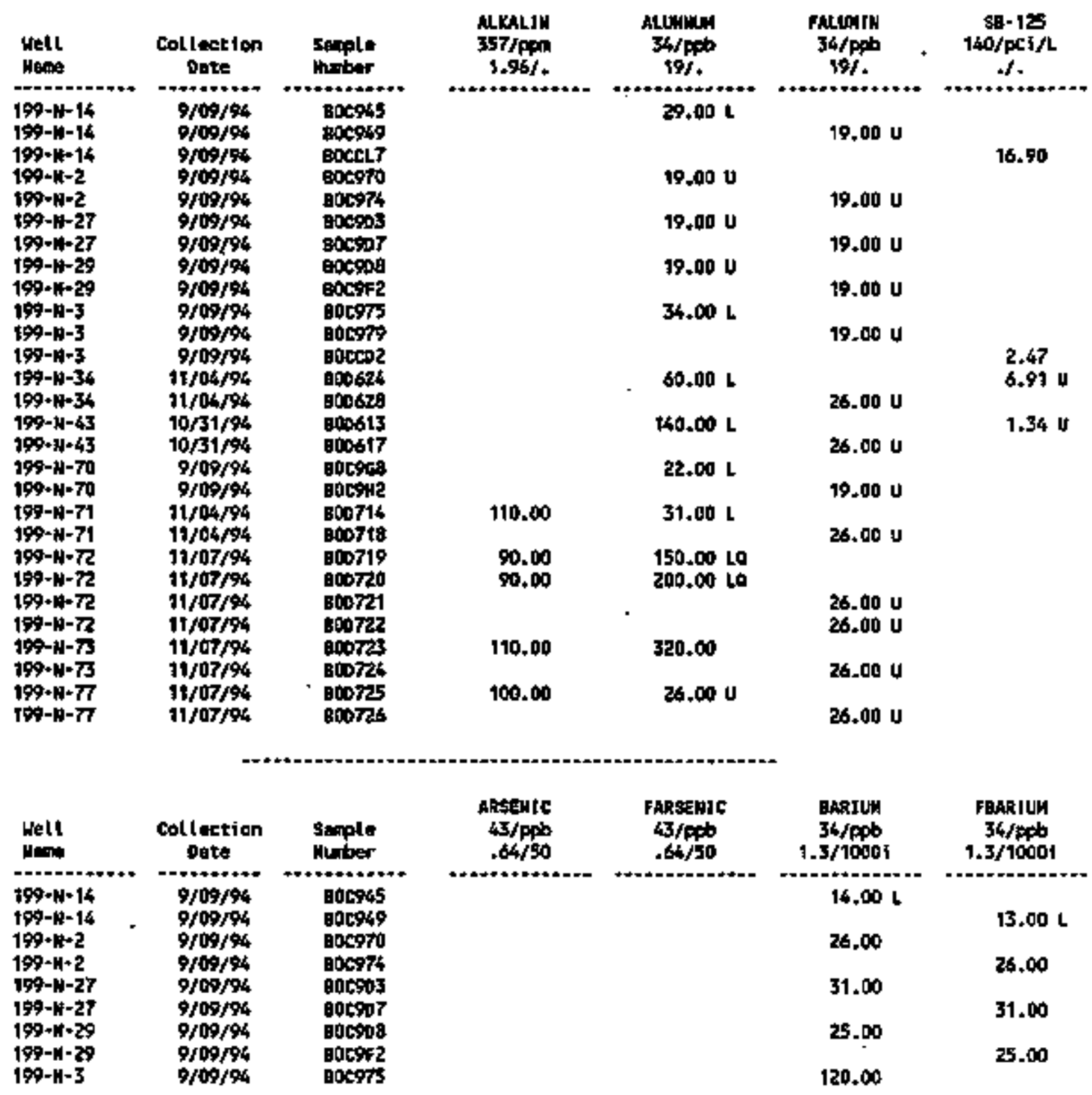


Table 4-4. Constituents with at Least One Detected Value for the I00-N RCRA Sjtes Data for Reporting Period Octaber 1 through December 31, 1994.

(sheet 2 of 9)

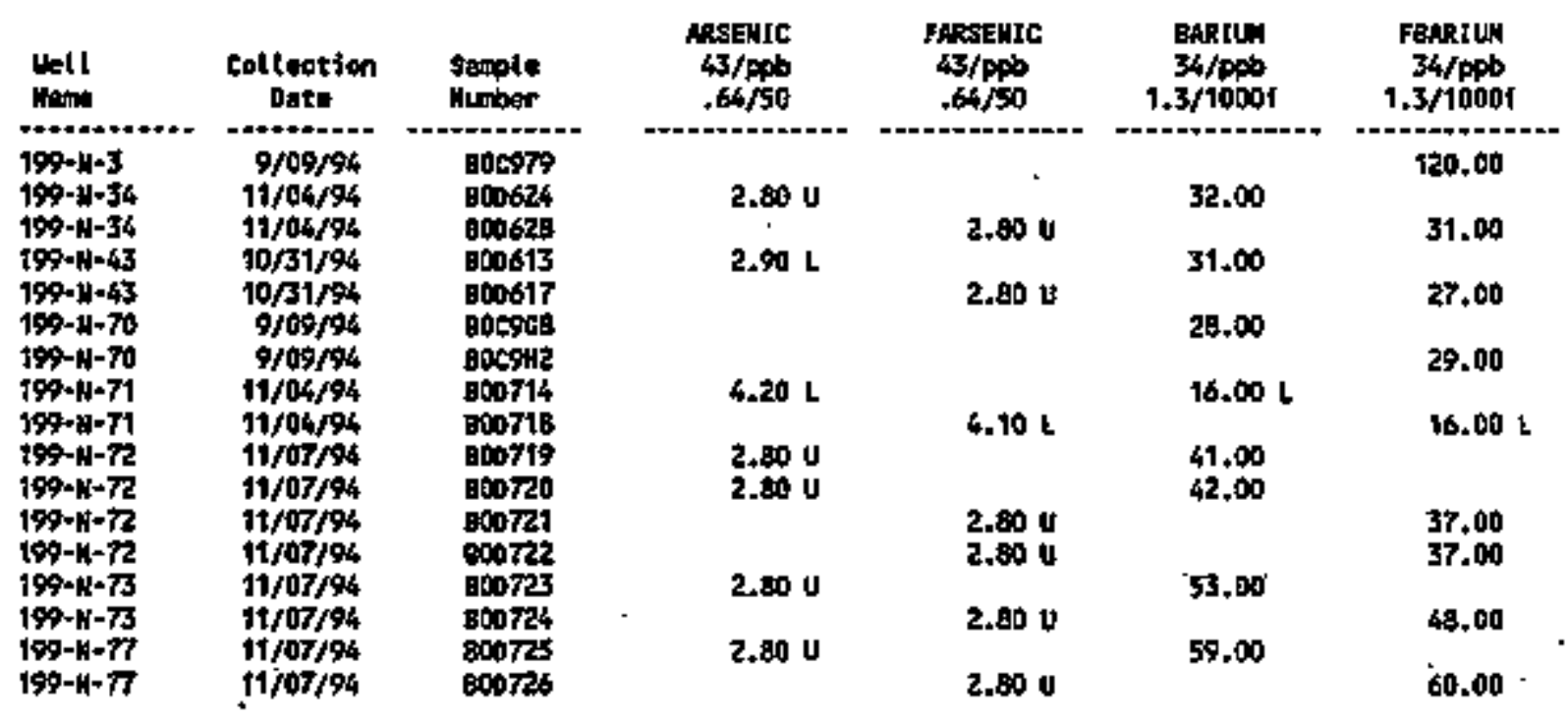

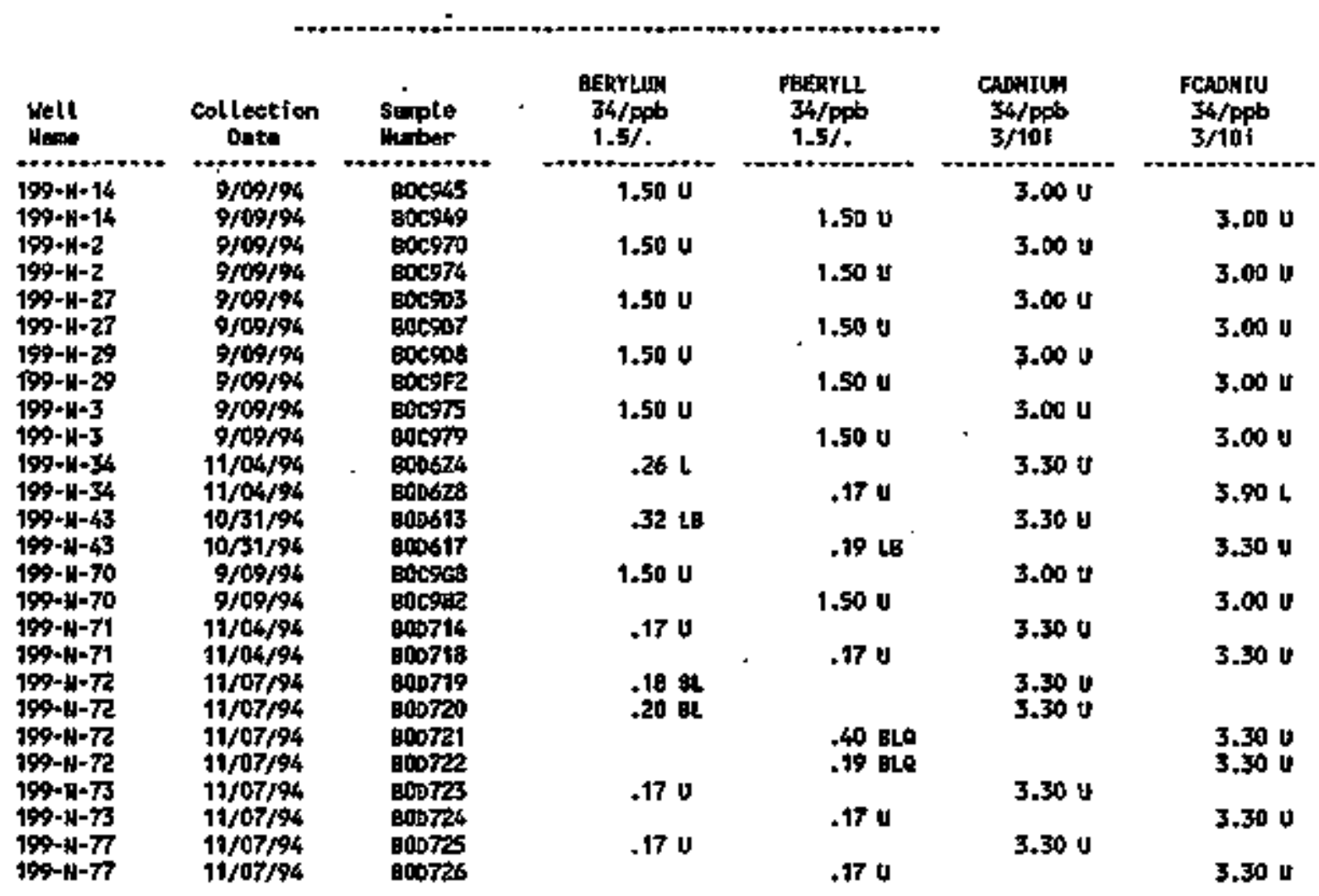


Table 4-4. Constituents with at Least one Dotected Value for the 100-N RCRA Sites Data for Reporting Period October 1 through December 31, 1994.

(sheet 3 of 9 )

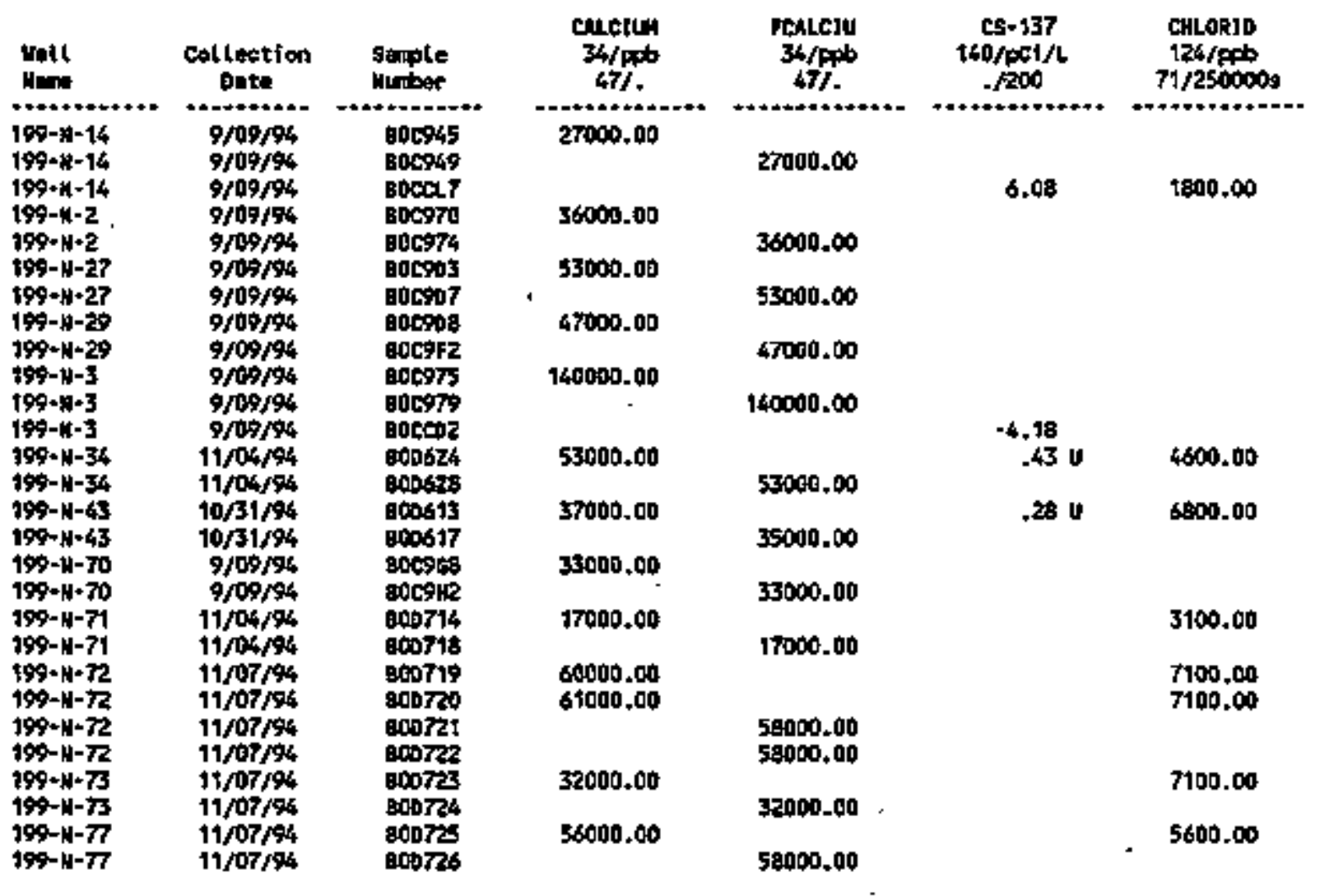


Table 4-4, Constituents with at Least One Detected Value for the 100-N RCRA Sites Data for Reporting Period October 1 through December 31, 1994. (sheet 4 of 9 )

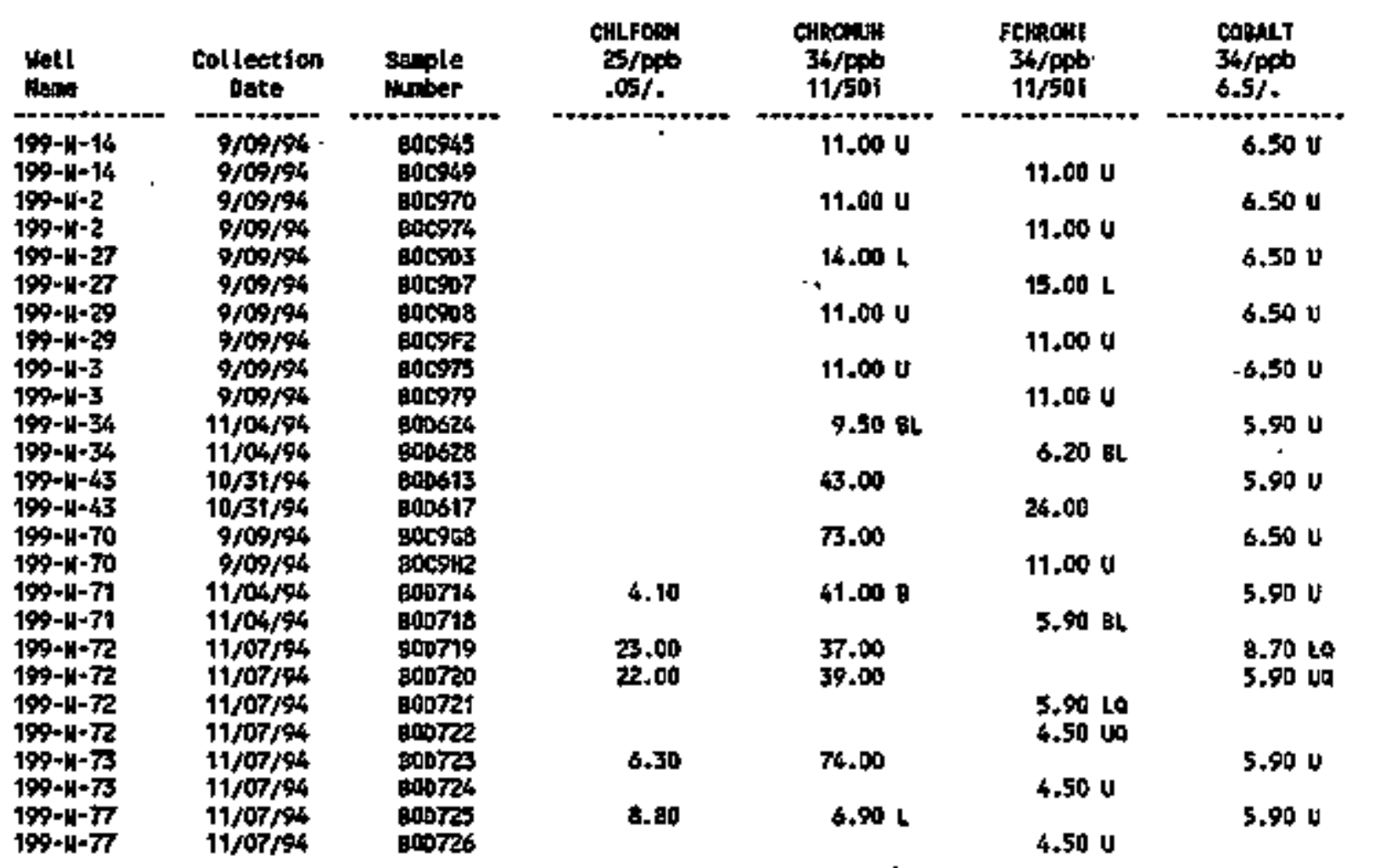

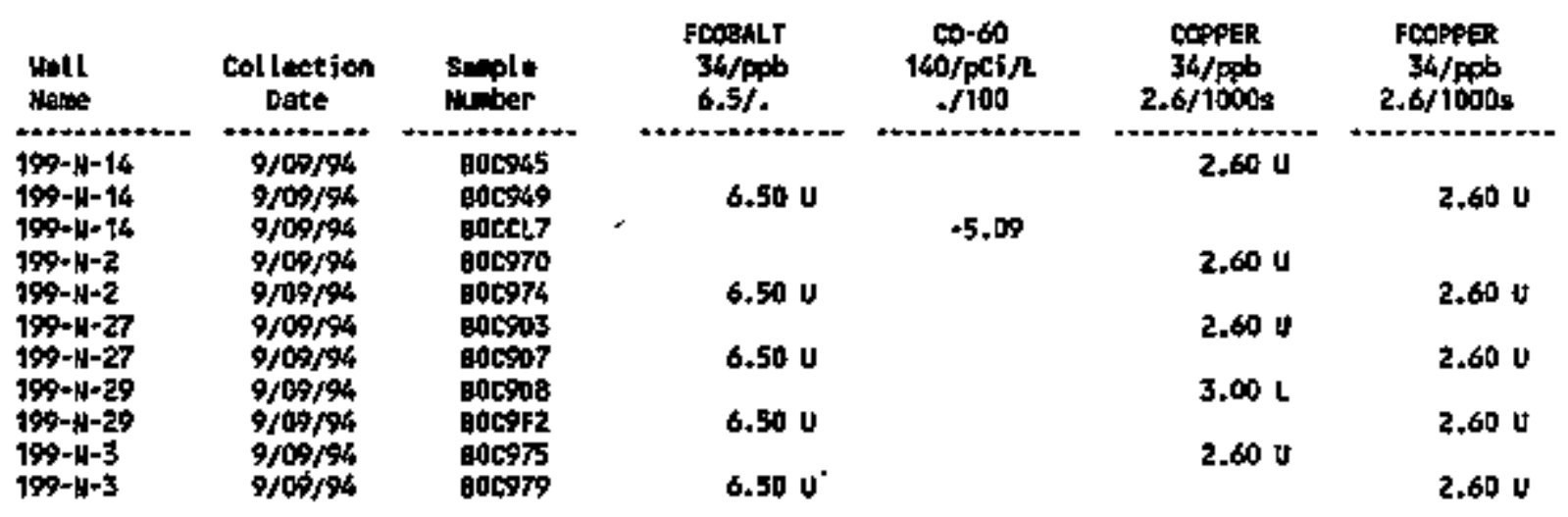


Table 4-4. Constituents with at Least One Detected Value for the 100-N RCRA Sites Data for Reporting Period October 1 through December 31, 1994.

(sheet 5 of 9 )

\begin{tabular}{|c|c|c|c|c|c|c|}
\hline $\begin{array}{l}\text { Well } \\
\text { Mente }\end{array}$ & $\begin{array}{l}\text { Collection } \\
\text { Date }\end{array}$ & $\begin{array}{l}\text { serple } \\
\text { Mugber }\end{array}$ & $\begin{array}{c}\text { FCobsitT } \\
34 / p \mathrm{pb} \\
6,5 \%\end{array}$ & $\begin{array}{c}10-60 \\
\mathrm{t} 60 / \mathrm{pci} / \mathrm{L} \\
+/ 100\end{array}$ & $\begin{array}{c}\text { COPPER } \\
3 / \text { ppb } \\
2.6 / 1000 \mathrm{~s}\end{array}$ & $\begin{array}{c}\text { FCOPPER } \\
34 / \mathrm{ppb} \\
2.6 / 1000 \mathrm{x}\end{array}$ \\
\hline 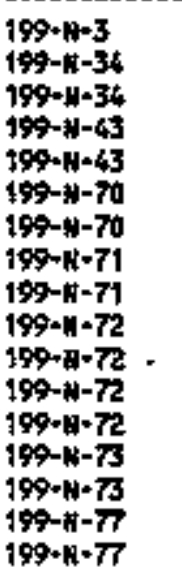 & $\begin{array}{r}9 / 09 / 94 \\
11 / 04 / 94 \\
11 / 04 / 94 \\
10 / 31 / 94 \\
10 / 31 / 94 \\
9 / 09 / 94 \\
9 / 07 / 94 \\
11 / 04 / 94 \\
11 / 04 / 94 \\
11 / 07 / 94 \\
11 / 07 / 94 \\
11 / 07 / 94 \\
11 / 07 / 94 \\
11 / 07 / 94 \\
11 / 07 / 94 \\
11 / 07 / 94 \\
11 / 07 / 94\end{array}$ & 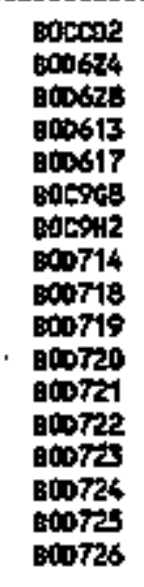 & $\begin{array}{l}5.90 \mathrm{U} \\
5.90 \mathrm{U} \\
6.50 \mathrm{U} \\
5.90 \mathrm{U} \\
5.90 \mathrm{U} \\
5.90 \mathrm{U} \\
5.90 \mathrm{U} \\
5.90 \mathrm{U}\end{array}$ & $\begin{array}{c}3.69 \\
10.10 \\
1.24\end{array}$ & $\begin{array}{l}4.10 \mathrm{U} \\
22.00 \\
2.60 \mathrm{U} \\
4.10 \mathrm{U} \\
4.60 \mathrm{~L} \\
5.60 \mathrm{~L} \\
5.70 \mathrm{~L} \\
4.10 \mathrm{U}\end{array}$ & $\begin{array}{l}4.10 \mathrm{U} \\
4.10 \mathrm{U} \\
3.50 \mathrm{~L} \\
4.10 \mathrm{U} \\
4.20 \mathrm{U} \\
4.70 \mathrm{U} \\
4.10 \mathrm{U} \\
4.10 \mathrm{U}\end{array}$ \\
\hline $\begin{array}{l}\text { Well } \\
\text { Mente . }\end{array}$ & $\begin{array}{l}\text { Collection } \\
\text { Date }\end{array}$ & $\begin{array}{l}\text { Sample } \\
\text { Muniver }\end{array}$ & $\begin{array}{l}\text { FUOR Lo } \\
\text { 124/ppb } \\
51 / 14001\end{array}$ & $\begin{array}{c}\text { ALPHK } \\
135 / \mathrm{DC1} / \mathrm{L} \\
. / 15\end{array}$ & $\begin{array}{c}\text { otta } \\
\text { 136/pit/L } \\
. / .\end{array}$ & $\begin{array}{c}t+0,4 \\
34 / 040 \\
18 / 3009\end{array}$ \\
\hline 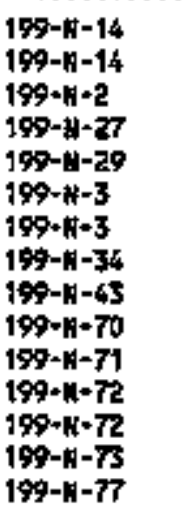 & $\begin{array}{c}9 / 09 / 94 \\
9 / 09 / 96 \\
9 / 09 / 94 \\
9 / 09 / 94 \\
9 / 09 / 94 \\
9 / 09 / 94 \\
9 / 09 / 94 \\
11 / 04 / 94 \\
10 / 31 / 94 \\
9 / 09 / 94 \\
11 / 04 / 94 \\
11 / 107 / 94 \\
11 / 07 / 94 \\
11 / 07 / 94 \\
11 / 07 / 94\end{array}$ & 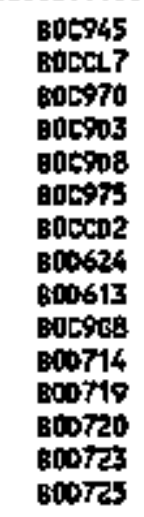 & $\begin{array}{l}500.00 \\
400.00 \\
500.00 \\
600.00 \\
600.00 \\
700.00 \\
900.00\end{array}$ & $\begin{array}{l}7.25 \\
1.94 \\
1.63\end{array}$ & $\begin{array}{r}1680.00 \\
65.10 \\
3.74\end{array}$ & $\begin{array}{r}510.00 \mathrm{a} \\
99.00 \mathrm{a} \\
120.00 \mathrm{a} \\
33.00 \mathrm{a} \\
280.00 \mathrm{a} \\
240.00 \\
940.00 \mathrm{a} \\
370.00 \mathrm{a} \\
190.00 \\
440.00 \mathrm{~B} \\
560.00 \mathrm{~g} \\
860.00 \mathrm{~g} \\
45.00 \mathrm{~B}\end{array}$ \\
\hline
\end{tabular}


Table 4-4. Constituents wtth at Least One Detected Value for the 100-N RCRA Sites Data for Reporting Period October 1 through December 31, 1994.

$$
\text { (sheet } 6 \text { of } 9 \text { ) }
$$

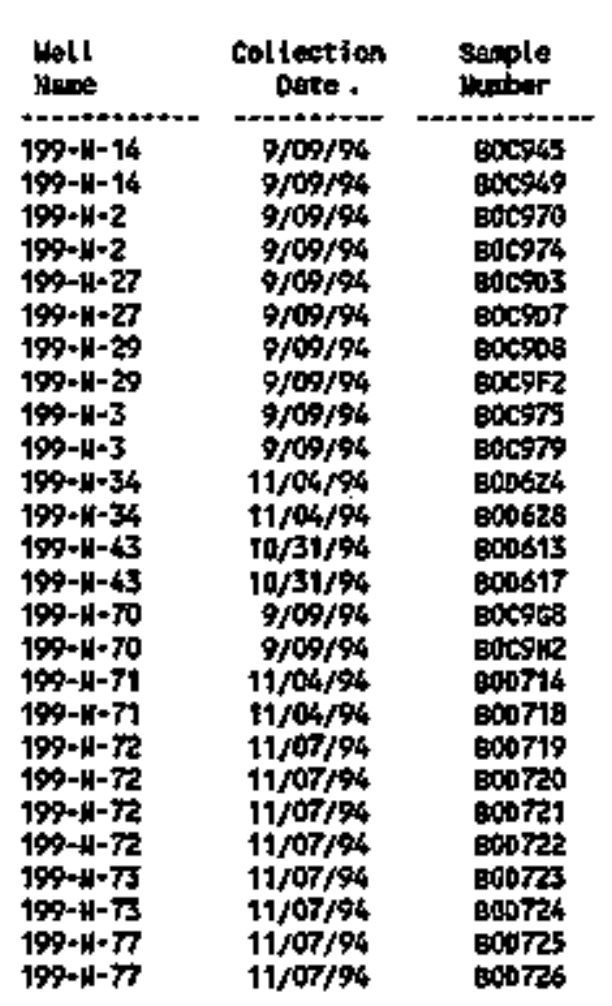

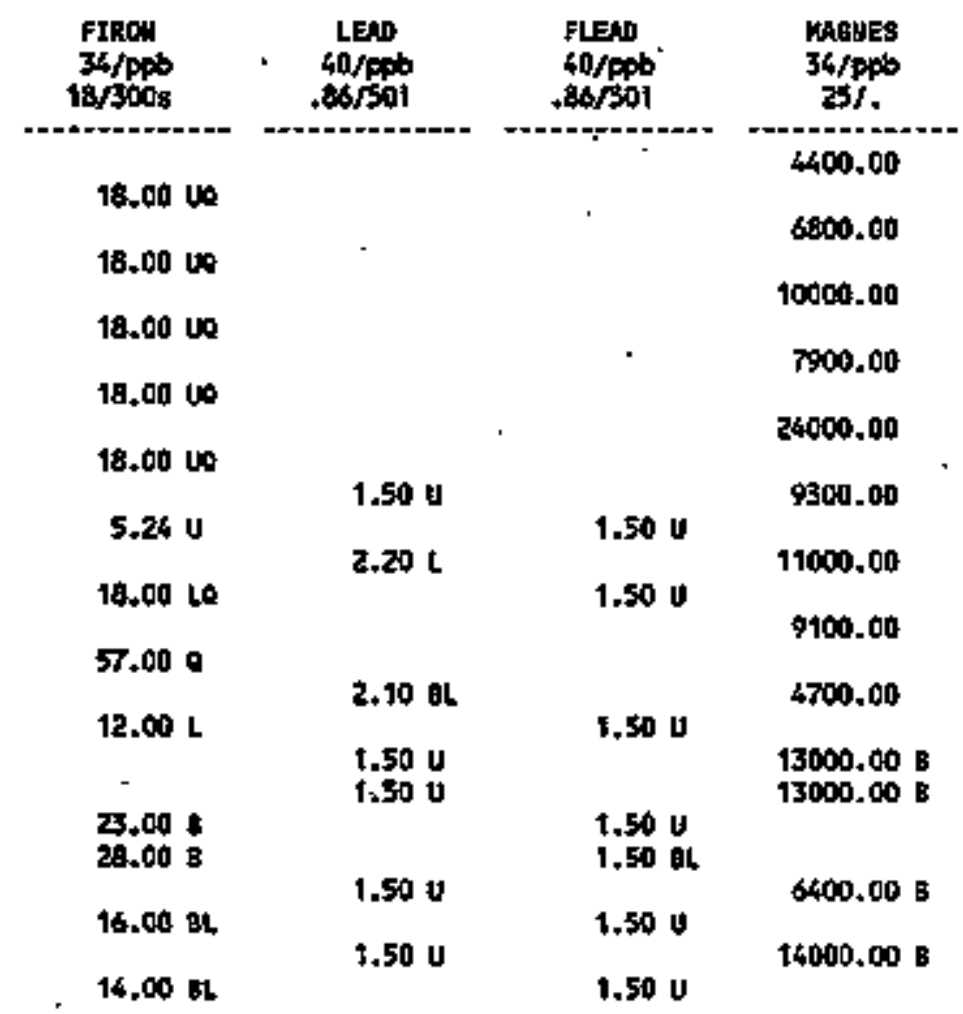

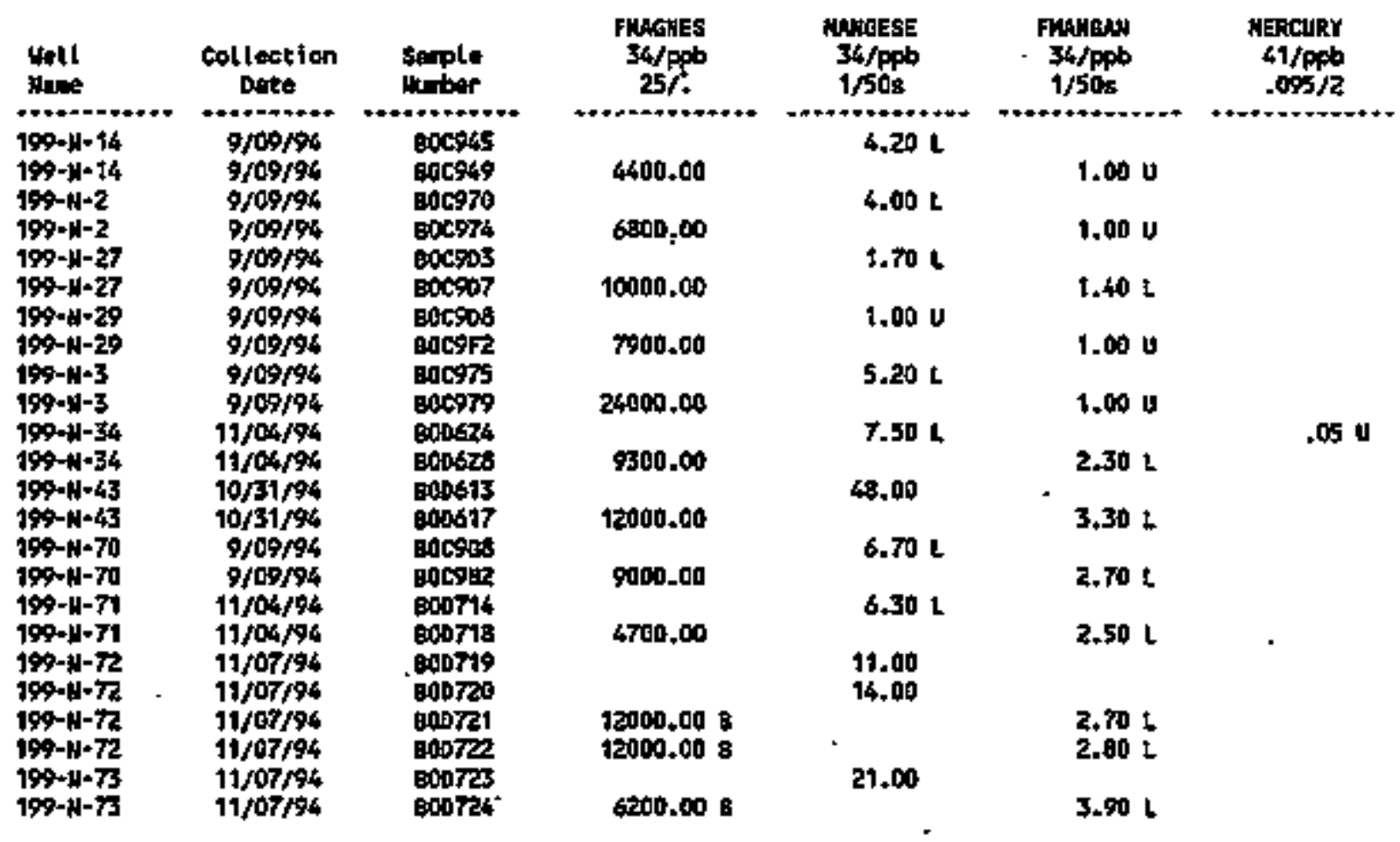


Table 4-4. Constituents with at Least One Detected Value for the 100-N RCRA Sites Data for Reporting Period October 1 through Decenber 31, 1994. (sheet 7 of 9)

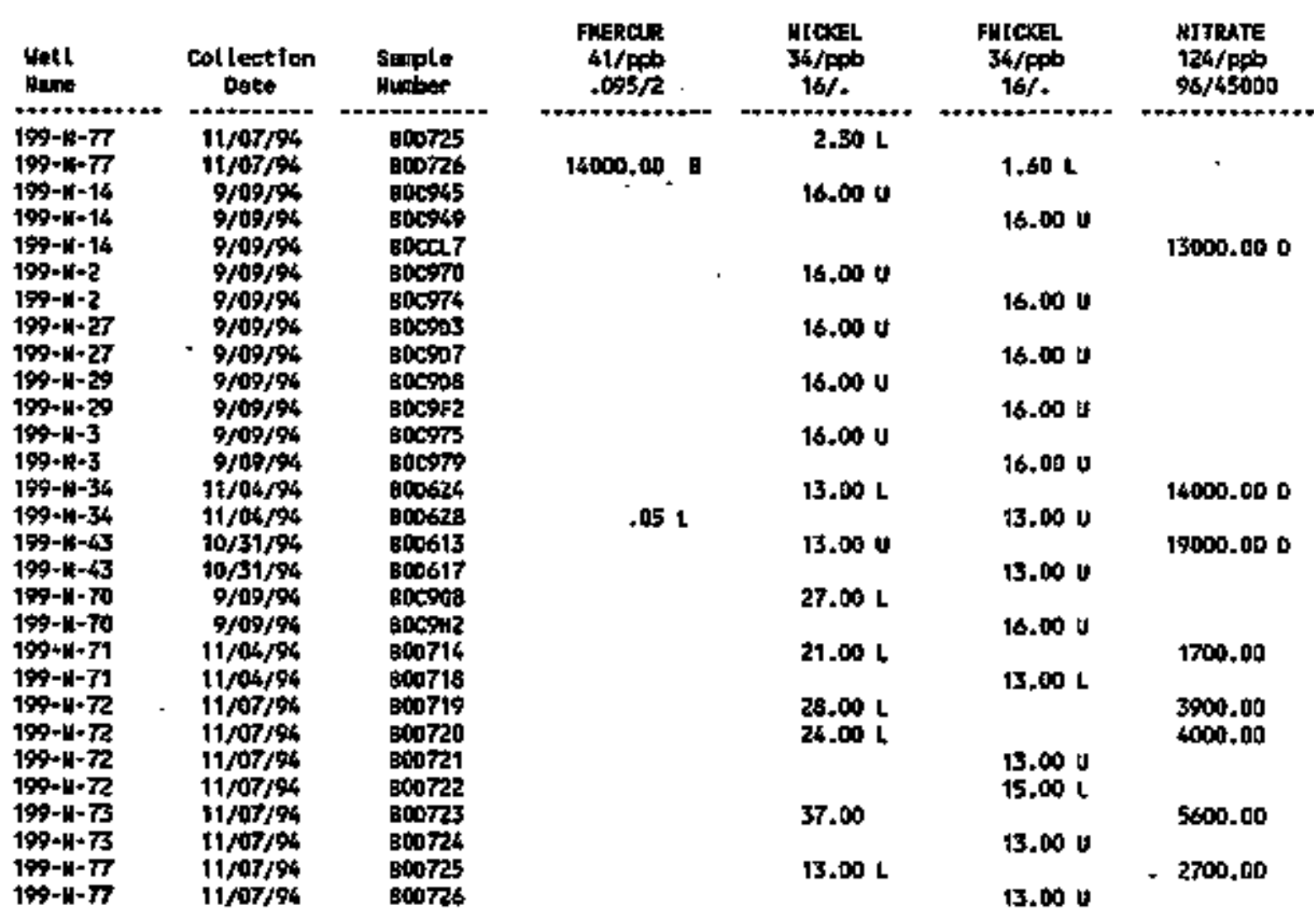


Table 4-4. Constituents with at Least One Detected Value for the 100-N RCRA Sites Data for Reporting Perlod

October 1 through December 31, 1994. (sheet 8 of 9)

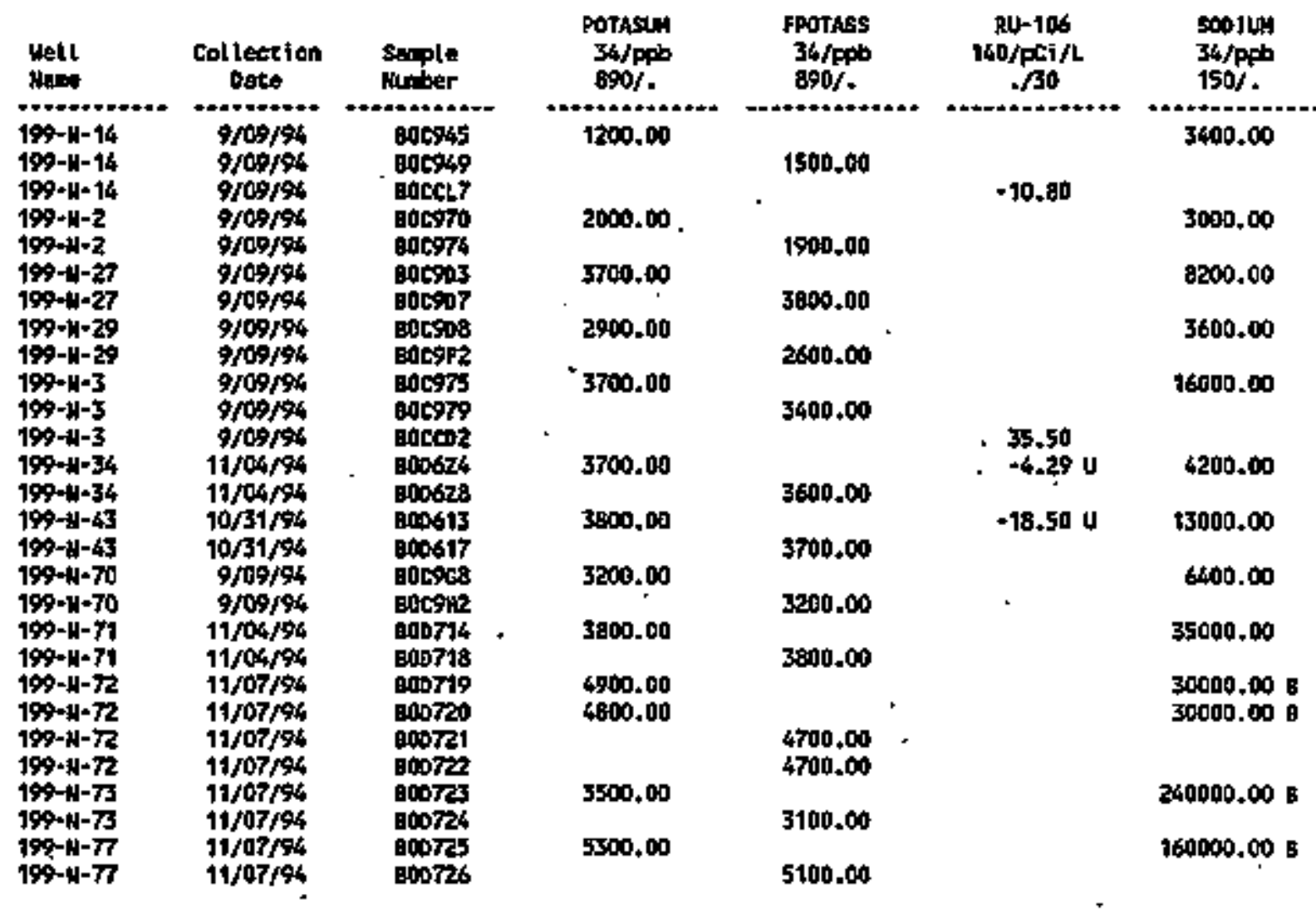

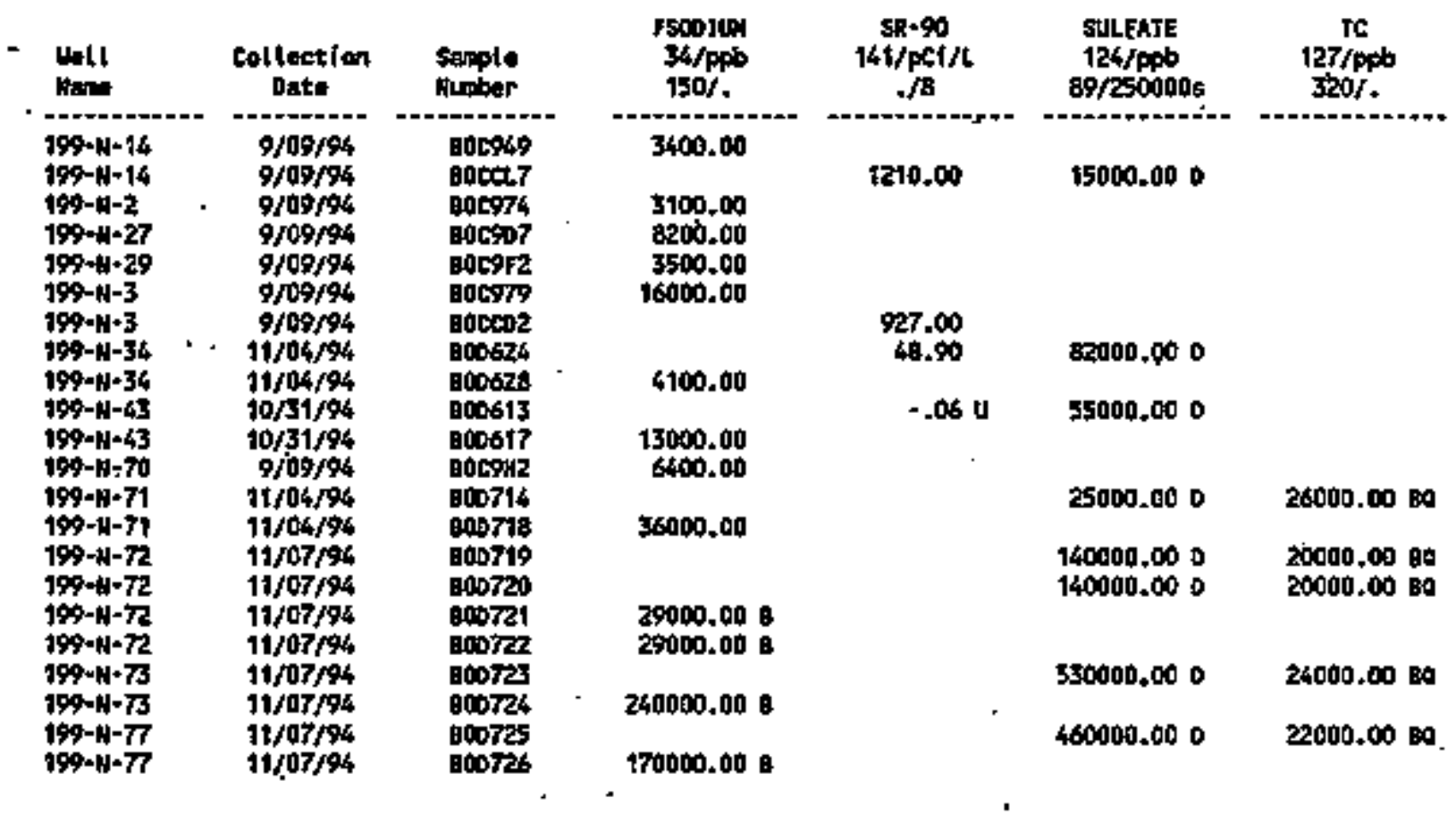


Table 4-4. Constituents with at Least One Detected Value for the 100-N RCRA Sttes Data for Reporting Period October 1 through Decenber 31, 1994. (sheet 9 of 9 )

\begin{tabular}{|c|c|c|c|c|c|c|}
\hline thell & $\begin{array}{c}\text { Coll betion } \\
\text { Date }\end{array}$ & $\begin{array}{l}\text { Semplo } \\
\text { Muniser }\end{array}$ & $\begin{array}{c}105 \\
65 / 0=0 \\
10 / 500 s\end{array}$ & $\begin{array}{c}\text { tRtT1til } \\
\text { 142/pci/l } \\
. / 20000\end{array}$ & $\begin{array}{c}\text { roago } \\
\text { 126/15iv } \\
.016 / .\end{array}$ & $\begin{array}{c}\text { URA!HIUM } \\
\text { 145/ppb } \\
+/ .\end{array}$ \\
\hline 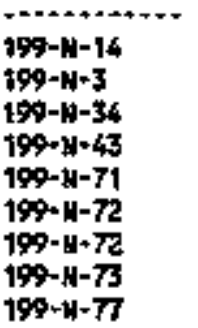 & $\begin{array}{l}9 / 09 / 94 \\
9 / 09 / 94 \\
11 / 04 / 94 \\
10 / 31 / 94 \\
11 / 04 / 94 \\
11 / 07 / 94 \\
11 / 07 / 94 \\
11 / 07 / 94 \\
11 / 07 / 94\end{array}$ & 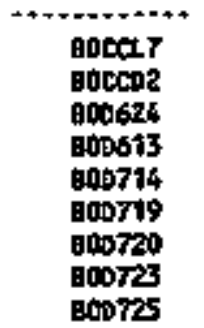 & $\begin{array}{l} \\
190.00 \\
380.00 \\
370.00 \\
910.00 \\
800.00\end{array}$ & $\begin{array}{l}63100.00 \\
20800.00 \\
23900.00 \\
29600.00\end{array}$ & $\begin{array}{r}2.10 \\
2.10 \\
9.100 \\
9.309 \\
14.000 \\
.730\end{array}$ & .32 \\
\hline
\end{tabular}

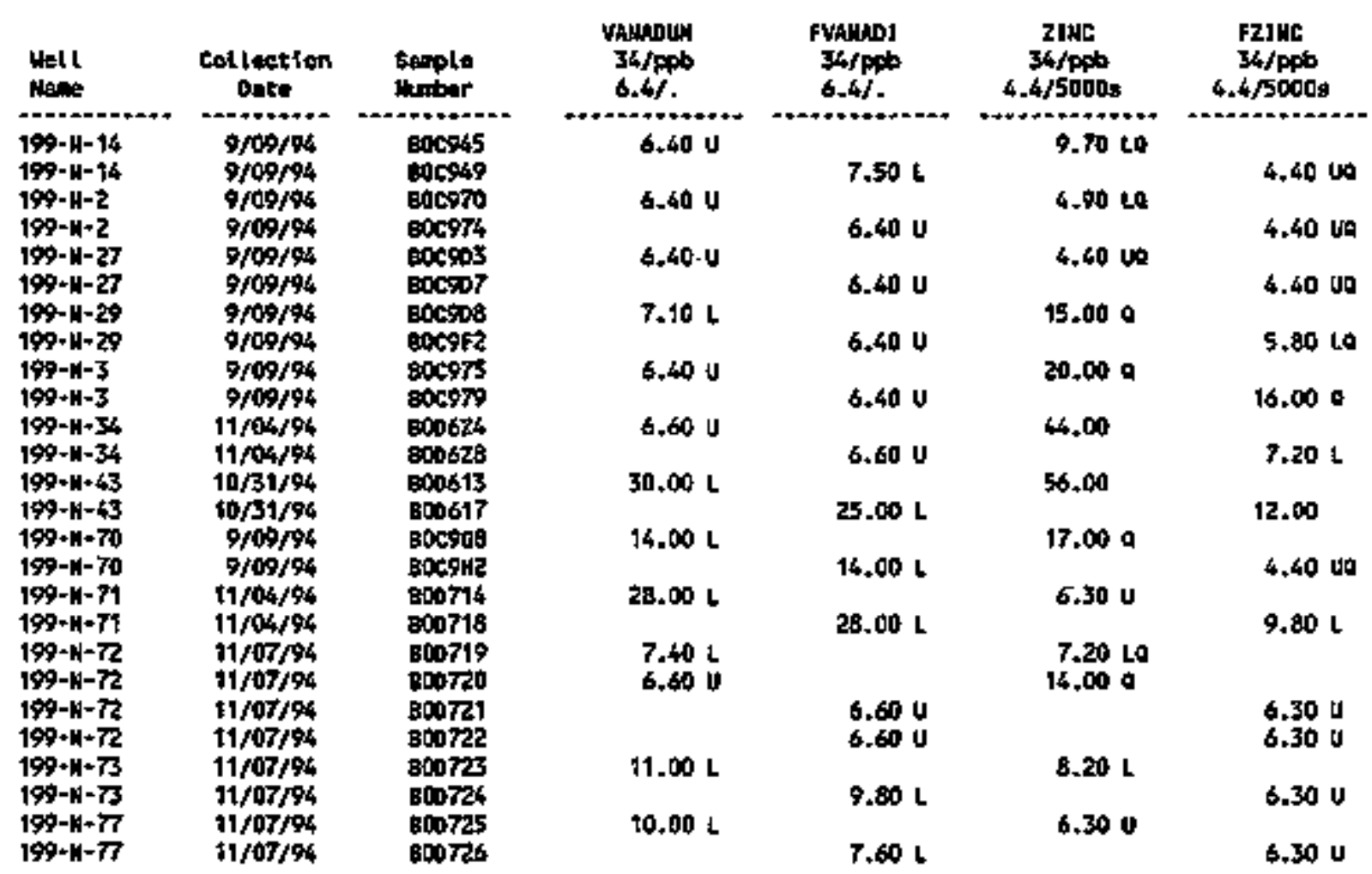

For explenstion of this toble, ses setion 1.4 of report. 
Table 4-5. Contamination Indicator Parameters for the 100-N RCRA Sites Data for Reporting Period October 1 through December 31, 1994. (sheet 1 of 1 )

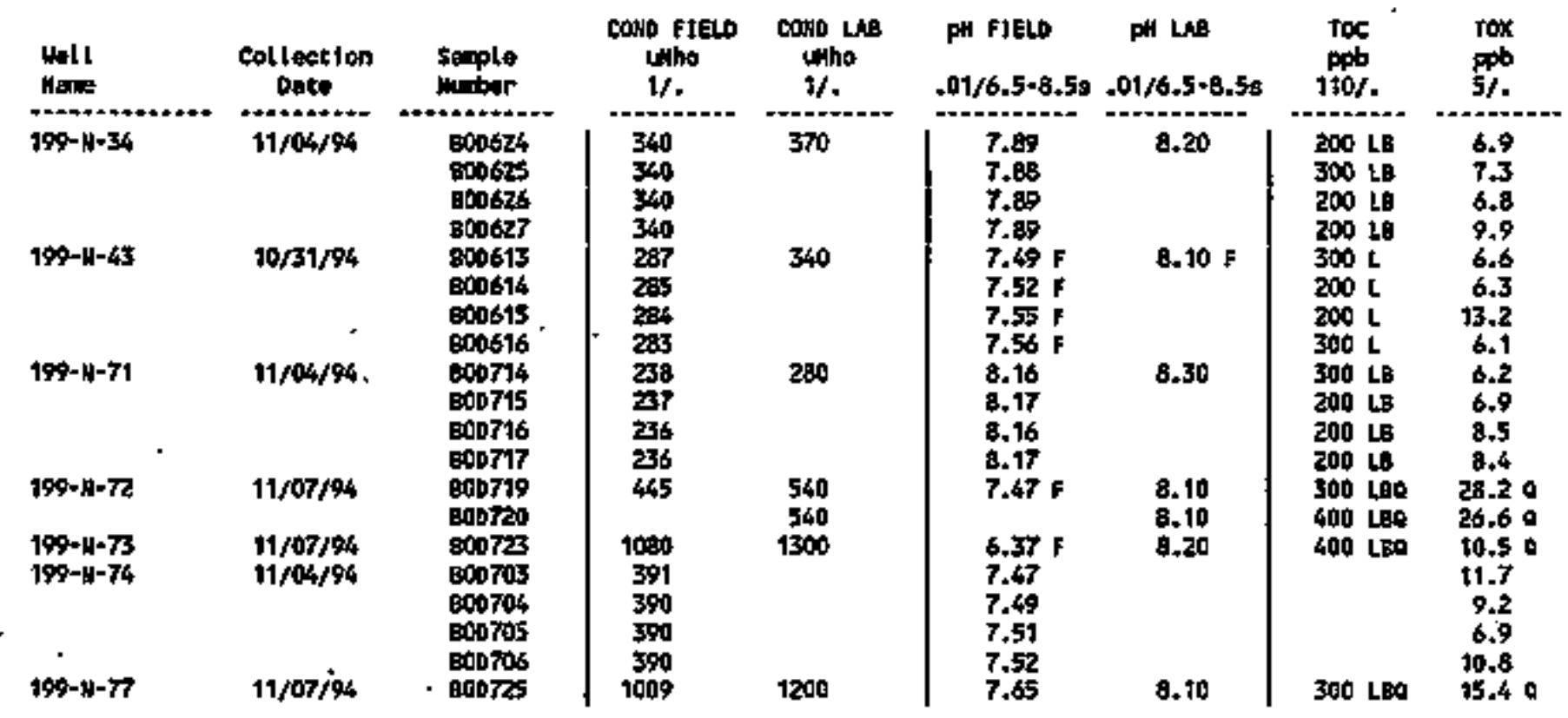

far explenstion of this table, ses section 1.4 of report. 
Table 4-6. Drinking Water Standards Exceeted for 100-N Area RCRA Sites, Fourth Quarter 1994.

\begin{tabular}{|c|c|}
\hline Constituent (Dis) & $\begin{array}{l}\text { Wells exceeding DUS } \\
\text { (number of samples) }\end{array}$ \\
\hline Field ph $(6.5-8.5)$ & $N-73^{a}$ \\
\hline Chromium, unfiltered samples (50 ppb) & $\begin{array}{l}1324-N / M A: N-72 . \\
1325-N: N-70\end{array}$ \\
\hline Chromium, filtered samples (50 ppb) & none. \\
\hline Iron, unfiltered samples (300 ppb) & $\begin{array}{l}1301-N: N-14 \\
1324-N / N A: N-72(2), N-73 \\
1325-N: N-43, N-70\end{array}$ \\
\hline Iron, filtered sample $(300 \mathrm{ppb})$ & none. \\
\hline Ruthen jum-106 (30 pCi/L) & 1301-N: $\mathrm{N}-3$. \\
\hline Sulfate $(250,000 \mathrm{ppb})$ & 1324-N/NA: $\mathrm{N}-73, \mathrm{~N}-77$. \\
\hline Strontium-90 (8 $\mathrm{pCi} / \mathrm{L})$ & $1301-\mathrm{N}: \mathrm{N}-14, \mathrm{~N}-3, \mathrm{~N}-34$ \\
\hline Tritfum $(20,000 \mathrm{pCl} / \mathrm{L})$ & $\begin{array}{l}1301-\mathrm{N}: \mathrm{N}-14, \mathrm{~N}-3, \mathrm{~N}-34 \\
1325-\mathrm{N}: \mathrm{N}-43 .\end{array}$ \\
\hline
\end{tabular}
submitted.

'Data are in question. Requests for Aralytical Data Evaluation were

DWS = drinking water standard. 
Tabie 4-7. Requests for Ana7yt1ca] Data Evaituation for the 100-A RCRA Sites, Fourth Quarter 1994.

\begin{tabular}{|c|c|c|c|c|}
\hline Well no. & $\begin{array}{c}\text { Sample } \\
\text { date }\end{array}$ & \multicolumn{1}{|c|}{ Constftuent } & \multicolumn{2}{|c|}{ Reason } \\
\hline \multicolumn{3}{|c|}{ 1325-N LWDF } \\
\hline $\mathrm{N}-43$ & $10 / 31 / 94$ & $\mathrm{pH}$ (1ab and f1e1d) & $\begin{array}{l}\text { Lab and field values do not } \\
\text { agree. } \begin{array}{l}\text { Insufficient historical } \\
\text { data to judge which 1s more } \\
\text { representat fve. }\end{array}\end{array}$ \\
\hline $\mathrm{N}-72$ & $11 / 7 / 94$ & $\mathrm{pH}$ (field) & $\begin{array}{l}\text { lower than historical trend; } \\
\text { lower than iaboratory pH. }\end{array}$ \\
\hline$N-73$ & $11 / 7 / 94$ & $\mathrm{pH}$ (field) & $\begin{array}{l}\text { Lower than historical trend; } \\
\text { lower than laboratory pH. }\end{array}$ \\
\hline
\end{tabular}

LWDF = Liquid Waste Disposal Facjlity. 
DOE/RL-94-36-4 .

This page tntentionaliy left b7ank. 


\section{D0E/RL-94-36-4}

\section{CONTENTS}

$5.0 \quad 216-B-3$ POND SYSTEN $\ldots \ldots \ldots \ldots \ldots$. . . . . . . . .

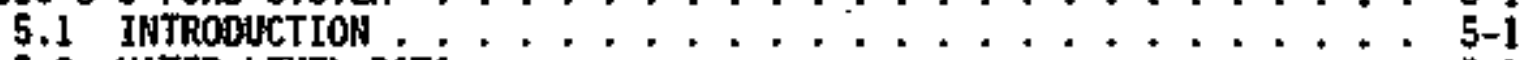

5.2 WATER LEVEL DATA $\ldots \ldots \ldots \ldots \ldots \ldots \ldots \ldots \ldots \ldots,{ }^{5-1}$

5.3 WATER CHEMISTRY DATA . . . . ............. 5-1 
DOE/RL-94-36-4

\section{LIST OF FIEURES}

5-1 216-8-3 Pond Groundwater Monftoring Hetwork . . . . . . . . 5-3

\section{LIST OF TABLES}

5-1 Monitoring Mell Purpose and Sampling Schedule for the 216-B-3 Pond Systen Hetwork . . . . . . . . . . . 5-4

5-2 RCRA Water Level Measurement Report 216-B-3 Pond System, Fourth Quarter $1994 \ldots . . \ldots$. . . . . . . . . . . . . . .

5-3 Constituent List and Sumpary of Results for the 216-8-3 Pond Data for Reporting Period October 1 through Decenber 31, 1994 . . . 5-9

5-4 Constituents with at Least One Detected Value for the 216-B-3 Pond Data for Reporting Period October 1 through December 31, 1994 . . . 5-12

5-5 Contamination Indicator Parameters for the 216-B-3 Pond Data for Reporting Perlod October 1 through December 31, 1994 . . . 5-16

5-6 Constituents Exceeding Drinking Water Standards for the 216-B-3 Pond System ................ 5-19 
DOE/RL-94-36-4

\title{
5.0 216-8-3 POND SYSTEM
}

\author{
D. B. Barnett \\ Westinghouse Hanford Company
}

\subsection{INTROOUCTION}

The 216-8-3 Pond System is located east of the 200 East Area and originally consisted of a main pond and three expansion ponds for wastewater disposal. Several ditches leading to the ponds and the main pond itself are no longer in use and are backfi11ed (Figures 1-1 and 5-1). These surface impoundments cover approximately 41.3 hectares (102 acres). Only the $3 \mathrm{C}$ expansion pond now receives wastewater. Table 5-1 lists the groundwater wells and their monitoring status.

The 216-B-3 Pond System groundwater monitoring me17 locations are shown in Figure 5-1. Detection monitoring began at the 216-B-3 Pond System in November 1988 and continued through June 1990, when assessment monitoring was scheduled to begin because of elevated levels of ToX in two-downgradient monitoring we11s (699-43-41E and 699-43-41F). Assessment monjtoring actually began in June 1991 when groundwater sampling on the Hanford Site resumed, following a hiatus, as described in the Groundwater Quality Assessment Plan for the 216-8-3 Pond Systen (Harris 1990). Thus far, no specific constituents have been denonstrably linked to the elevated ToX results.

\subsection{WATER LEVE DATA}

Water levels were measured monthly, and once during sample events, in all wells during the October through December 1994 pertod. Table 5-2 lists the results of the water level measurements. Results for some wells are outside of the expected range of water levels for those wells, and may be in error. The suspected measurements are in welis $299-\bar{E} 32-4,699-40-40 \mathrm{~B}, 699-41-4 Z$, 699-42-37, 699-42-40A, 699-43-416, and 699-43-42J.

\subsection{MATER CHEHISTRY DATA}

Al] weIls scheduled for the October through December 1994 sampling were sampled during October, except upgradient we11s 299-E18-1 and 299-E32-4, which were sampted in December. Any results for the pertod that are unavailable as of the writing of this report will be reported in future guarterly reports.

The $l$ ist of constituents for which data were received and the sumary of results availabje are given in Table 5-3. The results of the available analyses, for constituents with at least one value above the MDL, are reported in Table 5-4. Results of analyses for CIPs are reported in Table 5-5. Explanations of data flags and qualifiers are given in Section 1.4:

Constituents exceeding OUS during the October through December 1994 period are listad in Table 5-6. The constituent list for this quarter 
inciades those requirod by regulations and compounds under investigation for links to TOX occurrence. Groundwater manttoring requirements and strategy for the 216-8-3 Pond System are described by Sweeney (1995).

A RADE has been submitted for a result of $24 \mathrm{ppb}$ diethyl phthalate in well 699-40-40B. This compound has not been previously detected in this well. This well also produced an elevated average result for ToX, but the diethyl phthalate cannot be directly correlated to ToX occurrence. A companion well, 699-40-40A, produced a result of $7.2 \mathrm{ppb}$ of tris-2-chloroethyl phosphate. A RADE was also subuitted for the trittum result in upgradient well 299-E18-1. This result is ten times higher than any previous tritium results' from this we11 and may be erroneous. 


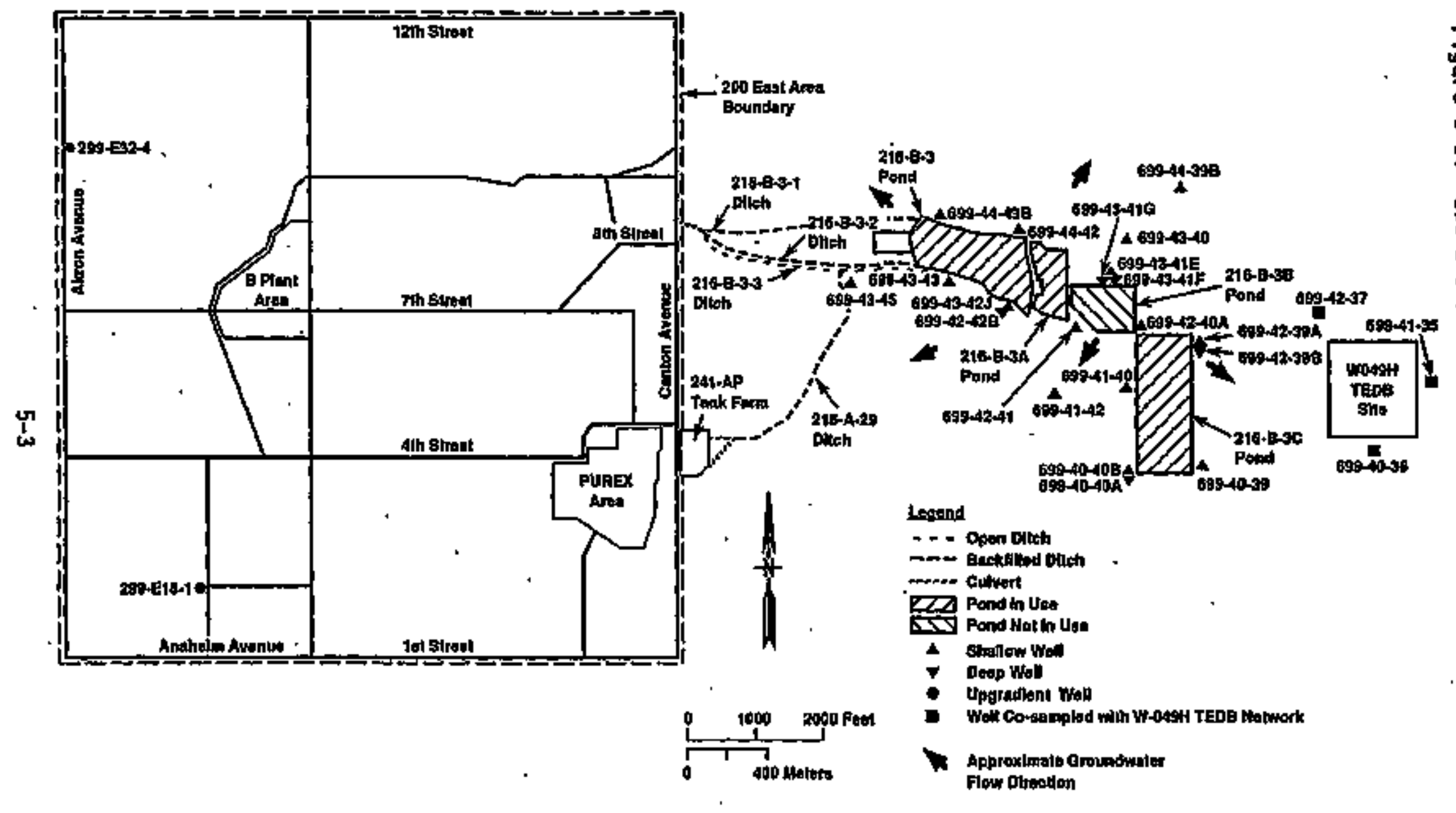

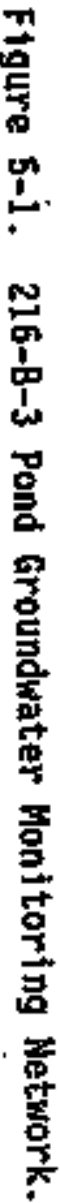

Hassatation 
Table 5-1. Honitoring Welt Purpose and Sampling Schedule for the 216-B-3 Pond System Network.

\begin{tabular}{|c|c|c|c|c|}
\hline Wel] no. & $\begin{array}{l}\text { Relative } \\
\text { position }\end{array}$ & Hydrogeologic unjt & $\begin{array}{l}\text { Sample } \\
\text { frequency }\end{array}$ & $\begin{array}{l}\text { Sample date, } \\
\text { 4th Qtr } 1994\end{array}$ \\
\hline 299-E18-1 & Upgradient & Hanford: Water Tab]e & Quarter]y & $12 / 01 / 94$ \\
\hline $299-E 32-4^{6}$ & Upgradient & Hanford: Water Table & Quarteriy & $12 / 13 / 94$ \\
\hline $699-40-36^{\circ}$ & Downgradient & Ringold: Top of Confined & Quarter]y & $10 / 27 / 94$ \\
\hline $699-40-39$ & Downgradient & R1ngold: Upper Confined & Quarter]y & $10 / 25 / 94$ \\
\hline $699-40-40 A$ & Downgradient & Ringold: Lower Confined & Quarteriy & $10 / 12 / 94$ \\
\hline $699-40-40 \mathrm{~B}$ & Downgradient & Ringold: Upper Confined & Quarterly & $10 / 14 / 94$ \\
\hline $699-41-35^{\circ}$ & Downgradient & Ringold: Top of Confined & Quarterly & $10 / 27 / 94$ \\
\hline $699-41-40$ & Downgradient & Ringold: Upper Confined & Quarterly & $10 / 24 / 94$ \\
\hline $699-41-42$ & Downgradient & Ringold: Top of Confined & Quarterly & $10 / 20 / 94$ \\
\hline $699-42-37^{c}$ & Downgradient & Ringold: Top of Confined & Quarterly & $10 / 27 / 94$ \\
\hline $699-42-39 A$ & Downgradient. & Ringold: Upper Confined & Quarteriy & $10 / 12 / 94$ \\
\hline $699-42-39$ & Downgradient & Ringold: Lower Conftined & Quarteriy & $10 / 12 / 94$ \\
\hline $699-42-40 A$ & Downgradient & Ringold: Upper Confined & Quarterly & $10 / 24 / 94$ \\
\hline $699-42-41$ & Downgradient & Ringold: Hater Table & Quarterly & $10 / 19 / 94$ \\
\hline $699-42-42 \mathrm{~B}$ & Downgradient & Ringold: Lower Unconfined & Quarteriy & $10 / 20 / 94$ \\
\hline $699-43-40$ & Downgradient & Ringold: Water Table & Quarteriy & $10 / 19 / 94$ \\
\hline $699-43-41 \mathrm{E}$ & Downgradient & R1ngold: Upper Confined & Quarterly & $10 / 19 / 94$ \\
\hline $699-43-41 F$ & Downgradient & Ringold: Lower Confined & Quarter7y & $10 / 20 / 94$ \\
\hline $699-43-416$ & Downgradient & Ringold: $\begin{array}{l}\text { Bottam of } \\
\text { Conflned }\end{array}$ & Quarterly & $10 / 19 / 94$ \\
\hline $699-43-42 J$ & Downgradient & Ringold: Water Table & Quarteriy & $10 / 20 / 94$ \\
\hline $699-43-43^{d}$ & Downgradient. & Hanford/Ringold: Water Table & Quarterly & $10 / 06 / 94$ \\
\hline $699-43-45^{d}$ & Downgradient & Hanford/Ringold: Water Table & quarterly & $10 / 06 / 94$ \\
\hline $699-44-398$ & Downgradjent & Ringold: Water Table & Quarter]y & $10 / 20 / 94$ \\
\hline $699-44-42$ & Downgradiant & Ringold: Water Tabla & Quarteriy & $10 / 20 / 94$ \\
\hline $699-44-43 B$ & bowngradient & Ringold: Water Table & Quarterly & $10 / 21 / 94$ \\
\hline
\end{tabular}

Well shared with the 2101-M Pond network.

Well shared with the Low-Level Haste Burial Grounds network.

Wells shared with the W-049H Treated Effluent Disposal Basin network.

We1 is shared with the 216-A-29 Ditch network. 
Table 5-2. RCRA Water Level Measurement Report. 216-8-3 Pond System, Fourth Quarter 1994.

(sheet 1 of 4)

\begin{tabular}{|c|c|c|c|c|c|c|}
\hline Weit & $\cdot$ & Date & $\begin{array}{l}\text { Depth to } \\
\text { water (ft) }\end{array}$ & & $\begin{array}{l}\text { Water } \\
\text { elevation } \\
\text { (ft) }\end{array}$ & $\begin{array}{l}\text { level } \\
\text { above msl } \\
\text { (m) }\end{array}$ \\
\hline 299-El8-1 & & $\begin{array}{l}10 / 27 / 94 \\
11 / 28 / 94 \\
12 / 01 / 94 \\
12 / 15 / 94\end{array}$ & $\begin{array}{l}318.89 \\
318.85 \\
318.99 \\
318.67\end{array}$ & ז & $\begin{array}{l}401.35 \\
401.39 \\
401.25 * \\
401.57\end{array}$ & $\begin{array}{l}122.33 \\
122.34 \\
122.30 \\
122.40\end{array}$ \\
\hline 299-E32-4 & & $\begin{array}{l}10 / 11 / 94 \\
12 / 13 / 94 \\
12 / 14 / 94\end{array}$ & $\begin{array}{l}284.79 \\
284.93 \\
284.53\end{array}$ & & $\begin{array}{l}401.09{ }^{\star} \\
400.95 *+ \\
401.35\end{array}$ & $\begin{array}{l}122.25 \\
122.21 \\
122.33\end{array}$ \\
\hline $699-40-36$ & & $\begin{array}{l}10 / 24 / 94 \\
10 / 27 / 94 \\
11 / 17 / 94 \\
12 / 12 / 94\end{array}$ & $\begin{array}{l}118.17 \\
117.90 \\
117.95 \\
118.03\end{array}$ & & $\begin{array}{l}410.75 \\
411.02^{\star} \\
410.97 \\
410.89\end{array}$ & $\begin{array}{l}125.20 \\
125.28 \\
125.26 \\
125.24\end{array}$ \\
\hline $699-40-39$ & & $\begin{array}{l}10 / 24 / 94 \\
10 / 25 / 94 \\
11 / 17 / 94 \\
12 / 12 / 94\end{array}$ & $\begin{array}{l}129.23 \\
129.14 \\
128.96 \\
129.29\end{array}$ & & $\begin{array}{l}412.61 \\
412.70^{*} \\
412.88 \\
412.55\end{array}$ & $\begin{array}{l}125.76 \\
125.79 \\
125.85 \\
125.75\end{array}$ \\
\hline $699-40-40 A$ & & $\begin{array}{l}10 / 12 / 94 \\
10 / 24 / 94 \\
11 / 17 / 94 \\
12 / 12 / 94\end{array}$ & $\begin{array}{l}129.87 \\
129.70 \\
129.50 \\
129.93\end{array}$ & & $\begin{array}{l}411.34 * \\
411.51 \\
411.71 \\
411.28\end{array}$ & $\begin{array}{l}125.38 \\
125.43 \\
125.49 \\
125.36\end{array}$ \\
\hline $699-40-40 B$ & . & $\begin{array}{l}10 / 14 / 94 \\
10 / 24 / 94 \\
11 / 17 / 94 \\
12 / 12 / 94\end{array}$ & $\begin{array}{l}129.45 \\
130.35 \\
130.16 \\
130.61\end{array}$ & $\cdot$ & $\begin{array}{l}412.73^{*}+ \\
411.83 \\
412.02 \\
411.57\end{array}$ & $\begin{array}{l}125.80 \\
125.53 \\
125.58 \\
125.45\end{array}$ \\
\hline $699-41-35$ & & $\begin{array}{l}10 / 24 / 94 \\
10 / 27 / 94 \\
11 / 17 / 94 \\
12 / 12 / 94\end{array}$ & $\begin{array}{r}108.66 \\
108.30 \\
108.37 \\
108.44\end{array}$ & & $\begin{array}{l}411.72 \\
412.08^{\star} \\
412.01 \\
411.94\end{array}$ & $\begin{array}{l}125.49 \\
125.60 \\
125.58 \\
125.56\end{array}$ \\
\hline $699-41-40$ & & $\begin{array}{l}10 / 24 / 94 \\
10 / 24 / 94 \\
11 / 17 / 94 \\
12 / 12 / 94\end{array}$ & $\begin{array}{l}130.17 \\
130.15 \\
129.93 \\
130.53\end{array}$ & & $\begin{array}{l}415.77 \\
415.79^{*} \\
416.01 \\
415.41\end{array}$ & $\begin{array}{l}126.73 \\
126.73 \\
126.80 \\
126.62\end{array}$ \\
\hline $699-41-42$ & & $\begin{array}{l}10 / 20 / 94 \\
10 / 24 / 94 \\
11 / 17 / 94\end{array}$ & $\begin{array}{l}231.55 \\
232.44 \\
232.33\end{array}$ & & $\begin{array}{l}412.36^{*} t \\
411.47 \\
411.58\end{array}$ & $\begin{array}{l}125.69 \\
125.42 \\
125.45\end{array}$ \\
\hline
\end{tabular}


Table 5-2. RCRA Water Level Measurement Report. 216-B-3 Pond System, Fourth Quarter 1994.

(sheet 2 of 4 )

\begin{tabular}{|c|c|c|c|c|}
\hline We]l & Date & $\begin{array}{l}\text { Depth to } \\
\text { water (ft) }\end{array}$ & $\begin{array}{l}\text { Hater } \\
\text { elevation } \\
\text { (ft) }\end{array}$ & $\begin{array}{l}\text { leve1 } \\
\text { above ms1 } \\
\text { (m) }\end{array}$ \\
\hline $699-41-42$ & $12 / 12 / 94$ & 232.86 & 411.05 & 125.29 \\
\hline $699-42-37$ & $\begin{array}{l}10 / 24 / 94 \\
10 / 27 / 94 \\
11 / 17 / 94 \\
12 / 12 / 94\end{array}$ & $\begin{array}{l}104.55 \\
204.98 \\
104.20 \\
104.35\end{array}$ & $\begin{array}{l}414.85 \\
314.42^{*}+ \\
415.20 \\
415.05\end{array}$ & $\begin{array}{r}126.45 \\
95.84 \\
126.55 \\
126.51\end{array}$ \\
\hline $699-42-39 A$ & $\begin{array}{l}10 / 12 / 94 \\
10 / 24 / 94 \\
11 / 17 / 94 \\
12 / 12 / 94\end{array}$ & $\begin{array}{l}139.85 \\
138.54 \\
138.30 \\
138.59\end{array}$ & $\begin{array}{l}418.29 * \\
419.60 \\
419.84 \\
419.55\end{array}$ & $\begin{array}{l}127.49 \\
127.89 \\
127.97 \\
127.88\end{array}$ \\
\hline $699-42-39 B$ & $\begin{array}{l}10 / 12 / 94 \\
10 / 24 / 94 \\
11 / 17 / 94 \\
12 / 12 / 94\end{array}$ & $\begin{array}{l}139.75 \\
138.97 \\
138.71 \\
138.93\end{array}$ & $\begin{array}{l}418.57^{*} \\
419.35 \\
419.61 \\
419.39\end{array}$ & $\begin{array}{l}127.58 \\
127.82 \\
127.90 \\
127.83\end{array}$ \\
\hline $699-42-40 A$ & $\begin{array}{l}10 / 24 / 94 \\
10 / 24 / 94 \\
11 / 17 / 94 \\
12 / 12 / 94\end{array}$ & $\begin{array}{l}123.64 \\
123.28 \\
122.58 \\
123.91\end{array}$ & $\begin{array}{l}421.89 *^{+} \\
422.25 \\
422.95 \\
421.62\end{array}$ & $\begin{array}{l}128.59 \\
128.70 \\
128.92 \\
128.51\end{array}$ \\
\hline $699-42-41$ & $\begin{array}{r}10 / 19 / 94 \\
10 / 24 / 94 \\
11 / 17 / 94 \\
12 / 12 / 94\end{array}$ & $\begin{array}{l}146.38 \\
146.67 \\
146.13 \\
147.07\end{array}$ & $\begin{array}{l}420.92^{*} \\
420.63 \\
421.17 \\
420.23\end{array}$ & $\begin{array}{l}12 \mathrm{~B} .30 \\
128.21 \\
128.37 \\
128.09\end{array}$ \\
\hline $699-42-426$ & $\begin{array}{l}10 / 20 / 94 \\
10 / 24 / 94 \\
11 / 17 / 94 \\
12 / 12 / 94\end{array}$ & $\begin{array}{l}166.50 \\
166.85 \\
166.71 \\
167.38\end{array}$ & $\begin{array}{l}416.73^{*} \\
416.38 \\
416.52 \\
415.85\end{array}$ & $\begin{array}{l}127.02 \\
126.91 \\
126.96 \\
126.75\end{array}$ \\
\hline $699-43-40$ & $\begin{array}{l}10 / 19 / 94 \\
10 / 24 / 94 \\
11 / 17 / 94 \\
12 / 12 / 94\end{array}$ & $\begin{array}{l}120.63 \\
120.91 \\
120.98 \\
121.61\end{array}$ & $\begin{array}{l}421.57^{\star} \\
421.29 \\
421.22 \\
420.59\end{array}$ & $\begin{array}{l}128.49 \\
128.41 \\
128.39 \\
128.20\end{array}$ \\
\hline $699-43-41 E$ & $\begin{array}{l}10 / 19 / 94 \\
10 / 24 / 94 \\
11 / 17 / 94 \\
12 / 12 / 94\end{array}$ & $\begin{array}{l}129.25 \\
129.90 \\
129.74 \\
130.33\end{array}$ & $\begin{array}{l}421.61 * \\
420.96 \\
421.12 \\
420.53\end{array}$ & $\begin{array}{l}128.51 \\
128.31 \\
128.36 \\
128.18\end{array}$ \\
\hline
\end{tabular}


Table 5-2. RCRA Hater Level Measurement Report 216-B-3 Pond System, Fourth Quarter 1994.

(sheet 3 of 4)

\begin{tabular}{|c|c|c|c|c|}
\hline Hel 1 & Date & $\begin{array}{l}\text { Depth to } \\
\text { water (ft) }\end{array}$ & $\begin{array}{l}\text { Hater } \\
\text { elevatjon } \\
\text { (tt) }\end{array}$ & $\begin{array}{l}\text { level } \\
\text { above ns] } \\
\text { (圆) }\end{array}$ \\
\hline $699-43-41 F$ & $\begin{array}{l}10 / 20 / 94 \\
10 / 24 / 94 \\
11 / 17 / 94 \\
12 / 12 / 94\end{array}$ & $\begin{array}{l}130.02 \\
129.98 \\
129.77 \\
130.40\end{array}$ & $\begin{array}{l}420.99 * \\
421.03 \\
421.24 \\
420.61\end{array}$ & $\begin{array}{l}128.32 \\
128.33 \\
128.39 \\
128.20\end{array}$ \\
\hline $699-43-416$ & $\begin{array}{r}10 / 19 / 94 \\
10 / 24 / 94 \\
11 / 17 / 94 \\
12 / 12 / 94\end{array}$ & $\begin{array}{l}133.67 \\
134.03 \\
134.02 \\
134.56\end{array}$ & $\begin{array}{l}417.67^{*}+ \\
417.31 \\
417.32 \\
416.78\end{array}$ & $\begin{array}{l}127.31 \\
127.20 \\
127.20 \\
127.03\end{array}$ \\
\hline $699-43-423$ & $\begin{array}{l}10 / 20 / 94 \\
10 / 24 / 94 \\
11 / 17 / 94 \\
12 / 12 / 94\end{array}$ & $\begin{array}{l}163.21 \\
163.70 \\
163.58 \\
164.34\end{array}$ & $\begin{array}{l}418.47^{*_{+}} \\
417.98 \\
418.10 \\
417.34\end{array}$ & $\begin{array}{l}127.55 \\
127.40 \\
127.44 \\
127.21\end{array}$ \\
\hline $699-43-43$ & $\begin{array}{l}10 / 06 / 94 \\
10 / 24 / 94 \\
11 / 17 / 94 \\
12 / 12 / 94\end{array}$ & $\begin{array}{l}164.75 \\
165.34 \\
165.28 \\
165.92\end{array}$ & $\begin{array}{l}414.62 \star \\
414.03 \\
414.09 \\
413.45\end{array}$ & $\begin{array}{l}126.38 \\
126.20 \\
126.21 \\
126.02\end{array}$ \\
\hline $\begin{array}{c}699-43-45 \\
.\end{array}$ & $\begin{array}{l}10 / 06 / 94 \\
10 / 24 / 94 \\
11 / 17 / 94 \\
12 / 12 / 94\end{array}$ & $\begin{array}{l}195.00 \\
195.01 \\
195.04 \\
195.17\end{array}$ & $\begin{array}{l}402.68 * \\
402.67 \\
402.64 \\
402.51\end{array}$ & $\begin{array}{l}122.74 \\
122.73 \\
122.72 \\
122.69\end{array}$ \\
\hline $699-44-39 B$ & $\begin{array}{l}10 / 20 / 94 \\
10 / 24 / 94 \\
11 / 17 / 94 \\
12 / 12 / 94\end{array}$ & $\begin{array}{l}91.46 \\
92.05 \\
92.01 \\
92.75\end{array}$ & $\begin{array}{l}421.94^{*}+ \\
421.35 \\
421.39 \\
420.65\end{array}$ & $\begin{array}{l}128.61 \\
128.43 \\
128.44 \\
128.21\end{array}$ \\
\hline $\begin{array}{c}699-44-42 \\
.\end{array}$ & $\begin{array}{l}10 / 20 / 94 \\
10 / 24 / 94 \\
11 / 17 / 94 \\
12 / 12 / 94\end{array}$ & $\begin{array}{l}159.00 \\
159.21 \\
159.13 \\
159.79\end{array}$ & $\begin{array}{l}420.22^{\circ} \\
420.01 \\
420.09 \\
419.43\end{array}$ & $\begin{array}{l}128.08 \\
128.02 \\
128.04 \\
127.84\end{array}$ \\
\hline $699-44-43 B$ & $\begin{array}{l}10 / 21 / 94 \\
10 / 24 / 94 \\
11 / 17 / 94 \\
12 / 12 / 94\end{array}$ & $\begin{array}{l}165.65 \\
165.83 \\
165.97 \\
166.20\end{array}$ & $\begin{array}{l}414.47^{*} \\
414.29 \\
414.15 \\
413.92\end{array}$ & $\begin{array}{l}126.33 \\
126.28 \\
126.23 \\
126.16\end{array}$ \\
\hline
\end{tabular}


Tab]e 5-2. RCRA Water Level Measurement Report 216-8-3 Pond System, Fourth Quarter 1994. (sheet 4 of 4 )

NOTES: 1. Water Tevel elevations are calculated by subtracting the measured depth-to-water from the surveyed elevation for the well.

2. Depth-to-water values are transeribed from fleld records.

3. Elevations marked with an $1 * 1$ were measured at the time of sampiting.

4. Elevation marked with a ' + ' are outside of the expected range, and are supected of error. 
Table 5-3. Constituent List and Summary of Results for the 216-B-3 Pond Data for Reporting Period October 1 through December 31, 1994.

(sheet 1 of 3 )

COMTAMIMATIOU IMDICAIOR PARNMETEAS

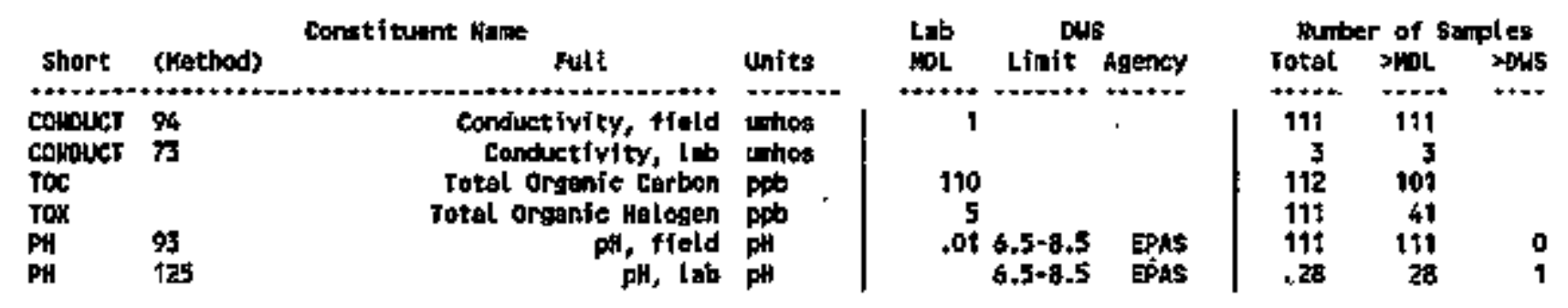

DR1NKIUS WATER PURAOTETERS

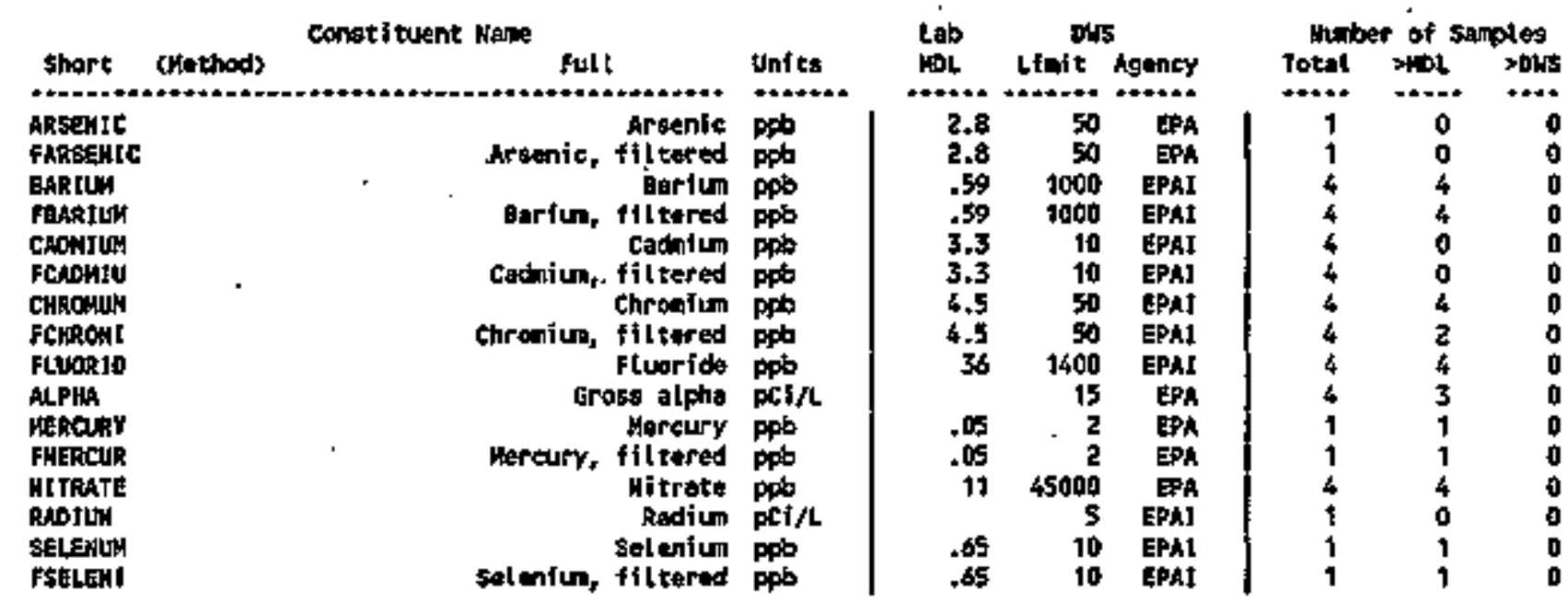

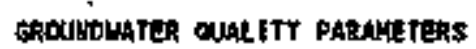

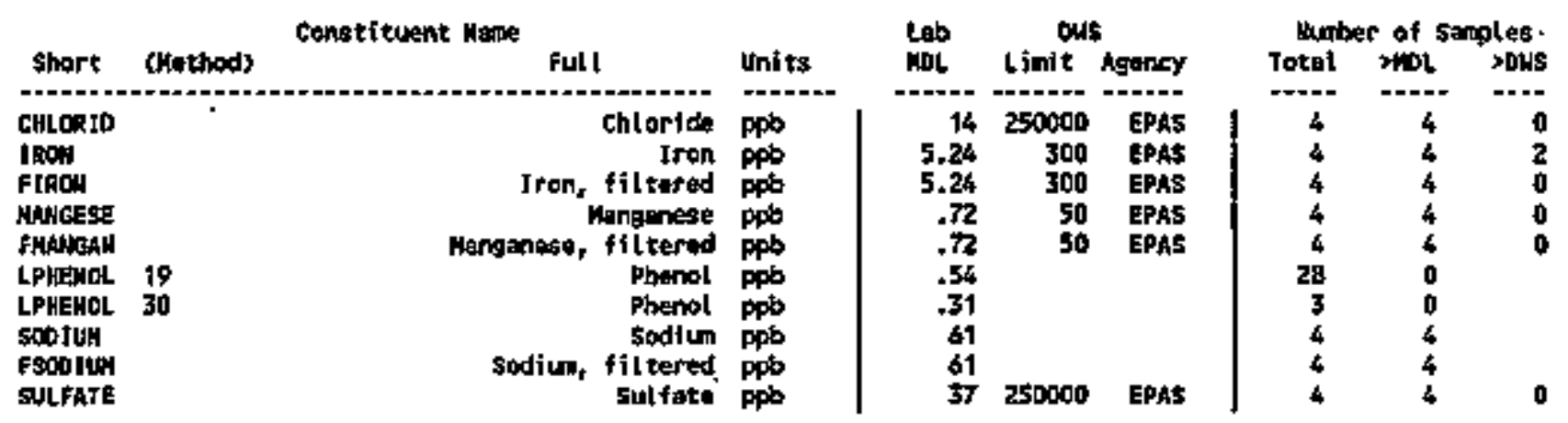


Table 5-3. Const1tuent Ltst and Summary of Results for the 216-B-3 Pond Data for Reporting Period October I through Decesuber 31, 1994.

(sheet 2 of 3 )

\$ITE SPECIf1C NH OTHER COWSTITUENTS

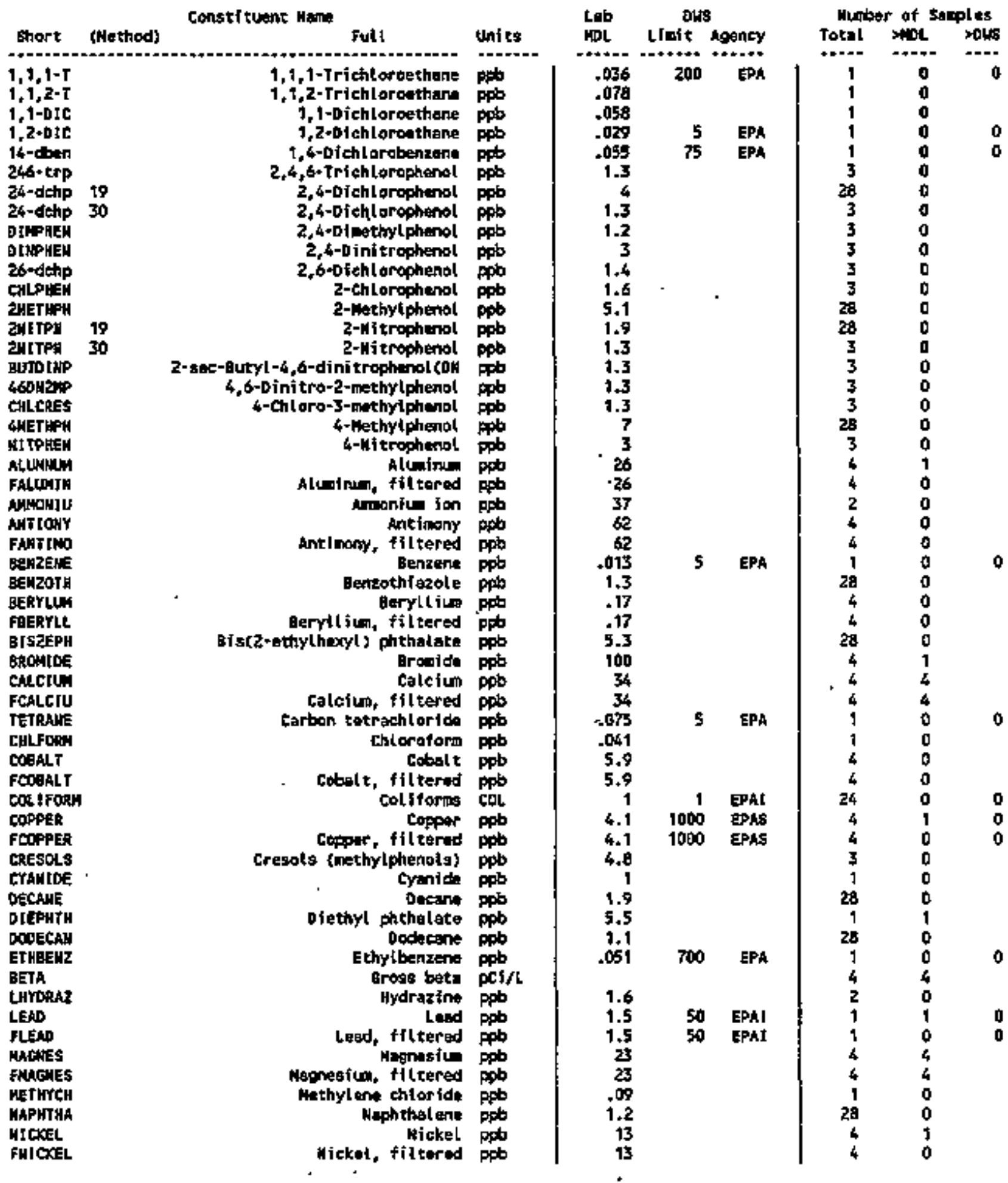


Table 5-3. Constituent List and Surmary of Results for the 216-B-3 Pond Data for Reporting Period October 1 through Decenber 31, 1994. (sheet 3 of 3 )

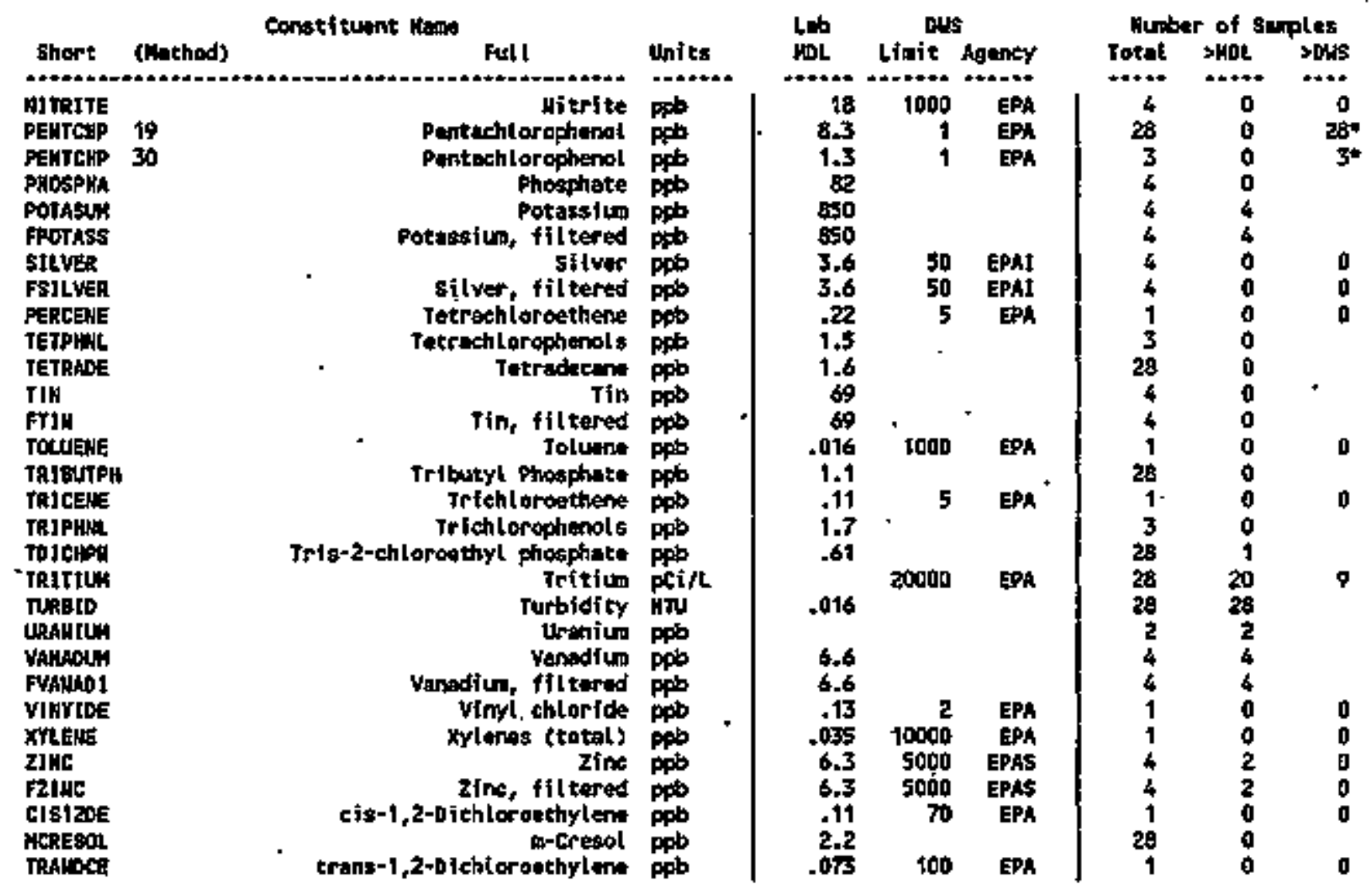

For explanation of this teble, see section $\mathbf{t . h}$ of report. 
Table 5-4. Constituents with at Least One Detected Value for the 216-B-3 Pond Data for Reporting Period October 1 through December 31, 1994. (sheet 1 of 4 )

\begin{tabular}{|c|c|c|}
\hline Well & $\begin{array}{c}\text { Coll lect ion } \\
\text { Daten }\end{array}$ & $\begin{array}{l}\text { semple } \\
\text { thuber }\end{array}$ \\
\hline $\begin{array}{l}299-E 18-1 \\
299-E 18-1 \\
299-E 32-4 \\
299-E 32-4 \\
699-43-43 \\
699-43-43 \\
699 \cdot 43-45 \\
699-43-45\end{array}$ & $\begin{array}{l}12 / 01 / 94 \\
12 / 01 / 94 \\
12 / 13 / 94 \\
12 / 13 / 94 \\
10 / 06 / 44 \\
10 / 06 / 94 \\
10 / 05 / 94 \\
10 / 06 / 94\end{array}$ & 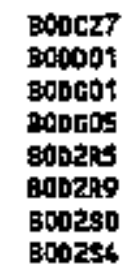 \\
\hline
\end{tabular}

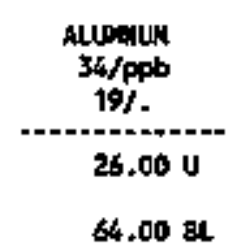

$26.00 \mathrm{U}$

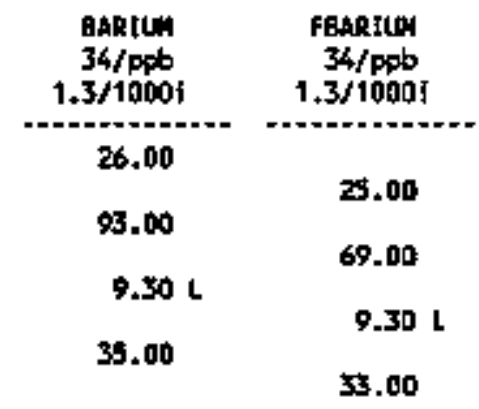

26.00 U

3.00

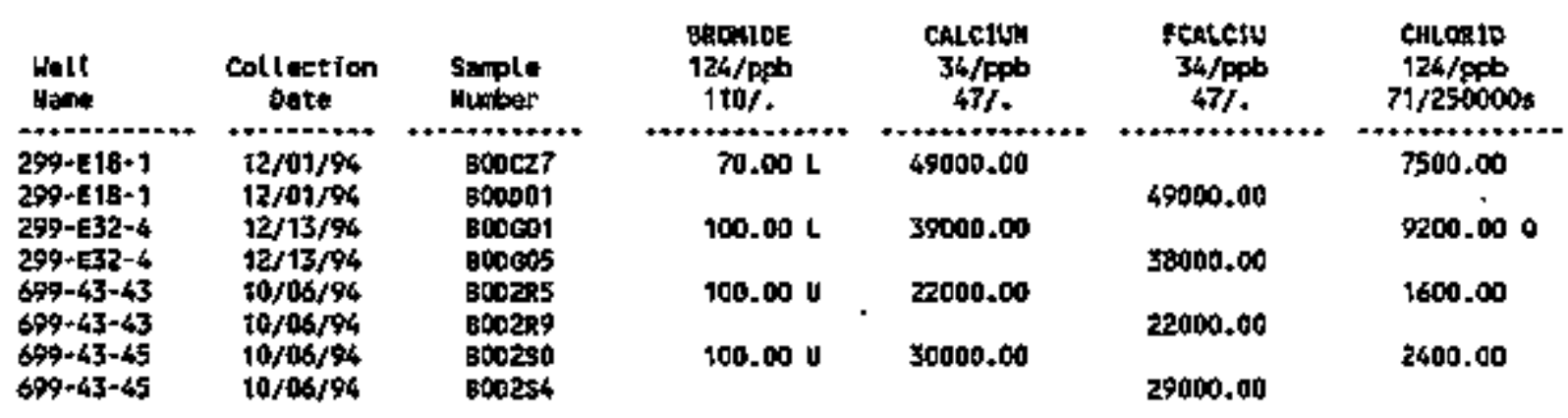

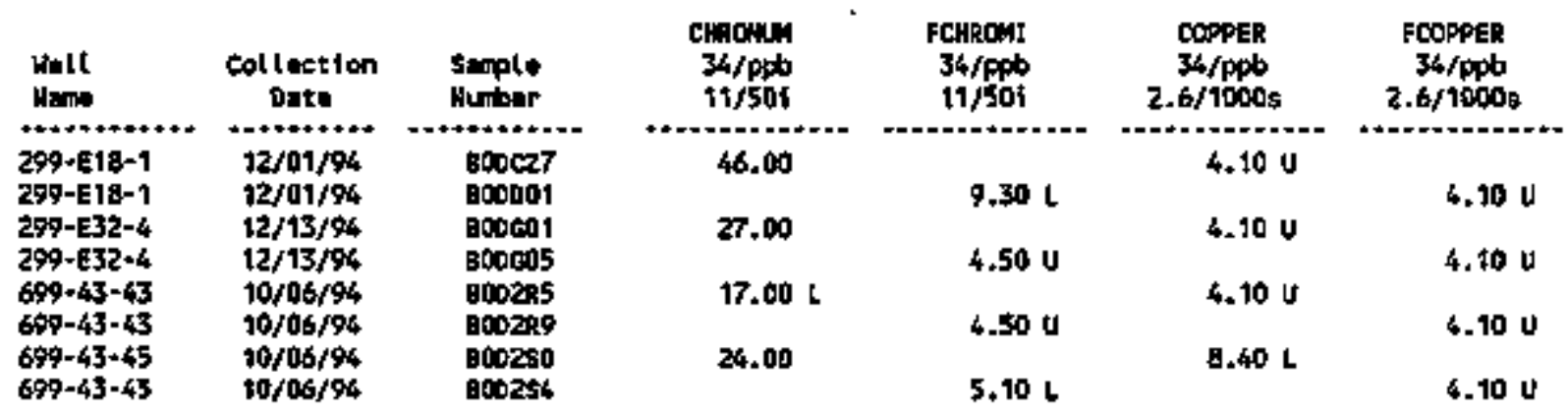

\begin{tabular}{|c|c|c|c|c|c|c|}
\hline Woll & $\begin{array}{c}\text { Eallection } \\
\text { Dete }\end{array}$ & $\begin{array}{l}\text { sanple } \\
\text { Mumber }\end{array}$ & $\begin{array}{l}\text { DIEPHTH } \\
19 / \mathrm{ppb} \\
5 / .\end{array}$ & $\begin{array}{l}\text { fluokto } \\
124 / \mathrm{ppb} \\
51 / 14001\end{array}$ & $\begin{array}{c}\text { ACFHA } \\
135 / \mathrm{ptI} / \mathrm{h} \\
. / 15\end{array}$ & $\begin{array}{c}\text { BETA } \\
136 / \mathrm{pCI} / \mathrm{L} \\
. /\end{array}$ \\
\hline $\begin{array}{l}299-E 18-1 \\
294-E 32-4 \\
699-40-40 B\end{array}$ & $\begin{array}{l}12 / 01 / 94 \\
12 / 13 / 94 \\
10 / 14 / 94\end{array}$ & $\begin{array}{l}\text { 800c27 } \\
\text { B00e01 } \\
\text { 800380 }\end{array}$ & 24.00 & $\begin{array}{l}600.00 \\
900.00\end{array}$ & $\begin{array}{l}4.33 \\
1.38\end{array}$ & $\begin{array}{r}8.93 \\
11.60\end{array}$ \\
\hline $\begin{array}{l}699-43-43 \\
699-43-45\end{array}$ & $\begin{array}{l}10 / 06 / 94 \\
10 / 06 / 94\end{array}$ & $\begin{array}{l}0002 \mathrm{k} 5 \\
000280\end{array}$ & & $\begin{array}{l}500.00 \\
700.00\end{array}$ & $2 . .32 \mathrm{v}$ & $\begin{array}{l}3.60 \\
3.41\end{array}$ \\
\hline
\end{tabular}


Table 5-4. Constituents with at Least One Detected Value for the 216-B-3 Pond Data for Reporting Perlod October 1 through December 31, 1994. (sheet 2 of 4 )

\begin{tabular}{|c|c|c|}
\hline $\begin{array}{l}\text { Matl } \\
\text { Mase }\end{array}$ & Collection & Segple \\
\hline $\begin{array}{l}299-18-1 \\
299-E 18-1 \\
799-632-4 \\
299-E 32-4 \\
699-43-43 \\
699-43-43 \\
699-43-45 \\
699-43-45\end{array}$ & $\begin{array}{l}12 / 01 / 94 \\
12 / 01 / 84 \\
12 / 13 / 94 \\
12 / 13 / 94 \\
10 / 06 / 94 \\
10 / 06 / 94 \\
10 / 06 / 94 \\
10 / 06 / 94\end{array}$ & 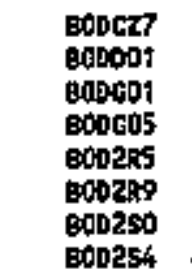 \\
\hline
\end{tabular}

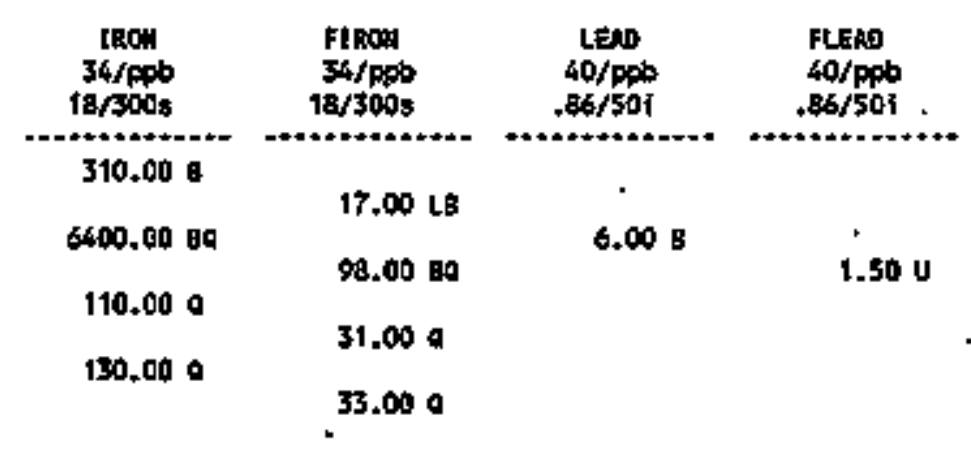

\begin{tabular}{|c|c|c|}
\hline Wetl & $\begin{array}{c}\text { Collection } \\
\text { Date }\end{array}$ & $\begin{array}{l}\text { 5erpl } \\
\text { thriber }\end{array}$ \\
\hline $\begin{array}{l}299-E 18-1 \\
299-E 18-1 \\
299-E 32-4 \\
299-E 32-4 \\
699-43-43 \\
699-43-43 \\
699-43-45 \\
699+43-45\end{array}$ & $\begin{array}{l}12 / 01 / 94 \\
12 / 01 / 94 \\
12 / 13 / 94 \\
12 / 13 / 94 \\
10 / 06 / 94 \\
10 / 06 / 94 \\
10 / 06 / 94 \\
10 / 06 / 94\end{array}$ & 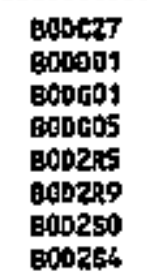 \\
\hline
\end{tabular}

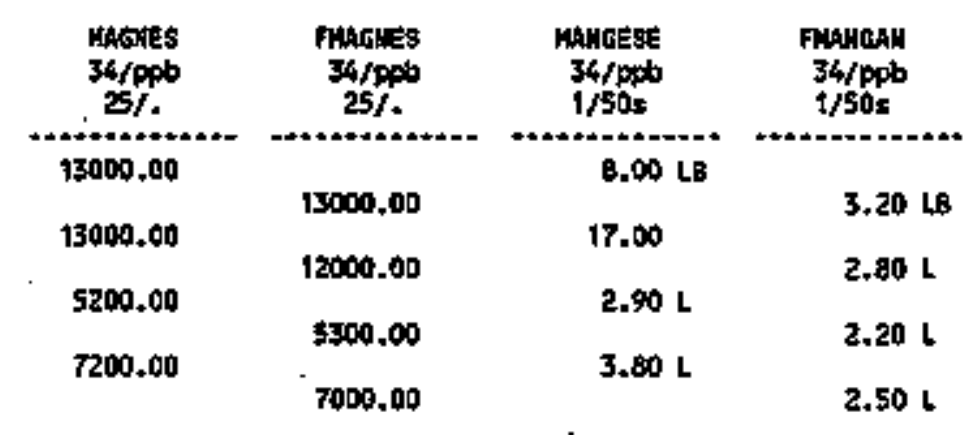

\begin{tabular}{|c|c|c|c|c|c|c|}
\hline \multirow[b]{2}{*}{ Watl } & & \multirow[b]{2}{*}{$\begin{array}{c}\text { HJCKEL } \\
\text { 34/ppb } \\
16 \%\end{array}$} & \multirow[b]{2}{*}{$\begin{array}{c}\text { FKICKEL } \\
34 / \mathrm{ppb} \\
16 / .\end{array}$} \\
\hline & $\begin{array}{c}\text { Callection } \\
\text { Date }\end{array}$ & $\begin{array}{l}\text { Salple } \\
\text { Wuber }\end{array}$ & $\begin{array}{l}\text { MERTRRY } \\
41 / p p b \\
.095 / 2\end{array}$ & $\begin{array}{l}\text { Trerour } \\
41 / \mathrm{ppb} \\
.095 / 2\end{array}$ & & \\
\hline $\begin{array}{l}299-E 18-1 \\
299-518-1 \\
299-832-4 \\
299-832-4 \\
699-43-43 \\
699-43-43 \\
699-43-45 \\
699-43-45\end{array}$ & $\begin{array}{l}12 / 01 / 94 \\
12 / 01 / 94 \\
12 / 13 / 94 \\
12 / 13 / 94 \\
10 / 06 / 94 \\
10 / 06 / 94 \\
10 / 06 / 94 \\
10 / 06 / 94\end{array}$ & 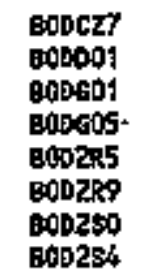 & $.11 \mathrm{gat}$ & .10 घLa & $\begin{array}{l}17.00 \mathrm{~L} \\
13.00 \mathrm{U} \\
13.00 \mathrm{v} \\
13.00 \mathrm{v}\end{array}$ & $\begin{array}{l}13.00 \mathrm{U} \\
13.00 \mathrm{U} \\
13.00 \mathrm{U} \\
\$ 3.00 \mathrm{U}\end{array}$ \\
\hline
\end{tabular}


Table 5-4. Constituents with at Least One Detected Value for the 216-B-3 Pond Data for Reporting Pariod Dctober 1 through December 31, 1994. (sheet 3 of 4 )

\begin{tabular}{|c|c|c|c|c|c|c|}
\hline $\begin{array}{l}\text { Wetl } \\
\text { Hame }\end{array}$ & $\begin{array}{l}\text { Collestion } \\
\text { Date }\end{array}$ & $\begin{array}{l}\text { Sample } \\
\text { Munbert }\end{array}$ & $\begin{array}{l}\text { UIJRATE } \\
124 / \mathrm{pPO} \\
96 / 45000\end{array}$ & $\begin{array}{l}\text { POTASUN } \\
34 / \mathrm{ppb} \\
890 / .\end{array}$ & $\begin{array}{l}\text { FPOTASS } \\
34 / \text { ppb } \\
\text { 990\%. }\end{array}$ & $\begin{array}{l}\text { SELEMDI } \\
48 / \mathrm{ppb} \\
1.4 / 10 \mathrm{i}\end{array}$ \\
\hline $\begin{array}{l}299-E 18-1 \\
299-E 18-1 \\
299-E 32-4 \\
299-E 32-4 \\
699-43-43 \\
699-43-43 \\
697-43-45 \\
699-43-45\end{array}$ & $\begin{array}{l}12 / 01 / 94 \\
12 / 01 / 94 \\
12 / 13 / 94 \\
12 / 13 / 94 \\
10 / 06 / 94 \\
10 / 06 / 94 \\
10 / 06 / 94 \\
10 / 06 / 94\end{array}$ & 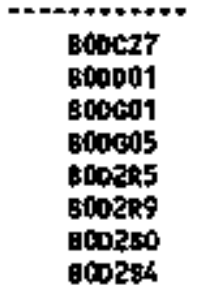 & $\begin{array}{l}14000.000 \\
27000.0009 \\
700.00 \\
1200.00\end{array}$ & $\begin{array}{l}5500.00 \\
6300.00 \\
4600.00 \\
6500.00\end{array}$ & $\begin{array}{l}5500,00 \\
6900,00 \\
4700,00 \\
400,00\end{array}$ & $4.40 \mathrm{~L}$ \\
\hline $\begin{array}{l}\text { Well } \\
\text { Wamm }\end{array}$ & $\begin{array}{c}\text { Collection } \\
\text { Datt }\end{array}$ & $\begin{array}{l}\text { Sanple } \\
\text { Nuber }\end{array}$ & $\begin{array}{l}\text { FSELEHA } \\
\text { 4E/ppb } \\
1,4 / 101\end{array}$ & $\begin{array}{l}500109 \\
34 / p 06 \\
150 / .\end{array}$ & $\begin{array}{l}\text { F500 14ג4 } \\
34 / p p b \\
150 / .\end{array}$ & $\begin{array}{c}\text { SHLLATE } \\
124 / \mathrm{gpb} \\
89 / 250000 \mathrm{~g}\end{array}$ \\
\hline $\begin{array}{l}299-E 18-1 \\
299-E 18-1 \\
299-532-4 \\
299-632-6 \\
697-43-43 \\
697-43-43 \\
699-43-45 \\
697-43-45\end{array}$ & $\begin{array}{l}12 / 01 / 94 \\
12 / 01 / 94 \\
12 / 13 / 94 \\
12 / 13 / 94 \\
10 / 06 / 94 \\
10 / 06 / 94 \\
10 / 06 / 94 \\
10 / 06 / 94\end{array}$ & 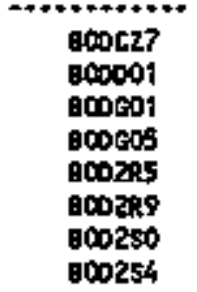 & $4.50 \mathrm{~L}$ & $\begin{array}{l}26000.00 \mathrm{~g} \\
26000.00 \mathrm{0} \\
5500.00 \\
8800.000\end{array}$ & $\begin{array}{l}* \ldots+\cdots \cdots+\cdots \\
26000.00 \mathrm{~s} \\
25000.00 \mathrm{a} \\
5500.00 \\
8600.00\end{array}$ & $\begin{array}{c}110000.000 \\
45000.000 \\
9900.00 \mathrm{D} \\
9700.00\end{array}$ \\
\hline
\end{tabular}

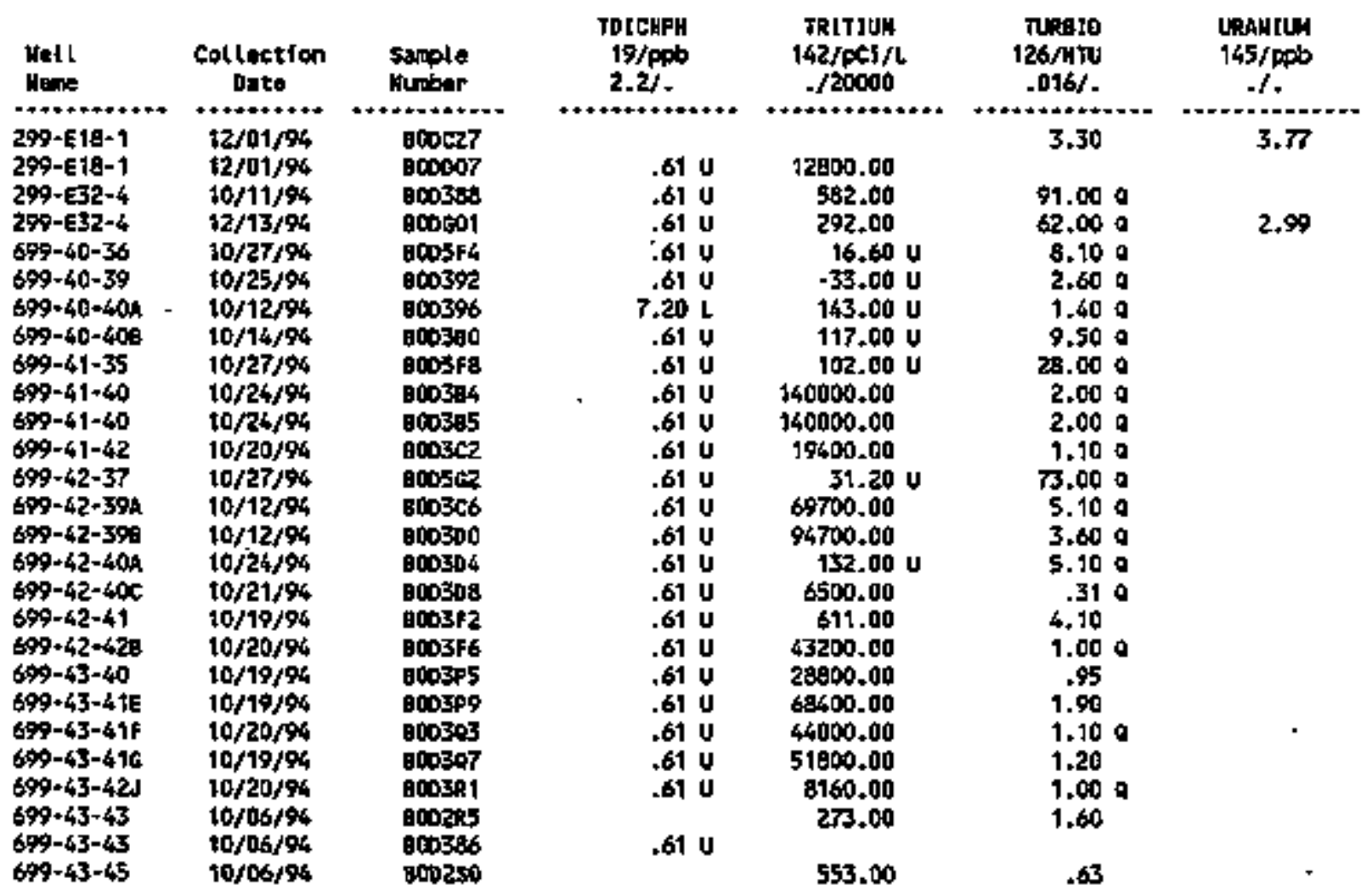


Table 5-4. Constituents with at Least One Detected Value for the 216-B-3 Pond Data for Reporting Period October 1 through December 31, 1994. (sheet 4 of 4 )

\begin{tabular}{|c|c|c|c|c|c|c|}
\hline $\begin{array}{l}\text { Wetl } \\
\text { Honle }\end{array}$ & $\begin{array}{l}\text { Col letetion } \\
\text { Date }\end{array}$ & $\begin{array}{l}\text { Sempl* } \\
\text { Mager }\end{array}$ & $\begin{array}{l}\text { TOtCGPH } \\
19 / \mathrm{ppl} \\
2.2 / .\end{array}$ & $\begin{array}{l}\text { TRLTILH } \\
142 / \% \mathrm{CI} / \mathrm{L} \\
. / 20000\end{array}$ & $\begin{array}{l}\text { TURAID } \\
\text { 126/11tu } \\
.016 / .\end{array}$ & $\begin{array}{c}\text { URAfitu } \\
145 / \mathrm{ppb} \\
.\end{array}$ \\
\hline $\begin{array}{l}699-63-45 \\
699+44-393 \\
697-44-42 \\
609-44-438\end{array}$ & $\begin{array}{l}10 / 05 / 94 \\
10 / 20 / 94 \\
10 / 20 / 94 \\
10 / 21 / 94\end{array}$ & $\begin{array}{l}\cos 387 \\
800375 \\
8003 \pi 9 \\
800353\end{array}$ & $\begin{array}{l}.61 \mathrm{y} \\
.61 \mathrm{U} \\
.61 \mathrm{U} \\
.61 \mathrm{U}\end{array}$ & $\begin{array}{c}-1 \% 6.00 \mathrm{U} \\
593.00 \\
15500.00\end{array}$ & $\begin{array}{r}1.700 \\
.649 \\
4.600\end{array}$ & \\
\hline $\begin{array}{l}\text { Hedt } \\
\text { Nemes }\end{array}$ & $\begin{array}{c}\text { Colletetion } \\
\text { Date }\end{array}$ & $\begin{array}{l}\text { 8exple } \\
\text { durber }\end{array}$ & $\begin{array}{l}\text { Vhildouls } \\
\text { 34/ppb } \\
6.4 / .\end{array}$ & $\begin{array}{l}\text { Fvallad } \\
34 / \mathrm{ppb} \\
6.4 /\end{array}$ & $\begin{array}{c}\text { 2IRC } \\
3 \% / \mathrm{ppb} \\
4,4 / 5000 \mathrm{~s}\end{array}$ & $\begin{array}{c}521 \mathrm{kx} \\
3 \$ / \mathrm{ppb} \\
4.4 / 5000 \mathrm{~s}\end{array}$ \\
\hline $\begin{array}{l}299 \cdot E 18-1 \\
299-E 18-1 \\
299-E 32-4 \\
299 \cdot E 32-4 \\
699-43-43 \\
699-43-45 \\
699 \cdot 43-45 \\
698-43-45\end{array}$ & $\begin{array}{l}12 / 01 / 94 \\
12 / 01 / 94 \\
12 / 13 / 94 \\
12 / 13 / 94 \\
10 / 06 / 94 \\
10 / 06 / 94 \\
10 / 06 / 94 \\
10 / 06 / 94\end{array}$ & 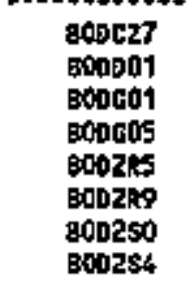 & $\begin{array}{l}9.00 \mathrm{~L} \\
39.00 \\
27.00 \mathrm{~L} \\
33.00\end{array}$ & $\begin{array}{l}11.00 \mathrm{~L} \\
19.00 \mathrm{~L} \\
26.00 \mathrm{~L} \\
29.00 \mathrm{~L}\end{array}$ & $\begin{array}{c}6.30 \mathrm{~V} \\
6.30 \mathrm{v} \\
21.00 \mathrm{v0} \\
21.00 \mathrm{g0}\end{array}$ & $\begin{array}{c}6.39 \mathrm{U} \\
6.30 \mathrm{y} \\
16.00 \mathrm{Bg} \\
17.00 \mathrm{Bd}\end{array}$ \\
\hline
\end{tabular}

For explention of this toble, see section 1.4 of report. 
Table 5-5. Contanination Indicator Parameters for the 216-8-3 Pond Data for Reporting Perlod October 1 through December 31, 1994. (sheet 1 of 3 )

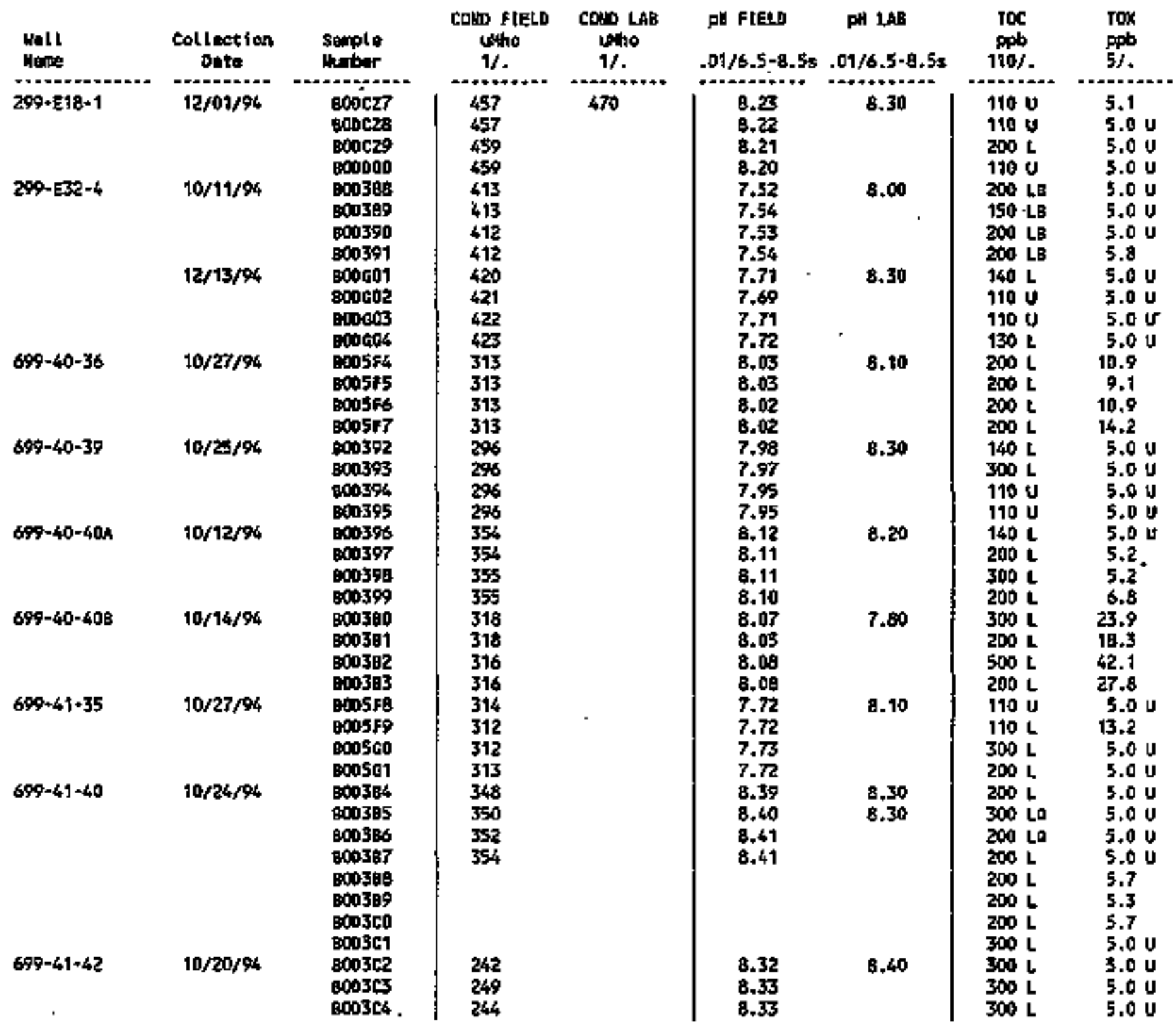


Table 5-5. Contamination Indicator Parameters for the 216-B-3 Pond Bata for Reporting Period October 1 through December 31, 1994.

(sheet 2 of 3 )

\begin{tabular}{|c|c|c|c|c|c|c|c|c|}
\hline $\begin{array}{l}\text { Noll } \\
\text { Mame }\end{array}$ & $\begin{array}{l}\text { Collection } \\
\text { Dote }\end{array}$ & $\begin{array}{l}\text { suple } \\
\text { number }\end{array}$ & $\begin{array}{c}\text { CON FIELO } \\
\text { Who } \\
1 \%\end{array}$ & $\begin{array}{l}\text { COND LAB } \\
\text { Whio } \\
1 \%\end{array}$ & $\begin{array}{l}\text { pis fiald } \\
.0 t / 6.5-B .5 s\end{array}$ & $\begin{array}{c}\text { PH LAB } \\
.01 / 6.5-8.5 s\end{array}$ & $\begin{array}{l}70 \mathrm{c} \\
\mathrm{ppb} \\
110 \%\end{array}$ & $\begin{array}{l}\mathrm{Ttsx} \\
\mathrm{ppb} \\
5 \%\end{array}$ \\
\hline $\begin{array}{l}699-41-42 \\
699-42-37\end{array}$ & $\begin{array}{l}10 / 20 / 98 \\
10 / 27 / 94\end{array}$ & $\begin{array}{l}600355 \\
000502 \\
000543 \\
000504 \\
000505\end{array}$ & $\begin{array}{l}246 \\
350 \\
349 \\
340 \\
347\end{array}$ & (. & $\begin{array}{l}8.33 \\
7.62 \\
7.64 \\
7.65 \\
7.66\end{array}$ & 8.70 & $\begin{array}{l}300 \mathrm{~L} \\
110 \mathrm{~L} \\
120 \mathrm{~L} \\
100 \mathrm{U} \\
110\end{array}$ & $\begin{array}{l}5.0 \mathrm{U} \\
5.0 \mathrm{U} \\
5.0 \mathrm{U} \\
7.4\end{array}$ \\
\hline $699-42-39 \mathrm{~A}$ & $10 / 12 / 94$ & $\begin{array}{l}\text { 1003c6 } \\
8003 c 7 \\
800368 \\
800309\end{array}$ & $\begin{array}{l}391 \\
37 \% \\
379 \\
390\end{array}$ & & $\begin{array}{l}7.55 \\
7.55 \\
7.54 \\
7.54\end{array}$ & 8.60 & $\begin{array}{l}200 \mathrm{~L} \\
200 \mathrm{~L} \\
200 \mathrm{~L} \\
200 \mathrm{~L}\end{array}$ & $\begin{array}{l}5.0 \mathrm{U} \\
5.0 \mathrm{U} \\
5.0 \mathrm{U} \\
5.0 \mathrm{U}\end{array}$ \\
\hline $699+42-39$ & $10 / 12 / 94$ & $\begin{array}{l}800300 \\
800301 \\
800302 \\
800503\end{array}$ & $\begin{array}{l}387 \\
389 \\
389 \\
397\end{array}$ & & $\begin{array}{l}7.76 \\
7.76 \\
7.77 \\
7.76\end{array}$ & 8.10 & $\begin{array}{l}200 \mathrm{~L} \\
360 \mathrm{~L} \\
300 \mathrm{~L} \\
200 \mathrm{~L}\end{array}$ & $\begin{array}{l}5.0 \mathrm{U} \\
5.0 \mathrm{U} \\
5.0 \mathrm{U} \\
5.0 \mathrm{U}\end{array}$ \\
\hline & $10 / 15 / 94$ & $\begin{array}{l}\text { 800300 } \\
000301 \\
000502 \\
0 \cos 303\end{array}$ & $\begin{array}{l}388 \\
388 \\
398 \\
389\end{array}$ & & $\begin{array}{l}7.76 \\
7.76 \\
7.77 \\
7.76\end{array}$ & 8.10 & $\begin{array}{l}200 \mathrm{~L} \\
300 \mathrm{~L} \\
300 \mathrm{~L} \\
200 \mathrm{~L}\end{array}$ & $\begin{array}{l}5.0 \mathrm{U} \\
5.0 \mathrm{U} \\
5.0 \mathrm{U} \\
5.0 \mathrm{U}\end{array}$ \\
\hline $690 \cdot 42 \cdot 40 \mathrm{k}$ & $10 / 24 / 94$ & $\begin{array}{l}000304 \\
8000305 \\
800306 \\
800307\end{array}$ & $\begin{array}{l}154 \\
155 \\
155 \\
134\end{array}$ & & $\begin{array}{l}8.23 \\
8.21 \\
8.20 \\
8.19\end{array}$ & 8.10 & $\begin{array}{l}600 \mathrm{~L} \\
600 \mathrm{~L} \\
600 \mathrm{~L} \\
700 \mathrm{~L}\end{array}$ & $\begin{array}{l}5.04 \\
5.6 \\
5.3\end{array}$ \\
\hline $690+42-40 \mathrm{C}$ & $10 / 21 / 94$ & 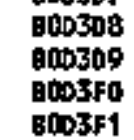 & $\begin{array}{l}342 \\
336 \\
334 \\
333\end{array}$ & & $\begin{array}{l}8.36 \\
8.28 \\
8.26 \\
\text { B.28 }\end{array}$ & 8.20 & $\begin{array}{l}200 \mathrm{~L} \\
200 \mathrm{~L} \\
200 \mathrm{~L} \\
200 \mathrm{~L}\end{array}$ & $\begin{array}{l}5.00 \\
5.0 \mathrm{U} \\
5.0 \mathrm{U} \\
5.0 \mathrm{U}\end{array}$ \\
\hline $699-42-41$ & $10 / 19 / 94$ & $\begin{array}{l}\text { B00352 } \\
\text { B003F3 } \\
B 00374 \\
800355\end{array}$ & $\begin{array}{l}150 \\
148 \\
148 \\
148\end{array}$ & & $\begin{array}{l}7.24 \\
7.26 \\
7.27 \\
7.31\end{array}$ & 8.10 & $\begin{array}{c}600 \mathrm{~L} \\
800 \mathrm{~L} \\
600 \mathrm{~L} \\
1000\end{array}$ & $\begin{array}{l}8.2 \\
8.3 \\
5.2 \\
5.00\end{array}$ \\
\hline$\triangle 99-42-42 B$ & $10 / 20 / 94$ & $\begin{array}{l}\text { B003F6 } \\
\text { Boosf7 } \\
600358 \\
\text { B003F9 }\end{array}$ & $\begin{array}{l}252 \\
252 \\
252 \\
252\end{array}$ & & $\begin{array}{l}7.97 \\
7.97 \\
7.97 \\
7.96\end{array}$ & 8.20 & $\begin{array}{l}400 \mathrm{~L} \\
200 \mathrm{~L} \\
200 \mathrm{~L} \\
200 \mathrm{~L}\end{array}$ & $\begin{array}{l}5.00 \\
5.00 \\
5.00 \\
5.00\end{array}$ \\
\hline $699-43-40$ & $10 / 18 / 94$ & $\begin{array}{l}\text { B003P5 } \\
\text { B003P6 } \\
8003 P 7 \\
\text { 8003P8 }\end{array}$ & $\begin{array}{l}244 \\
242 \\
242 \\
242\end{array}$ & & $\begin{array}{l}8.11 \\
8.11 \\
8.10 \\
8.03\end{array}$ & 8.10 & $\begin{array}{l}600 \mathrm{~L} \\
500 \mathrm{~L} \\
400 \mathrm{~L} \\
400 \mathrm{~L}\end{array}$ & $\begin{array}{l}9.9 \\
9.3 \\
7.8\end{array}$ \\
\hline $699 \cdot 43 \cdot 41 E$ & $10 / 19 / 94$ & $\begin{array}{l}B 003 P 9 \\
800390 \\
800301 \\
800302\end{array}$ & $\begin{array}{l}313 \\
312 \\
311 \\
3197\end{array}$ & & $\begin{array}{l}8.28 \\
8.26 \\
9.25 \\
8.24\end{array}$ & 8.60 & $\begin{array}{l}400 \mathrm{~L} \\
200 \mathrm{~L} \\
300 \mathrm{~L} \\
400 \mathrm{~L}\end{array}$ & $\begin{array}{r}5.5 \\
6.9 \\
6.6 \\
11.5\end{array}$ \\
\hline 699-43-41f & $10 / 20 / 96$ & $\begin{array}{l}000303 \\
100304\end{array}$ & 260 & & $\begin{array}{l}\text { B. } 43 \\
\text { B.15. }\end{array}$ & 8.20 & $\begin{array}{l}200 \mathrm{~L} \\
200 \mathrm{~L}\end{array}$ & $\begin{array}{l}5.7 \\
5.0 \mathrm{U}\end{array}$ \\
\hline
\end{tabular}


Table 5-5. Contantination Ind1cator Parameters for the 216-8-3 Pond Data for Reporting Perlod October 1 through December 31 , 1994.

(sheet 3 of 3 )

\begin{tabular}{|c|c|c|c|c|c|c|c|c|}
\hline $\begin{array}{l}\text { Notl } \\
\text { Uene }\end{array}$ & $\begin{array}{c}\text { Col lection } \\
\text { Dotes }\end{array}$ & $\begin{array}{l}\text { Salpiple } \\
\text { Nuber }\end{array}$ & $\begin{array}{c}\text { COND FJELD } \\
\text { Uitho } \\
1 / .\end{array}$ & $\begin{array}{c}\text { COMD LAB } \\
\text { who } \\
1 / .\end{array}$ & $\begin{array}{l}\text { PH FJELO } \\
.01 / 6.5-8.58\end{array}$ & $\begin{array}{l}\text { PA LAB } \\
.0 t / 6.5-8.5 \mathrm{~s}\end{array}$ & $\begin{array}{c}\text { TOC } \\
p p b \\
110 / .\end{array}$ & $\begin{array}{l}\text { Tox } \\
\text { ppob } \\
5 \%\end{array}$ \\
\hline $699+43-41 F$ & $10 / 20 / 94$ & $\begin{array}{l}600305 \\
000306\end{array}$ & $\begin{array}{l}81 \\
249\end{array}$ & & $\begin{array}{l}8.11 \\
8.11\end{array}$ & & $\begin{array}{l}200 \mathrm{~L} \\
300 \mathrm{LB}\end{array}$ & $\begin{array}{l}5.00 \\
5.0 \mathrm{U}\end{array}$ \\
\hline $699-43+416$ & $10 / 19 / 24$ & $\begin{array}{l}600307 \\
6000308 \\
6000309 \\
0003720\end{array}$ & $\begin{array}{l}270 \\
269 \\
269 \\
268\end{array}$ & & $\begin{array}{l}7.82 \\
7.80 \\
7.77 \\
7.76\end{array}$ & 8.20 & $\begin{array}{l}200 \mathrm{E} \\
200 \mathrm{~L} \\
300 \mathrm{E} \\
200 \mathrm{~L}\end{array}$ & $\begin{array}{l}5.0 \mathrm{u} \\
5.0 \mathrm{U} \\
5.0 \mathrm{U} \\
5.0 \mathrm{uf}\end{array}$ \\
\hline $699-43-42 \mathrm{~J}$ & $10 / 20 / 94$ & 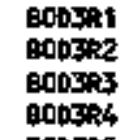 & $\begin{array}{l}273 \\
274 \\
274 \\
274\end{array}$ & r & $\begin{array}{l}8.20 \\
8.20 \\
8.20 \\
8.20\end{array}$ & 8.40 & $\begin{array}{l}500 \mathrm{LB} \\
300 \mathrm{LB} \\
300 \mathrm{LB} \\
\mathbf{3 0 0} \mathrm{LB}\end{array}$ & $\begin{array}{l}5.0 \mathrm{U} \\
5.3 \\
5.0 \mathrm{U} \\
5.1\end{array}$ \\
\hline $699.43-43$ & $10 / 00 / 94$ & $\begin{array}{l}\text { Bopzas } \\
0002 R 6 \\
8002 R 7 \\
0002 k 8 \\
800386\end{array}$ & $\begin{array}{l}214 \\
210 \\
208 \\
207\end{array}$ & 180 & $\begin{array}{l}7.94 \\
7.92 \\
7.92 \\
7.92\end{array}$ & 8.30 & $\begin{array}{l}600 \mathrm{~L} \\
600 \mathrm{~L} \\
300 \mathrm{~L} \\
600 \mathrm{~L}\end{array}$ & $\begin{array}{l}5.0 \mathrm{U} \\
5.0 \mathrm{U} \\
5.0 \mathrm{U} \\
5.0 \mathrm{U}\end{array}$ \\
\hline $\begin{array}{l}697-43-45 \\
.\end{array}$ & 10/06/94 & $\begin{array}{l}600250 \\
600251 \\
600252 \\
600253 \\
600357\end{array}$ & $\begin{array}{l}248 \\
248 \\
248 \\
249\end{array}$ & 240 & $\begin{array}{l}7.84 \\
7.86 \\
7.84 \\
7.88\end{array}$ & 8.20 & $\begin{array}{l}400 \mathrm{~L} \\
400 \mathrm{~L} \\
400 \mathrm{~L} \\
500 \mathrm{~L}\end{array}$ & $\begin{array}{l}5.0 \mathrm{U} \\
5.0 \mathrm{U} \\
5.0 \mathrm{U} \\
5.0 \mathrm{U}\end{array}$ \\
\hline $699-44-398$ & $10 / 20 / 54$ & 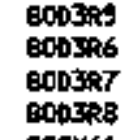 & $\begin{array}{l}181 \\
179 \\
178 \\
179\end{array}$ & & $\begin{array}{l}7.61 \\
7.61 \\
7.61 \\
7.61\end{array}$ & 8.30 & $\begin{array}{l}1000 \mathrm{~B} \\
8010 \mathrm{LB} \\
6010 \mathrm{LB} \\
600 \mathrm{LB}\end{array}$ & $\begin{array}{l}8.4 \\
5.0 \text { ut } \\
7.2 \\
7.1\end{array}$ \\
\hline $689+44-42$ & $9 / 09 / 94$ & 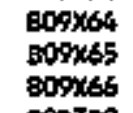 & $\begin{array}{l}230 \\
229 \\
226\end{array}$ & & $\begin{array}{l}8.28 \\
8.28 \\
8.24\end{array}$ & & & \\
\hline & $10 / 20 / \%$ & $\begin{array}{l}8003199 \\
600350 \\
600351 \\
000352\end{array}$ & $\begin{array}{l}188 \\
190 \\
190 \\
190\end{array}$ & & $\begin{array}{l}8.16 \\
8.15 \\
8.15 \\
8.15\end{array}$ & 8.30 & $\begin{array}{l}600 \mathrm{LB} \\
500 \mathrm{LB} \\
700 \mathrm{LB} \\
600 \mathrm{LB}\end{array}$ & $\begin{array}{l}7.0 \\
6.4 \\
6.6 \\
5.5\end{array}$ \\
\hline $699-44-438$ & $10 / 21 / 94$ & $\begin{array}{l}600383 \\
600354 \\
600355 \\
600356\end{array}$ & $\begin{array}{l}277 \\
276 \\
276 \\
276\end{array}$ & & $\begin{array}{l}8.13 \\
8.13 \\
\mathrm{~g} .13 \\
\mathrm{~g} .13\end{array}$ & 8.30 & $\begin{array}{l}300 \mathrm{~L} \\
300 \mathrm{~L} \\
400 \mathrm{~L} \\
300 \mathrm{~L}\end{array}$ & $\begin{array}{l}5.0 \mathrm{U} \\
5.0 \mathrm{U} \\
5.0 \mathrm{U} \\
5.0 \mathrm{U}\end{array}$ \\
\hline
\end{tabular}

For explanation of uhis table, sea section 3.4 of report. 
Tabie 5-6. Constituents Exceeding Orinking Water Standards for the 216-8-3 Pond System for Reporting Period October 1 through December $31,1994$.

\begin{tabular}{|l|c|l|}
\hline \multicolumn{1}{|c|}{ Constituent } & $\begin{array}{c}\text { DWS } \\
\text { (units) }\end{array}$ & Welis with resuTts exceeding DWS \\
\hline $\begin{array}{l}\text { Iron (unfiltered } \\
\text { samples) }\end{array}$ & $\begin{array}{c}300 \\
\text { (ppb) }\end{array}$ & $299-E 18-1^{4}, 699-E 32-4^{\mathrm{B}}$ \\
\hline Tritium & 20,000 & $299-E 18-1,699-41-40,699-42-39 A$, \\
& $(\mathrm{pCi} / \mathrm{L})$ & $699-42-39 \mathrm{~B}, 699-42-42 \mathrm{~B}, 699-43-40$, \\
& & $699-43-41 \mathrm{E}, 699-43-41 \mathrm{~F}, 699-43-416$ \\
\hline
\end{tabular}

"A Jaboratory qualifier or data flag is associated with this result. DWS - drinking water standard. 
DOE/RL-94-36-4

This page intentionaliy left blank. 
DOOE/RL-94-36-4

\section{CONTENSS}

$6.0216-\mathrm{A}-29 \mathrm{DITCH}$

6.1 INTRODUCTIOH

6.2 WATER LEVEL MEASUREMENTS

6-1

6.3 WATER CHEMISTRY DATA

6-2 
DOE/RL-94-36-4

LIST OF FIGURES

6-1 We11 Location Map for the 216-A-29 Ditch . . . . . . . . . . 6-3

\section{LIST OF TABLES}

6-1 Monitoring Well Purpose and Sampling Schedule for the 2i16-A-29 Ditch ................ . . . . . .

6-3 Constituent List and Summary of Results for the 216-A-29 Ditch Data for Reporting Period October 1 through December 31 , 1994 . . . 6-9

6-4 Constituents with at least One Detected Value for the 216-A-29 Ditch Data for Reporting Period 0ctober I through Decerber 31, 1994 . . . 6-12

6-5 Contamination Indicator Parameters for the 216-A-29 Ditch Data for Reporting Period October 1 through December 31, 1994 . . 6-23 


\title{
6.0 216-A-29 DITCH
}

\author{
P. B. Freeman \\ Hestinghouse Hanford Company
}

\subsection{INTROOUCTION}

The 216-A-29 Bitch (A-29 Ditch) is located immedlately east of the 200 East Area in the central portion of the Hanford Site (Figure 6-1). From 1955 to July 1991, this unlined ditch recejved effiuent from the Plutonium-Uranium Extraction (PUREX) Plant chemical sewer line and conducted it to the 216-B-3-3 Oitch and the B Pond System. After July 15, 1991, the effluent to the A-29 Ditch was rerouted to the PUREX cooling water line, the ditch was backfilled, and the site was graded and stabilized to neet Milestone M-17-10 of the Hanford Federa? Facility Agreement and Consent Order (Tri-Party Agreement) (Ecology et al. 1992).

Groundwater beneath the A-29 Ditch has been monttored by a RCRA groundwater monitoring network since 1988 because of earlier discharges of caustic and potentially hazardous chemjcals to the ditch. The A-29 Ditch is currentiy in an interim-status groundwater quality assessment nonitoring program because of elevated specific conductance levels in one monitoring well (299-E25-35) (Chou et a1. 1990).

The RCRA groundwater monitoring network consists of 3 upgradient and 10 downgradient wel1s. Mine additional wells in the surrounding area are used to provide supplementary data for the groundwater qual tyy assessment investigation. Figure 6-1 shows the locations of the wells used to monitor groundwater quality for the A-29 Ditch. Table 6-1 presents pertinent data about these wells.

\subsection{MATER LEVEL MEASUREHENTS}

Water levels are measured monthly in the 13 wells comprising the A-29 Ditch wonitoring network, and are measured quarterly in the 9 additional wel1s that provide supplemental analytical data for the groundwater quality assessment investigation. Water level measurements are also recorded whenever the wells are sampled.

Water 1eve1 measurements obtained during this quarter are presented in Table 6-2. Several inconsistencies were noted when recent data were compared to the water levels prevfously measured. Hater levels reported for wel1 299-E26-13 on October 12, well 299-E26-28 on October 18, and we11 299-E25-34 on 0ctober 19 fel1 outside of the expected ranges. Inspection of the fieid report sheets does not reveal any data transcription or retrieval errors. These data are flagged in Table 6-2. 
DOE/RL-94-36-4

\subsection{WATER CHEMISTRY DATA}

Groundwater beneath the A-29 Dttch is sampled quarterly in accordance with the groundwater qua]1ty assessment plan (Chou et a1. 1990). The constituent Jist and summary of results are provided in Table 6-3. Reported analytical data are included in Tables 6-4 and 6-5. Table 6-4 provides a list of the weils and analytical results for chenica] constftuents with at least one detected value above the HoL. In many cases, the detected values are from wel1s that are monitored to provide comparative regional data for the groundwater quality assessment investigation. Results of analyses for CIPs are reported in Table 6-5. Prevjousily nonreported and rereported analytical data for the A-29 groundwater monitoring network are included in the tables in this report.

One well (299-E25-42) had turbioity data results that were greater than 5 NTUS. RADEs have been submitted for this wel1. Data assoctated with these high-turbidity values may be affected. High turbidity is a laboratory interferant for aany analytical techniques; therefore being able to separate good from suspect data resuits is unlikely.

Two other wells had individual constituent results that do not match the historical trends. Anmoniug lon was ldentified at the 200-ppb jevel in well 299-E25-28 in the A-29 D1tch detection network. Coba1t-60 was identified in we11 299-E25-36 in the A-29 01tch supplemental network. This is the first. sampling period where data results for these constituents have been above the laboratory contractual 1imits. RADEs have been submitted for this weil.

Constituents reported in concentrations that exceed the DWS for the A-29 Bitch network we11s include unfittered chromium in welis 299-E25-32P, 299-E24-34, 299-E25-35, 299-E25-42, 299-E25-43, 299-E26-12, and 299-E256-13; unftltered iron th we]ls 299-E,25-32P, 299-E24-34, 299-E25-35, 299-E25-42, $299-E 25-43,299-E 25-47,299-E 25-48$, and 299-E256-13; and tritiun in wells 299-E25-32P, 299-E25-35, 299-E25-47, and 299-E25-42.

Constituents reported in concentrations that exceed the DWS at the wells that provide regional data for the A-29 Ditch groundwater suplemental investigation include unfiltered chroritum in wells 299-E17-15, 299-E17-20, 299-E25-11, 299-E25-31, and 299-E25-36; unfiltered iron in we11s 299-17-15, 299-E17-20, 299-E25-11, 299-E25-21 and 299-E25-31; and tritium in welt's 299-E17-15, 299-E17-20, 299-E25-11, 299-E25-I8, 299-E25-19, and 299-E25-20, and $\mathrm{pH}$ in well 299-E25-21.

Wells with detections of Co-60 and I-129 are cosampled with the A-36B and A-10 networks. Discussion of these detections are contained in Sections 7 and 8, respectively.

Data are flagged in Tables 6-4 and 6-5 according to the explanation found in Sections 1.2 and 1.4. 


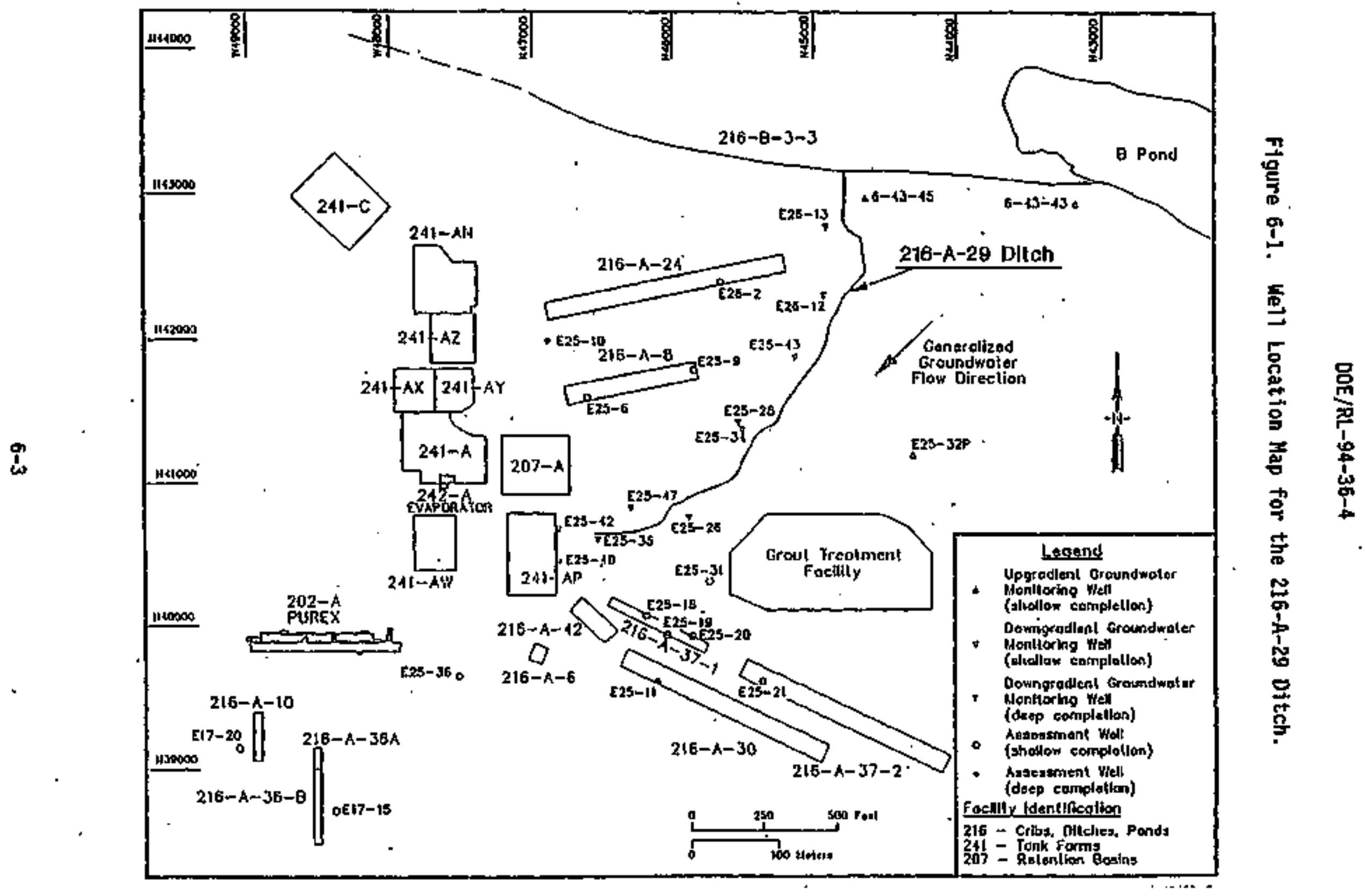


Table 6-1. Monitoring Well Purpose and Sampling Schedule for the 216-A-29 Ditch. (2 sheets)

\begin{tabular}{|c|c|c|c|c|}
\hline Mell no. & $\begin{array}{l}\text { Relative } \\
\text { position }\end{array}$ & Hydrogeologic unit & $\begin{array}{c}\text { Säple } \\
\text { frequency }\end{array}$ & $\begin{array}{l}\text { Sampie date, } \\
\text { 4th Qtr } 1994\end{array}$ \\
\hline $699-43-43^{b}$ & Upgradjent & $\begin{array}{l}\text { Hanford: Hater } \\
\text { Table }\end{array}$ & Quarterly & $10 / 06 / 94$ \\
\hline $699-43-45^{b}$ & Upgradient & $\begin{array}{l}\text { Hanford: Water } \\
\text { Table }\end{array}$ & quarterly & $10 / 06 / 94$ \\
\hline 299-E25-32P & Downgradient & $\begin{array}{l}\text { Hanford: Water } \\
\text { Table }\end{array}$ & Quarterly & $10 / 11 / 94$ \\
\hline $299-E 25-26$ & Downgradient & Hanford: Mid-Depth & Quarterly & $10 / 19 / 94$ \\
\hline 299-E25-29 & Downgradient & Ringold: Deep & Quàrterly & $10 / 18 / 94$ \\
\hline $299-E 25-34$ & Downgradient & $\begin{array}{l}\text { Hanford: Hater } \\
\text { Table }\end{array}$ & Quarterly & $10 / 19 / 94$ \\
\hline 299-E25-35 & Downgradient & $\begin{array}{l}\text { Hanford: Hater } \\
\text { Table }\end{array}$ & Quarterly & $10 / 19 / 94$ \\
\hline $299-E 25-42$ & Downgradient: & $\begin{array}{l}\text { Hanford: Water } \\
\text { Table }\end{array}$ & Quarterly & $10 / 11 / 94$ \\
\hline $299-E 25-43$ & Downgradtent & $\begin{array}{l}\text { Hanford: Water } \\
\text { Table }\end{array}$ & Quarterly & $10 / 11 / 94$ \\
\hline $299-E 25-47$ & Downgradlent & $\begin{array}{l}\text { Hanford: Water } \\
\text { Table }\end{array}$ & Quarterly & $10 / 12 / 94$ \\
\hline $299-E 25-48$ & Downgradient & $\begin{array}{l}\text { Hanford: Water } \\
\text { Table }\end{array}$ & Quarteriy & $10 / 12 / 94$ \\
\hline 299-E26-12 & Downgradient & $\begin{array}{l}\text { Hanford: Water } \\
\text { Tabte }\end{array}$ & Quarterly & $10 / 11 / 94$ \\
\hline 299-E25-I3 & Downgradient & $\begin{array}{l}\text { Hanford: Water } \\
\text { Table }\end{array}$ & Quarterly & $10 / 12 / 94$ \\
\hline $299-E 17-15^{c_{4}}$ & Downgradient & $\begin{array}{l}\text { Ringold: Water } \\
\text { Table }\end{array}$ & Quarter1y & $12 / 06 / 94$ \\
\hline $299-E 17-20^{d} \cdot c$ & Downgradient & $\begin{array}{l}\text { Ringold: Water } \\
\text { Table }\end{array}$ & Quarterly & $10 / 14 / 94$ \\
\hline $299-E 25-11^{\bullet}$ & Downgradient & $\begin{array}{l}\text { Undifferentiated } \\
\text { sedioents: } \\
\text { Mid-Depth }\end{array}$ & Quarterly & $10 / 13 / 94$ \\
\hline $299-E 25-18^{e}$ & Downgradient & $\begin{array}{l}\text { Undifferentiated } \\
\text { sediments: } \\
\text { Mid-Depth }\end{array}$ & Quarterly & $10 / 18 / 94$ \\
\hline
\end{tabular}


Table 6-1. Monitoring Well Purpose and Sampling Schiedule for the 216-A-29 Ditch. (2 sheets)

\begin{tabular}{|c|c|c|c|c|}
\hline Hell no. & $\begin{array}{l}\text { Relative } \\
\text { postition }\end{array}$ & Hydrogeologft unft $t^{a}$. & $\begin{array}{c}\text { Sample } \\
\text { frequency }\end{array}$ & $\begin{array}{l}\text { Sample date, } \\
\text { 4th Qtr } 1994\end{array}$ \\
\hline $299-E 25-19^{\circ}$ & Downgradient & $\begin{array}{l}\text { Undtfferentiated } \\
\text { sediments: } \\
\text { Mid-Depth }\end{array}$ & Quarterly & $10 / 18 / 94$ \\
\hline $299-E 25-20^{\circ}$ & Downgradient & $\begin{array}{l}\text { Undifferentiated } \\
\text { sediments: } \\
\text { Mid-Depth }\end{array}$ & Quarterly & $10 / 21 / 94$ \\
\hline $299-E 25-21^{e}$ & Downgradient & $\begin{array}{l}\text { Hanford: Water } \\
\text { Table }\end{array}$ & Quarterly & $10 / 18 / 94$ \\
\hline $299-E 25-31^{\circ}$ & Downgradient & $\begin{array}{l}\text { Hanford: Water } \\
\text { Table }\end{array}$ & Quarterly & $10 / 12 / 94$ \\
\hline $299-E 25-36^{d . e}$ & Downgradient & $\begin{array}{l}\text { Ringold: hater } \\
\text { Table }\end{array}$ & Quarterly & $10 / 13 / 94$ \\
\hline
\end{tabular}

Hydrogeologic units include the sandy gravels of the Hanford formation and the silty sands of the Ringold Formation.

Wells shared with the 216-B-3 Pond monitoring network.

Clells shared with the 216-A-36B monitoring network.

Wells shared with the 216-A-10 monitoring network.

Wells used for supplemental groundwater quality. 
Table 6-2. RCRA Water Level Measurenent Report 216-A-29 Ditch - Fourth Quarter 1994. (sheet 1 of 3)

\begin{tabular}{|c|c|c|c|c|}
\hline Well & Date & $\begin{array}{l}\text { Depth to } \\
\text { water (ft) }\end{array}$ & $\begin{array}{l}\text { Water } \\
\text { elevation } \\
\text { (ft) }\end{array}$ & $\begin{array}{l}\text { level } \\
\text { above ms } 1 \\
\text { (田) }\end{array}$ \\
\hline \multicolumn{5}{|c|}{ Wells in the Monjtoring System } \\
\hline $299-E 25-26$ & $\begin{array}{l}10 / 19 / 94 \\
10 / 24 / 94 \\
11 / 17 / 94 \\
12 / 12 / 94\end{array}$ & $\begin{array}{l}266.91 \\
266.64 \\
266.66 \\
266.54\end{array}$ & $\begin{array}{l}401.61 * \\
401.88 \\
401.86 \\
401.98\end{array}$ & $\begin{array}{l}122.41 \\
122.49 \\
122.49 \\
122.52\end{array}$ \\
\hline $299-E 25-28$ & $\begin{array}{l}10 / 18 / 94 \\
10 / 24 / 94 \\
11 / 17 / 94 \\
12 / 12 / 94\end{array}$ & $\begin{array}{l}260.85 \\
260.21 \\
260.17 \\
260.48\end{array}$ & $\begin{array}{l}401.59{ }^{*}+ \\
402.23 \\
402.27 \\
401.96\end{array}$ & $\begin{array}{l}122.40 \\
122.60 \\
122.61 \\
122.52\end{array}$ \\
\hline 299-E25-32P & $\begin{array}{l}10 / 11 / 94 \\
10 / 24 / 94 \\
11 / 17 / 94 \\
12 / 13 / 94\end{array}$ & $\begin{array}{l}267.99 \\
267.91 \\
267.80 \\
268.28\end{array}$ & $\begin{array}{l}402.05 * \\
402.13 \\
402.24 \\
401.76\end{array}$ & $\begin{array}{l}122.54 \\
122.57 \\
122.60 \\
122.46\end{array}$ \\
\hline $\begin{array}{c}\text { 299-E25-34 } \\
.\end{array}$ & $\begin{array}{l}10 / 19 / 94 \\
10 / 24 / 94 \\
11 / 17 / 94 \\
12 / 12 / 94\end{array}$ & $\begin{array}{l}261.72 \\
260.78 \\
260.68 \\
260.75\end{array}$ & $\begin{array}{l}401.15^{\star}+ \\
402.09 \\
402.19 \\
402.12\end{array}$ & $\begin{array}{l}122.27 \\
122.56 \\
122.59 \\
122.57\end{array}$ \\
\hline $299-E 25-35$ & $\begin{array}{l}10 / 19 / 94 \\
10 / 24 / 94 \\
11 / 17 / 94 \\
12 / 12 / 94\end{array}$ & $\begin{array}{l}272.77 \\
272.65 \\
272.69 \\
272.75\end{array}$ & $\begin{array}{l}401.62^{\star} \\
401.74 \\
401.70 \\
401.64\end{array}$ & $\begin{array}{l}122.41 \\
122.45 \\
122.44 \\
122.42\end{array}$ \\
\hline $299-E 25-42$ & $\begin{array}{l}10 / 11 / 94 \\
10 / 24 / 94 \\
11 / 17 / 94 \\
12 / 12 / 94\end{array}$ & $\begin{array}{l}281.56 \\
281.42 \\
281.51 \\
281.56\end{array}$ & $\begin{array}{l}401.73 * \\
401.87 \\
401.78 \\
401.73\end{array}$ & $\begin{array}{l}122.45 \\
122.49 \\
122.46 \\
122.45\end{array}$ \\
\hline $299-E 25-43$ & $\begin{array}{l}10 / 11 / 94 \\
10 / 24 / 94\end{array}$ & $\begin{array}{l}247.42 \\
247.63\end{array}$ & $\begin{array}{l}402.47^{*} \\
402.26\end{array}$ & $\begin{array}{l}122.67 \\
122.61\end{array}$ \\
\hline $299-E 25-47$ & $\begin{array}{l}10 / 12 / 94 \\
10 / 24 / 94 \\
11 / 17 / 94\end{array}$ & $\begin{array}{l}271.62 \\
271.68 \\
271.77\end{array}$ & $\begin{array}{l}402.15^{*} \\
402.09 \\
402.00\end{array}$ & $\begin{array}{l}122.58 \\
122.56 \\
122.53\end{array}$ \\
\hline $299-E 25-48$ & $\begin{array}{l}10 / 12 / 94 \\
10 / 24 / 94 \\
11 / 17 / 94\end{array}$ & $\begin{array}{l}280.50 \\
280.29 \\
280.44\end{array}$ & $\begin{array}{l}401.81 * \\
402.02 \\
401.87\end{array}$ & $\begin{array}{l}122.47 \\
122.54 \\
122.49\end{array}$ \\
\hline
\end{tabular}


Table 6-2. RCRA Water Level Measurement Report 216-A-29 Ditch - Fourth Quarter 1994. (sheet 2 of 3 )

Well Date $\quad \begin{gathered}\text { Depth to } \\
\text { water (ft) }\end{gathered} \quad$\begin{tabular}{c}
$\begin{array}{c}\text { Water level } \\
\text { elevation above ms1 } \\
(\mathrm{ft})\end{array}$ \\
\hline
\end{tabular}

Hells in the Monitoring System

\begin{tabular}{|c|c|c|c|c|}
\hline $299-626-12$ & $\begin{array}{l}10 / 11 / 94 \\
10 / 24 / 94 \\
11 / 17 / 94 \\
12 / 12 / 94\end{array}$ & $\begin{array}{l}228.25 \\
228.34 \\
228.20 \\
228.59\end{array}$ & $\begin{array}{l}402.49 * \\
402.40 \\
402.54 \\
402.15\end{array}$ & $\begin{array}{l}122.68 \\
122.65 \\
122.69 \\
122.58\end{array}$ \\
\hline 299-E26-13 & $\begin{array}{l}10 / 12 / 94 \\
10 / 24 / 94 \\
11 / 17 / 94 \\
12 / 12 / 94\end{array}$ & $\begin{array}{l}204.15 \\
202.68 \\
202.57 \\
202.70\end{array}$ & $\begin{array}{l}400.87 *_{+} \\
402.34 \\
402.45 \\
402.32\end{array}$ & $\begin{array}{l}122.19 \\
122.63 \\
122.67 \\
122.63\end{array}$ \\
\hline $699-43-43$ & $\begin{array}{l}10 / 06 / 94 \\
10 / 24 / 94 \\
11 / 17 / 94 \\
12 / 12 / 94\end{array}$ & $\begin{array}{l}164.75 \\
165.34 \\
165.28 \\
165.92\end{array}$ & $\begin{array}{l}414.62^{\star} \\
414.03 \\
414.09 \\
413.45\end{array}$ & $\begin{array}{l}126.38 \\
126.20 \\
126.21 \\
126.02\end{array}$ \\
\hline \multirow[t]{2}{*}{$699-43-45$} & $\begin{array}{l}10 / 06 / 94 \\
10 / 24 / 94 \\
11 / 17 / 94 \\
12 / 12 / 94\end{array}$ & $\begin{array}{l}195.00 \\
195.01 \\
195.04 \\
195.17\end{array}$ & $\begin{array}{l}402.68 * \\
402.67 \\
402.64 \\
402.51\end{array}$ & $\begin{array}{l}122.74 \\
122.73 \\
122.72 \\
122.69 .\end{array}$ \\
\hline & \multicolumn{3}{|c|}{ Supplemental Kells for Monitoring } & 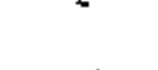 \\
\hline 299-E17-15 & $\begin{array}{l}12 / 06 / 94 \\
12 / 12 / 94\end{array}$ & $\begin{array}{l}321.65 \\
321.24\end{array}$ & $\begin{array}{l}400.13 * \\
400.54\end{array}$ & $\begin{array}{l}121.96 \\
122.08\end{array}$ \\
\hline $299-E 17-20$ & $\begin{array}{l}10 / 14 / 94 \\
12 / 12 / 94\end{array}$ & $\begin{array}{l}318.70 \\
317.80\end{array}$ & $\begin{array}{l}400.53^{*} \\
401.43\end{array}$ & $\begin{array}{l}122.08 \\
122.36\end{array}$ \\
\hline 299-E25-I1 & $\begin{array}{l}10 / 13 / 94 \\
12 / 21 / 94\end{array}$ & $\begin{array}{l}279.56 \\
279.92\end{array}$ & $\begin{array}{l}401.72 * \\
401.36\end{array}$ & $\begin{array}{l}122.44 \\
122.33\end{array}$ \\
\hline 299-E25-18 & $\begin{array}{l}10 / 18 / 94 \\
12 / 21 / 94\end{array}$ & $\begin{array}{l}277.85 \\
277.58\end{array}$ & $\begin{array}{l}401.20^{*} \\
401.47\end{array}$ & $\begin{array}{l}122.29 \\
122.37\end{array}$ \\
\hline 299-E25-19 & $\begin{array}{l}10 / 18 / 94 \\
12 / 21 / 94\end{array}$ & $\begin{array}{l}275.95 \\
275.88\end{array}$ & $\begin{array}{l}401.25^{*} \\
401.32\end{array}$ & $\begin{array}{l}122.30 \\
122.32\end{array}$ \\
\hline $299-\mathrm{E} 25-20$ & $10 / 12 / 94$ & 274.96 & $401.34 *$ & 122.33 \\
\hline
\end{tabular}


Table 6-2. RCRA Hater Leve1 Measurement Report 216-A-29 Ditch - Fourth Quarter 1994.

(sheet 3 of 3)

\begin{tabular}{|c|c|c|c|c|}
\hline Well & Date & $\begin{array}{l}\text { Depth to } \\
\text { water (ft) }\end{array}$ & $\begin{array}{l}\text { Water } \\
\text { elevation } \\
\text { (ft) }\end{array}$ & $\begin{array}{l}\text { level } \\
\text { above ns] } \\
\text { (n) }\end{array}$ \\
\hline \multicolumn{5}{|c|}{ Supplemental Wells for Monitoring } \\
\hline 299-E25-20 & $12 / 21 / 94$ & 275.01 & 401.29 & 122.31 \\
\hline 299-E25-21 & $10 / 18 / 94$ & 275.49 & $401.78 *$ & 122.46 \\
\hline 299-E25-31 & $10 / 12 / 94$ & 272.55 & $402.09 *$ & 122.56 \\
\hline 299-E25-36 & $\begin{array}{l}10 / 13 / 94 \\
12 / 12 / 94\end{array}$ & $\begin{array}{l}305.96 \\
306.03\end{array}$ & $\begin{array}{l}401.43 * \\
401.36\end{array}$ & $\begin{array}{l}122.36 \\
122.33\end{array}$ \\
\hline $299-E 25-6$ & $12 / 21 / 94$ & 259.46 & 398.85 & 121.57 \\
\hline 299-E25-9 & $12 / 21 / 94$ & 255.51 & 399.35 & 121.72 \\
\hline 299-E26-2 & $12 / 21 / 94$ & 233.64 & $+\quad 401.66$ & 122.43 \\
\hline
\end{tabular}

MOTES: 1. Water level elevations are calculated by subtracting the wasured depth-to-water from the surveyed eievation for the wall.

2. Depth-to-water values are transcribed from field records.

3. E7evations marked with an '*' were measured at the time of sampling.

4. Elevations marked with a ' + ' are outside of the expected range, and are suspected of error. 
Table 6-3. Constituent List and Stamary of Results for the 216-A-29 Ditch Data for Reporting Period October 1 through December 31, 1994.

(sheet 1 of 3 )

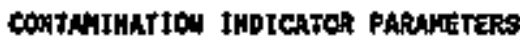

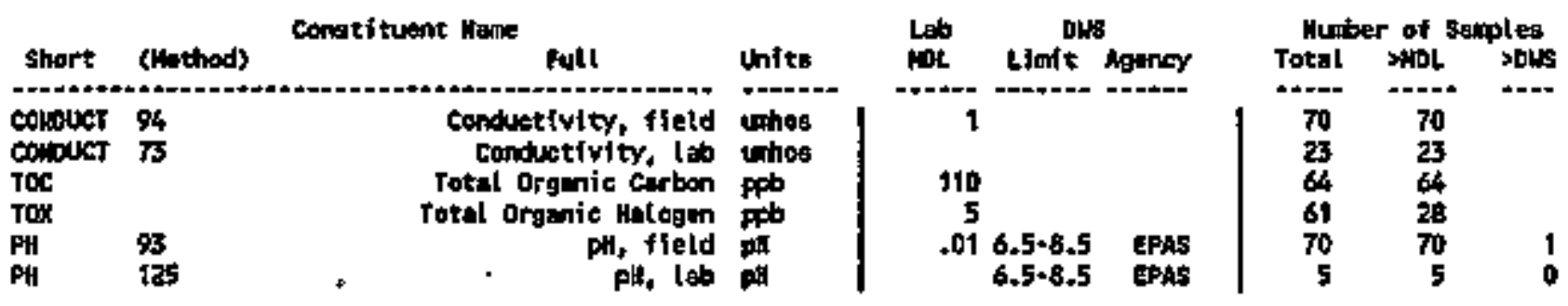

\section{DRIGKING WRTER PARUCTERS}

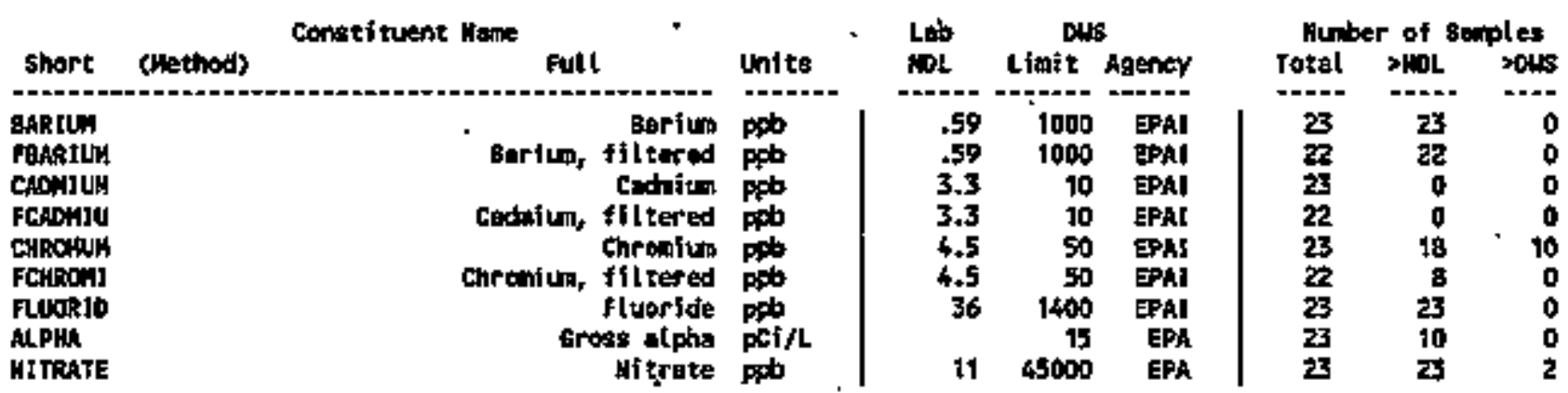

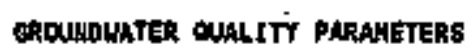

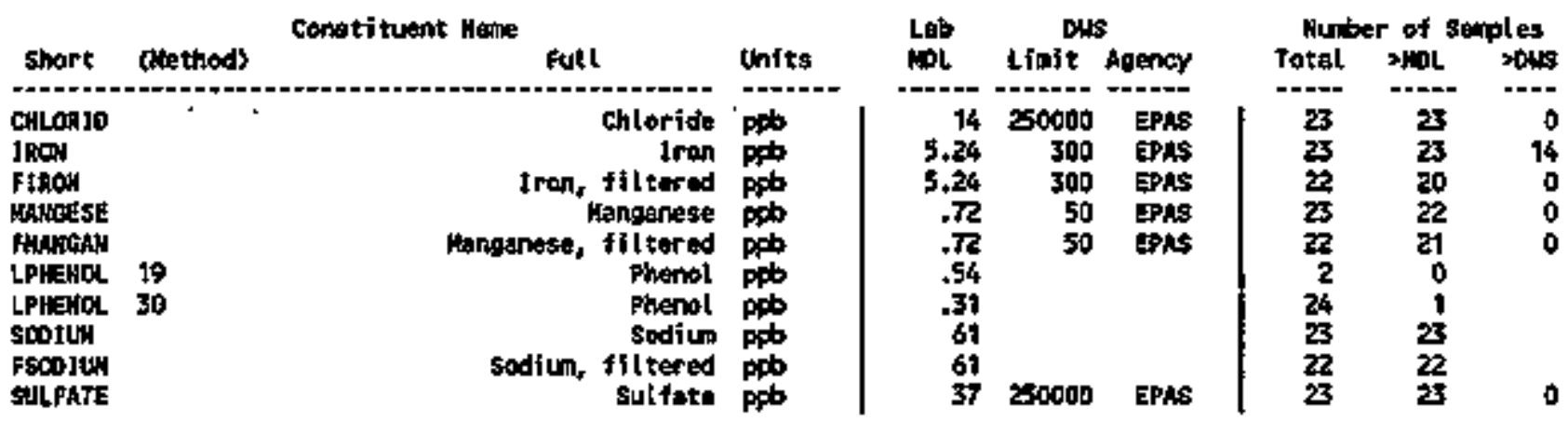


Table 6-3. Constituent List and Summary of Results for the 216-A-29 Ditch Data for Reporting Period October 1 through December 31, 1994. (sheet 2 of 3 )

S1TE SPECIFIC AHD OTHER COHSTITUIERS

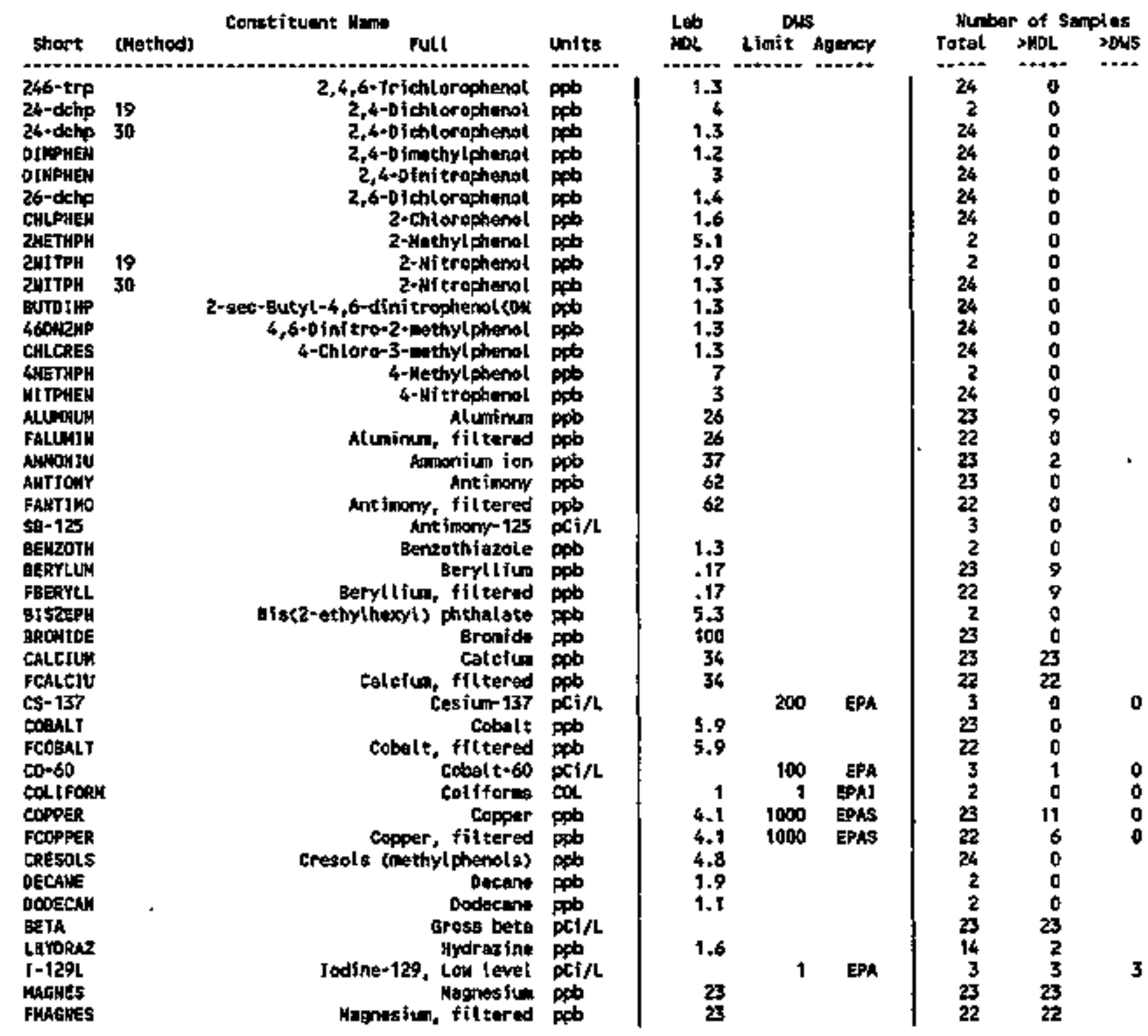


Table 6-3. Constituent List and Sumpary of Results for the 216-A-29 Ditch Data for Reporting Period October 1 through December 31, 1994.

(sheet 3 of 3 )

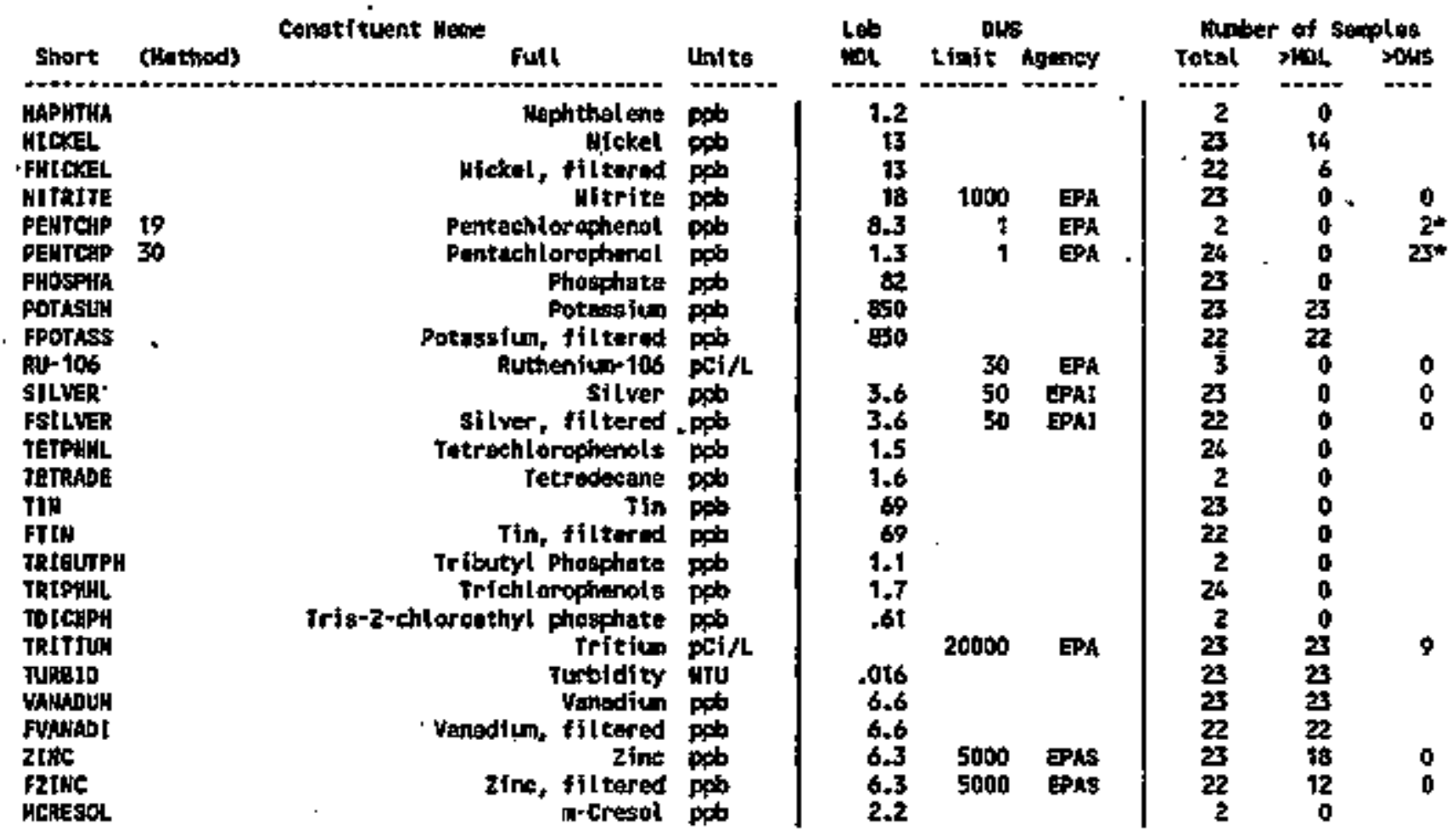

Far explangtion of this tuble, ses section 1.4 of report. 
Tab]e 6-4. Constituents with at Least One Detected Vajue for the 216-A-29 Dftch Data for Reporting Period October 1 through December 31, 1994.

(sheet 1 of 11)

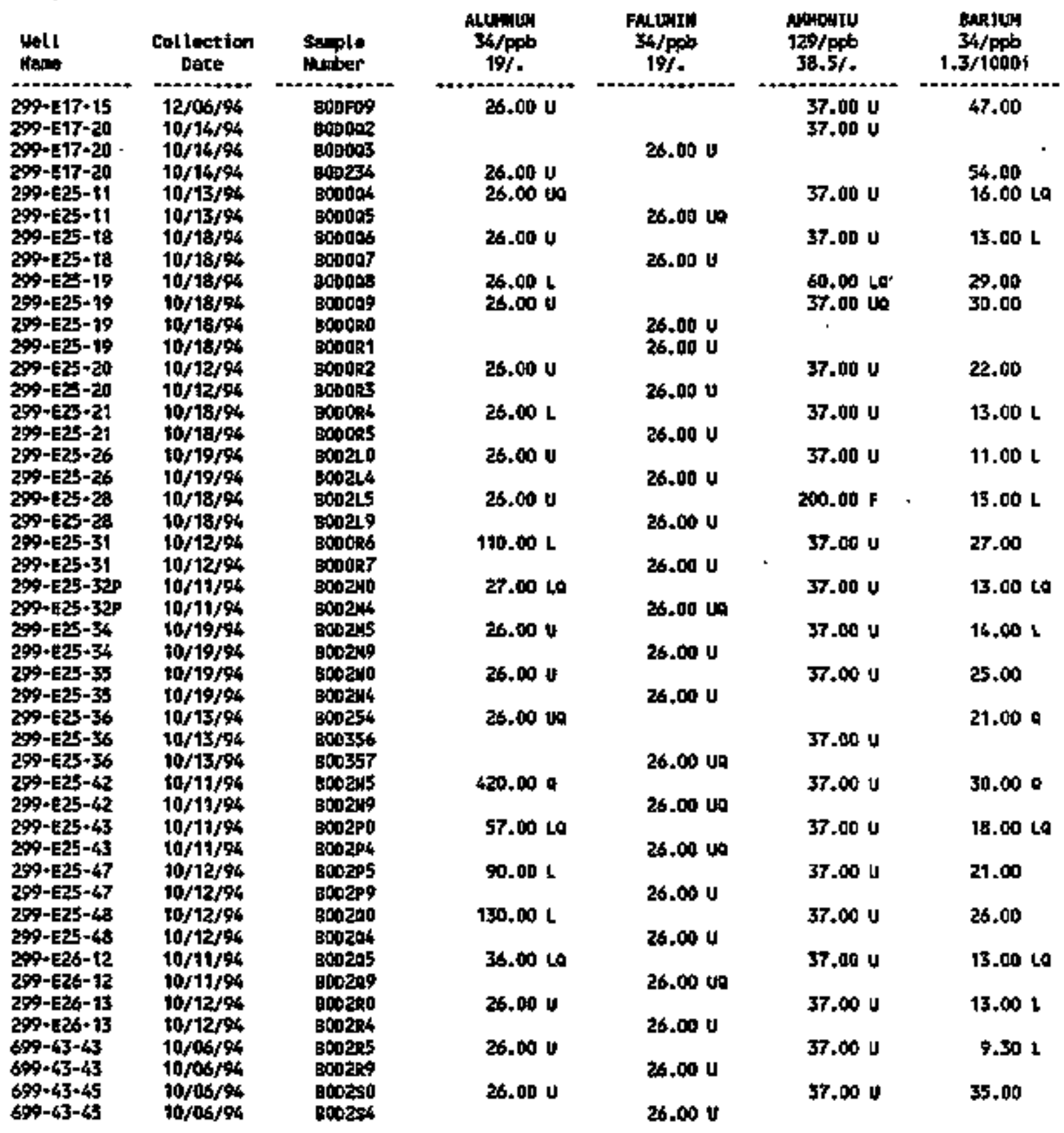


Table 6-4. Constituents with at Least One Detected Value for the 216-A-29 Ditch Data for Reporting Period -October 1 through December 31, 1994. (sheet 2 of 11)

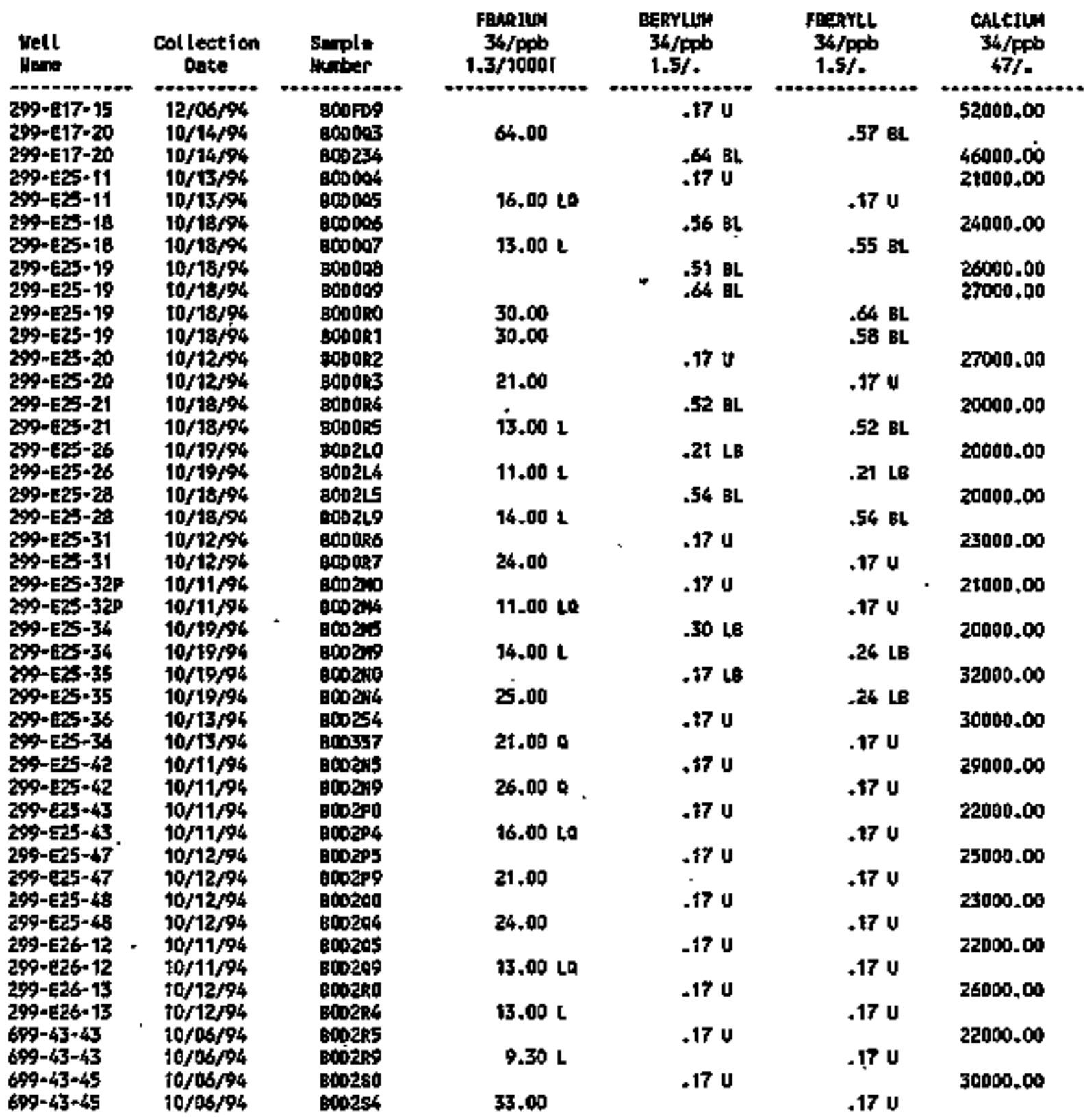


Table 6-4. Constftuents with at Least One Detected Value for the 216-A-29 Ditch Data for Reporting Period Detober 1 through December 31, 1994. (sheet 3 of 11 )

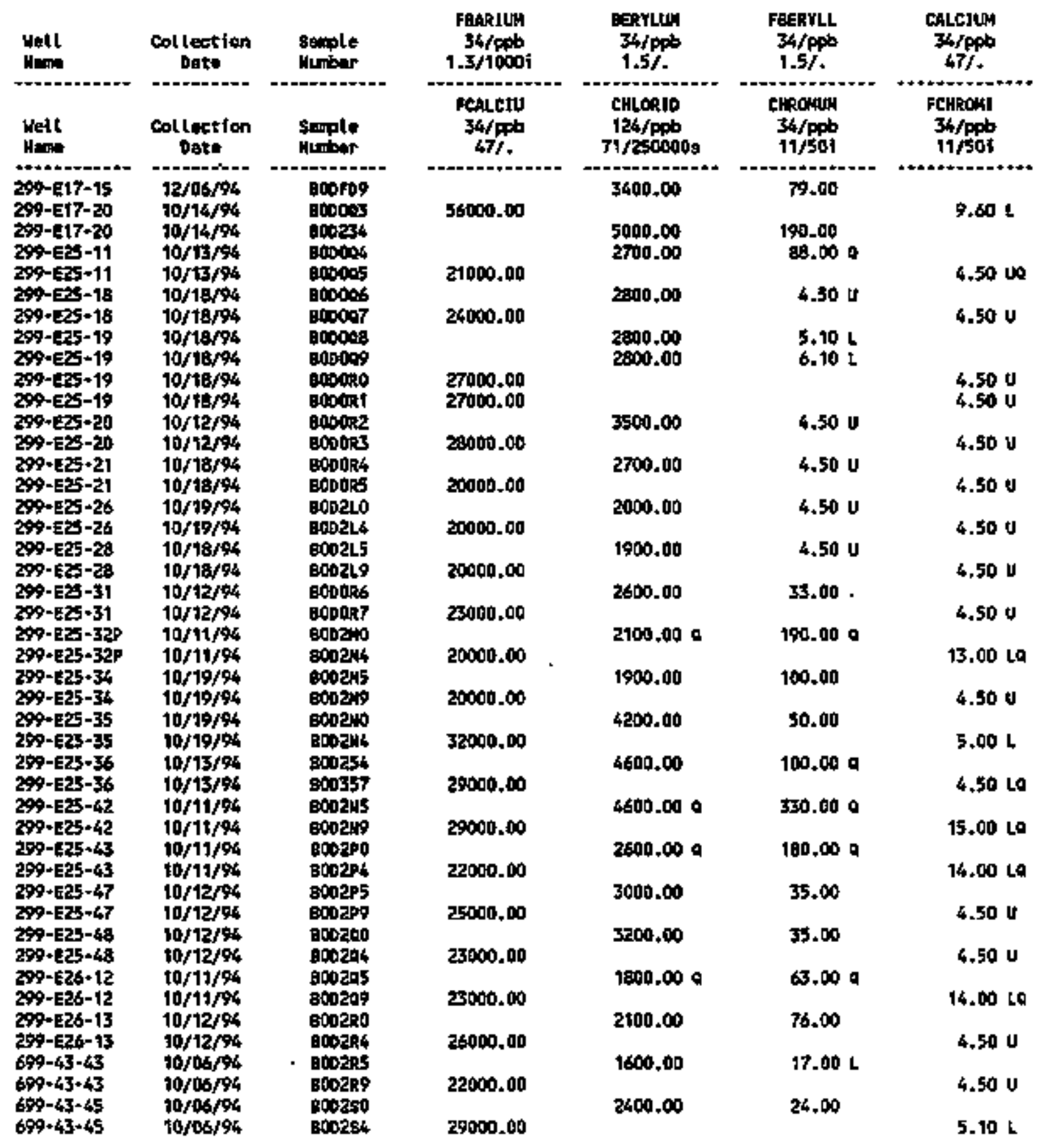


Tạble 6-4. Constituents with at Least One Detected Value for the 216-A-29 Ditch Data for Reporting Period October 1 through December 31, I994.

(sheet 4 of II)

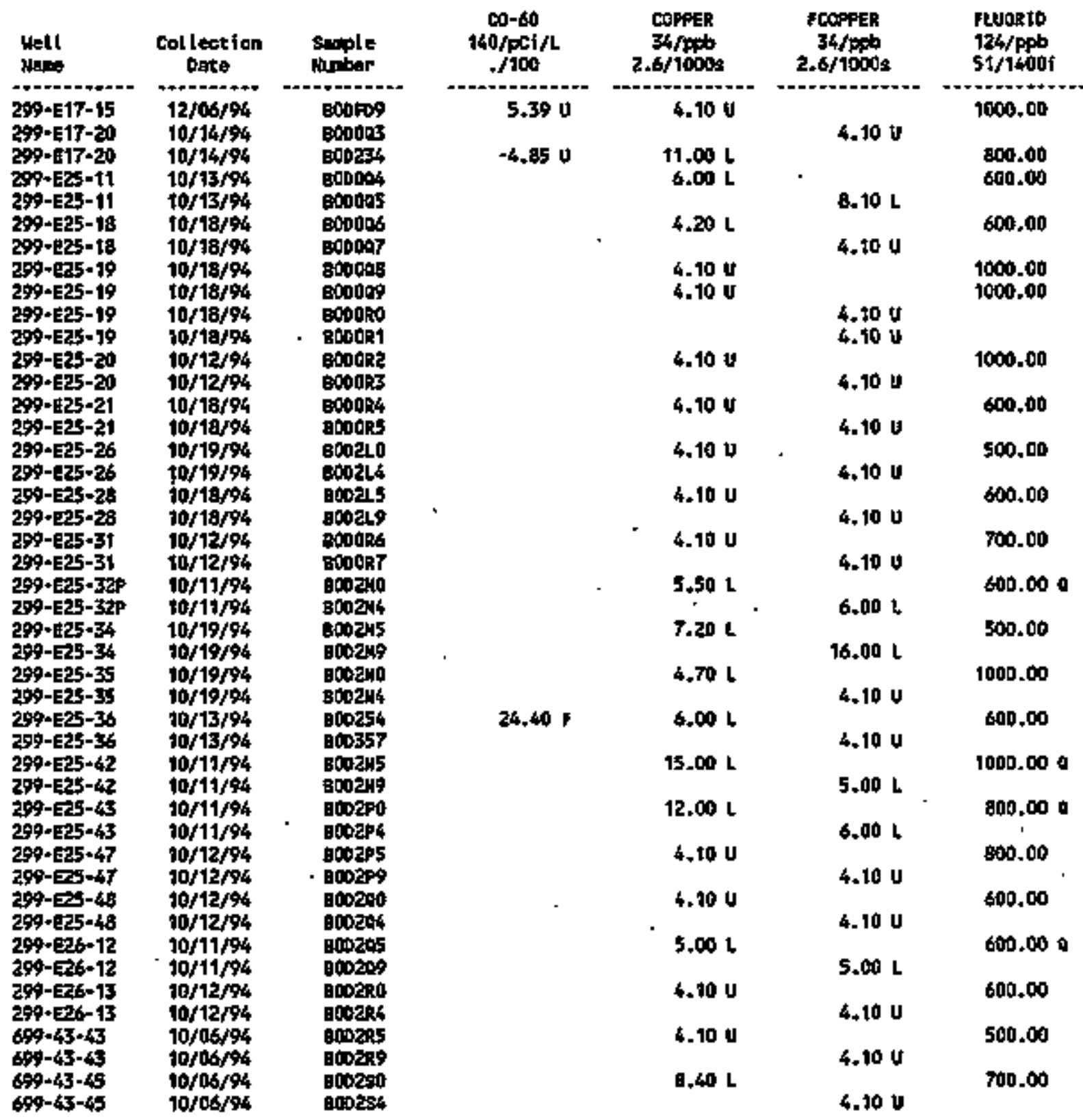


Table 6-4. Constituents with at Least One Detected Value for the 216-A-29 Ditch Data for Reporting Pertod October 1 through December 31, 1994.

(sheet 5 of 11)

\begin{tabular}{|c|c|c|c|c|c|c|}
\hline $\lim _{\text {leme }}$ & $\begin{array}{l}\text { Col lection } \\
\text { pate }\end{array}$ & $\underset{\text { Suple }}{\text { Suber }}$ & $\begin{array}{c}\text { ALPHA } \\
\text { 135/PCIA } \\
\text { of15 }\end{array}$ & 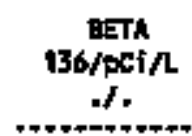 & 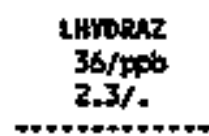 & $\begin{array}{c}{[-129} \\
139 / \mathrm{pCi} / \mathrm{L} \\
-/ 1\end{array}$ \\
\hline 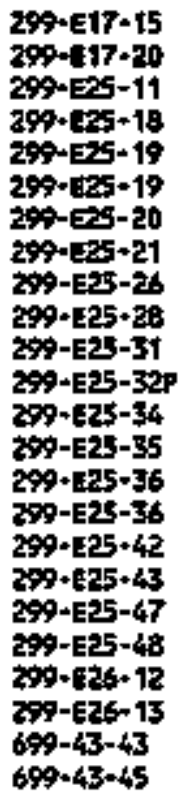 & $\begin{array}{l}12 / 06 / 94 \\
10 / 14 / 94 \\
10 / 13 / 94 \\
10 / t 8 / 94 \\
10 / 18 / 94 \\
10 / 18 / 94 \\
10 / 12 / 94 \\
10 / 18 / 94 \\
10 / 19 / 94 \\
10 / 18 / 94 \\
10 / 12 / 94 \\
10 / 11 / 94 \\
10 / 19 / 94 \\
10 / 19 / 94 \\
10 / 13 / 94 \\
10 / 13 / 94 \\
10 / 11 / 94 \\
10 / 11 / 94 \\
50 / 12 / 94 \\
10 / 12 / 94 \\
10 / 11 / 94 \\
10 / 12 / 94 \\
10 / 04 / 94 \\
10 / 06 / 94\end{array}$ & 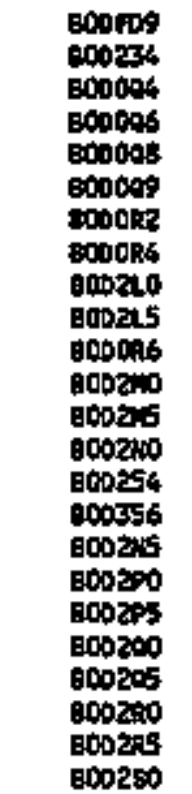 & 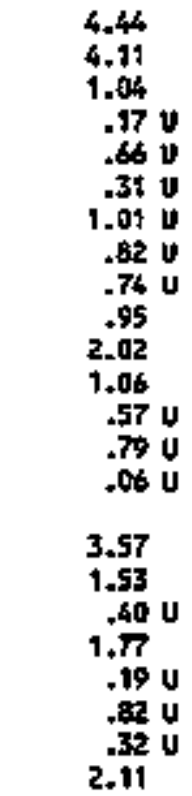 & $\begin{array}{l}92.60 \\
77.60 \\
7.83 \\
6.15 \\
6.27 \\
8.69 \\
4.50 \\
4.06 \\
3.03 \\
4.17 \\
7.06 \\
3.01 \\
4.37 \\
7.60 \\
5.13 \\
7.26 \\
5.92 \\
6.17 \\
4.43 \\
3.72 \\
3.23 \\
3.60 \\
3.41\end{array}$ & $\begin{array}{l}1.60 \mathrm{U} \\
1.60 \mathrm{U} \\
\\
1.60 \mathrm{U} \\
1.60 \mathrm{U} \\
1.60 \mathrm{U} \\
\\
1.60 \mathrm{U} \\
7.00 \mathrm{~L} \\
1.60 \mathrm{U} \\
1.60 \mathrm{U} \\
1.60 \mathrm{U} \\
1.60 \mathrm{U} \\
1.60 \mathrm{U} \\
1.60 \mathrm{U} \\
1.60 \mathrm{U}\end{array}$ & $\begin{array}{l}9.25 \\
8.94\end{array}$ \\
\hline
\end{tabular}


Table 6-4. Constituents' with at Least One Detected Value for the 216-A-29 Ditch Data for Reporting Pertod October 1 through December 31, 1994. (sheet 6 of 11)

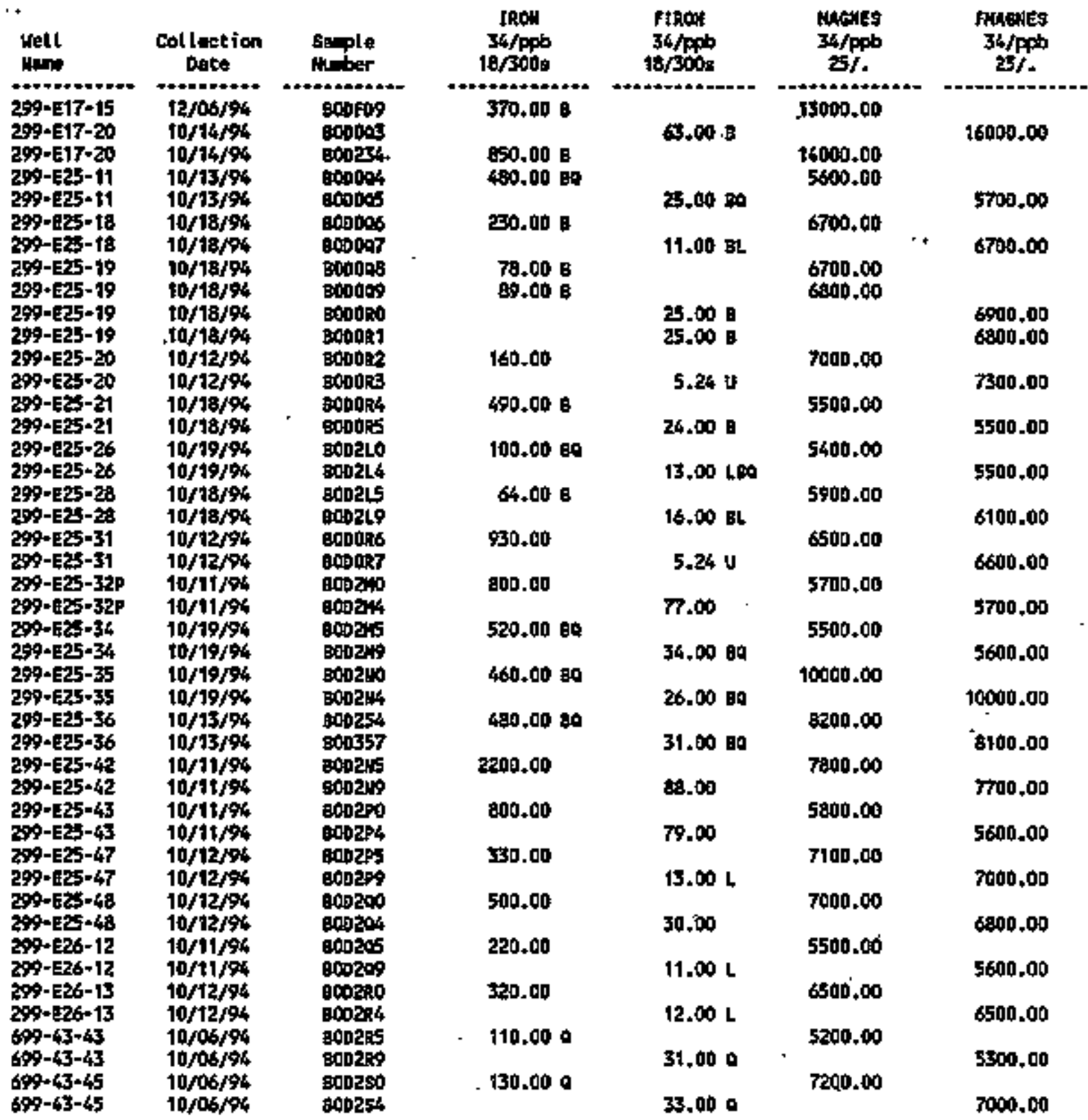


Table 6-4. Constituents with at Least One Detected Value for the 216-A-29 Djtch Data for Reporting Period

October 1 through December 31, 1994.

(sheet 7 of 11)

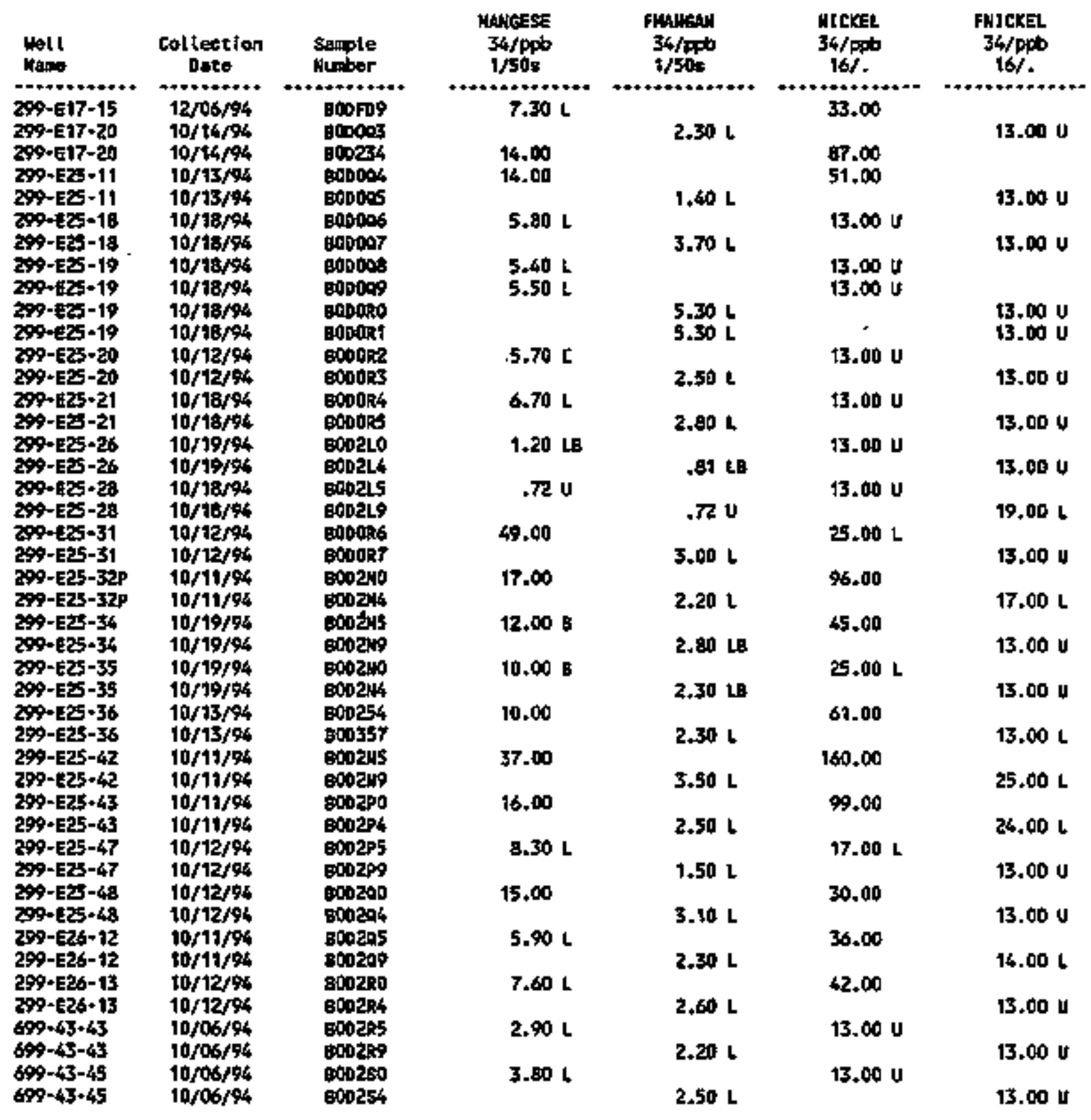


Table 6-4. Constituents with at Least One Detected Value for the 216-A-29 D1tch Data for Reporting Perlod October 1 through Decenber 31, 1994. (sheet 8 of 11)

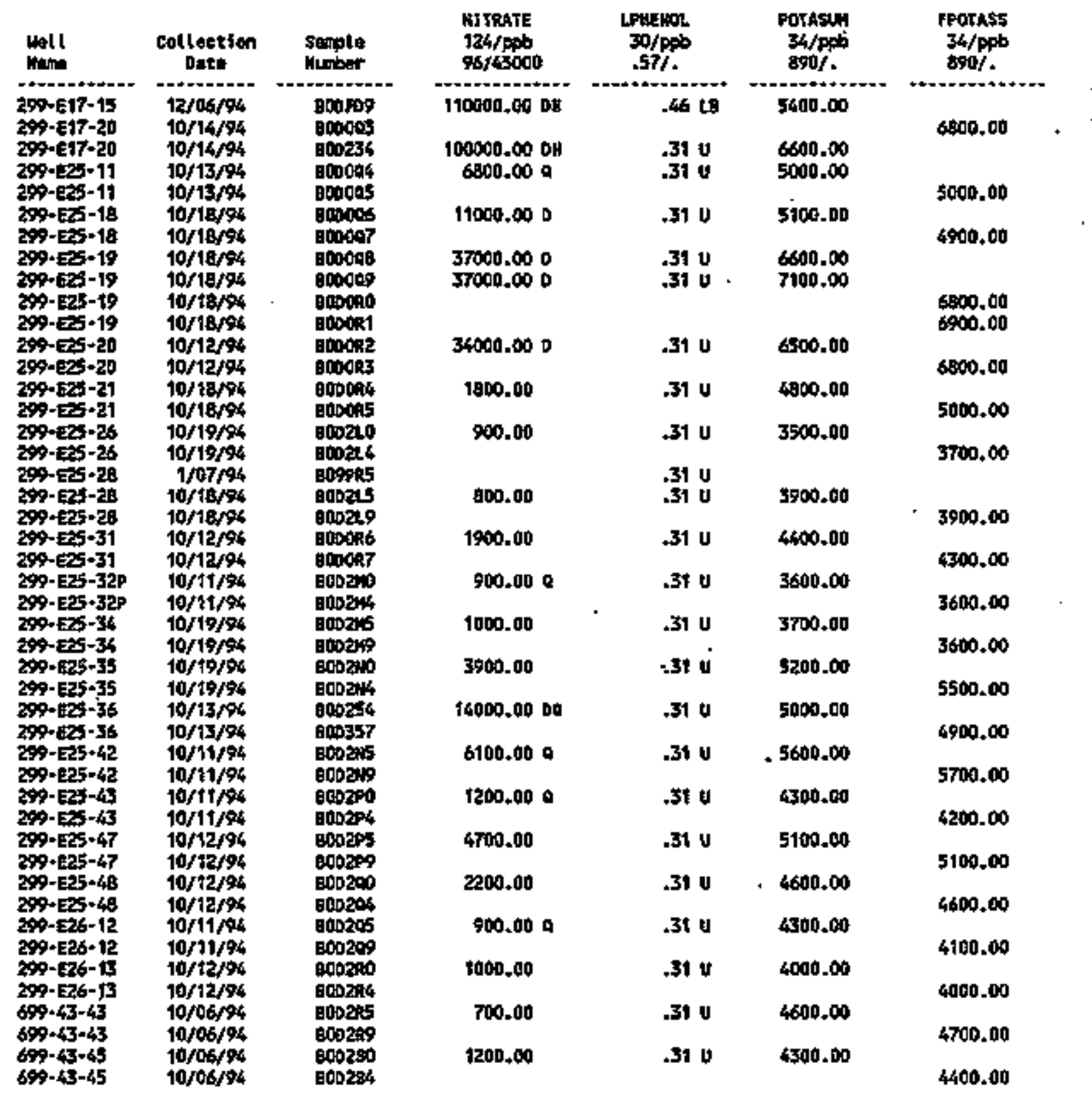


Table 6 4. Constituents with at least One Detected Value for the 216-A-29 Ditch Data for Reporting Period

October 1 through Decenber 31, 1994. (sheet 9 of 11)

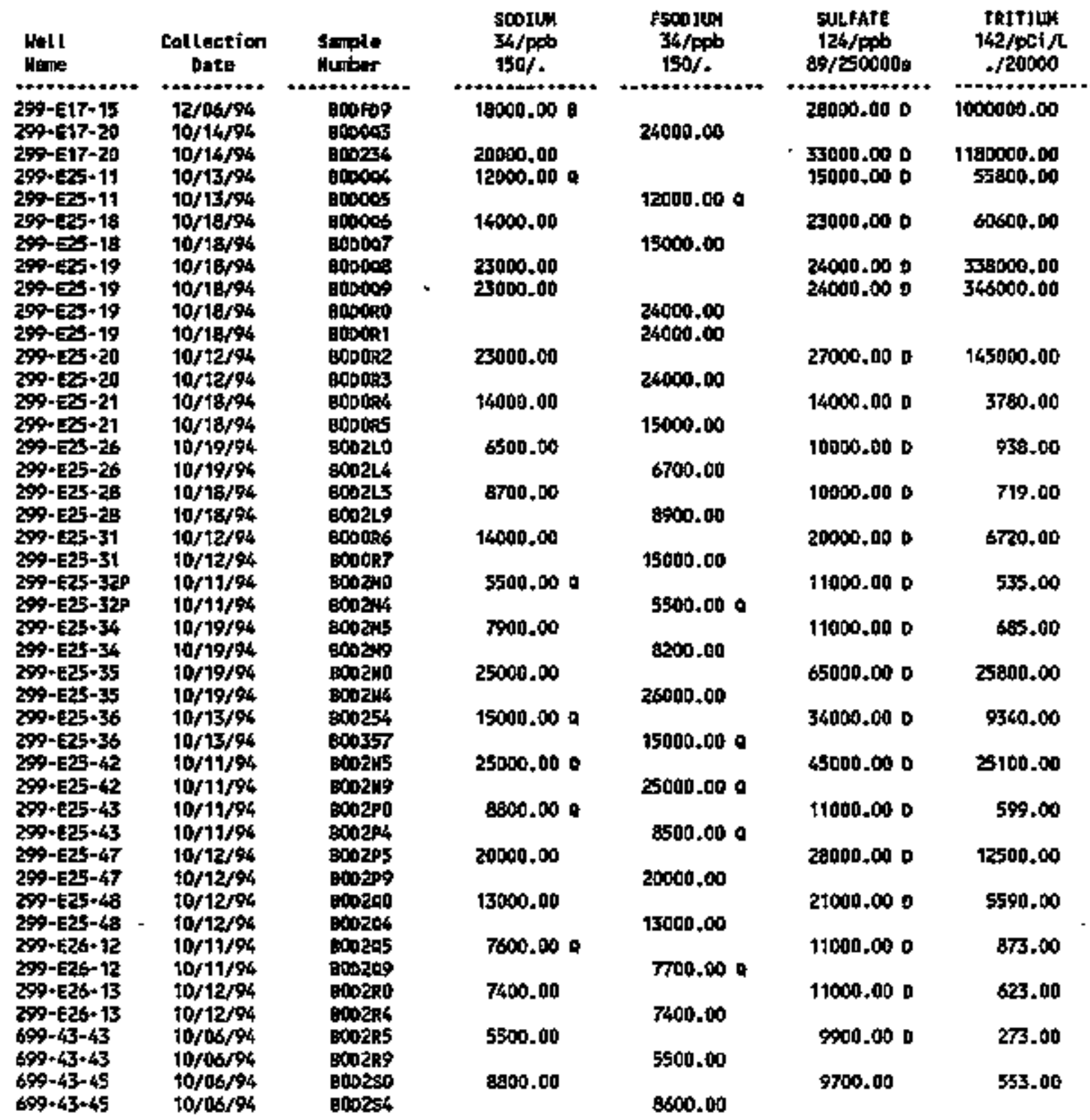


Table 6-4. Constituents with at Least One Detected Va7ue for the 216-A-29 Ditch Data for Reporting Perlod October 1 through December 31, 1994. (sheet 10 of 11)

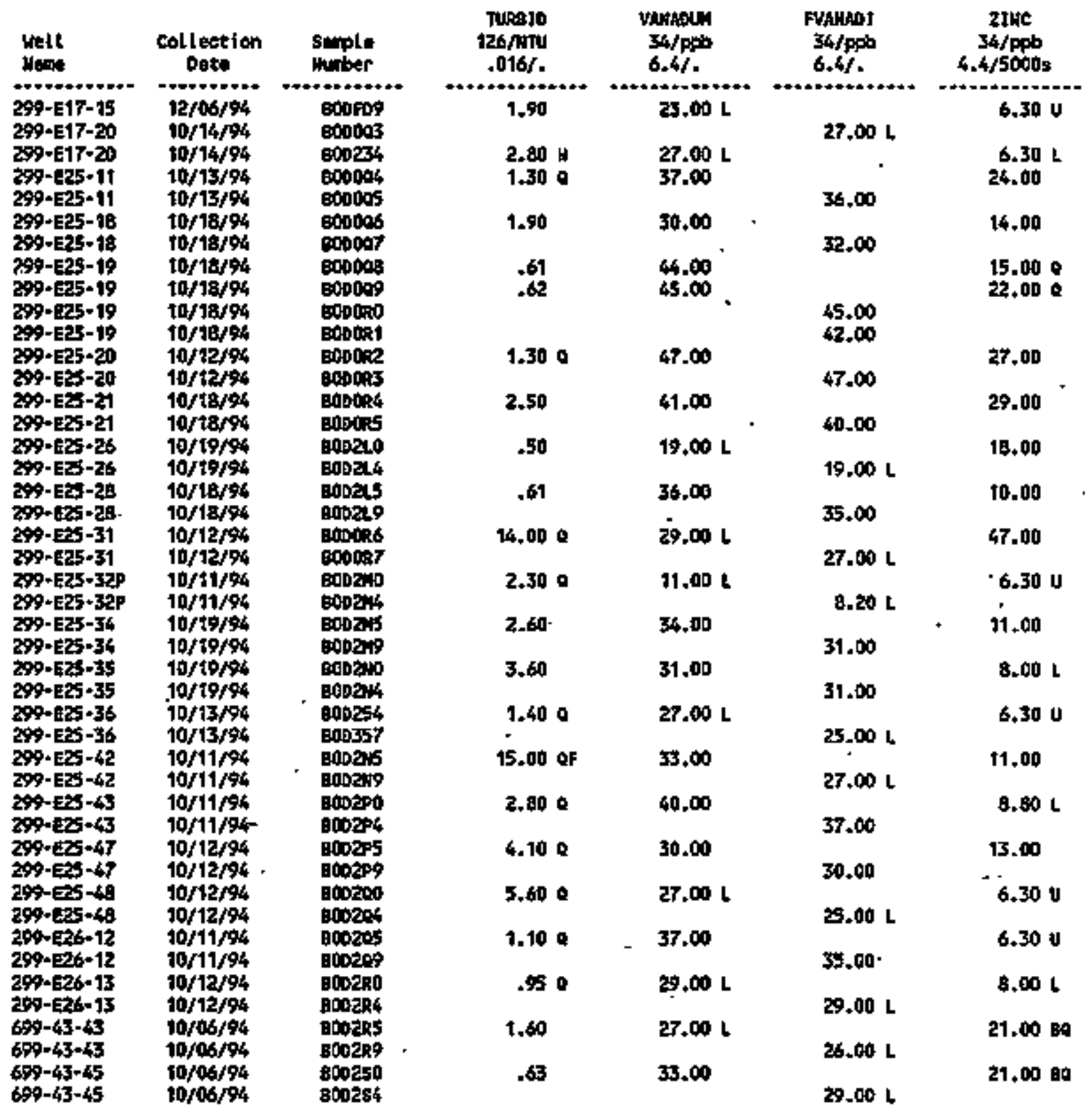


Table 6-4. Constituents with at Least One Detected Value for the 216-A-29 Ditch Data for Reporting Perjod October 1 through December 31, 1994.

(sheet 11 of 11 )

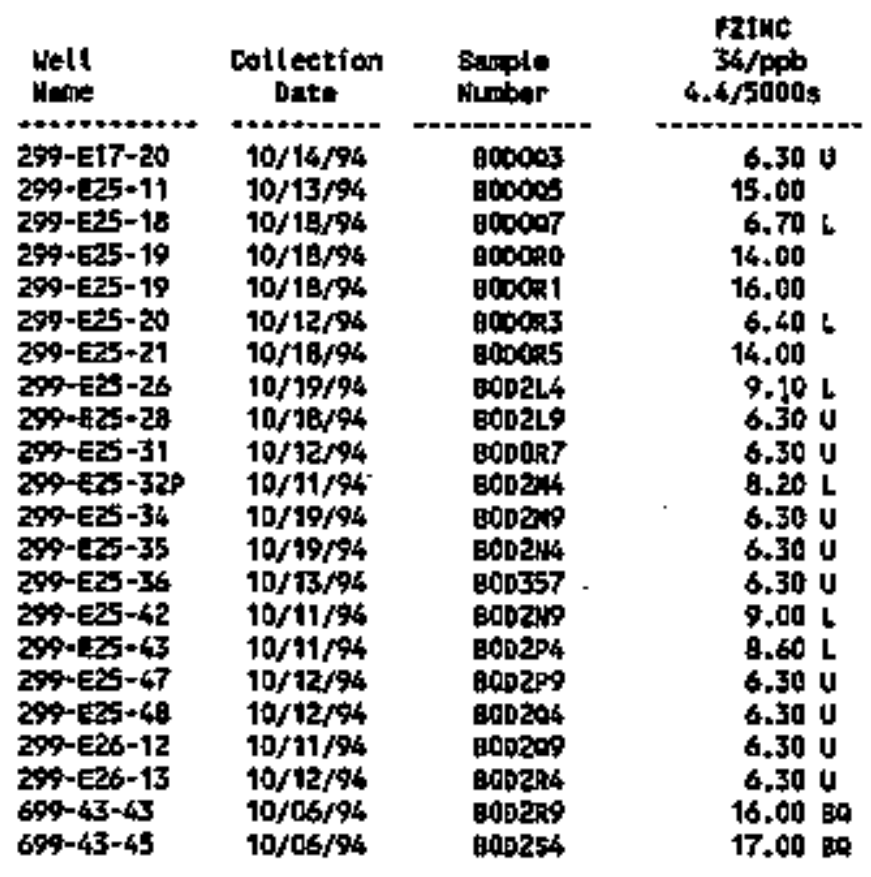

For exptanation of this tuble, ses Section 1.4 of report. 


\section{DOE/RL-94-36-4}

Table 6-5. Contamination Indicator Parameters for the 216-A-29 Ditch

Data for Reporting Period October 1 through December 31, 1994. (sheet 1 of 2)

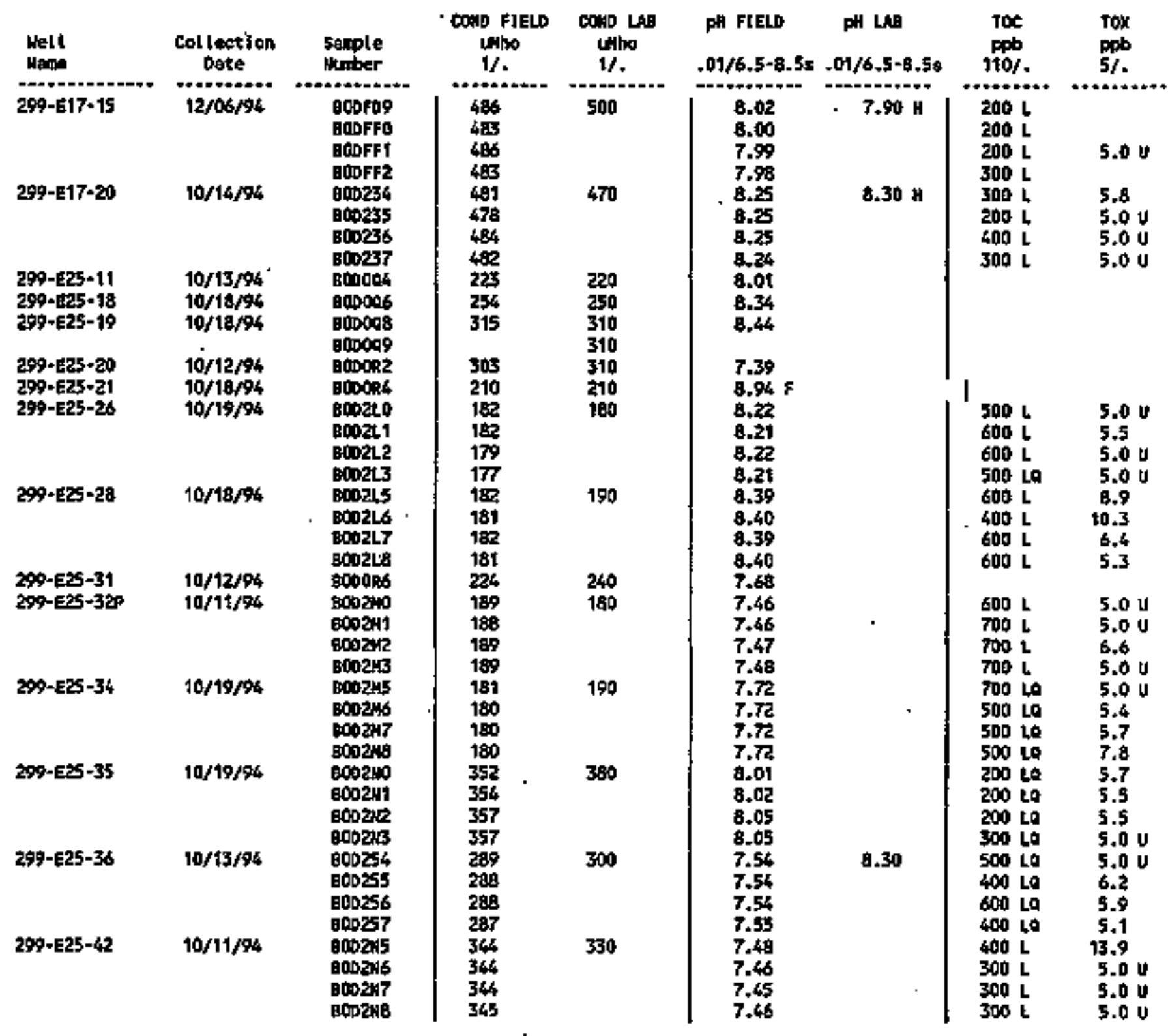


Table 6-5. Contamination Indicator Parameters for the 216-A-29 Ditch Data for Reporting Period October i through December 31, 1994. (sheet 2 of 2)

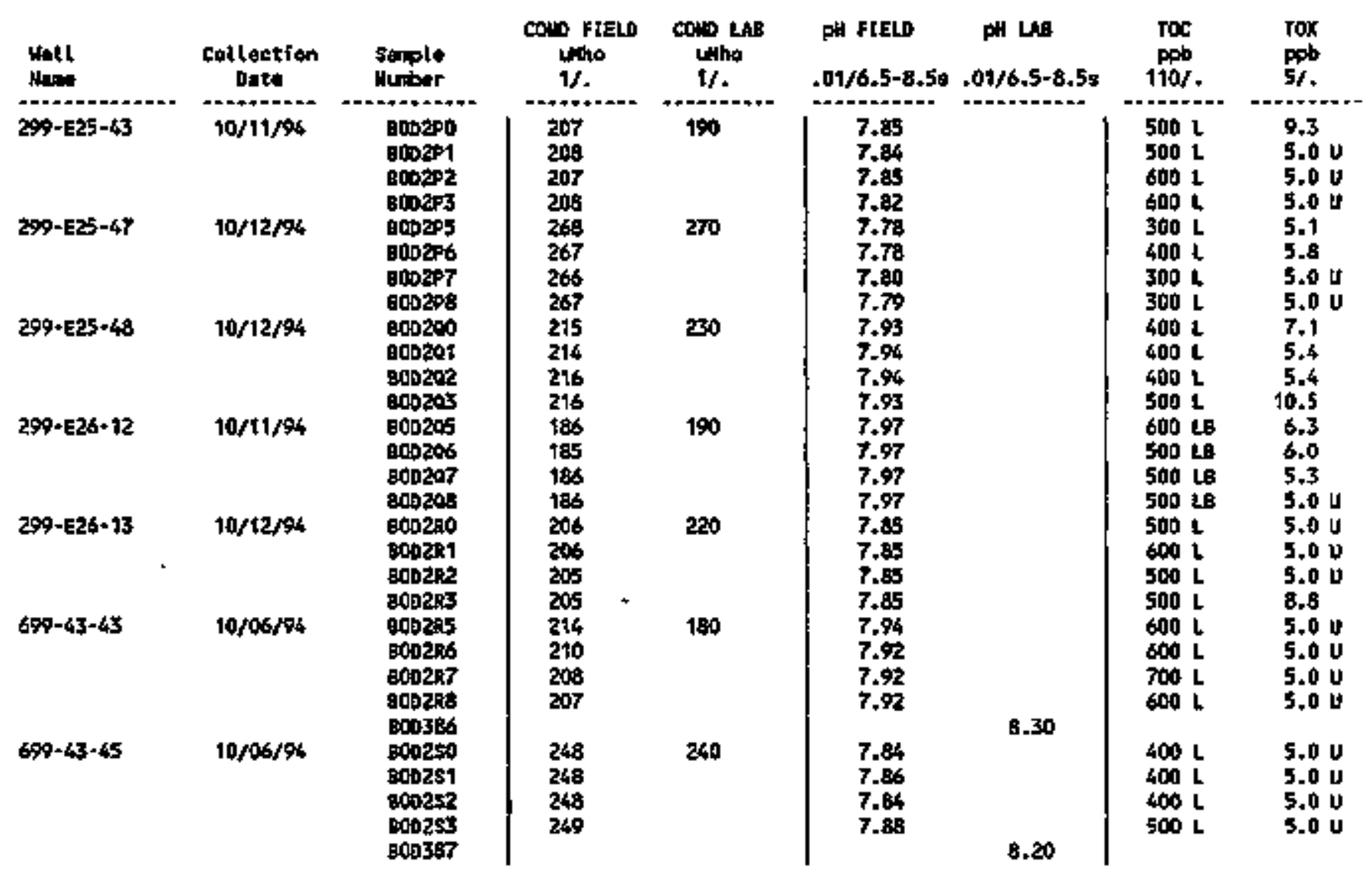

For explenstion of this teble, see Setion $t .4$ of report. 
DOE/RL-94-36-4

CONTENTS

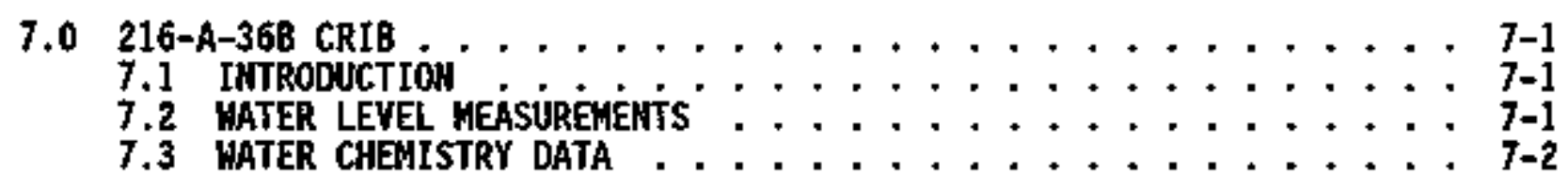


7-1 We11 Location Map for the 216-A-36B Cr1b ........... 7-3

\section{LIST OF TABLES}

7-1 Well Identification and Sampling Schedule for the 216-A-36B Crib

Groundwater Monitoring Network ...... 7-4

7-2 RCRA Water Level Measurement Report 216-A-36B Crib, Fourth Quarter $1994 \ldots \ldots$ 7-5

7-3 Constituent List and Summary of Results for the 216-A-36B Crib Data for Repart ing Period October I through December 31, 1994 . . 7-6

7-4. Constituents with at Least One Detected Value for the 216-A-36B Crib Data for Reporting Period October 1 through December 31, 1994 . . . 7-9

7-5 Contanination Indicator Paraneters for the $216-\mathrm{A}-36 \mathrm{~B} \mathrm{Crib}$ Data for Reporting Pertod October 1 through December 31, 1994 . . 7-12

7-6 Orinking Water Standards Exceeded in the 216-A-36B Cib Groundwater Monitoring Network................. 7-13 


\title{
7.0 216-A-36B CRIB
}

\author{
H. H. Eorington \\ Westinghouse Hanford Company
}

\subsection{IHTRODUCTIOH}

The 216-A-36B Crib (A-36B Crib) is a retired liquid waste disposal facility for the PUREX PIant. The crib is located in the 200 East Area approximately $365 \mathrm{~m}(1,200 \mathrm{ft}$ ) south of the PUREX Plant (Figures 1-1 and 7-1). The A-36B Crib is the southernmost 152 a (500 ft) of a crib originally known as the 216-A-36 Crib. The anmonia scrubber distillate waste stream that resulted from PUREX operations was discharged to the crtb to percolate through the soil column. The original crib received liquid effiuent from. September 1965 to March 1966 . Because a substantial inventory of radionuclides was disposed of in the crib and was assuned to have infiltrated the sediments near the inlet of the crib, a grout curtatn was emplaced to partition the crib into two sections, known as $A$ and B. Discharge to the A-36B Crib section resumed in March 1966 and continued until 1972 when the crib was temporarily removed from service. The crib was placed back in service in November 1982 and operated until it was petired in 0ctober 1987.

Ammonia scrubber distillate effluent disposed of in the A-36B Crib was the condensate from the fuel-rod decladding operation. In this process, the zircalloy cladding was removed from the irradiated fuel by dissolving the cladding in a boiling solution of ammonium fluoride and anmonium nitrate. Waste stream constjtuents are known to have included radionuclides (tritium, strontium-90, cesfun-137, ruthenium-106, cobalt-60, and uranium) and hazardous substances (ammonitur, fiuoride, and nitrate).

In accordance with the Hanford Federal Facility Agreement and Consent Order (Tri-Party Agreement) (Ecology et al. 1994), Mi lestone M-20-34, RCRA closure/post-closure plans for the A-36B Crib are to be submitted to Ecology and the EPA in March 1996. An interin-status detection-level RCRA groundwater monitoring network has been in pTace at the A-36B Crib since May 1988.

The RCRA groundwater monitoring system consists of five downgradient wells and three upgradient wells, as listed in Table 7-1. This is a change from the previous reporting period then the wonitoring network consisted of s1x downgradient wel1s and one upgradient well. The Groundwater Monttoring Plan for A-36B has been revised. The two upgradient we1ls from the 216-A-10 Crib monjtoring network, 299-E24-18 and 299-E25-36, have been added as upgradient wells and 299-E17-5 is no longer sampled and is used for water level information only.

We1l locations for the A-36B monitoring network are shown in Figure 7-1. CIP critical wans were calculated using four quarters of data from September 1988 to June 1989. As shown in Table 7-1, data from well 299-El7-15 are also used by the 216-A-29 Ditch groundwater quality assessment. 


\subsection{MATER LEVEL HEASUREMENTS}

Water leve] measurements are made quarterly and during seralannual RCRA sample collection. Water level data collected during the october through December 1994 perjod are reported in Table 7-2. Three wells have water 1evel measurements that are out of the expected range. These measurements occurred at the time of sampling.

\subsection{MATER CHENISTRY DATA}

Groundwater samples were collected frow the 216-A-36B $\mathrm{Cr} 1 \mathrm{~b}$ monitoring network during October and December 1994. The constituent 1 ist and sumary of results are provided in Table 7-3. Constituents with at least one value above the MDL for all avallable dat are presented in Table 7-4. Table 7-5 11sts the results of the CIPs. Data flags are discussed in Section 1.2 and 1.4 .

No CIP replicate average exceeded the calculated critical mean during the reporting period. A RADE was subaitted for field $\mathrm{pH}$ results from well 299-E17-19. The fjeld measurements taken in quadrupjicate for this well ranged between 6.67 and 6.75 , whije the lab pH for one of these samples was 8.I. Past field pH measurements for this well have been between 7.5 and 8.0 .

Constituents exceeding the drinking water standards during the reporting period were jodine-129, tritium, chrosium, nitrate, and iron. For details on specific wells, see Table $7-6$.

Samples from we11 299-E17-9 were misplaced in the samplers holding area for 55 days before they were sent to the laboratory for analysis. Therefore, not all of the sample results are contained in this report and holding times were missed for laboratory $\mathrm{pH}$, laboratory conductivity, turbidity, anions, and phenols. A fieTd analys is was done of $\mathrm{pH}$, conductivity, and nitrate the jast week of January 1995 to isse as comparison results. This will be discussed in the January-Harch 1995 quarterly report when the rest of the data have been reported. 


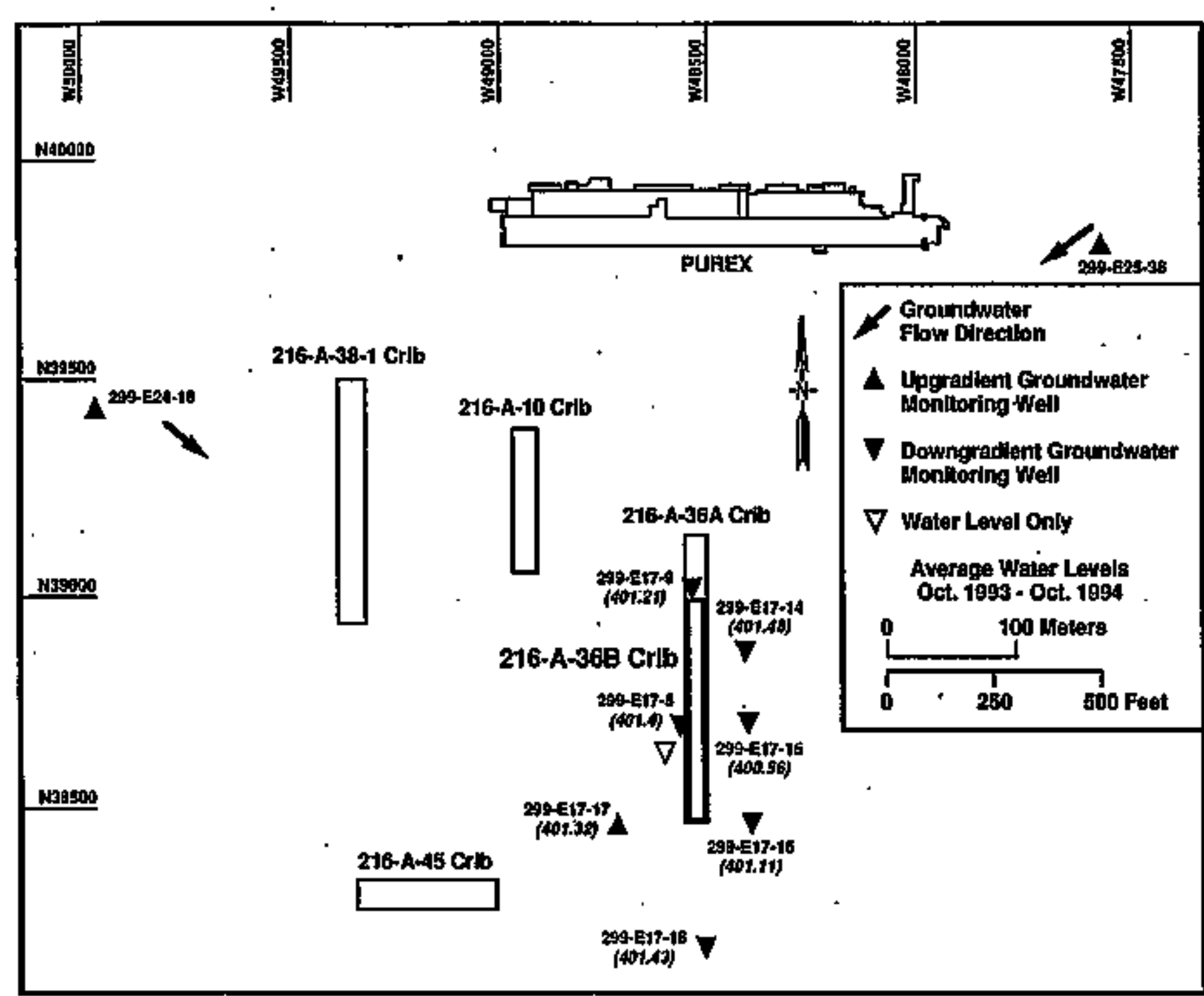

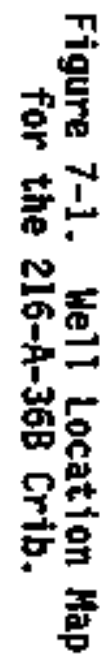

H94t10.10.12 
Table 7-1. Well Identification and Sampling Schedule for the 216-A-36B Crib Groundwater Monitoring Network.

\begin{tabular}{|c|c|c|c|c|}
\hline $\begin{array}{c}\text { Wel1 } \\
\text { no- } \\
(299-)\end{array}$ & $\begin{array}{l}\text { Relative } \\
\text { position }\end{array}$ & Hydrogeologic, unit & $\begin{array}{c}\text { Sample } \\
\text { frequency }\end{array}$ & $\begin{array}{l}\text { Sample date, } \\
\text { 4th Qtr } 1994\end{array}$ \\
\hline E]7-17 & Upgradient & Ringold: Water Table & Semiannually & $12 / 6 / 94$ \\
\hline E24-17 & Upgradtent & Ringold: Water Tab]e & Semiannual 1y & $10 / 14 / 94$ \\
\hline $\mathrm{E} 25-36^{\circ}$ & Upgradient. & Ringold: Water Table & Semfannual1y & $10 / 13 / 94$ \\
\hline E17-5 & Downgrad lent & Ringold: Hater Table & $\begin{array}{c}\text { water leve1 } \\
\text { only }\end{array}$ & - \\
\hline El7-9 & Downgradient & Ringold: Hater Table' & Semiannual 1y & $12 / 7 / 94$ \\
\hline E17-14 & Downgradient & Ringold: Water Table & Semiannua $11 y$ & $12 / 6 / 94$ \\
\hline$E 17-15^{9}$ & Downgradient & Ringold: Water Tabla & Semi annua\}ly & $12 / 6 / 94$ \\
\hline$E 17-16$ & Downgradient & Ringold: Water Table & Semi annuałly & $12 / 6 / 94$ \\
\hline E17-18 & Downgradient & Ringold: Nater Table & Semiannually & $12 / 7 / 94$ \\
\hline
\end{tabular}

We11 shared with the 216-A-29 Ditch network. 
Tab7e 7-2. RCRA Water Level Measurement Report 216-A-36B Crib, Fourth Quarter 1994.

\begin{tabular}{|c|c|c|c|c|}
\hline We?1 & Date & $\begin{array}{l}\text { Depth to } \\
\text { water (ft) }\end{array}$ & $\begin{array}{l}\text { Yater } \\
\text { elevation } \\
\text { (ft) }\end{array}$ & $\begin{array}{l}\text { Jeve? } \\
\text { above ns1 } \\
\text { (in) }\end{array}$ \\
\hline 299-E17-14 & $\begin{array}{l}12 / 06 / 94 \\
12 / 12 / 94\end{array}$ & $\begin{array}{l}320.88 \\
320.80\end{array}$ & $\begin{array}{l}401.30 * \\
401.38\end{array}$ & $\begin{array}{l}122.32 \\
122.34\end{array}$ \\
\hline 299-E17-15 & $\begin{array}{l}12 / 06 / 94 \\
12 / 12 / 94\end{array}$ & $\begin{array}{l}321.65 \\
321.24\end{array}$ & $\begin{array}{l}400.13^{\star} \\
400.54\end{array}$ & $\begin{array}{r}121.96 \\
-122.08\end{array}$ \\
\hline 299-E17-I6 & $\begin{array}{l}12 / 06 / 94 \\
12 / 12 / 94\end{array}$ & $\begin{array}{l}319.48 \\
319.47\end{array}$ & $\begin{array}{l}401.10^{\star} \\
401.11\end{array}$ & $\begin{array}{l}122.26 \\
122.26\end{array}$ \\
\hline 299-E17-I7 & $\begin{array}{l}12 / 05 / 94 \\
12 / 12 / 94\end{array}$ & $\begin{array}{l}320.13 \\
318.64\end{array}$ & $\begin{array}{l}399.79 *_{+} \\
401.28\end{array}$ & $\begin{array}{l}121.86 \\
122.31\end{array}$ \\
\hline 299-E17-18 & $\begin{array}{l}12 / 07 / 94 \\
12 / 12 / 94\end{array}$ & $\begin{array}{l}320.76 \\
319.36\end{array}$ & $\begin{array}{l}399.89{ }^{*}+ \\
401.29\end{array}$ & $\begin{array}{l}121.89 \\
122.31\end{array}$ \\
\hline 299-E17-5 & $12 / 12 / 94$ & 317.86 & $400.83^{\circ}$ & 122.17 \\
\hline 299-E17-9 & $\begin{array}{l}12 / 07 / 94 \\
12 / 12 / 94\end{array}$ & $\begin{array}{l}316.87 \\
316.52\end{array}$ & $\begin{array}{l}400.77^{\star} \\
401.12\end{array}$ & $\begin{array}{l}122.15 \\
122.26\end{array}$ \\
\hline 299-E24-18 & $\begin{array}{l}10 / 14 / 94 \\
12 / 12 / 94\end{array}$ & $\begin{array}{l}400.75 \\
317.93\end{array}$ & $\begin{array}{l}318.53^{\star}+ \\
401.35\end{array}$ & $\begin{array}{r}97.09 \\
122.33\end{array}$ \\
\hline 299-E25-36 & $\begin{array}{l}10 / 13 / 94 \\
12 / 12 / 94\end{array}$ & $\begin{array}{r}305.96 \\
306.03\end{array}$ & $\begin{array}{l}401.43^{*} \\
401.36\end{array}$ & $\begin{array}{l}122.36 \\
122.33\end{array}$ \\
\hline
\end{tabular}

NOTES: 1. Water level elevations are calculated by subtracting the measured depth-to-water from the surveyed elevation for the well.

2. Depth-to-water values are transcribed from field records.

3. Elevations marked with an $1 * 1$ were measured at the time of sampling.

4. Elevation marked with a ' + ' are outside of the expected range, and are supected of error. 
Table 7-3. Constituent List and Sumaary of Results for the 216-A-36B Crib Data for Reporting Period October I through December 31, 1994.

(sheet 1 of 3)

COHTAMJMATICN IMDJCATOR PARAVETERS

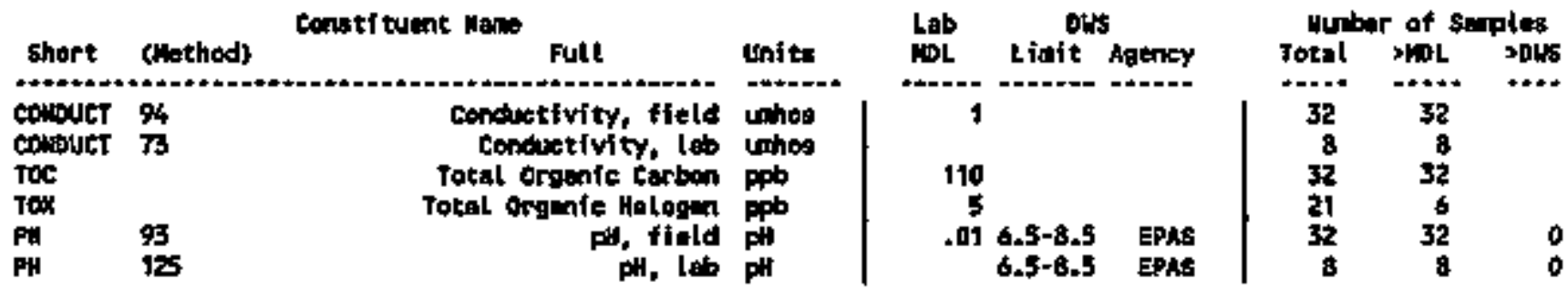

DRTHKIMC MTER PARAHETERS

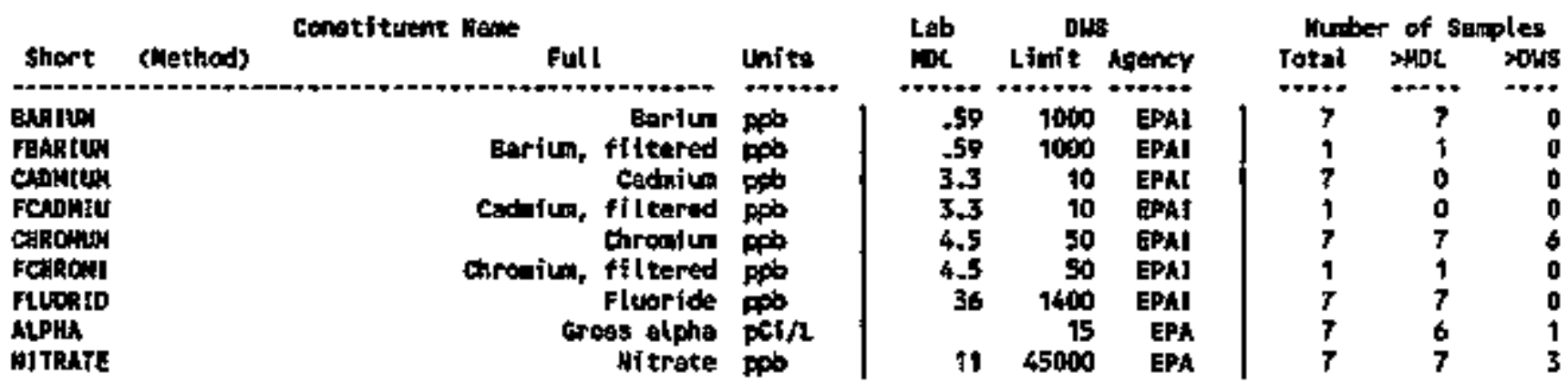

CROUMDHATER DUALITY PARANETERS

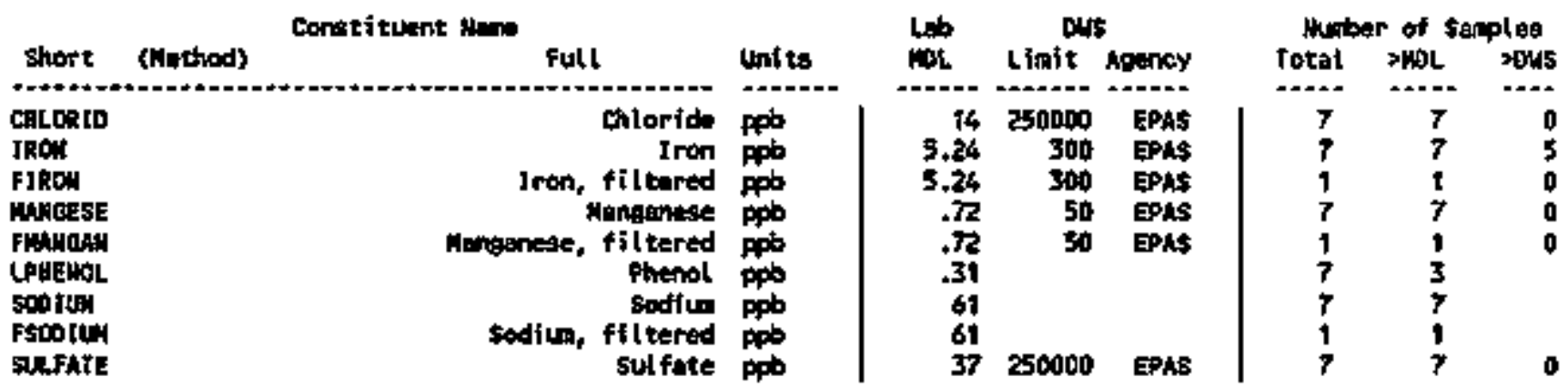


-Table 7-3, Constituent List and Sulamary of Results for the 216-A-36B Crib Data for Reporting Perjod October 1 through December 31, 1994. (sheet 2 of 3 )

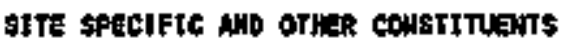

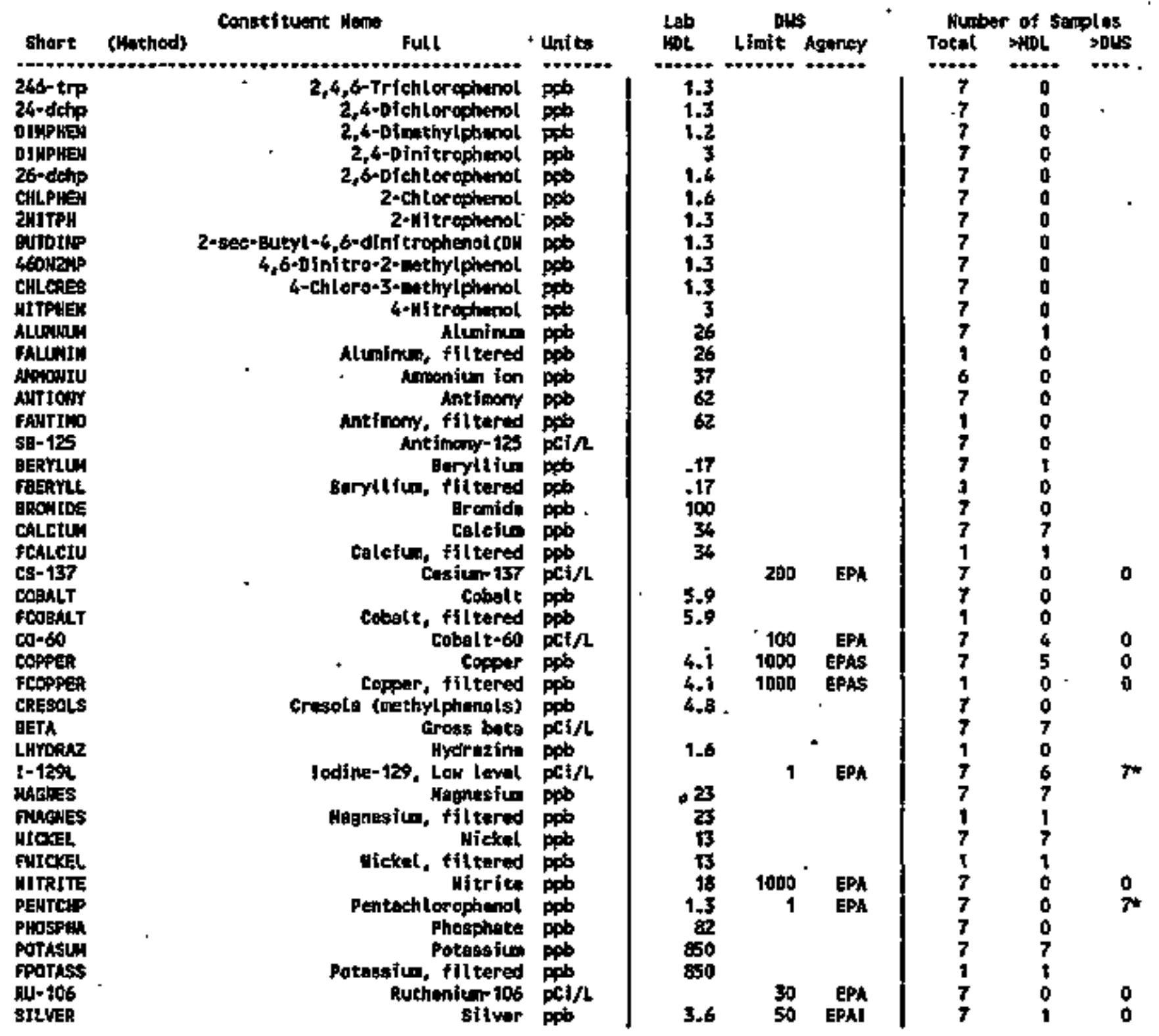


DOE /RL-94-36-4

Table 7-3. Constituent List and Summary of Results for the 216-A-36B Crib Data for Reporting Period October 1 through December 31, 1994. (sheet 3 of 3)

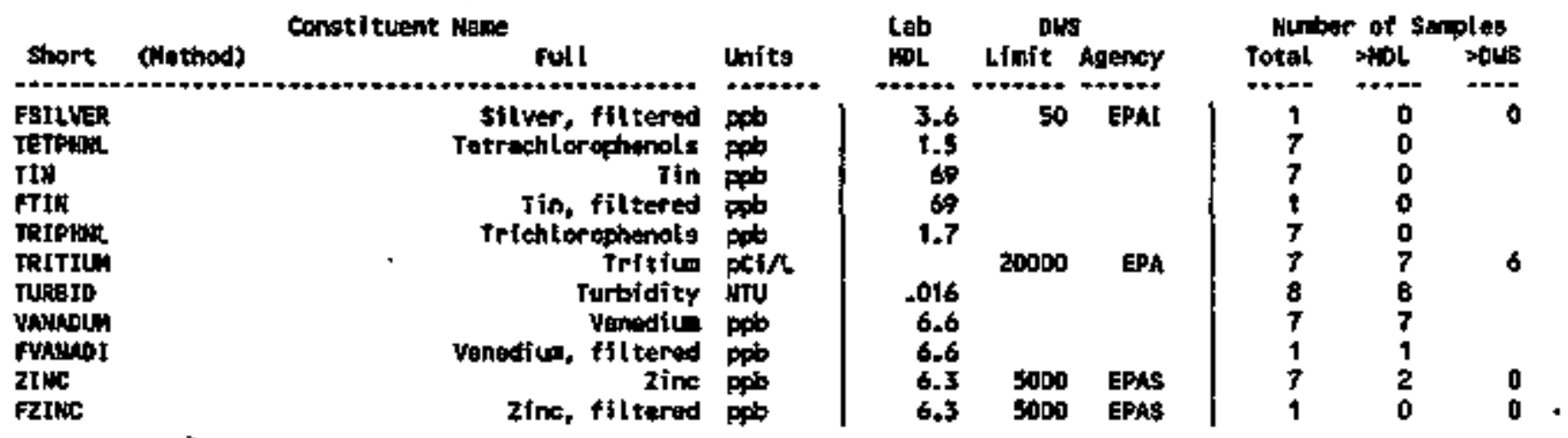

For explanation of this table, sees station 1.4 of report.

7-8 
Table 7-4. Constituents with at Least One Detected Value for the 216-A-36B Crib Data for Reporting Period

October 1 through December 31, 1994.

(sheet 1 of 3 )

\begin{tabular}{|c|c|c|c|c|c|c|}
\hline Helt & $\begin{array}{c}\text { collection } \\
\text { Doto }\end{array}$ & $\begin{array}{l}\text { Serpte } \\
\text { Humber }\end{array}$ & $\begin{array}{c}\text { ALUPALA } \\
\text { 36/pFb } \\
19 \% .\end{array}$ & 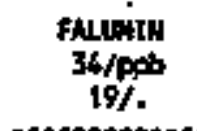 & $\begin{array}{c}\text { BARIUw } \\
\text { 34/ppb } \\
\text { t.3/1000i }\end{array}$ & $\begin{array}{c}\text { FBARIUN } \\
34 / \text { ppo } \\
1.3 / 10009\end{array}$ \\
\hline $\begin{array}{l}299-E 17-14 \\
299-E 17-15 \\
299-E 17-16 \\
29 \%-E 17-17 \\
299-E 17-18 \\
299-E 24-18 \\
299-E 25-36 \\
299-E 25-36\end{array}$ & $\begin{array}{l}12 / 06 / 94 \\
12 / 06 / 94 \\
12 / 06 / 94 \\
12 / 06 / 94 \\
12 / 97 / 94 \\
10 / 14 / 94 \\
10 / 43 / 44 \\
10 / 13 / 94\end{array}$ & 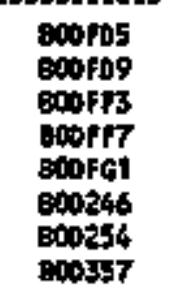 & $\begin{array}{l}39.00 \mathrm{U} \\
26.00 \mathrm{U} \\
26.00 \mathrm{U} \\
26.00 \mathrm{U} \\
26.00 \mathrm{U} \\
26.00 \mathrm{U} \\
26.00 \mathrm{U}\end{array}$ & 26.00 & $\begin{array}{l}53.00 \\
47.00 \\
39.000 \\
36.00 \\
37.000 \\
51.00 \\
21.000\end{array}$ & 21.000 \\
\hline
\end{tabular}

HAII
Hano
$299-E 17-14$
$299-E 17-15$
$299-E 17-16$
$299-E 17-17$
$299-E 17-18$
$299-E 24-18$
$299-E 25-36$
$299-E 25-36$

\begin{tabular}{|c|c|c|c|}
\hline $\begin{array}{c}\text { Cot lestion } \\
\text { Dato }\end{array}$ & $\begin{array}{l}\text { Sample } \\
\text { Uumber }\end{array}$ & $\begin{array}{l}\text { GERYLUA } \\
34 / \mathrm{pPb}^{\circ} \\
.1 .5 \%\end{array}$ & $\begin{array}{c}\text { PaEkTLL } \\
34 / \mathrm{Ppb} \\
\text { t.5/. }\end{array}$ \\
\hline $\begin{array}{l}12 / 06 / 44 \\
12 / 06 / 94 \\
12 / 06 / 94 \\
12 / 06 / 94 \\
12 / 07 / 94 \\
10 / 14 / 44 \\
10 / 43 / 44 \\
10 / 13 / 94\end{array}$ & 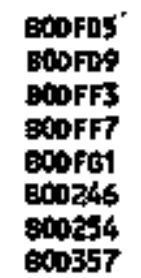 & $\begin{array}{l}.17 \mathrm{u} \\
.17 \mathrm{U} \\
.17 \mathrm{U} \\
.17 \mathrm{u} \\
.17 \mathrm{u} \\
.37 \mathrm{U} \\
.17 \mathrm{U}\end{array}$ & (17. \\
\hline
\end{tabular}

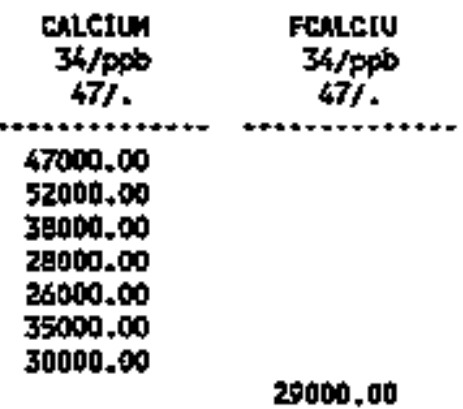

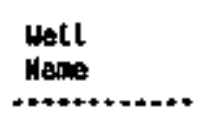

799-E17-14

299-E17-15

299-E17-16

$299-E 17-17$

299-E17-19

299-E24-18

299-E25-36

299-E25-36

\begin{tabular}{|c|c|}
\hline $\begin{array}{c}\text { Coltection } \\
\text { oete }\end{array}$ & $\begin{array}{l}\text { serpte } \\
\text { Member }\end{array}$ \\
\hline $\begin{array}{l}12 / 06 / 94 \\
12 / 06 / 94 \\
12 / 05 / 94 \\
12 / 06 / 94 \\
12 / 07 / 94 \\
10 / 14 / 94 \\
10 / 13 / 94 \\
10 / 13 / 94\end{array}$ & 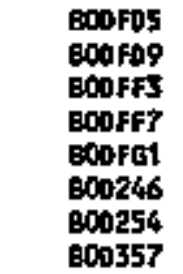 \\
\hline
\end{tabular}

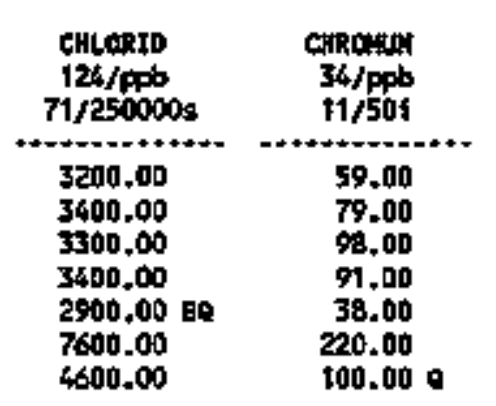

\begin{tabular}{|c|c|}
\hline $\begin{array}{l}\text { FCHDont } \\
34 / \text { pot } \\
11 / 50 \mathrm{t}\end{array}$ & $\begin{array}{c}00-60 \\
140 / \mathrm{psi}^{-1 / L} \\
.1100\end{array}$ \\
\hline & $\begin{array}{c}11.10 . \\
5.39 \mathrm{U} \\
15.30 \\
5.62 \mathrm{U} \\
9.67 \\
2.13 \mathrm{U} \\
24.40 \mathrm{~F}\end{array}$ \\
\hline
\end{tabular}

\begin{tabular}{|c|c|c|}
\hline Want & $\begin{array}{c}\text { Collection } \\
\text { Dete }\end{array}$ & $\begin{array}{l}\text { Sample } \\
\text { iknotper }\end{array}$ \\
\hline $\begin{array}{l}299-E 17-14 \\
299-E 17-15 \\
299-E 17-16 \\
299-E 17-17 \\
299-E 17-18 \\
299-E 24-18 \\
298-E 25-36 \\
299-E 25-36\end{array}$ & $\begin{array}{l}12 / 06 / 94 \\
12 / 06 / 94 \\
12 / 06 / 94 \\
12 / 06 / 94 \\
12 / 07 / 94 \\
10 / 14 / 44 \\
10 / 13 / 94 \\
10 / 13 / 96\end{array}$ & 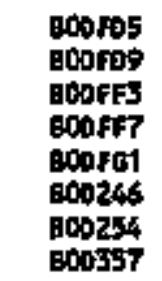 \\
\hline
\end{tabular}

\begin{tabular}{|c|c|c|}
\hline $\begin{array}{c}\text { CDPPJ } \\
34 / 4 \text { pb } \\
2.6 / 100104\end{array}$ & $\begin{array}{c}\text { FCOPPER } \\
34 / p \times b \\
2.6 / 1000 \mathrm{~s}\end{array}$ & 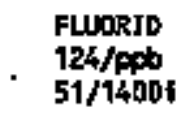 \\
\hline $\begin{array}{r}9.00 \mathrm{~L} \\
4.10 \mathrm{U} \\
4.10 \mathrm{U} \\
5.30 \mathrm{~L} \\
6.40 \mathrm{~L} \\
15.00 \mathrm{~L} \\
6.00 \mathrm{~L}\end{array}$ & & $\begin{array}{r}1000.09 \\
1000.00 \\
300.00 \\
1000.00 \\
600.00 \\
900.00 \\
600.00\end{array}$ \\
\hline
\end{tabular}

ALPtA
$135 /$ PCi/L
.115
4.26
4.44
3.95
4.45
4.92
16.40
$.06 \mathrm{U}$

$4.10 \mathrm{U}$ 
Tab1e 7-4. Constituents with at Least One Detected Value for the 216-A-36B Crtb Data for Reporting Period

October 1 through December 31, 1994. (sheet 2 of 3 )

\begin{tabular}{|c|c|c|c|c|c|c|}
\hline Wall & $\begin{array}{l}\text { Collection } \\
\text { Date }\end{array}$ & $\begin{array}{l}\text { Semple } \\
\text { Wholpar }\end{array}$ & $\begin{array}{c}\text { BeTA } \\
\text { I36/PCI/A } \\
. /\end{array}$ & $\begin{array}{c}1-129 \\
139 / p c i / L \\
/ / 1\end{array}$ & $\begin{array}{c}\text { JRON } \\
34 / \mathrm{ppob}^{\circ} \\
16 / 3000\end{array}$ & $\begin{array}{l}\text { FIROH } \\
\text { 34/ppo } \\
18 / 300 \%\end{array}$ \\
\hline $\begin{array}{l}299+E 17-14 \\
299-E 17-15 \\
299-E 17-18 \\
299-E 17-17 \\
299-E 17-18 \\
299-E 24-18 \\
299-E 45-36 \\
299-E+5-36\end{array}$ & $\begin{array}{l}12 / 06 / 94 \\
12 / 06 / 94 \\
12 / 06 / 94 \\
12 / 06 / 94 \\
12 / 07 / 94 \\
10 / 14 / 94 \\
10 / 13 / 94 \\
10 / 13 / 94\end{array}$ & 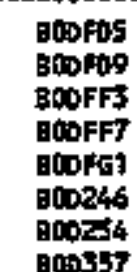 & $\begin{array}{l}88.60 \\
92.60 \\
62.90 \\
14.30 \\
21.90 \\
26.70 \\
5.13\end{array}$ & $\begin{array}{l}9.15 \\
9.75 \\
6.11 \\
5.996 \\
4.79 \\
2.66 \\
1.20\end{array}$ & $\begin{array}{l}270.00 \mathrm{~B} \\
370.00 \mathrm{~B} \\
440.00 \mathrm{~B} \\
390.00 \mathrm{~g} \\
190.00 \mathrm{8} \\
870.00 \mathrm{00} \\
480.00 \mathrm{B0}\end{array}$ & 31 to $\mathrm{po}$ \\
\hline
\end{tabular}

\begin{tabular}{l} 
Well \\
Heme \\
\hdashline $299-E 17+14$ \\
$299-E 17-15$ \\
$25 \%-E 17-56$ \\
$299-E 17-17$ \\
$299-E 17-18$ \\
$299-E 24-18$ \\
$299+E 25-36$ \\
$259-E 25-36$
\end{tabular}

\begin{tabular}{|c|c|}
\hline $\begin{array}{c}\text { Collestion } \\
\text { Date }\end{array}$ & $\begin{array}{l}\text { Sanple } \\
\text { Hunber }\end{array}$ \\
\hline $\begin{array}{l}12 / 06 / 94 \\
12 / 06 / 94 \\
12 / 01 / 94 \\
12 / 06 / 94 \\
12 / 07 / 94 \\
10 / 14 / 94 \\
10 / 13 / 94 \\
10 / 13 / 46\end{array}$ & 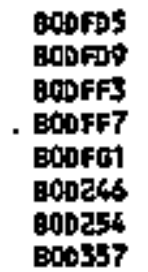 \\
\hline
\end{tabular}

\begin{tabular}{cc} 
AUCHES & $\begin{array}{c}\text { FMAGHES } \\
34 / \mathrm{ppb} \\
34 / F \mathrm{~b}\end{array}$ \\
$25 /$. & $25 /$. \\
\hline 15000.00 & \\
13000.00 & \\
11000.00 & \\
8300.00 & \\
7700.00 & \\
11000.00 & \\
8200.00 &
\end{tabular}

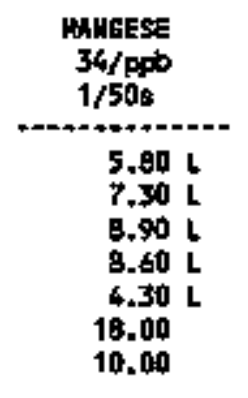

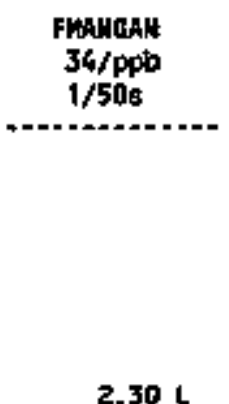

\begin{tabular}{|c|c|c|}
\hline Lith & Collection & sepple \\
\hline $\begin{array}{l}299-E 17-14 \\
299-E 17-15 \\
299-E 17-16 \\
299-E 17-17 \\
299-E 17-18 \\
299-E 24-18 \\
299-E 25-36 \\
299-E 25-36\end{array}$ & $\begin{array}{l}12 / 06 / 94 \\
12 / 06 / 94 \\
12 / 6 / 46 \\
12 / 06 / 94 \\
12 / 07 / 94 \\
10 / 14 / 44 \\
10 / 13 / 94 \\
10 / 13 / 94\end{array}$ & $\begin{array}{l}\text { BODFD5 } \\
\text { B00FD9 } \\
\text { B00FF3 } \\
\text { B00FF7 } \\
800 F B 1 \\
\text { 800246 } \\
\text { B00254 } \\
800357\end{array}$ \\
\hline
\end{tabular}

347006
$16 /$.
$23.00 \mathrm{~L}$
33.00
54.00
34.00
30.00
100.00
61.00
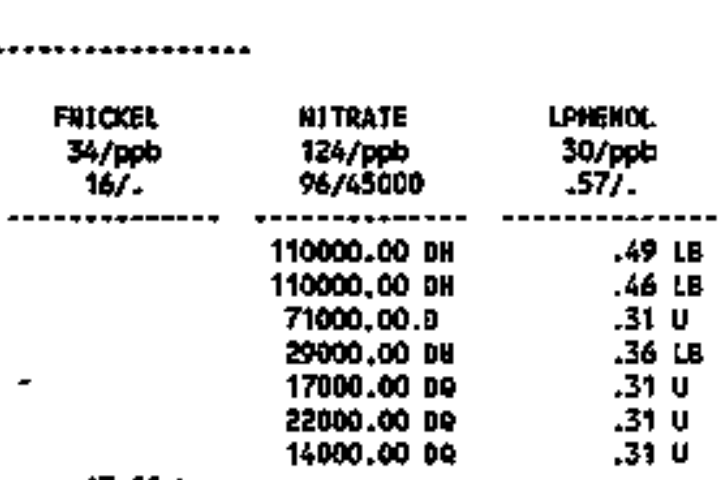

$13.00 \mathrm{~L}$

\begin{tabular}{|c|c|c|}
\hline $\begin{array}{l}\text { Uall } \\
\text { Mans }\end{array}$ & $\begin{array}{c}\text { colilection } \\
\text { Dete }\end{array}$ & $\begin{array}{l}\text { Sample } \\
\text { Huiber }\end{array}$ \\
\hline $\begin{array}{l}299-E 17-14 \\
299-E 17-15 \\
299-E 17-16 \\
299-E 17-17 \\
299-E 17-18 \\
299-624-18 \\
299-E 75-36 \\
299-675+36\end{array}$ & $\begin{array}{l}12 / 06 / 94 \\
12 / 06 / 94 \\
12 / 06 / 94 \\
12 / 06 / 94 \\
12 / 07 / 94 \\
10 / 14 / 94 \\
10 / 13 / 94 \\
10 / 13 / 94\end{array}$ & 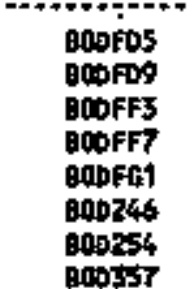 \\
\hline
\end{tabular}

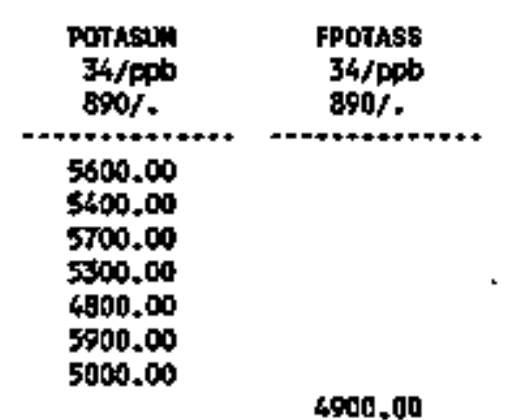

81LVER
$34 / \mathrm{ppb}$
$3.4 / 501$
$3.70 \mathrm{~L}$
$3.60 \mathrm{U}$
$3.60 \mathrm{U}$
$3.60 \mathrm{U}$
$3.60 \mathrm{U}$
$3.60 \mathrm{U}$
$3.60 \mathrm{U}$

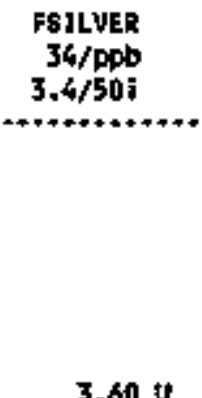


Table 7-4. Constituents with at Least One Detected Value for the 216-A-36B Crib Data for Reporting Period October 1 through December 31, 1994. (sheet 3 of 3 )

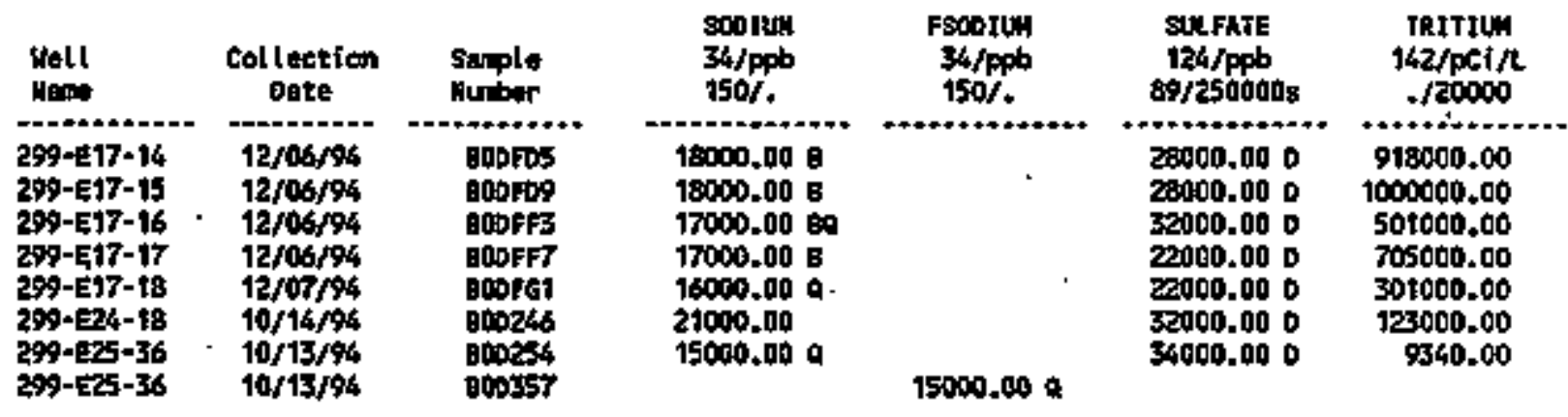

\begin{tabular}{|c|c|c|c|c|c|c|}
\hline Woll & $\begin{array}{c}\text { Collection } \\
\text { Date }\end{array}$ & $\begin{array}{l}\text { senple } \\
\text { humbir }\end{array}$ & 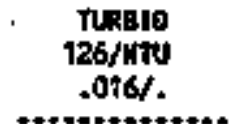 & $\begin{array}{l}\text { Wunpu } \\
34 / 17 \% \\
6.4 / 7\end{array}$ & $\begin{array}{l}\text { FWaind } \\
34 / \mathrm{ppb} \\
6.4 /\end{array}$ & $\begin{array}{c}\text { zInc } \\
34 / \mathrm{Pob}^{\circ} \\
4,4 / 5000 \%\end{array}$ \\
\hline $\begin{array}{l}299-E 17-14 \\
299-E 17-15 \\
299-E 27-16 \\
299-E t 7-17 \\
299-E t 7-18 \\
299-E 17-9 \\
294-E 24-18 \\
299-625-36 \\
299-E 25-36\end{array}$ & $\begin{array}{l}12 / 06 / 94 \\
12 / 06 / 94 \\
12 / 06 / 94 \\
12 / 06 / 94 \\
12 / 07 / 94 \\
12 / 07 / 94 \\
10 / 14 / 94 \\
10 / 13 / 94 \\
10 / 13 / 94\end{array}$ & 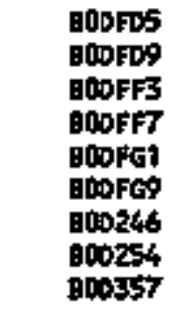 & $\begin{array}{l}1.10 \\
1.90 \\
1.50 \mathrm{a} \\
1.20 \\
1.50 \mathrm{a} \\
2.10 \mathrm{aH} \\
1.40 \mathrm{0} \\
1.40 \mathrm{a}\end{array}$ & $\begin{array}{c}24.00 \mathrm{~L} \\
23.00 \mathrm{~L} \\
27.00 \mathrm{~L} \\
25.00 \mathrm{~L} \\
27.00 \mathrm{~L} \\
21.00 \mathrm{~L} \\
27.00 \mathrm{~L} \\
.\end{array}$ & $25.00 \mathrm{~L}$ & $\begin{array}{l}6.30 \mathrm{U} \\
6.30 \mathrm{U} \\
6.30 \mathrm{U} \\
0.30 \mathrm{U} \\
7.90 \mathrm{~L} \\
8.40 \mathrm{~L} \\
6.30 \mathrm{U}\end{array}$ \\
\hline
\end{tabular}

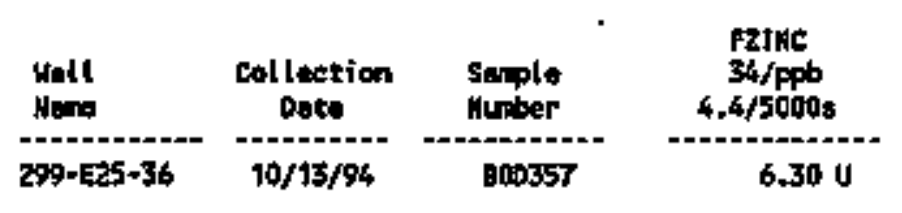

For explandien of this table, see sectlon 1.4 of report. 
Table 7-5. Contamination Indicator Parameters for the 216-A-36B Crib Data for Reporting Period October 1 through December 31, 1994.

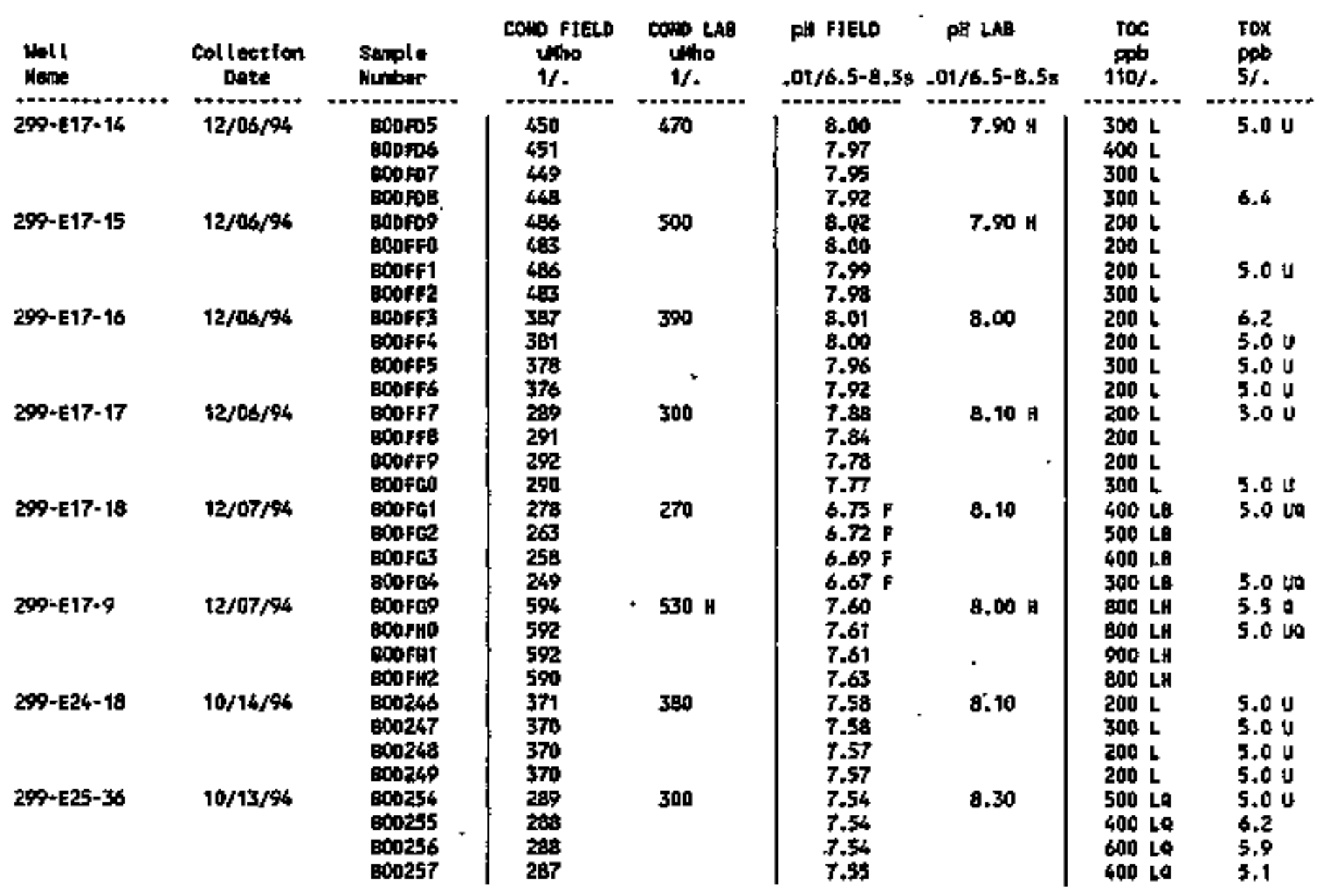

For explanation of this table, see sectian 1.4 of raport. 


$$
\text { DOE/RL-94-36-4 }
$$

Table 7-6. Drinking Water Standards Exceeded in the 216-A-36B Cib Groundwater Monitoring Network.

\begin{tabular}{|c|c|}
\hline Constituent (OWS) & Wel1s exceeding OWS (299-) \\
\hline Chromium, unfiltered samples (50pp) & E17-14, E17-15, E17-16, E17-17 \\
\hline Iron, unfiltered samples (300 ppb) & E17-15, E17-16, E17-17 \\
\hline Nitrate (45,000 ppb) & E17-14, E17-15, E17-16 \\
\hline Tritfum (20,000 pCi/L) & E17-14, E17-15, E17-16, E17-17, \\
\hline E17-18 \\
\hline Iodine-129(1 pCi/L) & E17-14, E17-15, E17-16, E17-17, \\
\hline
\end{tabular}


DOE/RL-94-36-4

This page intentionaliy left bTank. 


\section{CONTENTS}

8.0 216-A-10 CRIB . . . . . . . . . . . . . 8-1

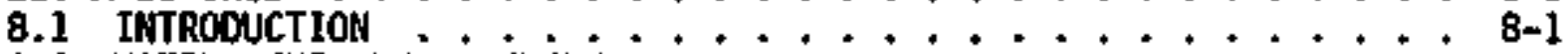

8.2 MATER LEVEL MEASUREMENTS $\ldots \ldots \ldots$

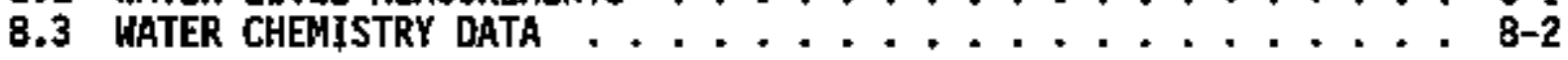


DOE/RL-94-36-4

LIST OF FIGURES

8-1. Well Location Map for the 216-A-10 Crib . . . . . . . . 8-3

\section{LIST OF TABLES}

B-1 Well Identification and 5ampling Schedule for the 216-A-10 Crib Groundwater Monitoring Network . . . . . . . . . . 8-4

B-2 - RCRA Water Level Measurement Report 216-A-10 Crib, Fourth Quarter $1994 \ldots . . . . . . . . . . .8-5$

8-3 Constituent List and Sammary of Result for the 216-A-10 Crib Data for Reporting Period October 1 through Decenber 31, 1994 ... 8- 8

8-4 Constituents with at Least One Detected Value for the 216-A-10 Crib Data for Reporting Period October 1 through Decenber 31, 1994 . . . 8-9

8-5 Contamination Indicator Paraneters for the $216-\mathrm{A}-10 \mathrm{Cr} i \mathrm{~b}$ Data for Reporting Period October 1 through Decenber 31, 1994 . . 8-13

8-6 Orinking Water Standards Exceeded in the 216-A-36B Crib Groundwater

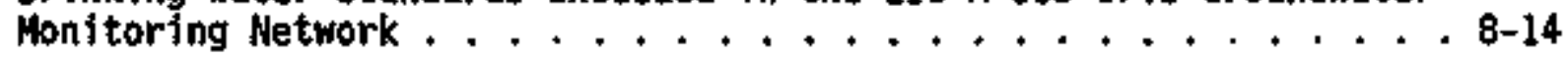


DOE/RL-94-36-4

\title{
8.0 216-A-10 CRIB
}

\author{
H. H. Edrington \\ Westinghouse Hanford Coupany
}

\subsection{INTRODUCTION}

The 216-A-10 Crib (A-10 $\mathrm{Cr}$ ib) is a retired liquid waste disposal facility that was used during the operation of the PUREX Plant. The crib is located in the 200 East Area approximately $120 \mathrm{~m}(400 \mathrm{ft})$ south of the PUREX P1 ant (Figures 1-1 and 8-1).

Severat waste streams, collectively referred to as the process distillate discharge, were disposed of in the A-10 Crib for soil-column disposal during PUREX P1ant operations. Process dist11]ate discharge waste was. first disposed of in the crib for 4 months during the 1956 PUREX startup. In 1961, the A-10 Crib replaced the 216-A-5 Crib and received PUREX effluent continuously until 1973. Periodic discharges were recejved in 1977, 1978, and 1981 . From 1982 to 1987, continuous effluent discharges to the A-10 Crjb were resumed. Effluent volumes from 1981 to 1986 averaged $1 \times 10^{5} \mathrm{~L}(2.6 \times$ $10^{6}$ gal) per year. In 1987, the A-10 Crib was removed from service and replaced by the 216-A-45 Crib.

The process distillate discharge waste stream sent to the A-10 Crib was characteristicaliy acidic and contained concentrated salts. Waste strean constituents included al iphatic hydrocarbon compounds, organic conplexants, and radionuclides (plutoniur, uranium, strontfum-90, cobait-60, cesfum-134 and -137, ruthenium-103 and -106 , and tritium).

In accordance with the Hanfond Federal Facility Agreement and Consent Order (Tri-Party Agreement) (Ecology et al. 1992), Mjlestone M-20-33, RCRA closure/post-closure plans for the A-10 Crib are to be submitted to Ecology and EPA in March 1996. An interim-státus detection-level RCRA groundwater monitoring network has been operational since November 1988.

The RCRA groundwater monitoring system consists of six downgradient wells and two upgradient wells, as 1isted in Table 8-1. Two wells in the 216-A-10 network are shared with the groundwater monitoring network for the A-29 Ditch facility. We11 locations are show in Figure 8-1. Background wonitoring was completed in the fourth quarter of 1989, following the first four quarters of groundwater sampiing.

\subsection{WATER LEVEL MEASUREMEMTS}

Hater lovel measurenents are made quarterly and during semfannual RCRA sample collection. Water level data collected during the october through Decenber 1994 period are reported in Tab]e 8-2. 


\subsection{MATER CHEMISTRY DATA}

Groundwater samples were collected from the 216-A-10 Crib nonjtoring network during October 1994 . The constituent list and summary of results are provided in Table 8-3. Constituents with at least one value above the MDL for all avallable data are presented in Table 8-4. Table 8-5 lists the results of the CIPS. Data flags are discussed in Sections 1.2 and 1.4 .

No CIP replicate average exceeded the calculated critical mean during the reporting period. A RAOE was submitted for unfiltered iron and turbidity results from well 299-E17-19. Results were several orders of magnitude higher than previous semi-annual sampling results. This well has been put on the list for remediation'for this year.

Constituents exceeding the drinking water standards during the reporting period were lodine-129, tritium, unfiltered chromium, nitrate, unfiltered manganese, gross alpha, and unfittered iron. For details on specific wells, see Table 8-6.

Ruthenium-106 was detected at $70.5 \mathrm{pCl} / \mathrm{L}$, which exceeds the drinking water standard of $30 \mathrm{pC} 1 / \mathrm{L}$. Based on the limit of detection (LOD) calculations in the 1993 RCRA Annual Report, ruthenium-106 has an LOD of approximately 130 to $150 \mathrm{pCj} / \mathrm{L}$. Therefore, the value of $70.5 \mathrm{pC} j / \mathrm{L}$ is not a meaningful value and is undetected. 


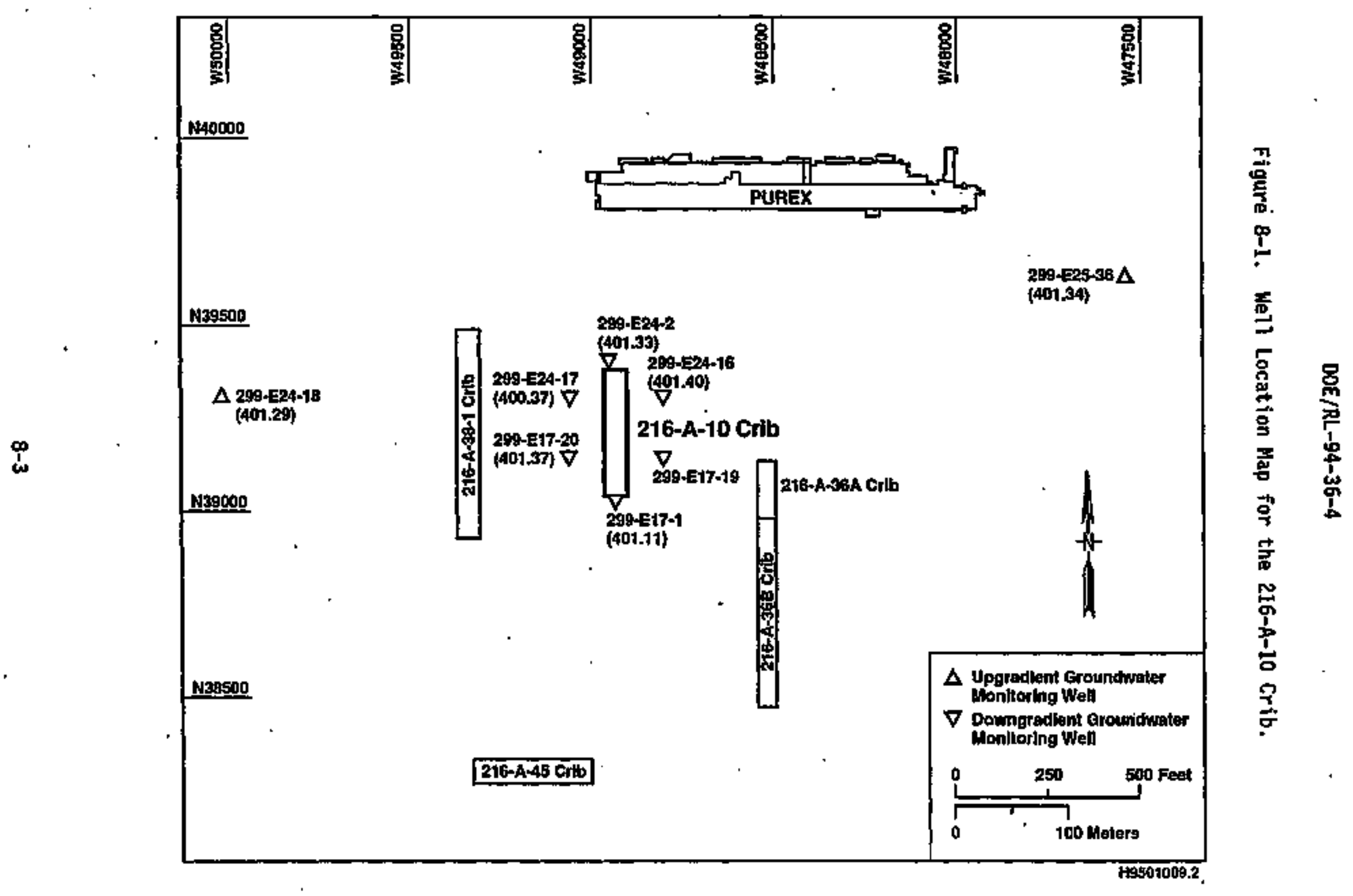


Table 8-1. Well Identification and Sampling Schedule for the 216-A-10 Crib Groundwater Monitoring Network.

\begin{tabular}{|c|c|c|c|c|}
\hline $\begin{array}{c}\text { We1] } \\
\text { no. } \\
\left(299_{-}\right)\end{array}$ & $\begin{array}{l}\text { Relative } \\
\text { position }\end{array}$ & Hydrogeologic unit & $\begin{array}{l}\text { Sample } \\
\text { frequency }\end{array}$ & $\begin{array}{l}\text { Sample date, } \\
\text { 4th Qtr } 1994\end{array}$ \\
\hline E24-18 & Upgradient & $\begin{array}{l}\text { Ringold: Water } \\
\text { Table }\end{array}$ & Semi annual ly & $10 / 14 / 94$ \\
\hline$E 25-36^{\circ}$ & Upgradient & $\begin{array}{l}\text { Ringold: Water } \\
\text { Table }\end{array}$ & Semt annualiy & $10 / 13 / 94$ \\
\hline E17-1 & Downgradient & $\begin{array}{l}\text { Ringold: Water } \\
\text { Table }\end{array}$ & Semiannually & $10 / 21 / 94$ \\
\hline E17-19 & Downgradient & $\begin{array}{l}\text { Ringold: Water } \\
\text { Table }\end{array}$ & Semi annual1y & $10 / 14 / 94$ \\
\hline$E 17-20^{\circ}$ & Downgradient & $\begin{array}{l}\text { Ringold: Water } \\
\text { Table }\end{array}$ & Semiannua11y & $10 / 14 / 94$ \\
\hline E24-2 & Downgradient & $\begin{array}{l}\text { Ringold: Water } \\
\text { Table }\end{array}$ & Semiannually & $10 / 14 / 94$ \\
\hline E24-16 & Downgradient & $\begin{array}{l}\text { Ringold: Water } \\
\text { - Table }\end{array}$ & Semi annuat $1 y$ & $10 / 13 / 94$ \\
\hline E24-17 & Downgradient & $\begin{array}{l}\text { Ringold: Hater } \\
\text { Table }\end{array}$ & Semiannualiy & $10 / 13 / 94$ \\
\hline
\end{tabular}

"Well sampled during period for 216-A-29 Ditch groundwater quality assessment. 
Table 8-2. RCRA Water Leve1 Measurement Report 216-A-10 Crib, Fourth Quarter 1994.

\begin{tabular}{|c|c|c|c|c|}
\hline Welt & Date & $\begin{array}{l}\text { Depth to } \\
\text { water (ft) }\end{array}$ & $\begin{array}{l}\text { Water } \\
\text { elevation } \\
(\mathrm{ft})\end{array}$ & $\begin{array}{l}\text { level } \\
\text { above msl } \\
\text { (m) }\end{array}$ \\
\hline 299-EI7-1 & $\begin{array}{l}10 / 21 / 94 \\
12 / 12 / 94\end{array}$ & $\begin{array}{l}318.68 \\
318.13\end{array}$ & $\begin{array}{l}400.49^{*}+ \\
401.04\end{array}$ & $\begin{array}{l}122.07 \\
122.24\end{array}$ \\
\hline 299-EI7-19 & $12 / 12 / 94$ & 318.49 & 400.84 & 122.18 \\
\hline $299-\mathrm{E} 17-20$ & $\begin{array}{l}10 / 14 / 94 \\
12 / 12 / 94\end{array}$ & $\begin{array}{l}318.70 \\
317.80\end{array}$ & $\begin{array}{l}400.53^{*} \\
401.43\end{array}$ & $\begin{array}{l}122.08 \\
122.36\end{array}$ \\
\hline 299-E24-16 & $\begin{array}{l}10 / 13 / 94 \\
12 / 12 / 94\end{array}$ & $\begin{array}{l}316.70 \\
316.89\end{array}$ & $\begin{array}{l}401.57^{*} \\
401.38\end{array}$ & $\begin{array}{l}122.40 \\
122.34\end{array}$ \\
\hline 299-E24-17 & $\begin{array}{l}10 / 13 / 94 \\
12 / 12 / 94\end{array}$ & $\begin{array}{l}318.27 \\
318.22\end{array}$ & $\begin{array}{l}400.42^{*} \\
400.47\end{array}$ & $\begin{array}{l}122.05 \\
122.06\end{array}$ \\
\hline 299-E24-18 & $\begin{array}{l}10 / 1.4 / 94 \\
12 / 12 / 94\end{array}$ & $\begin{array}{l}400.75 \\
317.93\end{array}$ & $\begin{array}{l}318.53^{*}+ \\
401.35\end{array}$ & $\begin{array}{r}97.09 \\
122.33\end{array}$ \\
\hline 299-E24-2 & $\begin{array}{r}10 / 14 / 94 \\
\cdot 12 / 12 / 94\end{array}$ & $\begin{array}{l}316.16 \\
315.96\end{array}$ & $\begin{array}{l}401.31^{*} \\
401.51\end{array}$ & $\begin{array}{l}122.32 \\
122.38\end{array}$ \\
\hline $299-E 25-36$ & $\begin{array}{l}10 / 13 / 94 \\
12 / 12 / 94\end{array}$ & $\begin{array}{l}305.96 \\
306.03\end{array}$ & $\begin{array}{l}401.43^{*} \\
401.36\end{array}$ & $\begin{array}{l}122.36 \\
122: 33\end{array}$ \\
\hline
\end{tabular}

NOTES: 1. Water level elevations are calculated by subtracting the measured depth-to-water from the surveyed elevation for the well.

2. Depth-to-water values are transcribed from field records.

3. Elevations marked with an ${ }^{* * 1}$ were measured at the time of sampling.

4. Elevation marked with a ' + ' are outside of the expected range, and are supected of error. 
Table 8-3. Constituent List and Sunmary of Results for the 216-A-10 Crib Data for Reporting Period October 1 through Decenber 31, 1994. (sheet 1 of 3 )

CONIAHIMATIOM IIDTChTOR PARAHETERS

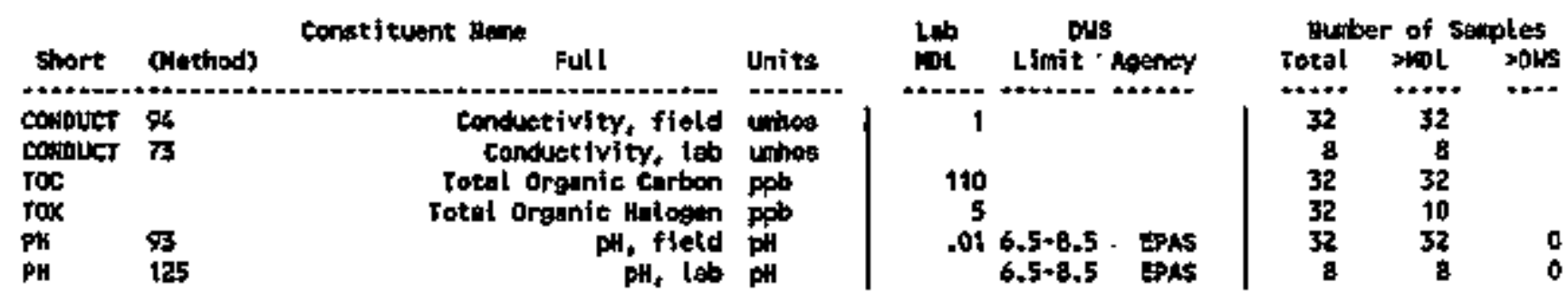

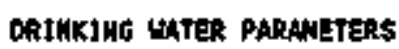

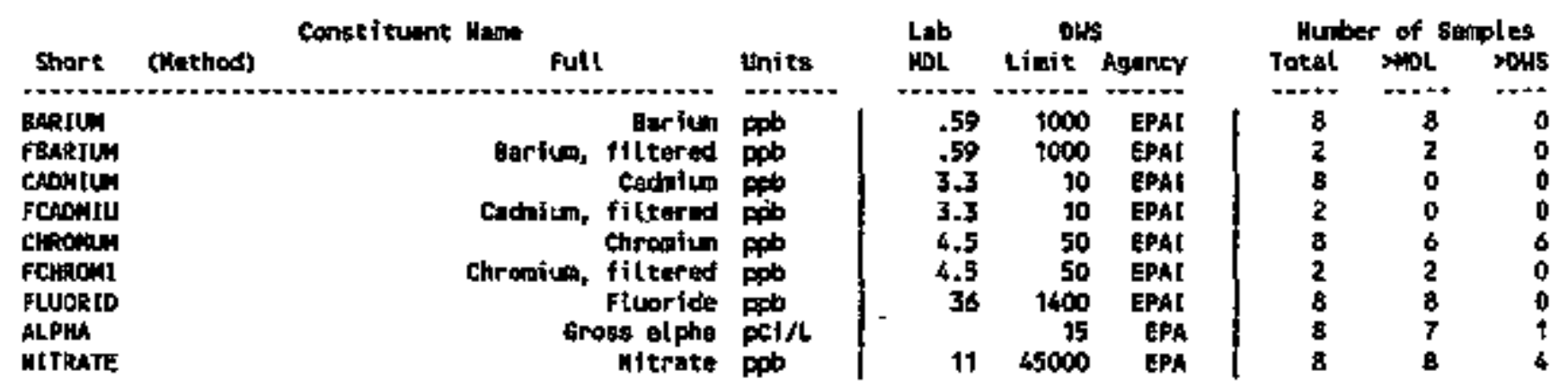

CROUNOWATER GUALITY PARANETEAS

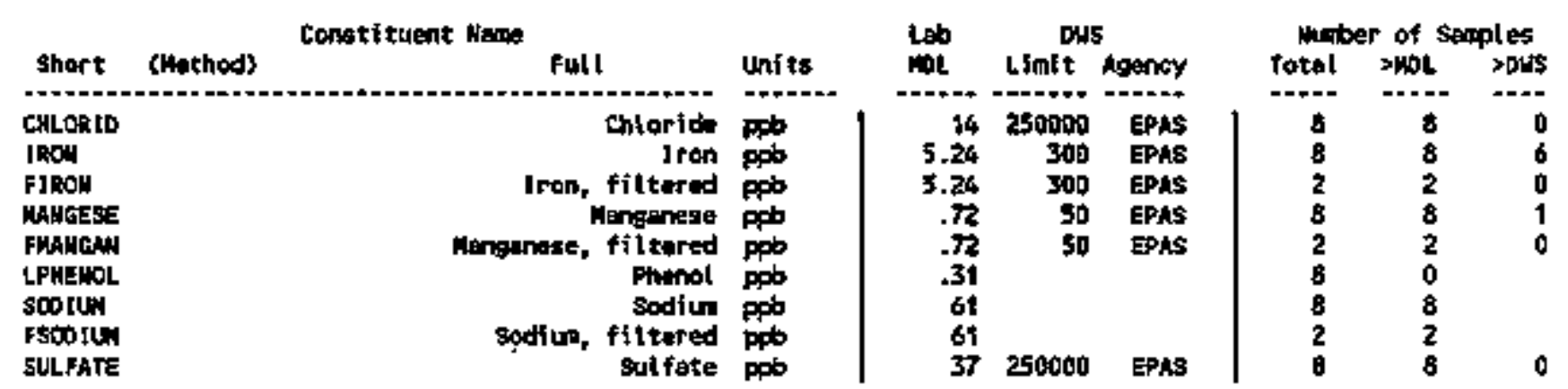


Table 8-3. Constituent List and Summary of Results for the 216-A-10 Crib Data for Reporting Period October 1 through December 31, 1994.

(sheet 2 of 3 )

SITE SPECIFIC AND QTHER CONSTITLEUTS

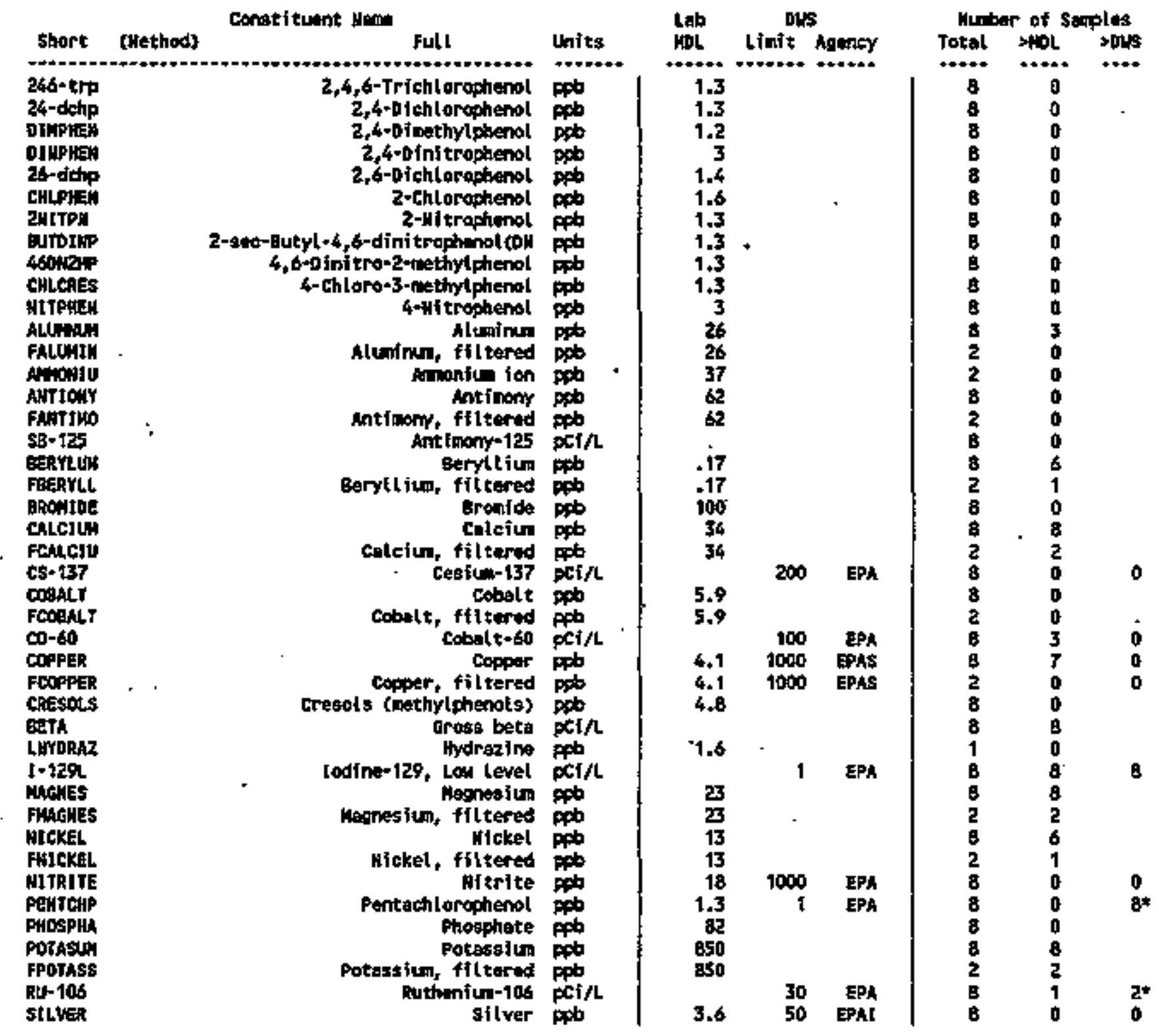


Tabje 8-3. Constituent List and Summary of Resu]ts for the 216-A-10 Crib Data for Reporting Period October 1 through December 31, 1994. (sheet 3 of 3 )

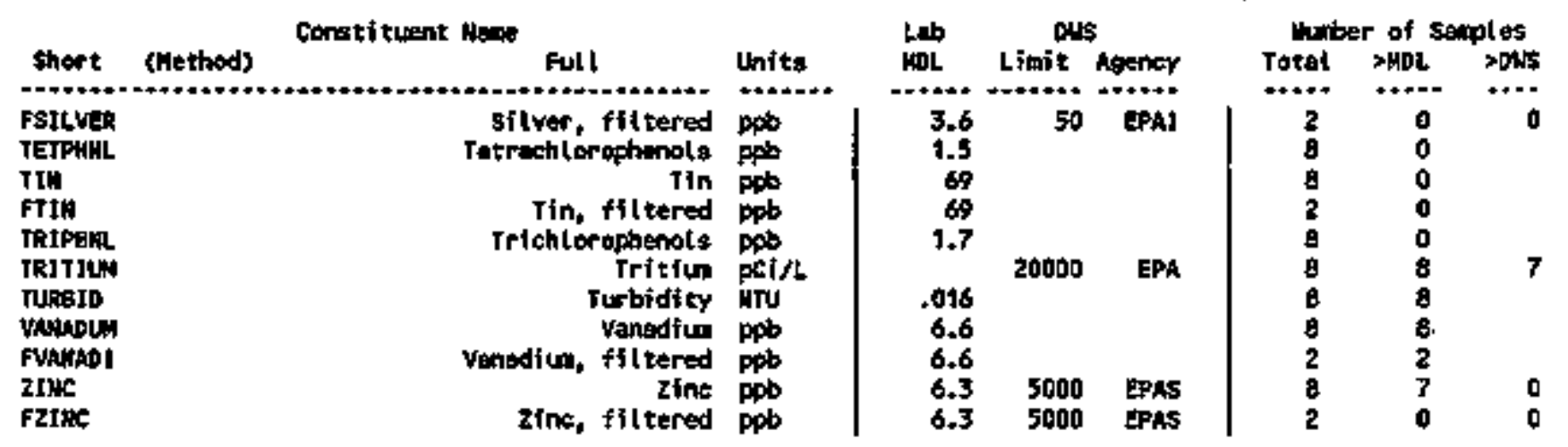

For explanation of this tubte, sat setion 1.4 of raport. 
Table 8-4. Constituents with at Least One Detected Va]ue for the 216-A-10 Crib Oata for Reporting Period

October 1 through December' 31, 1994.

. . (sheet 1 of 4)

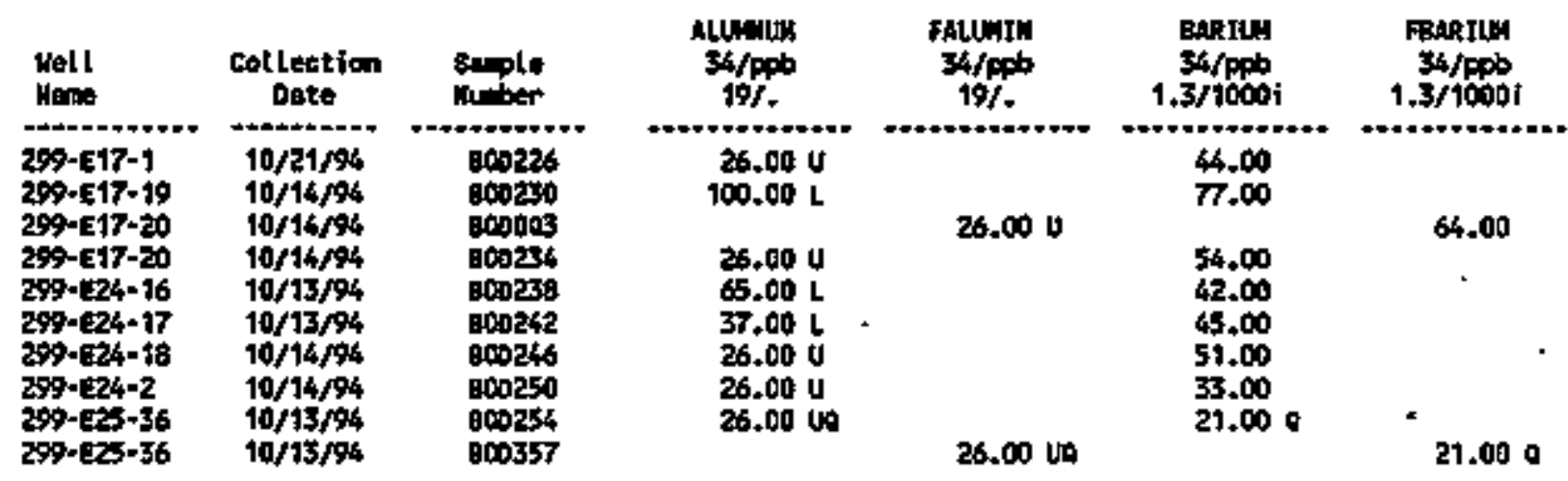

\begin{tabular}{|c|c|c|c|c|c|c|}
\hline $\begin{array}{l}\text { Whill } \\
\text { Haming }\end{array}$ & $\begin{array}{l}\text { Colleteion } \\
\text { Date }\end{array}$ & Stele & $\begin{array}{l}\text { Benrttut } \\
34 / \mathrm{ppb} \\
1.5 /\end{array}$ & $\begin{array}{c}\text { FBaftht } \\
34 / \mathrm{cpb} \\
1.3 / .\end{array}$ & $\begin{array}{c}\text { chilctid } \\
34 / \mathrm{Fph} \\
47 / .\end{array}$ & $\begin{array}{c}\text { FCALCIU } \\
34 / 1++5 \\
47 /\end{array}$ \\
\hline $\begin{array}{l}299-E 17-1 \\
299-E 17-19 \\
299-E 17-20 \\
299-E 17-20 \\
299-E 24-16 \\
299-E 24-17 \\
299-E 24-16 \\
299-624-2 \\
299-E 27-36 \\
299-E 26-36\end{array}$ & $\begin{array}{l}10 / 21 / 94 \\
10 / 14 / 94 \\
10 / 14 / 94 \\
10 / 16 / 94 \\
10 / 13 / 94 \\
10 / 13 / 94 \\
10 / 14 / 94 \\
10 / 14 / 94 \\
10 / 13 / 94 \\
10 / 13 / 94\end{array}$ & $\begin{array}{l}800226 \\
800230 \\
800003 \\
800234 \\
100238 \\
800242 \\
800246 \\
800250 \\
600254 \\
100357\end{array}$ & $\begin{array}{l}.61 \mathrm{~L} \\
.53 \mathrm{BL} \\
.64 \mathrm{dL} \\
.33 \mathrm{~L} \\
.17 \mathrm{U} \\
.37 \mathrm{~L} \\
.21 \mathrm{~L} \\
.17 \mathrm{U}\end{array}$ & $.17 \mathrm{v}$ & $\begin{array}{r}47000.00 \\
35000.00 \\
4000.00 \\
35000.00 \\
35000.00 \\
35000.00 \\
36000.00 \\
30000.00\end{array}$ & 56000.00 \\
\hline
\end{tabular}

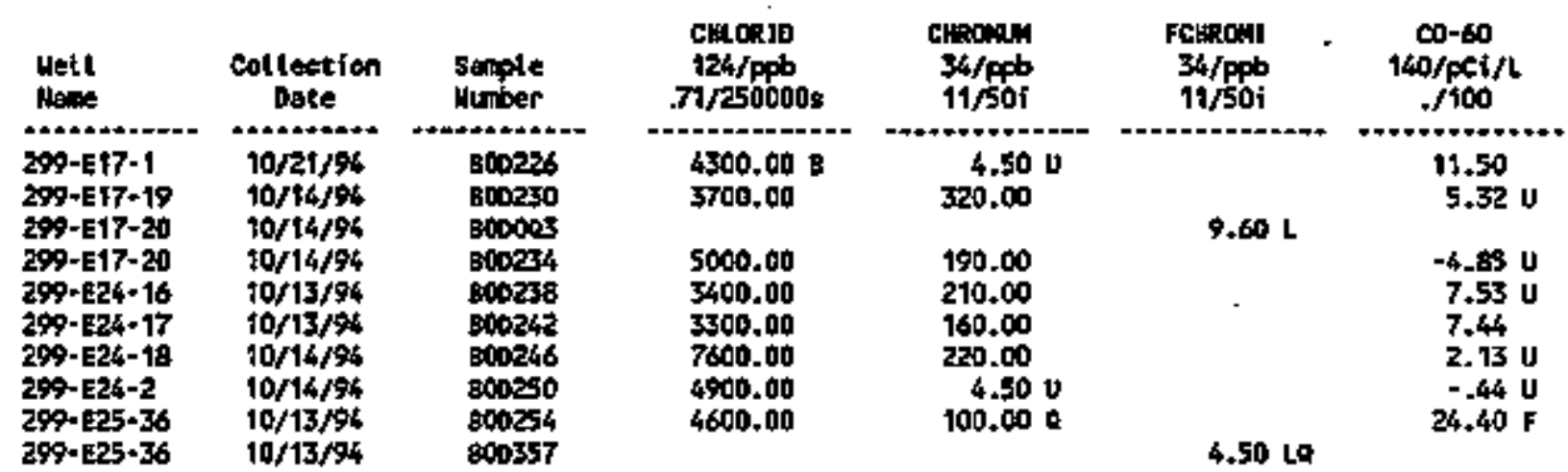


Table 8-4. Constftuents with at Least One Detected Value for the 216-A-10 Crib Data for Reporting Period

October 1 through Decenber 31, 1994.

(sheet 2 of 4)

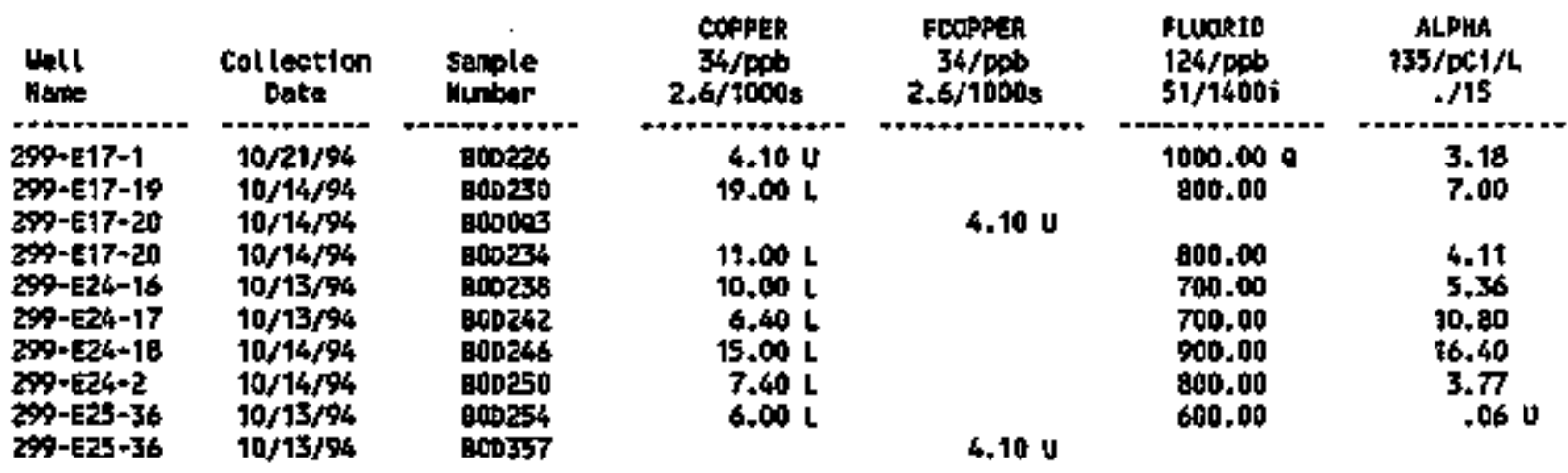

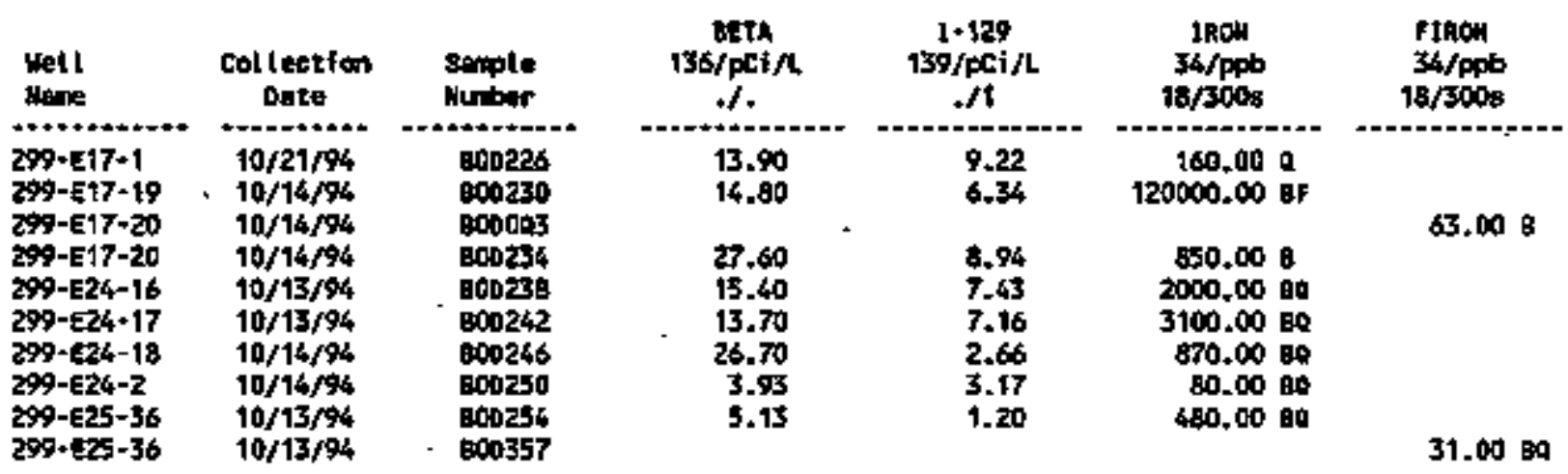

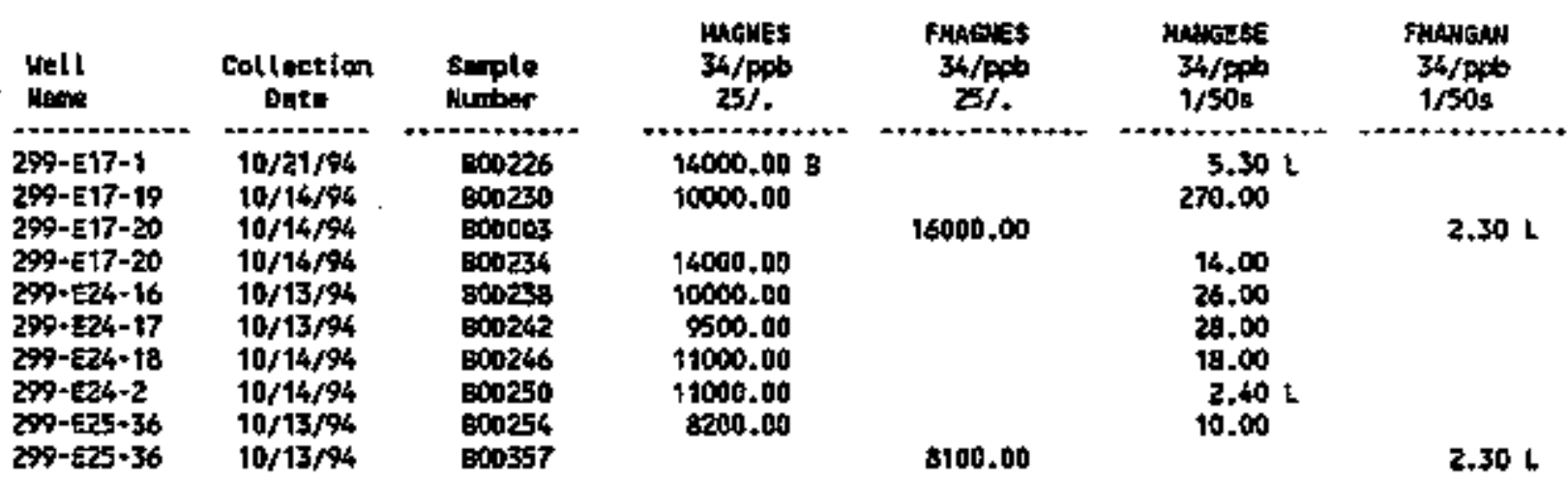


Table 8-4. Constftuents with at Least One Detected Value for the 216-A-10 Crib Data for Reporting Period

October 1 through December 31, 1994. (sheet 3 of 4 )

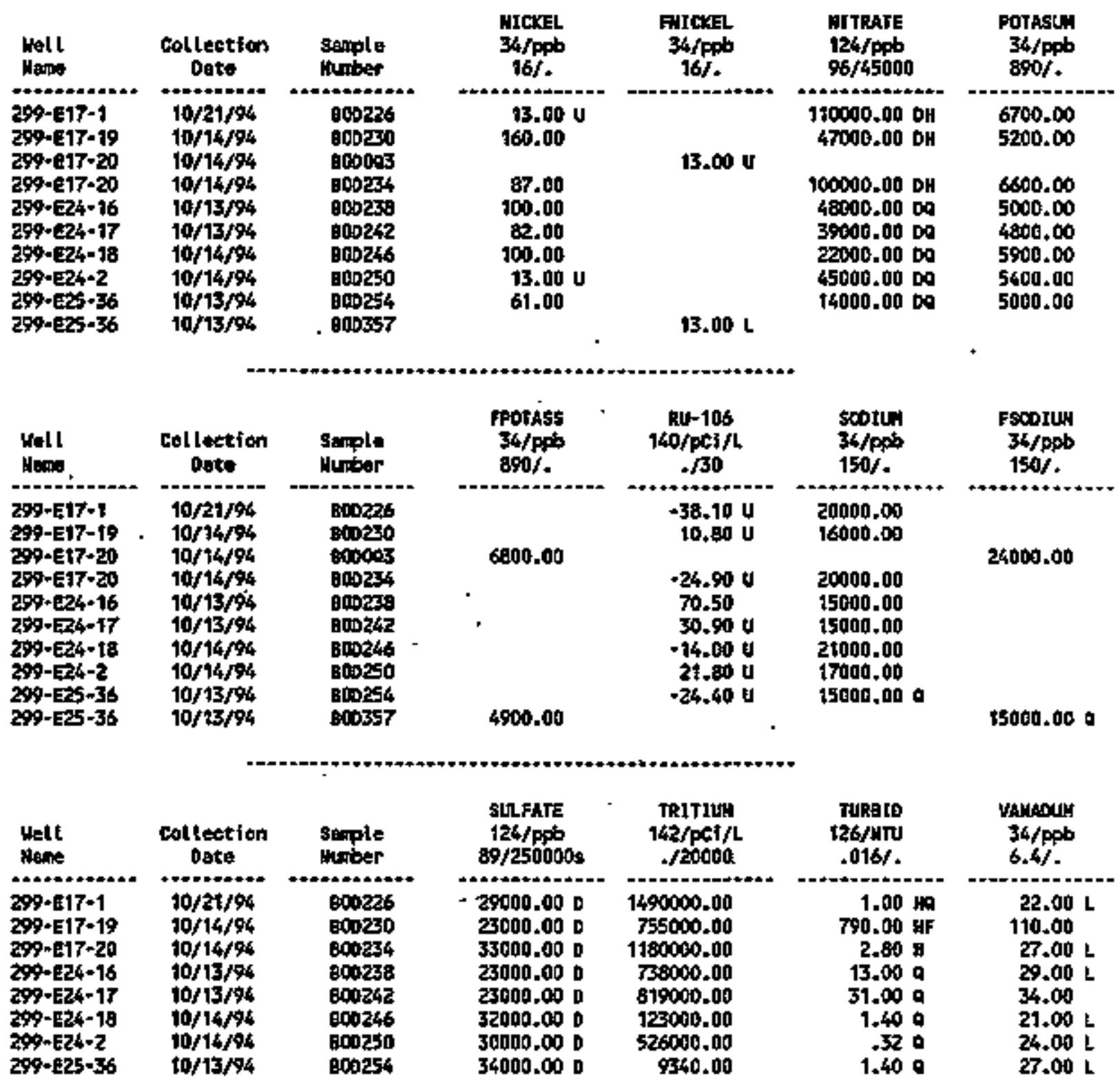


Table 8-4. Constituents with at Least One Detected Value for the 216-A-IO Crib Data for Reporting Period October 1 through December 31, 1994. (sheet 4 of 4 )

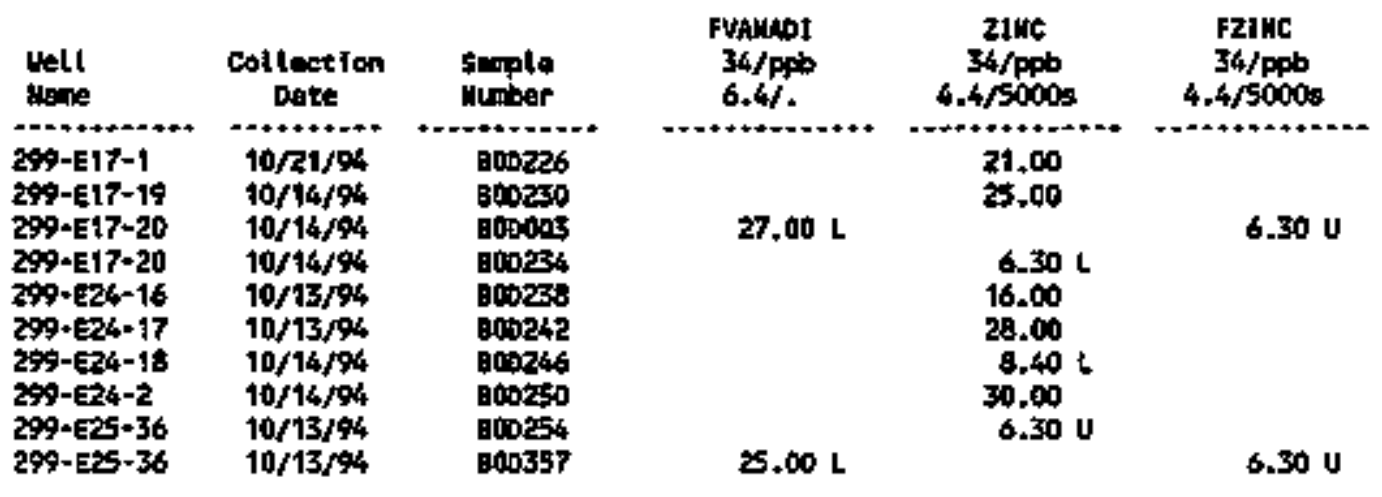

For explanation of this table, set \$ection 1,4 of report. 
Table 9-5. Contanination Indicator Paralieters for the $216-\mathrm{A}-10 \mathrm{Crib}$ Oata for Reporting Pertod October 1 through December 31, 1994.

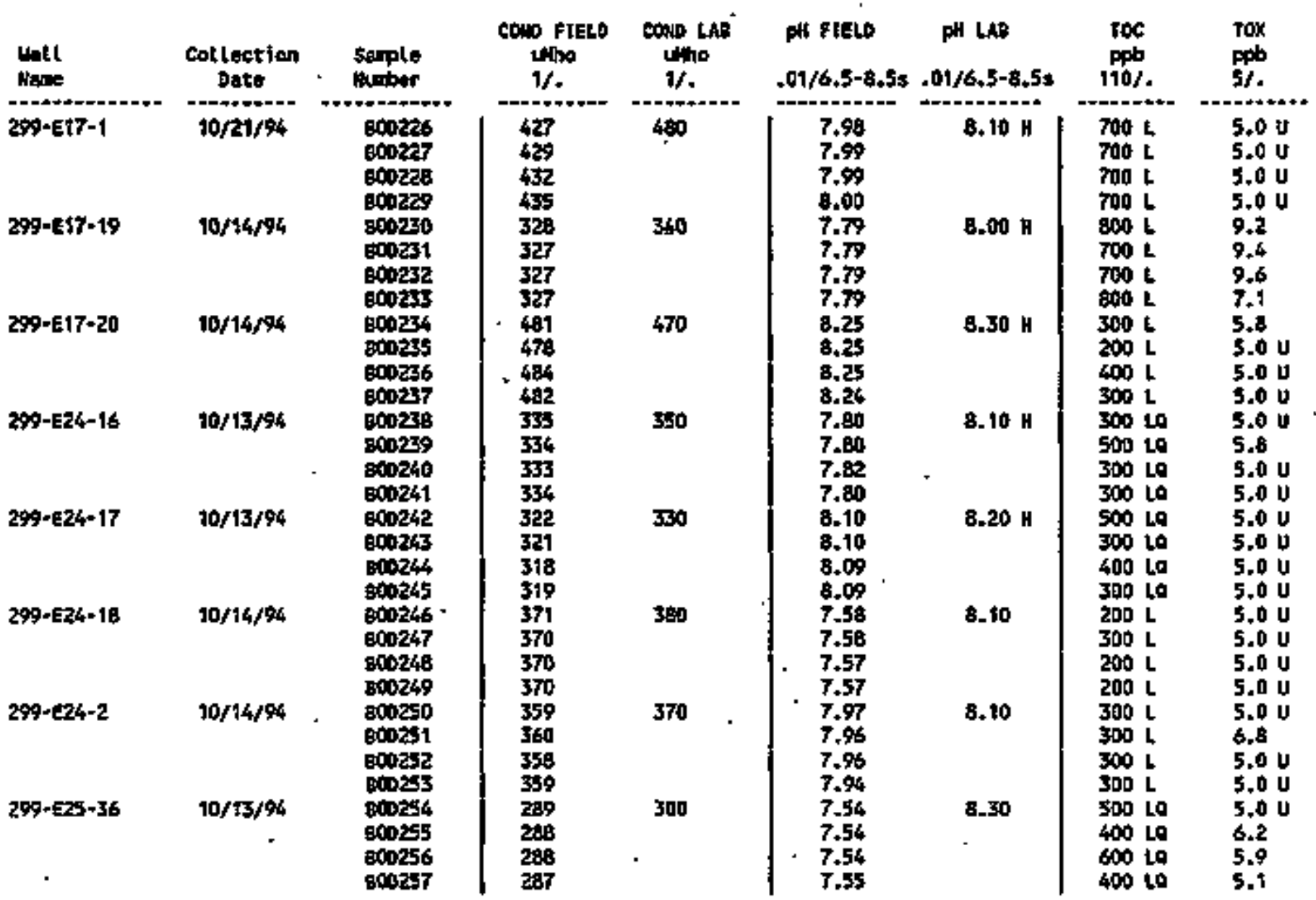

For mplanation of this table, sen suction 1.4 of repart. 
Table 8-6. Brtnking Water Standards Exceeded in the 216-A-36B Crib Groundwater Monitoring Hetwork.

\begin{tabular}{|c|c|}
\hline Constituent (OWS) & Wells exceeding OWS (299-) \\
\hline Chrogtium, unfiltered (50ppb) & E17-19, E17-20, E24-16, E24-17, \\
\hline Iron, unf11 tered (300 ppb) & E17-19, E17-20, E24-16, E24-17, \\
\hline E24-18, E25-36 \\
\hline Nitrate (45,000 ppb) & E17-19, E17-20, E24-16, E17-1 \\
\hline Tritium (20,000 pCj/L) & Al1 wel1s in network \\
\hline Gross Alpha (15 pCi/L) & E24-18 \\
\hline Manganese, unfiltered (50 ppb) & E17-19 \\
\hline Iodine-129 (1 pCi/L) & Al1 wel1s in network \\
\hline
\end{tabular}


DOE/RL-94-36-4

\section{CONTERTS}

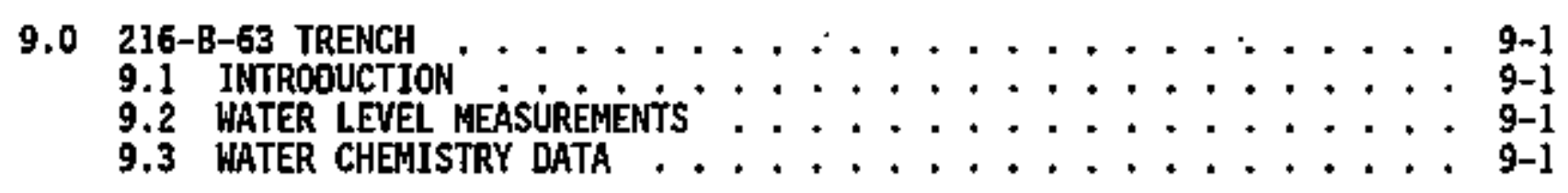




\section{LIST OF FIGLRES}

9-I Me11 Location Map for the 216-8-63 Trench. . . . . . . . 9-3

\section{LIST OF TABLES}

9-1 Monitoring Well Purpose and Sampling schedule for the 2]6-B-63 Jrench Network . . . . . . . . . . . . . . 9-4

9-2 RCRA Water Level Measurement Report 216-8-63 Trench,

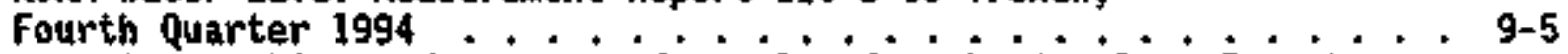

9-3 Constituent List and Summary of Resul ts for the 216-8-63 Trench Data for Reporting Period October 1 through December 31, 1994 . . . 9-7

9-4 Constituents with at Least One Detected Value for the 216-B-63 Trench Data for Reporting Period October I through December 31, 1994

9-5 Contamination Indicator Parabeters for the 216-8-63 Trench Data for Reporting Period October 1 through December 31, 1994 . . . 9-18 


\title{
9.0 216-B-63 TRENCH
}

\author{
h. D. Sweeney \\ Westinghouse Hanford Company
}

\subsection{INTROBUCTIOH}

A RCRA interim-status detection-level groundwater monitoring network is being established for the 216-B-63 Trench. The 216-B-63 Trench received wastewater containing hazardous waste and radioactive materials frow the B P1 ant located in the 200 East Area (Figures 1-1 and 9-1). Liquid effluent discharge. to the 216-B-63 Trench ceased in February 1992.

The 216-B-63 Trench was an open, unlined earthen trench that was in service from March 1970 until February 1992. The trench received $4.0 \times 10^{5}$ to $1.5 \times 10^{6} \mathrm{~L} /$ day $(100,000$ to $400,000 \mathrm{gal} /$ day) of liquid effluent fron the B P1ant chemtcal sewer, none of whtch is now designated as dangerous waste. The effluent was a mixture of steam condensate and raw water. Bocumented hazardous discharges occurred from 1970 to October 1985 and consisted of aqueous sulfuric actid and sodium hydroxide solutions exceeding the pH jimits of 2.0 and 12.5, respectively. Radioactive soils were dredged from the trench in August 1970. In 1985, physical controts, radiation monitors, and operating procedures were modified to avoid inadvertent discharge of chemicals or radioactive substances to the wastewater stream. The trench has been subsequentiy backfllied and stabitzed and the diversion box leading to jt has been filled with concrete.

Four quarters of groundwater background sampling have been cainpleted for all the wells in the network. Background sampling is done to estabi ish background water quality, in accordance with the requirements outlined in 40 CFR 265 Interin-5tatus Indficator-Evaluation Groundwater Monitoring Plan for the 216-6-63 Trench (PNL 1989). - The 12 we11s within the network are now sampled semiannually. . Based on the present groundwater fiou direction, seven of the wells are downgradient. and five are upgradient of the trench (see Figure 9-1). The purpose of the weils, their sampling schedule, and the dates sampled are sumbarized tn Table 9-1'. As indicated in Table 9-1, some wells in the 216-8-63 Trench network are shared with the Low-Level Burial Grounds (LLBG) and single-shell tanks (SST) groundwater monitoring networks.

\subsection{MATER LEYEL MEASUREMENTS}

Water level measurements obtained during the October 1 through Deceraber 31 , 1994 period are presented in Table 9-2. Water measurements in woll 299-E33-36 indicate water levels lower than in nearby weils in the network. Reasons for the apparent discrepancy are still under investigation.

\subsection{WATER CHENISTRY DATA}

Chentstry data received before the cutoff date for this report are reported in Tables 9-3 through 9-5. Concentrations of unfiltered chromium and 
iron in some wells continue to fluctuate above the DHS for these constituents. Analysis of filtered sanples for these parameters yields concentrations well below the OWS. Iodine-129 is also above DWS for this quarter, but the values do not exceed historical trends for these wells. A statistical evaluation has been completed for indjcator parameters for the 216-8-63 Trench. A pH exceedance was noted during sampling in well 299-E33-36. The pH measurements were obtained durfing sampling with field-grade instrumentation. It is possible that these readings are in error because they are not consistent with the historical trend. This well has been resampled and the verification analysis will be performed by a laboratory to determine the validity of the data. An explanation of data flags can be found in Section 1.4. 


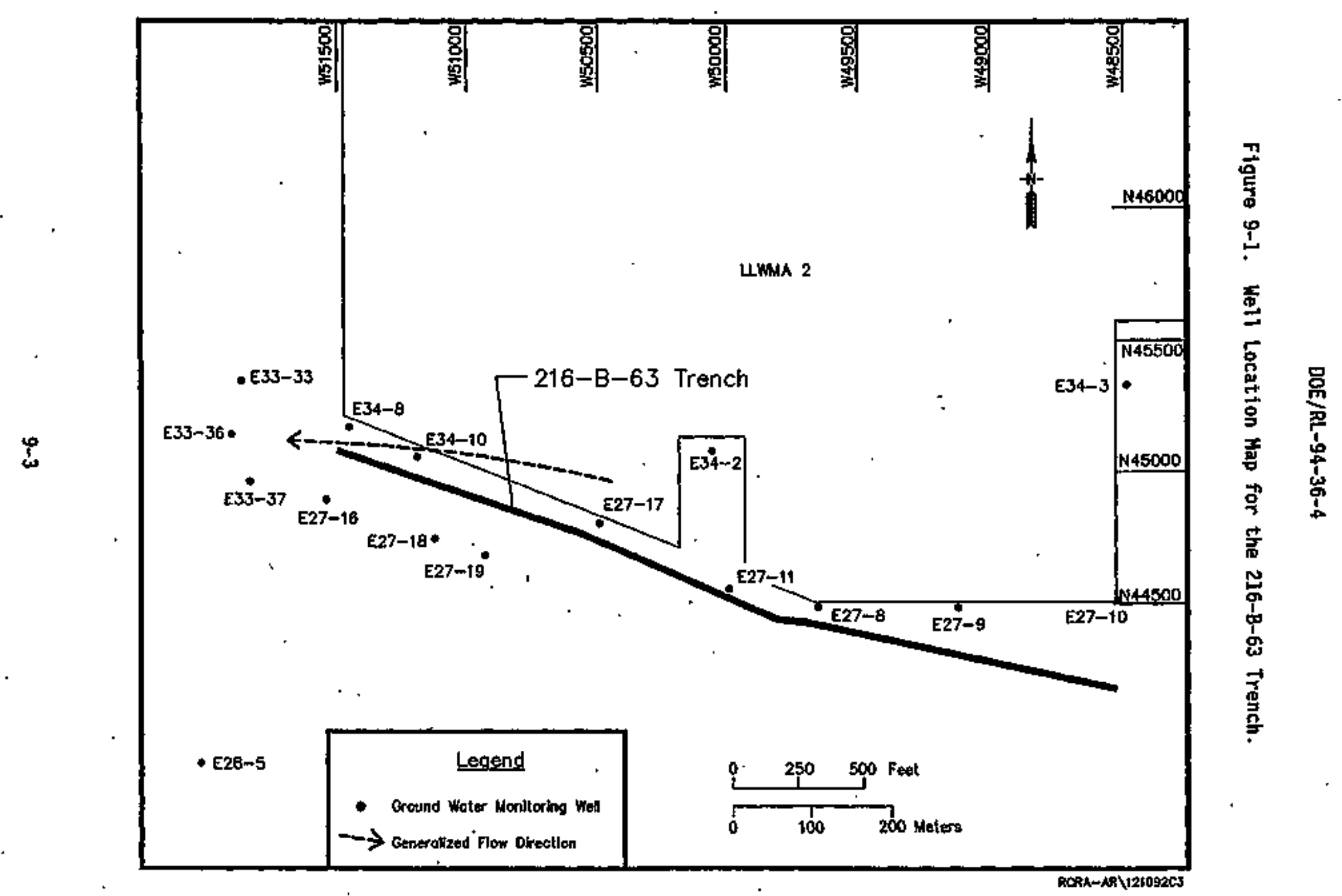


Table 9-1. Monitoring Well Purpose and Sampling Schedule for the 216-8-63 Trench Network.

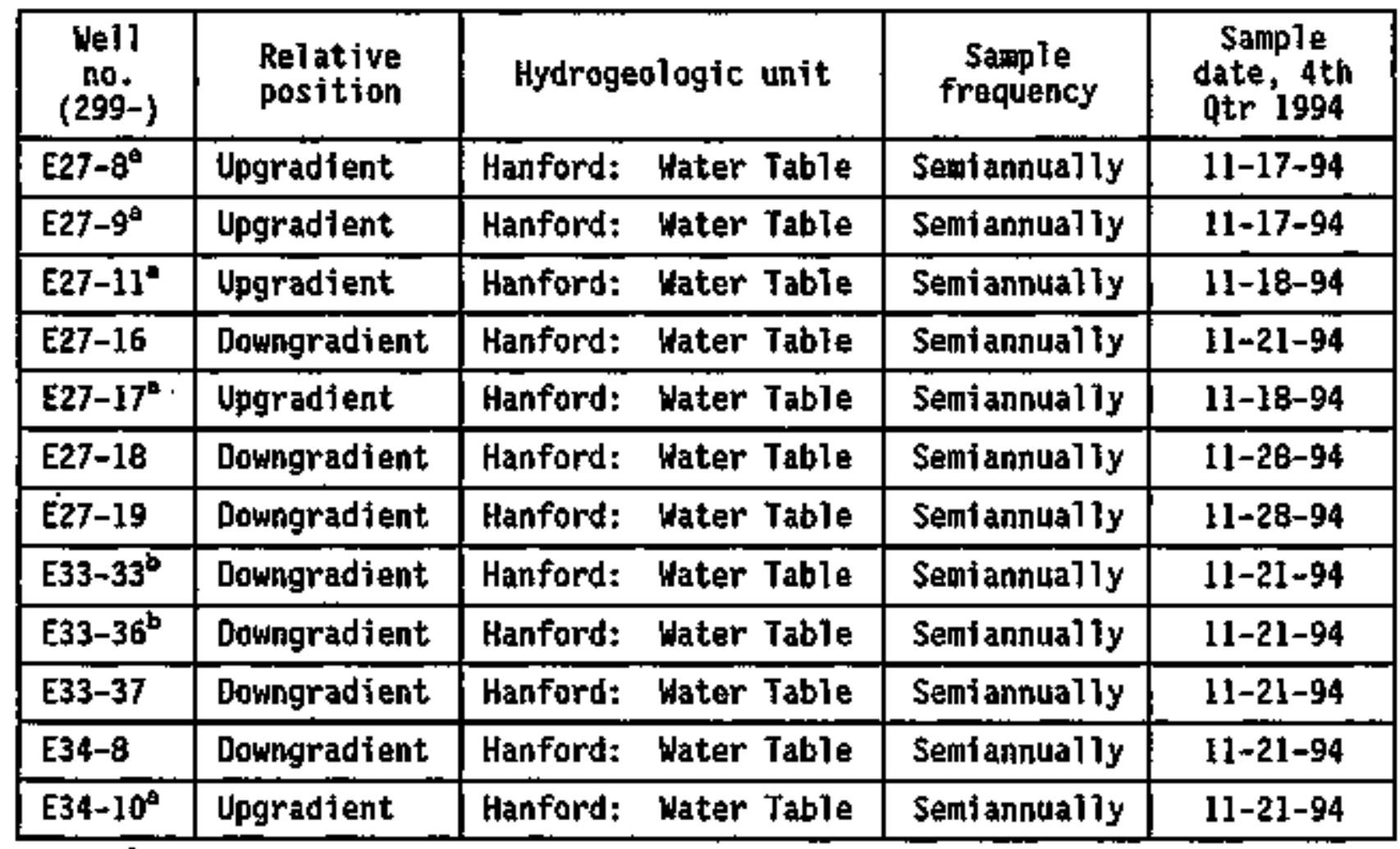

abell shared with the Low-Level Burial Grounds facility network.

Well shared with the stingle-sheli tanks facility network. 
Table 9-2, RCRA Water Leve\} Measurement Report 216-B-63 Trench, Fourth Quarter 1994.

(sheet I of 2)

\begin{tabular}{|c|c|c|c|c|}
\hline WeIl & Date & $\begin{array}{l}\text { Depth to } \\
\text { water (ft) }\end{array}$ & $\begin{array}{l}\text { Water } \\
\text { elevation } \\
\text { (ft) }\end{array}$ & $\begin{array}{l}\text { level } \\
\text { above ms] } \\
\text { (m) }\end{array}$ \\
\hline \multicolumn{5}{|c|}{ Wells Monitoring the Top of the Unconfined Aquifer } \\
\hline $299-E, 27-11$ & $\begin{array}{l}11 / 18 / 94 \\
12 / 14 / 94\end{array}$ & $\begin{array}{l}241.89 \\
241.88\end{array}$ & $\begin{array}{l}401.40^{*} \\
401.41\end{array}$ & $\begin{array}{l}122.35 \\
122.35\end{array}$ \\
\hline 299-E27-16 & $\begin{array}{l}11 / 21 / 94 \\
12 / 21 / 94\end{array}$ & $\begin{array}{l}251.00 \\
250.95\end{array}$ & $\begin{array}{l}401.13^{\star} \\
401.18\end{array}$ & $\begin{array}{l}122.26 \\
122.28\end{array}$ \\
\hline 299-E27-17 & $\begin{array}{l}11 / 18 / 94 \\
12 / 14 / 94\end{array}$ & $\begin{array}{l}233.00 \\
232.94\end{array}$ & $\begin{array}{l}401.72^{\star} \\
401.78\end{array}$ & $\begin{array}{l}122.44 \\
122.46\end{array}$ \\
\hline 299-E27-18 & $11 / 28 / 94$ & 248.35 & $401.80^{*}$ & 122.47 \\
\hline 299-E27-19 & $11 / 28 / 94$ & 249.70 & $401.18^{*}$ & 122.28 \\
\hline 299-E27-8 & $\begin{array}{l}11 / 17 / 94 \\
12 / 14 / 94\end{array}$ & $\begin{array}{l}236.73 \\
236.28\end{array}$ & $\begin{array}{l}401.10^{*} \\
401.55\end{array}$ & $\begin{array}{l}122.26 \\
122.39\end{array}$ \\
\hline $299-E, 27-9$ & $\begin{array}{l}11 / 17 / 94 \\
12 / 14 / 94\end{array}$ & $\begin{array}{l}227.66 \\
227.50\end{array}$ & $\begin{array}{l}401.55 * \\
401.71\end{array}$ & $\begin{array}{l}122.39 \\
122.44\end{array}$ \\
\hline 299-E33-33 & $\begin{array}{l}10 / 12 / 94 \\
1 ? / 15 / 94 \\
11 / 21 / 94 \\
12 / 09 / 94\end{array}$ & $\begin{array}{l}238.81 \\
238.65 \\
238.94 \\
238.74\end{array}$ & $\begin{array}{l}401.58 \\
401.74 \\
401.45 * \\
401.65\end{array}$ & $\begin{array}{r}122.40 \\
122.45 \\
-\quad 122.36 \\
122.42\end{array}$ \\
\hline 299-E33-36 & $\begin{array}{l}11 / 21 / 94 \\
12 / 21 / 94\end{array}$ & $\begin{array}{l}248.25 \\
246.76\end{array}$ & $\begin{array}{l}398.42^{*} \\
399.91\end{array}$ & $\begin{array}{l}121.44 \\
121.89\end{array}$ \\
\hline 299-E33-37 & $\begin{array}{l}11 / 21 / 94 \\
12 / 21 / 94\end{array}$ & $\begin{array}{l}251.54 \\
251.71\end{array}$ & $\begin{array}{l}401.47^{*} \\
401.30\end{array}$ & $\begin{array}{l}122.37 \\
122.32\end{array}$ \\
\hline 299-E34-10 & $\begin{array}{r}11 / 21 / 94 \\
12 / 14 / 94\end{array}$ & $\begin{array}{l}238.10 \\
238.01\end{array}$ & $\begin{array}{l}401.67^{\star} \\
401.76\end{array}$ & $\begin{array}{l}122.43 \\
122.46\end{array}$ \\
\hline 299-E34-8 & $11 / 21 / 94$ & 241.82 & $398.70^{*}$ & 121.52 \\
\hline
\end{tabular}


Table 9-2. RCRA Water Level Measureant Report 216-B-63 Trench, Fourth Quarter 1994. (sheet 2 of 2)

MOTES: 1 . Water level elevations are calculated by subtracting the measured depth-to-water from the surveyed el evation for the well.

2. Depth-to-water values are transcribed from fjeld records.

3. Elevations marked with an $1 *$ ' were measured at the time of sampling.

4. Elevations marked with a ' + ' are outside of the expected range, and are suspected of error. 
Table 9-3. Constituent List and Summary of Results for the 216-B-63 Trench Data for Reporting Period October 1 through December 31, 1994.

(sheet 1 of 4 )

\section{CONTNAJHATION IMDICATOA PARANETERS}

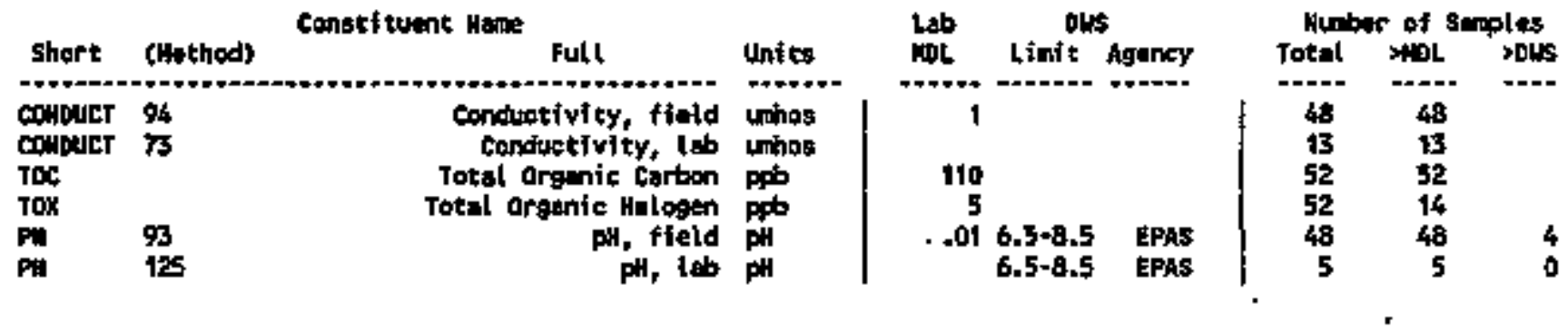

DRJMKLWG WHTER PARAYTERS

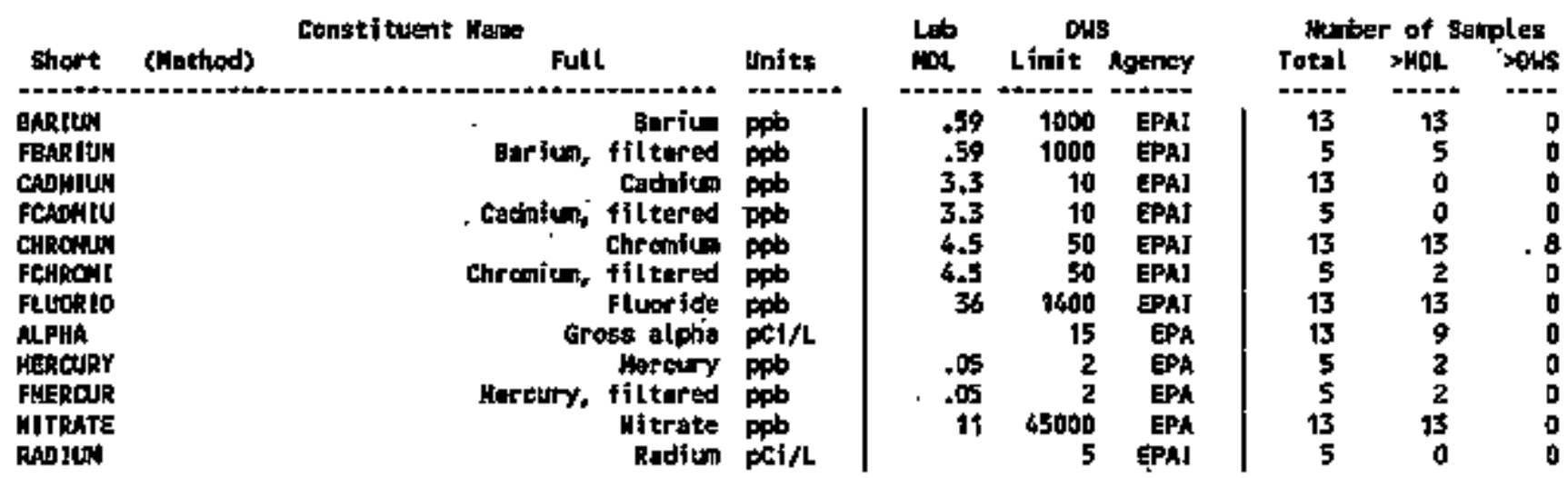

GROMDUWAER CUALJTY PARARETERS

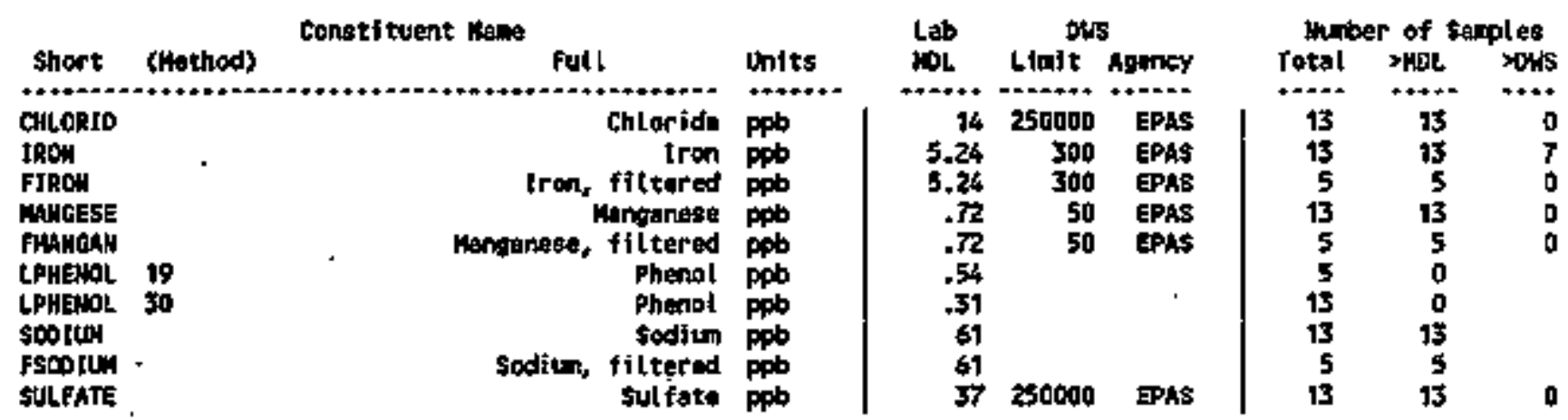


Table 9-3. Constituent List and Summary of Results for the 216-B-63 Trench Data for Reporting Period October 1 through December 31, 1994. (sheet 2 of 4 )

S1TE SPEG1FIC AND OTHER COHSTITUEATS

\begin{tabular}{|c|c|c|c|c|c|c|c|c|c|}
\hline & & Constituant the & & Lab & OLt5 & & Min & $r$ of 5 & ples \\
\hline Short & (Hethod) & full I & Unt ts & Hot & L数i $t$ & Agency & Total & 3LL & sous \\
\hline$\ddot{1}, 1, j \cdot T$ & - & 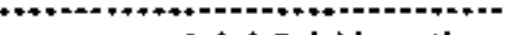 & $=--$ & 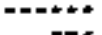 & $=+\cdots$ & $+\infty+$ & $\cdots+$ & $=+\infty=0$ & $\cdots$ \\
\hline $\begin{array}{l}1,1,3-T \\
1,1,2-T\end{array}$ & & $1,1,1-$ Trichloctethane & pplo & .036 & 200 & EPA & 13 & 3 & 0 \\
\hline $\begin{array}{l}1,1,2-1 \\
1,1-010\end{array}$ & & 1,1,2-Trichloe enthen & $p$ & .078 & & & 13 & 0 & \\
\hline $\begin{array}{l}1,1-01 c \\
1,2-01 c\end{array}$ & & 1,1-bichlorathen & ppb & .058 & & & 13 & 0 & \\
\hline $\begin{array}{l}1,2-b 1 c \\
14-d b e n\end{array}$ & & 1,2-bichlor outhere & pqb & .029 & 5 & EPA & 13 & 0 & 0 \\
\hline 14-04-tip & & 2,4,6-6-Trichlorophenol & pos & 1.3 & 13 & te & $\begin{array}{l}13 \\
13\end{array}$ & 0 & $\mathbf{0}$ \\
\hline 24 -dens & 19 & 3,4 -ofechtorophenol & Ppb & 4 & & & 5 & 0 & \\
\hline $26-d a h p$ & 30 & 2,4-0 lehilorophenal & ppb & 1.3 & & & 13 & 0 & \\
\hline Drapkit & & $2,4-0$ rnethylphanol & Pps & 1.2 & & & 13 & 0 & \\
\hline 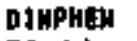 & & $2,4-03 n t$ trophenol & PFb & 3 & & & 13 & 0 & \\
\hline 76-dichp. & & 2,6-0ichlorophenol & ppb & 1.4 & & & 13 & 0 & \\
\hline CHLPHEX & & 2-Whlorophenol & ppb & 1.6 & & & 13 & 0 & \\
\hline 2HETHPH & & 2-Wethylphenol & ppb & 5.1 & & & 5 & $\mathbf{0}$ & \\
\hline Ziv'TFH & 19 & 2-133 trophenol & pab & 1.9 & & & 5 & 0 & \\
\hline 2:JTPH & 30 & 2-Nt trophenol & efb & 1.3 & & & 13 & 0 & \\
\hline EUTDLAP & & 2+9ec-Butyl-4,6-dinitraphenol cou & apb & 1.3 & & & 13 & 0 & \\
\hline 460112NP & & 4,6-0initro-2-nethylphenol & ppb & 1.3 & & & 13 & () & \\
\hline CELARES & & 4-Chloro-3-nethylphenol & pob & $\mathbf{t . 3}$ & & & 13 & 0 & \\
\hline 4METHPH & & 4+Wothylithenol & pab & 7 & & & 5 & $\mathbf{0}$ & \\
\hline NITPHEN & & $4=H$ i trophiqnol & ph & 3 & & & 13 & $\mathbf{0}$ & \\
\hline 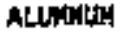 & & Aluminan & Fpb & 26 & & & 13 & 10 & \\
\hline FHLUAlN & & Aluninum, fíltared & ppt & 26 & & & 5 & 0 & \\
\hline AHTIOAT & & Antinony & $p p b$ & 62 & & & 13 & 0 & \\
\hline FANTINO & & mitimory, filterad & pob & 62 & & & 5 & 0 & \\
\hline $58 \cdot 125$ & & Ant iromy-125 & $\begin{array}{l}\mathrm{pCt} / \mathrm{h} \\
\mathrm{pob}\end{array}$ & nets & & & $\begin{array}{l}2 \\
5\end{array}$ & $\begin{array}{l}0 \\
0\end{array}$ & \\
\hline $\begin{array}{l}\text { AR1016 } \\
\text { ART1221 }\end{array}$ & & $\begin{array}{l}\text { Aroclor-1016 } \\
\text { Aroclor-122t }\end{array}$ & $\begin{array}{l}\text { ppo } \\
\text { ppb }\end{array}$ & .052 & & & 5 & 0 & \\
\hline AR 1232 & & Aroclor- 1232 & ppb & .093 & & & 5 & 0 & \\
\hline ARt24t & & Aroctor-1242 & $\mathrm{ppb}$ & .068 & & & 5 & 0 & \\
\hline ARt2 48 & & Aroclor-1248 & ppb & .055 & & & 5 & 0 & \\
\hline $\operatorname{AR1254}$ & & Aroclor-1254 & Dob & .051 & & & 5 & 0 & \\
\hline AR 1260 & & Aroclor+1260 & pob & .058 & & & 5 & 0 & \\
\hline BEuLPre & & Bẹnzenne & ppb & .013 & 5 & EPh & 13 & 0 & $\mathbf{0}$ \\
\hline BENZOT & & senzothiezole & pob & 1,3 & & & 5 & 0 & \\
\hline GERTLUA & & enryllism & Fpb & .17 & & & $\mathbf{1 3}$ & 6 & \\
\hline FBERYLL & & Beryl lfin, filtered & Fpb & .17 & & & 5 & 3 & \\
\hline as-7 & & Beryll ium- 7 & pCi/l & & & & 2 & 0 & \\
\hline BIS2EPH & & Bis(z-ethylhexyl) phthalate & ppb & 5.3 & & & 5 & 0 & \\
\hline BRONLOE & & Bronite & $a$ & too & & & $\$ 3$ & 0 & \\
\hline CALCLLN & & Calciun & Fpb & 34 & & & ts & 13 & \\
\hline FCALCIU & & Calciun, filtered & ipb & 34 & & & 5 & 5 & \\
\hline $\begin{array}{l}\text { TETRANE } \\
\text { CEPR=144 }\end{array}$ & & $\begin{array}{l}\text { Cerbon tetrachloride } \\
\text { Ceriug/Prageodyitu. } 144\end{array}$ & pit & .075 & 5 & EPA & $\begin{aligned} 13 \\
2\end{aligned}$ & $\begin{array}{l}1 \\
0\end{array}$ & $\mathbf{0}$ \\
\hline & & क) & & & & & & u & \\
\hline
\end{tabular}


Tab]e 9-3. Constituent List and Summary of Resu]ts for the 216-B-63 Trench Data for Reporting Period October I through December 31, 1994. (sheet 3 of 4)

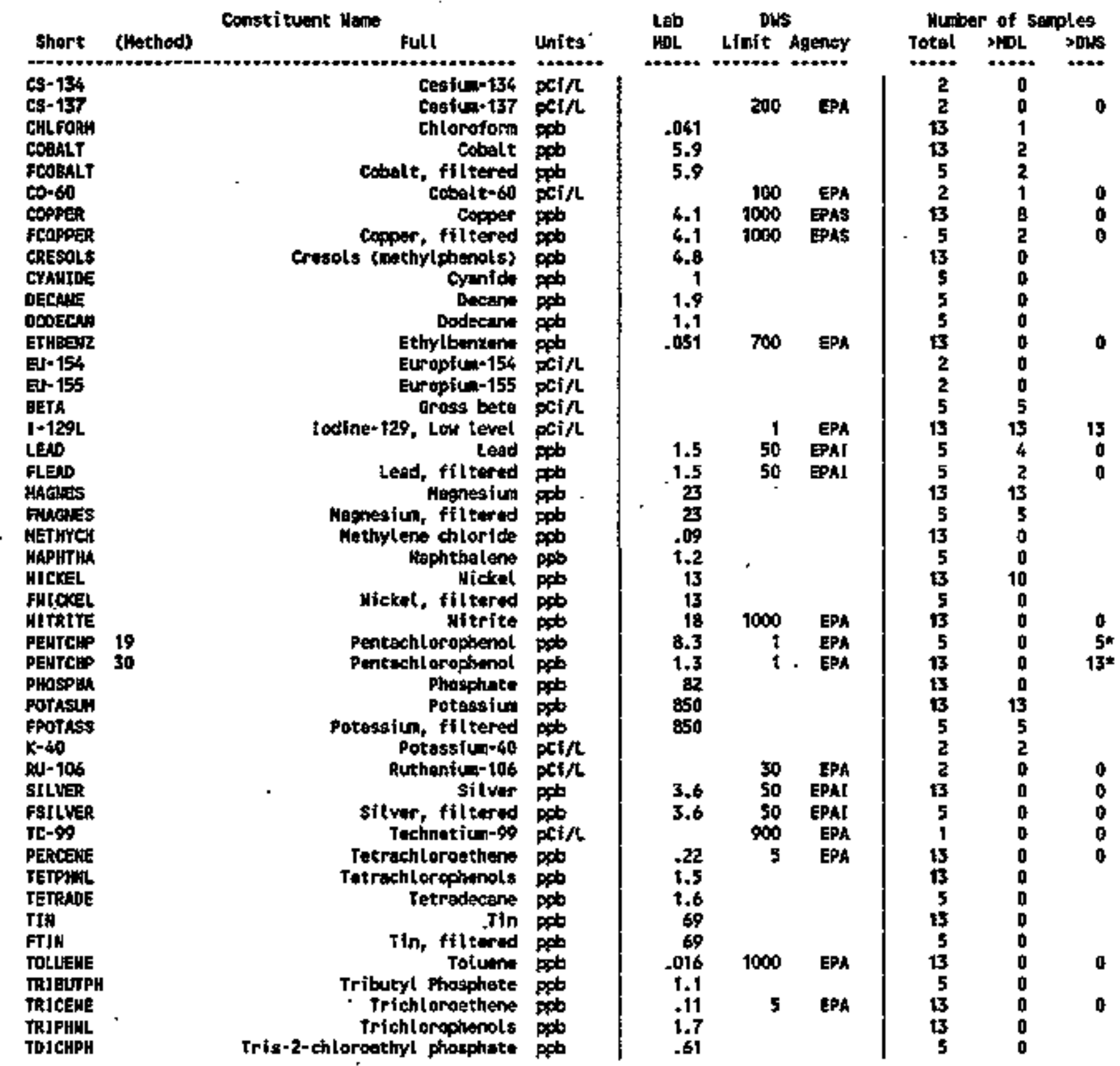


Table 9-3. Constituent List and Sumuary of Results for the 216-8-63 Trench. Data for Reporting Pertod October 1 through December 31, 1994.

(sheet 4 of 4 )

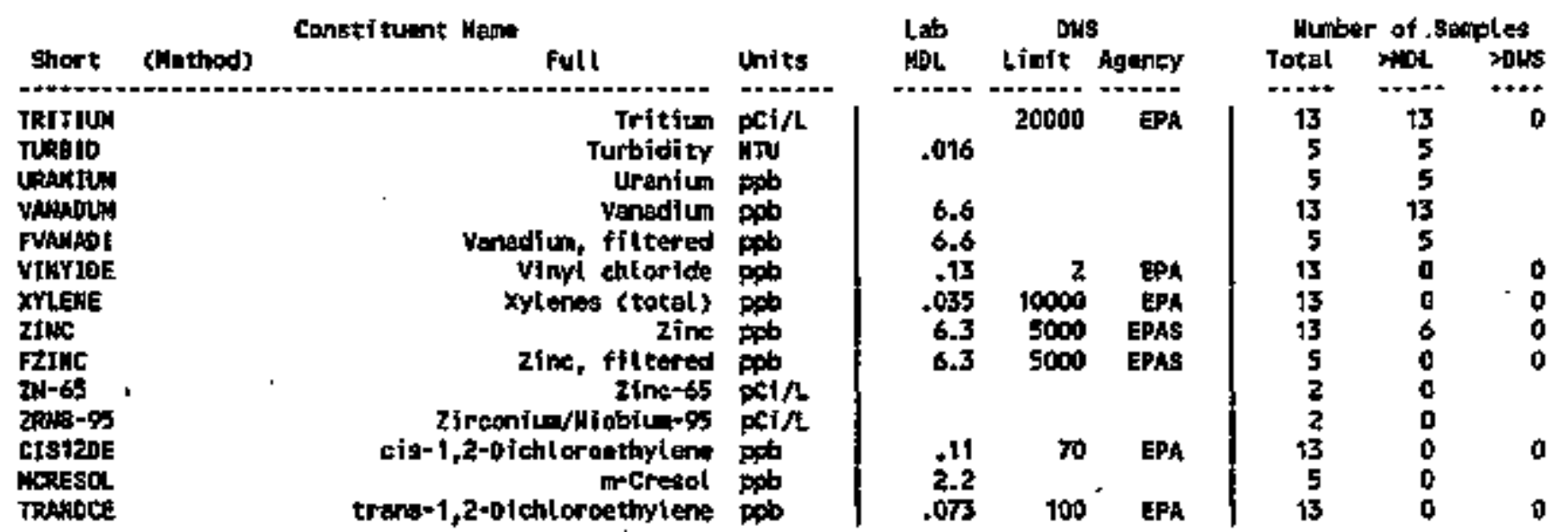

Far explanation of this table, sect section 1.4 of report. 
Table 9-4. Constituents with at Least One Detected Value for the 216-8-63 Trench Data for Reporting Period

October 1 through Decenber 31, 1994. (sheet 1 of 7)

\begin{tabular}{|c|c|c|c|c|c|c|}
\hline Mell & $\begin{array}{l}\text { Collection } \\
\text { Date }\end{array}$ & $\begin{array}{l}\text { Stuple } \\
\text { Murver }\end{array}$ & $\begin{array}{r}1,1,1-\mathrm{T} \\
25 / \mathrm{pbb} \\
.22 / 200\end{array}$ & $\begin{array}{c}\text { Mlswim } \\
\text { 36/ppb } \\
19 / .\end{array}$ & $\begin{array}{c}\text { FALMiIn } \\
34 / \text { FH } \\
19 \% .\end{array}$ & $\begin{array}{c}\text { Barrum } \\
34 / \mathrm{ppb}^{\mathrm{b}} \\
1.3 / 1000 \mathrm{i}\end{array}$ \\
\hline 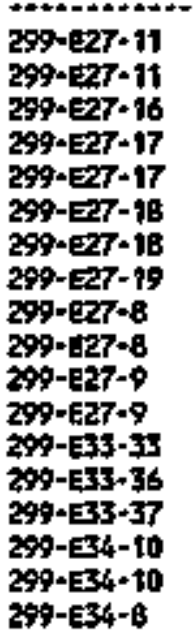 & $\begin{array}{l}11 / 18 / 94 \\
11 / 18 / 94 \\
11 / 21 / 94 \\
11 / 18 / 94 \\
11 / 18 / 94 \\
11 / 28 / 94 \\
11 / 28 / 94 \\
11 / 28 / 94 \\
11 / 17 / 94 \\
111 / 77 / 94 \\
11 / 17 / 94 \\
11 / 17 / 94 \\
11 / 21 / 94 \\
11 / 21 / 94 \\
11 / 21 / 94 \\
11 / 21 / 94 \\
11 / 21 / 94 \\
11 / 21 / 94\end{array}$ & 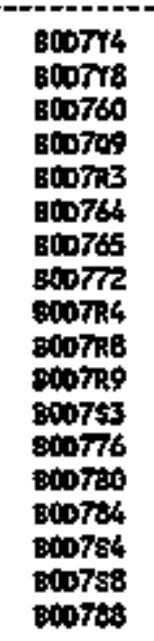 & $\begin{array}{l}.04 \mathrm{v} \\
.04 \mathrm{v} \\
.04 \mathrm{v} \\
.06 \mathrm{z} \\
.051 \\
.05 \mathrm{i} \\
.04 \mathrm{v} \\
.04 \mathrm{v} \\
.04 \mathrm{v} \\
.04 \mathrm{v} \\
.04 \mathrm{v} \\
.04 \mathrm{v} \\
.04 \mathrm{v}\end{array}$ & $\begin{array}{l}78.00 \mathrm{~L} \\
46.00 \mathrm{~L} \\
26.00 \mathrm{U} \\
29.00 \mathrm{~L} \\
26.00 \mathrm{U} \\
32.00 \mathrm{~L} \\
26.00 \mathrm{U} \\
35.00 \mathrm{~L} \\
32.00 \mathrm{~L} \\
37.00 \mathrm{~L} \\
51.00 \mathrm{l} \\
54.00 \mathrm{~L} \\
41.00 \mathrm{~L}\end{array}$ & $\begin{array}{c}26.00 \mathrm{v} \\
26.00 \mathrm{v} \\
26.00 \mathrm{v}\end{array}$ & $\begin{array}{l}39.00 \\
29.00 \\
33.00 \\
35.00 \\
35.00 \\
43.00 \\
6.00 \\
23.00 \\
31.00 \\
33.00 \\
37.00 \\
38.00 \\
37.00\end{array}$ \\
\hline Mell & $\underset{\substack{\text { colluction } \\
\text { Dato }}}{ }$ & $\begin{array}{l}\text { Sespte } \\
\text { Metibar }\end{array}$ & $\begin{array}{l}\text { FBARIUM } \\
\text { 34/ppb } \\
1.3 / 1000 i\end{array}$ & $\begin{array}{c}\text { EERYun } \\
34 / \mathrm{pDO} \\
\text { t.5/. }\end{array}$ & $\begin{array}{l}\text { FBERYRL } \\
\text { 34/ppb } \\
1.5 /\end{array}$ & $\begin{array}{c}\text { CALCIU } \\
34 / \mathrm{ppb}^{2} \\
47 /\end{array}$ \\
\hline 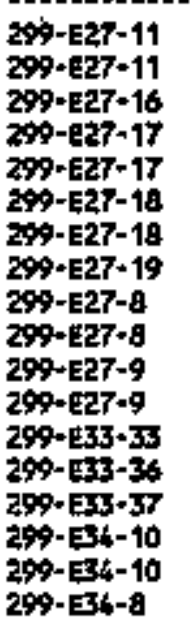 & $\begin{array}{l}11 / 18 / 94 \\
11 / 18 / 94 \\
11 / 21 / 94 \\
11 / 18 / 94 \\
11 / 18 / 94 \\
11 / 24 / 94 \\
11 / 28 / 94 \\
11 / 28 / 4 \\
11 / 17 / 94 \\
11 / 17 / 94 \\
11 / 17 / 94 \\
11 / 17 / 94 \\
11 / 21 / 94 \\
11 / 21 / 94 \\
11 / 21 / 94 \\
11 / 21 / 94 \\
11 / 21 / 94 \\
11 / 21 / 94\end{array}$ & 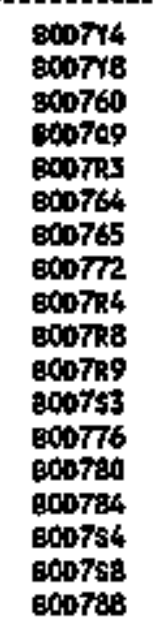 & $\begin{array}{l}25.00 \\
25.00\end{array}$ & $\begin{array}{l}.27 \mathrm{~L} \\
.291 \\
.43 \mathrm{~L} \\
.17 \mathrm{U} \\
.17 \mathrm{U} \\
.17 \mathrm{U} \\
.17 \mathrm{U} \\
.17 \mathrm{U} \\
.17 \mathrm{U} \\
.28 \mathrm{~L} \\
.21 \mathrm{~L} \\
.21 \mathrm{~L} \\
.17 \mathrm{U}\end{array}$ & $\begin{array}{r}.17 \mathrm{U} \\
.34 \mathrm{~L}\end{array}$ & $\begin{array}{l}38000.00 \\
30000.00 \\
39000.00 \\
31000.00 \\
31000.00 \\
35000.00 \\
35000.00 \\
36000.00 \\
31000.00 \\
29000.00 \\
28000.00 \\
38000.00 \\
32000.00\end{array}$ \\
\hline
\end{tabular}


Table 9-4. Constituents with at Least One Detected Value for the 216-8-63 Trench Data for Reporting Period october 1 through December 31, 1994.

(sheet 2 of 7 )

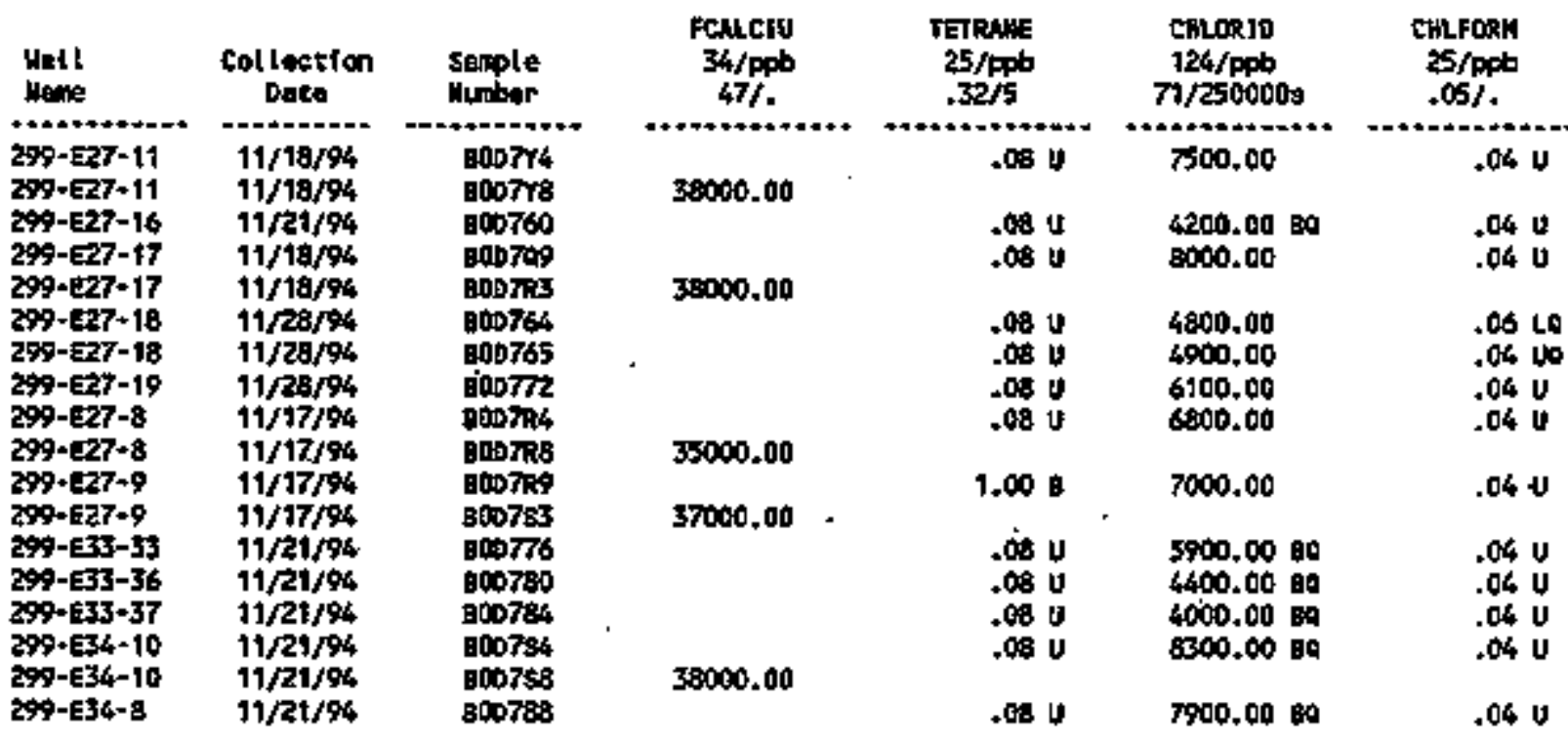

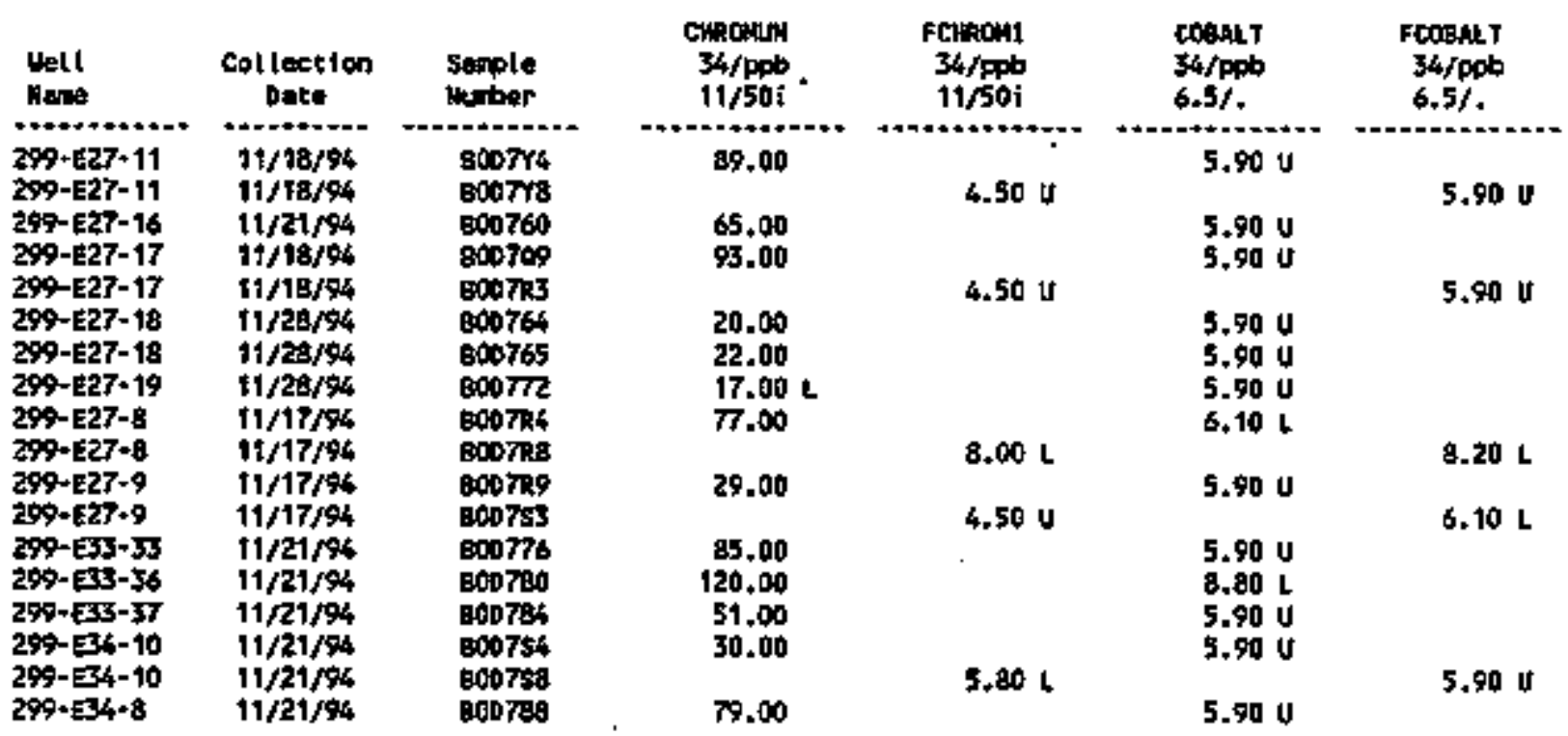


Table 9-4. Constituents with at Least One Betected Value for the 216-8-63 Trench Data for Reporting Period October 1 through December 31, 1994. (sheet 3 of 7)

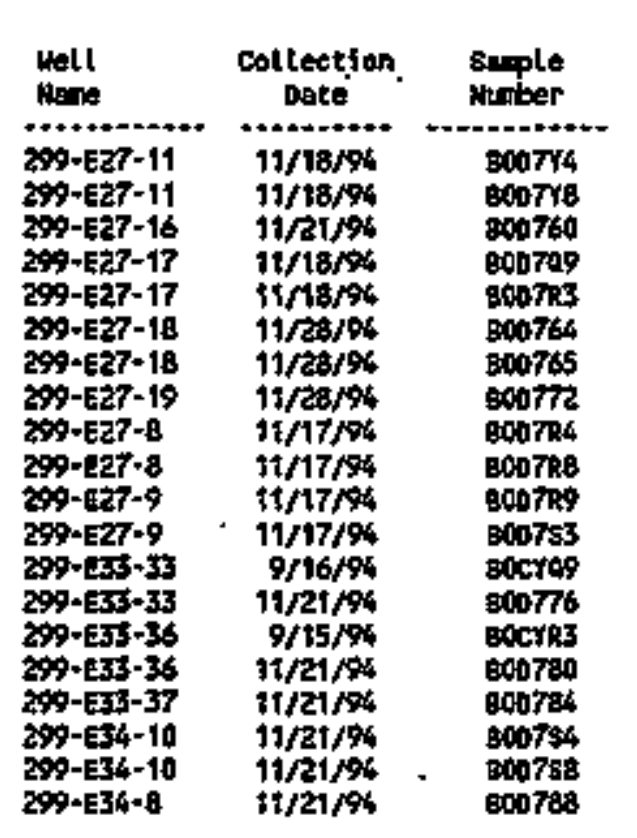

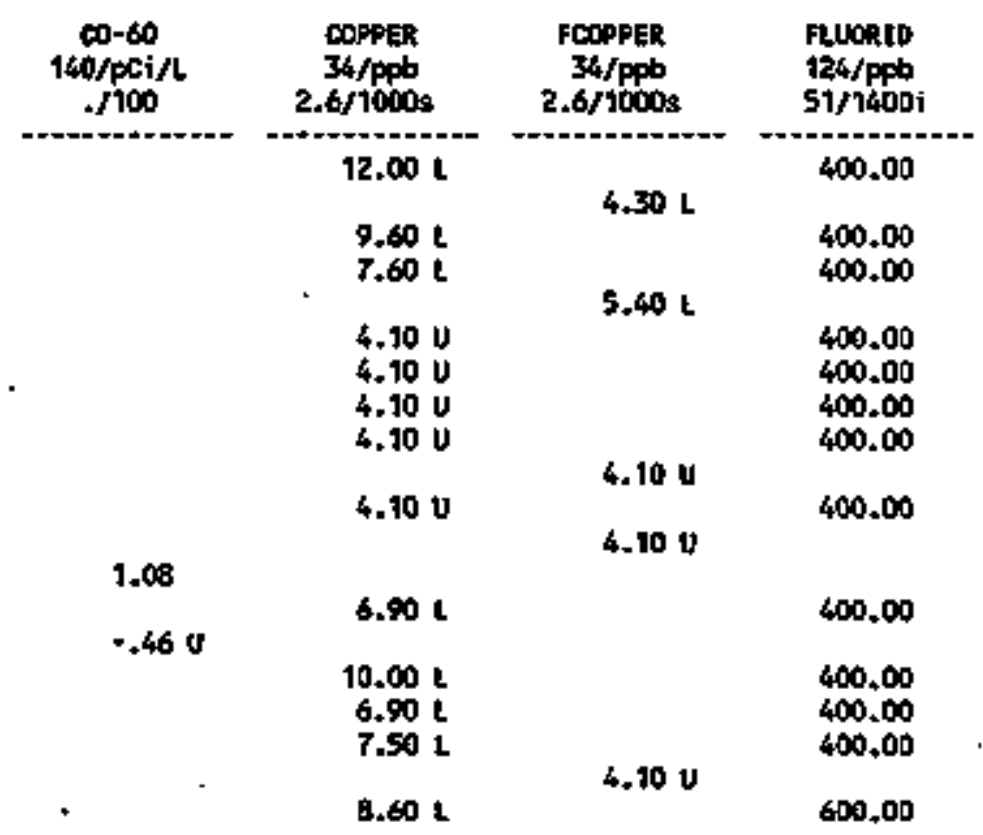

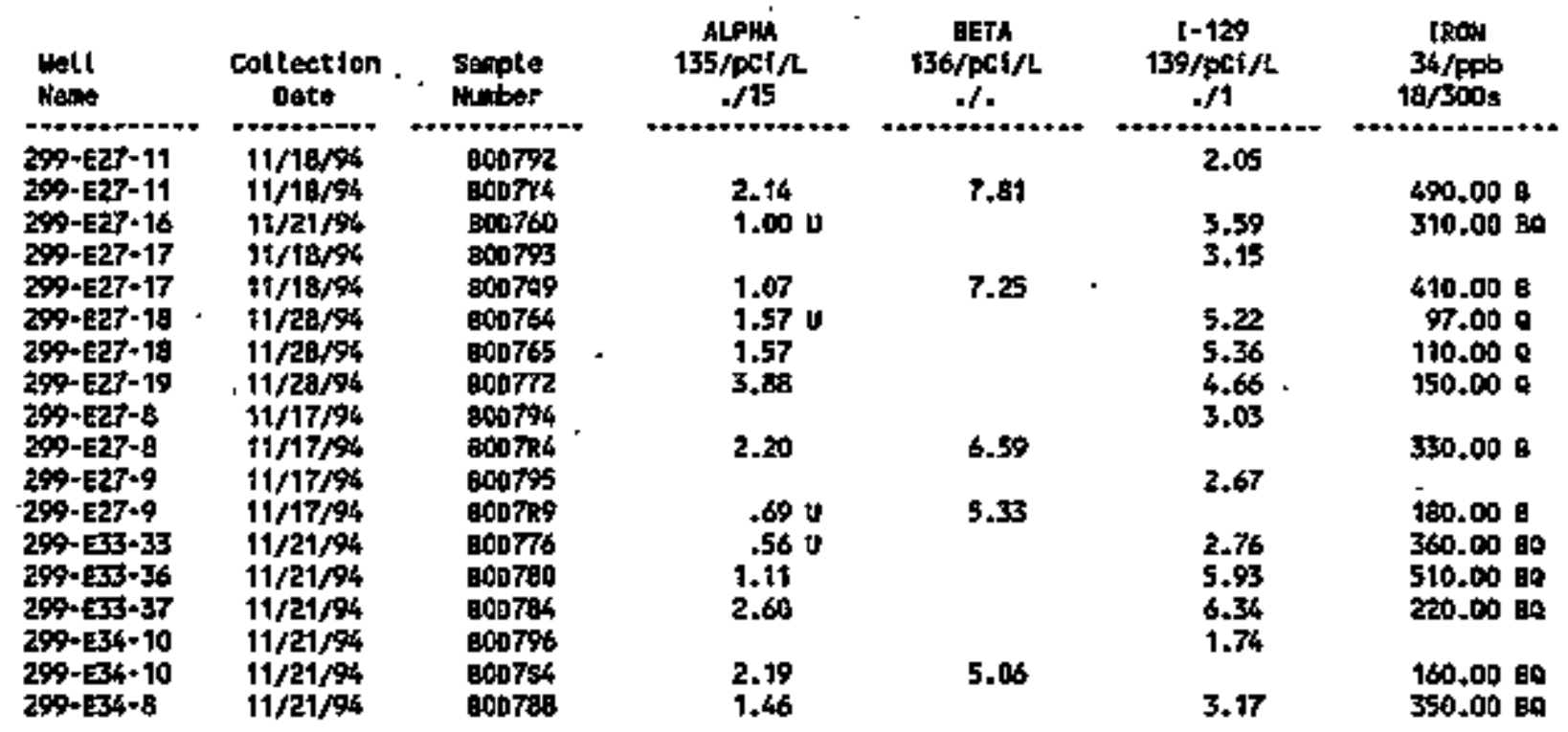


Table 9-4. Constjtuents with at Least One Detected Value for the 216-B-63 Trench Data for Reporting Period

October 1 through December 31, 1994.

(sheet 4 of 7 )

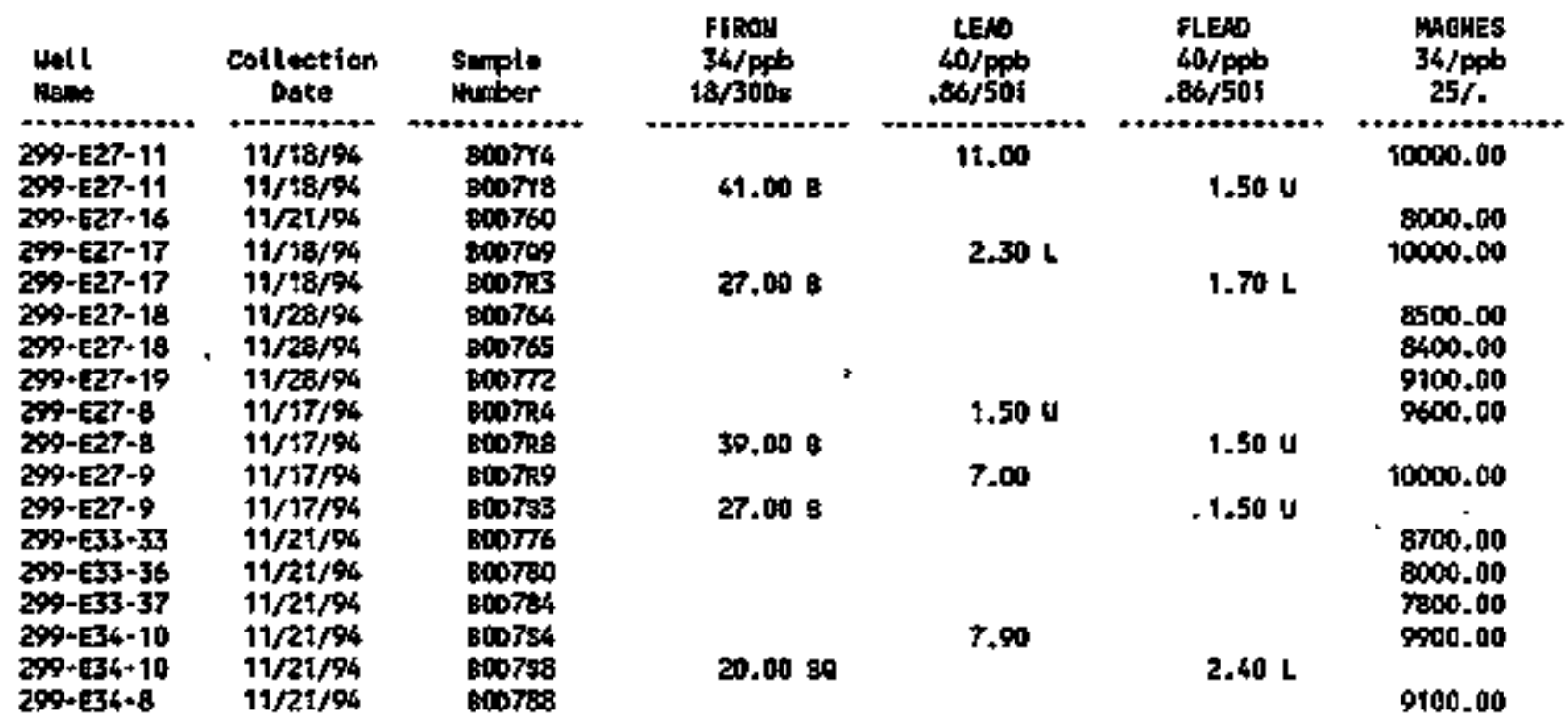

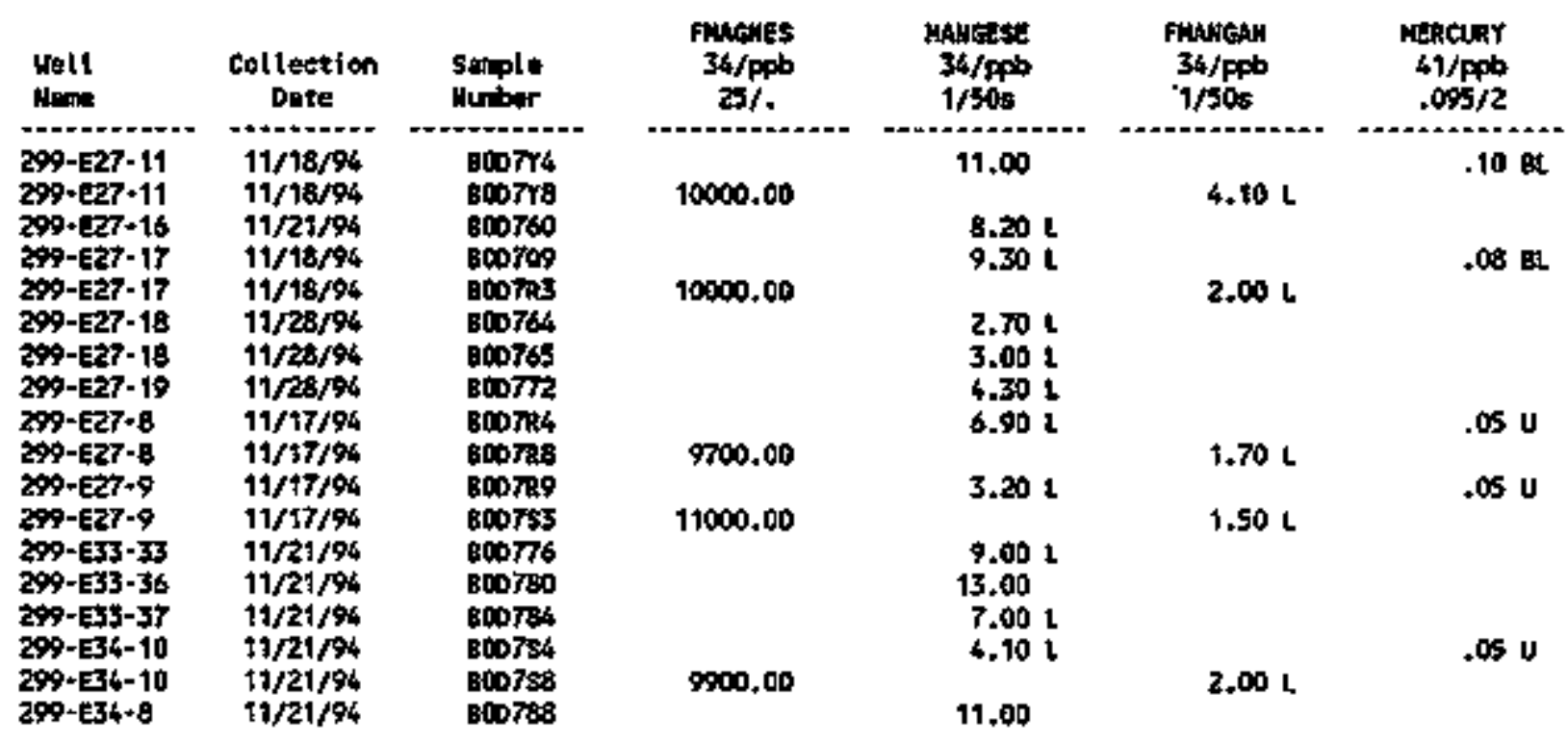


Table 9-4. Constituents with at Least One Detected Value for the 216-B-63 Trench Data for Reporting Period October 1 through Decenber 31, 1994.

(sheet 5 of 7 )

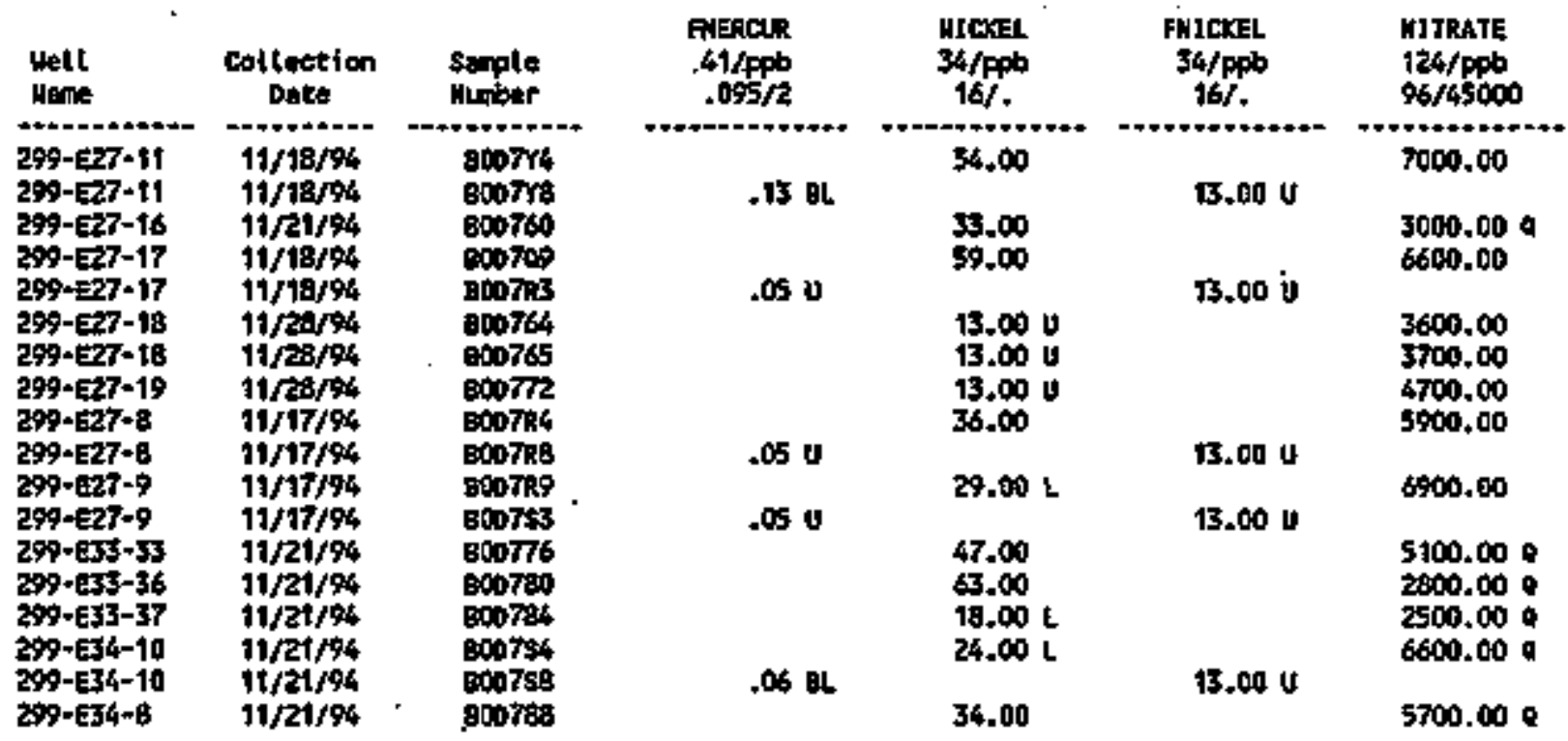

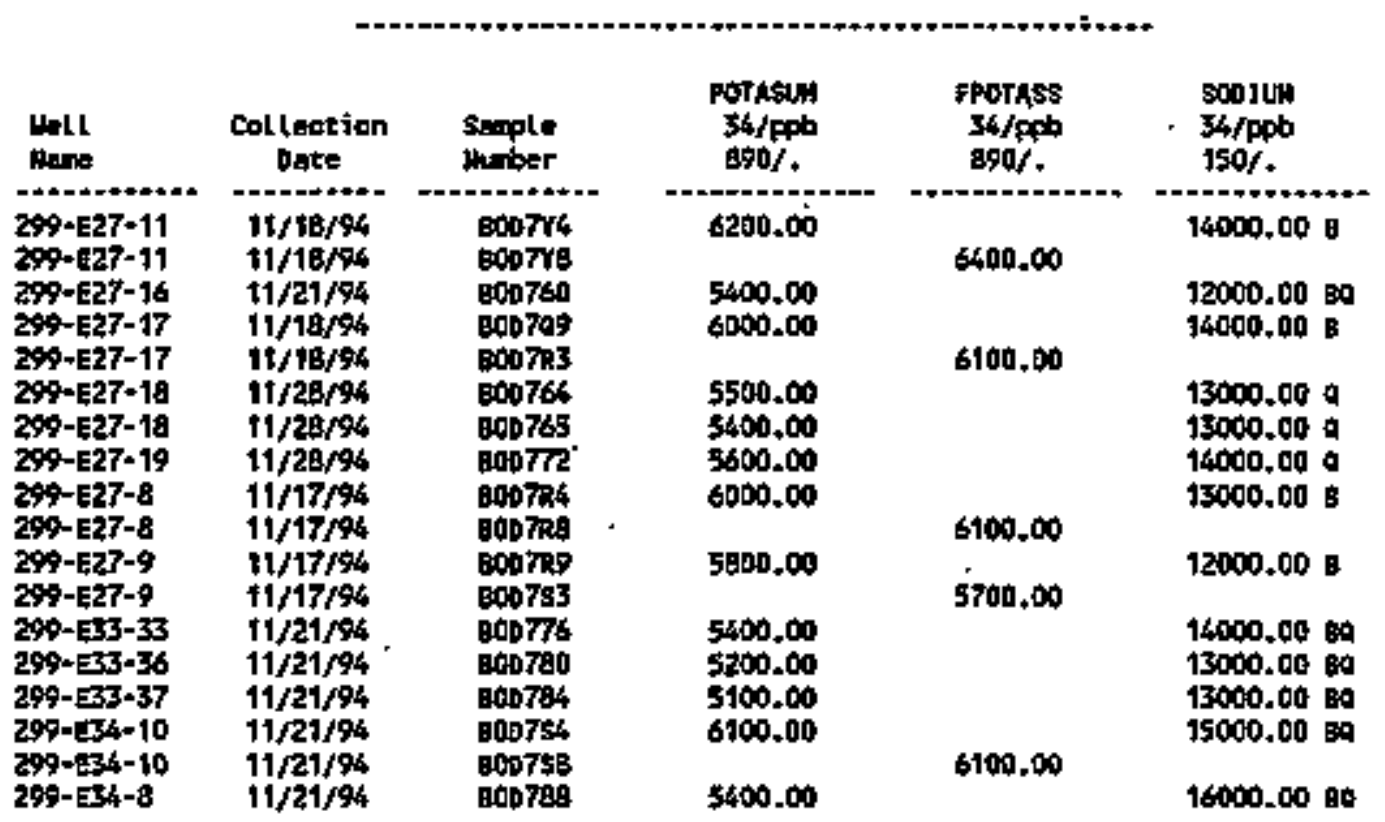


Table 9-4. Constituents with at Least One Detected Value for the 216-B-63 Trench Data for Reporting Period October 1 through December 31, 1994. (sheet 6 of 7 )

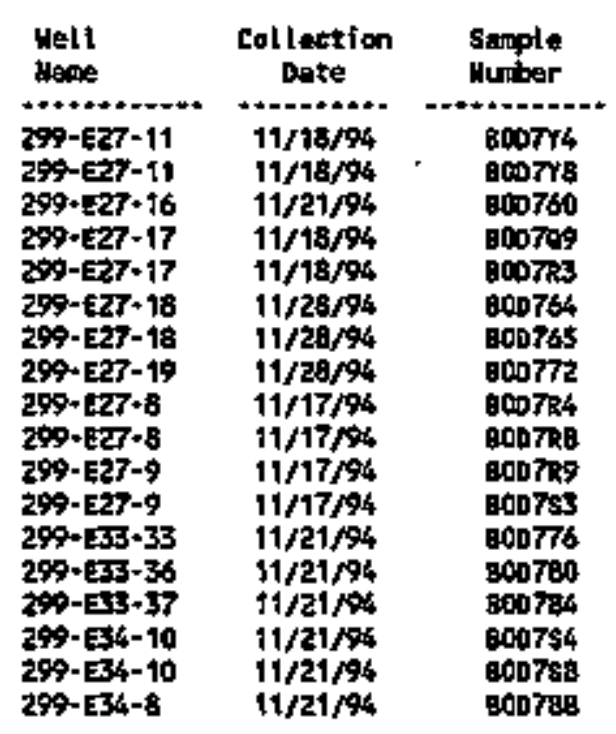

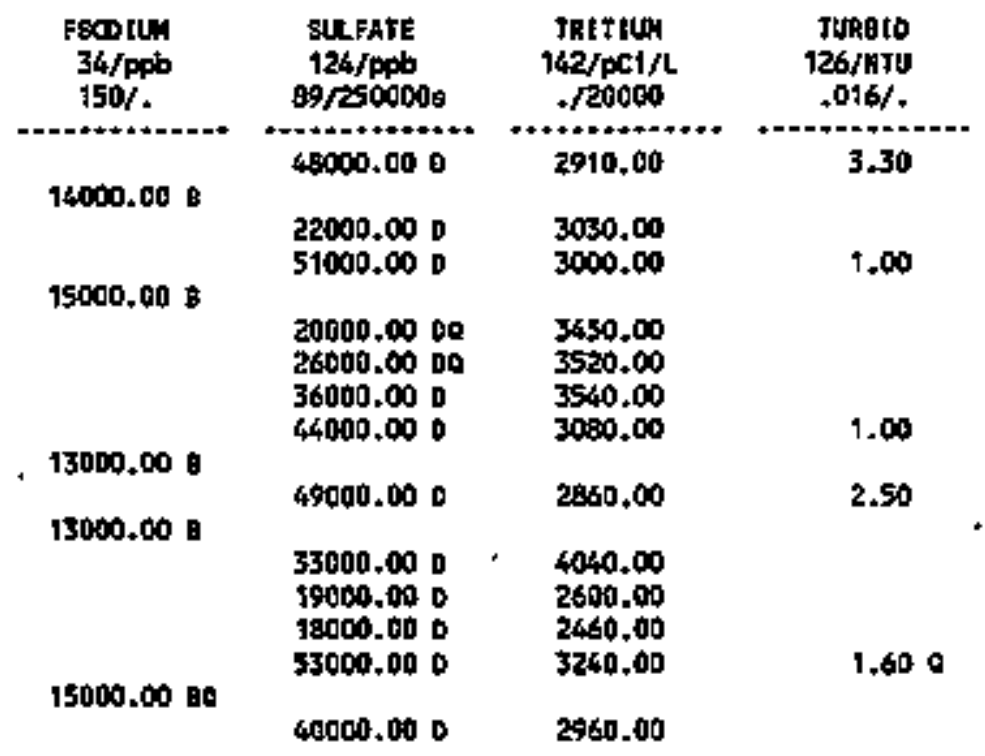

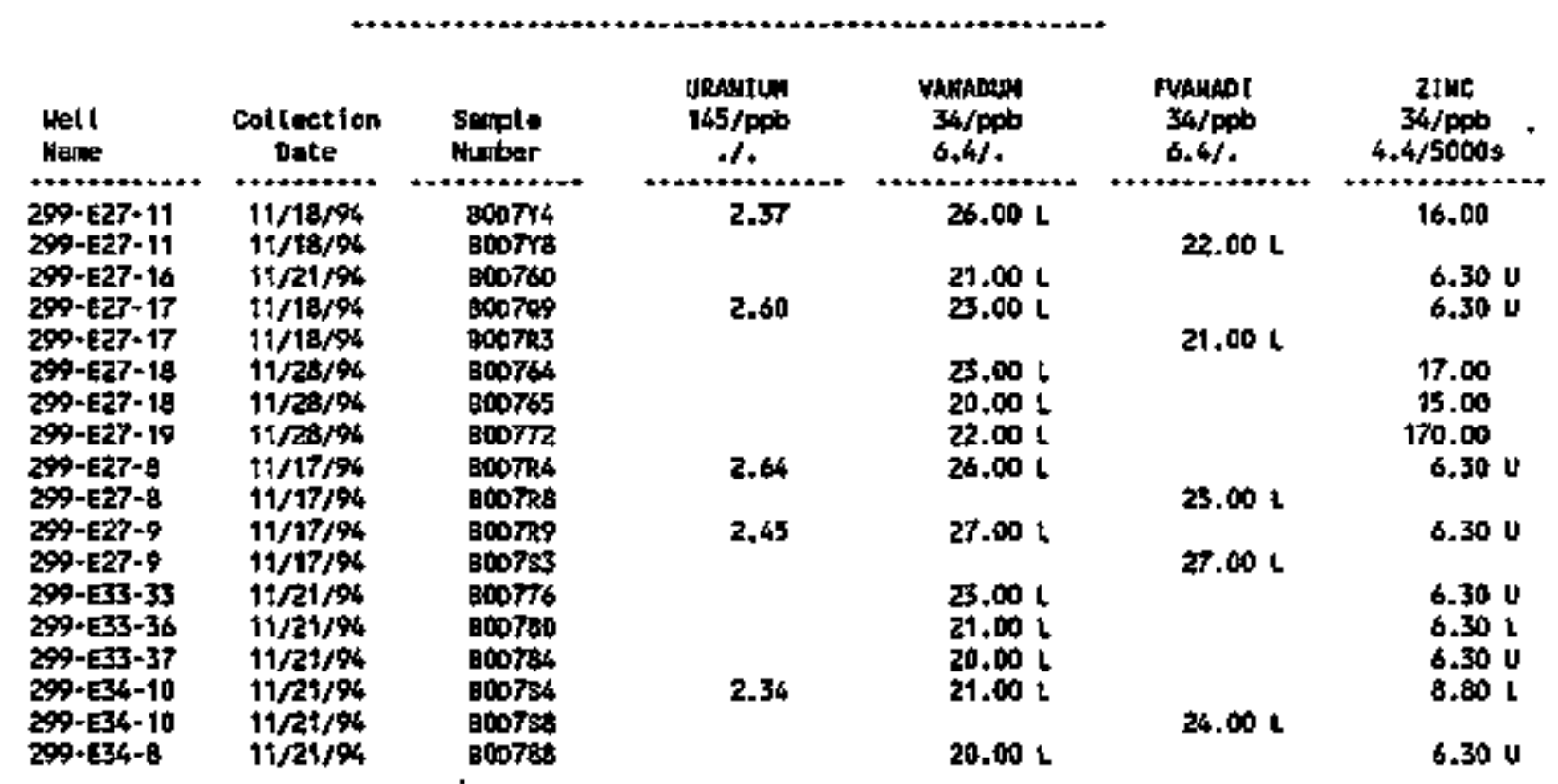


Table 9-4. Constituents with at Least One Detected Value for the 216-B-63 Trench. Data for Reporting Period October 1 through December 31, 1994. (sheet 7 of 7 )

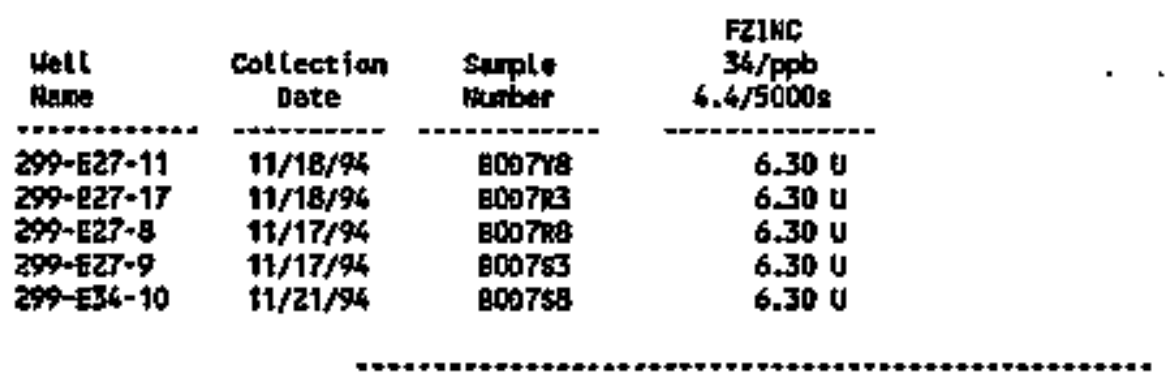

For expleration of thio table, see section $1 . \overline{4}$ of report. 
Table 9-5.. Contamination Indicator Parameters for the 216-B-63 Trench Data for Reporting Period October 1 through December 31, 1994. (sineet 1 of 2)

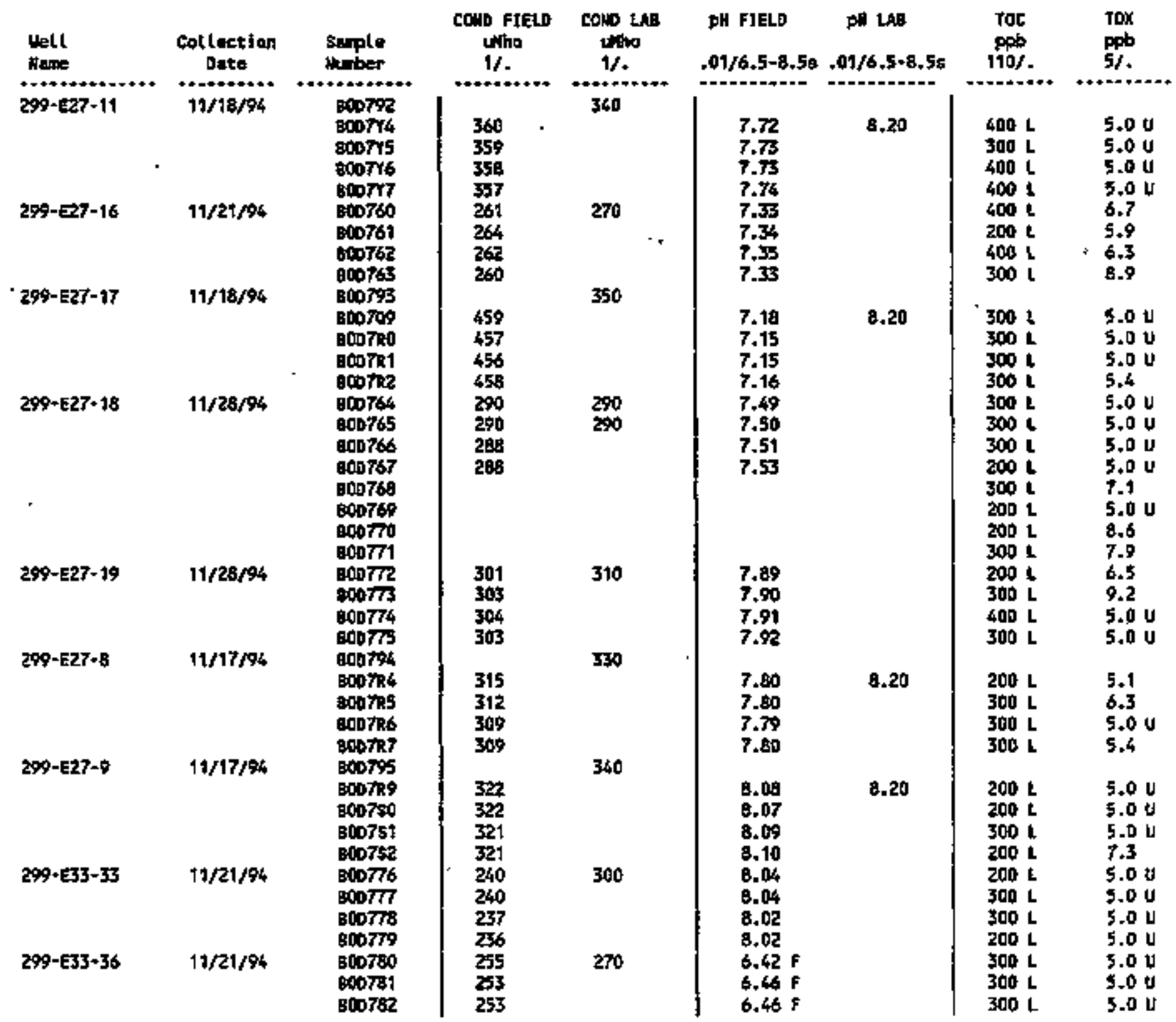


Tab]e 9-5. Contamination Indicator Parameters for the 216-B-63 Trench Data for Reporting Period October 1 through December 31, 1994. (sheet 2 of 2)

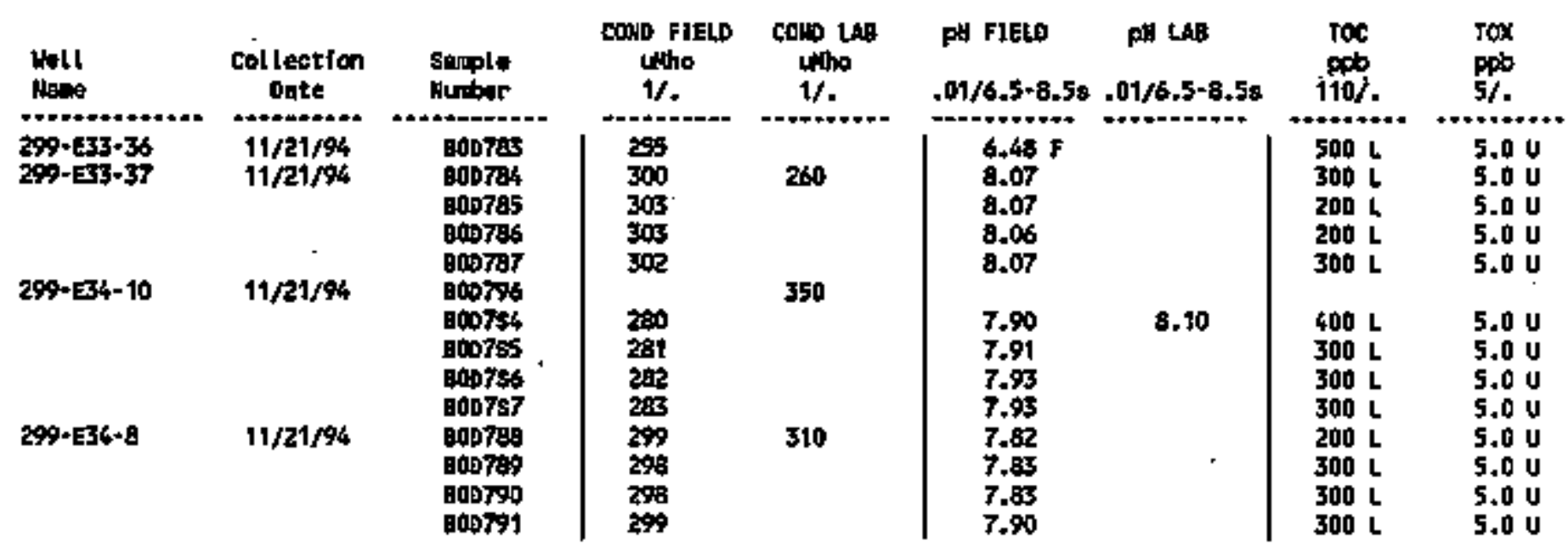

For explention of this table, set Section 1,4 of report. 
DOE/RL-94-36-4

This page intentionatly left blank. 


\section{COATEEMTS}

$10.0216-5-10$ POND AND DITCH .................... 10-1 10.1 IMTRODUCTION ...................... 10-1

10.2 WATER LEVEL MEASUREMENTS $\ldots \ldots \ldots$

10.3 WATER CHEMISTRY DATA ........................ 
DOE/RL-94-36-4

LIST OF FIGURES

10-1 Well Location Map for the 216-S-10 Facility .......... . 10-4

\section{LIST OF TABLES}

10-1 Monitoring We11 Purpose and Sampling Schedule for the 216-S-10 Facility ....... 10-5

10-2 RCRA Hater Leve1 Measurement Report 216-S-10 Facility, Fourth Quarter $1994 \ldots \ldots . \ldots . \ldots 10-6$

10-3 Constituent List and Summary of Resujts for the $21 \dot{6}^{\circ}-\dot{S}-10$ Facil ity Data for Reporting Perjod October I through Decealber 31, 1994 . . . 10-7

10-4 Constituents with at Least One Detected Value for the 216-5-10 Facility Data for Reporting Period October 1 through December 31, $1994 \ldots \ldots$. . . . . . . 10

10-5 Contamination Indicator Parameters for the 216-S-10 Faciitity Data for Reporting Period October 1 through Decenber 31, 1994 . . 10-14 
D0E/RL-94-36-4

\title{
10.0 216-S-10 POND AND DITCH
}

\author{
J. W. Lindberg \\ Nestinghouse Hanford Company
}

\subsection{INTRODUCTIOA}

The 216-5-10 Pond and Ditch (S-10 Facility) is located south-southwest of the 200 Hest Area, directly outside the perimeter fence. The S-10 Facility received wastewater frow the Reduction-oxidation (REDOX) P1ant (202-S Building) located in the 200 West Area. In the past, wastewater disposed of at the S-10 factitity has contained hazardous waste and radioactive materials. Since 1985, physical controls and operating procedures have been modified to avoid inadvertent discharge of hazardous and radioactive substances to the rastewater strean. The facility has been monitored under interiw status since August 1991. In October 1991, the effiluent waste strean, including the water source, to the 216-S-10 Ditch was deactivated. Because the $5-10$ Factlity is not expected to receive addttional hazardous and radioactive substances, the facility will be closed in accordance with WAC 173-303-610 (final status).

The 5-10 Facjifty initjally comprised an open, unlined ditch $1.8 \mathrm{~m}(6 \mathrm{ft})$ deep, $1.2 \mathrm{~m}(4 \mathrm{ft})$ wide at the bottom, and $686 \mathrm{~m}(2,250 \mathrm{ft})$ long. In addition, an open, unTined percolation pond, approximately 2 hectares (5 acres) in size, was constructed at the southwest end of the ditch, and was active during part of the tifme the ditch was receiving waste.

The 216-S-IO Ditch began receiving wastawater from the REDOX P1ant in August 1951, and the 216-S-10 Pond was dug and placed in service in February 1954. In 0etober 1985, the 216-\$-10 Pond and portions of the 216-S-10 Ditch were decommissioned, backfilled, and stabilized. The remaining portion of the 216-S-10 Ditch (approximately $594 \mathrm{~m}$ [1,950 ft]) received nonhazardous, nonregulated waste from the 202-\$ Building chemical sewer until deactivation in October 1991. This waste stream comprised cooling water, stean condensate, water tower overfiow, and drain effluent. These source streams had been routed so that they would not come into contact with hazardous materials.

Releases of hazardous materials and constituents to the S-10 facil tity are pooriy documented. Radioact ive waste was reportedly disposed to the \$-10 Facility as a result of contaminated floor and sewer drains at the REDOX Plant. Chemical releases were documented in 1954 and 1983, and known constituents are $\mathrm{A}]\left(\mathrm{MO}_{3}\right)_{3}, \mathrm{NaNO}_{3}, \mathrm{MaOH}, \mathrm{Na}_{3} \mathrm{PO}_{4}, \mathrm{HaF}$, $\mathrm{HaCl}$, and $\mathrm{K}_{2} \mathrm{Cr}_{2} \mathrm{O}_{7}$.

During 1991 the annua] volume of effluent discharged to the 216-S-10.01tch was between $1.9 \times 10^{8}$ and $5.7 \times 10^{8} \mathrm{~L}\left(5.0 \times 10^{7}\right.$ and $1.5 \times 10^{8} \mathrm{gal}$ ). This volume of discharge created a local ized recharge mound and an associated perched water table directly below the receiving (north) end of the ditch. This recharge mound has since disstpated, and the perched water zone has been dry since shortly after the ditch was deactivated. 
Groundwater beneath the S-I0 Facjitty is sampled semiannually in accordance with the Interim-Status Groundwater Nonitoring Plan for the 216-S-10 Pond and Ditch (UHC 1990a). The site is currently in a detectionlevel indicator parameters evaluation program.

The monjtoring network currently consists of two upgradient wells, three downgradient wells, and one downgradient well that monitors the base of the unconfined aquifer (Figure 10-1). Table 10-1 1ists the wells and their sampling status. Ail of the wells now have at least four quarters of analytical data. The results of the background statistical calculations for the upgradient monitoring wells (based on the first four quartters of analytical data) were presented in the Annual Report for RCRA Groundwater Honitoring Projects at Hanford site Facilities for 1993 (DOE-RL 1994).

\subsection{MATER LEVEL MEASUREMENTS}

Water leve] measurements obtatned during the fourth quarter of calendar year 1994 are presented in Table 10-2. Hater levels are measured at al1 wells at the time of sampling. Also, quarterly water Tevel measurements are made at six wells at the S-10 Facility jncluding 299-W26-7, 299-W26-8, 299-W26-9, 299-426-10, 299-W26-12, and 299-W27-2.

\subsection{WATER CHEMISTRY DATA}

Groundwater sampiing during the fourth quarter of calendar year 1994 included the five wells of the monitoring network that are screened in the upper portion of the unconfined aquifer (299-\$26-7, 8, 9, 10, and 12) (Figure 10-1). They were sampled on Decewber 7, 1994. The sixth wel1 (299-W27-2) (Figure 10-1) was not sampled In tilse to make the deadline for inclusion in the results for the fourth quarter. Results for this well will be reported in the first quarter report for 1995. Well 299-W27-2 monttors the base of the unconfined aquifer, and results are not used for statistical evaluation. Avallable data for the reporting period october 1 to December 31, 1994 are shown in Jables 10-3 through 10-5.

Drinking water parameters, groundwater qualfty parameters, and sitespecific and other constituents (Table 10-3) did not exceed OwSs except for unfiltered chromium in two upgradient we7]s, filtered chromium in one upgradient wel1, unfiltered iron in four wells (two upgradient and two downgradient), and unfiltered manganese in two downgradient wells (TabTe 10-4). High levels of chronium, iron, and manganese have been reported in the past in Hanford we11s and are assumed to be artifacts of well construction or the use of stainless steel well screens. The unusualiy high concentrations of aluminum in well 299-W26-10 probably indicate partjculates in the sample, which suggests high well turbidity.

Indicator parameters did not exceed their critjcal range or mean values in the fourth quarter of 1994 except for field-measured $\mathrm{pH}$ values from wells 299-W26-8 (an upgradient we11) and 299-W26-10 (a downgradient we11) (Table 10-5). These field measurements are suspected to be in error because they are not consistent with laboratory $\mathrm{pH}$ measurements, and they are not 
DOE/RL-94-36-4

consistent with the historical trend. The wells will be resampled. A RADE has been submitted for these data. 


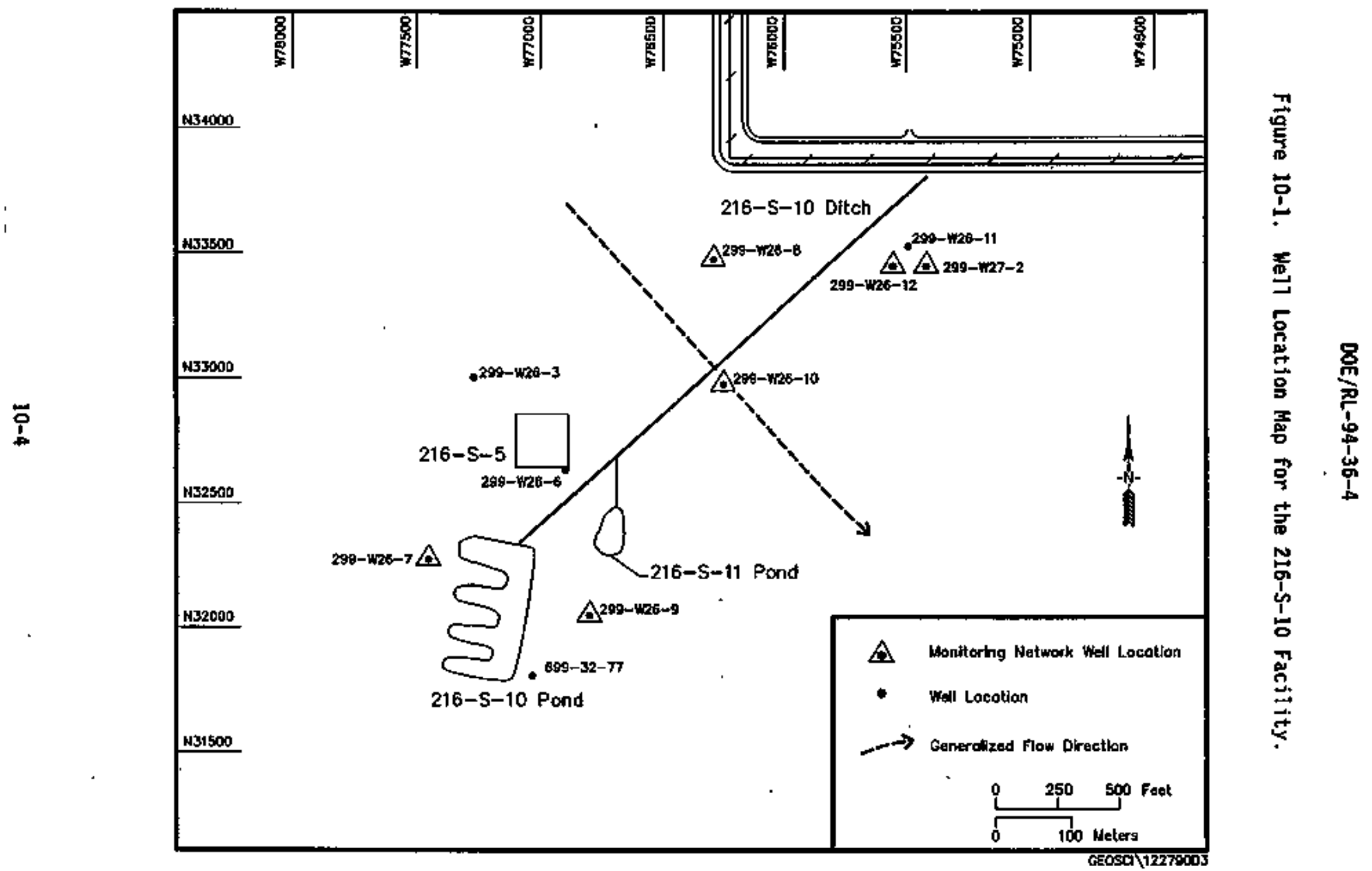


Table 10-1. Monjtoring Well Purpose and Sampling schedule for the 216-S-10 Facil ity.

\begin{tabular}{|l|l|l|l|l|}
\hline $\begin{array}{c}\text { We11 } \\
\text { no. } \\
\text { (299-) }\end{array}$ & $\begin{array}{c}\text { Re1ative } \\
\text { position }\end{array}$ & \multicolumn{1}{|c|}{ Hydrogeologic unit } & . $\begin{array}{c}\text { Sampling } \\
\text { frequency }\end{array}$ & $\begin{array}{c}\text { Last } \\
\text { sampling } \\
\text { date }\end{array}$ \\
\hline W26-7 & Upgradient & Ringold: Water Table & Semiannua71y & $12 / 07 / 94$ \\
\hline W26-8 & Upgradient & Rtngold: Water Table & Semiannually & $12 / 07 / 94$ \\
\hline W26-9 & Downgradient & Rtngo7d: Water Tab]e & Semiannually & $12 / 07 / 94$ \\
\hline W26-10 & Downgradient & Ringold: Water TabTe & Semiannually & $12 / 07 / 94$ \\
\hline W26-12 & Downgradient & Ringold: Water Table & Semiannually & $12 / 07 / 94$ \\
\hline W27-2 & Downgradient & $\begin{array}{l}\text { Ringold: Base of } \\
\text { Unconf ined Aquifer }\end{array}$ & Semiannua7ly & $06 / 20 / 94$ \\
\hline
\end{tabular}


Table 10-2. RCRA Water level Measurement Report 216-S-10 Facility, Fourth Quarter 1994.

\begin{tabular}{lllll}
\hline Wel1 & Date & $\begin{array}{c}\text { Depth to } \\
\text { water (ft) }\end{array}$ & $\begin{array}{c}\text { Water 1eve1 } \\
\text { elevation above ms } \\
\text { (ft) }\end{array}$ \\
\hline We17s Mon1toring the Top of the Unconfined Aquifer \\
(m)
\end{tabular}

NOTES: 1. Water level elevations are calculated by subtracting the measured depth-to-water from the surveyed elevation for the wet?.

2. Depth-to-water values are transcribed fron field records.

3. Elevations marked with an ' $\neq$ " were measured at the time of sampling.

4. Elevations marked wth a ' + ' are outside of the expected range, and are suspected of error. 
Table 10-3. Constituent List and Sumary of Results for the 216-S-10 Facility Data for Reporting Period October 1 through December 31, 1994.

(sheet 1 of 3)

CEITRH]MATION JHDICATOR PARANETERS

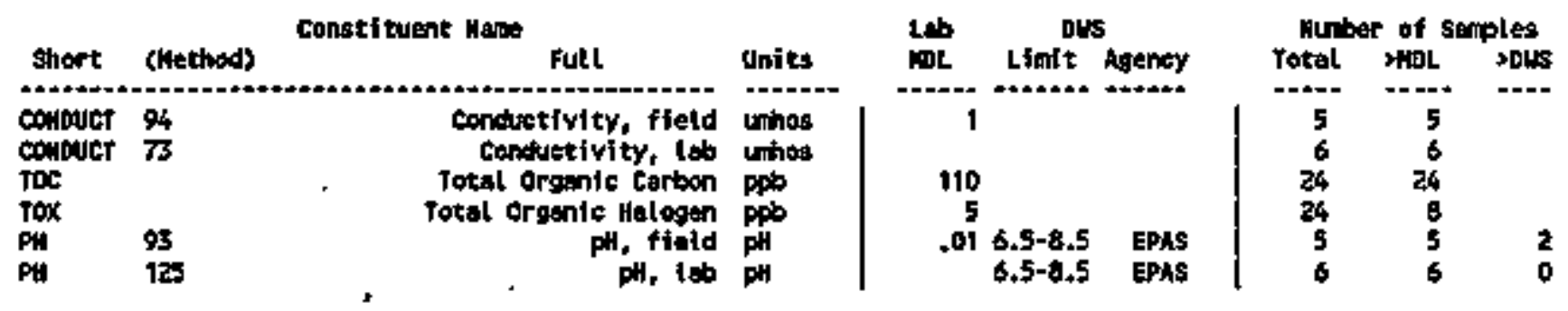

DRJHKLW UATER PARAYETERS

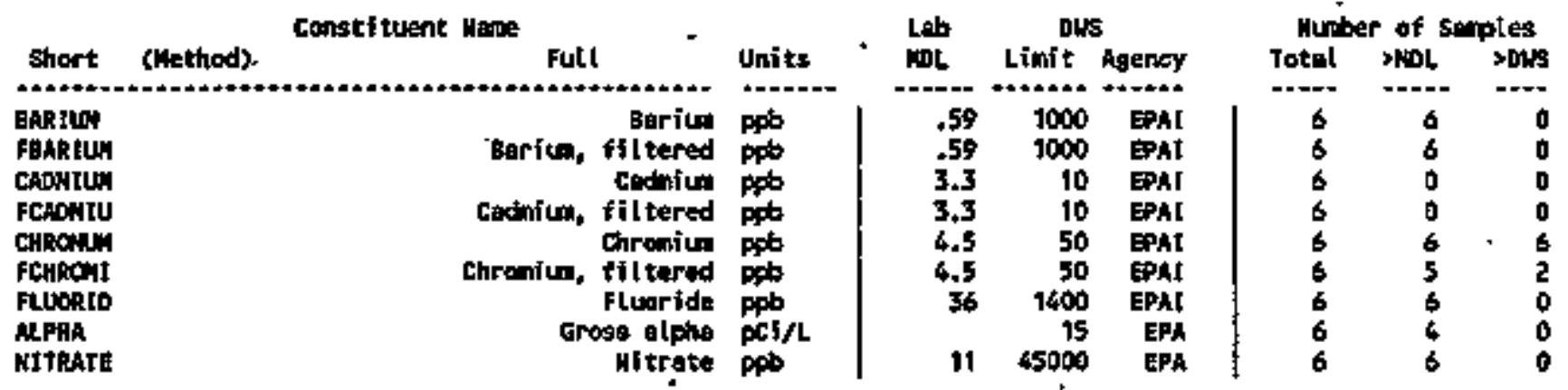

GROMDUKTER GULIIY PARNIETERS

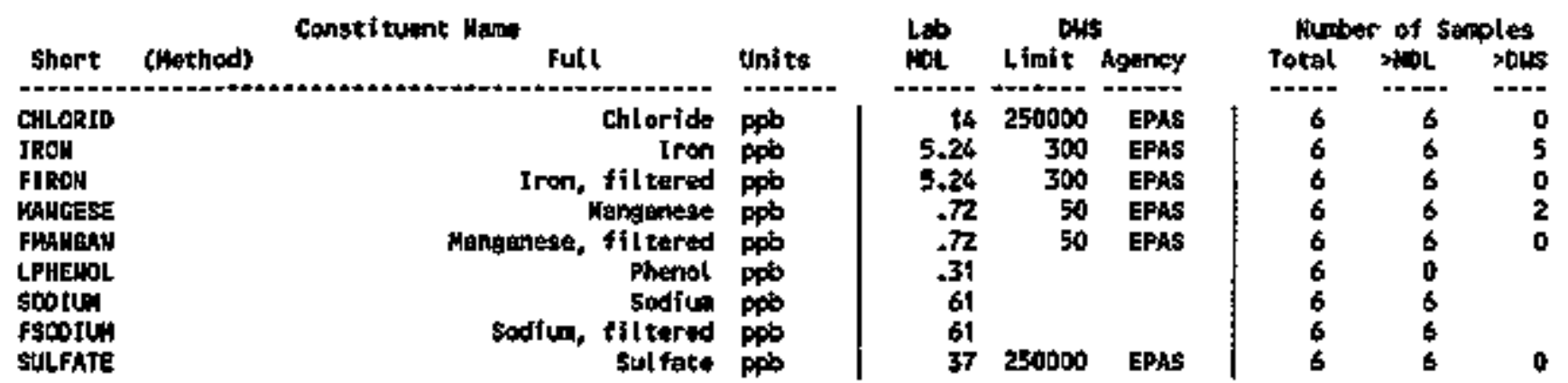


Table 10-3. Constituent List and Sumary of Results for the 216-S-10 Facility Data for Reporting Period October 1 through Decennber 31, 1994.

(sheet 2 of 3 )

SITE SPECIFIC ANG OYHRR COUSTITUENTS

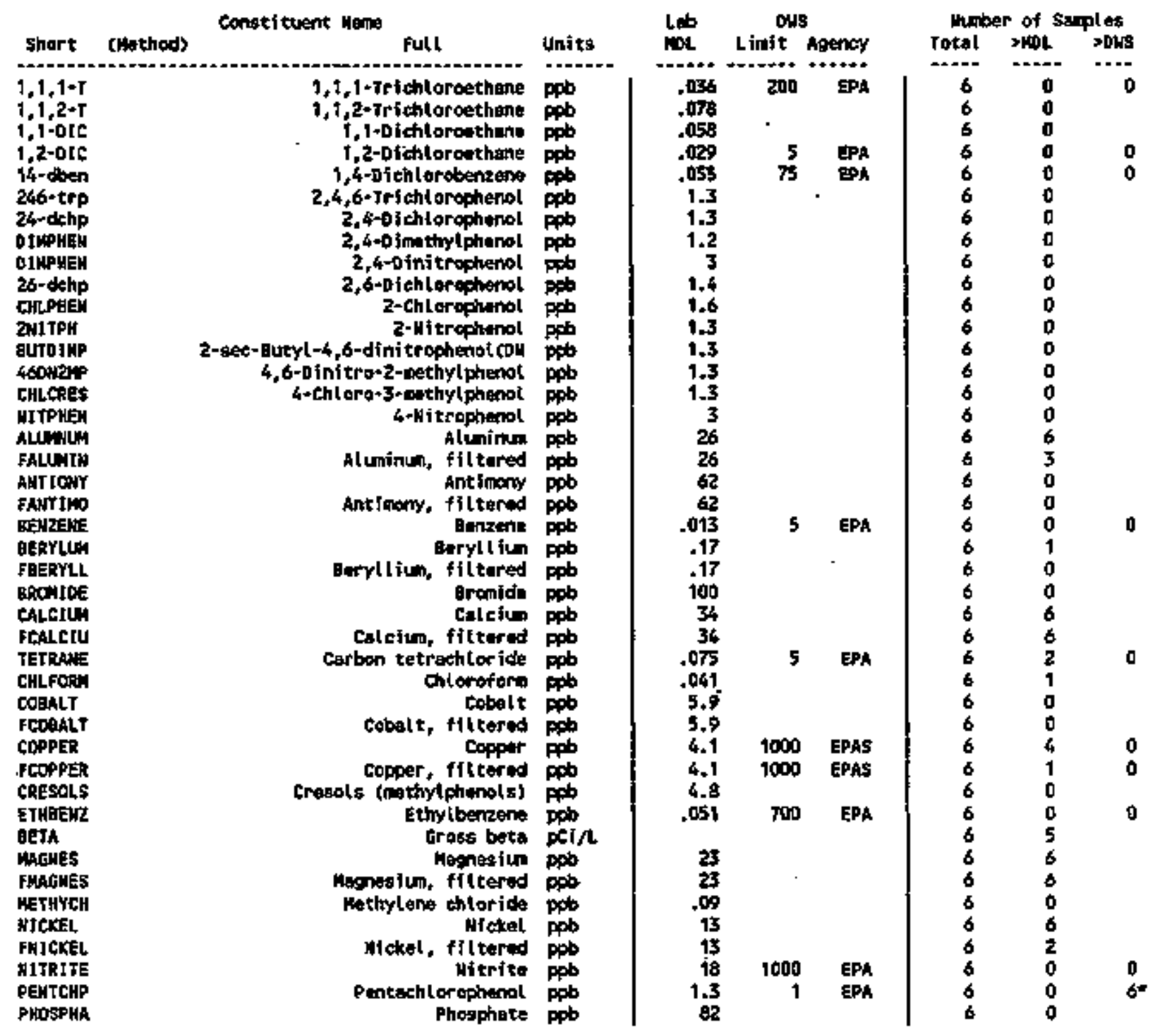


Table 10-3. Constituent List and Strmary of Results for the 216-\$-10 Facility Data for Reporting Period October 1 through December 31, 1994. (sheet 3 of 3 )

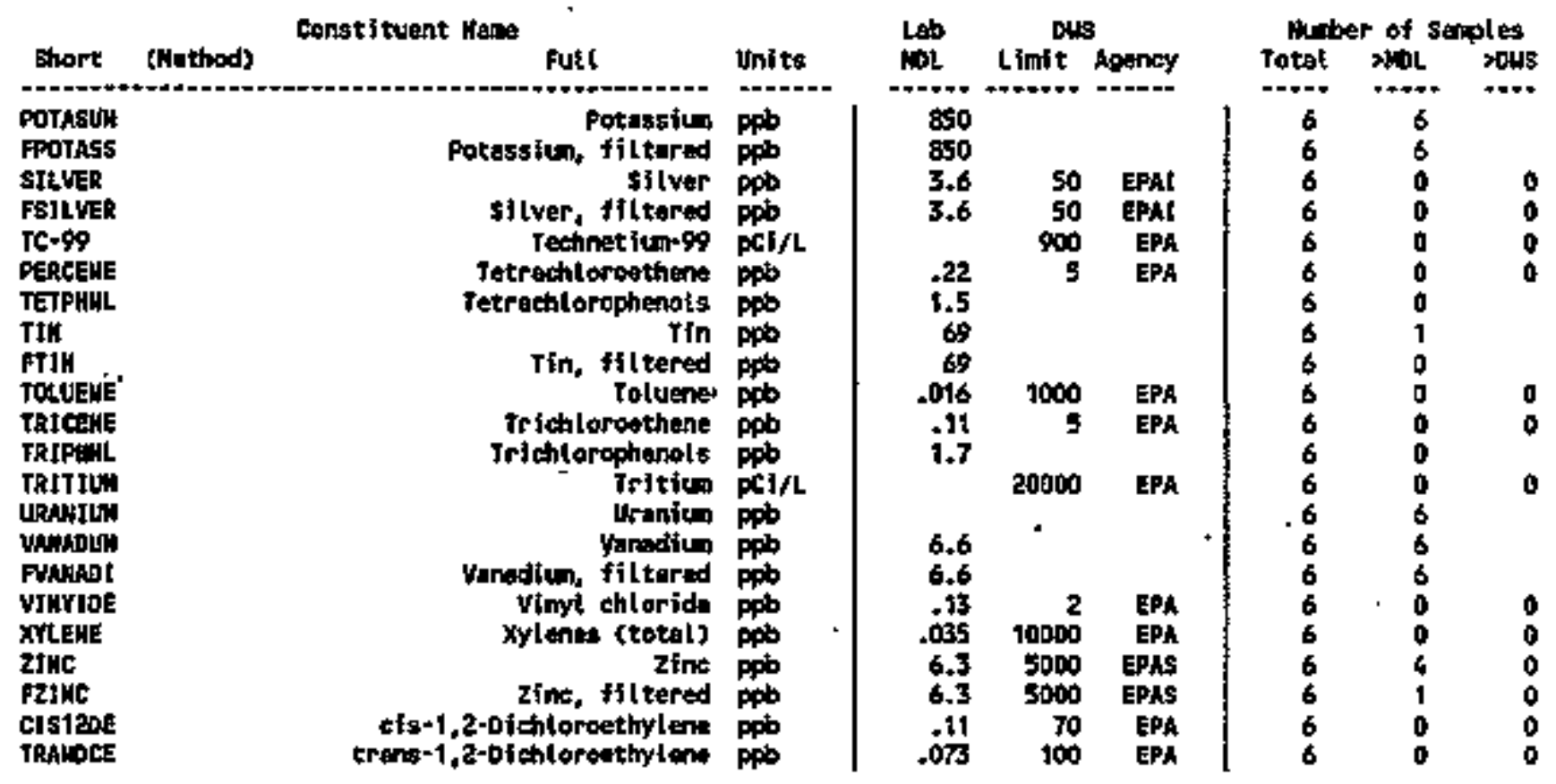

For explenstion of this table, gee Sertion 1.4 of report. 
Table 10-4. Constituents with at Least One Detected Value for the 216-S-10 Facility Data for Reporting Period October 1 through December 31, 1994.

(sheet 1 of 4)

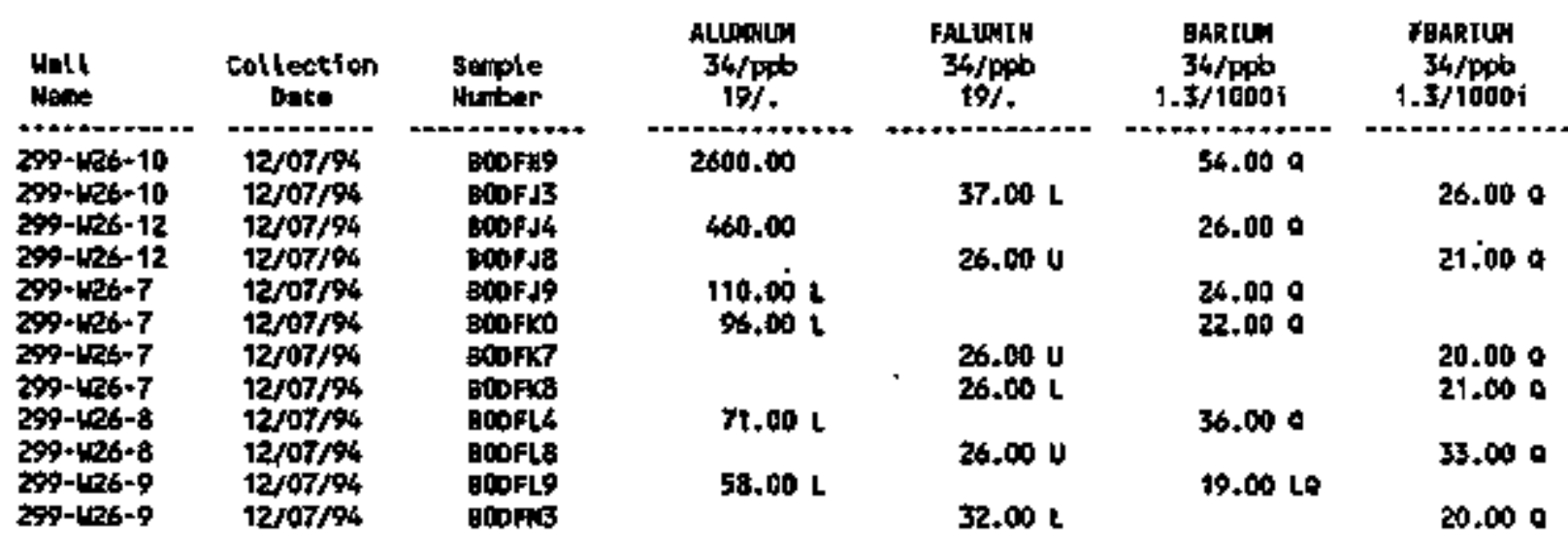

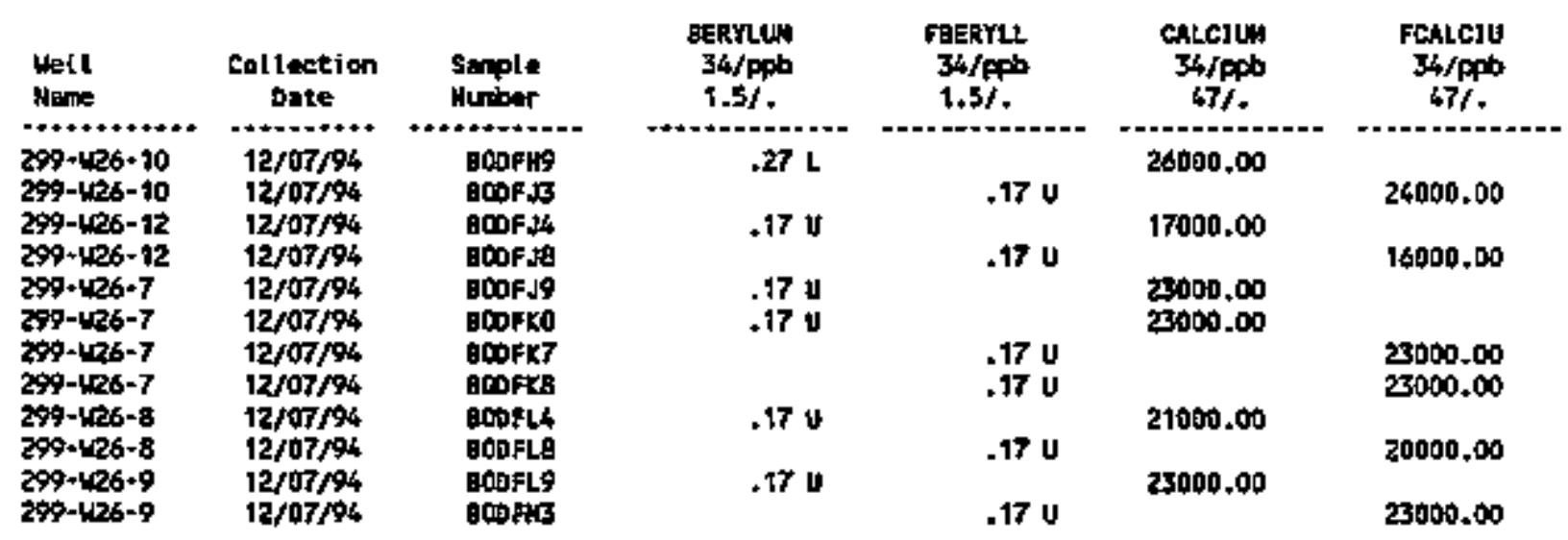

\begin{tabular}{|c|c|c|c|c|c|c|}
\hline $\begin{array}{l}\text { Woll } \\
\text { Nenese }\end{array}$ & $\begin{array}{l}\text { Collection } \\
\text { Date }\end{array}$ & $\begin{array}{l}\text { Sample } \\
\text { Munber }\end{array}$ & $\begin{array}{l}\text { TETRANE } \\
\text { 25/\%सb } \\
.32 / 5\end{array}$ & $\begin{array}{c}\text { CHLOR ID } \\
126 / \text { ppob } \\
71 / 250000 \mathrm{~s}\end{array}$ & $\begin{array}{c}\text { CHLFOSi } \\
\text { 25/gpt } \\
.05 \% .\end{array}$ & $\begin{array}{l}\text { CHRCWM } \\
34 / p^{b} \\
11 / 100\end{array}$ \\
\hline $\begin{array}{l}299-126-10 \\
299-126-12 \\
299-126-7 \\
299-1126-7 \\
299+126-8 \\
299+126-9\end{array}$ & $\begin{array}{l}12 / 07 / 94 \\
12 / 07 / 94 \\
12 / 07 / 94 \\
12 / 07 / 94 \\
12 / 07 / 94 \\
12 / 07 / 94\end{array}$ & $\begin{array}{l}\text { BODFHS } \\
\text { BODF } \\
\text { BODFJS } \\
\text { BODFKD } \\
\text { BCOFLL } \\
\text { BCOFLS }\end{array}$ & $\begin{array}{r}.64 \mathrm{t} \\
.08 \mathrm{v} \\
.08 \mathrm{v} \\
.08 \mathrm{v} \\
2.00 \\
.08 \mathrm{v}\end{array}$ & $\begin{array}{l}5900.00 \mathrm{Bg} \\
1600.00 \mathrm{Bg} \\
5700.00 \mathrm{g0} \\
5600.00 \mathrm{BO} \\
3100.00 \mathrm{B0} \\
6500.00 \mathrm{BO}\end{array}$ & $\begin{array}{l}.04 \mathrm{U} \\
.28 \mathrm{~L} \\
.04 \mathrm{U} \\
.06 \mathrm{U} \\
.04 \mathrm{U} \\
.06 \mathrm{U}\end{array}$ & $\begin{array}{r}74.00 \\
78.00 \\
250.00 \\
230.00 \\
340.00 \\
58.00\end{array}$ \\
\hline
\end{tabular}


Table 10-4. Constituents with at Least One Detected Value for the 216-\$-10 Facility Data for Reporting Period October 1 through December 31, 1994. (sheet 2 of 4 )

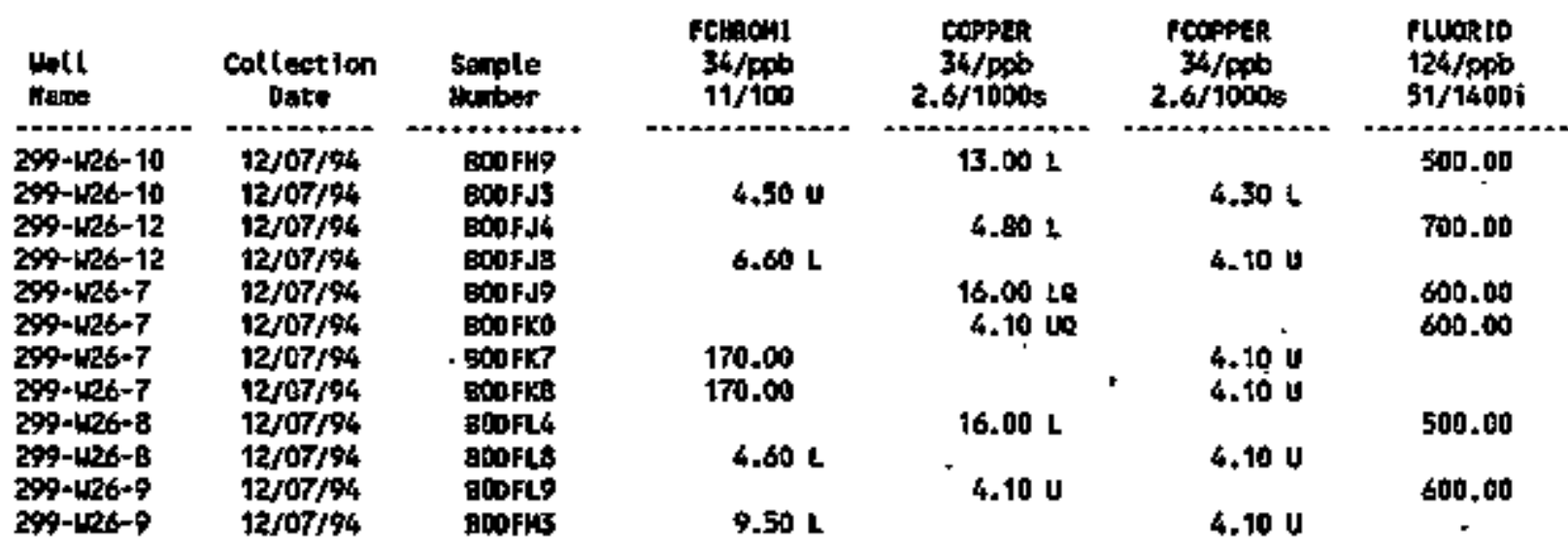

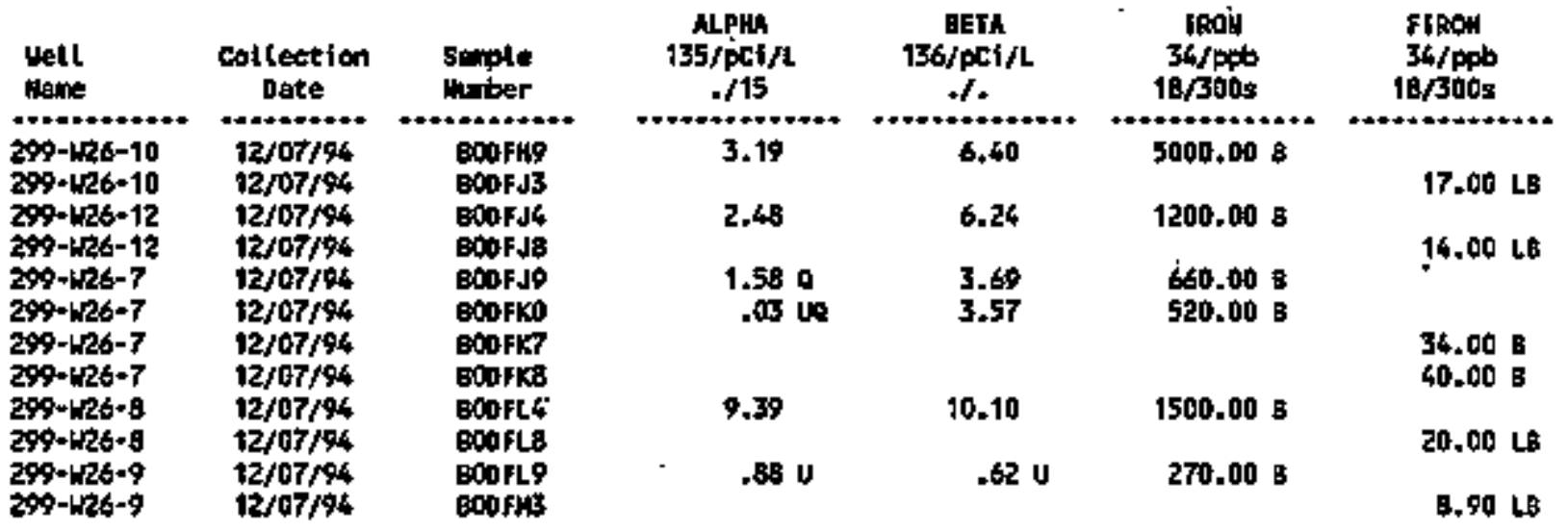

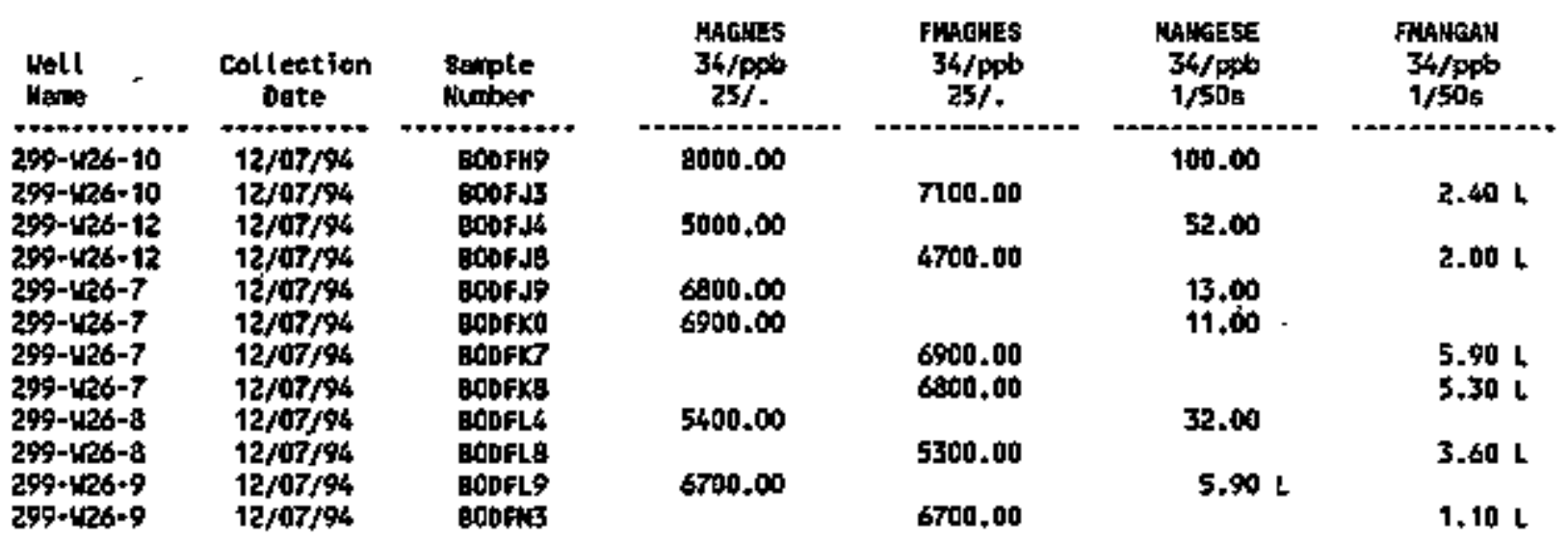


Table 10-4. Constituents with at Least One Detected Value for the 216-5-10 Facility Data for Reporting Period October 1 through Decenber 31, 1994. (sheet 3 of 4 )

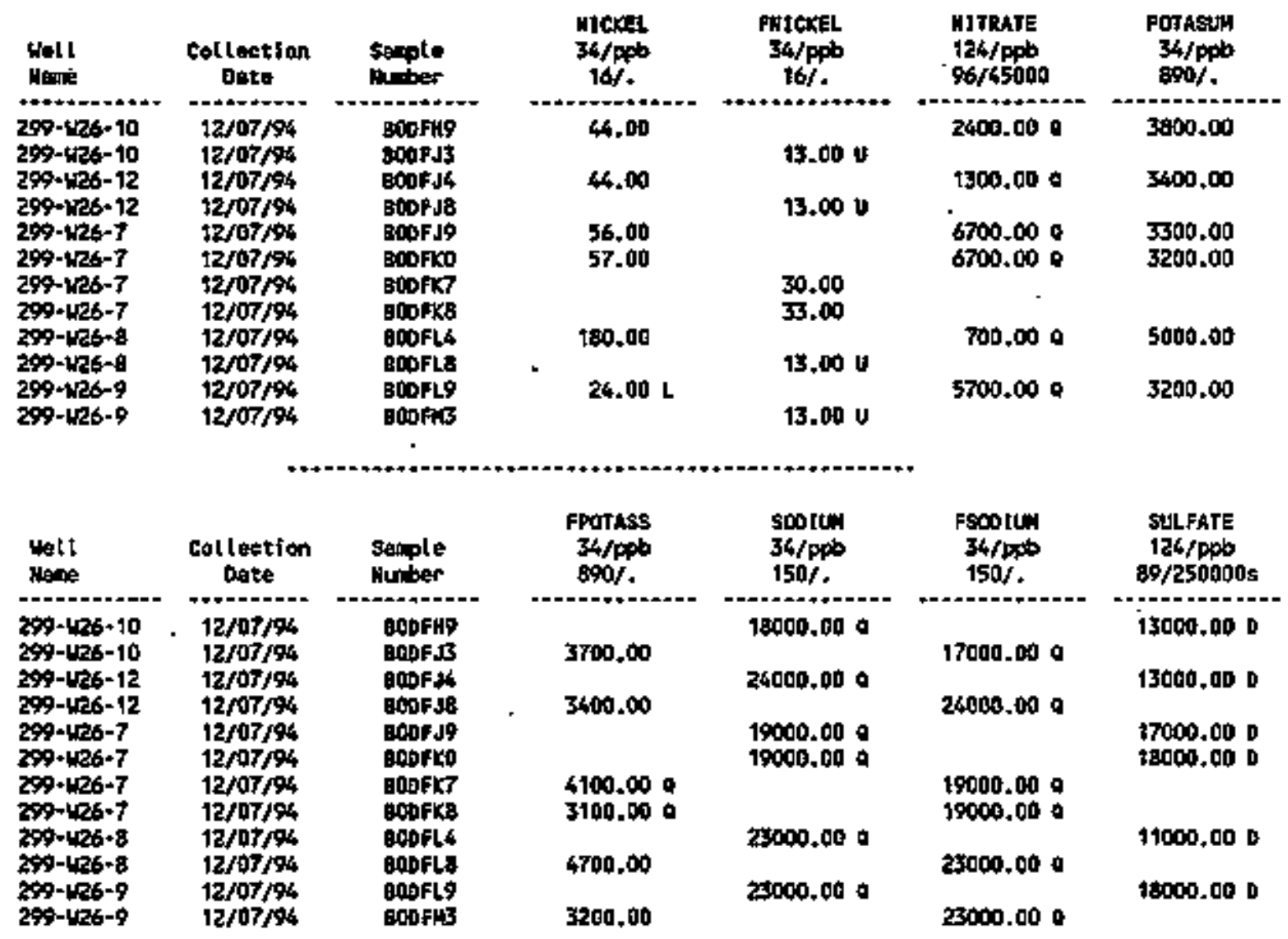

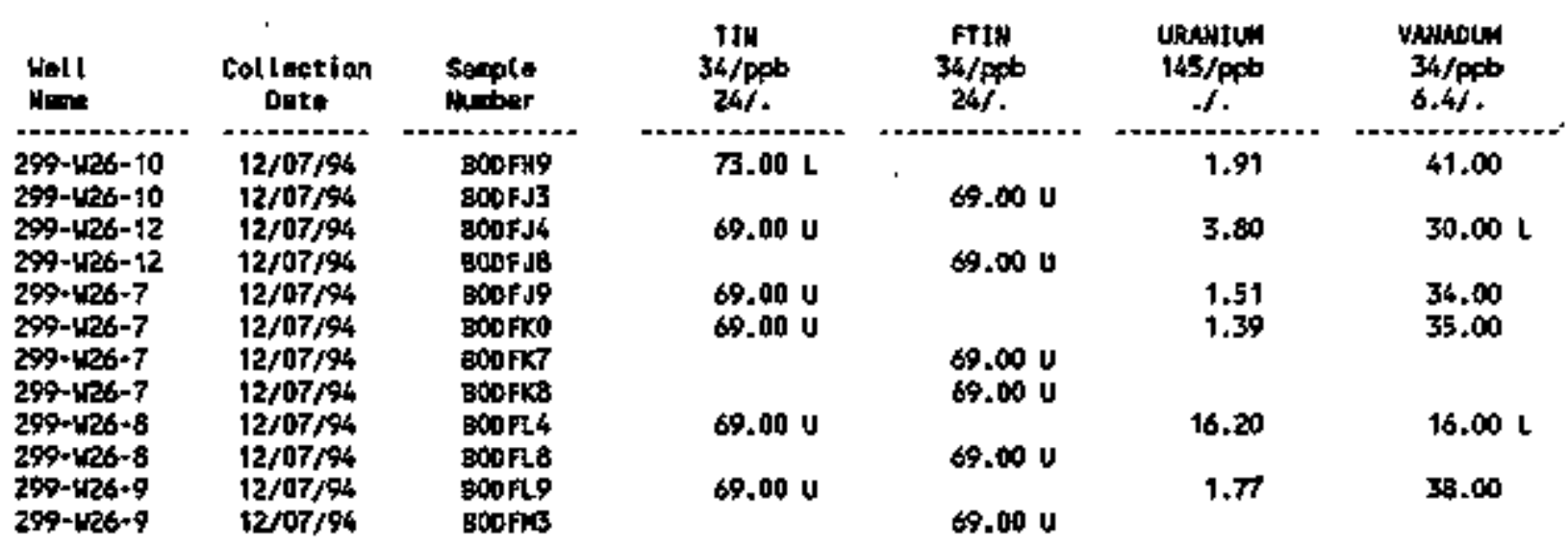


Tab7e 10-4. Constituents with at Least 0ne Detected Value for the 216-5-10 Facility Data for Reporting Period October 1 through December 31, 1994. (sheet 4 . of 4 )

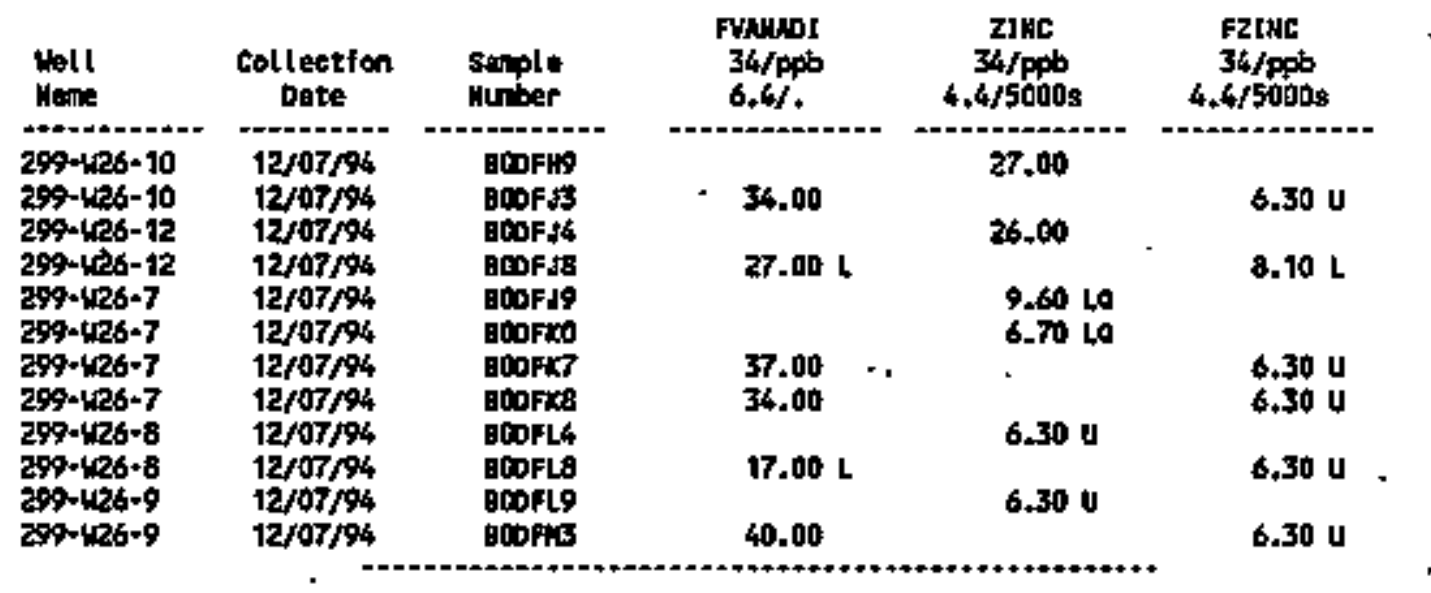

For toplantion of this table, get Section 1,4 of rapart. 
Table 10-5. Contamination Indicator Parameters for the 216-5-10 Facitity Data for Reporting Period Dctober 1 through December 31, 1994.

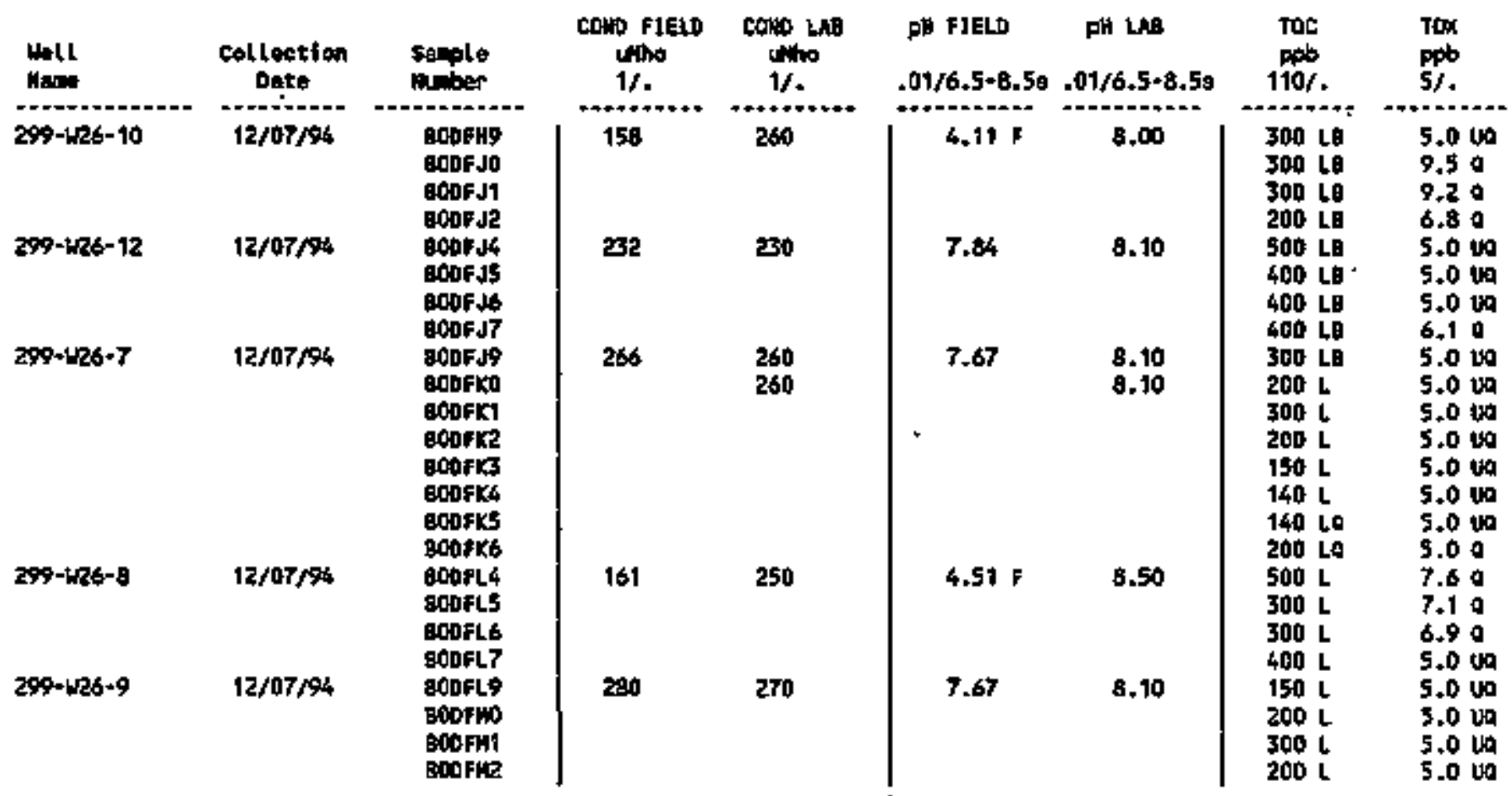


DOE/RL-94-36-4

\section{CONTENTS}

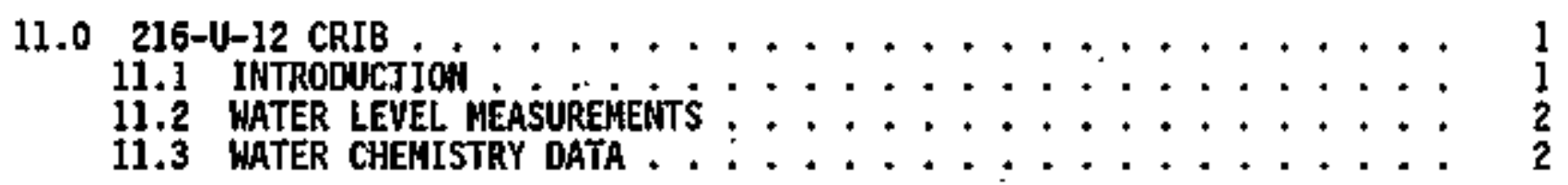




\section{DOE/RL-94-36-4}

\section{LIST OF FIEURES}

11-1 Well Location Map for 216-v-12 Crib . . . . . . . . . . . 3 3

\section{LIST OF TABLES}

11-1 Monitaring Mell Purpose and Sampting Schedule for the 216-U-12 Crib .................. 4

11-2 RCRA Water Leve] Measurement Report 216-U-12 Crib, Fourth Quarter $1994 \ldots \ldots$

11-3 Constituent List and Sumaary of Resuits for the $216-\mathrm{j}-\mathrm{i} 2$ Crib Data for Report ing Perfod October 1 through Decenber 31, 1994 . . 6

11-4 Constituents with at least one Detected Value for the 216-U-12 crib Data for Reporting Period October 1 through Decenber 31, 1994 . . 9

11-5 Contamination Indjcator Paraneters for the 216-U-12 Crib Data for Reporting Period October 1 through December 31, 1994 . . . I3 


\title{
$11.0 \cdot 216-\mathbb{U}-12$ CRIB
}

\author{
B. A. Viltiams \\ Westinghouse. Hanford Company
}

\subsection{INTROOUCTIOH}

The 216-U-12 crib (U-12 crib) is located approximately $609 \mathrm{~m}(1,998 \mathrm{ft}$ ) south of the U Plant in the 200 Nest Area (see Figure 1-I). The crib is an unlined, gravel-bottom percolation crib that is 3 by $30.5 \mathrm{~m}$ (10 by $100 \mathrm{ft}$ ) wide and 4 ( $13 \mathrm{ft}$ ) deep. The crib has a plastic barrier cover and is backfilled with the original excavated soil. A vitrified clay distribution pipe buried in the gravel dispersed the effluent across the botton of the crib.

A RCRA interim-status assessment-7eve1 groundwater monitoring network was established for the U-12 Crib in 1993 (WHC 1993a). The U-12 Crib received wastewater from the U Plant in the 200 Hest Area from April 1960 until February I988, when it was rep]aced by the 216-U-17 Crib. Wastewater disposed of in the $\mathrm{U}-12 \mathrm{Cr}$ b contafned hazardous waste and radioactive materials. The DDE, Richl and operations offtce (RL) has proposed that the facility be closed under RCRA interin status (40 CFR 265). This closure will be coordinated with the CERCLA Past Practíce activity (200 UP-2 Operable Unit cleanup).

The waste was composed of effluent from the $U$ Plant and included 291-U-1 Stack dratnage and process condensate from the 224-U Building. The U-12 Crib received this waste stream from April 1960 unti1 1972, when it was deactivated. The U-12 Crib-was react fvated in Hovember 1981 and received U-P\}ant waste until it was permanently retired in February 1988. An average of over $1,000,000 \mathrm{~L}(264,200 \mathrm{gal})$ of effluent was disposed of in the crib during each year of its active life. The U-12 Crib has received low-level radioactive waste that is known to have included chemicals such as nitric acid in addition to plutonfum, strontifum, ruthenium, and uraniun. In 1985, physicai controls and operating procedures were modified to avoid inadvertent discharge of hazardous chemfca]s to the wastewater stream. Hydrogeologic evaluation of the crib in 1983 indicated radioactive contamination to a depth of a least $43 \mathrm{~m}(140 \mathrm{ft})$. The depth to water beiou the crib is approximately $72 \mathrm{~m}(236 \mathrm{ft})$.

The RCRA Interim-Status Groundwater Monitoring Plan for the 216-U-12 Crib (WHC 1990b) presents the program used to establish the background levels for the CIPs. The docuinent summarizes the available data for the disposal facility. In accordance with the plan, four RCRA groundwater ronftoring wells were installed in 1990. The monitoring network consisted of one upgradient and three downgradient we11s (Figure 11-1).

The sanple results for the fourth quarter of 1994 represent the fourteenth calendar quarter of RCRA data collected at the U-12 Crib. Data collected during the third quarter of 1992 from downgradient wells 299-422-41 and 299-W22-42 showed elevated specific conductivity. Verification sampling and analysis during that and subsequent quarters corroborated these findings. Currently the U-12 Crib groundwater. monitoring weIl network is sampied quarterly for assessment monitoring in accordance with the Interim-Status Groundwater Quafity Assessment Plan for the 216-U-12 Crib (WHC 1993a). Monthly sampling has been conducted since November 1993 for the fleld parameters $\mathrm{pH}$ and specific conductivity. The 
data from the monthly sampling will be reported as they become available.

Two extsting wells, 299-w22-22 and 299-w22-23, were remediated for inclusion in the monitoring network, but well 299-w22-22 has been dropped from the network because of sampling complications. The new assessment well, 699-36-70A, was added to the network and sampied for the first tive on January 3, 1995. Table 11-1 lists the network wel1s and their sarpling status during the reporting period; currently the network consists of six welis.

\subsection{WATER LEVEL MEASURENENTS}

Water levels in each network well are measured at least quarterly and when water samples are coliected. Hater level measurements obtained during the quarter are presented in Table 11-2.

\subsection{WATER CHEMISTRY DATA}

Groundwater samples were collected in Decenber 1994 from all network wells. A11 data currentiy avaj]able from this and previous unreported sampling periods are show in Tables 11-3 through 11-5. Table 11-3 includes the constituent list and a summary of results. Tables $11-4$ and 11-5 show results for specific constituents. Analytical data unavaliable at the time this report was prepared will be included in future quarterly reports.

Spectfic conductivity in downgradient wells 299-W22-41 and 299-W22-42 continues to exceed the critical mean of $437 \mu$ mho/cm. Results for nitrate (ONS $=45,000 \mathrm{ppb}$ ), the principal constituent suspected of causing elevated conductivity, are 130,000 and $520,000 \mathrm{ppb}$, respectively. Technetium-99 also had elevated results in these two wells, which is consistent with previous data. Filtered chromium, jron, and manganese are reported above the DWS in we11 299W22-23. These values are being investigated. Most of the network well-s had values exceeding the DWS for unfiltered iron and chronium. Carbon tetrachloride w111 not be reported in future quarterly reports, but it should be noted that this constituent has been consistently above or at the DWS in all network wells. An explanation of any data flags can be found in Sections 1.2 and 1.4. 


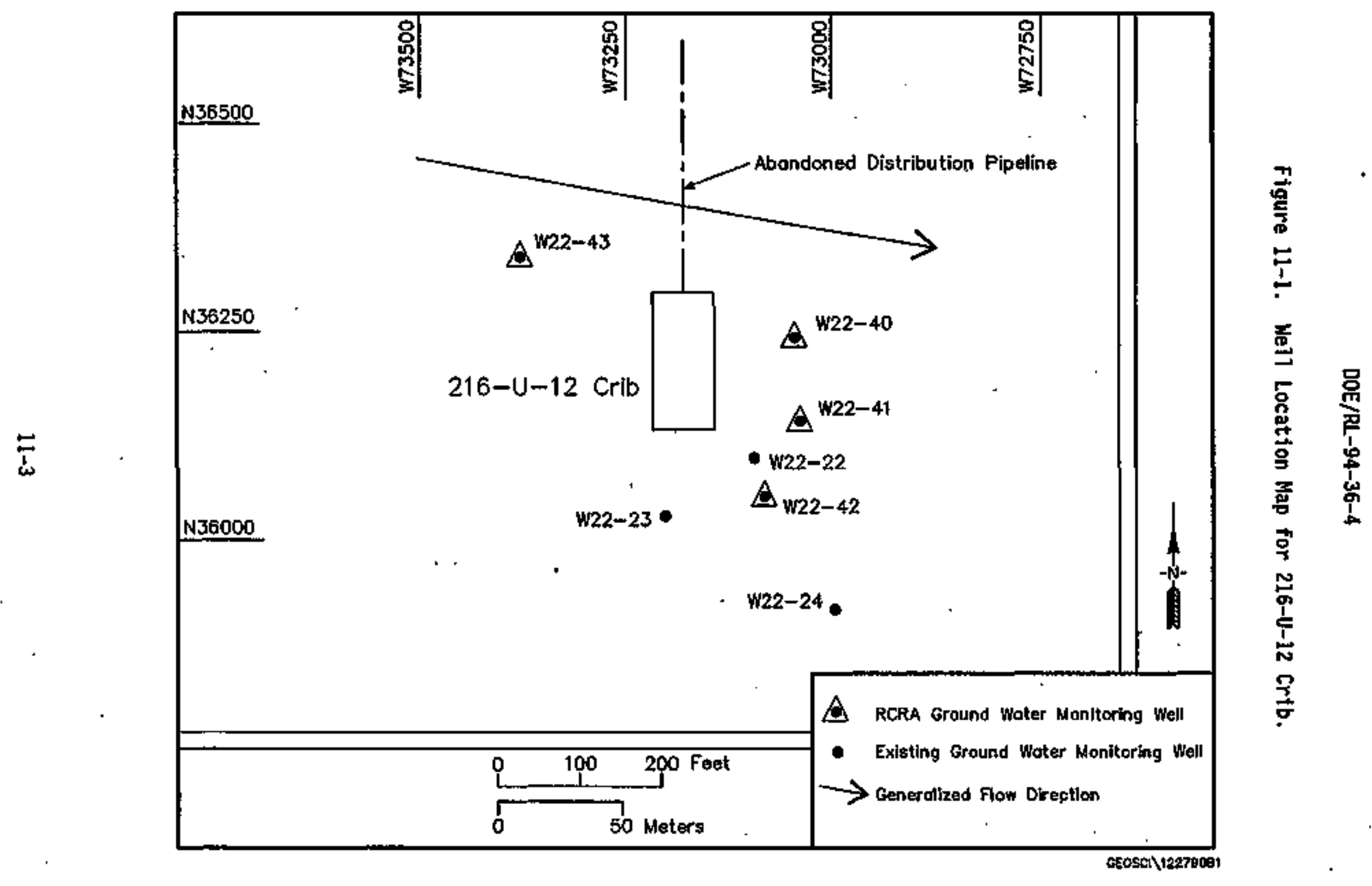


Table 11-1. Monitoring Weit Purpose and Sampling Schedule for the 216-14-12 crib.

\begin{tabular}{||l|l|l|l|l||}
\hline $\begin{array}{c}\text { Well no. } \\
(229-)\end{array}$ & $\begin{array}{c}\text { Relative } \\
\text { position }\end{array}$ & Hydrogeologic unjt & $\begin{array}{c}\text { Sample } \\
\text { frequency }\end{array}$ & $\begin{array}{c}\text { Sample } \\
\text { date, } \\
\text { 4th Qtr } \\
1994\end{array}$ \\
\hline W22-43 & Upgradient & $\begin{array}{l}\text { Ringold: Water } \\
\text { Table }\end{array}$ & Quarterly & $12 / 15 / 94$ \\
\hline W22-40 & Downgradient & $\begin{array}{l}\text { Ringold: Water } \\
\text { Table }\end{array}$ & Quarterly & $12 / 15 / 94$ \\
\hline W22-41 & Downgradient & $\begin{array}{l}\text { Ringold: Water } \\
\text { Table }\end{array}$ & Quarterly & $12 / 15 / 94$ \\
\hline w22-42 & Oommgradient & $\begin{array}{l}\text { Ringold: Water } \\
\text { Table }\end{array}$ & Quarterly & $12 / 15 / 94$ \\
\hline $699-36-70 A$ & Downgradient & $\begin{array}{l}\text { Ringold: Water } \\
\text { Tabie }\end{array}$ & Quarterly & $1 / 03 / 95$ \\
\hline W22-23 & Upgradient & $\begin{array}{l}\text { Ringold: Water } \\
\text { Table }\end{array}$ & Quarterly & $12 / 15 / 94$ \\
\hline
\end{tabular}

Note: Hydrogeologic units include the sandy gravels of the Hanford formation and silty sands of the Ringold Formation. 
Table 11-2. RCRA Water Level Measurement Report 216-U-12 Crib, Fourth Quarter 1994.

\begin{tabular}{|c|c|c|c|c|}
\hline Well & Date & $\begin{array}{c}\text { Depth to } \\
\text { water (ft) }\end{array}$ & $\begin{array}{l}\text { Water } \\
\text { elevation } \\
\text { (ft) }\end{array}$ & $\begin{array}{l}\text { Jeve] } \\
\text { above ms1 } \\
\text { (m) }\end{array}$ \\
\hline \multicolumn{5}{|c|}{ Wells Honitoring the Top of the Unconfined Aquifer } \\
\hline 299-W22-22 & $11 / 01 / 94$ & 234.22 & $455.83 *$ & 138.94 \\
\hline 299-W22-23 & $12 / 15 / 94$ & 236.27 & $454.49 *$ & 138.51 \\
\hline 299-\$22-40 & $\begin{array}{l}12 / 15 / 94 \\
12 / 20 / 94\end{array}$ & $\begin{array}{l}237.75 \\
238.04\end{array}$ & $\begin{array}{l}454.48^{*} \\
454.19\end{array}$ & $\begin{array}{l}138.53 \\
138.44\end{array}$ \\
\hline $299-\$ 22-41$ & $\begin{array}{l}11 / 01 / 94 \\
12 / 15 / 94 \\
12 / 20 / 94\end{array}$ & $\begin{array}{l}237.34 \\
237.14 \\
237.58\end{array}$ & $\begin{array}{l}454.40^{*} \\
454.60^{\star} \\
454.16\end{array}$ & $\begin{array}{l}138.50 \\
138.56 \\
138.43\end{array}$ \\
\hline 299-W22-42 & $\begin{array}{l}11 / 01 / 94 \\
12 / 15 / 94 \\
12 / 20 / 94\end{array}$ & $\begin{array}{l}236.82 \\
236.90 \\
237.32\end{array}$ & $\begin{array}{l}454.60^{*} \\
454.26^{*} \\
454.10^{2}\end{array}$ & $\begin{array}{l}138.56 \\
138.46 \\
138.41\end{array}$ \\
\hline 299-w22-43. & $\begin{array}{l}11 / 01 / 94 \\
12 / 15 / 94 \\
12 / 20 / 94\end{array}$ & $\begin{array}{l}236.07 \\
236.25 \\
236.41\end{array}$ & $\begin{array}{l}455.28^{*} \\
455.10^{*} \\
454.94\end{array}$ & $\begin{array}{l}138.77 \\
138.71 \\
138.67\end{array}$ \\
\hline $699-36-70 A$ & $\begin{array}{l}10 / 11 / 94 \\
10 / 16 / 94 \\
10 / 21 / 94 \\
11 / 02 / 94\end{array}$ & $\begin{array}{l}262.10 \\
260.95 \\
262.00 \\
256.00\end{array}$ & $\begin{array}{l}443.33^{\star} \\
444.58^{*} \\
443.43^{\star} \\
449.43^{*}+\end{array}$ & $\begin{array}{r}135.13 \\
135.51 \\
. \quad 135.16 \\
136.99\end{array}$ \\
\hline
\end{tabular}

NOTES: 1. Water level elevations are calculated by subtracting the measured depth-to-water from the surveyed elevation for the well.

2. Depth-to-water values are transcribed from field records.

3. ETevations marked with an ' $*$ ' were measured at the time of sampling.

4. Elevations marked with a ' + ' are outside of the expected range, and are suspected of error. 
Table 11-3. Constituent List and Summary of Results for the 216-U-12 Crib Data for Reporting Period October 1 through December 31, 1994. (sheet 1 of 3)

COMTAMFUATICN IADICATOR PARAHETERS

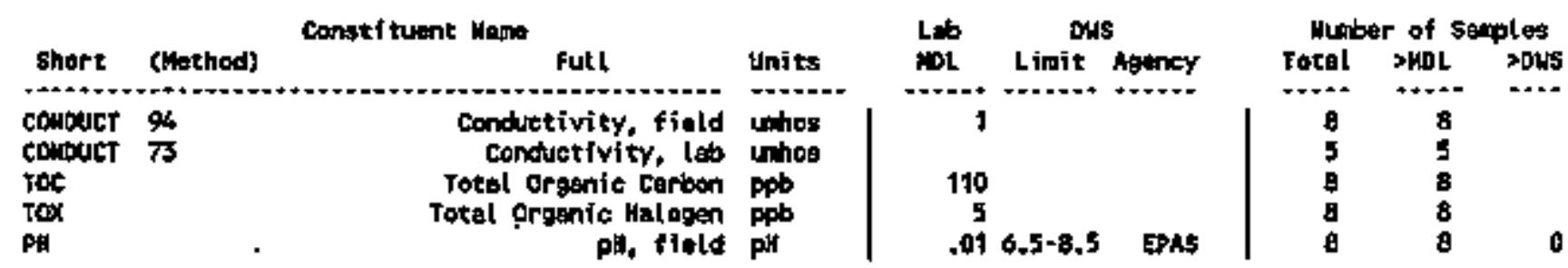

ORTOKILC WATER PARALTTERS

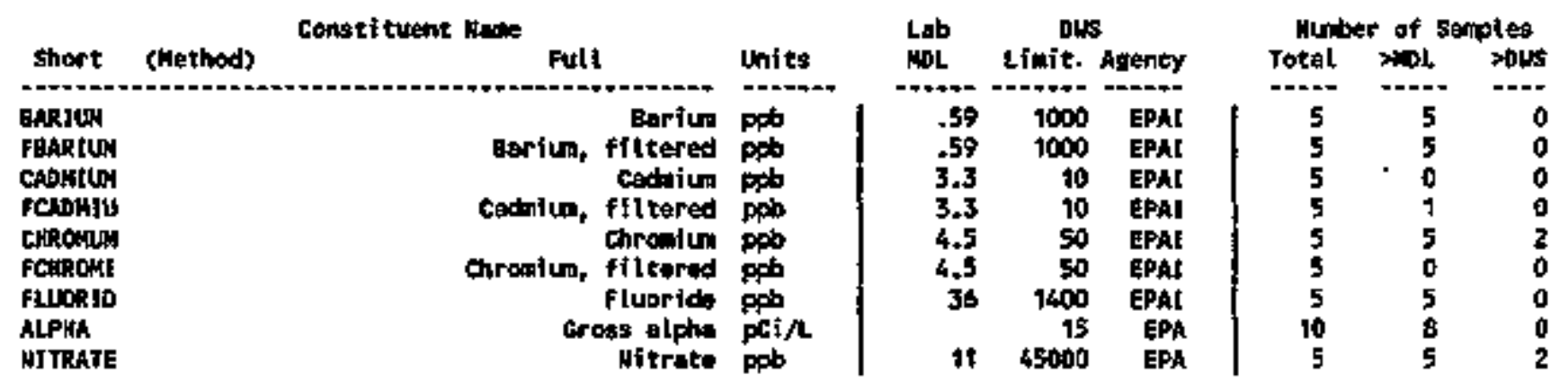

GROMDWATER QUALITY PARAMETERS

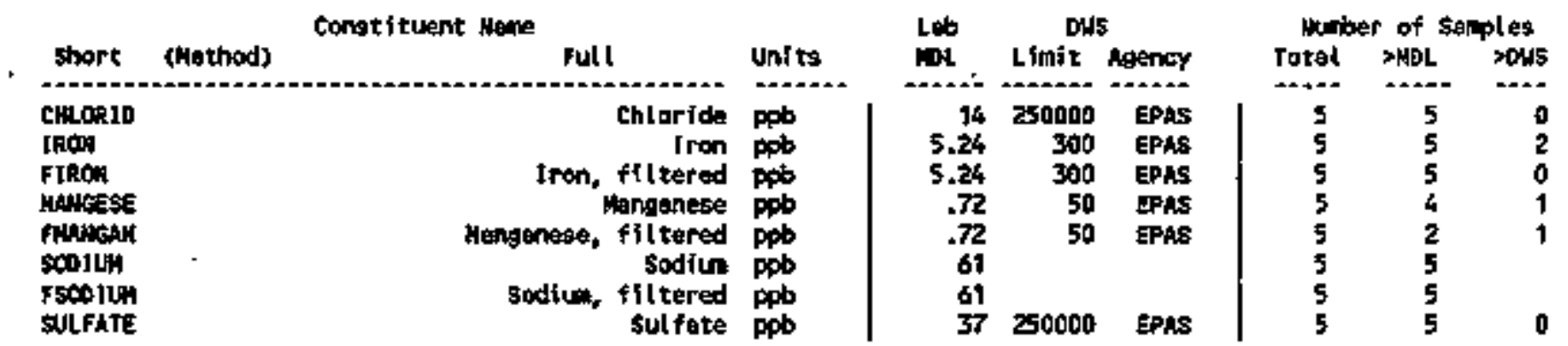


Tab]e 11-3. Constituent List and Summary of Results for the 216-(J-12 Crib Data for Reporting Period October 1 through December 31, 1994.

(sheet 2 of 3 )

stTe spectfic and othek const thueists

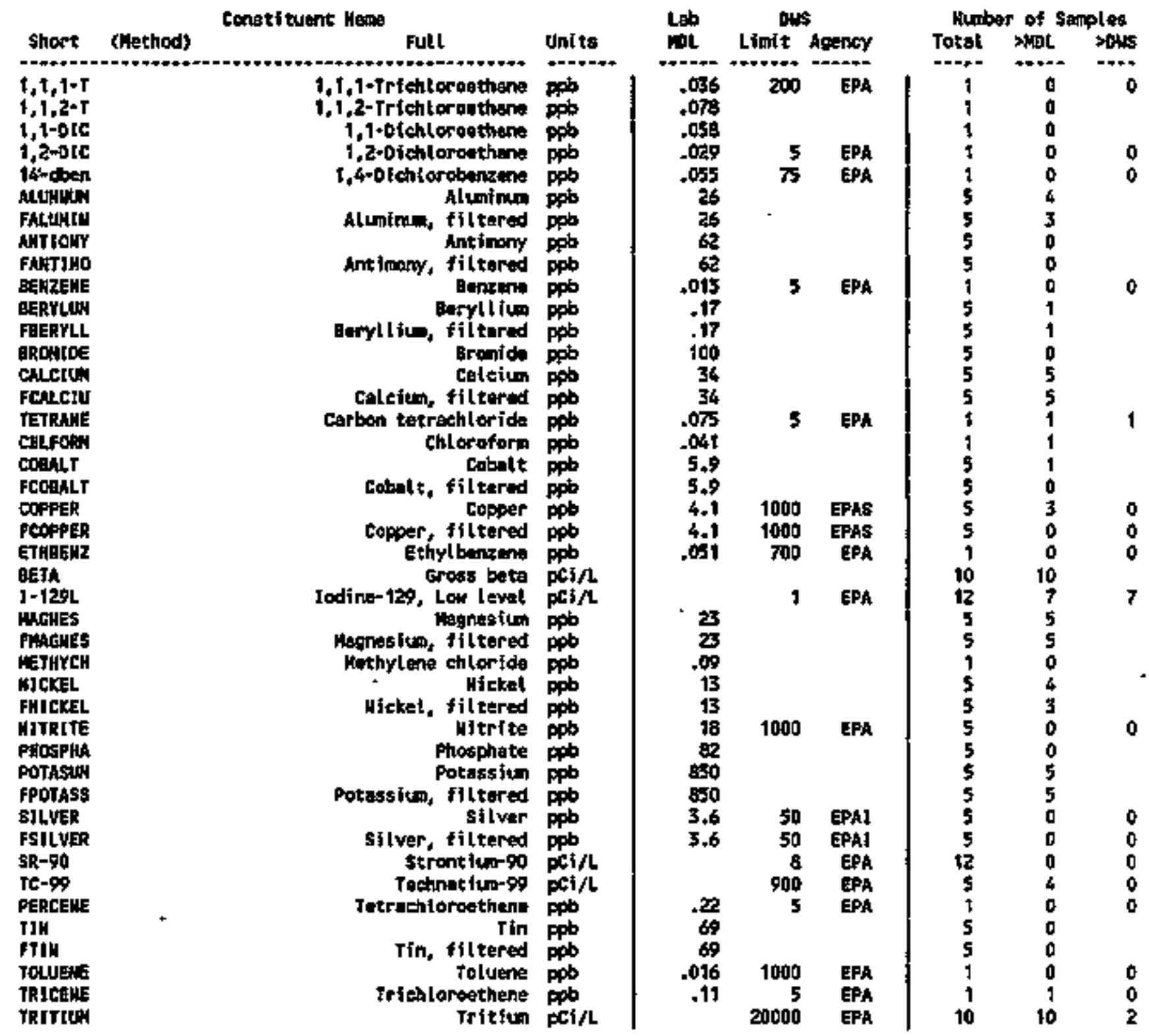


Table 11-3. Constituent List and Summary of Results for the 216-U-12 Crib Data for Reporting Period October 1 through December 31, 1994. (sheet 3 of 3 )

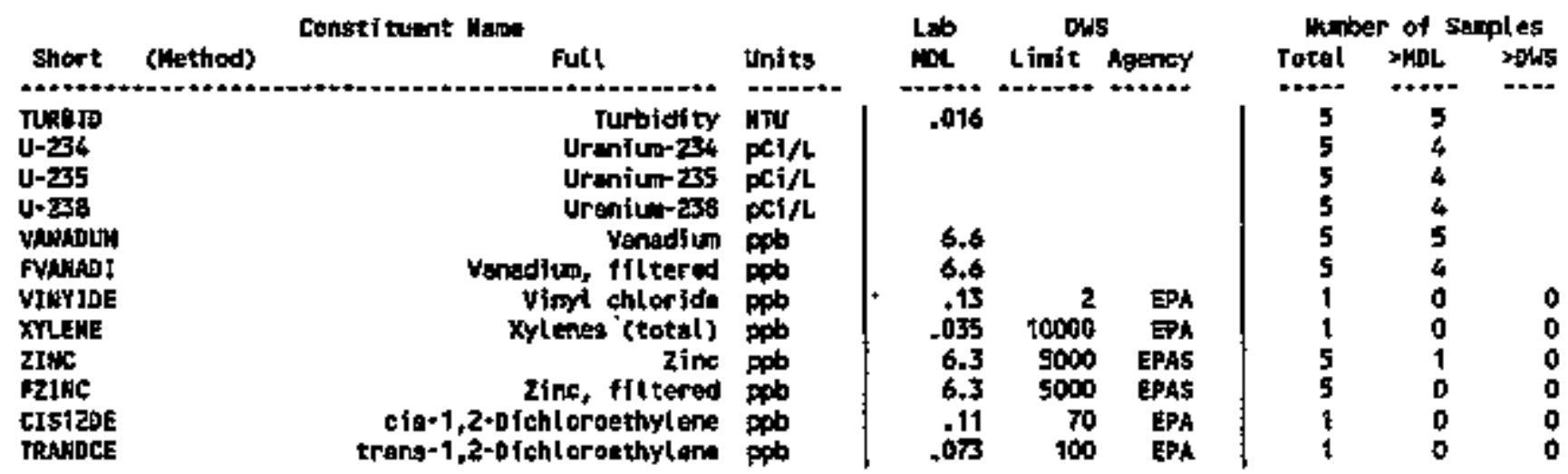

for explanatian of this table, sin setion 1.4 of report. 
Table 11-4. Constftuents with at Least One Detected. Value for the 216-U-12 Crib Data for Reporting Perjod October I through December 31, 1994. (sheet 1 of 4)

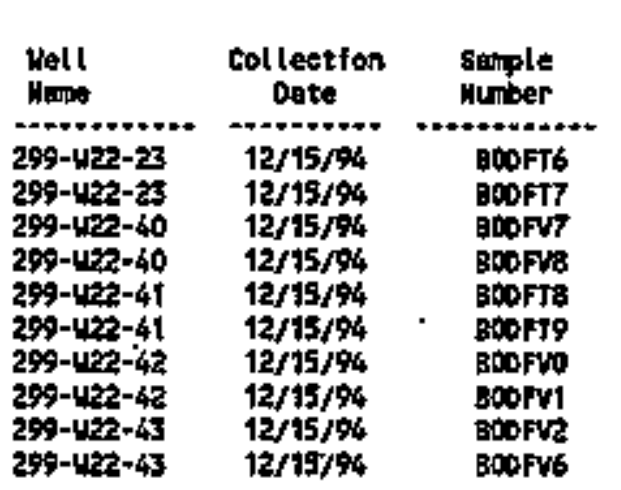

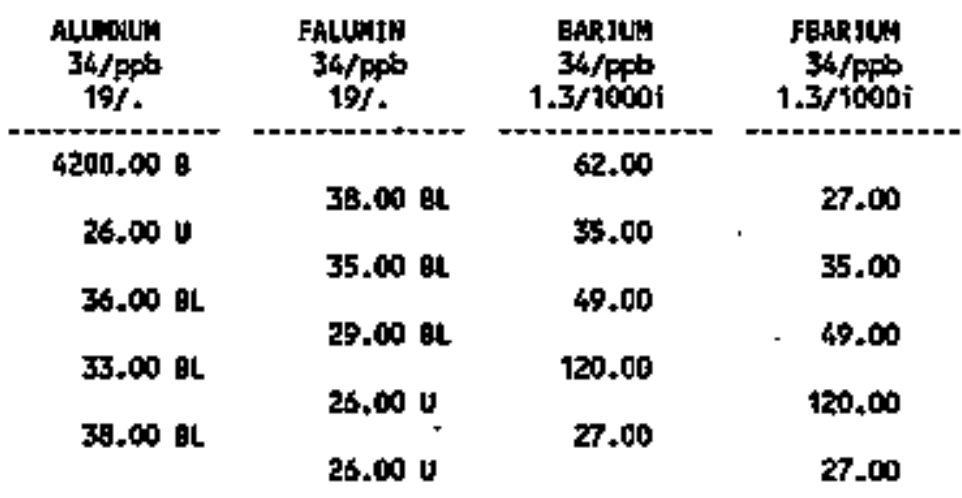

well
Hewe
$\ldots 99-1122-23$
$299-122-23$
$299-122-23$
$299-122-40$
$299-122-40$
$299-122-41$
$299-122-41$
$299-122-41$
$799-122-42$
$299-122-42$
$299-122-43$
$2922-43$

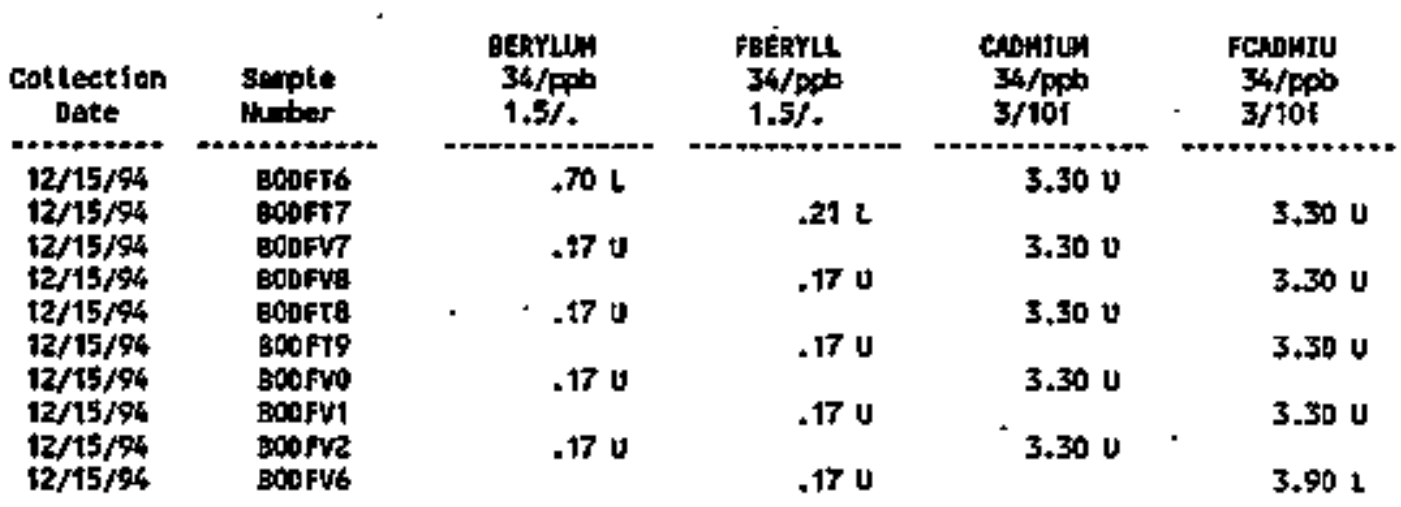

well
Hante
$299-122-23$
$299-122-23$
$299-122-40$
$290-422-40$
$299-422-40$
$299-422-41$
$299-422-41$
$299-4122-42$
$299-422-42$
$299-122-43$
$299-422-43$

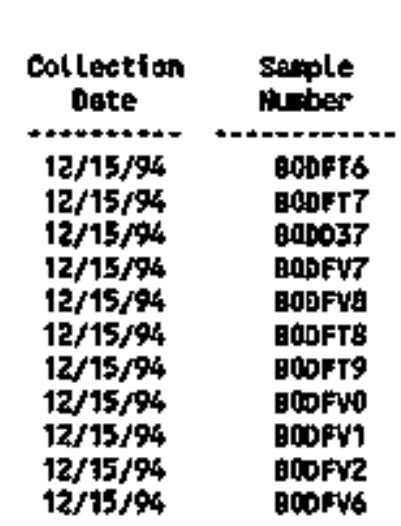

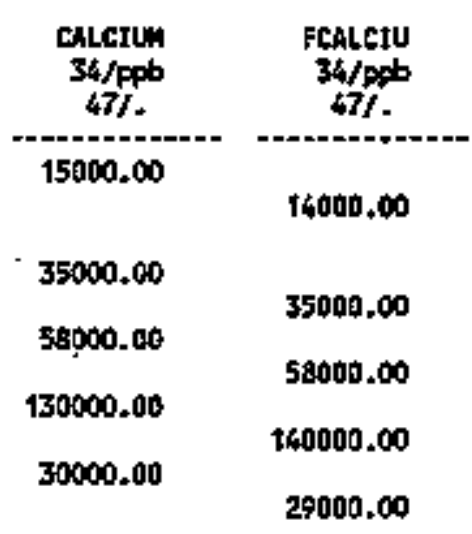

\begin{tabular}{|c|c|}
\hline 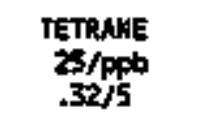 & $\begin{array}{c}\text { CALOR to } \\
\text { \$24/ppb } \\
71 / 2500005\end{array}$ \\
\hline & 17000.0000 \\
\hline \multirow[t]{3}{*}{10.00} & . 17000.0000 \\
\hline & 94000,0000 \\
\hline & \\
\hline
\end{tabular}


Table 11-4. Const tuents with at Least One Detected Value for the 216-U-12 Crib Data for Reporting Period October 1 through Decenber 31, 1994. (sheet 2 of 4)

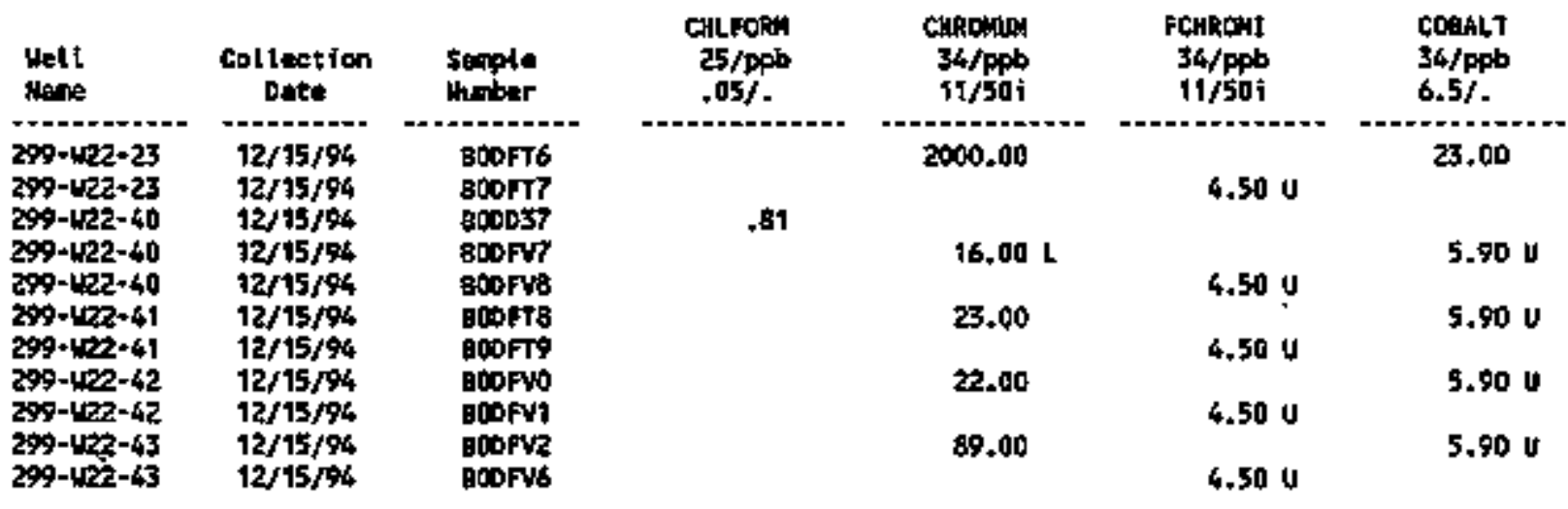

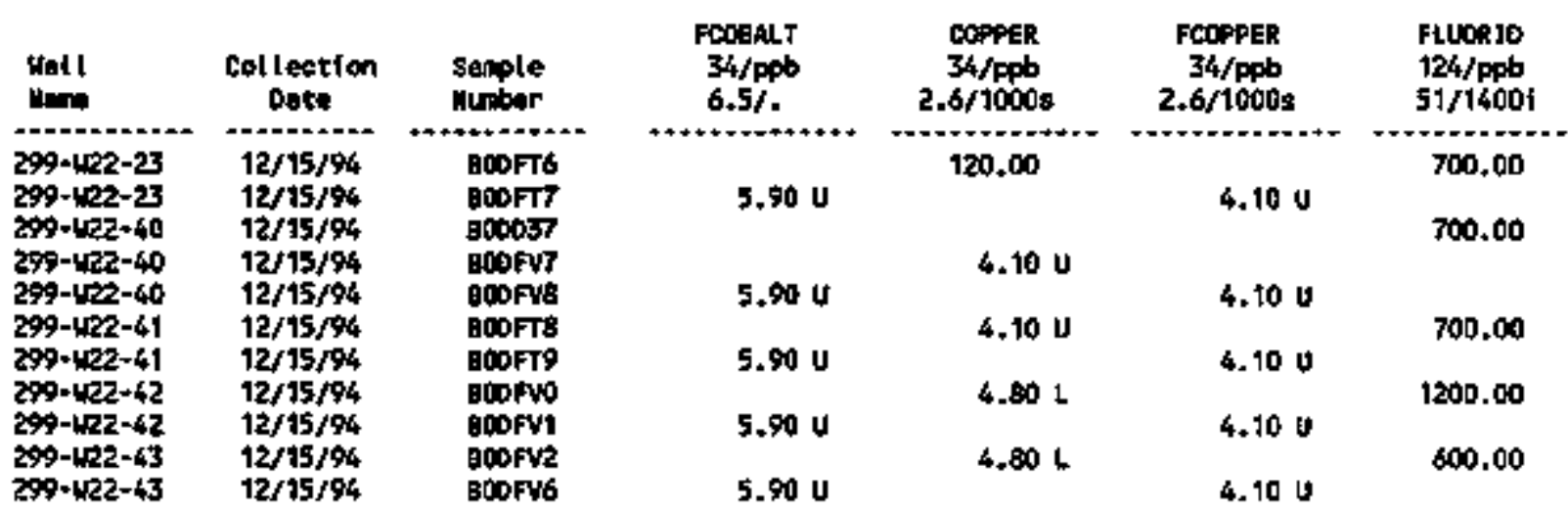

\begin{tabular}{|c|c|c|c|c|c|c|}
\hline Wod I & $\begin{array}{c}\text { Collection } \\
\text { Dat: }\end{array}$ & $\begin{array}{l}\text { Sarple } \\
\text { Munber }\end{array}$ & $\begin{array}{c}\text { AlPak } \\
135 / \mathrm{pCI} / \mathrm{L} \\
. / 15\end{array}$ & $\begin{array}{c}\text { BETA } \\
\text { t36/PC } 5 / \mathrm{L} \\
. . / .\end{array}$ & $\begin{array}{c}{[-129} \\
139 / \mathrm{pci} / \mathrm{t} \\
. / 1\end{array}$ & $\begin{array}{c}\text { trow } \\
34 / \mathrm{ppb} \\
18 / 300 \mathrm{~s}\end{array}$ \\
\hline $\begin{array}{l}299-122-22 \\
299-122-22 \\
299-422-23 \\
299-w 22-23 \\
299-422-40 \\
299-1222+40\end{array}$ & $\begin{array}{r}9 / 26 / 94 \\
9 / 26 / 94 \\
9 / 22 / 94 \\
12 / 15 / 94 \\
9 / 23 / 94 \\
12 / 15 / 94\end{array}$ & 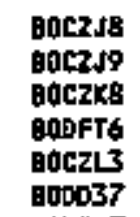 & $\begin{array}{l}+.14 \\
2.34 \\
2.310 \\
2.98\end{array}$ & $\begin{array}{r}5.18 \\
6.66 \\
7.29 \\
10.70\end{array}$ & $\begin{array}{l}1.930 \\
1.420 \\
9.37 \\
7.37 \\
.190\end{array}$ & $88000.00 \mathrm{a}$ \\
\hline $\begin{array}{l}299-w 22-40 \\
299-w 22-61 \\
299-w 22-41 \\
299-w 22-42 \\
299-w 22-42 \\
299-w 22-43 \\
299-w 22-43\end{array}$ & $\begin{array}{r}12 / 15 / 94 \\
9 / 23 / 94 \\
12 / 15 / 94 \\
9 / 25 / 94 \\
12 / 15 / 94 \\
9 / 22 / 94 \\
12 / 15 / 94\end{array}$ & 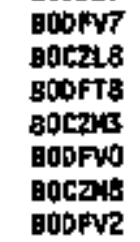 & $\begin{array}{l}.38 \text { U } \\
5.36 \\
3.30 \\
4.05 \\
2.210 \\
1.52\end{array}$ & $\begin{array}{r}21.00 \\
20.00 \\
28.80 \\
59.50 \\
7.97 \\
9.42\end{array}$ & $\begin{array}{l}-.35 u \\
.39 \\
=.12 \mathrm{U} \\
5.83 \\
8.08 \\
.11 \mathrm{u} \\
1.14 \mathrm{U}\end{array}$ & $\begin{array}{l}100.00 \mathrm{~B} \\
120.00 \mathrm{~B} \\
100.00 \mathrm{~B} \\
400.00 \mathrm{~s}\end{array}$ \\
\hline
\end{tabular}


Table 11-4. Constituents with at Least One Detected Value for the 216-U-12 Crib Dața for Reporting Period Octoier 1 through December 3!, 1994.

(sheet 3 of 4 )

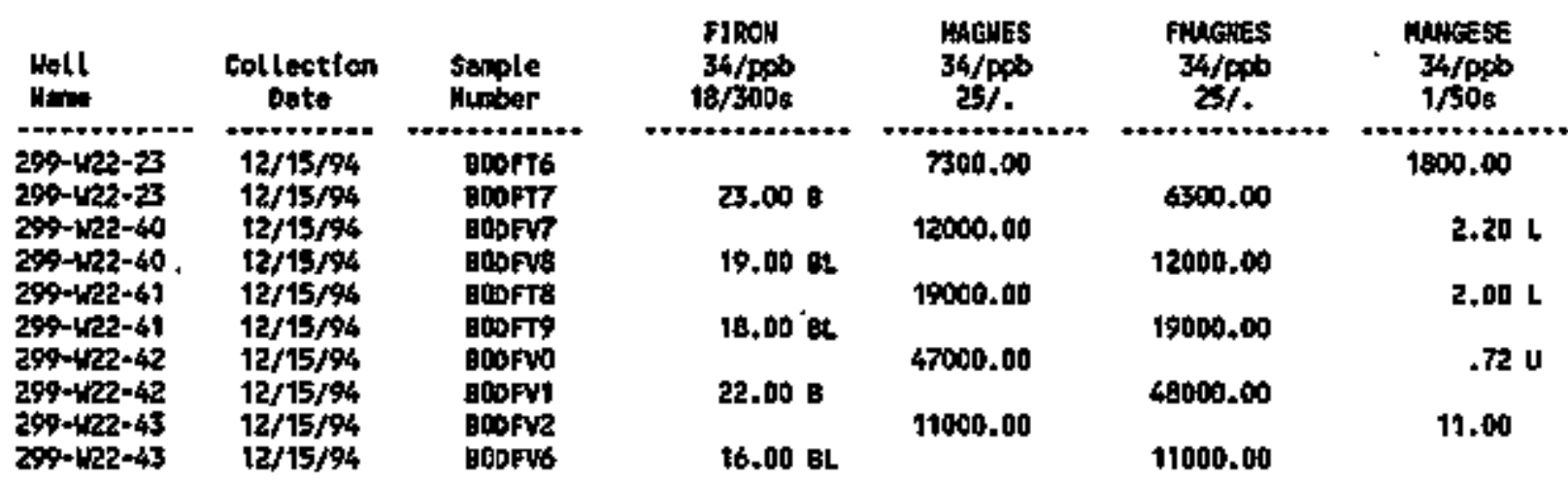

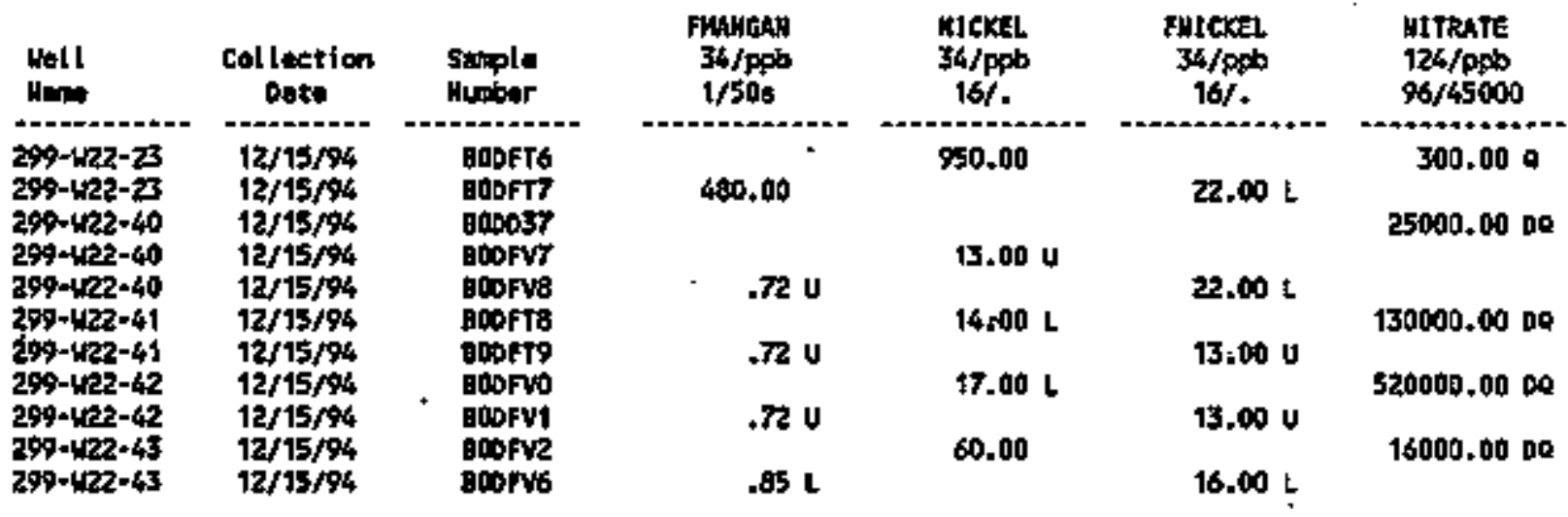

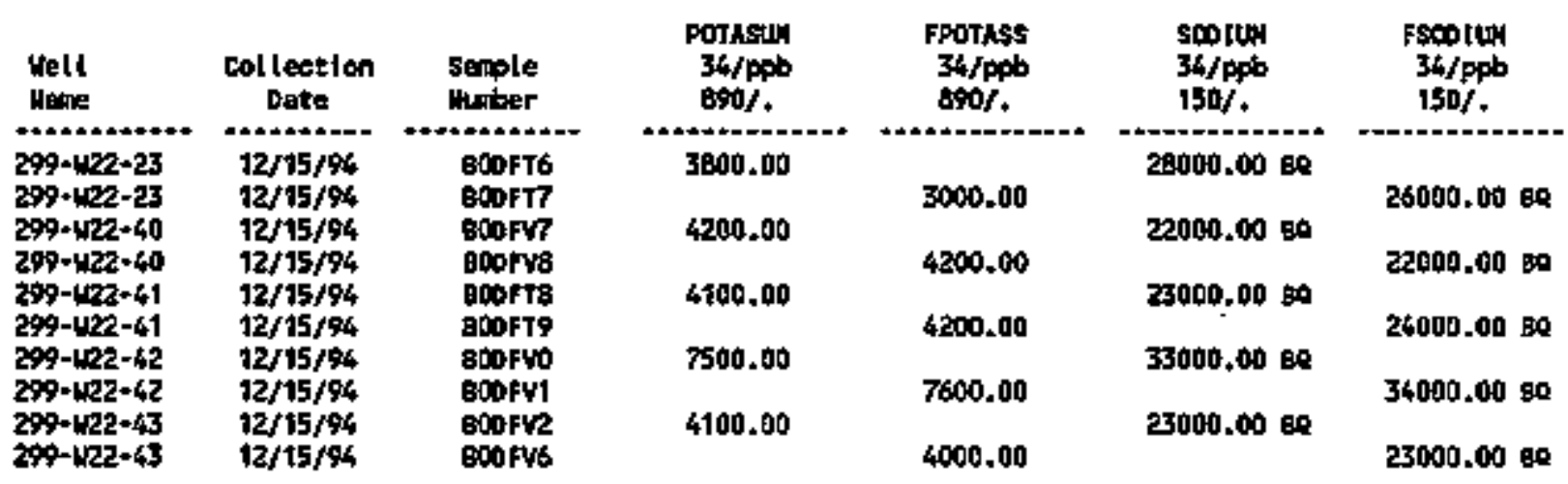


Table 11-4. Constituents with at Least One Detected Value for the 216-U-12 Crib Data for Reporting Period October 1 through Decenber 31, 1994. (sheet 4 of 4 )

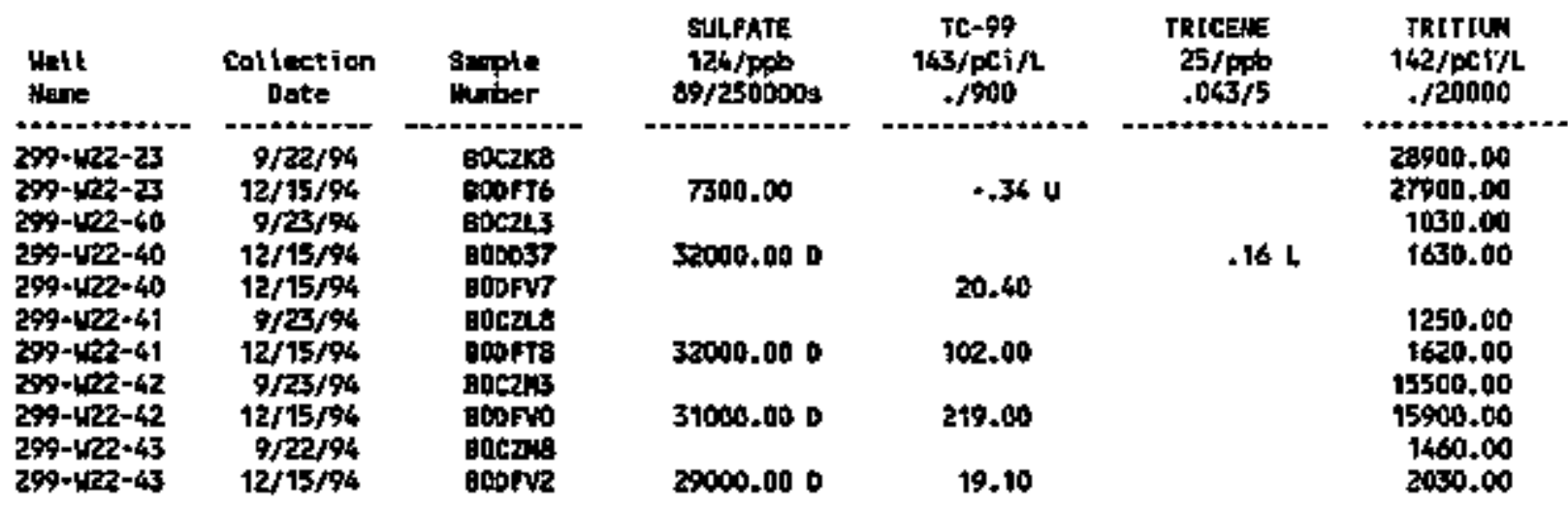

\begin{tabular}{|c|c|c|c|c|c|c|}
\hline Hell & $\begin{array}{c}\text { Col lection } \\
\text { oate }\end{array}$ & $\begin{array}{l}\text { sample } \\
\text { Huter }\end{array}$ & $\begin{array}{c}\text { TUREJd } \\
126 / \mathrm{ATU} \\
.016 / .\end{array}$ & $\begin{array}{c}1-234 \\
148 / p \times 1 / 1 \\
. /\end{array}$ & $\begin{array}{c}U \cdot 235 \\
149 / p C \mid / t \\
. /\end{array}$ & $\begin{array}{c}U \cdot 238 \\
146 / p C 1 / L \\
. /\end{array}$ \\
\hline $\begin{array}{l}299-122-23 \\
299-422+40 \\
299-122-41 \\
298-422-42 \\
294-422-43\end{array}$ & $\begin{array}{l}12 / 15 / 94 \\
12 / 15 / 94 \\
12 / 15 / 94 \\
12 / 15 / 94 \\
12 / 15 / 94\end{array}$ & 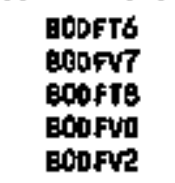 & $\begin{array}{r}290.009 \\
1.109 \\
1.200 \\
1.100 \\
1.200\end{array}$ & $\begin{array}{l}.31 \\
1.98 \\
. .02 \\
2.13 \\
1.10\end{array}$ & $\begin{array}{c}.08 \\
.07 \\
. .01 \mathrm{u} \\
.05 \\
.04\end{array}$ & $\begin{array}{c}.13 \\
1.46 \\
-.0014 \\
1.54 \\
.85\end{array}$ \\
\hline
\end{tabular}

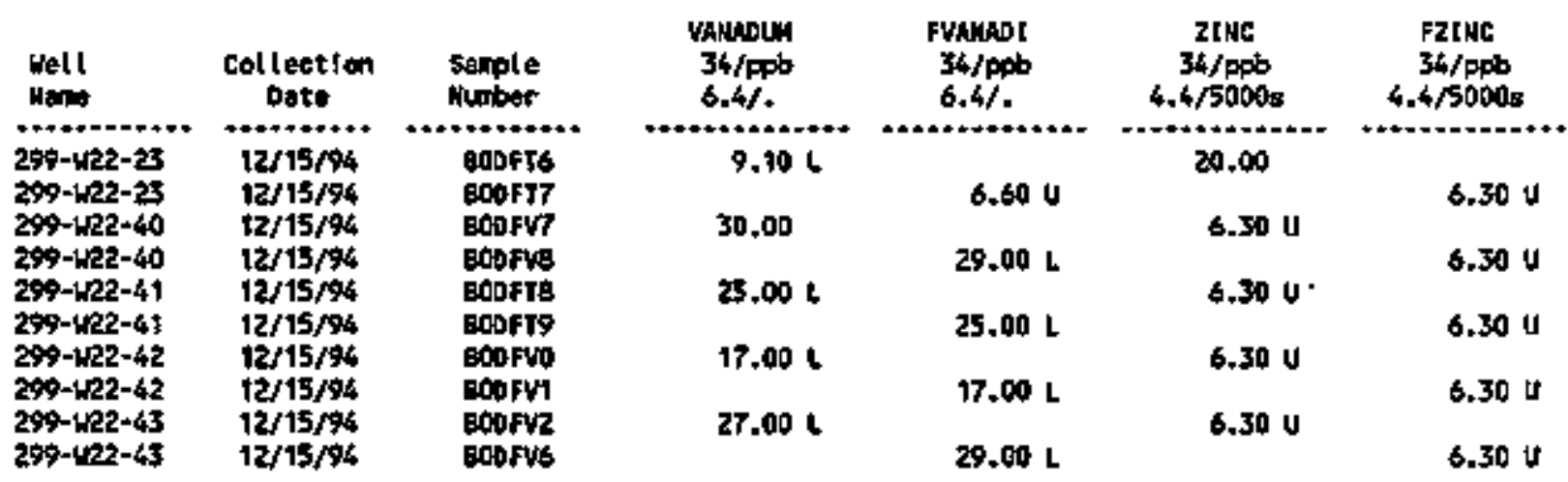

For atplenstion of this table, set Section 1.4 of report. 
Table 11-5. Contamination Indicator Parameters for the 216-U-12 Crib Data for Reporting Perjod october 1 through December 31, 1994. (sheet 1 of 1 )

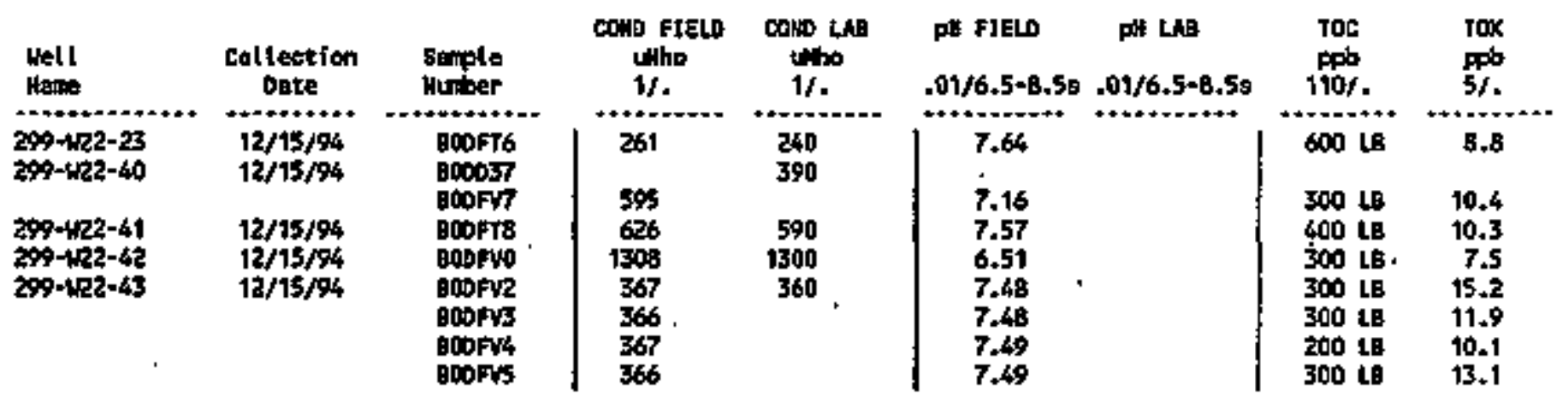

For explonotion of this table, sec section 1.4 of report. 
DOE/RL-94-36-4

This page intentfonally left blank. 


\section{CONTENTS}

12.0 LIQUID EFFLUENT RETENTIOA FACILITY $\ldots \ldots \ldots \ldots \ldots \ldots$ 12.1 INTROOUCTION

12.2 WATER LEVEL MEASUREMEATS

12.3 WATER CHEMISTRY DATA 
DOE/RL-94-36-4

LIST OF FIGURES

12-1 Well Location Map for the Liquid Effluent Retention Facility . . . 2

\section{LIST OF TABLES}

12-1 Honitoring Welt Purpose and Samping Schedule for the Liquid Effluent Retention Facility Metwork ...... 3

12-2 RCRA Water Level Measurement Report LERF, fourth Quarter 1994: . . 4 


\title{
12.0 LIQUID EFFLUENT RETENTION FACILITY
}

\author{
H. 0 . Sweeney \\ Westinghouse Hanford Company
}

\subsection{INTRODUCTION}

The Liquid Effluent Retention Facility (LERF) was constructed to provide Interim storage of 242-A Evaporator process condensate effluent suspected of containing listed and dangerous (ammonia) waste constituents. Construction of the LERF began in May 1990. The LERF, classified as a surface impoundment for mixed waste storage, will be permftted as a RCRA TSD facility. The evaporator was shut down in 1989 and restarted on Apri1 14, 1994. The LERF basins currently serve as temporary storage for evaporator process condensate unti 1 the Effluent Treatment Facility is operational.

The factlity is classified as a surface impoundment and was originally planned to have four basins arranged side by side. Four excavations were made; only three of the four basins are lined and are currently scheduled for use. The basins are located immedjately east of the northeast corner of the 200 East Area. The liquid effluent may contain spent nonhalogenated solvents (F003 and F005, WAC 173-303-9004, "Dangerous Waste Source List"), namely acetone, methyl ethyl ketone, and methyl isobutyl ketone. The ammonia content in the liquid effluent may aiso justify handling the offiuent as a dangerous waste.

An interim-status, detection-1evel groundwater monitaring network was installed around the LERF in 1990 in accordance with the Interim Status Groundwater Monitoring P7an for the 200 East Area Liquid Effluent Retention Facilfty (UHC 1991). Four quarters of background groundwater sampling and analys is were completed. Three downgradient wells and one upgradient welt constitute the monitoring network. Figure 12-1 shows the well locations. Table 12-1 provides the last sampling date and frequency and identifies the upgradient and downgradient groundwater monitoring wells. Table 12-2 shows current water table elevations for three of the four monitoring wells.

\subsection{KATER LEVEL MEASUREMETTS}

Water levels are measured quarterly and when groundwater samples are collected at each of the four wells in the network. Water level measurements obtained during the quarter are presented in Table 12-2. We11 299-E26-11 was inadvertantty skipped during the fourth quarter. Water level measurements for this well will be taken during the next sheduled sampling event. The water level in well 299-E26-9 has been below the level where conventiona] equipment could operate properly. However, recently, a slight rise in water level has been suficient to allow for conventional sampling.

\subsection{WATER CHEHISTRY DATA}

Wells in the LERF groundwater monitoring network are sampled semiannually. Samples were not taken from the wells during this quarter. 


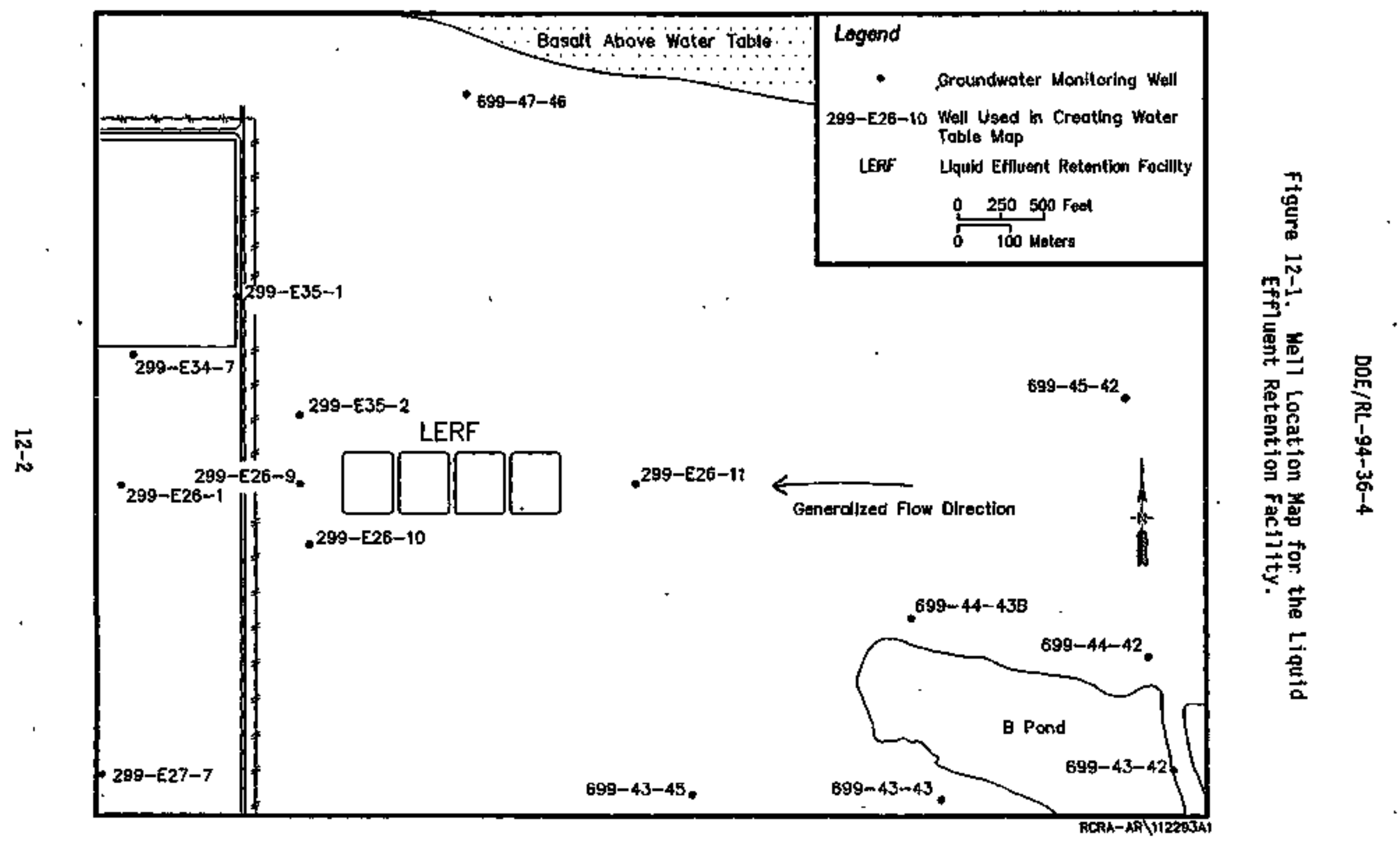


Table 12-1. Monitoring WeTl Purpose and Sampling Schedule for the liquid Effluent Retention Facility Network.

\begin{tabular}{|c|l|l|c|c|}
\hline $\begin{array}{c}\text { Well no. } \\
(299-)\end{array}$ & $\begin{array}{c}\text { Relative } \\
\text { position }\end{array}$ & Hyórogeologic untt & $\begin{array}{c}\text { Sample } \\
\text { frequency }\end{array}$ & $\begin{array}{c}\text { Sample date } \\
\text { 4th Qtr 1994 }\end{array}$ \\
\hline E26-9 & Downgradient & Hanford: Water Table & Semi annually & Not Sampled \\
\hline E26-10 & Downgradient & Hanford: Water Table & Semi annually & Not Sampled \\
\hline E26-11 & Upgradient & Hanford: Water Table & Semiannually & Not Sampled \\
\hline E35-2 & Downgradient & Hanford: Water Table & Sewl annually & Not Sampled \\
\hline
\end{tabular}


Table 12-2. RCRA Water Level Measurement Report LERF, Fourth Quarter 1994.

\begin{tabular}{lllll}
\hline Wel1 & Date & $\begin{array}{c}\text { Depth to } \\
\text { water (ft) }\end{array}$ & $\begin{array}{c}\text { Water level } \\
\text { elevation above ms } \\
(\mathrm{ft})\end{array}$ & (m) \\
\hline $299-E 26-10$ & $12 / 14 / 94$ & 199.51 & 401.96 & 122.52 \\
$299-E 26-9$ & $12 / 14 / 94$ & 200.91 & 401.98 & 122.52 \\
$299-E 35-2$ & $12 / 14 / 94$ & 200.11 & 402.25 & 122.61 \\
\hline
\end{tabular}

"NOTES: 1. Water level elevations are calculated by subtracting the measured depth-to-water from the surveyed elevation for the weil.

2. Depth-to-water values are transcrtbed from field records.

3. Elevations marked $w f$ th an $^{t *}$ ' were measured at the time of sampling.

4. Elevation marked with $a$ ' + ' are outside of the expected range, and are supected of error. 
DOE/RL-94-36-4

CONTENTS

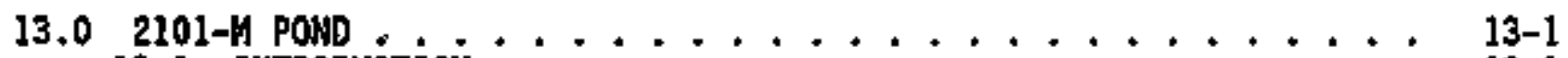

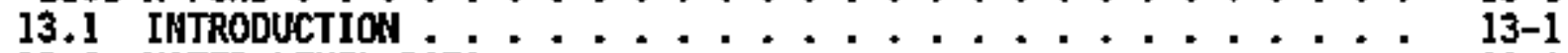
13.2 UATER LEVEL DATA . . . . . . ......... 13-1 13.3 MATER CHEMISTRY DATA ...................... 13-1 
DOE/RL-94-36-4

LIST OF FIGURES

13-1 Well Location Map for the 2101-W Pond ........... 13-3

\section{LIST OF TABLES}

13-1 Monitoring We1l Purpose and Sampling Schedule for the 2101-M Pond ................ 13-4

13-2 RCRA Water Level Measurement Report 2101-M Pond - Fourth Quarter 1994 .......... . 13-5

13-3 Constituent List and Summary of Resuits for the 2101-M Pond Data for Report ing Period October 1 through Becember 31,1994 . . 13-6

13-4 Constituents with at Least One Detected Value for the 2101-H Pond Data for Reporting Perlod October I through December 31, 1994 .. 13-9

13-5 Contamination Ind feator Parameters for the 2101-H Pond

Data for Reporting Pertod October 1 through December 31, 1994 . 13-12 


\title{
13.0 2101-H PONB
}

\author{
E. C. Thornton \\ Westinghouse Hanford Company
}

\subsection{IHTRODUCTIOH}

The 2101-M Pond is a U-shaped, unlined trench located in the 200 East Area. It has received wastewater from the 2101-M Building heating and air conditionting system since 1953. In 1981, Basalt Waste Isolation Project laboratories were plumbed into the discharge line from the 2101-M Building to the 2101-M Pond. From 1981 until mid-1985, these laboratories may have discharged dangerous waste into the 2101-H Pond. The most important chemicals used in the 2101-M Jaboratories were barium chloride and hydrochloric and nitric acids; they are assumed to have been disposed of in laboratory drajus connected to the 2101-M Pond.

The groundwater monitoring network for the 2101-M Pond consists of one upgradient (299-E18-1) and three domgradient (299-E18-2, 299-E18-3, and 299-E18-4) wells installed in the uppermost portion of the unconfined aquifer (Figure 13-1). HeÎl 299-E18-1 is ai so used as a background monjtoring well for the 216-B-3 Pond Systen.

Groundwater sampling began in August. 1988 for the 2101-M Pond and was conducted quarterly until August 1989. to estabt ish background values. Semiannual indicator parameter evaluation gonitoring was initiated in Hovember 1989 under RCRA interim-status regutations and is ongoing (Chamness et al. 1989). Table 13-1 provides information on the relative position of each well in the network, the hydrogeologic unit monitored, the sampling frequency, and the latest sampiing date.

The 2101-M Pond will be clean-closed by the end of the year. It is antfcipated that no further groundwater monitoring activities will be conducted at this location after clean-closure is certified, except for sampling of well 299-E18-1 in support of the 216-B-3 Pond System.

\subsection{MATER LEVEL DATA}

Water level measurements were performed in the October through December 1994 period for alj four wells. The data are presented in Table 13-2. These measurements include routine monthly measurements plas measurements performed at the time of sampling. Water levels have been corrected for vertical deviations for each welj based on inclinometer measurements.

\subsection{WATER CHEYISTRY DATA}

Semiannual groundwater sampling was conducted for the fourth quarter of 1994 on December 1, 1994, for the monitoring wells at 2101-M Pond. A11 available water chemistry data for this quarter are reported in Tables 13-3 
through 13-5. Data are flagged in Tables 13-4 and 13-5 according to the explanation provided fn Sections 1.2 and 1.4 .

The replicate averages for the contamination indicator parameters spectfic conductance, $\mathrm{pH}$, TOC, and TOX did not exceed the critical means for the 2101-M Pond. Weil 299-E18-4 was also sampled on October 19, 1994, for verification of a TOX exceedance associated with sampling conducted in December 1993. Laboratory results associated with the verffication samples were below the critical mean, indicating that the previous exceedance probabiy does not reflect an impact of 2101-H Pond on groundwater quality. As in previous quarters, the specific conductance values for upgradient well 299-E18-1 were elevated relative to the three downgradient wells. This appears to result frow the dilution of groundwater in the viclnity of the downgradient wells in response to the drainage of water from the $2101-M$ Pond (Thornton 1995).

Unfiltered chromlum concentrations for well 299-E18-4 and unf11tered iron concentrations for 299-E18-1 and 299-E18-4 were above the OWSs, but unfi]tered values for these metals were much lower and below the OWSs. Turbidity values slightly higher than 3 NTUs were also reported for these wells. This suggests that the higher metal concentrations associated with the unfiltered samples are related to the presence of solid debris incorporated into the samples (e.g., sediment particles or corrosion products). 
Figure 13-1. Hell Location Map for the 2101-M Pond.

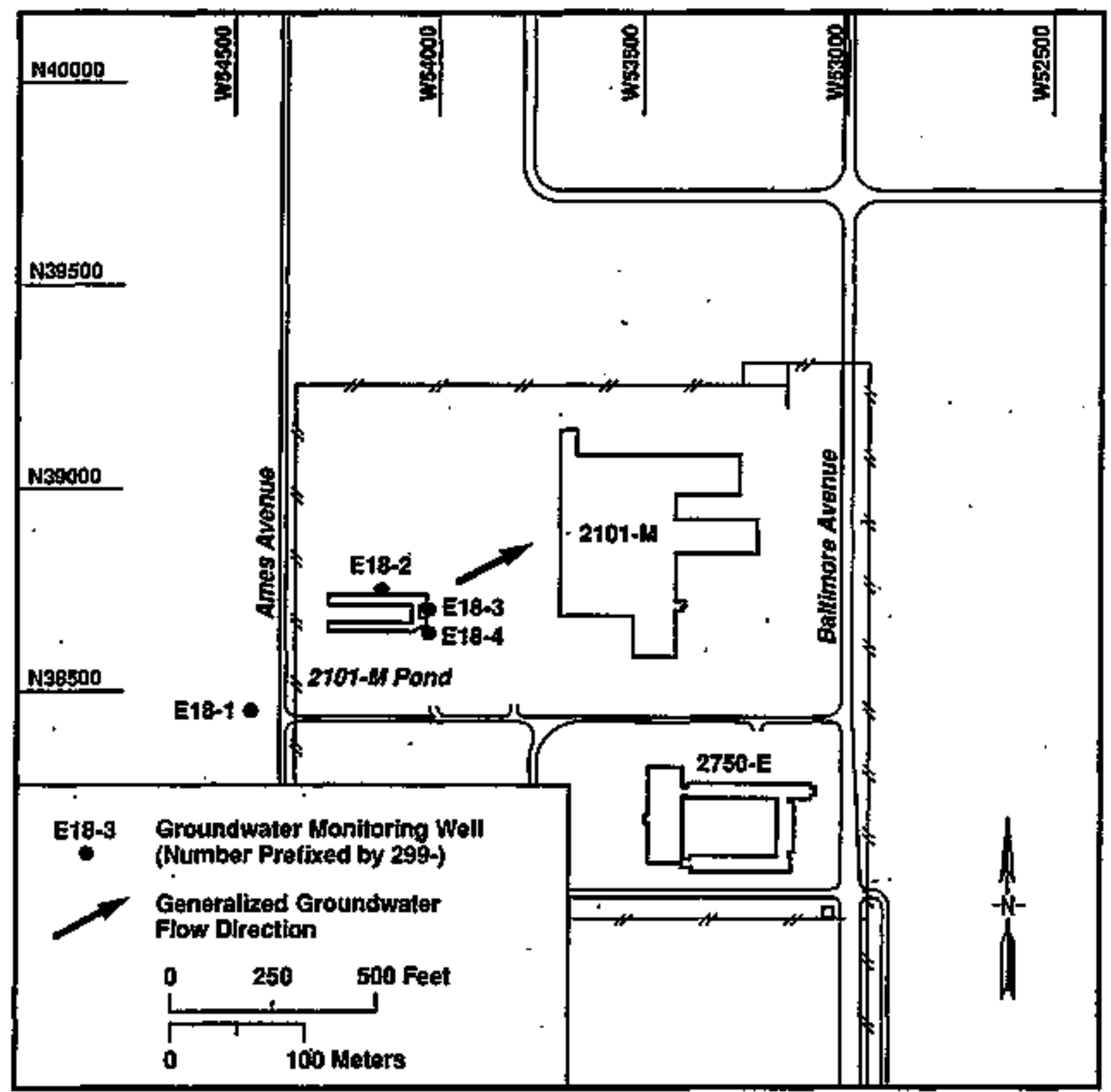


DOE/RL-94-36-4

Table 13-1. Monitoring Yell Purpose and Sampling Schedule for the 2101-H Pond.

\begin{tabular}{|c|c|l|c|c|}
\hline $\begin{array}{c}\text { Well no. } \\
(299-)\end{array}$ & $\begin{array}{c}\text { Relative } \\
\text { position }\end{array}$ & Hydrogeologic unit & $\begin{array}{c}\text { Sample } \\
\text { frequency }^{\circ}\end{array}$ & $\begin{array}{c}\text { Sample date } \\
\text { 4th Qto 1994 }\end{array}$ \\
\hline E18-1 & Upgradient & $\begin{array}{l}\text { Hanford/Ringold: } \\
\text { Water Table }\end{array}$ & Quarterly $^{\mathrm{b}}$ & $12 / 01 / 94$ \\
\hline E18-2 & Downgradient & $\begin{array}{l}\text { Hanford/Ringold: } \\
\text { Water Table }\end{array}$ & Semiannually & $12 / 01 / 94$ \\
\hline E18-3 & Downgradient & $\begin{array}{l}\text { Hanford/Ringold: } \\
\text { Water Table }\end{array}$ & Semiannually & $12 / 01 / 94$ \\
\hline El8-4 & Downgradient & $\begin{array}{l}\text { Hanford/Ringold: } \\
\text { Water Table }\end{array}$ & Semiannually & $12 / 01 / 94$ \\
\hline
\end{tabular}

"The hydrogeologic unit monitored at this site is a muddy, sandy gravel to a slightly muddy, gravelly sand over the screened interval from approximately 92 to $101 \mathrm{~m}$ ( 302 to $331 \mathrm{ft}$ ) below top of casing. This interval is interpreted to be near the base of the Hanford formation and the top of the Ringold Formation.

We 11 299-E18-1 is sampled on a quarterly basis because it is also designated as an upgradient we 17 for the 216-8-3 Pond System.

$13-4$ 
Table 13-2. RCRA Nater Level Measurement Report 2101-M Pond - Fourth Quarter 1994.

\begin{tabular}{|c|c|c|c|c|c|}
\hline \multicolumn{2}{|l|}{ Ne11 } & Date & $\begin{array}{l}\text { Depth to } \\
\text { water (ft) }\end{array}$ & \multicolumn{2}{|c|}{$\begin{array}{l}\text { Water leve? } \\
\text { elevation above ms\} } \\
\text { (ft) }\end{array}$} \\
\hline 299-E18-1 & & $\begin{array}{l}10 / 27 / 94 \\
11 / 28 / 94 \\
12 / 01 / 94 \\
12 / 15 / 94\end{array}$ & $\begin{array}{l}318.89 \\
318.85 \\
318.99 \\
318.67\end{array}$ & $\begin{array}{l}.401 .41 \\
401.45 \\
401.31 * \\
401.63\end{array}$ & $\begin{array}{l}122.35 \\
122.36 \\
122.32 \\
122.42\end{array}$ \\
\hline 299-E18-2 & & $\begin{array}{l}10 / 27 / 94 \\
11 / 28 / 94 \\
12 / 01 / 94 \\
12 / 15 / 94\end{array}$ & $\begin{array}{l}319.96 \\
320.07 \\
320.06 \\
319.94\end{array}$ & $\begin{array}{l}401.47 \\
401.36 \\
401.37^{\star} \\
401.49\end{array}$ & $\begin{array}{l}122.37 \\
122.33 \\
122.34 \\
122.37\end{array}$ \\
\hline 299-E18-3 & & $\begin{array}{l}10 / 27 / 94 \\
11 / 28 / 94 \\
12 / 01 / 94 \\
12 / 15 / 94\end{array}$ & $\begin{array}{l}320.59 \\
320.56 \\
320.81 \\
320.54\end{array}$ & $\begin{array}{l}401.48 \\
401.51 \\
401.26^{*} \\
401.53\end{array}$ & $\begin{array}{l}122.37 \\
122.38 \\
122.30 \\
122.39\end{array}$ \\
\hline 299-E18-4 & & $\begin{array}{l}10 / 19 / 94 \\
10 / 27 / 94 \\
11 / 28 / 94 \\
12 / 01 / 94 \\
12 / 15 / 94\end{array}$ & $\begin{array}{l}319.65 \\
320.09 \\
320.03 \\
320.00 \\
320.04\end{array}$ & $\begin{array}{l}401.92^{\star} \\
401.48 \\
401.54 \\
401.57^{\star} \\
401.53\end{array}$ & $\begin{array}{l}122.51 \\
122.37 \\
122.39 \\
122.40 \\
122.39\end{array}$ \\
\hline WOTES: 1. & \multicolumn{5}{|c|}{$\begin{array}{l}\text { 1. Water level levations are calculated by subtracting the } \\
\text { weasured depth-to-water from the surveyed elevation for } \\
\text { the wej1. Vertical deviation correction factors based on } \\
\text { inclinometer measurements are: }+0.06 \mathrm{ft} \text { for } 299-E 18-1 \text {, } \\
+0.22 \mathrm{ft} \text { for } 299-E 18-2 \text {, }+0.03 \mathrm{ft} \text { for } 299-E 18-3 \text {, and }+0.0 \mathrm{ft} \\
\text { for } 299-E 18-4 \text { (Chamness et al. 1990). } \\
\text { 2. Depth-to-water values are transcrtbed from field records. } \\
\text { 3. Elevations marked with an ' } t \text { ' were measured at the time of } \\
\text { sampling. } \\
\text { 4. Elevation marked with a ' }+ \text { ' are outside of the expected } \\
\text { range, and are suspected of error. }\end{array}$} \\
\hline
\end{tabular}


Table 13-3. Constituent List and Summary of Results for the 2101-M Pond Data for Reporting Period October I through December 31, 1994.

(sheet 1 of 3)

CONTNLWTION IROICATOR PARAMETERS

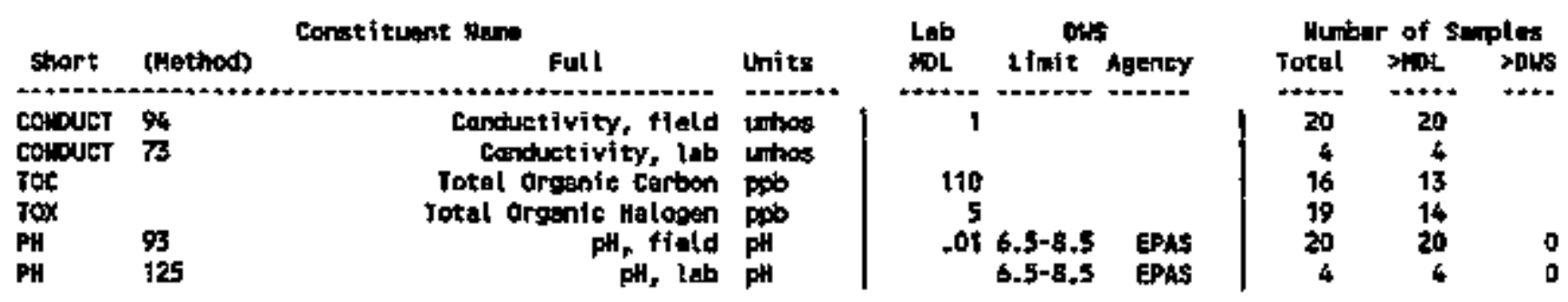

ORJMKTHG WATER PAMvicteRs

\begin{tabular}{|c|c|c|c|c|c|c|c|c|c|c|}
\hline & & Constituint Merse & & & طbا & DL: & & Her: & of of $s$ & ples \\
\hline shart & (Hethod) & Ful & & Units & WDt & Linit & Agancy & Totat & YHDL & SDUS \\
\hline & & & 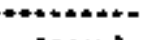 & & 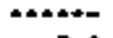 & 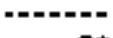 & $=-$ & $+-\infty$ & $=-\pi$ & $=-0$ \\
\hline ARFEMIC & & & Arsenic & pps & 2.8 & 50 & EPh & 4 & 3 & 0 \\
\hline FARSENTC & & Artente, & flltered & Ppb & 2.8 & 50 & EPA & 4 & 3 & 0 \\
\hline BAR [U, & & & Beriva & bp & .59 & 1000 & EPAI & 4 & 4 & 0 \\
\hline FoAktUi &. & Barfun, & isltered & ppo & 59 & 1000 & EPAI & 4 & 4 & 0 \\
\hline CHONIUI & & & tactudu & ppos & 3.3 & 10 & ЕРАI & 4 & 0 & 0 \\
\hline FCADilU & & Codiniur, & tiltered & $D D$ & 3.3 & 10 & EPAI & 4 & 9 & $?$ \\
\hline CHECNM & & & Chromiun & pob & 4.5 & 50 & EPAI & 4 & 4 & 1 \\
\hline FChotint & & Dhroolun, & filtered & B & 4.5 & 50 & EPAI & 4 & 2 & 0 \\
\hline $\begin{array}{l}\text { Fluarto } \\
\text { ALPHs }\end{array}$ & & or & $\begin{array}{l}\text { Fltroride } \\
\text { oss alphe }\end{array}$ & $p_{p+i / L}$ & 36 & $\begin{array}{r}1400 \\
15\end{array}$ & $\begin{array}{r}\text { EPA! } \\
\text { EPA }\end{array}$ & 4 & $\begin{array}{l}4 \\
3\end{array}$ & $\begin{array}{l}0 \\
0\end{array}$ \\
\hline AITRATE & & & Ai trate & pob & 11 & 45000 & EPA & 4 & 4 & 0 \\
\hline SELENM & & & Solentun & $m$ & .65 & 10 & EPAt & 4 & 1 & ? \\
\hline FSELEAI & & Sateriun, & tiltered & $p p \phi$ & .65 & 10 & EPh] & 4 & 1 & a \\
\hline
\end{tabular}

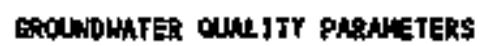

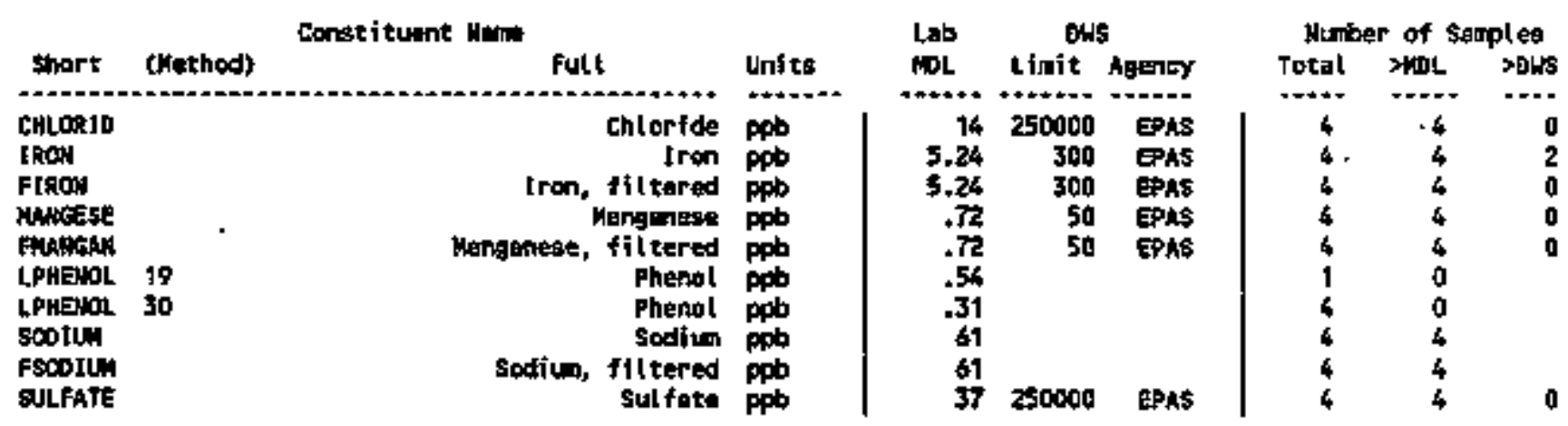


Table 13-3. Constituent List and Surmary of Results for the 2101-M Pond Data for Reporting Period October 1 through December 31, I9g4.

(sheet 2 of 3 )

SITE SPACIFJC AND OTHER CONSTJTIENTS

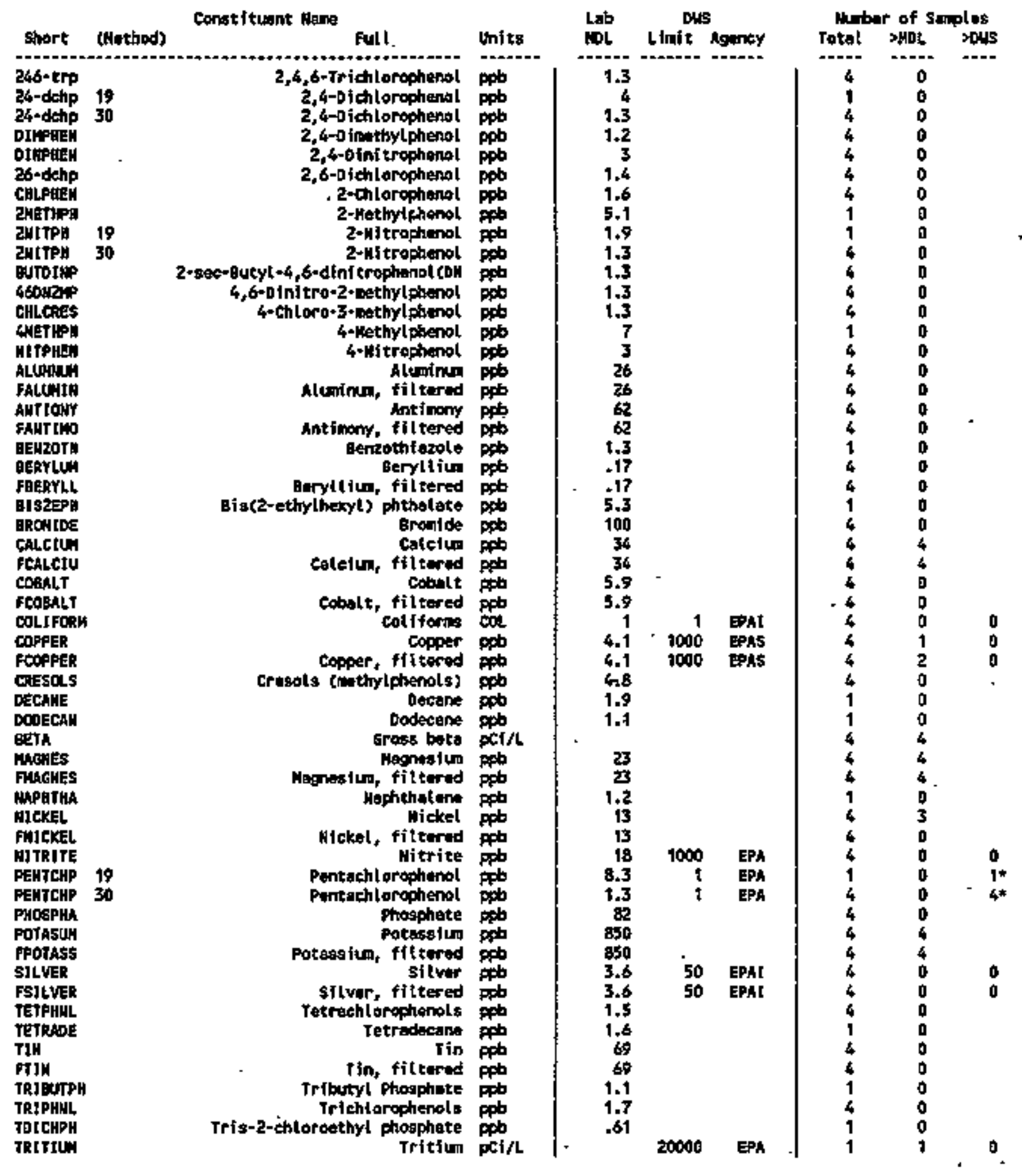


TabTe 13-3. Constituent List and Summary of Results for the 2101-M Pond Data for Reporting Period October 1 through Decenber 31, 1994. (sheet 3 of 3)

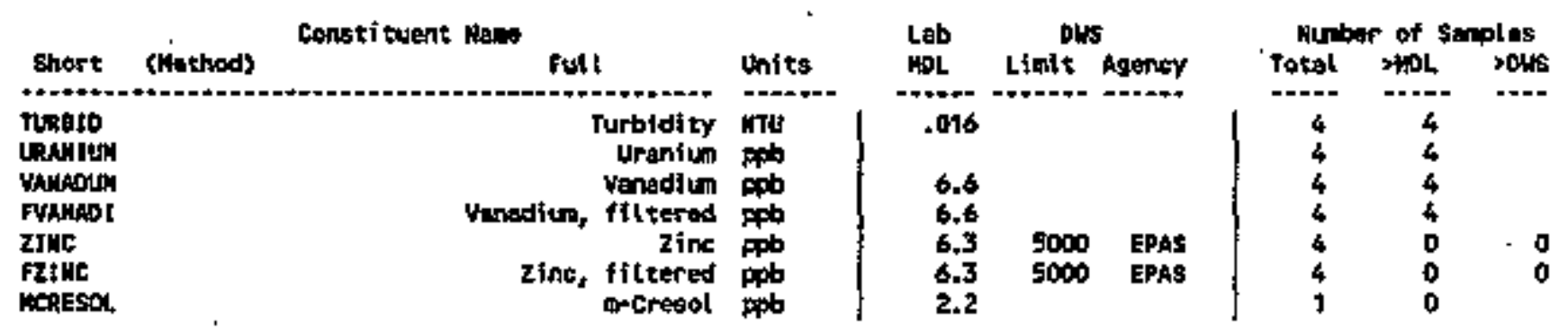

for explanetion of this rable, see section 1.4 of report. 
Table 13-4. Constituents with at Least One Detected Value for the 2101-M Pond Data for Reporting Perlod October 1 through Decenber 31, 1994. (sheet 1 of 3)

\begin{tabular}{|c|c|c|c|c|c|c|}
\hline wet & $\begin{array}{c}\text { Cotlection } \\
\text { otete }\end{array}$ & $\begin{array}{l}\text { stiple } \\
\text { singer }\end{array}$ & $\begin{array}{l}\text { ARseind } \\
43 / \mathrm{ppb} \\
.64 / 50\end{array}$ & $\begin{array}{l}\text { FAnSEnIC } \\
43 / \mathrm{ppb} \\
.64 / 50\end{array}$ & 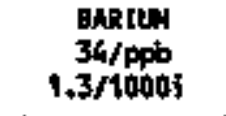 & $\begin{array}{c}\text { FBAR Itti } \\
\text { 36/ppb } \\
\text { t } 3 / 10001\end{array}$ \\
\hline $\begin{array}{l}299-E 18-1 \\
299-E 18-1 \\
299-E 18-2 \\
299-E 18-2 \\
299-E 18-3 \\
299-E 58-3 \\
299-E t 8-4 \\
299-E \$ 8-6\end{array}$ & $\begin{array}{l}12 / 01 / 94 \\
12 / 01 / 94 \\
12 / 01 / 94 \\
12 / 01 / 94 \\
12 / 01 / 94 \\
12 / 01 / 94 \\
12 / 01 / 94 \\
12 / 01 / 94\end{array}$ & 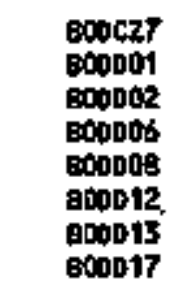 & $\begin{array}{l}2.40 \mathrm{~V} \\
5.40 \\
11.00 \\
12.00\end{array}$ & $\begin{array}{r}2.80 \mathrm{~V} \\
5.30 \\
10.00 \\
11.00\end{array}$ & $\begin{array}{l}26.00 \\
53.00 \\
53.00 \\
56.00\end{array}$ & $\begin{array}{l}25.00 \\
52.00 \\
52.00 \\
56.00\end{array}$ \\
\hline $\begin{array}{l}\text { Yell } \\
\text { Plame }\end{array}$ & $\begin{array}{c}\text { Collection } \\
\text { Date }\end{array}$ & sample & $\begin{array}{c}\text { EALCrum } \\
34 / p+6 \\
47 \%\end{array}$ & $\begin{array}{c}\text { FCALCId } \\
34 / \mathrm{pp} \$ \\
47 /\end{array}$ & $\begin{array}{l}\text { CHLQRIO } \\
\text { 124/ppb } \\
71 / 250006\end{array}$ & $\begin{array}{l}\text { Chrowand } \\
34 / p \text { pt } \\
11 / 501\end{array}$ \\
\hline $\begin{array}{l}299+E 18-1 \\
299-E 18-1 \\
299-E 18-2 \\
299-E 18-2 \\
299-E 18-3 \\
299+E 18-3 \\
299-E 18-4 \\
299-E 18-4\end{array}$ & $\begin{array}{l}12 / 01 / 94 \\
12 / 01 / 94 \\
12 / 01 / 94 \\
12 / 01 / 94 \\
12 / 01 / 94 \\
12 / 01 / 94 \\
12 / 01 / 94 \\
12 / 01 / 94\end{array}$ & 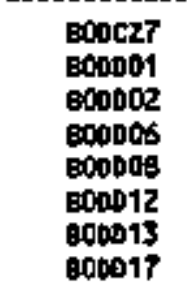 & $\begin{array}{l}49000.00 \\
29000.00 \\
27000.00 \\
29000.00\end{array}$ & $\begin{array}{l}49000.00 \\
29000.00 \\
26000.00 \\
29900.00\end{array}$ & $\begin{array}{c}7500.00 \\
9200.00 \\
10000.000 \\
8800.00\end{array}$ & $\begin{array}{l}45.00 \\
42.00 \\
32.00 \\
52.00\end{array}$ \\
\hline
\end{tabular}

\begin{tabular}{|c|c|c|c|c|c|c|}
\hline Holl & $\begin{array}{c}\text { Collectian } \\
\text { Date }\end{array}$ & $\begin{array}{l}\text { sample } \\
\text { Hurber }\end{array}$ & $\begin{array}{l}\text { FChinoid } \\
34 / \mathrm{Fph} \\
11 / 501\end{array}$ & $\begin{array}{c}\text { coppat } \\
34 / p q b \\
2.6 / 1000 \mathrm{~s}\end{array}$ & $\begin{array}{c}\text { FCOPPER } \\
34 / 0 \mathrm{pb} \\
\text { 2.6/10000 }\end{array}$ & $\begin{array}{l}\text { Fuantb } \\
124 / \mathrm{ppb} \\
\text { st/1400i }\end{array}$ \\
\hline $\begin{array}{l}299-E 18-1 \\
299-E 18-1 \\
299-E 18-2 \\
299-E 18-2 \\
299-E 18-3 \\
299-E 18-3 \\
299-E 18-4 \\
299-E 18-4\end{array}$ & $\begin{array}{l}12 / 11 / 94 \\
12 / 01 / 94 \\
12 / 01 / 94 \\
12 / 01 / 94 \\
12 / 01 / 94 \\
12 / 01 / 94 \\
12 / 01 / 94 \\
12 / 01 / 94\end{array}$ & 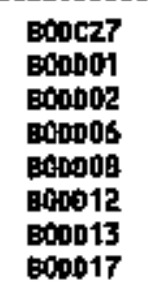 & $\begin{array}{l}9.30 \mathrm{~L} \\
4.50 \mathrm{U} \\
4.50 \mathrm{U} \\
5.20 \mathrm{~L}\end{array}$ & $\begin{array}{l}4.10 \mathrm{U} \\
4.30 \mathrm{LB} \\
4.10 \mathrm{~V} \\
4.10 \mathrm{U}\end{array}$ & $\begin{array}{l}4.10 \mathrm{v} \\
7.40 \mathrm{LB} \\
4.10 \mathrm{U} . \\
4.80 \mathrm{Lg}\end{array}$ & $\begin{array}{r}600.00 \\
-700.00 \\
.400 .00 \\
500.00\end{array}$ \\
\hline
\end{tabular}

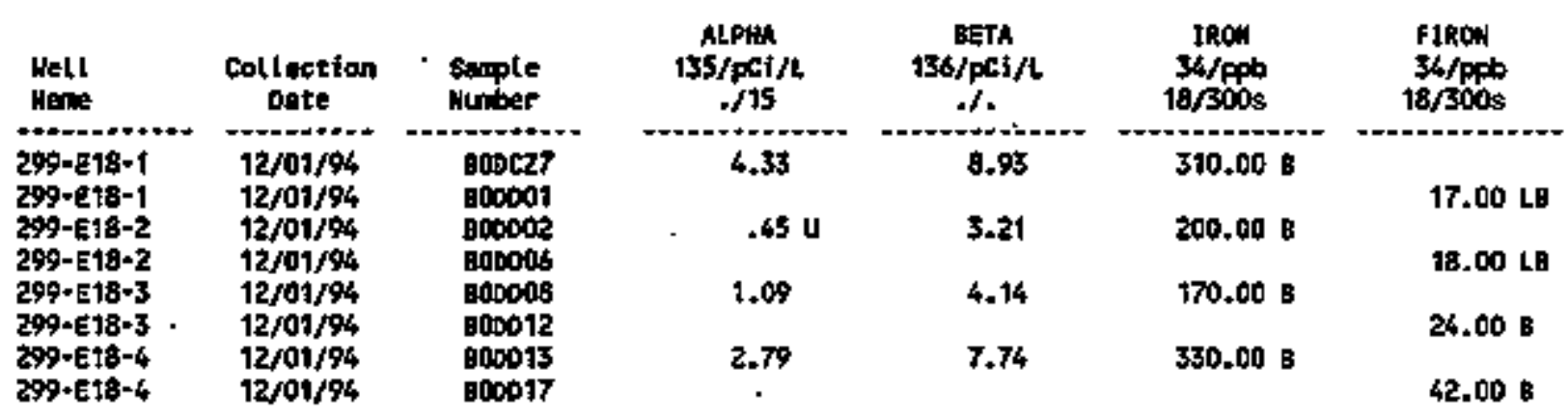


Table 13-4. Constituents with at Least One Detected Value for the 2101-M Pond Data for Reporting Period October 1 through Deceaber 31, 1994.

(sheet 2 of 3 )

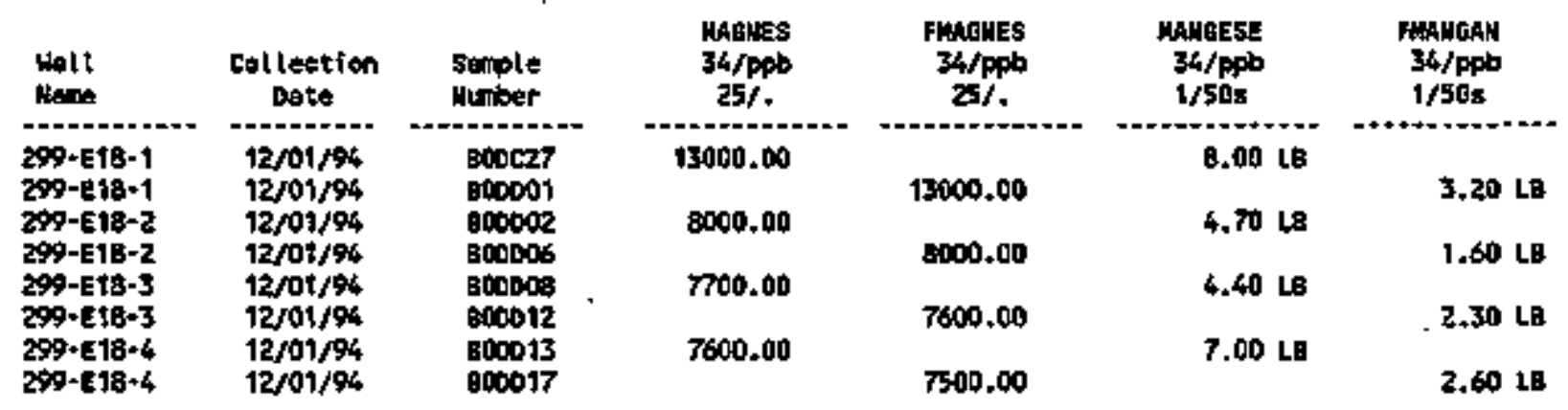

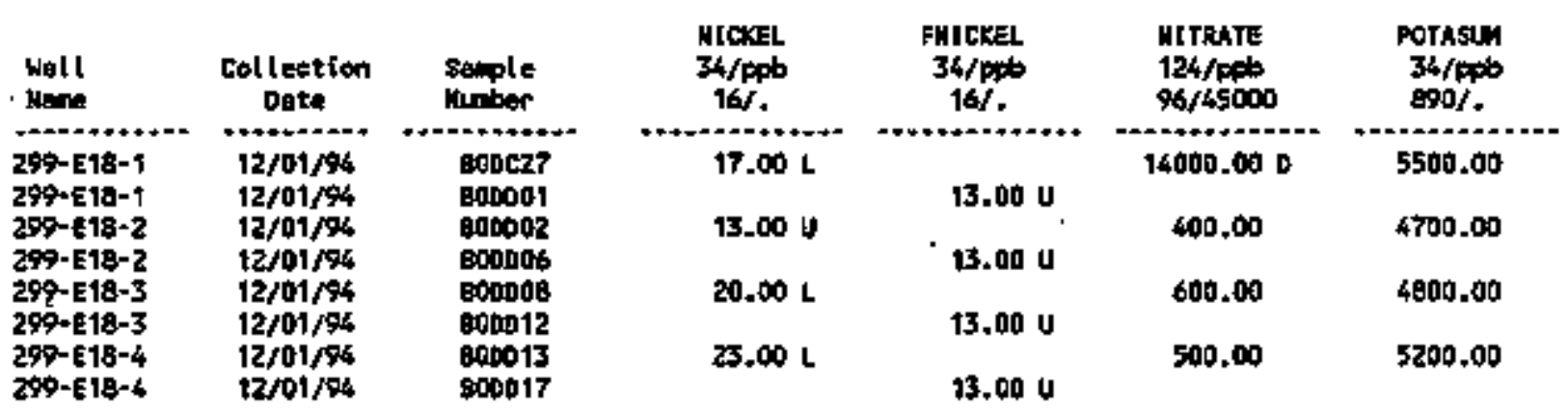

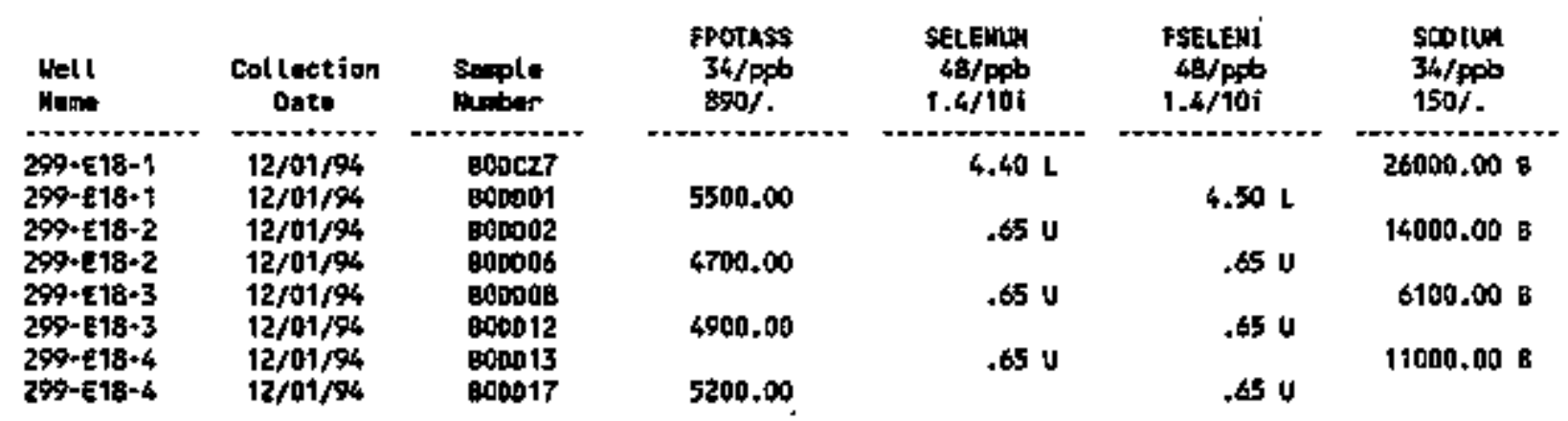

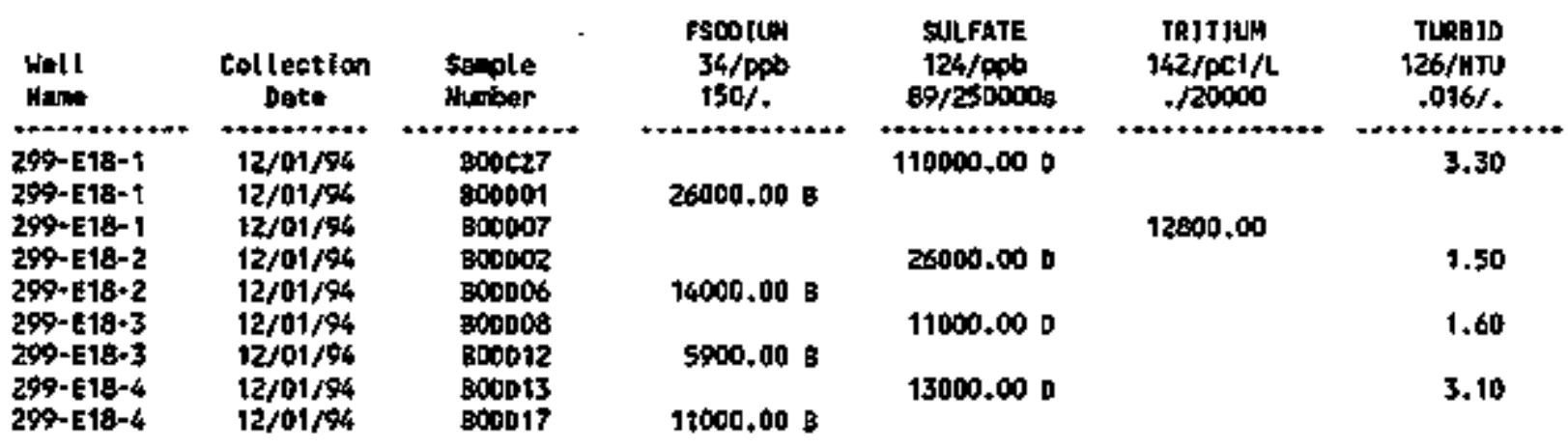


Tabie 13-4. Constjtuents with at Least One Detected Value for the 2101-M Pond Data for Reporting Period October 1 through December 31, 1994. (sheet 3 of 3)

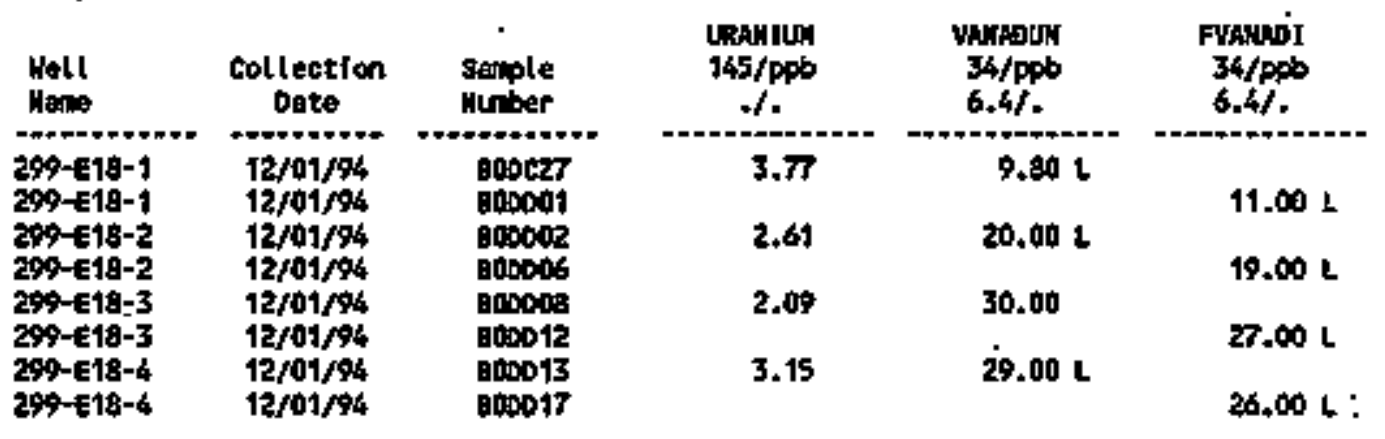

For explanation of this tople, set section 1.4 of report. 
Tab7e 13-5. Contamination Indicator Parameters for the 2101-M Pond Data for Reporting Period October 1 through December 31, 1994.

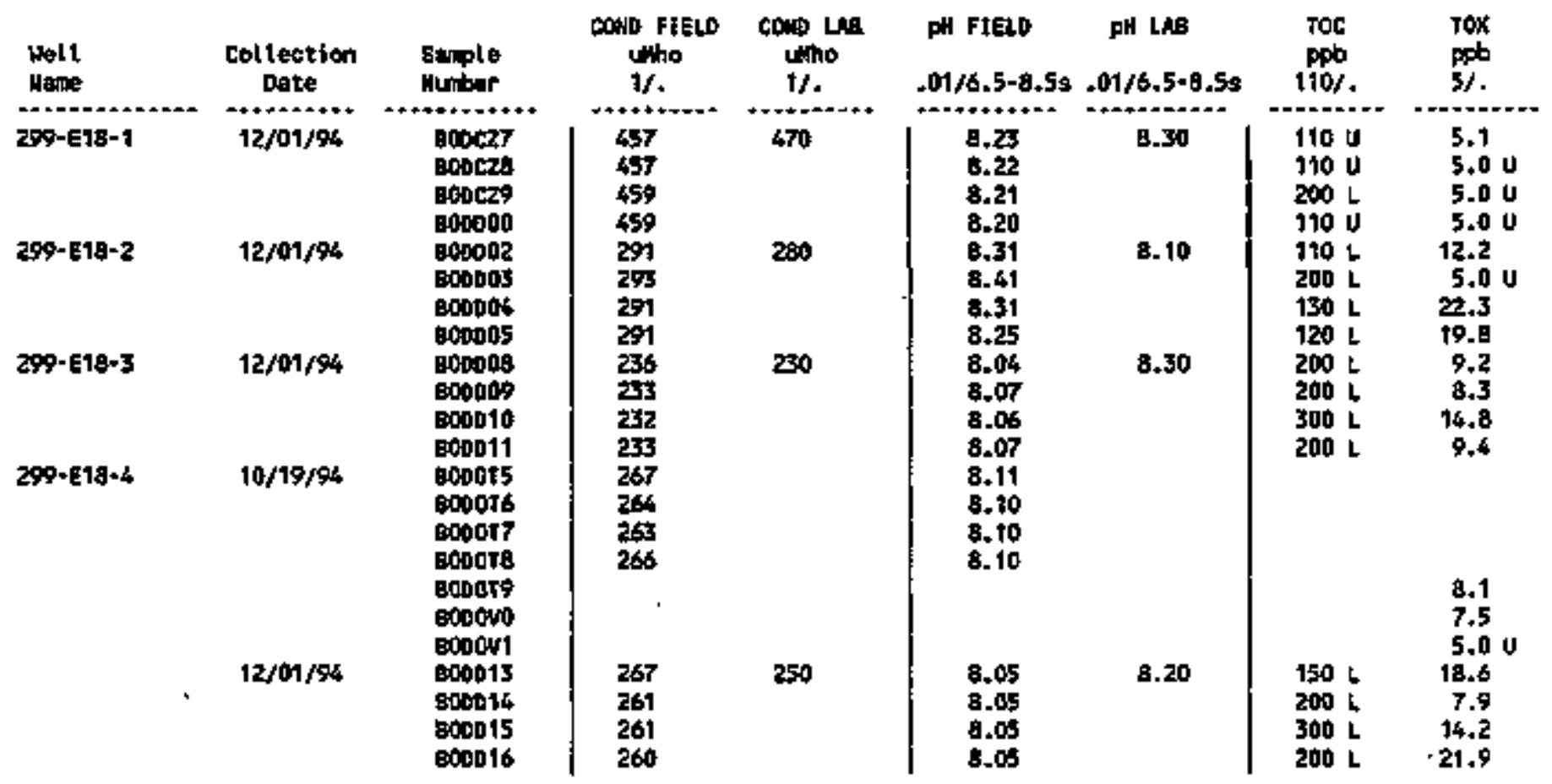




\section{DOE/RL-94-36-4}

\section{COMTENTS}

14.0 LON-LEVEI BURIAL GROUNOS $\ldots \ldots \ldots \ldots \ldots \ldots \ldots$

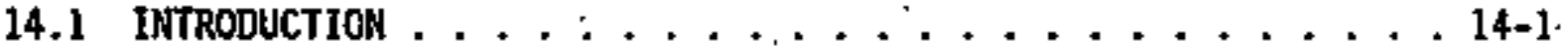

14.2 WATER LEVEL MEASUREMENTS . ................ . . . . .

14.3 MATER CHEMISTRY OATA ......................... 


\section{LIST OF FIGULES}

14-1 Well Location Map for Low-Level Waste Managenent Area 1 . . . . . . 14-3

I4-2 Well Location Map for Low-Level Waste Management Area $2 . . . . . .14-4$

14-3 Well Location Map for Low-Level Waste Management Area $3 . . . . .14-5$

14-4 Wel1 Location Map for Low-Level Waste Management Area 4 . . . . . . 14-6

14-5 WeT1 Location Map for Low-Level Waste Managewent Area $5 . . . . .14-7$

\section{LIST OF TABLES}

14-1 Low-LeveT Burial Grounds . . . . . . . . . . . . . . . . 14-8

14-2 Monitoring Ke11 Purpose and Sampling Schedule

for Low-Leve1 Naste Hanagement Area 1 . . . . . . . . . 14-9

14-3 Monitoring We11 Purpose and Sampling Schedule

for Low-Leve] Waste Management Area $2 \ldots . . . . .14-10$

14-4 Monitoring Well Purpose and Sampling Schedule for Low-Leve] Haste Management Area 3 ... . . . . . . 14-11

14-5 Monitoring Hell Purpose and Sampling Schedule for Low-Leve1 Naste. Management Area 4 . . . . . . . . . . 14-12

14-6 Monitoring Well Purpose and Sampling Schedule for Low-Leve1 Waste Management Area $5 \ldots \ldots$ I4-13

14-7 RCRA Water Level Measurement Report LLWMA 1, Fourth Quarter 1994

14-8 RCRA Water Level Measurement Report LLLWMA 2, Fourth Quarter 1994

14-9 RCRA Water Level Measurement Report LLMMA 3, Fourth Quarter 1994

14-10 RCRA Water Level Measurement Report LLWMA 4, Fourth Quarter 1994

14-11 RCRA Water Leve] Measurement Report LLWMA 5, Fourth Quarter 1994

14-12 Constituent l.fst and Summary of Results for the Low-Level Waste

Management Area 1 Oata for Reporting Period

October 1 through December 31, 1994

14-13 Constituents with at Least One Detected Valce for the Low-Level

Waste Managentent Area I Data for Reporting Period

October 1 through December 31, 1994 ........ . 14-26

14-14 Contamination Indicator Parameters for the Low-Level Waste

Management Area 1 Data for Reporting Pertod

October 1 through December 31, 1994 ........... 14-35

14-16 Constituents with at Least One Detected Value for the Lor-Level

Waste Management Area 2 Data for Reporting Period

October 1 through December $31,1994 \ldots 14-40$

14-17 Contamination Indicator Parameters for the Low-Level Waste

Management Area 2 Data for Reporting Period

October ! through December 31, 1994 .......... . 14-48

14-18 Constituent List and Summary of Results for the Low-Level

Waste Management Area 3 Data for Reporting Period

October 1 through December 31, 1994

14-19 Constituents with at Least One Detected Value for the Low-Level

Waste Management Area 3 Data for Reporting Period

October 1 through December $31,1994 \ldots . . . . . .14-53$

14-20 Contamination Indicator Parameters for the Low-Level Waste

Management Area 3 Data for Reporting Period

October 1 through Decemer 31, 1994 


\section{LIST OF TABLES (cont)}

14-21 Constituent List and Sumbary of Results for the Low-Leve1

Haste Managenent Area 4 Data for Reporting Period

October 1 through Decenther 31, 1994 . . . . . 14-57

14-22 Constituents with at Least One Detected Va]ue for the Low-Level

Waste Managewent Area 4 Data for Reporting Period

October 1 through Decenber 31, 1994 . . . . . . . . . . . 14-60

14-23 Contamination Indicator Parameters for the Low-Level Waste

Management Area 4 Data for Reporting Period

October 1 through December 31, 1994 . . . . . . . . . . 14-69

14-24 Constituent List and Summary of Resu]ts for the Low-Level

Haste Management Area 5 Data for Reporting Period

October 1 through December 31, $1994 \ldots$. . . . . . . . . . 14-71

14-25 Constituents with at least one Detected Value for the Low-Level

Waste Hanagement Area 5 Data for Reporting Period

October 1 through December 31, $1994 \ldots$ 14-72

14-26 Contamination Indicator Parameters for the Low-Level Haste

Management Area 5 Data for Reporting Period

October 1 through December 31, 1994 ............... 14-73

14-27 Hater Qual ity Standards Exceeded at Low-Leve?

Waste Management Area 1. . . . . . . . .. . . . . . . . . . 14-73

14-2B Water Qual ity Standards Exceeded at Low-Leve1

Waste Management Area $2 \ldots \ldots$. . . . . . . . . . . . 14-74

14-29 Water Quality Standards Exceeded at Low-Leve]

Waste Management Area 3 . . . . . . . . . . . . . . 14-74

14-31 Water Quality Standards Exceeded at Low-Level

Waste Management Area 5 .................. 14-75 
DOE/RL-94-36-4

This page intentionaliy left blank. 
DOE/RL-94-36-4

\title{
14.0 LON-LEYEL BURIAL GROUNDS
}

\author{
R. B. Nercer \\ Westinghouse Hanford Company
}

\subsection{INTROBICTION}

The 200 Areas LLBG consist of five Low-Leve1 Waste Management Areas (LLWMA). LLWMA-1 and LLWMA-2 are 7ocated in the 200 East Area and LLWMA-3, LLWMA-4, and LLWMA-5 are located in the 200 West Area- (Figures 14-1 through 14-5). A description of the waste contained in each burial ground 1s provided in Table 14-1.

The groundrater monitoring program for the LLBG began wt th the installation of 35 monitoring wells in 1987. Additional wells have been installed in subsequent years. The present monitoring networks are listed in Tables 14-2 through 14-6.

Semiannual detection level sampling is being conducted at LLWMA-1, LLWMA-2, LLWMA-4, and LLWMA-5. Background values are being reestabtished for LLWMA-3 because recently installed upgradient wells have significantly changed the monitoring network. Seglannual sampling is being conducted at LLWHA-3 with additional quarteriy sampling for CIPs at the shallow upgradient wells.

A carbon tetrachloride punp-and-treat demonstration project is continuing this quarter near LLWMA-4. Groundwater is being extracted from we11 299-W18-1 and injected into well 299-418-4 after treatment. Groundwater levels are being monttored with transducers in the extraction and injection wells and in 299-W18-2, 299-W18-5, and 299-W18-24. Well 299-W18-24 is an upgradient monitoring we]T for LLWHA-4. Ho igpacts on the LLWMA-4 monitoring network have been noted to date.

\subsection{HATER LEVEL MEASUREMEMTS}

In addition to measurements taken at the time of sampling, water levels were measured quarterly at the LLBG. Water level measurements are presented in Tables 14-7 through 14-11. Additional water level measurements are reported for some wells if they are monitored for other projects. Suspected errors in the water level measurements occurred at several wells this quarter, as indicated in the tables. Hater level measurements were not taken at we11 299-W6-5 because of an obstructed casing; the casing in wel1 299-46-5 was crimped during installation. Although remedial action enlarged the casing to permit the installation of the pump, the well does not have sufficient space to measure water levels, and additional well remediation may not be posstble without damaging the casing. Wel1 299-E34-4 has been dry since 1t was constructed. .Hater levels are no longer measured in wells 299-E34-6 and 299-E35-1 because they have fallen below the bottom of the screens. three of these wells were completed to the top of basalt. The LLWMA-2 monitoring network continues to meet the requirement of at least one upgradient and three downgradient monttoring wells. 


\subsection{WATER CHENISTRY DATA}

Groundwater samples were collected as scheduled at the LLBG. Samples were analyzed for CIPS, Interim primary drinking water parameters, water quality parameters, and site-specific parameters. Results of analyses are ifsted in Tables 14-12 through 14-26. These tables a]so contain any previousiy unreported data and data that are being rereported because of changes in the data qualifier flags.

Additional samples were collected at some LLWWA-3 and LLWMA-5 groundwater monitoring wells for iodine-129, strontilut-90, technetjum-99, and total dissolved solids. These samples are tn support of groundwater quality assessinent at the $T$ and TX-TY SST farms. Iodjne-129 samples were also collected at some wells in LLWMA-2 for the B-63 Trench RCRA prograa. Results for these analyses are reported in the data tables in this section.

The replicate averages for field ph were below the critical range established for LLWNA-4 in upgradient well 299-\$15-16. Resampiing was not scheduled because the laboratory vaiue was within the critical range. RADEs have been initlated on the field results. The replicate averages of other indicator parameters did not exceed the criticaT means at LLMMA-I, LLLMMA-2, or LLIMA-4.

Analytical resuits flagged with a ' $Q$ ' are associated with suspect QC data. Data qualifier and data flags are discissed $1 \mathrm{n}$ more detail in Sections 1.2 and 1.4 , respectively. Results are flagged with an ' $F$ ' if a RADE has been issued because the results did not follow the past trends.

Tables 14-27 through 14-30 list the water quality standards that were exceeded for the data reported this quarter. Unfiltered metals accounted for most of the entries in these tabies. Standards were not exceeded for the related filtered samples. 
Figure 14-1. Well Location Map for Low-Level Waste Management Area 1.

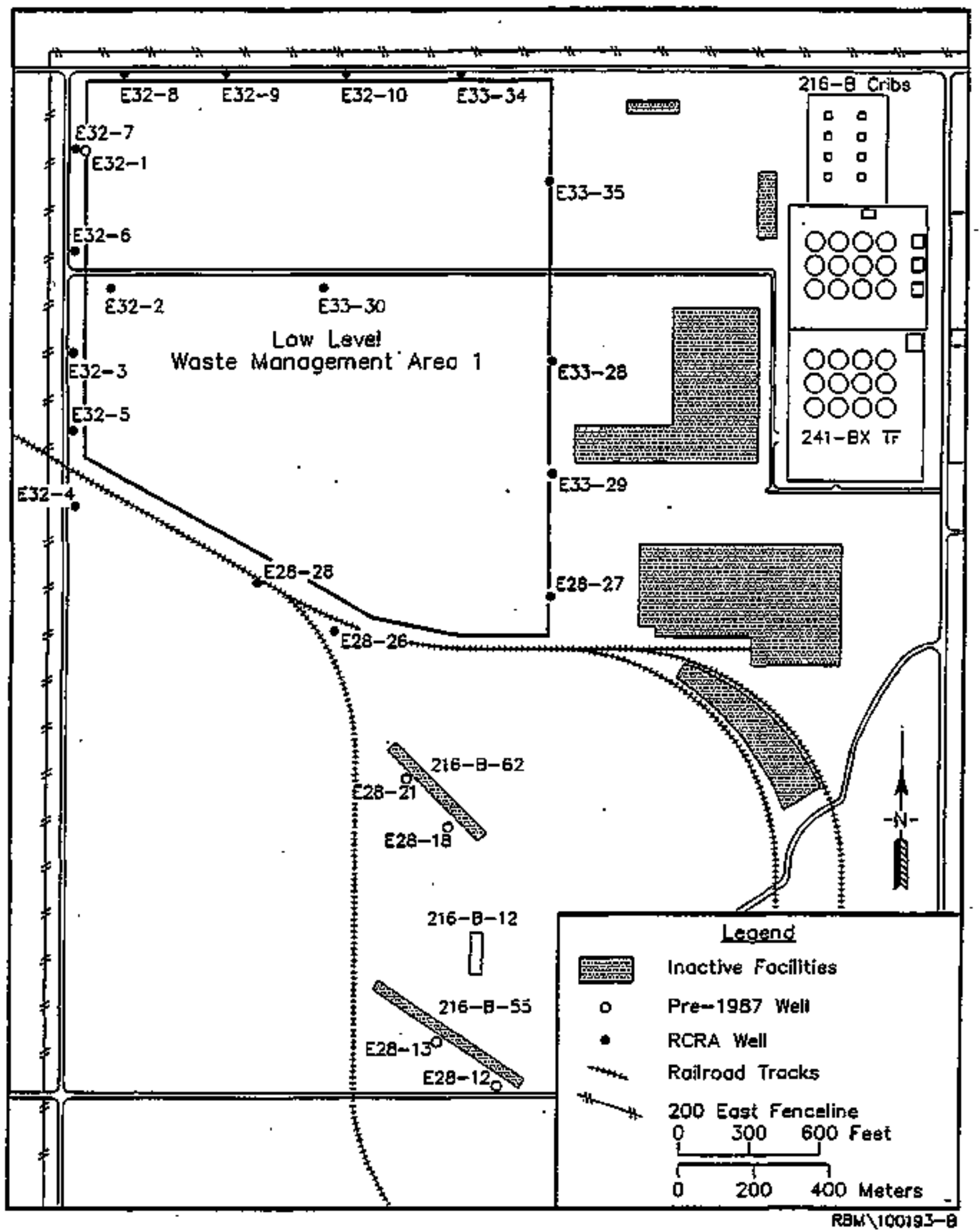




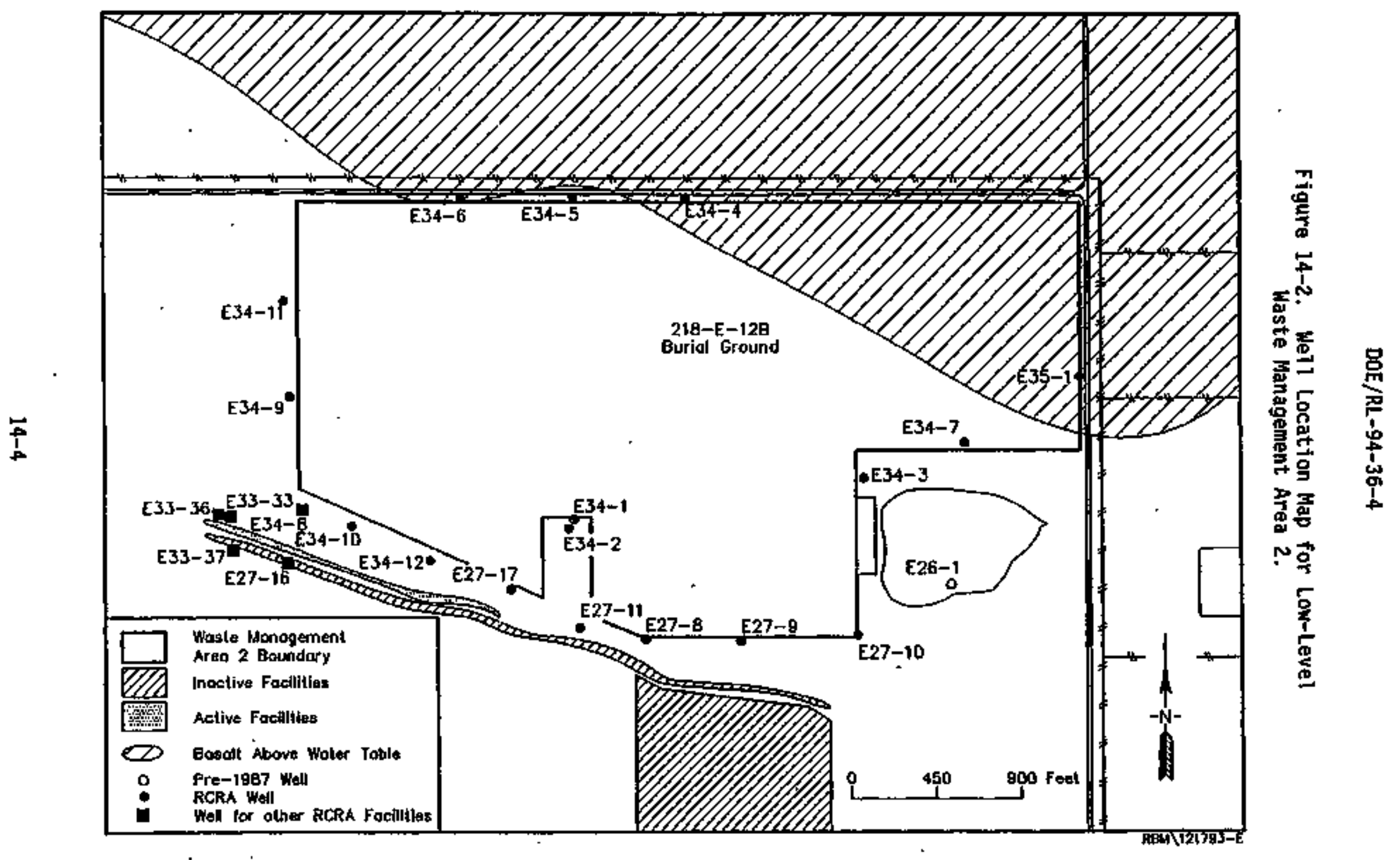




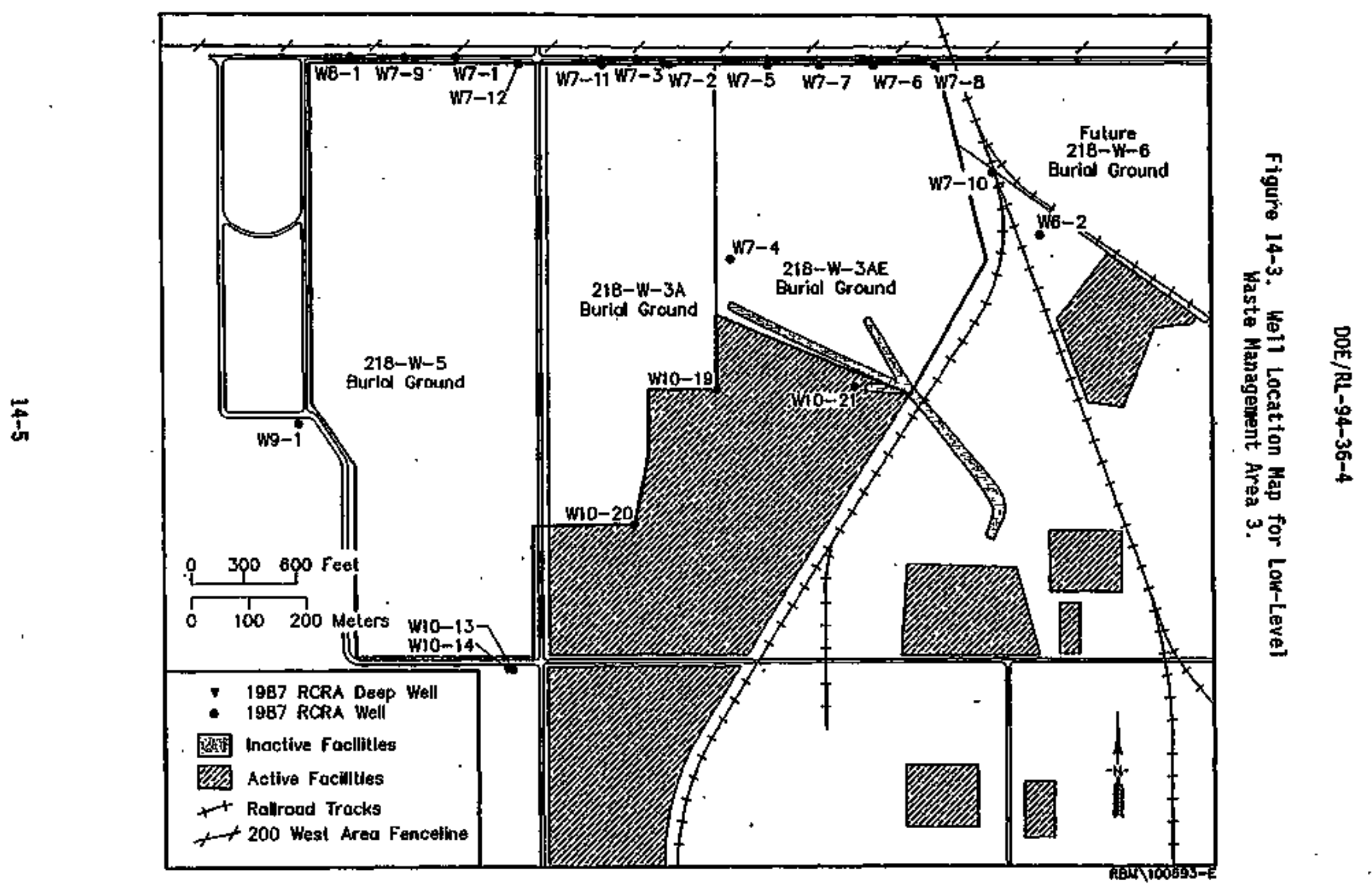


Figure 14-4. Mell Location Map for Low-Level Waste Management Area 4.

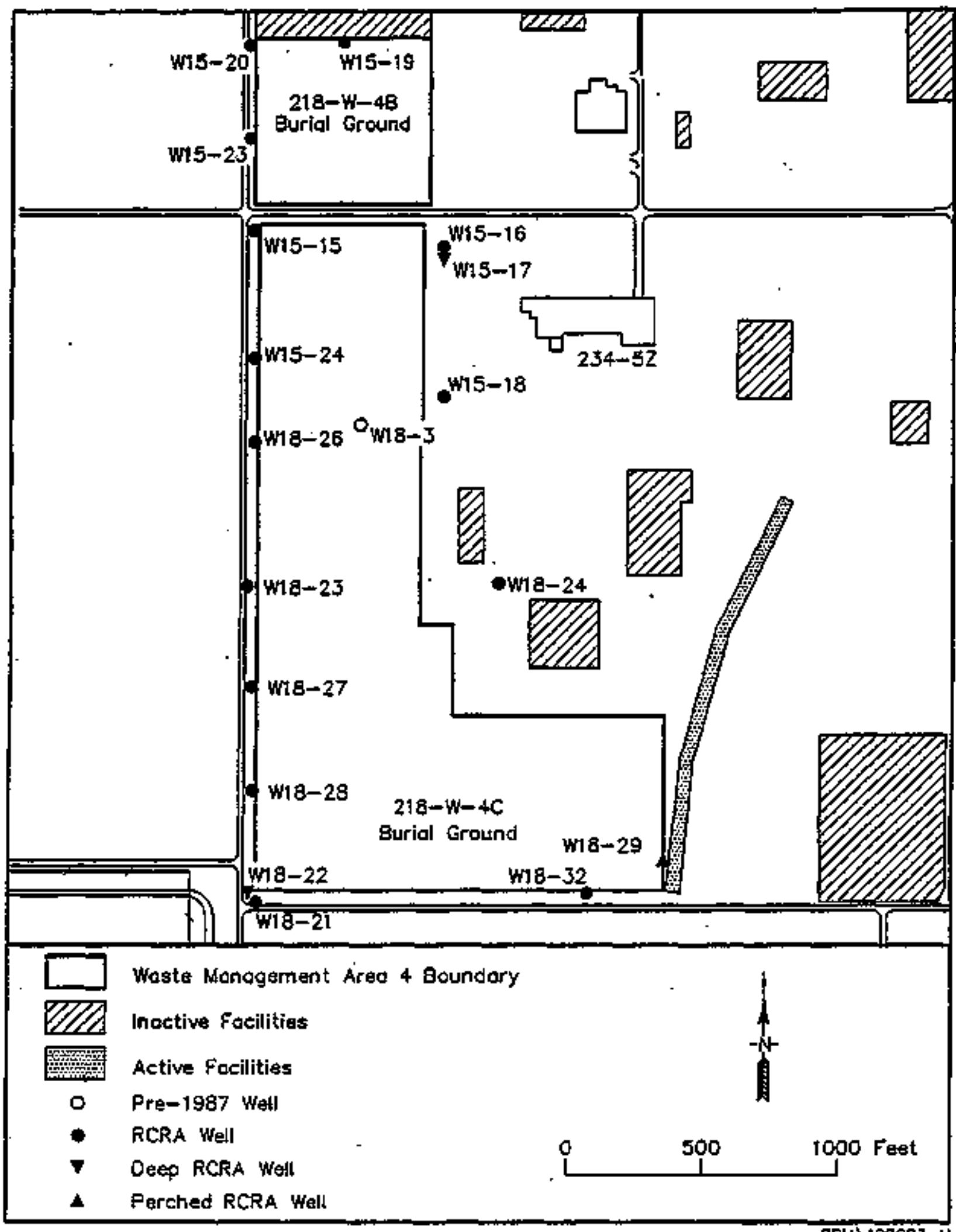




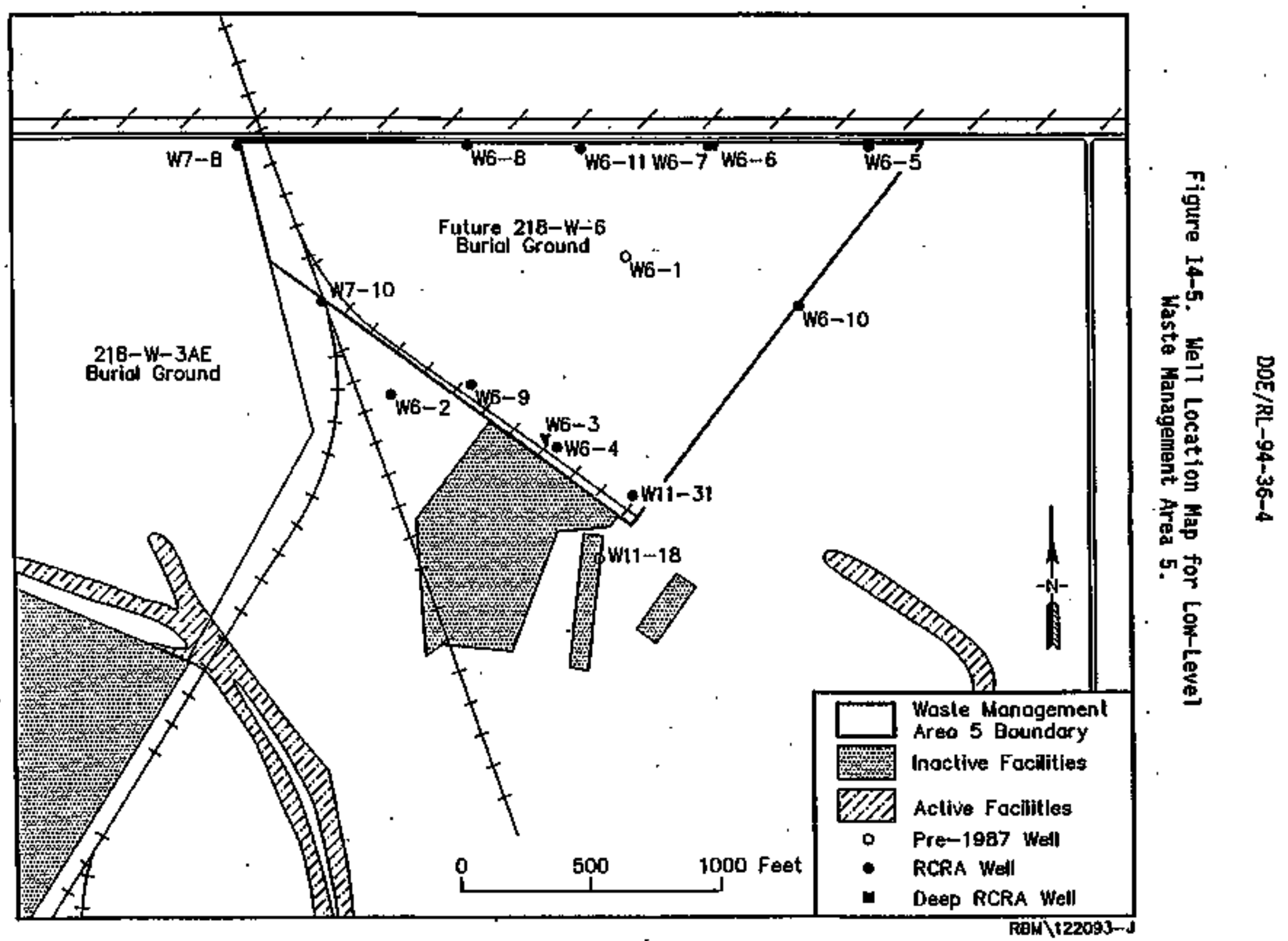


DOE/RL-94-36-4

Table 14-1. Low-Level Burial Grounds.

\begin{tabular}{|c|c|c|c|}
\hline LLWMA- & Burial ground & First use & Contents \\
\hline 1 & $218-E-10$ & 1960 & $\begin{array}{l}\text { Oragoff material } \\
\text { Faf\}ed equiptont } \\
\text { Mixed waste }\end{array}$ \\
\hline 2 & $218-E-12 B$ & 1968 & $\begin{array}{l}\text { Miscellaneous dry waste } \\
\text { Transuranic waste } \\
\text { Submarine reactor compartments }\end{array}$ \\
\hline \multirow[t]{3}{*}{3} & $218-W-3 A$ & 1970 & $\begin{array}{l}\text { Ion exchange resins } \\
\text { Failed equipment }\end{array}$ \\
\hline & $218-W-3 A E$ & 1981 & $\begin{array}{l}\text { Low-level waste } \\
\text { Low-level mixed waste }\end{array}$ \\
\hline & $218-h-5$ & 1986 & $\begin{array}{l}\text { Low-level waste } \\
\text { Low-level mixed waste }\end{array}$ \\
\hline \multirow[t]{2}{*}{4} & $218-4-4 B$ & 1968 & $\begin{array}{l}\text { Mixed waste } \\
\text { Retrievable transuranic waste }\end{array}$ \\
\hline & $218-H-4 C$ & 1978 & $\begin{array}{l}\text { Transuranic waste } \\
\text { Mixed waste } \\
\text { Low-Tevel waste }\end{array}$ \\
\hline 5 & $218-W-6$ & $N / A$ & Hone to date \\
\hline
\end{tabular}

N/A - not applicable. 
Table 14-2. Monftoring We1] Purpose and Sampling Schedule for Low-Level Waste Management Area 1.

\begin{tabular}{|c|c|c|c|c|}
\hline $\begin{array}{c}\text { We11 no. } \\
(299-)\end{array}$ & $\begin{array}{l}\text { Relative } \\
\text { position }\end{array}$ & Hydrogeologic unit & $\begin{array}{c}\text { Sample } \\
\text { interval }\end{array}$ & $\begin{array}{l}\text { Sample date, } \\
\text { 4th Qtrr } 1994\end{array}$ \\
\hline$E 28-26^{87}$ & Upgradient & Hanford: Water Table & Semiannuatiy & $12 / 12 / 94$ \\
\hline$E 28-27^{87}$ & Upgradient & Hanford: Water Table & Semtannually & $12 / 12 / 94$ \\
\hline$E 28-28^{90}$ & Upgradient & Hanford: Water Table & Semiannualiy & $12 / 12 / 94$ \\
\hline$E 32-2^{67}$ & Downgradient & Hanford: Water Table & Semtannually & $12 / 13 / 94$ \\
\hline $\mathrm{E} 32-3^{87}$ & Downgradient & hanford: Water Table & Semiannuat1y & $12 / 13 / 94$ \\
\hline$E 32-4^{97}$ & Upgradient & Hanford: Water Table & Semiannualiy & $\begin{array}{l}10 / 11 / 94^{*} \\
12 / 13 / 94\end{array}$ \\
\hline$E 32-5^{89}$ & Bowngradient & Hanford: Hater Table & Semiannual\}y & $12 / 13 / 94$ \\
\hline E32-691 & Downgradient & Hanford: Water Table & Semiannualiy & $12 / 12 / 94$ \\
\hline $\mathrm{E} 32-7^{91}$ & Downgradient & Hanford: Water Table & Semiannual1y & $12 / 12 / 94$ \\
\hline$E 32-8^{91}$ & Downgradtent & Hanford: Water Table & Semiannual]y & $12 / 12 / 94$ \\
\hline$E 32-9^{91}$ & Downgradient & Hanford: Mater Table & Semiannua71y & $12 / 13 / 94$ \\
\hline$E 32-10^{92}$ & Downgradient & Hanford: Water Table & Sẹmíannually & $12 / 13 / 94$ \\
\hline$E 33-28^{87}$ & Upgradient & Hanford: Hater Table & Semiannua] $7 y$ & $12 / 12 / 94$ \\
\hline$E 33-29^{87}$ & Upgradient & Hanford: Water Tabie & Semiannually & $12 / 12 / 94$ \\
\hline$E 33-30^{87}$ & Downgradient & Hanford: Water Tab]e & Semiannuaj1y & $12 / 14 / 94$ \\
\hline $633-34^{50}$ & Downgradient & Hanford: Hater Table & Semiannual]y & $12 / 14 / 94$ \\
\hline $633-35^{90}$ & Upgradient & Hanford: Mater Table & Semiannuaj1y & $12 / 12 / 94$ \\
\hline
\end{tabular}

Note: Superscript following well number indicates year of installation. Sampled for B-Pond. 
Table 14-3. Monitoring Well Purpose and Sampling Schedule . for Low-Level Waste Management Area 2.

\begin{tabular}{|c|c|c|c|c|}
\hline $\begin{array}{c}\text { Hell no. } \\
(299-)\end{array}$ & $\begin{array}{l}\text { Relative } \\
\text { position }\end{array}$ & Hydrogeologic unit & $\begin{array}{l}\text { Sample } \\
\text { interval }\end{array}$ & $\begin{array}{l}\text { Sample date, } \\
4 \text { th Qtr } 1994\end{array}$ \\
\hline$E 27-8^{97}$ & Downgradient & Hanford: Water Table & Seatiannually & $11 / 17 / 94$ \\
\hline$E 27-9^{87}$ & Downgradient & Hanford: Hater Table & Seniannualiy & $11 / 17 / 94$ \\
\hline$E 27-10^{87}$ & Upgradjent & Hanford: Water Table & Sent annual ly & $12 / 13 / 94$ \\
\hline$E 27-11^{89}$ & Downgradtent & Hanford: Water Table & Sept annually & $11 / 18 / 94$ \\
\hline$E 27-17^{91}$ & Downgradtent & Hanford: Water Table & Sepiannually & $11 / 17 / 94$ \\
\hline$E 34-2^{87}$ & Downgradient & Hanford: Water TabTe & Sent annual $7 y$ & $11 / 21 / 94$ \\
\hline$E 34-3^{87}$ & Upgradient & Hanford: Water Table & Semi annuat $1 y$ & $11 / 21 / 94$ \\
\hline $\mathrm{E3}^{34-4^{87}}$ & Upgradient & Hanford: Water Table & HA & Dry \\
\hline$E 34-5^{87}$ & Upgradient & Hanford: Water Table & Seraiannually & $11 / 18 / 94$ \\
\hline$E 34-6^{87}$ & Upgradient & Hanford: Water Table & HA & Dry \\
\hline$E 34-7^{89}$ & Upgradtent & Hanford: Water Table & Semiannually & $11 / 18 / 94$ \\
\hline$E 34-9^{91}$ & Dowingradient & Hanford: Water Table & Semiannually & $11 / 28 / 94$ \\
\hline$E 34-10^{91}$ & Domgradient & Hanford: Water Table & Semjannua 11y & $11 / 21 / 94$ \\
\hline$E 34-11^{92}$ & Downgradient & Hanford: Water Table & Semiannua $11 y$ & $11 / 28 / 94$ \\
\hline$E 34-12^{92}$ & Downgradient & Hanford: Water Table & Semiannually & $11 / 28 / 94$ \\
\hline$E 35-1^{89}$ & Upgradient & Hanford: Water Table & NA & Ory \\
\hline
\end{tabular}

Notes: Superscript following well number indicates year of installation. MA = not available. 
Table 14-4. Monjtoring Welt Purpose. and Sampling Schedule for Low-Level Waste Management Area 3.

\begin{tabular}{|c|c|c|c|c|}
\hline $\begin{array}{l}\text { Well no. } \\
\left(299_{-}\right)\end{array}$ & $\begin{array}{l}\text { Refative } \\
\text { posfition }\end{array}$ & Hydrogeologtc unit & $\begin{array}{c}\text { Sawple } \\
\text { interval }\end{array}$ & $\begin{array}{l}\text { Sample date, } \\
\text { 4th Qtr } 1994\end{array}$ \\
\hline W6- $2^{87 \pi}$ & Downgradient & Ringold: Water Table & Semiannual1y & $\begin{array}{l}11 / 30 / 94^{\mathrm{b}} \\
12 / 14 / 94^{\mathrm{c}}\end{array}$ \\
\hline $17-1^{87}$ & Downgradient & Ringold: Water Table & Semfannua\}1y & Not Sampled \\
\hline $47-2^{87}$ & Downgradient & Ringold: Water Table & Semfannua]1y & Hot Satipled \\
\hline $47-3^{87}$ & Downgradient & Ringold: Deep & Semiannually & Not Sampled \\
\hline $47-4^{87}$ & Downgradient & Ringold: Water Table & Semiannually & Not Sampled \\
\hline $47-5^{87}$ & Downgradient & Ringo]d: Water Tab]e & Sect annually & Hot Sampled \\
\hline $47 \div 6^{87}$ & Downgradient & Ringold: Water Table & Sem1 annuality & Hot Sampled \\
\hline$W 7-7^{89}$ & Downgradjent & Ringold: Water Table & Semlannualiy & Hot Sampled \\
\hline W7- $-8^{89}$ & Downgradient & Ringold: Water TabTe & Semiannually & Hot Sampled \\
\hline W7-990 & Downgradient & Ringald: Water Table & Semi annually & Hot Sampled \\
\hline$W 7-10^{500 m}$ & Downgradient & Ringold: Water Table & Semiannualiy & Hot Sampled \\
\hline$W 7-11^{91}$ & Downgradient & Ringold: Water Table & Semt annual1y & Hot Sampled \\
\hline$W 7-12^{91}$ & Downgradient & Ringold: Water Table & Serifannuality & Not Sampled \\
\hline $48-1^{87}$ & Downgradient & Ringo]d: Water Table & Seml annual iy & $11 / 30 / 94^{\mathrm{b}}$ \\
\hline$W 9-1^{57}$ & Upgradient & Ringold: Water Table & Quarterly ${ }^{\mathbf{a}}$ & $12 / 14 / 94$ \\
\hline W10-13 ${ }^{87}$ & Upgradient & Ringold: Water Table & Quarterly $\mathrm{y}^{\mathrm{a}}$ & $12 / 14 / 94$ \\
\hline W10-14 & Upgradient & Ringold: Deep & Semiannually & Not Sampled \\
\hline$W 10-19^{92}$ & Upgradient & Ringold: Water Table & Quarterly" & $12 / 14 / 94$ \\
\hline $110-20^{93}$. & Upgradient & Ringold: Water Table & Quarterly" & $12 / 15 / 94$ \\
\hline $410-21^{93}$ & Upgradient & Ringold: Water Table & Quarterly & $12 / 14 / 94$ \\
\hline
\end{tabular}

Notes: Superscript following well number indicates year of installation. Deep indicates well monitors bottom of the unconfined aquifer.

- Semiannual sampling with additional CIP sampling quarterly.

m Well monitors multiple LLMMA's.

- Resampling for field indicator parameters.

- Sampled for Single Shell Tanks. 
Table 14-5. Monitoring Well Purpose and Sampling Schedule for Low-l.eve? Waste Management Area 4.

\begin{tabular}{|c|c|c|c|c|}
\hline $\begin{array}{c}\text { Well no. } \\
(299-)\end{array}$ & $\begin{array}{l}\text { Relative } \\
\text { position }\end{array}$ & Hydrogeologic unit & $\begin{array}{c}\text { Sample } \\
\text { Interval }\end{array}$ & $\begin{array}{l}\text { Sample date, } \\
\text { 4th Qtr } 1994\end{array}$ \\
\hline $415-15^{87}$ & Downgradient & Ringold: Hater Table & Semiannually & $11 / 10 / 94$ \\
\hline $415-16^{87}$ & Upgradient & Ringold: Mater Table & Semiannual1y & $11 / 08 / 94$ \\
\hline W15-17 & Upgradient & Ringold: Deep & Seatannually & $11 / 09 / 94$ \\
\hline $115-18^{87}$ & Upgradient & Ringold: Water Table & Semiannually & $11 / 08 / 94$ \\
\hline W15-19 & Downgradient & Ringold: Kater Table & Seasiannual1y & $11 / 30 / 94$ \\
\hline W15-20 & Downgradient & Rtngold: Water Table & Semfannula1]y & $11 / 11 / 94$ \\
\hline W15-23 & Downgradient & Ringold: Water Table & Semiannua\}1y & $11 / 11 / 94$ \\
\hline H15-2489. & Downgradient & Ringold: Water Table & Semiannuaj1y & $11 / 10 / 94$ \\
\hline $\mathrm{W} 18-21^{87}$ & Downgradient & Ringold: Water Table & Semiannua11y & $11 / 28 / 94$ \\
\hline W18-2297 & Downgradjent & Ringold: Deep & Semiannua]1y & $11 / 29 / 94$ \\
\hline $118-23^{87}$ & Downgradient & Rjngold: Water Table & Semiannually & $11 / 11 / 94$ \\
\hline $\mathrm{H} 18-24^{87}$ & Upgradient & Ringold: Water Table & Semíannual1y & $11 / 08 / 94$ \\
\hline W18-26 & Downgradient & Ringold: Water Table & Semiannual1y & $11 / 28 / 94$ \\
\hline W18-27 & Downgradient & Ringold: Water Table & Semiannually & $11 / 28 / 94$ \\
\hline$W 18+28^{91}$ & Downgradient & Ringold: Hater Table & Semiannual1y & $11 / 28 / 94$ \\
\hline N18-29: & Upgradient & Hanford: Perched & Semiannually & Dry \\
\hline $\mathrm{W} 18-32^{92}$ & Upgradient & Ringold: Hater Table & Semiannual1y & $11 / 09 / 94$ \\
\hline
\end{tabular}

Notes: Superscript following well number indicates year of installation. Deep indicates well monitors bottom of the unconfined aquifer. 
Table 14-6. Manitoring Well Purpose and Sampling Schedule for Low-Leve1 Haste Management Area 5.

\begin{tabular}{|c|c|c|c|c|}
\hline $\begin{array}{c}\text { Well no. } \\
(299-)\end{array}$ & $\begin{array}{l}\text { Relative } \\
\text { position }\end{array}$ & Hydrogeologic unit & $\begin{array}{c}\text { Sample } \\
\text { interval }\end{array}$ & $\begin{array}{l}\text { Sample date, } \\
\text { 4th Qtr } 1994\end{array}$ \\
\hline $46-2^{87 \pi}$ & Upgradient & Ringold: Water Table & Semianneally & $\begin{array}{l}11 / 30 / 94^{b} \\
12 / 14 / 94^{c}\end{array}$ \\
\hline $46-3^{91}$ & Upgradtent & Ringold: Deep & Semtannualiy & Not Sampled \\
\hline $46-4^{91}$ & Upgradtent & Ringold: Water Table & Semtannualiy & $12 / 14 / 94^{c}$ \\
\hline$\sqrt{6-5^{91}}$ & Downgradient & Ringold: Water Tabla & Semiannually & Not Sampled \\
\hline$W 6-6^{91}$ & Downgradient & Ringold: Deep & Semfannually & Hot Sampled \\
\hline$N 6-7^{91}$ & Downgradient & Ringold: Hater Table & Seniannually & Not Sampled \\
\hline W6-891 & Downgradient & Ringold: Water Table & Semiannually & Hot Sampled \\
\hline $46-9^{92}$ & Upgradient & Ringold: Water Table & Semi annual1y & $12 / 13 / 94^{\circ}$ \\
\hline $46-10^{92}$ & Upgradient & Ringold: Nater Table & Semiannually & $12 / 14 / 94^{c}$ \\
\hline $46-11^{92}$ & Downgradient & Ringold: Water Table & Semt annual ly & Not Sampled \\
\hline$W 6-12^{92}$ & Downgradient & Ringold: Water Table & Semiannualiy & Hot Sampled \\
\hline$W 7-10^{900 \times 1}$ & Upgradient & Ringold: Water Table & Sepiannualiy & Not Sarapled \\
\hline$W 11-31^{92}$ & Upgradient & Ringold: Water Table & Semiannually & $12 / 14 / 94^{c}$ \\
\hline
\end{tabular}

Hotes: Superscript following we1l number indicates year of installation. Deep indicates well monjtors botton of the unconftned aquifer.

- He11 monitors mutiple LLMMAs.

- Resampling for field indicator parameters.

c Sampled for Single Shell Tanks. 
Tab]e 14-7. RCRA Hater Level Measurenent Report LLWMA 1, Fourth Quarter 1994.

(sheet 1 of 2)

\begin{tabular}{|c|c|c|c|c|}
\hline Well & Date & $\begin{array}{l}\text { Depth to } \\
\text { water (ft) }\end{array}$ & $\begin{array}{l}\text { Water } \\
\text { elevation } \\
\text { (ft) }\end{array}$ & $\begin{array}{l}\text { level } \\
\text { above msl } \\
\text { (m) }\end{array}$ \\
\hline \multicolumn{5}{|c|}{ LLMA-1 Mel1s Monttoring the Top of the Unconfined Aquifer } \\
\hline $299-E 28-26$ & $\begin{array}{l}12 / 12 / 94 \\
12 / 14 / 94\end{array}$ & $\begin{array}{l}285.95 \\
285.78\end{array}$ & $\begin{array}{l}401.31 * \\
401.48\end{array}$ & $\begin{array}{l}122.32 \\
122.37\end{array}$ \\
\hline 299-E28-27 & $\begin{array}{l}12 / 12 / 94 \\
12 / 14 / 94\end{array}$ & $\begin{array}{l}279.08 \\
278.93\end{array}$ & $\begin{array}{l}401.50 * \\
401.65\end{array}$ & $\begin{array}{l}122.38 \\
122.42\end{array}$ \\
\hline $299-E 28-28$ & $\begin{array}{l}12 / 12 / 94 \\
12 / 14 / 94\end{array}$ & $\begin{array}{l}285.50 \\
285.25\end{array}$ & $\begin{array}{l}401.05^{\star} \\
401.30\end{array}$ & $\begin{array}{l}122.24 \\
122.32\end{array}$ \\
\hline $299-E 32-10$ & $\begin{array}{l}12 / 13 / 94 \\
12 / 14 / 94\end{array}$ & $\begin{array}{l}236.70 \\
236.36\end{array}$ & $\begin{array}{l}401.18^{*} \\
401.52\end{array}$ & $\begin{array}{l}122.28 \\
122.38\end{array}$ \\
\hline 299-E32-2 & $\begin{array}{l}12 / 13 / 94 \\
12 / 14 / 94\end{array}$ & $\begin{array}{l}269.15 \\
268.82\end{array}$ & $\begin{array}{l}400.91 * \\
401.24\end{array}$ & $\begin{array}{l}122.20 \\
122.30\end{array}$ \\
\hline 299-E32-3 & $\begin{array}{l}12 / 13 / 94 \\
12 / 14 / 94\end{array}$ & $\begin{array}{l}275.31 \\
275.22\end{array}$ & $\begin{array}{l}401.20 * \\
401.29\end{array}$ & $\begin{array}{l}122.29 \\
122.31\end{array}$ \\
\hline 299-E,32-4 & $\begin{array}{l}10 / 11 / 94 \\
12 / 13 / 94 \\
12 / 14 / 94\end{array}$ & $\begin{array}{l}284.79 \\
284.93 \\
284.53\end{array}$ & $\begin{array}{l}401.09 * \\
400.95 * \\
401.35 t\end{array}$ & $\begin{array}{l}122.25 \\
122.21 \\
122.33\end{array}$ \\
\hline $299-E 32-5$ & $\begin{array}{l}12 / 13 / 94 \\
12 / 14 / 94\end{array}$ & $\begin{array}{l}281.22 \\
280.50\end{array}$ & $\begin{array}{l}400.92^{\star}+ \\
401.64\end{array}$ & $\begin{array}{l}122.20 \\
122.42\end{array}$ \\
\hline 299-E32-6 & $\begin{array}{l}12 / 12 / 94 \\
12 / 14 / 94\end{array}$ & $\begin{array}{l}265.95 \\
265.90\end{array}$ & $\begin{array}{l}401.50 * \\
401.55\end{array}$ & $\begin{array}{l}122.38 \\
122.39\end{array}$ \\
\hline 299-E32-7 & $\begin{array}{l}12 / 12 / 94 \\
12 / 14 / 94\end{array}$ & $\begin{array}{l}256.99 \\
256.91\end{array}$ & $\begin{array}{l}401.38^{*} \\
401.46\end{array}$ & $\begin{array}{l}122.34 \\
122.37\end{array}$ \\
\hline 299-E32-8 & $\begin{array}{l}12 / 12 / 94 \\
12 / 14 / 94\end{array}$ & $\begin{array}{l}244.18 \\
244.15\end{array}$ & $\begin{array}{l}401.36 * \\
401.39\end{array}$ & $\begin{array}{l}122.33 \\
122.34\end{array}$ \\
\hline 299-E32-9 & $\begin{array}{l}12 / 13 / 94 \\
12 / 14 / 94\end{array}$ & $\begin{array}{l}241.96 \\
241.33\end{array}$ & $\begin{array}{l}401.37 * \\
402.00+\end{array}$ & $\begin{array}{l}122.34 \\
122.53\end{array}$ \\
\hline $299-E 33-28$ & $\begin{array}{l}12 / 12 / 94 \\
12 / 14 / 94\end{array}$ & $\begin{array}{l}262.98 \\
262.85\end{array}$ & $\begin{array}{l}401.25^{*} \\
401.38\end{array}$ & $\begin{array}{l}122.30 \\
122.34\end{array}$ \\
\hline
\end{tabular}


Table 14-7. RCRA Nater Leve] Measurement Report LLWMA 1, Fourth Quarter 1994. (sheet 2 of 2)

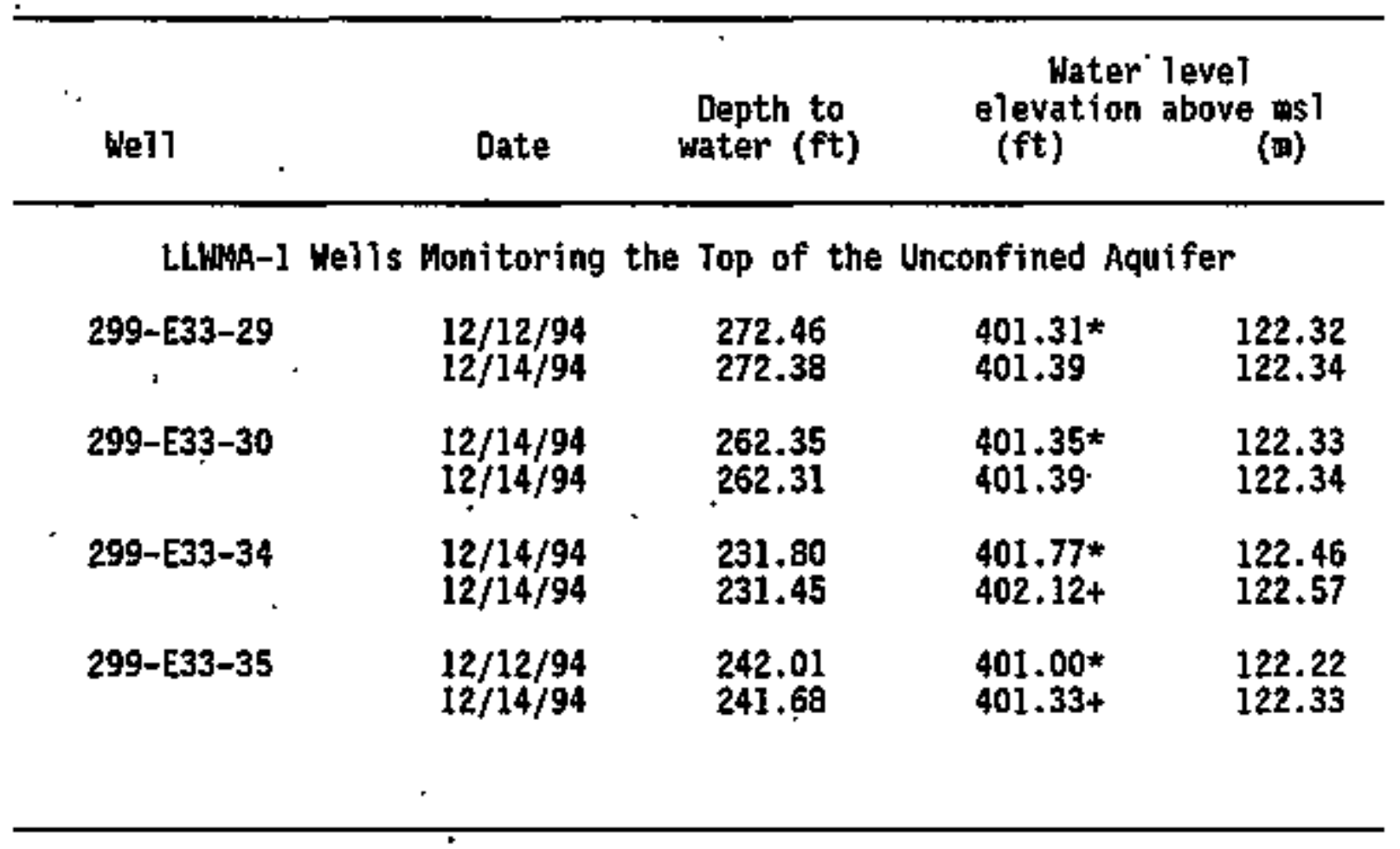

NOTES: 1. Water level elevations are calculated by subtracting the measured depth-to-water fron the surveyed elevation for the well.

2. Depth-to-water values are transcribed from field records.

3. Elevations marked with an '*' were measured at the time of sampiing.

4. Elevations marked with a ' + ' are outside of the expected range, and are suspected of error. 
Table 14-8. RCRA Nater Level Measurement Report LLMMA 2, Fourth Quarter 1994. (sheet 1 of 2)

\begin{tabular}{|c|c|c|c|c|}
\hline Well & Date & $\begin{array}{l}\text { Depth to } \\
\text { water (ft) }\end{array}$ & $\begin{array}{l}\text { Water } \\
\text { elevation } \\
\text { (ft) }\end{array}$ & $\begin{array}{l}1 \\
\text { (e) } m s 1\end{array}$ \\
\hline \multicolumn{5}{|c|}{ LLWA-2 Wells Monitoring the Top of the Unconfined Aquifer } \\
\hline 299-E27-10 & $\begin{array}{l}12 / 13 / 94 \\
12 / 14 / 94\end{array}$ & $\begin{array}{l}223.00 \\
225.50\end{array}$ & $\begin{array}{l}401.47^{*} \\
398.97+\end{array}$ & $\begin{array}{l}122.37 \\
121.61\end{array}$ \\
\hline $299-E 27-11$ & $\begin{array}{l}11 / 18 / 94 \\
12 / 14 / 94\end{array}$ & $\begin{array}{l}241.89 \\
241.88\end{array}$ & $\begin{array}{l}401.40^{*} \\
401.41\end{array}$ & $\begin{array}{l}122.35 \\
122.35\end{array}$ \\
\hline 299-E27-17 & $\begin{array}{l}11 / 18 / 94 \\
12 / 14 / 94\end{array}$ & $\begin{array}{l}233.00 \\
232.94\end{array}$ & $\begin{array}{l}401.72^{\star} \\
401.78\end{array}$ & $\begin{array}{l}122.44 \\
122.46\end{array}$ \\
\hline 299-E27-8 & $\begin{array}{l}11 / 17 / 94 \\
12 / 14 / 94\end{array}$ & $\begin{array}{l}236.73 \\
236.28\end{array}$ & $\begin{array}{l}401.10^{*} \\
401.55\end{array}$ & $\begin{array}{l}122.26 \\
122.39\end{array}$ \\
\hline $299-E 27-9$ & $\begin{array}{l}11 / 17 / 94 \\
12 / 14 / 94\end{array}$ & $\begin{array}{l}227.66 \\
227.50\end{array}$ & $\begin{array}{l}401.55^{*} \\
401.71\end{array}$ & $\begin{array}{l}122.39 \\
122.44\end{array}$ \\
\hline 299-E34-10 & $\begin{array}{l}11 / 21 / 94 \\
12 / 14 / 94\end{array}$ & $\begin{array}{l}238.10 \\
238.01\end{array}$ & $\begin{array}{l}401.67^{*} \\
401.76\end{array}$ & $\begin{array}{l}122.43 \\
122.46\end{array}$ \\
\hline 299-E,34-11 & $\begin{array}{l}11 / 28 / 94 \\
12 / 14 / 94\end{array}$ & $\begin{array}{l}216.27 \\
216.21\end{array}$ & $\begin{array}{l}401.68 * \\
401.74\end{array}$ & $\begin{array}{l}122.43 \\
122.45\end{array}$ \\
\hline $299-E 34-12$ & $\begin{array}{l}11 / 28 / 94 \\
12 / 14 / 94\end{array}$ & $\begin{array}{l}237.28 \\
237.24\end{array}$ & $\begin{array}{l}401.55^{\star} \\
401.59\end{array}$ & $\begin{array}{l}122.39 \\
122.40\end{array}$ \\
\hline 299-E34-2 & $\begin{array}{l}11 / 21 / 94 \\
12 / 13 / 94\end{array}$ & $\begin{array}{l}229.30 \\
229.25\end{array}$ & $\begin{array}{l}401.50^{*} \\
401.55\end{array}$ & $\begin{array}{l}122.38 \\
122.39\end{array}$ \\
\hline 299-E34-3 & $\begin{array}{l}11 / 21 / 94 \\
12 / 14 / 94\end{array}$ & $\begin{array}{l}209.75 \\
209.81\end{array}$ & $\begin{array}{l}401.77^{\star} \\
401.71\end{array}$ & $\begin{array}{l}122.46 \\
122.44\end{array}$ \\
\hline 299-E34-5. & $\begin{array}{l}11 / 18 / 94 \\
12 / 13 / 94\end{array}$ & $\begin{array}{l}190.02 \\
188.13\end{array}$ & $\begin{array}{l}400.77^{\star} * \\
402.66\end{array}$ & $\begin{array}{l}122.15 \\
122.73\end{array}$ \\
\hline 299-E34-9 & $\begin{array}{l}11 / 28 / 94 \\
12 / 14 / 94\end{array}$ & $\begin{array}{l}227.08 \\
226.98\end{array}$ & $\begin{array}{l}401.61 * \\
401.71\end{array}$ & $\begin{array}{l}122.41 \\
122.44\end{array}$ \\
\hline
\end{tabular}




\section{DOE/RL-94-36-4}

Table 14-8. RCRA Nater Level Measurement Report LLWMA 2, Fourth Quarter 1994.

(sheet 2 of 2)

NOTES: 1. Nater level elevations are calculated by subtracting the measured depth-to-water from the surveyed elevation for the well.

2. Depth-to-water values are transeribed from field records.

3. Elevations marked with an ' $\star{ }^{t}$ were measured at the time of sampling.

4. Elevations marked with a ' + ' are outside of the expected range, and are suspected of error. 
Table 14-9. RCRA Water Level Measurement Report LLMA 3, Fourth Quarter 1994.

(sheet 1 of 2)

Wel1

Date

Depth to

water ( $f t$ )

Water level e]evation above ms 1

(ft)

(m)

LLWMA-3 Nells Monjtoring the Top of the Unconfined Aquifer

\begin{tabular}{|c|c|c|c|c|}
\hline 299-W10-13 & $12 / 14 / 94$ & 237.77 & $461.27^{*_{+}}$ & 140.60 \\
\hline $299-W 10-19$ & $\begin{array}{l}12 / 14 / 94 \\
12 / 14 / 94\end{array}$ & $\begin{array}{l}223.74 \\
223.46\end{array}$ & $\begin{array}{l}459.25+ \\
459.53 *\end{array}$ & $\begin{array}{l}139.98 \\
140.06\end{array}$ \\
\hline 299-W6-2 & $\begin{array}{l}11 / 30 / 94 \\
12 / 14 / 94 \\
12 / 14 / 94\end{array}$ & $\begin{array}{l}235.86 \\
236.02 \\
235.77\end{array}$ & $\begin{array}{l}456.59 * \\
456.43 \\
456.68 *\end{array}$ & $\begin{array}{l}139.17 \\
139.12 \\
139.20\end{array}$ \\
\hline 299-w7-1 & $12 / 14 / 94$ & 233.49 & 457.22 & 139.36 \\
\hline $299-W 7-10$ & $12 / 14 / 94$ & 233.85 & 455.81 & 138.93 \\
\hline 299-47-11 & $12 / 14 / 94$ & 224.54 & 456.91 & 139.27 \\
\hline 299-W7-12 & $12 / 14 / 94$ & 230.74 & 457.19 & 139.35 \\
\hline $299+N 7-2$ & $12 / 14 / 94$ & 219.47 & 456.12 & 139.03 \\
\hline 299-W7-4 & $12 / 14 / 94$ & 213.87 & 458.05 & 139.61 \\
\hline 299-W7-5 & $12 / 14 / 94$ & 217.41 & 455.64 & 138.88 \\
\hline $299-W 7-6$ & $12 / 14 / 94$ & 223.14 & 455.50 & 138.84 \\
\hline $299-47-7$ & $12 / 14 / 94$ & 219.26 & 455.68 & 138.89 \\
\hline $299-W 7-9$ & $12 / 14 / 94$ & 234.38 & 457.71 & 139.51 \\
\hline 299-W8-1 & $\begin{array}{l}11 / 30 / 94 \\
12 / 14 / 94\end{array}$ & $\begin{array}{l}243.48 \\
243.62\end{array}$ & $\begin{array}{l}457.85 * \\
457.71\end{array}$ & $\begin{array}{l}139.55 \\
139.51\end{array}$ \\
\hline 299-W9-1 & $12 / 14 / 94$ & 277.41 & $460.32 *$ & 140.31 \\
\hline 299-W10-20 & $\begin{array}{l}12 / 14 / 94 \\
12 / 15 / 94\end{array}$ & $\begin{array}{l}227.56 \\
222.43\end{array}$ & $\begin{array}{l}459.98 \\
465.11{ }^{*}\end{array}$ & $\begin{array}{l}140.20 \\
141.77\end{array}$ \\
\hline $299-W 10-21$ & $12 / 14 / 94$ & 215.69 & $458.37^{*}$ & 139.71 \\
\hline
\end{tabular}


Table 14-9. .RCRA Water Level Heasurement Report

LLLWA 3, Fourth Quarter 1994.

(sheet 2 of 2)

\begin{tabular}{|c|c|c|c|c|}
\hline We11 & Date & $\begin{array}{l}\text { Bepth to } \\
\text { water (ft) }\end{array}$ & $\begin{array}{l}\text { Water } \\
\text { e]evatior } \\
\text { (ft) }\end{array}$ & $\begin{array}{l}\text { leve1 } \\
\text { above ns1 } \\
\begin{array}{l}\text { (a) }\end{array}\end{array}$ \\
\hline \multicolumn{5}{|c|}{ LLMA-3.Wells Monitoring the } \\
\hline 299-W10-14 & $12 / 14 / 94$ & 239.04 & 460.39 & 140.33 \\
\hline \multirow[t]{2}{*}{$299-17-3$} & $12 / 14 / 94$ & 221.03 & 455.11 & 138.72 \\
\hline & & & & . \\
\hline
\end{tabular}

NOTES: 1. Water level elevations are calculated by subtracting the measured depth-to-water from the surveyed elevation for the well.

2. Depth-to-water values are transcribed from field records.

3. Elevations marked with an $" * 1$ were measured at the time of sartpling.

4. Elevations marked with a ' + ' are outside of the expected range, and are suspected of error. 
Table 14-10. RCRA Water Leve1 Measurement Report LLWHA 4, Fourth Quarter 1994. (sheet 1 of 2)

\begin{tabular}{|c|c|c|c|c|}
\hline We1\} & Date & $\begin{array}{l}\text { Depth to } \\
\text { water (ft) }\end{array}$ & $\begin{array}{l}\text { Water } \\
\text { elevation } \\
(\mathrm{ft})\end{array}$ & $\begin{array}{l}\text { level } \\
\text { above msl } \\
\text { (m) }\end{array}$ \\
\hline \multicolumn{5}{|c|}{ LLWWA-4 We]ls Monitoring } \\
\hline $299-W 15-15$ & $11 / 10 / 94$ & 236.53 & $461.43 *$ & 140.64 \\
\hline $299-N 15-16$ & $\begin{array}{l}11 / 08 / 94 \\
12 / 14 / 94\end{array}$ & $\begin{array}{l}222.24 \\
222.63\end{array}$ & $\begin{array}{l}462.65 * \\
462.26\end{array}$ & $\begin{array}{l}141.02 \\
140.90\end{array}$ \\
\hline $299-W 15-18$ & $\begin{array}{l}11 / 08 / 94 \\
12 / 14 / 94\end{array}$ & $\begin{array}{l}223.40 \\
223.57\end{array}$ & $\begin{array}{l}462.31^{*} \\
462.14\end{array}$ & $\begin{array}{l}140.91 \\
140.86\end{array}$ \\
\hline 299-N15-19 & $\begin{array}{l}11 / 30 / 94 \\
12 / 14 / 94\end{array}$ & $\begin{array}{l}229.62 \\
229.31\end{array}$ & $\begin{array}{l}461.98 * \\
462.29+\end{array}$ & $\begin{array}{l}140.81 \\
140.91\end{array}$ \\
\hline $299-W 15-20$ & $\begin{array}{l}11 / 11 / 94 \\
12 / 14 / 94\end{array}$ & $\begin{array}{l}237.30 \\
236.57\end{array}$ & $\begin{array}{l}461.06^{*+} \\
461.79\end{array}$ & $\begin{array}{l}140.53 \\
140.75\end{array}$ \\
\hline 299-W15-23 & $\begin{array}{l}11 / 11 / 94 \\
12 / 14 / 94\end{array}$ & $\begin{array}{l}237.89 \\
237.74\end{array}$ & $\begin{array}{l}461.60^{*} \\
461.75\end{array}$ & $\begin{array}{l}140.70 \\
140.74\end{array}$ \\
\hline $299-W 15-24$ & $\begin{array}{l}11 / 10 / 94 \\
12 / 14 / 94\end{array}$ & $\begin{array}{l}237.63 \\
237.67\end{array}$ & $\begin{array}{l}461.74^{\star} \\
461.70\end{array}$ & $\begin{array}{l}140.74 \\
140.73\end{array}$ \\
\hline 299-W18-21 & $\begin{array}{l}11 / 28 / 94 \\
12 / 14 / 94\end{array}$ & $\begin{array}{l}207.22 \\
206.90\end{array}$ & $\begin{array}{l}461.40^{\star} \\
461.72\end{array}$ & $\begin{array}{l}140.63 \\
140.73\end{array}$ \\
\hline $299-N 18-23$ & $\begin{array}{l}11 / 11 / 94 \\
12 / 14 / 94\end{array}$ & $\begin{array}{l}234.96 \\
235.05\end{array}$ & $\begin{array}{l}461.85^{*} \\
461.76\end{array}$ & $\begin{array}{l}140.77 \\
140.74\end{array}$ \\
\hline $299-W 18-26$ & $\begin{array}{l}11 / 28 / 94 \\
12 / 14 / 94\end{array}$ & $\begin{array}{l}237.49 \\
237.26\end{array}$ & $\begin{array}{l}461.56 \star \\
461.79\end{array}$ & $\begin{array}{l}140.68 \\
140.75\end{array}$ \\
\hline $299-N 18-27$ & $\begin{array}{l}11 / 28 / 94 \\
12 / 14 / 94\end{array}$ & $\begin{array}{l}228.61 \\
228.45\end{array}$ & $\begin{array}{l}461.64^{*} \\
.461 .80\end{array}$ & $\begin{array}{l}140.71 \\
140.76\end{array}$ \\
\hline 299-W18-28 & $\begin{array}{l}11 / 28 / 94 \\
12 / 14 / 94\end{array}$ & $\begin{array}{l}218.25 \\
217.98\end{array}$ & $\begin{array}{l}461.74^{\star} \\
462.01\end{array}$ & $\begin{array}{l}140.74 \\
140.82\end{array}$ \\
\hline 299-W18-32 & $\begin{array}{l}11 / 09 / 94 \\
12 / 14 / 94\end{array}$ & $\begin{array}{l}139.18 \\
214.57\end{array}$ & $\begin{array}{l}537.47^{\star}+ \\
462.08\end{array}$ & $\begin{array}{l}163.82 \\
140.84\end{array}$ \\
\hline
\end{tabular}


Table 14-10. RCFA Water Levei Measurement Report LLWMA 4, Fourth Quarter 1994. (sheet 2 of 2)

\begin{tabular}{|c|c|c|c|}
\hline Helt & Date & $\begin{array}{l}\text { Depth to } \\
\text { water (ft) }\end{array}$ & $\begin{array}{l}\text { Water level } \\
\text { elevation above msi } \\
\text { (ft) }\end{array}$ \\
\hline
\end{tabular}

LLWHA-4 Wells Monitoring the Bottom of the Unconfined Aquifer 299-พ15-17 $11 / 09 / 94$ 221.86 $12 / 14 / 94 \rightarrow 222.58$ $462.78^{*_{4}}$ 462.06 141.06 140.84

299-W18-22

$11 / 29 / 94$ $12 / 14 / 94$

207.16

207.20
$461.33^{*}$

461.29
140.61 140.60

MOTES: I. Water level elevations are calculated by subtracting the measured depth-to-water from the surveyed elevation for the we11.

2. Depth-to-water values are transcribed from field records.

3. Elevations marked with an ' $*$ ' were measured at the time of sampling.

4. Elevations earked with a ' $t$ ' are outside of the expected range, and are suspected of error. 
Table 14-11. RCRA Water Leve] Measurument Report LLWMA 5, Fourth Quarter 1994.

\begin{tabular}{|c|c|c|c|c|}
\hline Well & Date & $\begin{array}{l}\text { Depth to } \\
\text { water (ft) }\end{array}$ & $\begin{array}{l}\text { Nater } \\
\text { eievation } \\
\text { (ft) }\end{array}$ & $\begin{array}{l}\text { level } \\
\text { above } \mathrm{ms} 1 \\
\text { (II) }\end{array}$ \\
\hline \multicolumn{5}{|c|}{ LLWMA-5 Mells Monitoring the Top of the Unconfined Aquifer } \\
\hline $299-411-31$ & $\begin{array}{l}12 / 13 / 94 \\
12 / 14 / 94\end{array}$ & $\begin{array}{l}250.56 \\
250.09\end{array}$ & $\begin{array}{l}456.30 \\
456.77 *+\end{array}$ & $\begin{array}{l}139.08 \\
139.22\end{array}$ \\
\hline 299-W6-10 & $\begin{array}{l}12 / 14 / 94 \\
12 / 14 / 94\end{array}$ & $\begin{array}{l}258.92 \\
258.92\end{array}$ & $\begin{array}{l}453.56 \\
453.56^{\star}\end{array}$ & $\begin{array}{l}138.25 \\
138.25\end{array}$ \\
\hline 299-w6-11 & $12 / 14 / 94$ & 249.13 & 453.73 & 138.30 \\
\hline 299-w6-12 & $12 / 14 / 94$ & 237.71 & 454.80 & 138.62 \\
\hline 299-W6-2 & $\begin{array}{l}11 / 30 / 94 \\
12 / 14 / 94 \\
12 / 14 / 94\end{array}$ & $\begin{array}{l}235.86 \\
236.02 \\
235.77\end{array}$ & $\begin{array}{l}456.59 * \\
456.43 \\
456.68^{*}\end{array}$ & $\begin{array}{l}139.17 \\
139.12 \\
139.20\end{array}$ \\
\hline $299-W 6-4$ & $\begin{array}{r}12 / 14 / 94 \\
12 / 14 / 94\end{array}$ & $\begin{array}{l}245.16 \\
245.15\end{array}$ & $\begin{array}{l}456.09 * \\
456.10\end{array}$ & $\begin{array}{l}139.02 \\
139.02\end{array}$ \\
\hline 299-W6-7 & $12 / 14 / 94$ & 257.37 & 452.91 & 138.05 \\
\hline 299-W6-9 & $12 / 13 / 94$ & 240.85 & $455.25^{\star}+$ & 138.76 \\
\hline $299-w 7-10$ & $12 / 14 / 94$ & 233.85 & 455.81 & 138.93 \\
\hline
\end{tabular}

LLWMA-5 Wells Monitoring the Bottow of the Unconfined Aquifer

$\begin{array}{lllll}299-W 6-3 & 12 / 14 / 94 & 244.07 & 455.76 & 138.92 \\ 299-W 6-6 & 12 / 14 / 94 & 257.17 & 452.82 & 138.02\end{array}$

NOTES: 1. Water Tevel elevations are calcolated by subtracting the measured depth-to-water from the surveyed elevation for . the well.

2. Depth-to-water values are transcribed from field records.

3. Elevations marked with an $* *$ were measured at the time of sampling.

4. Elevations marked with a ' $f$ ' are outside of the expected range, and are suspected of error. 
Table 14-12. Constjtuent List and Submary of Results for the Low-Leve] Haste Management Area 1 Data for Reporting Period

October 1 through Decenter 31, 1994.

(sheet 1 of 3 )

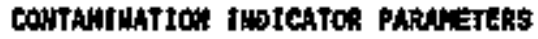

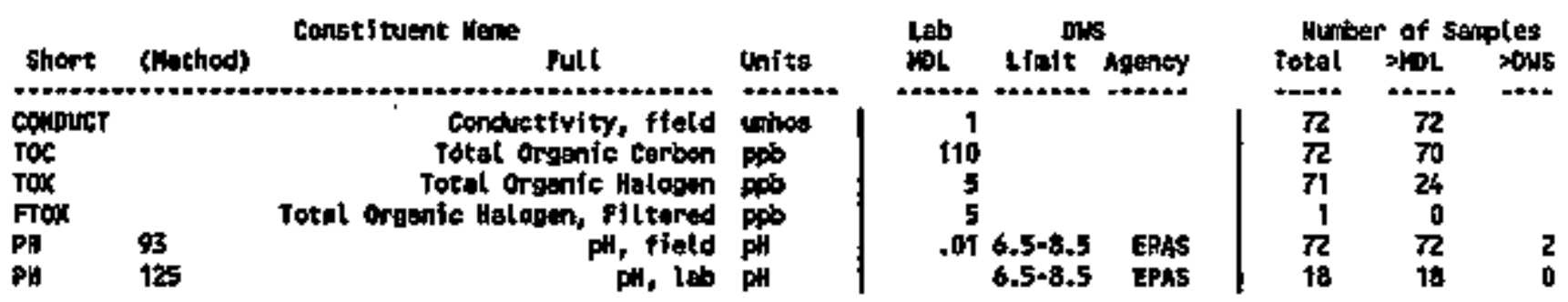

\section{DEINKLW WATER PARALETERS}

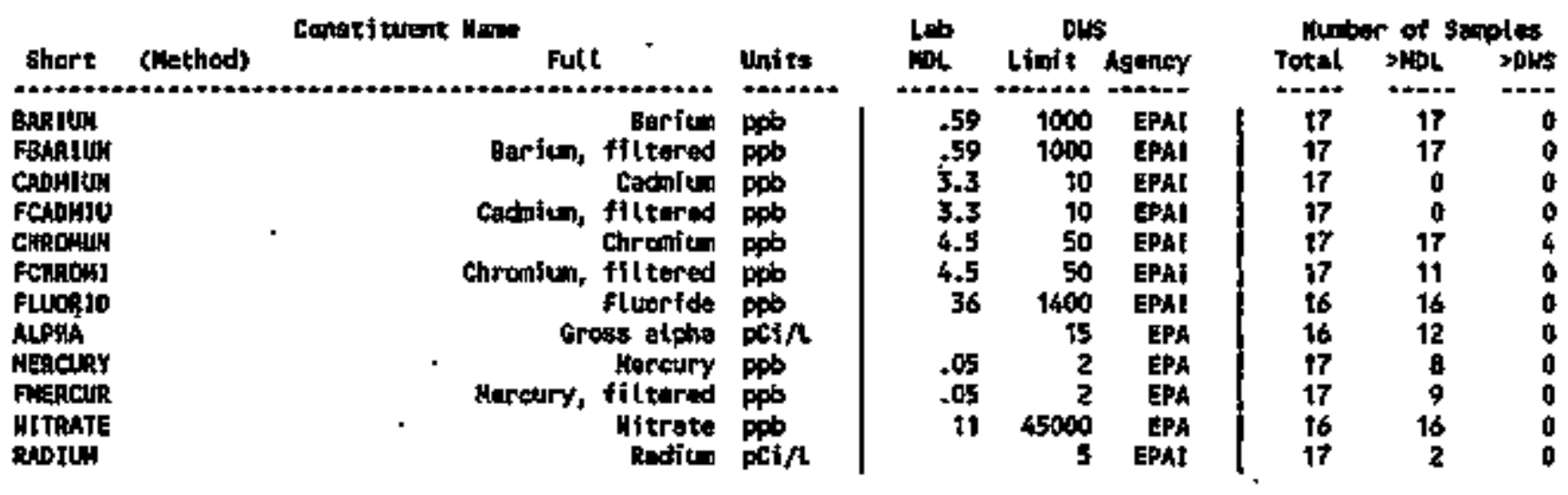

\section{GRCUNDATER CUhLITY PARAHETERS}

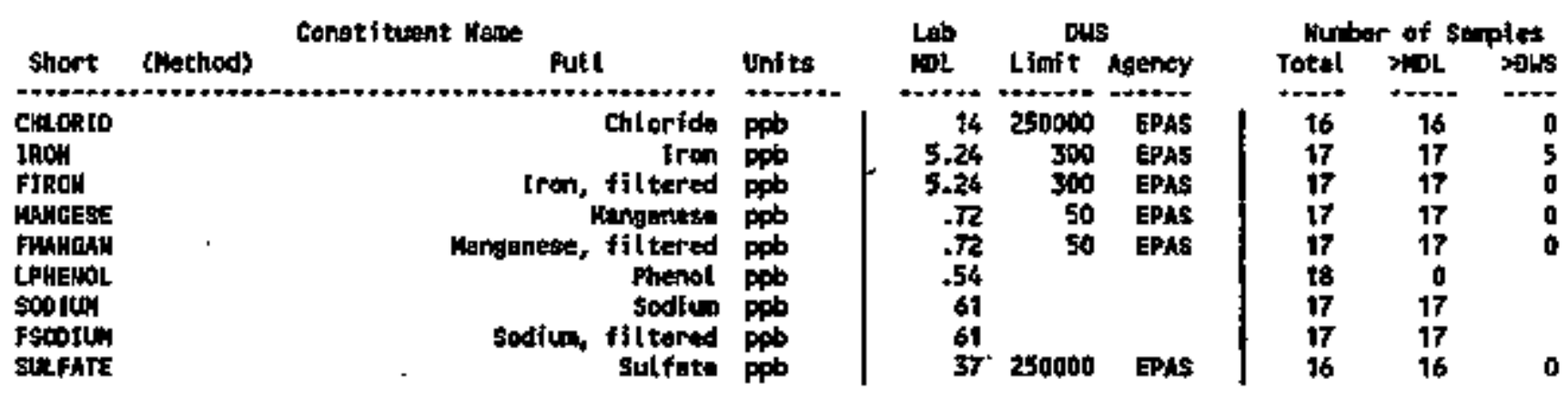


Table 14-12. Constituent List and Summary of Results for the Low-Leve1 Waste Management Area I Data for Reporting Period

October 1 through December 31, 1994.

(sheet 2 of 3 )

SITE SPEC1FIC AN DTHER COHSTITUENTS

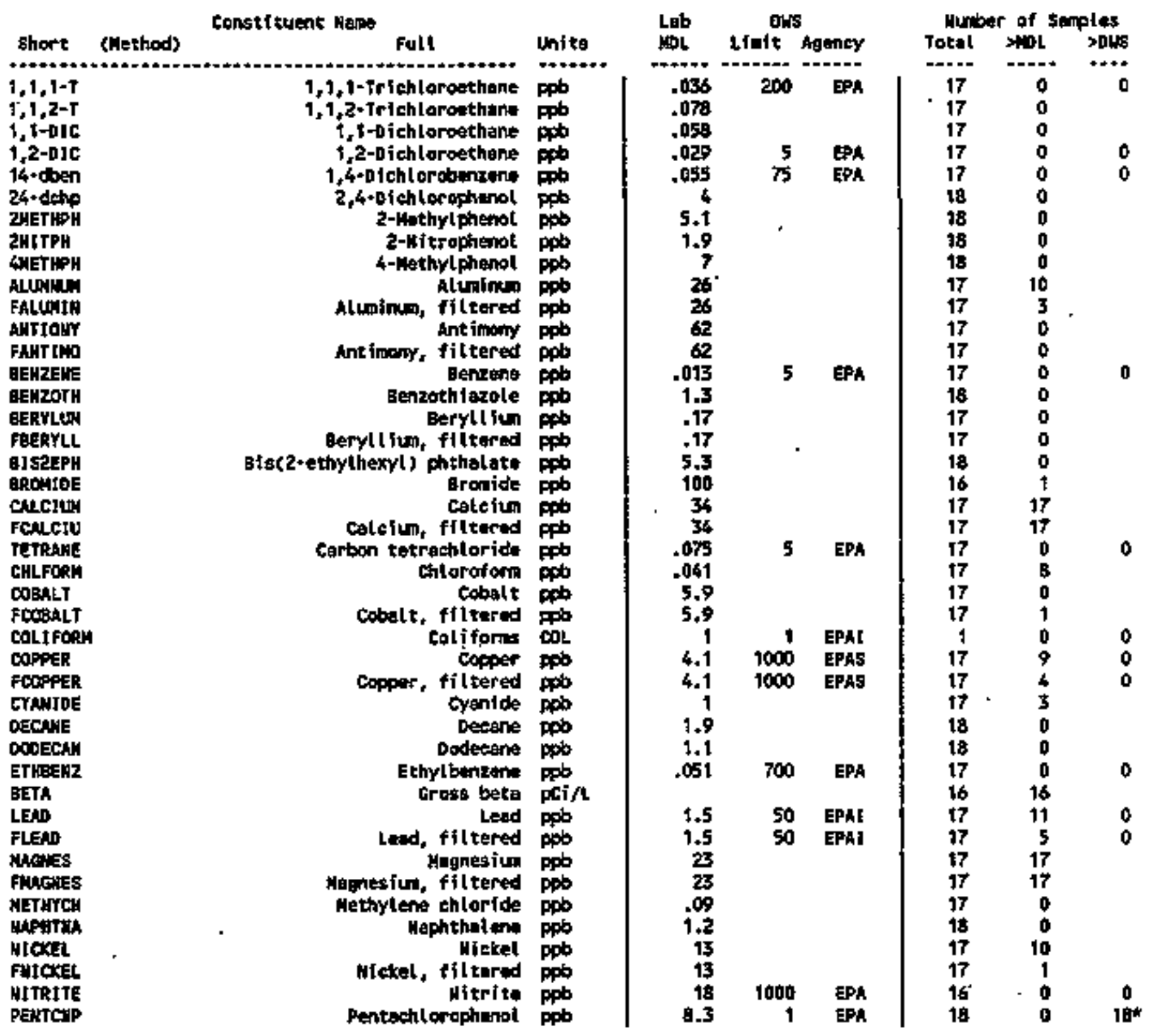


Table 14-12. Constituent List and Summary of Results for the Low-LeveT Waste Management Area 1 Data for Reporting Period

October I through December 31, 1994. (sheet 3 of 3 )

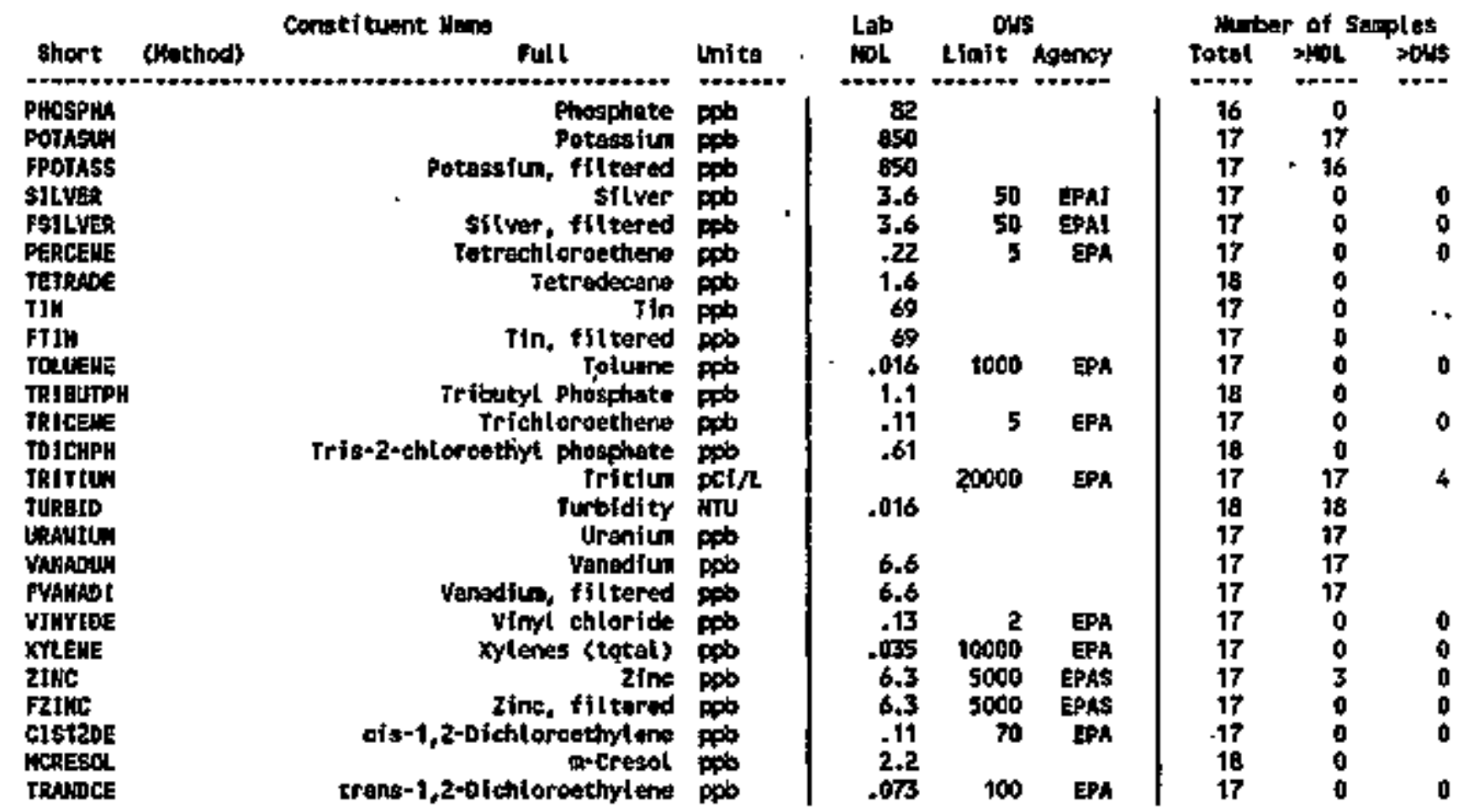

For explanution of thjs zeblo, see settion 1.4 of report. 
Table 14-13. Constituents with at Least One Detected Value for the Low-Level Waste Management Area 1 Data for Reporting Period

October : through December 31, 1994. (sheet I of 9)

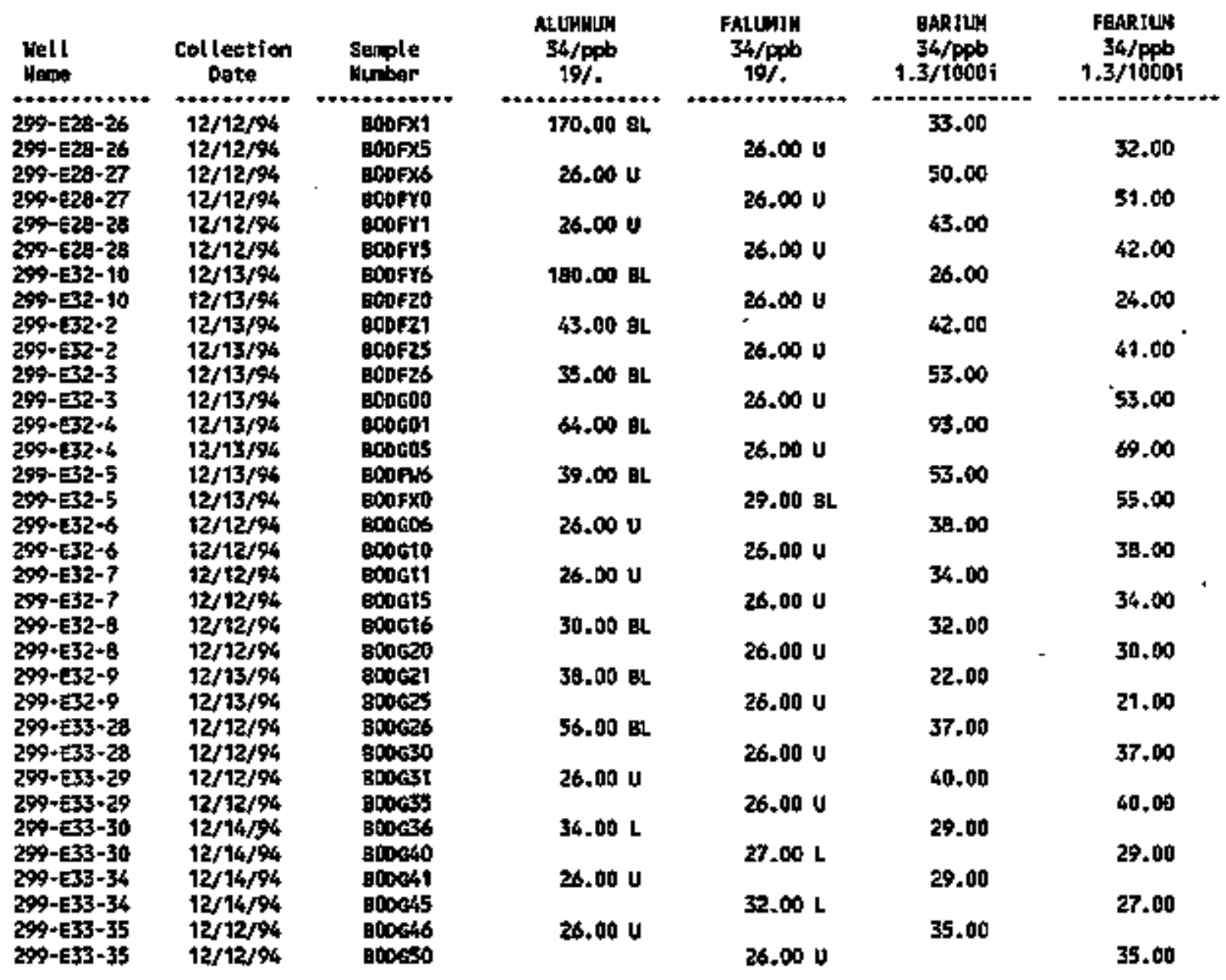

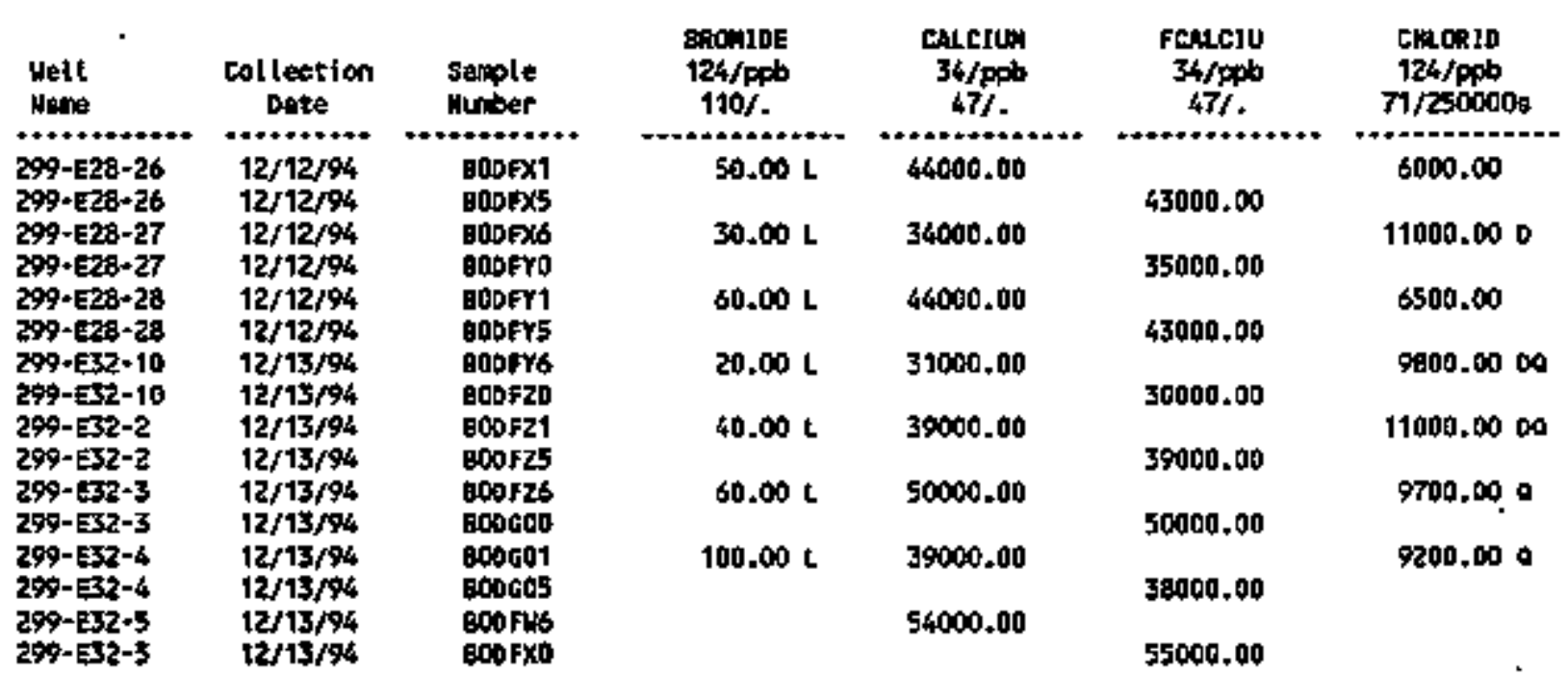


Table 14-13. Constituents with at Least One Detected Value for the Low-Level Waste Management Area 1 Data for Reporting Period October 1 through December 31, 1994.

(sheet 2 of 9 )

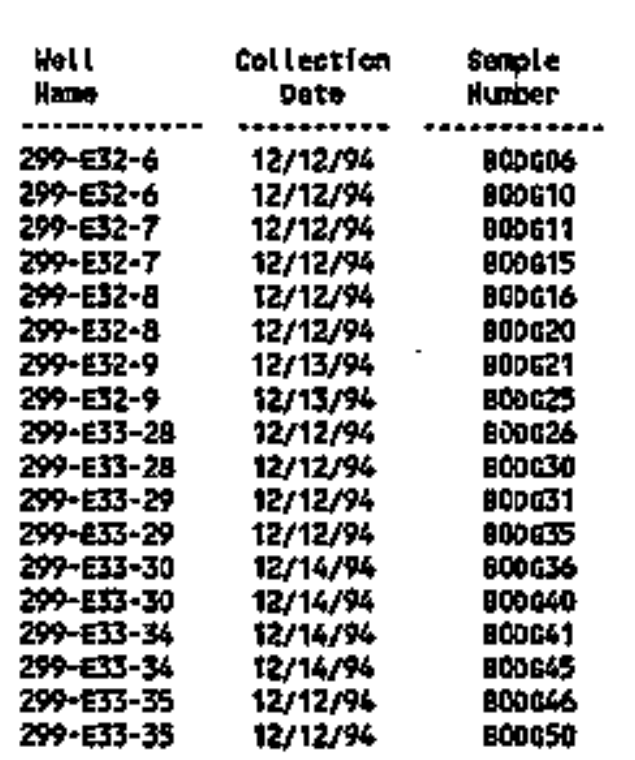

$\begin{array}{cc}\begin{array}{c}\text { EROHIDE } \\ \text { t24/pob } \\ 710 / .\end{array} & \begin{array}{c}\text { CALCIUH } \\ 34 / \mathrm{ppb} \\ 47 / .\end{array} \\ 30.00 \mathrm{~L} & 36000.00 \\ 14.00 \mathrm{~L} & 30000.00 \\ 14.00 \mathrm{~L} & 31000.00 \\ 12.00 \mathrm{~L} & 31000.00 \\ 20.00 \mathrm{~L} & 31000.00 \\ 14.00 \mathrm{~L} & 31000.00 \\ 20.00 \mathrm{~L} & 31000.00 \\ 20.00 \mathrm{~L} & 37000.00 \\ 20.00 \mathrm{~L} & 31000.00\end{array}$

$\begin{gathered}\text { FCALEIU } \\ 34 / F P b \\ 47 / .\end{gathered}$
36000.00
30000.00
31000.00
30060.00
31000.00
31000.00
31000.00
36000.00
31000.00

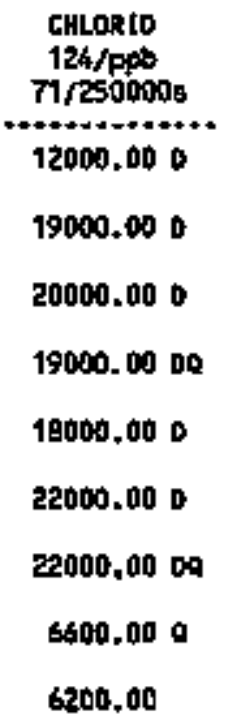

Hell

$299-E 26-26$

299-E28-26

$299-629-27$

$290-629-27$

299-E28-28

299-eZ2-28

299-E32-10

$299-E 32-10$

$299-132+2$

$29 \%-6+2-2$

299-E32-3

299-E32-3

$299-1732-4$

299-E자-4

$299-532-5$

$249-632-5$

299-E-12-6

299-E32-6

299- 5 - $32-7$

299-준-7

299-E준-8

299-E32-8

299-e32-9

299-톤여

299-E33-28

$258-633-28$

299-est-29

299-E33-29

294-E3-30

299-주3-30

$299-633-34$

299-E33-34

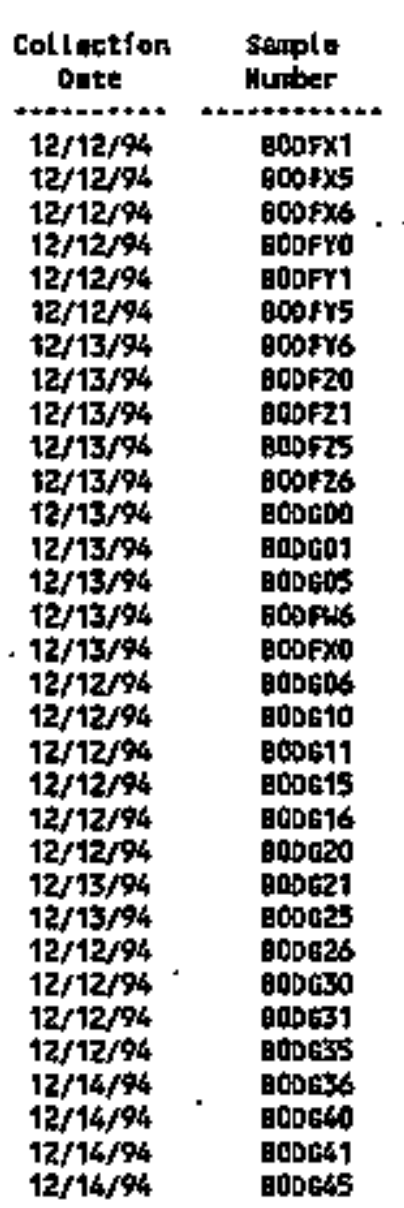

\begin{tabular}{|c|c|}
\hline $\begin{array}{l}\text { chLFORN } \\
3 / \text { pob } \\
05 / .\end{array}$ & $\begin{array}{r}\text { CHEC:14 } \\
34 / \mathrm{Fh} \\
11 / 50 i\end{array}$ \\
\hline $.04 \mathrm{kx}$ & 140.00 \\
\hline $.04 \mathrm{tx}$ & 48.00 \\
\hline $.04 \mathrm{U}$ & $12.00 \mathrm{~L}$ \\
\hline $20 \mathrm{~L}$ & 33.00 \\
\hline $.17 \mathrm{~L}$ & 32.00 \\
\hline $.08 \mathrm{~L}$ & 23.00 \\
\hline $.04 \mathrm{~V}$ & 27.00 \\
\hline $.04 \mathrm{~V}$ & $\$ 9.00$ \\
\hline .040 & 64.00 \\
\hline .64 & 17.00 \\
\hline$-06 v X$ & $\$ 2.00$ \\
\hline .63 & 100.00 \\
\hline $.89 \times$ & 23.00 \\
\hline $.88 \times$ & 23.00 \\
\hline. .494 & 55.00 \\
\hline $.20 \mathrm{l}$ & 24.00 \\
\hline
\end{tabular}

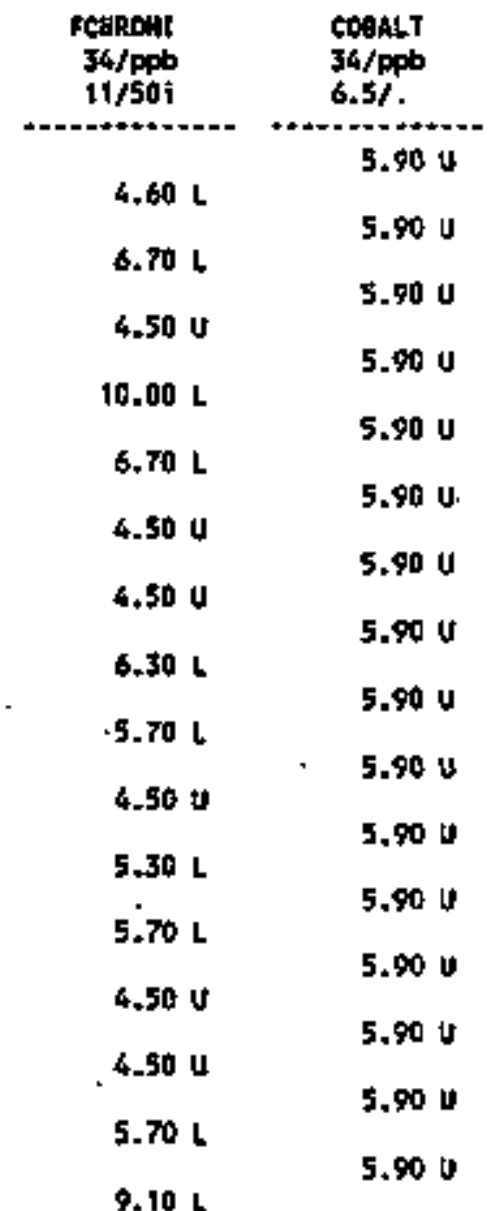


Table 14-13. Constituents with at Least One Detected Value for the Low-Level Waste Management Area 1 Data for Reporting Period October 1 through December 31, 1994. (sheet 3 of 9)

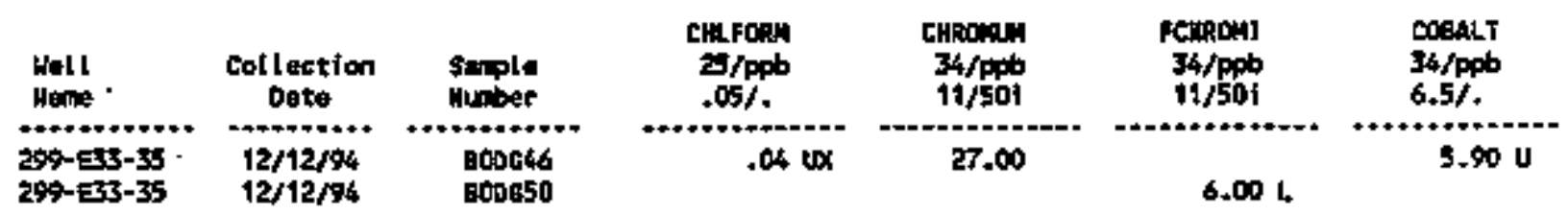

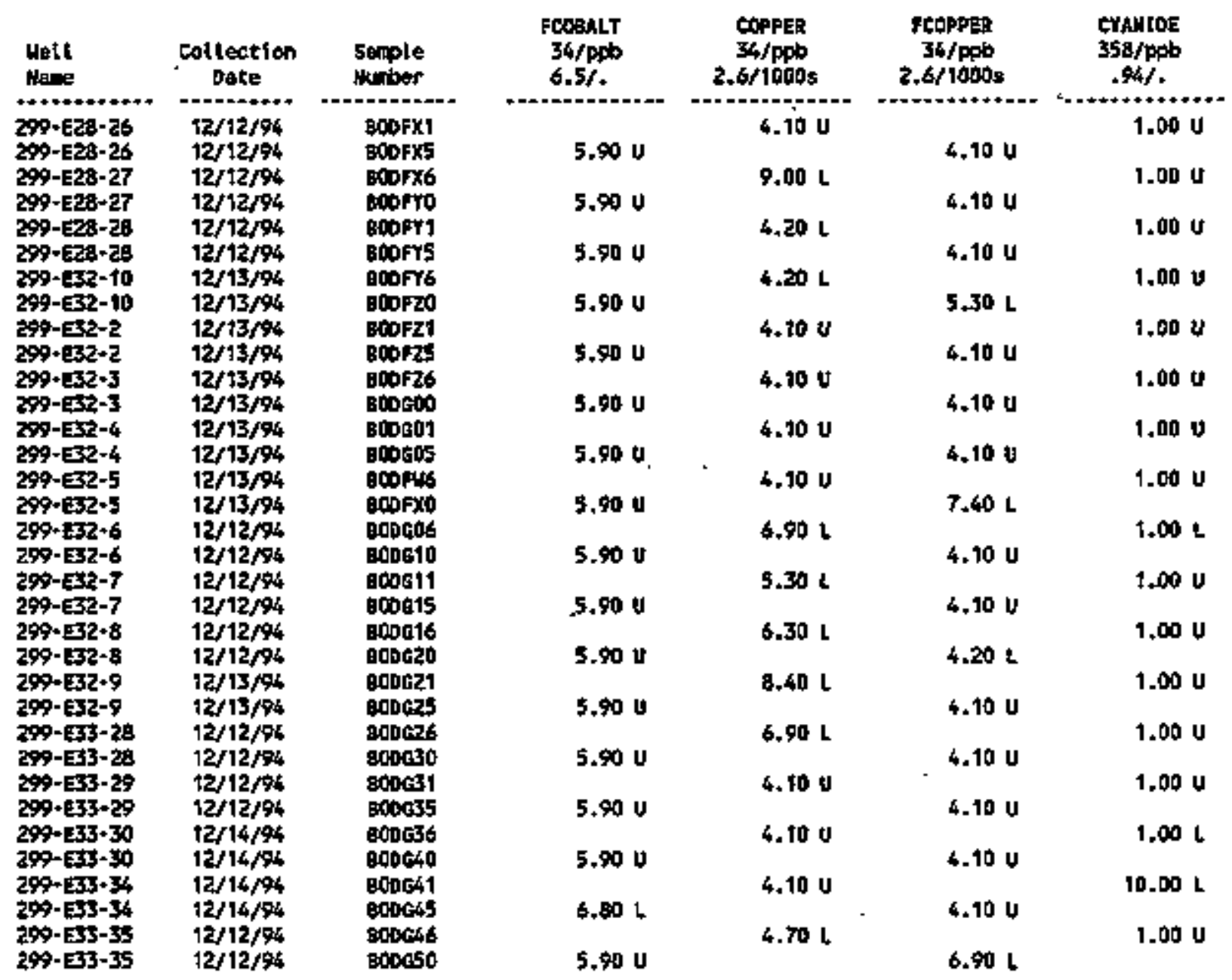

\begin{tabular}{|c|c|c|c|c|c|c|}
\hline Lelt & $\begin{array}{c}\text { Cottectian } \\
\text { Date }\end{array}$ & $\begin{array}{l}\text { Suple } \\
\text { Nutber }\end{array}$ & $\begin{array}{l}\text { FLuoR to } \\
\text { 124/ppo } \\
51 / 1400 \mathrm{i}\end{array}$ & $\begin{array}{c}\text { ALPHR } \\
135 / \mathrm{pCi} / \mathrm{L} \\
\text {,f15 }\end{array}$ & $\begin{array}{c}\text { BETA } \\
\text { 136/pCi/L } \\
+K\end{array}$ & $\begin{array}{c}\text { JRON } \\
34 / \mathrm{pos} \\
18 / 300 \mathrm{~s}\end{array}$ \\
\hline $\begin{array}{l}299-\mathrm{e} 28-26 \\
299+\mathrm{E} 28-27 \\
299-628-28 \\
299-632-10 \\
299-E 32-2 \\
299-632-3 \\
299-E 32-4\end{array}$ & $\begin{array}{l}12 / 12 / 94 \\
12 / 12 / 94 \\
12 / 12 / 94 \\
12 / 13 / 96 \\
12 / 13 / 94 \\
12 / 13 / 94 \\
12 / 13 / 94\end{array}$ & 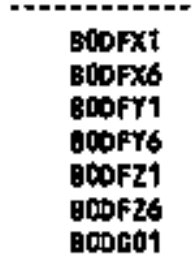 & $\begin{array}{l}400.00 \\
600.00 \\
500.00 \\
500.00 \\
800.00 \\
600.00 \\
700.00\end{array}$ & $\begin{array}{r}7.91 \\
7.74 \\
11.70 \\
1.99 \\
6.90 \\
11.80 \\
1.38\end{array}$ & $\begin{array}{r}10.50 \\
13.30 \\
9.19 \\
34.90 \\
13.50 \\
12.60 \\
11.60\end{array}$ & $\begin{array}{r}860.0060 \\
210.00 \mathrm{00} \\
61.00 \mathrm{60} \\
460.00 \mathrm{60} \\
140.00 \mathrm{60} \\
86.00 \mathrm{B0} \\
6400.00 \mathrm{00}\end{array}$ \\
\hline
\end{tabular}


Table 14-13. Constjtuents with at Least One Detected VaTue for the Low-Level Waste Management Area 1 Data for Reporting Period October 1 through Decenber 31, 1994. (sheet 4 of 9)

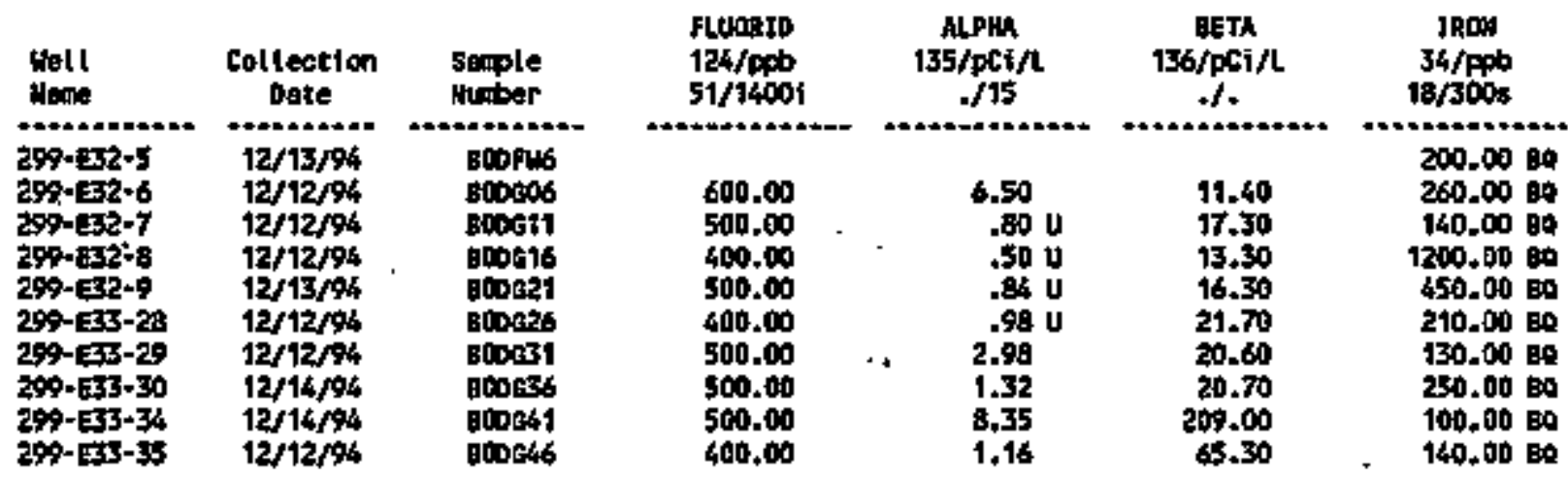

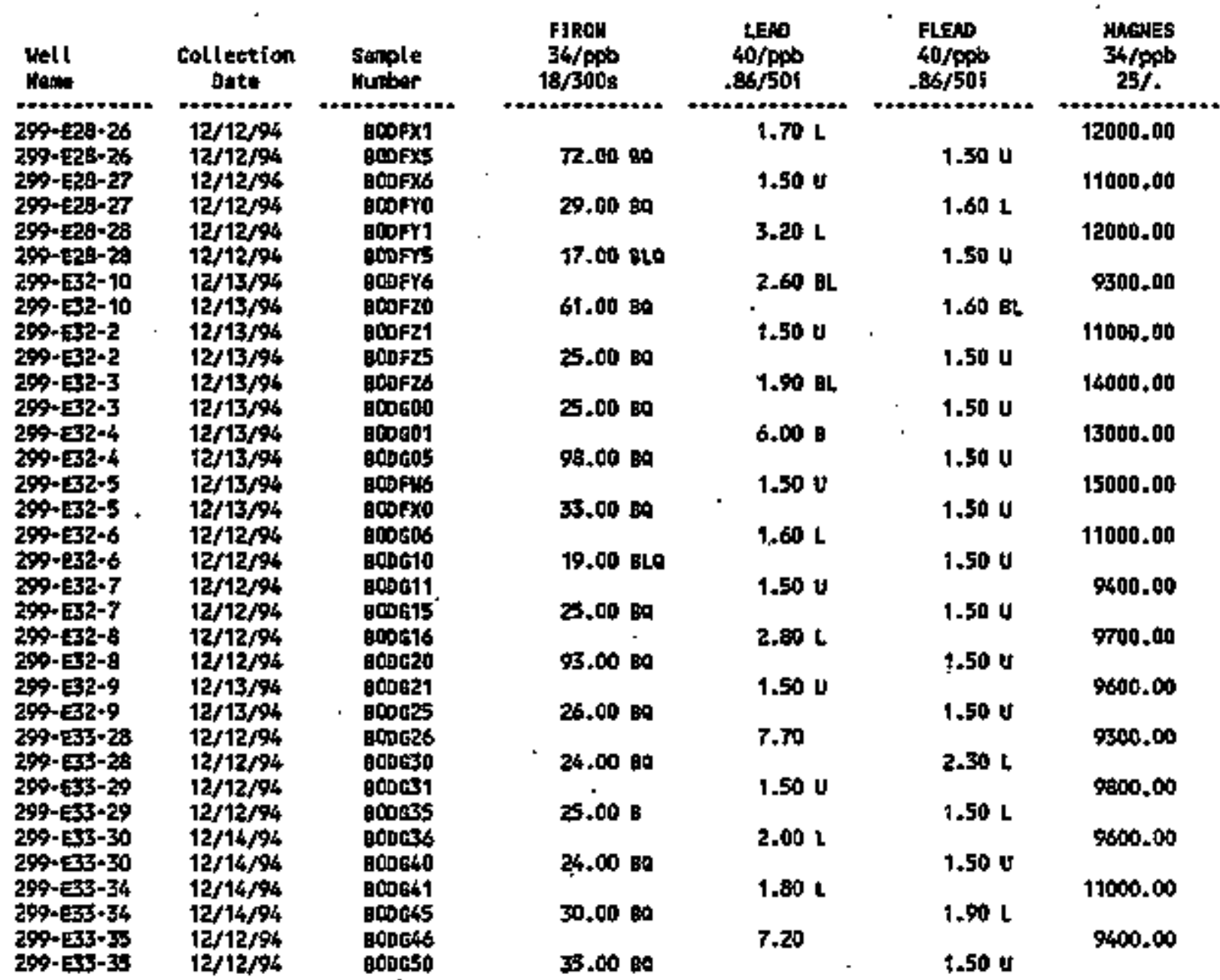


Table 14-13. Constituents with at Least One Detected. Value for the Low-Level Waste Management Area 1 Data for Reporting Period

October 1 through Decenber 31, 1994.

(sheet 5 of 9)

\begin{tabular}{|c|c|c|c|c|c|c|}
\hline Lelt & $\begin{array}{c}\text { Cotlection } \\
\text { Dote }\end{array}$ & $\begin{array}{l}\text { sample } \\
\text { Henter }\end{array}$ & $\begin{array}{l}\text { FWAGHES } \\
34 / \mathrm{ppb} \\
25 \% .\end{array}$ & $\begin{array}{c}\text { MNIGESE } \\
34 / \mathrm{ppb} \\
\text { t/509 }\end{array}$ & $\begin{array}{c}\text { Funwas } \\
34 / \mathrm{ppb} \\
1 / 509\end{array}$ & $\begin{array}{l}\text { HERCURY } \\
41 / p p b 6 \\
.095 / 2\end{array}$ \\
\hline $290+529-26$ & $12 / 12 / 96$ & BODFX1 & .... & 16.00 & 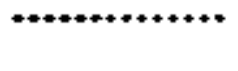 & .0s to \\
\hline $299-628-26$ & $12 / 12 \pi 94$ & B00Fx5 & 11000,00 & & $2.10 \mathrm{~L}$ & \\
\hline $\begin{array}{l}299-E 28-27 \\
299+E 28-27\end{array}$ & $\begin{array}{l}12 / 12 / 44 \\
12 / 12 / 04\end{array}$ & $\begin{array}{l}\text { BobFx6 } \\
\text { BOofro }\end{array}$ & t1800, o0 & $5.30 \mathrm{~L}$ & 20 & D U \\
\hline $299-628-28$ & $12 / 12 / 94$ & siorrt & 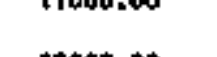 & 3.606 & & as \\
\hline $\begin{array}{l}299-E 28-28 \\
299-E 32-10\end{array}$ & $\begin{array}{l}12 / 12 / 94 \\
12 / 13 / 46\end{array}$ & $\begin{array}{l}\text { BODFY5 } \\
\text { BDOFYS }\end{array}$ & 120000 & 9.50 L & $3,30 \mathrm{~L}$ & .10 BLO \\
\hline $299-532-10$ & $12 / 13 / 94$ & BLPET & $9200+100$ & & $5.90 \mathrm{~L}$ & \\
\hline $\begin{array}{l}29 \%-E 32-2 \\
299-E 32-2\end{array}$ & $12 / 13 / 94$ & $\begin{array}{l}\text { BCOFz1 } \\
\text { BODF25 }\end{array}$ & 11000.00 & $2.60 \mathrm{~L}$ & 1401 & $.13 \mathrm{BLO}$ \\
\hline $209-132-3$ & $12 / 13 / 94$ & cotezes & 11000000 & $1.50 \mathrm{~L}$ & $1+960$ & .09 a<co \\
\hline $\begin{array}{l}298-6927-3 \\
299--32-4\end{array}$ & $\begin{array}{l}12 / 13 / 94 \\
12 / 13 / 44\end{array}$ & $\begin{array}{l}800000 \\
\operatorname{Bopec01}\end{array}$ & 14000.00 & 17.00 & $1.60 \mathrm{~L}$ & .t1 BEO \\
\hline $\begin{array}{l}299-132-4 \\
299-E 32-5\end{array}$ & & 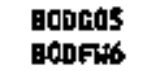 & 12000.00 & & $2.80 \mathrm{l}$ & \\
\hline $\begin{array}{l}299-532-5 \\
299-E 2-6\end{array}$ & $\begin{array}{l}12 / 13 / 94 \\
12 / 12 / 54\end{array}$ & $\begin{array}{l}\text { 8005EX0 } \\
\text { 1001506 }\end{array}$ & $150,00.00$ & $8.60 \mathrm{~L}$ & $1.40 \mathrm{~L}$ & os uo \\
\hline $298-122-6$ & $12 / 12 / 84$ & 800610 & 11000.00 & & 1.80 & \\
\hline $\begin{array}{l}299-E 32-7 \\
299-E 32-7\end{array}$ & $\begin{array}{l}12 / 12 / 94 \\
12 / 12 / 94\end{array}$ & $\begin{array}{l}800011 \\
800015\end{array}$ & $\$ 400.00^{\circ}$ & $3.50 \mathrm{~L}$ & 2.601 & 05 \\
\hline $\begin{array}{l}290-132-8 \\
299-E 32-8\end{array}$ & $12 / 12 / 94$ & 0001616 & $9700, \infty 0$ & 8,806 & 29 & .0500 \\
\hline 299-Ex2-9 & $12 / 13 / 94$ & BotoG21 & (א. & 12.00 & 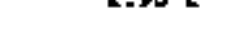 & .11 BLO \\
\hline $\begin{array}{l}299-E 32-9 \\
299+E 33-28\end{array}$ & $\begin{array}{l}12 / 13 / 94 \\
12 / 12 / \%\end{array}$ & $\begin{array}{l}80,0025 \\
600026\end{array}$ & 9500.00 & $5.20 \mathrm{~L}$ & $4.00 \mathrm{~L}$ & 150 \\
\hline 299-E33-28 & $12 / 12 / 9 \%$ & 800050 & 9300.00 & & $1.30 \mathrm{~L}$ & \\
\hline $\begin{array}{l}299-E 33-29 \\
299-E 33-29\end{array}$ & $\begin{array}{l}12 / 12 / 94 \\
12 / 12 / 94\end{array}$ & $\begin{array}{l}800631 \\
800635\end{array}$ & 9900,10 & $3.50 \mathrm{~L}$ & $1.30 \mathrm{~L}$ & .05 Uq \\
\hline $290-133-50$ & $12 / 14 / 94$ & 900036 & & $5.30 \mathrm{~L}$ & & .11 BLO \\
\hline $\begin{array}{l}299-673-39 \\
299- \pm 33-34\end{array}$ & $\begin{array}{l}12 / 14 / 94 \\
12 / 14 / 48\end{array}$ & $\begin{array}{l}8000000 \\
800041\end{array}$ & 9900,00 & $2.10 \mathrm{~L}$ & 1 & 13 BLO \\
\hline $33-34$ & $12 / 14 / 94$ & 800045 & 11000.00 & & $.95 \mathrm{~L}$ & \\
\hline$\frac{29-033-35}{299-033-35}$ & $\begin{array}{l}12 / 12 / 94 \\
12 / 12 / 96\end{array}$ & $\begin{array}{l}\text { B001646 } \\
B 00550\end{array}$ & 0500 & 3.766 & & 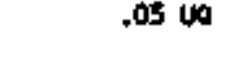 \\
\hline & 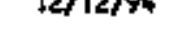 & & & & & \\
\hline
\end{tabular}


Table I4-13. Constjtuents with at Least One Detected Value for the Low-Level Haste Management Area 1 Data for Reporting Period Dctober 1 through December 31, 1994. (sheet 6 of 9 )

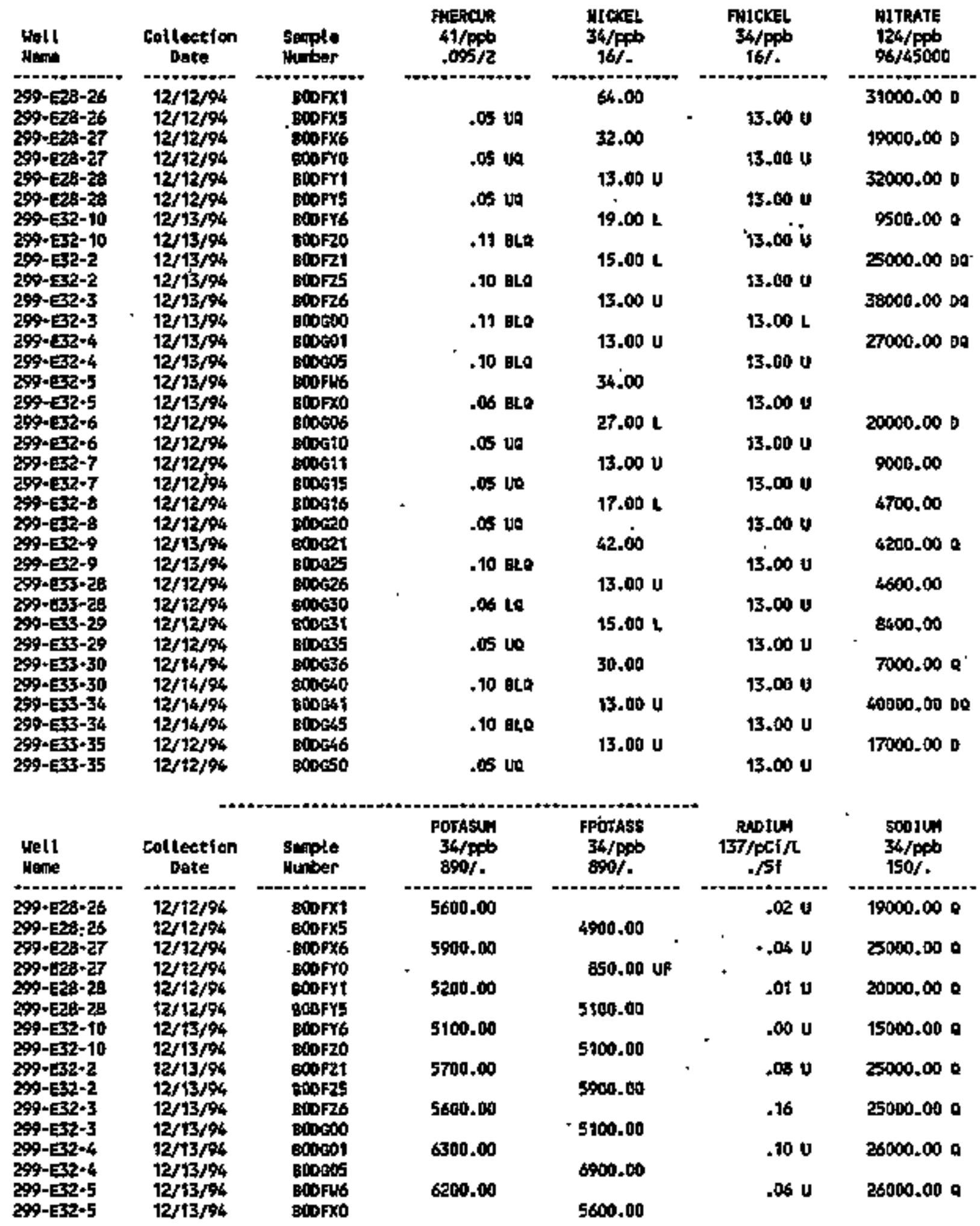


Table 14-13. Constituents with at Least One Detected Value for the Low-Level Waste Management Area 1 Data for Reporting Period

October 1 through December 31, 1994.

(sheet 7 of 9)

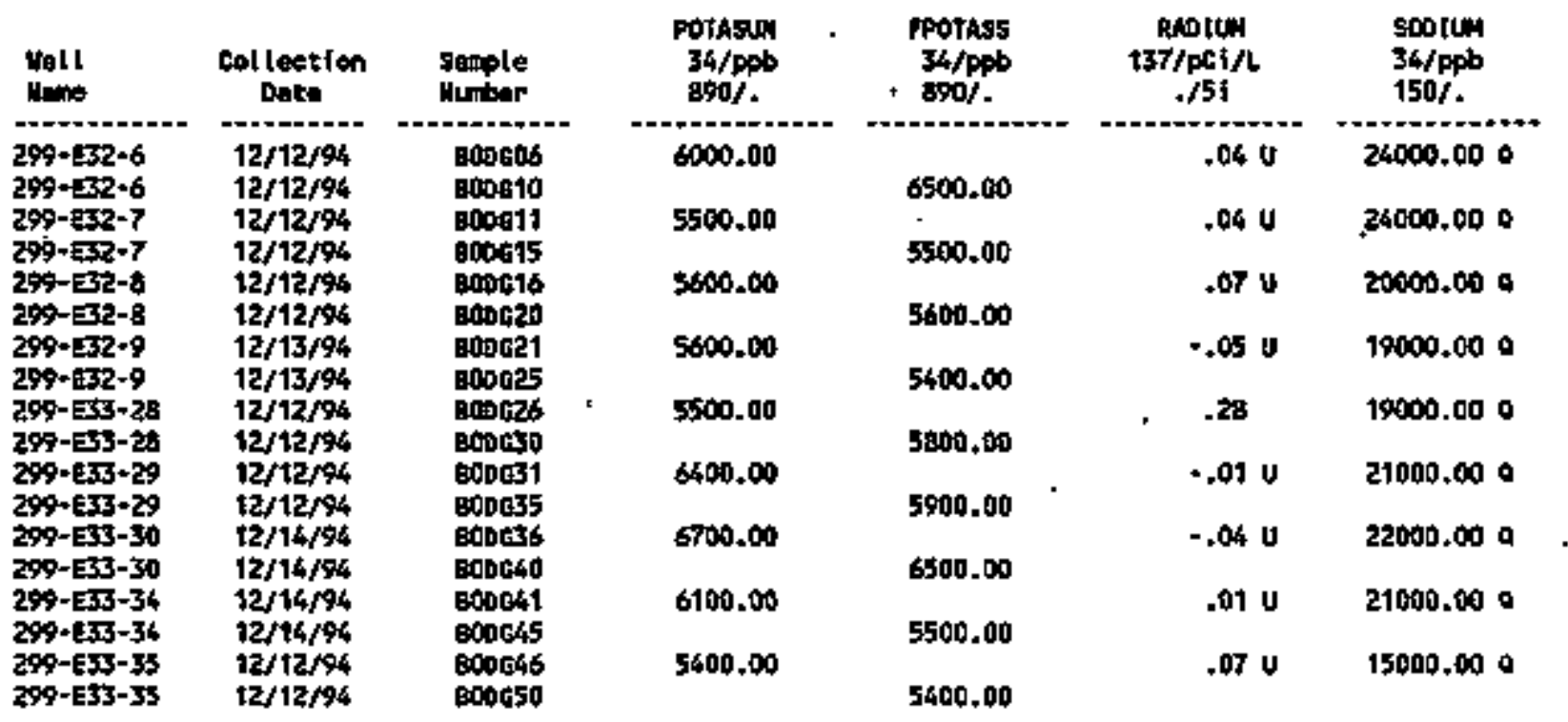

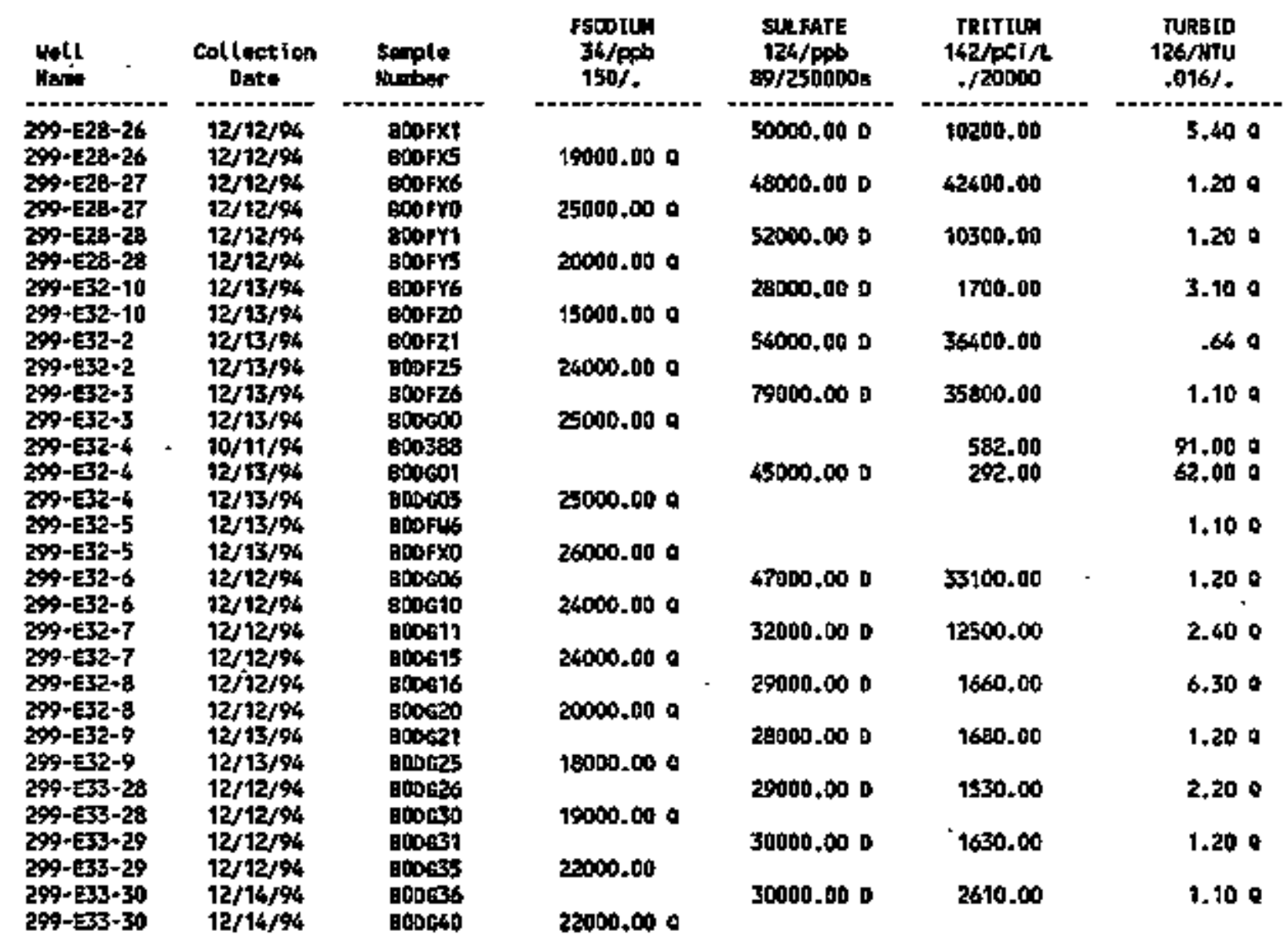


Table 14-13. Constituents with at Least one Detected Value for the Low-Level Waste Management Area 1 Data for Reporting Pertod

October 1 through December 31, 1994. (sheet 8 of 9)

\begin{tabular}{|c|c|c|c|c|c|c|}
\hline $\begin{array}{l}\text { Well } \\
\text { Wame }\end{array}$ & $\begin{array}{c}\text { Colteotion } \\
\text { Dote }\end{array}$ & Striple & $\begin{array}{c}\text { Fsobjutil } \\
34 / \mathrm{ppb} \\
150 / .\end{array}$ & $\begin{array}{c}\text { SULFATE } \\
\text { 124/Fpb } \\
\text { a9/2500019 }\end{array}$ & $\begin{array}{c}\text { TR1T Iw } \\
\text { t42/pef/L } \\
. / 20000\end{array}$ & $\begin{array}{c}\text { TURBID } \\
\text { 126/4DU } \\
.016 / .\end{array}$ \\
\hline $\begin{array}{l}299 \cdot E 33-36 \\
299-E 35-36 \\
299-E 33-35 \\
299-E 33-35\end{array}$ & $\begin{array}{l}12 / 14 / 94 \\
12 / 14 / 94 \\
12 / 12 / 44 \\
12 / 12 / 94\end{array}$ & $\begin{array}{l}\text { E00061 } \\
\text { goposs } \\
\text { gopest } \\
\text { sonoso }\end{array}$ & $\begin{array}{l}20000.000 \\
15000.000\end{array}$ & $\begin{array}{l}32000.000 \\
24000.005\end{array}$ & $\begin{array}{l}5740.00 \\
2780.00\end{array}$ & $\begin{array}{c}.520 \\
+ \\
1.409\end{array}$ \\
\hline
\end{tabular}

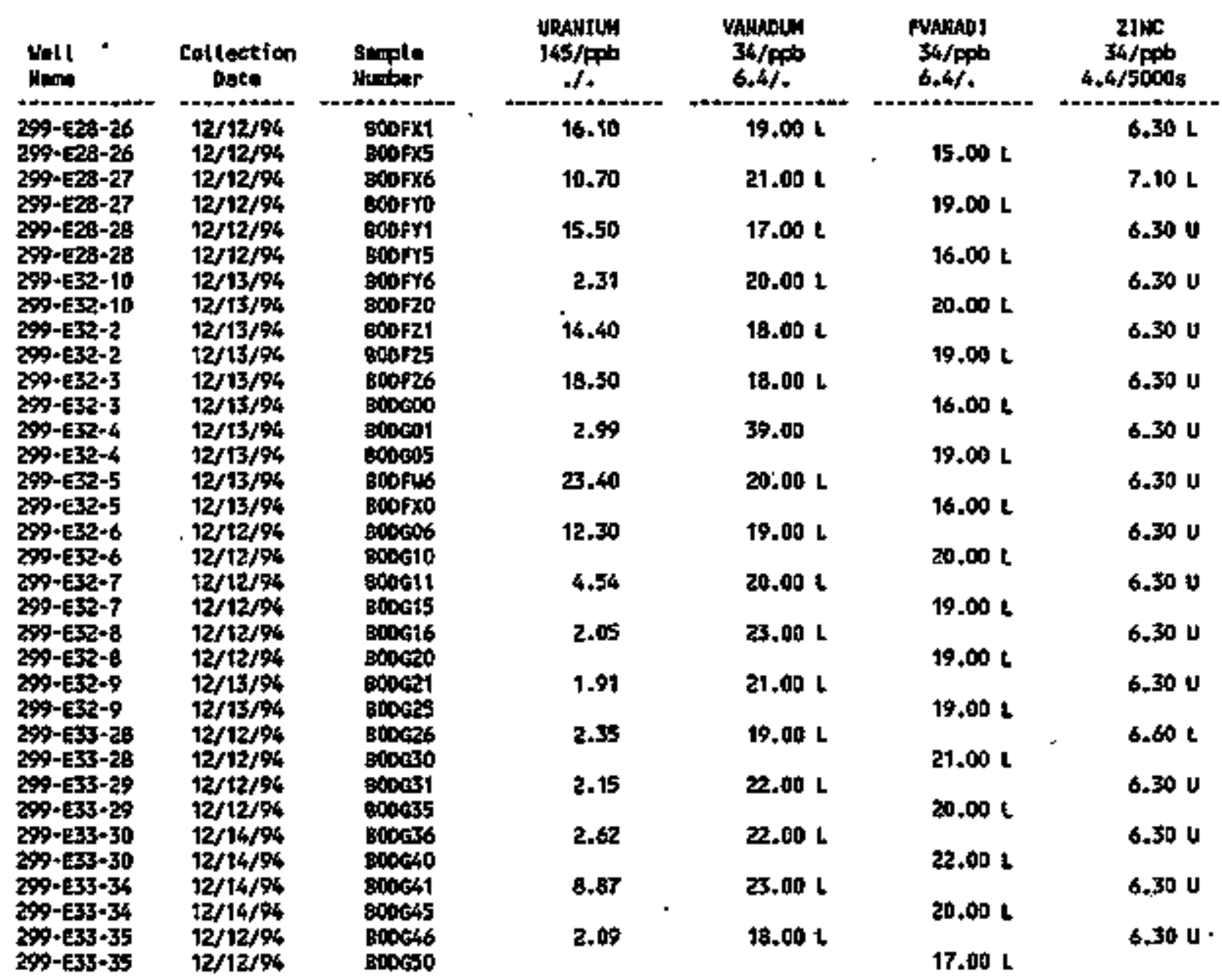


Table I4-13. Constituents with at Least One Detected Value for the Low-Level Waste Hanagement Area 1 Data for Reporting Period

October I through December 31, 1994. (sheet 9 of 9 )

\begin{tabular}{|c|c|c|c|c|}
\hline Helt & $\begin{array}{c}\text { Coltectjor } \\
\text { Qote }\end{array}$ & $\begin{array}{l}\text { 8nple } \\
\text { Therber }\end{array}$ & $\begin{array}{c}12110 \\
\text { 34/pep } \\
4.4 / 50000\end{array}$ & \\
\hline 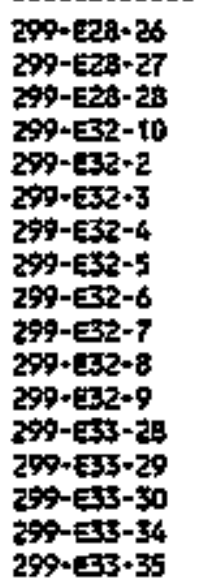 & $\begin{array}{l}12 / 12 / 94 \\
12 / 12 / 94 \\
12 / 12 / 94 \\
12 / 13 / 94 \\
12 / 13 / 94 \\
12 / 13 / 94 \\
12 / 13 / 94 \\
12 / 13 / 94 \\
12 / 12 / 94 \\
12 / 12 / 94 \\
12 / 12 / 94 \\
12 / 13 / 94 \\
12 / 12 / 94 \\
12 / 12 / 94 \\
12 / 14 / 94 \\
12 / 14 / 94 \\
12 / 12 / 94\end{array}$ & 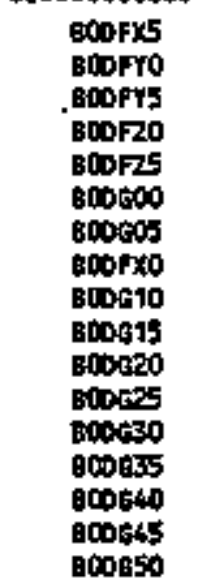 & $\begin{array}{l}6.30 \mathrm{U} \\
6.30 \mathrm{U} \\
6.30 \mathrm{U} \\
6.30 \mathrm{U} \\
6.30 \mathrm{U} \\
6.30 \mathrm{U} \\
6.30 \mathrm{U} \\
6.30 \mathrm{U} \\
6.30 \mathrm{U} \\
6.30 \mathrm{U} \\
6.30 \mathrm{U} \\
6.30 \mathrm{U} \\
6.30 \mathrm{U} \\
6.30 \mathrm{U} \\
6.30 \mathrm{U} \\
6.30 \mathrm{U} \\
6.30 \mathrm{U}\end{array}$ & r \\
\hline
\end{tabular}

Far explanation of this table, sep section 1.4 of report. 
Table 14-14. Contamination Indicator Parameters for the Low-Leve]

Waste Management Area I Data for Reporting Period

October 1 through December 31, 1994.

(sheet 1 of 2)

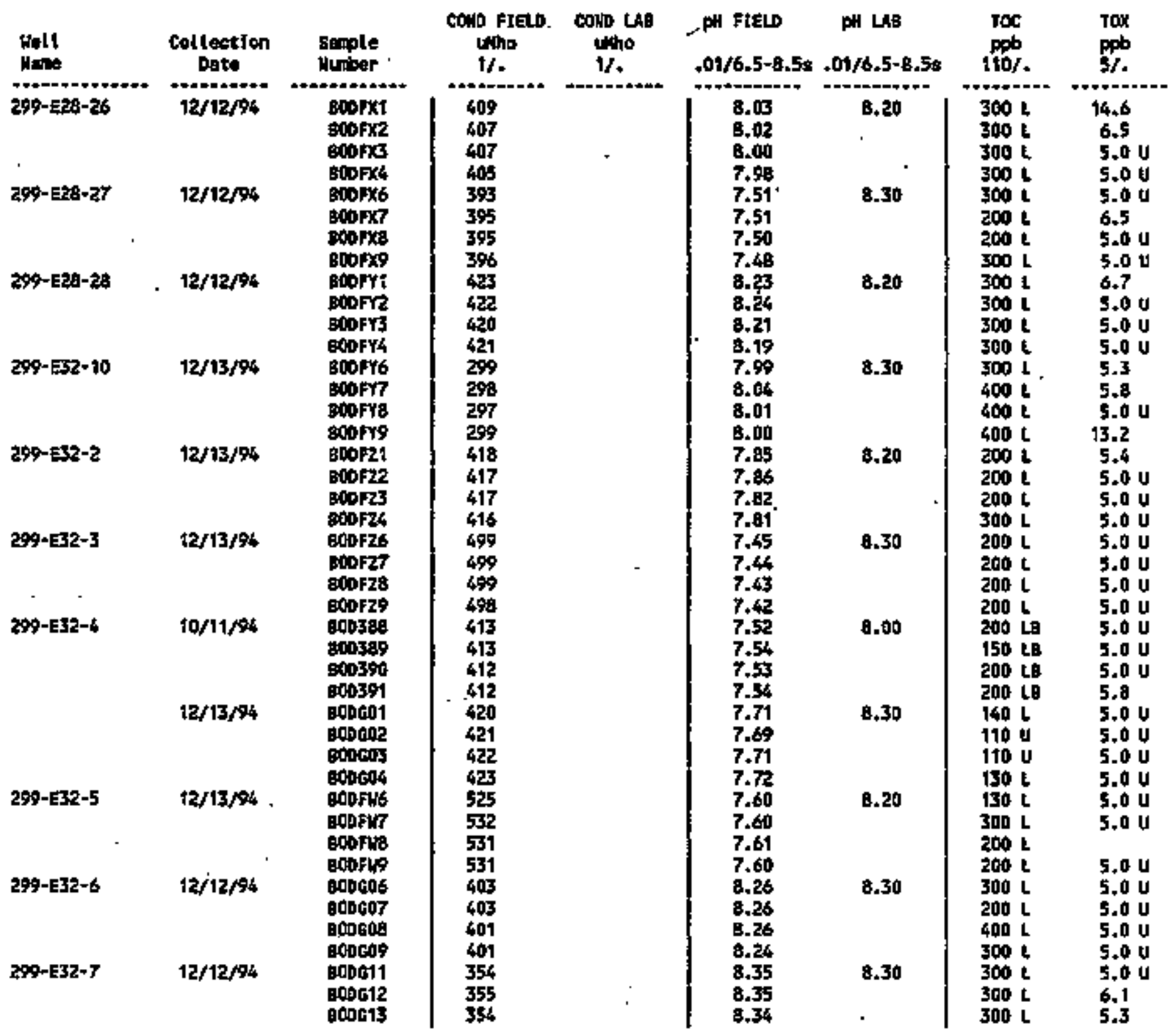


Table 14-14. Contamination Indicator Parameters for the Low-Leve1 Waste Management Area I Data for Reporting Period

October 1 through December 31, 1994.

(sheet 2 of 2)

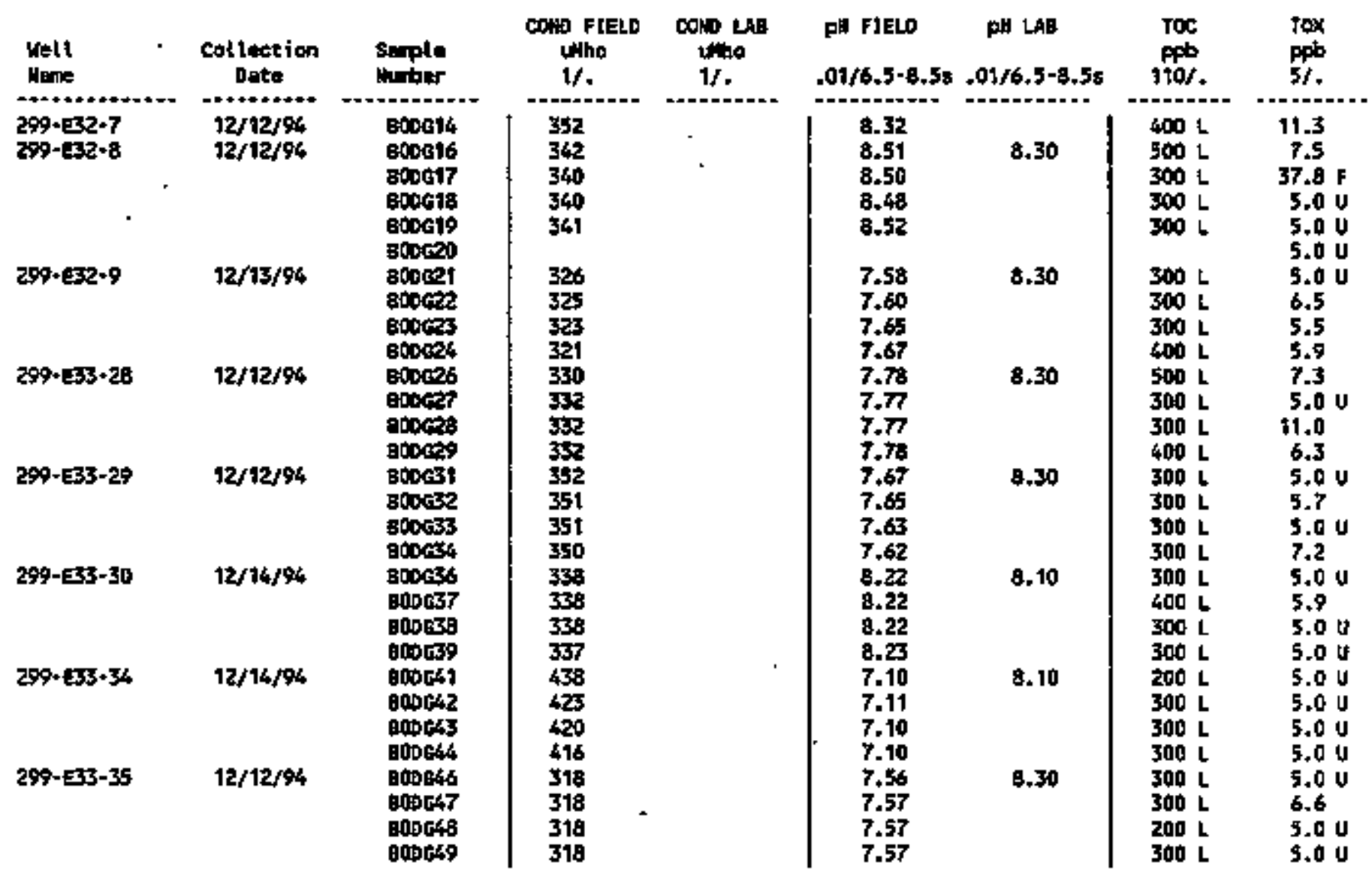

For explention of this table, ste section 1.4 af report. 
Table 14-15. Constituent List and Summary of Results for the Low-Level Waste Hanagement Area 2 Data for Reporting Period

October I through December 31, 1994.

(sheet 1 of 3)

COWTANIMKTION INOJCATOR PARAMETERS

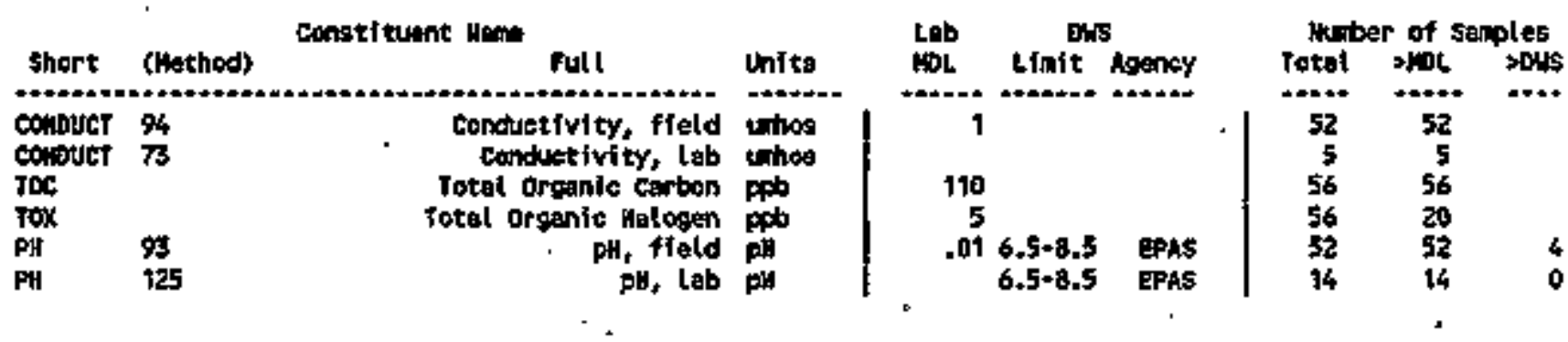

DRIMKINO WATER PARAHETERS

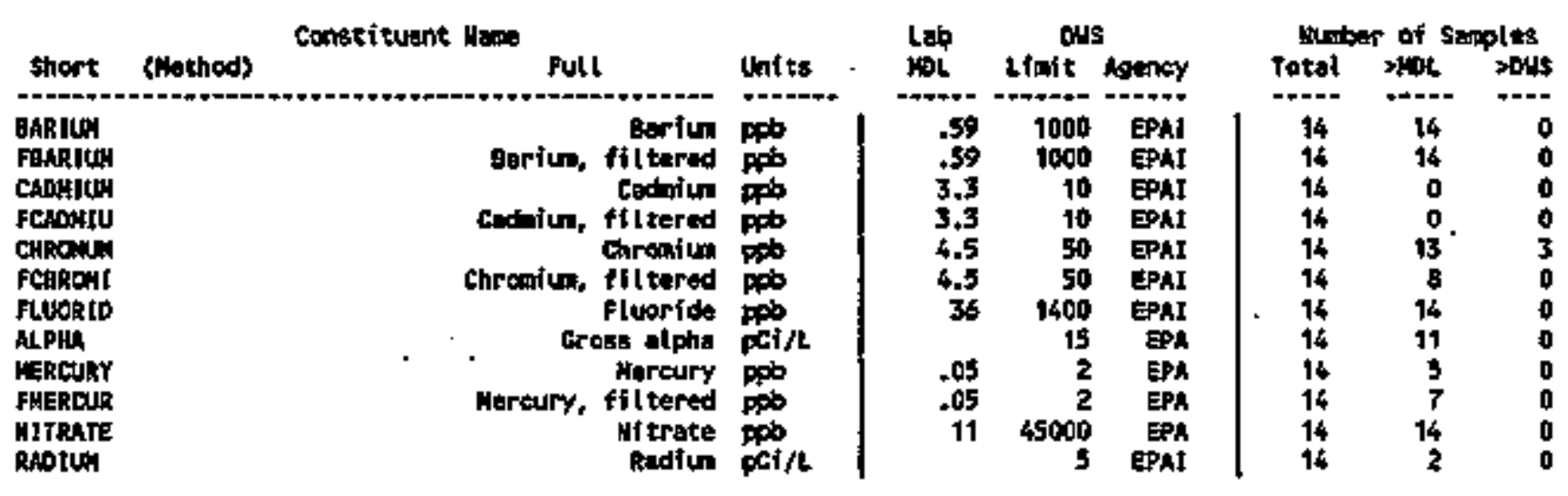

GROUNOWTER OUALITI PARAYETEKS

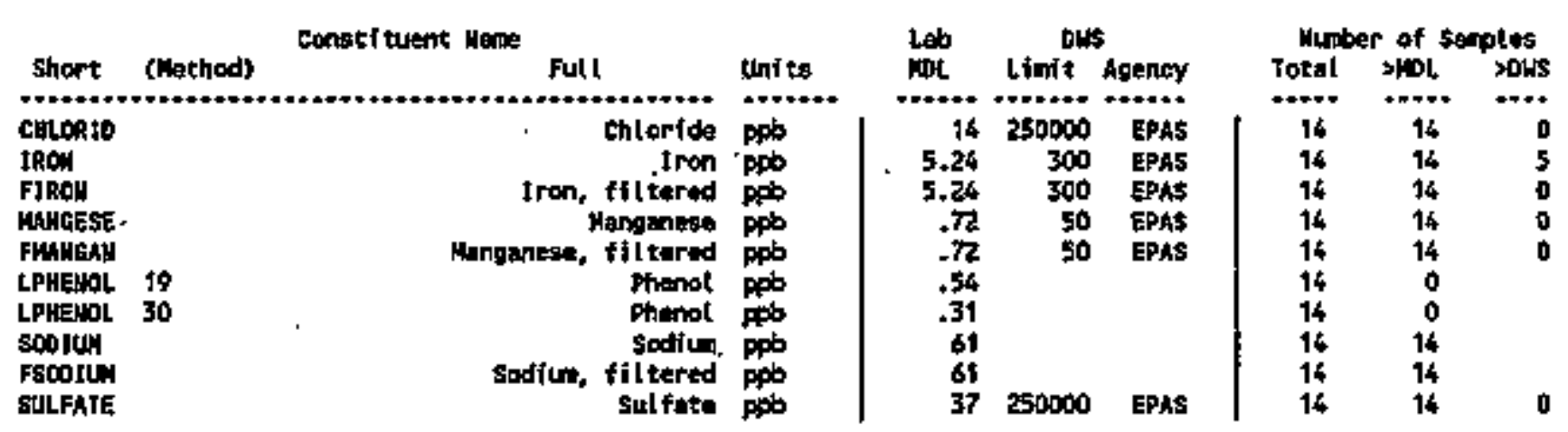


Table 14-15. Constituent List and Summary of Results for the Low-Level Waste Management Area 2 Data for Reporting Period

October 1 through December 31, 1994. (sheet 2 of 3 )

SIJE SOECIFIC AND OTHER CONSTITUENTS

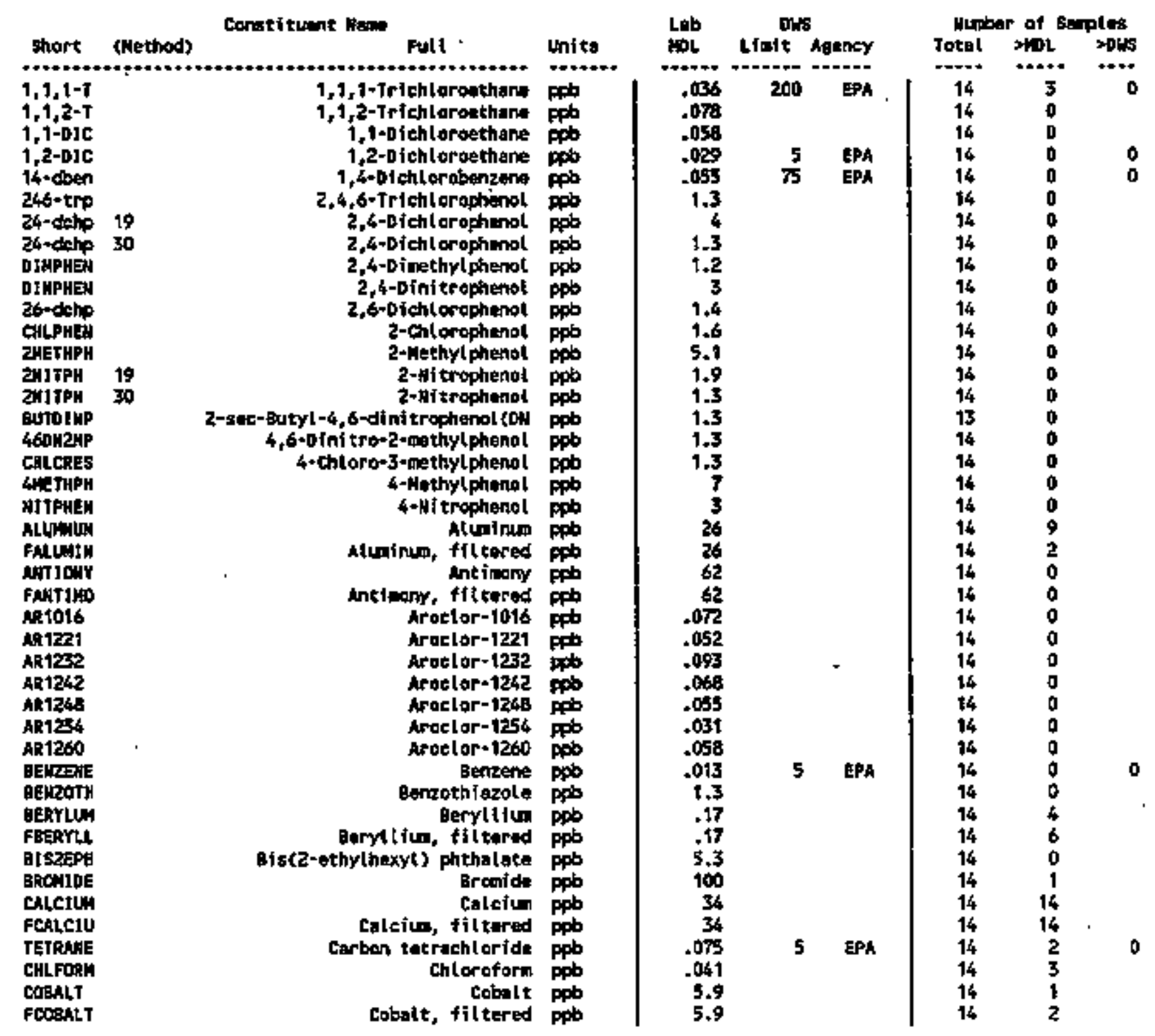


Table 14-15. Constituent List and Summary of Results for the Low-Leve] Waste Management Area 2 Data for Reporting Period

October 1 through December 31, 1994.

(sheet 3 of 3 )

\begin{tabular}{|c|c|c|c|c|c|c|c|c|c|}
\hline & & Congtituant Wamo & & Lab & Out & & Met & ir of $s$ & \\
\hline Shart & (Whthod) & Fult & Wnits & $\mathrm{HOL}$ & Lînitt & Agincy & Total & >HOL & salis \\
\hline apper & & Cootsr & $\mathbf{m b}$ & $4-t$ & 1000 & EFAS & th & 7 & $\overline{0}$ \\
\hline FCOPPER & - & copper, ftltered & ppo & 4,1 & 1000 & EPAS & 14 & 2 & 0 \\
\hline CRESOLLS & & Crasols (nathylphenols) & pob & 4.8 & & & 14 & 0 & \\
\hline CYHNTCE & & Cyanid & Pph & 1 & & & 14 & 0 & \\
\hline DEChIE & & pegarts & ppb & 1.9 & & & 14 & 0 & \\
\hline DoOEd & & Dodlectine & ppb & 1,1 & & & 14 & 0 & \\
\hline ETHВEИZ & & Ethylbenzene & pob & .051 & 700 & EPA & 14 & $\mathbf{0}$ & 0 \\
\hline 日ETh & $r$ & Grass beta & pill & & & & 16 & 14 & \\
\hline $\begin{array}{l}1-129 L \\
\text { LES }\end{array}$ & 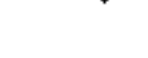 & loding-12\%, Lon level & $\mathrm{PCI}$ & 5 & 1 & EPA & $=\frac{5}{14}$ & 5 & 5 \\
\hline FtEAO & & land, ftltereat & pop & 1.9 & 50 & EPAI & 14 & 7 & 9 \\
\hline MUEUES & & Magnistum & into & $\mathbf{z}$ & & & 16 & 14 & \\
\hline FWh,DLES & & Hognesiur, fittered & PFb & 23 & & & 16 & 14 & \\
\hline GETHYCH & & Matbylene thorids & $\mathbf{F p b}$ & .09 & & & 14 & $\mathbf{0}$ & \\
\hline MAPHTH & & Maphthal ene & ppb & 1.2 & & & 16 & $\mathbf{0}$ & \\
\hline HICKF년 & & Aieckel & $D+b$ & 13 & & & 14 & 11 & \\
\hline FHICXEL & & -Nickel, filtered & pob & 13 & & . & 14 & 2 & \\
\hline $\begin{array}{l}\text { MITRITE } \\
\text { PEuTCFP }\end{array}$ & & Mitrite & pob & 18 & $\$ 000$ & EPA & 14 & 0 & 0 \\
\hline $\begin{array}{l}\text { PENTCCPP } \\
\text { PENTCHP }\end{array}$ & 19 & Pentach L or paphenol & b & 8.3 & 1 & Eph & 14 & 0 & 14* \\
\hline PHOSPKA & & Pentechl brophenol & Fpo & 1.5 & & & & 0 & $14^{\star}$ \\
\hline PDTASIN & & $\begin{array}{l}\text { Phosphote } \\
\text { Pataseiun }\end{array}$ & 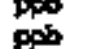 & 850 & & & $\begin{array}{l}14 \\
14\end{array}$ & 14 & \\
\hline fpotass & & Potassiun, filterid & pob & 850 & & & 14 & 14 & \\
\hline SILVER & & silver & pob. & 3.6 & 50 & EPAI & 14 & 0 & 0 \\
\hline F9ILVEF & & sitver, filtered & Pph & 3.6 & 50 & EPA1 & 14 & 0 & $\mathbf{0}$ \\
\hline PERteine & & Tetrachiloroethene & pob & .22 & 5 & EPA & 14 & 0 & $\mathbf{0}$ \\
\hline TETPHALL & & Totrachlor ophenots & pos & 1.5 & & & 14 & a & \\
\hline Tetaune & & Iotredecosin & $p$ & 1.6 & & & 14 & 0 & \\
\hline & & Tin & pho & 69 & & & 14 & $\cdots 0$ & \\
\hline $\begin{array}{l}\text { FTld } \\
\text { Folveng }\end{array}$ & & Tin, filtered & pob & .016 & 1000 & EPA & $\begin{array}{l}14 \\
14\end{array}$ & 0 & 0 \\
\hline TRIBUTPH & & Irifutyl Phosphte & pots & 1.1 & & & 1 & 0 & \\
\hline TRICENE & & Trichlorputhene & pot & .11 & 5 & EPA & 1 & 0 & 0 \\
\hline T月IPH!H & & Triehl orophenols & $p$ & 1.7 & & ? & & 0 & \\
\hline Tolchoㅂ & & Tris-2-chlaroethyl phouphtst & pob &. .81 & mant & & $10+2+$ & 0 & \\
\hline $\begin{array}{l}\text { ThItIUA } \\
\text { TURBID }\end{array}$ & & Tritius & pCI/ & ots & 3100 & . ЕР & 16 & 12 & १ \\
\hline GRANIU & & $\begin{array}{l}\text { Whition } \\
\text { Ureniut }\end{array}$ & ph & $=010$ & & & 14 & 14 & \\
\hline VALADU & - & Varidiun & pos & 6.5 & & & 14 & 14 & \\
\hline FANULI & & vanadium, fitcered & pob & 6.6 & & & & 14 & \\
\hline Vinftbe & & Vimpt chtorids & pob & 13 & 2 & EPA & 16 & 0 & 0 \\
\hline $\begin{array}{l}\text { XYLEile } \\
\text { zINC }\end{array}$ & & $\begin{array}{r}\text { Xylente (total) } \\
\text { zinc }\end{array}$ & pob & $\begin{array}{r}.035 \\
6,3\end{array}$ & $\begin{array}{r}10000 \\
5000\end{array}$ & $\begin{array}{l}\text { EPA } \\
\text { EPAS }\end{array}$ & $\begin{array}{l}14 \\
14\end{array}$ & $\begin{array}{l}0 \\
5\end{array}$ & 0 \\
\hline Fatik & & zinc, fi & pos & 6.3 & 5000 & EPAS. & 1 & 3 & 0 \\
\hline CIS120E & & 8-1,2-bichl oraethyten: & & $=11$ & 70 & EPA & 1 & 0 & 0 \\
\hline TRANACE & & trans-1,2-Dicht & $\infty$ & .073 & 100 & $E P A$ & 14 & 0 & 0 \\
\hline
\end{tabular}

For explantion of this table, ste section 1,4 of report. 
Table 14-16. Constituents with at Least One Detected Value for the Low-Level Waste Managenent Area 2 Data for Reporting Perjod

october 1 through December 31, 1994.

(sheet 1 of 8)

\begin{tabular}{|c|c|c|c|c|c|c|}
\hline $\begin{array}{l}\text { Wel I } \\
\text { Rane? }\end{array}$ & $\begin{array}{c}\text { Collection } \\
\text { Date }\end{array}$ & suple & $\begin{array}{r}1,1,1-\mathrm{T} \\
28 / \mathrm{ppb} \\
.2 \mathrm{e} / 200\end{array}$ & $\begin{array}{c}\text { АLшиан } \\
34 / \text { pot } \\
19 / .\end{array}$ & 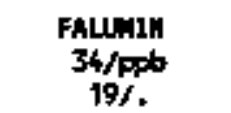 & $\begin{array}{r}\text { BARIUn } \\
34 / 460 \\
1,3 / 10001\end{array}$ \\
\hline$n+\cdots+\cdots+\cdots$ & $+++++\infty+\infty+\infty$ & $+\ldots+\ldots+\cdots+\cdots$ & $++\cdots+\cdots+\cdots+\cdots+\infty$ & $\cdots$ & $*$ & -- \\
\hline $\begin{array}{l}29-E 27-10 \\
299+E-27+10\end{array}$ & $12 / 13 / 44$ & $\begin{array}{l}600702 \\
800706\end{array}$ & $.04 v$ & 26.00 U & $26.00 \mathrm{U}$ & 26.00 \\
\hline $\begin{array}{l}299-E 27-11 \\
299-E 27-11\end{array}$ & $\begin{array}{l}11 / 18 / 94 \\
11 / 18 / 94\end{array}$ & $\begin{array}{l}600744 \\
800748\end{array}$ & $.04 \mathrm{~V}$ & 78.001 & & 39.00 \\
\hline $\begin{array}{l}299-E 27-17 \\
299-E 27-17\end{array}$ & $\begin{array}{l}11 / 18 / 94 \\
11 / 48 / 94\end{array}$ & $\begin{array}{l}80070 \% \\
\operatorname{se07} 3\end{array}$ & $.04 U$ & 26.0 U U & & 33.00 \\
\hline $\begin{array}{l}299+[27-8 \\
299-[27-8\end{array}$ & $\begin{array}{l}11 / 17 / 94 \\
11 / 17 / 94\end{array}$ & $\begin{array}{l}\text { B007R4 } \\
\text { B007R8 }\end{array}$ & $.06 \mathrm{U}$ & $26.00 \mathrm{U}$ & & 25.00 \\
\hline $\begin{array}{l}759-E 77-9 \\
79-E 77=9\end{array}$ & $\begin{array}{l}11 / 17 / 94 \\
11 / 17 / 94\end{array}$ & $\begin{array}{l}\text { Bontrog } \\
\cos 753\end{array}$ & $.04 \mathrm{~V}$ & $35.010 \mathrm{~L}$ & $26.00 \mathrm{U}$ & 23,00 \\
\hline $\begin{array}{l}299-E 34-10 \\
299-E 34-10\end{array}$ & $\begin{array}{l}11 / 21 / 94 \\
11 / 21 / 96\end{array}$ & $\begin{array}{l}800754 \\
800758\end{array}$ & $.04 \mathrm{U}$ & 54.001 & $26.00 \mathrm{U}$ & 38.00 \\
\hline $\begin{array}{l}299+34-11 \\
299-234-11\end{array}$ & $\begin{array}{l}11 / 28 / 94 \\
11 / 28 / 94\end{array}$ & $\begin{array}{l}00759 \\
00073\end{array}$ & $.04 \mathrm{U}$ & . $30.00 \mathrm{~L}$ & $32.00 \mathrm{~L}$ & 34.00 \\
\hline $\begin{array}{l}2 \%-634-12 \\
259-634-12\end{array}$ & $\begin{array}{l}11 / 28 / 94 \\
11 / 28 / 94\end{array}$ & $\begin{array}{l}000774 \\
000715\end{array}$ & .091 & $\begin{array}{l}100.00 \mathrm{~L} \\
1+0.00 \mathrm{~L}\end{array}$ & & $\begin{array}{l}68.00 \\
69.00\end{array}$ \\
\hline $\begin{array}{l}2 \$ 9-E 34-12 \\
2 \% 9-E 4-12\end{array}$ & $\begin{array}{l}11 / 28 / 94 \\
11 / 28 / 96\end{array}$ & $\begin{array}{l}\text { Bootriz } \\
\text { Btorvs }\end{array}$ & & & $\begin{array}{l}26.00 \mathrm{U} \\
26.00 \mathrm{U}\end{array}$ & \\
\hline $\begin{array}{l}299+[346-2 \\
259-634-2\end{array}$ & $\begin{array}{l}11 / 21 / 94 \\
11 / 21 / 94\end{array}$ & $\begin{array}{l}\text { sotorv4 } \\
\text { sobrvs }\end{array}$ & $.04 \mathrm{U}$ & $55.00 \mathrm{~L}$ & $37.00 \mathrm{~L}$ & 36.00 \\
\hline $\begin{array}{l}249-E 34-3 \\
249-E 34-3\end{array}$ & $\begin{array}{l}11 / 21 / 40 \\
11 / 21 / 94\end{array}$ & $\begin{array}{l}\text { Btoris } \\
\text { Botoris }\end{array}$ & $.04 \mathrm{U}$ & 26.004 & $26.00 \mathrm{U}$ & 48,00 \\
\hline $\begin{array}{l}299-E 34-5 \\
290-E 34-5\end{array}$ & $\begin{array}{l}11 / 18 / 94 \\
11 / 18 / 94\end{array}$ & 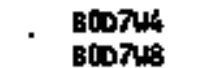 & $.04 \mathrm{U}$ & $26.00 \mathrm{~L}$ & $26.00 \mathrm{U}$ & 27.00 \\
\hline $\begin{array}{l}299 \cdot 134+7 \\
799 \cdot 134+7\end{array}$ & $\begin{array}{l}11 / 18 / 94 \\
11 / 18 / 94\end{array}$ & $\begin{array}{l}\text { B007ng } \\
\text { B007x }\end{array}$ & $.06 \mathrm{U}$ & $67.00 \mathrm{~L}$ & $26.00 \mathrm{t}$ & 35.00 \\
\hline $\begin{array}{l}257-E 34-9 \\
2 \$ 7-E 34-9\end{array}$ & $\begin{array}{l}11 / 28 / 94 \\
11 / 28 / 94\end{array}$ & $\begin{array}{l}000 \times \times 4 \\
8007 \times 8\end{array}$ & .084 & $26,00 \mathrm{t}$ & 26.000 & 45.00 \\
\hline
\end{tabular}

\begin{tabular}{|c|c|c|c|c|c|c|}
\hline $\begin{array}{l}\text { Well } \\
\text { Name }\end{array}$ & $\begin{array}{c}\text { Colletion } \\
\text { Date }\end{array}$ & $\begin{array}{l}\text { Sellple · } \\
\text { Munber }\end{array}$ & $\begin{array}{c}\text { FEARJuH } \\
\text { 34/ppb } \\
1,3 / \text { todot }\end{array}$ & $\begin{array}{c}\text { BERYLLH } \\
34 / \mathrm{ppb} \\
1.5 / .\end{array}$ & $\begin{array}{c}\text { FEERYLL } \\
34 / \mathrm{Fpb} \\
1.5 \%\end{array}$ & $\begin{array}{l}\text { ERONIOE } \\
124 / \text { peb } \\
110 \%\end{array}$ \\
\hline $299-E 27-10$ & $12 / 13 / 94$ & Booros & & .970 & & $30.00 \mathrm{~L}$ \\
\hline $\begin{array}{l}299+[27+10 \\
299+[27+11\end{array}$ & $\begin{array}{l}12 / 13 / 94 \\
11 / 18 / 96\end{array}$ & 800796 & 26.00 & & $.17 \mathrm{U}$ & \\
\hline $259-627-11$ & $11 / 18 / 94$ & - GDOTY8 & 39.00 & +276 & $.29 \mathrm{~L}$ & $100.00 \mathrm{U}$ \\
\hline $299-627+17$ & $11 / 18 / 94$ & 800709 & & $.43 \mathrm{~L}$ & & $100.00 \mathrm{U}$ \\
\hline $\begin{array}{l}259-e x 7-17 \\
60+07-8\end{array}$ & $11 / 18 / 94$ & B100723 & 33.00 & & $.58 \mathrm{~L}$ & \\
\hline $209-627-8$ & $11 / 17 / 94$ & 800728 & $\mathbf{3 . 0 0}$ & .180 & 170 & 700.000 \\
\hline $\begin{array}{l}299-E 27-9 \\
299-E 27-9\end{array}$ & $11 / 17 / 94$ & $\begin{array}{l}8007299 \\
\text { Bon753 }\end{array}$ & $x, 00$ & $.17 \mathrm{v}$ & & $100.00 \mathrm{~V}$ \\
\hline $299-154-10$ & $11 / 21 / 94$ & B00754 & 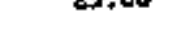 & .212 & $.34 \mathrm{~L}$ & $100.00 \mathrm{U}$ \\
\hline $299-E 34-10$ & $11 / 21 / 96$ & $8007 \div 8$ & 37,00 & & ע & \\
\hline $299-E 34-11$ & $11 / 28 / 94$ & B00759 & & $.17 \mathrm{~V}$ & & $80.00 \mathrm{~L}$ \\
\hline $\begin{array}{l}299-E 34-11 \\
299-E 34-12\end{array}$ & $11 / 28 / 94$ & BcosT3 & 33.00 & 170 & . $17 \mathrm{U}$ & $60.00 \mathrm{~L}$ \\
\hline $299-234-12$ & $11 / 28 / 94$ & D007TS & & $.17 \mathrm{U}$ & & $50.00 \mathrm{l}$ \\
\hline $\begin{array}{l}299-E 34-12 \\
799-E 34-17\end{array}$ & $11 / 28 / 94$ & atoriv2 & 67.00 & & $.17 \mathrm{U}$ & \\
\hline $299-e 34-2$ & $11 / 21 / 94$ & BCorN4 & & $.17 \mathrm{U}$ & & $100.00 \mathrm{U}$ \\
\hline $34-2$ & $11 / 21 / 94$ & Bcons & 37.00 & & .351 & \\
\hline $\begin{array}{l}298-134-3 \\
299--14-3\end{array}$ & $\begin{array}{l}11 / 21 / 94 \\
11 / 21 / 96\end{array}$ & $\begin{array}{l}\text { 8c007v9 } \\
\text { Bconsts }\end{array}$ & 48.00 & .17 U & & $100.00 \mathrm{U}$ \\
\hline & & & & & . 170 & \\
\hline
\end{tabular}


Table 14-16. Constituents with at Least One Detected Va] ue for the Low-Level Waste Nanagement Area 2 Data for Reporting Period

October 1 through December 31, 1994. (sheet 2 of 8)

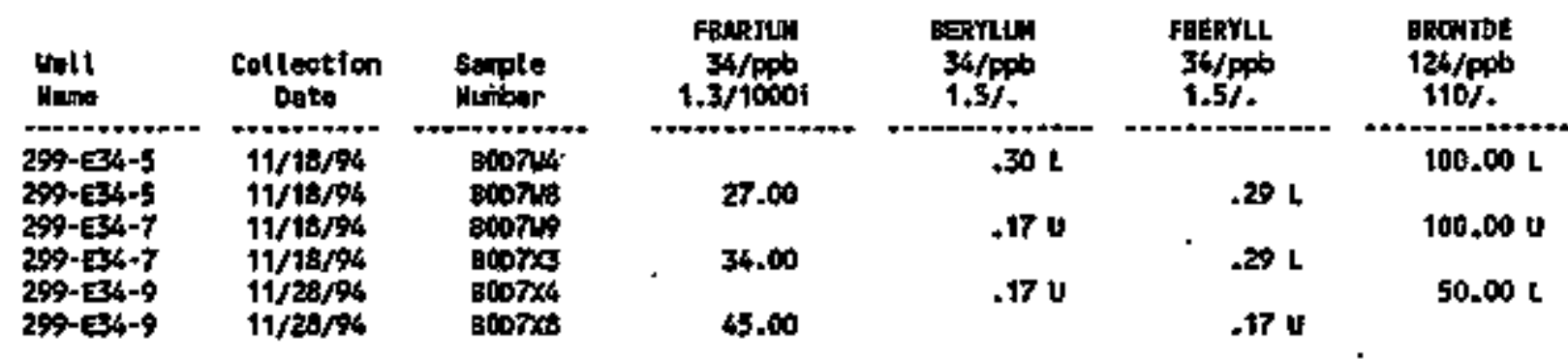

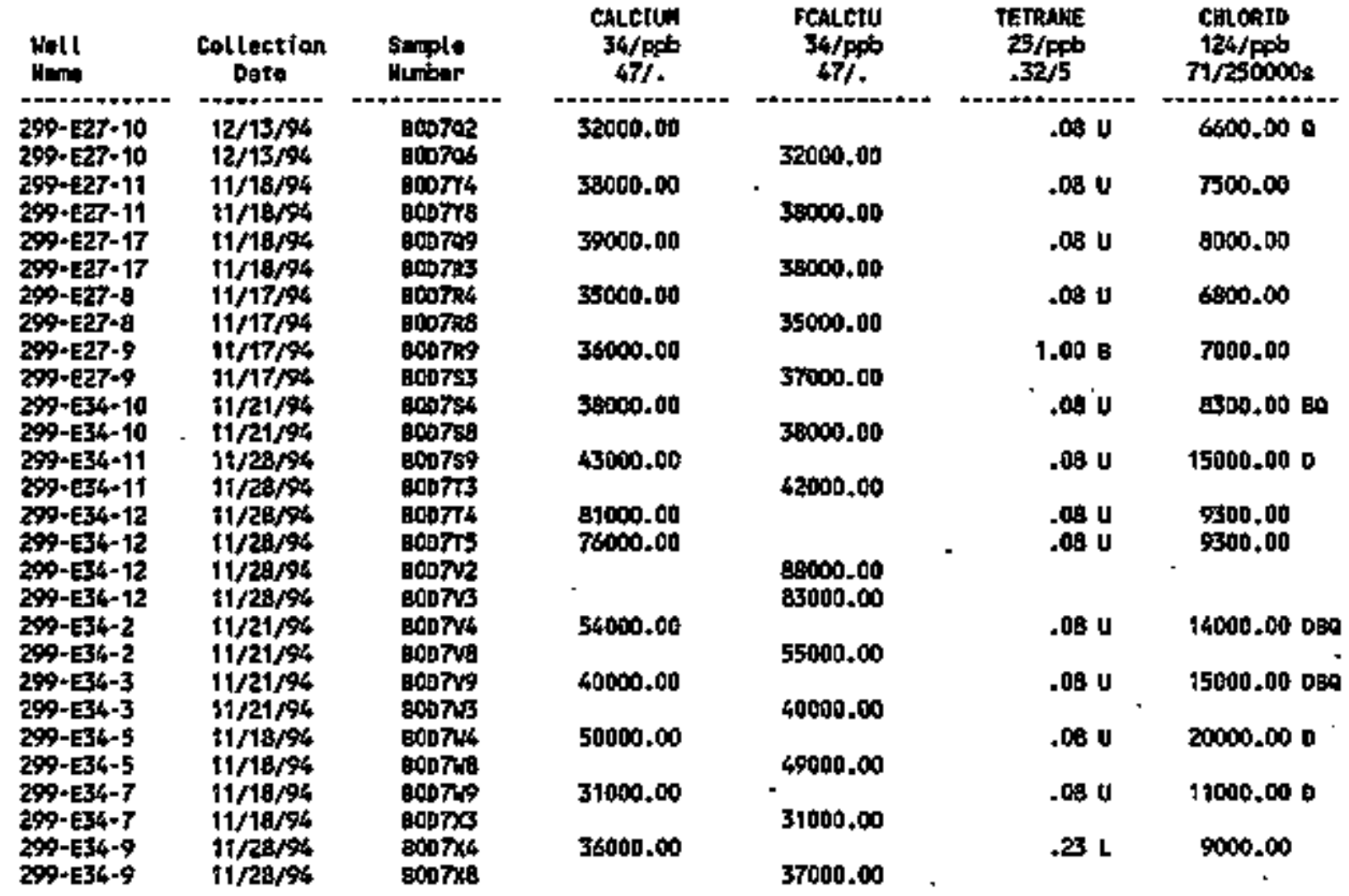


Table 14-16. Constituents with at Least One Detected Value for the Low-Level Waste Management Area 2 Data for Reporting Perjod

October 1 through Decenaber 31, 1994. (sheet 3 of 8 )

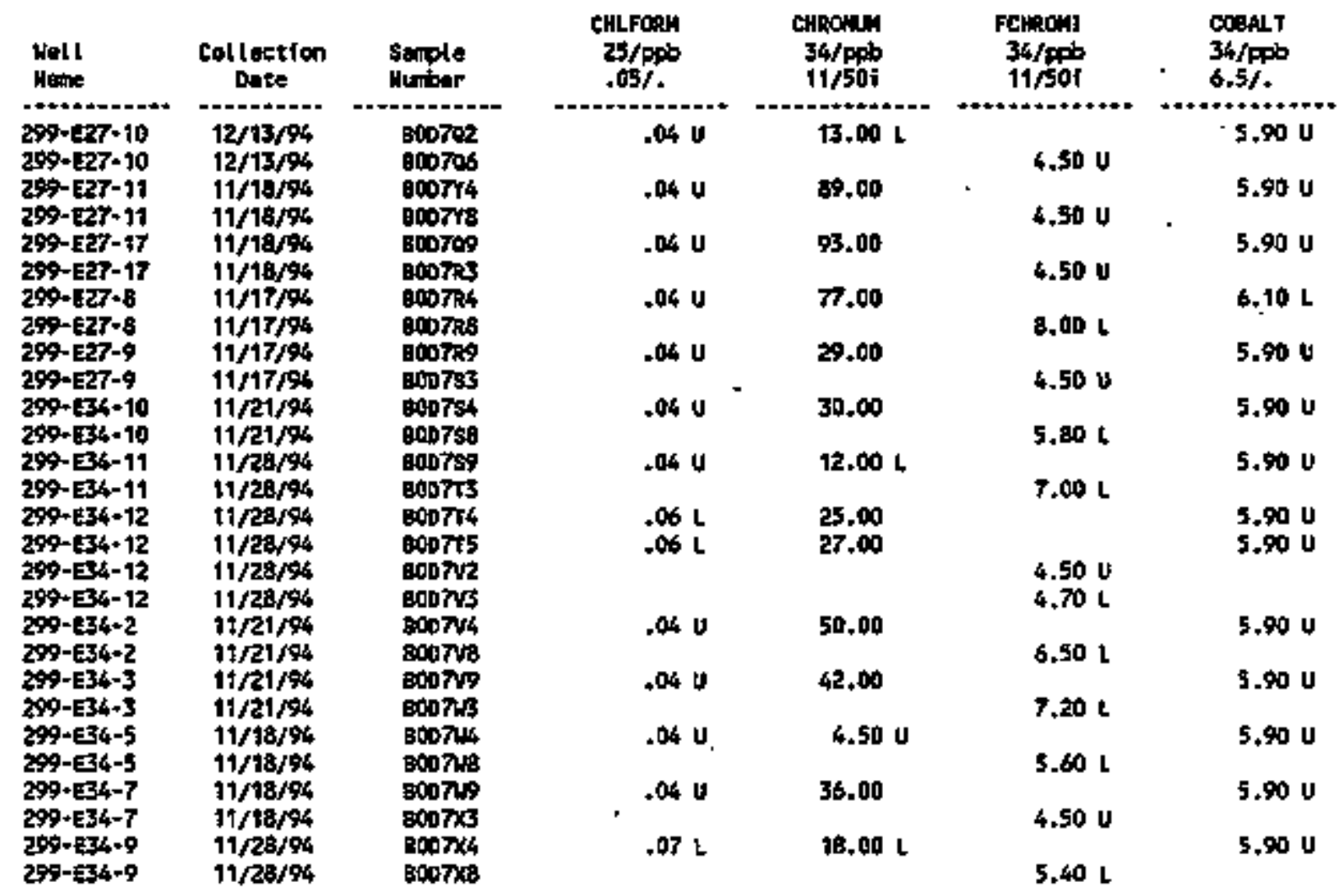

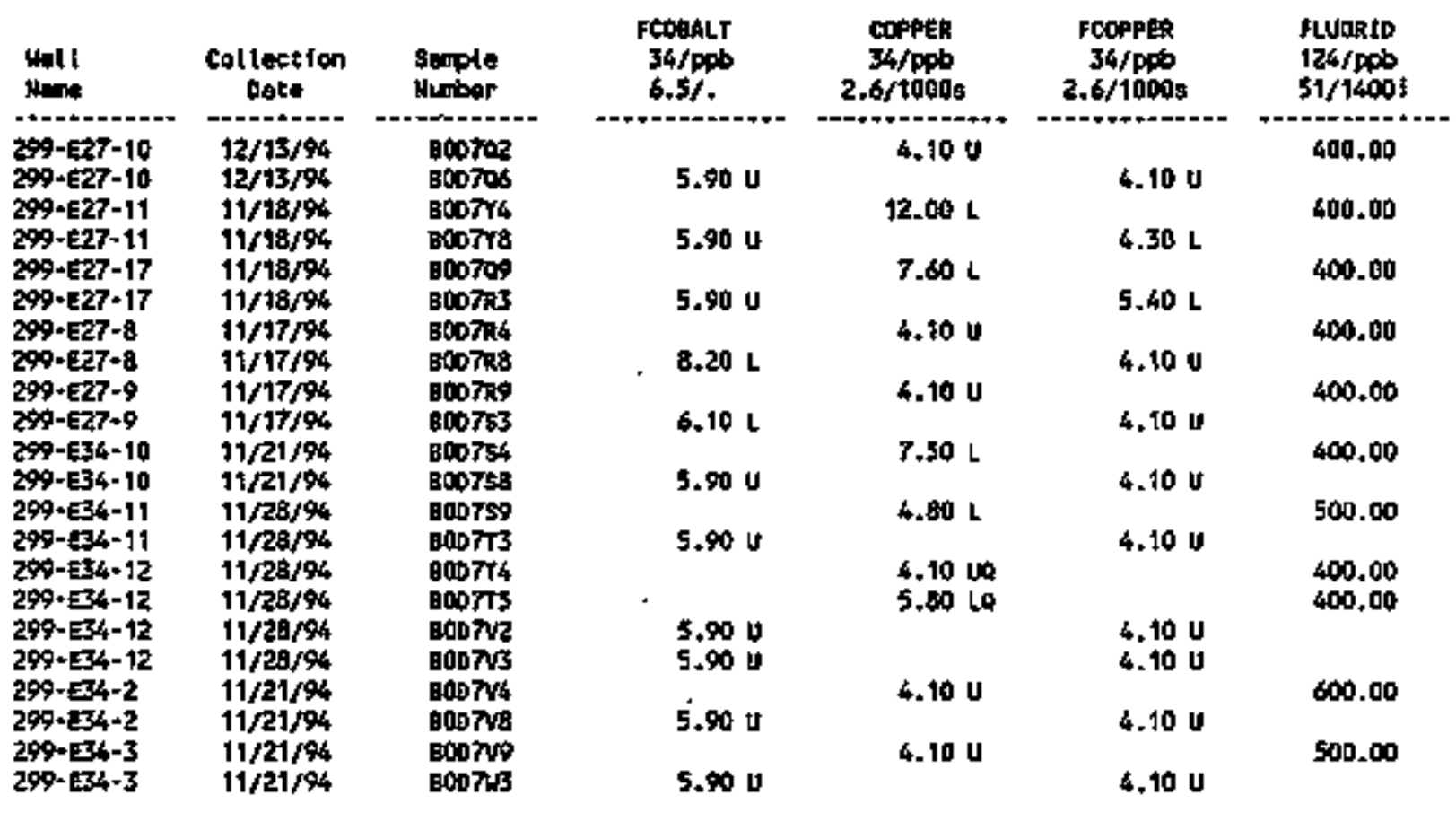


Table 14-16. Constituents with at Least One Detected Value for the Low-Level Waste Management Area 2 Data for Reporting Period October 1 through December 31, 1994.

(sheet 4 of 8)

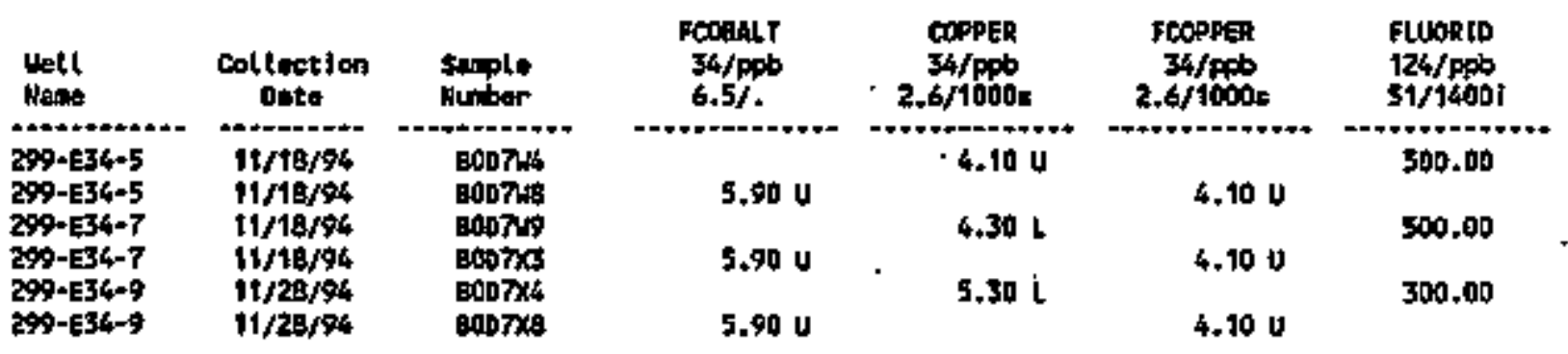

\begin{tabular}{|c|c|c|c|c|c|c|}
\hline Wetl & $\underset{\text { Date }}{\text { Coltuction }}$ & $\begin{array}{l}\text { Salplo } \\
\text { mintor }\end{array}$ & 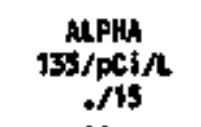 & $\begin{array}{c}\text { OETA } \\
136 / \mathrm{DCI} / \mathrm{L} \\
. /\end{array}$ & $\begin{array}{c}t-129 \\
139 / \not C 1 / L \\
. / 1\end{array}$ & $\begin{array}{c}\text { IROA } \\
34 / \mathrm{CPb} \\
18 / 300 \mathrm{~s}\end{array}$ \\
\hline $\begin{array}{l}299-E 27-10 \\
299-E 27-11\end{array}$ & $\begin{array}{l}t 2 / 13 / 94 \\
t 1 / 18 / 94\end{array}$ & $\begin{array}{l}100702 \\
800792\end{array}$ & $\$ .00$ & 5,53 & 2.05 & 74.0060 \\
\hline $299-E 27-11$ & 11/t8/94 & $\begin{array}{l}\text { 8007r6 } \\
\text { 000793 }\end{array}$ & 2.14 & 7.81 & & $490.00 \mathrm{~B}$ \\
\hline $\begin{array}{l}299-627-17 \\
299-627-8\end{array}$ & $\begin{array}{l}11 / 18 / 94 \\
11 / 17 / 94\end{array}$ & $\begin{array}{l}000709 \\
000794\end{array}$ & 1.07 & 7.25 & 3.03 & $410.00 \mathrm{E}$ \\
\hline $\begin{array}{l}299-E 27-8 \\
299-E 27-9\end{array}$ & $\begin{array}{l}11 / 17 / 94 \\
11 / 17 / 94\end{array}$ & $\begin{array}{l}000724 \\
000795\end{array}$ & 2.20 & 6.59 & & 330.008 \\
\hline $\begin{array}{l}299-E 27 \cdot 9 \\
299-E 34-10\end{array}$ & & $\begin{array}{l}8007 \pi 9 \\
800796\end{array}$ & $.69 \mathrm{U}$ & 5.33 & & 180.008 \\
\hline $\begin{array}{l}299-E 34-10 \\
299-E 4-17 \\
299-E 4-12 \\
299-E 34+12 \\
299-E 34-2 \\
299-E 34-3 \\
299-E 44-5 \\
299-E 4-7 \\
299-E 4-9\end{array}$ & $\begin{array}{l}11 / 21 / 94 \\
11 / 29 / 94 \\
11 / 29 / 94 \\
11 / 29 / 94 \\
11 / 21 / 94 \\
11 / 21 / 94 \\
11 / 18 / 94 \\
11 / 19 / 94 \\
11 / 28 / 44\end{array}$ & 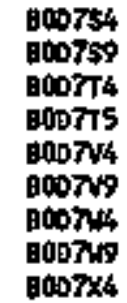 & $\begin{array}{l}2.19 \\
4.46 \\
3.73 \\
3.28 \\
3.01 \\
.42 \mathrm{u} \\
4.20 \\
.78 \mathrm{U} \\
2.45\end{array}$ & $\begin{array}{r}5.06 \\
7.48 \\
9.31 \\
10.00 \\
5.79 \\
7.89 \\
9.48 \\
7.27 \\
11.50\end{array}$ & & $\begin{array}{r}160.0080 \\
73.008 \\
340.000 \\
350.000 \\
210.0060 \\
180.0080 \\
30.00 \mathrm{E} \\
270.008 \\
83.000\end{array}$ \\
\hline
\end{tabular}

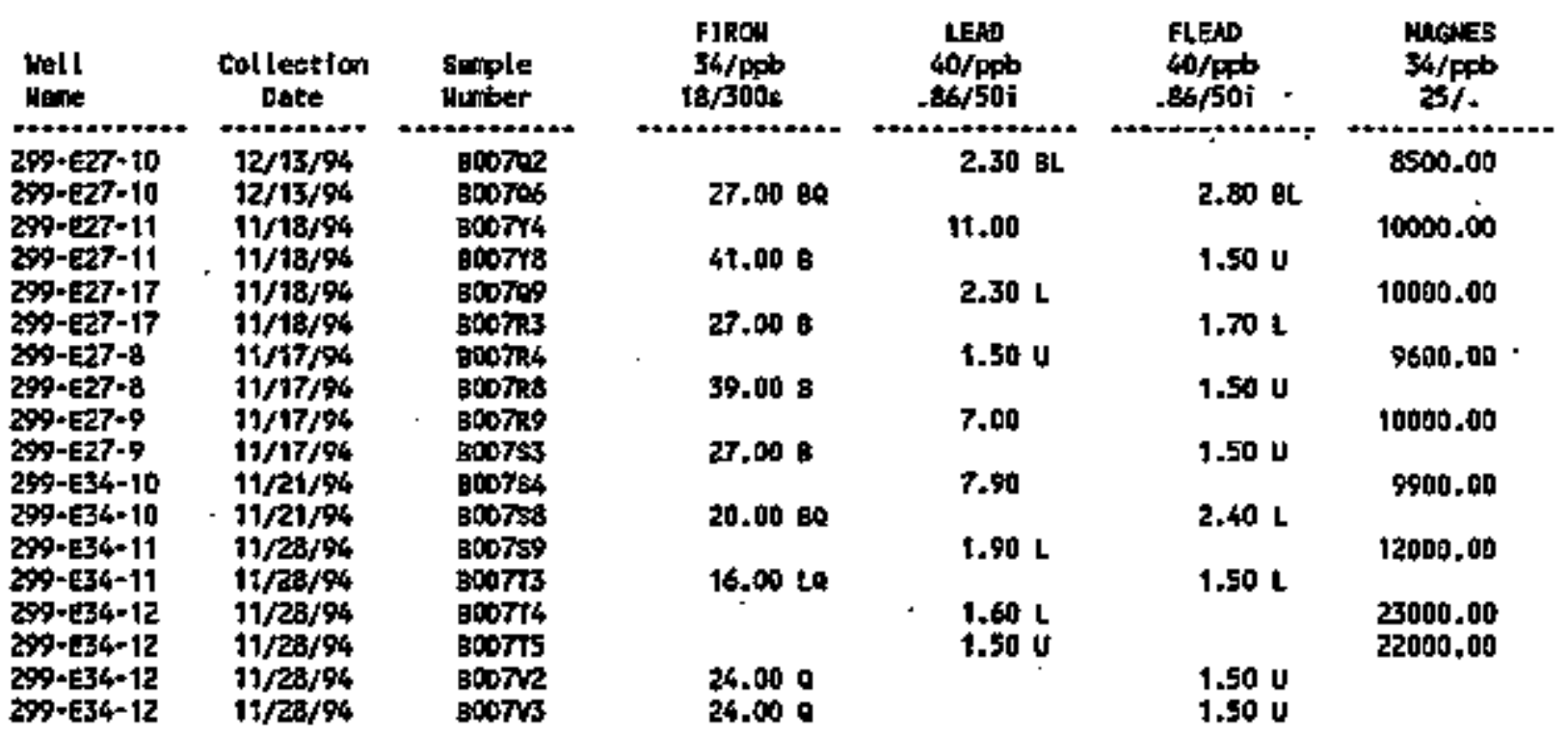


Table 14-16. Constjtuents with at Least One Detected Value for the Low-Level Waste Management Area 2 Data for Reporting Period October 1 through Decenber 31, 1994.

(sheet 5 of 8 )

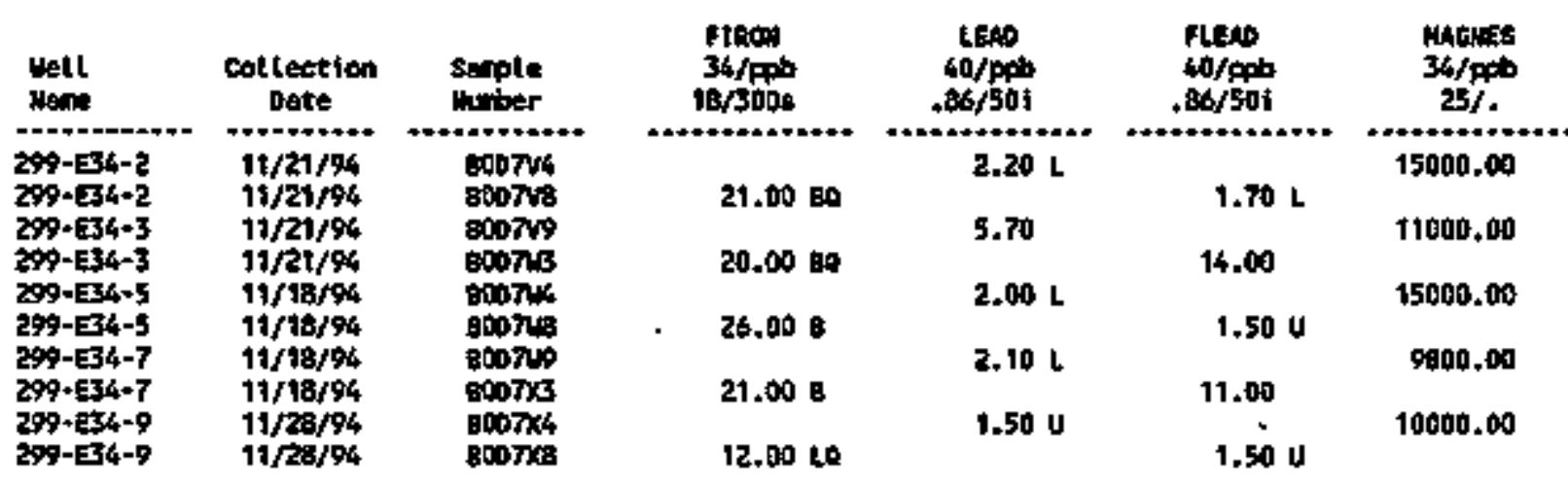

\begin{tabular}{|c|c|c|c|c|c|c|}
\hline $\begin{array}{l}\text { Woll } \\
\text { Merne }\end{array}$ & $\begin{array}{l}\text { Col lection } \\
\text { Date }\end{array}$ & $\begin{array}{l}\text { sample } \\
\text { Humber }\end{array}$ & 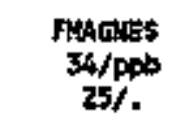 & $\begin{array}{l}\text { Milinese } \\
34 / \mathrm{ppb} \\
1 / 50 \mathrm{~s}\end{array}$ & $\begin{array}{l}\text { FNANGW } \\
\text { 34/Ppb } \\
1 / 59 \mathrm{~s}\end{array}$ & $\begin{array}{l}\text { MERCURY } \\
41 / \mathrm{ppb} \\
.095 / 2\end{array}$ \\
\hline $\begin{array}{l}299-E 27-10 \\
299+E 27+10 \\
299-E 27-11 \\
299-E 27-11 \\
299-E 27-17 \\
299-E 27-17 \\
299-E 27-8 \\
299-E 27-8 \\
299-E 27-9 \\
299-E 27-9 \\
299-E 34-10 \\
299-E 34-10 \\
299-E 34-11 \\
299-E 34-11 \\
299-E 34-12 \\
299-E 34-12 \\
299-E 34-12 \\
299-E 34-12 \\
299-E 34-2 \\
299-E 34-2 \\
299-E 34-3 \\
299-E 34-3 \\
299-E 34-5 \\
299-E 34-5 \\
299-E 34-5 \\
299-E 34-7 \\
299-E 34-9 . \\
299-E 34-9\end{array}$ & $\begin{array}{l}12 / 13 / 94 \\
12 / 13 / 94 \\
11 / 18 / 94 \\
11 / 14 / 94 \\
11 / 18 / 94 \\
11 / 18 / 94 \\
11 / 17 / 94 \\
11 / 17 / 94 \\
11 / 17 / 94 \\
11 / 17 / 94 \\
11 / 21 / 94 \\
11 / 21 / 94 \\
11 / 28 / 94 \\
11 / 28 / 94 \\
11 / 28 / 94 \\
11 / 28 / 94 \\
11 / 25 / 94 \\
11 / 28 / 94 \\
11 / 21 / 94 \\
11 / 21 / 94 \\
11 / 21 / 94 \\
11 / 21 / 94 \\
11 / 18 / 94 \\
11 / 18 / 94 \\
11 / 18 / 94 \\
11 / 18 / 94 \\
11 / 28 / 94 \\
11 / 28 / 94\end{array}$ & 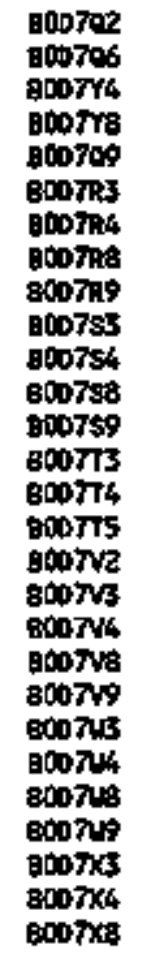 & $\begin{array}{r}8500.00 \\
10000.00 \\
100000.00 \\
9700.00 \\
11000.00 \\
9900.00 \\
12000.00 \\
23000.00 \\
21000.00 \\
15000.00 \\
17000.00 \\
25000.00 \\
9900.00 \\
10000.00\end{array}$ & $\begin{array}{l}2.20 \mathrm{~L} \\
11.00 \\
9.30 \mathrm{~L} \\
6.90 \mathrm{~L} \\
3.20 \mathrm{~L} \\
4.10 \mathrm{~L} \\
2.80 \mathrm{~L} \\
7.90 \mathrm{~L} \\
7.60 \mathrm{~L} \\
4.40 \mathrm{~L} \\
4.90 \mathrm{~L} \\
7.20 \mathrm{~L} \\
7.20 \mathrm{~L} \\
2.50 \mathrm{~L}\end{array}$ & $\begin{array}{l}2.20 \mathrm{~L} \\
4.10 \mathrm{~L} \\
2.00 \mathrm{~L} \\
1.70 \mathrm{~L} \\
1.50 \mathrm{~L} \\
2.00 \mathrm{~L} \\
2.10 \mathrm{~L} \\
2.70 \mathrm{~L} \\
2.80 \mathrm{~L} \\
1.80 \mathrm{~L} \\
2.10 \mathrm{~L} \\
6.40 \mathrm{~L} \\
3.30 \mathrm{~L} \\
1.20 \mathrm{~L}\end{array}$ & $\begin{array}{l}.11 \mathrm{BL} \\
.0 \mathrm{BL} \\
.08 \mathrm{BL} \\
.05 \mathrm{U} \\
.05 \mathrm{0} \\
.05 \mathrm{U} \\
.05 \mathrm{v} \\
.05 \mathrm{U} \\
.05 \mathrm{U} \\
.05 \mathrm{BL} \\
.05 \mathrm{tt} \\
.05 \mathrm{U} \\
.08 \mathrm{BL} \\
.05 \mathrm{~J}\end{array}$ \\
\hline
\end{tabular}


Table 14-16. Constituents with at least One Detected Value for the Low-Level Waste Management Area. 2 Data for Reporting Period October 1 through. December 31, 1994.

(sheet 6 of 8 )

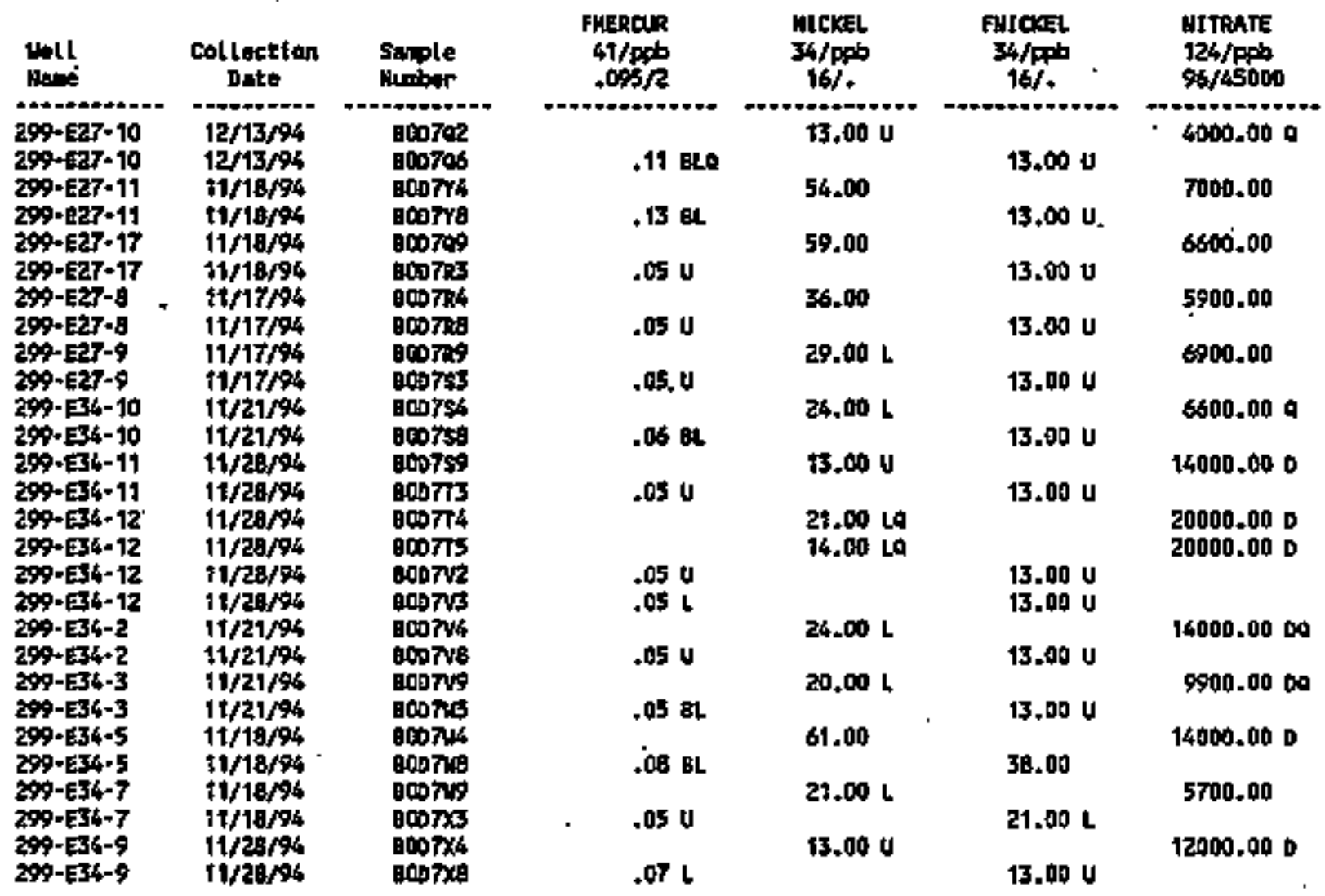

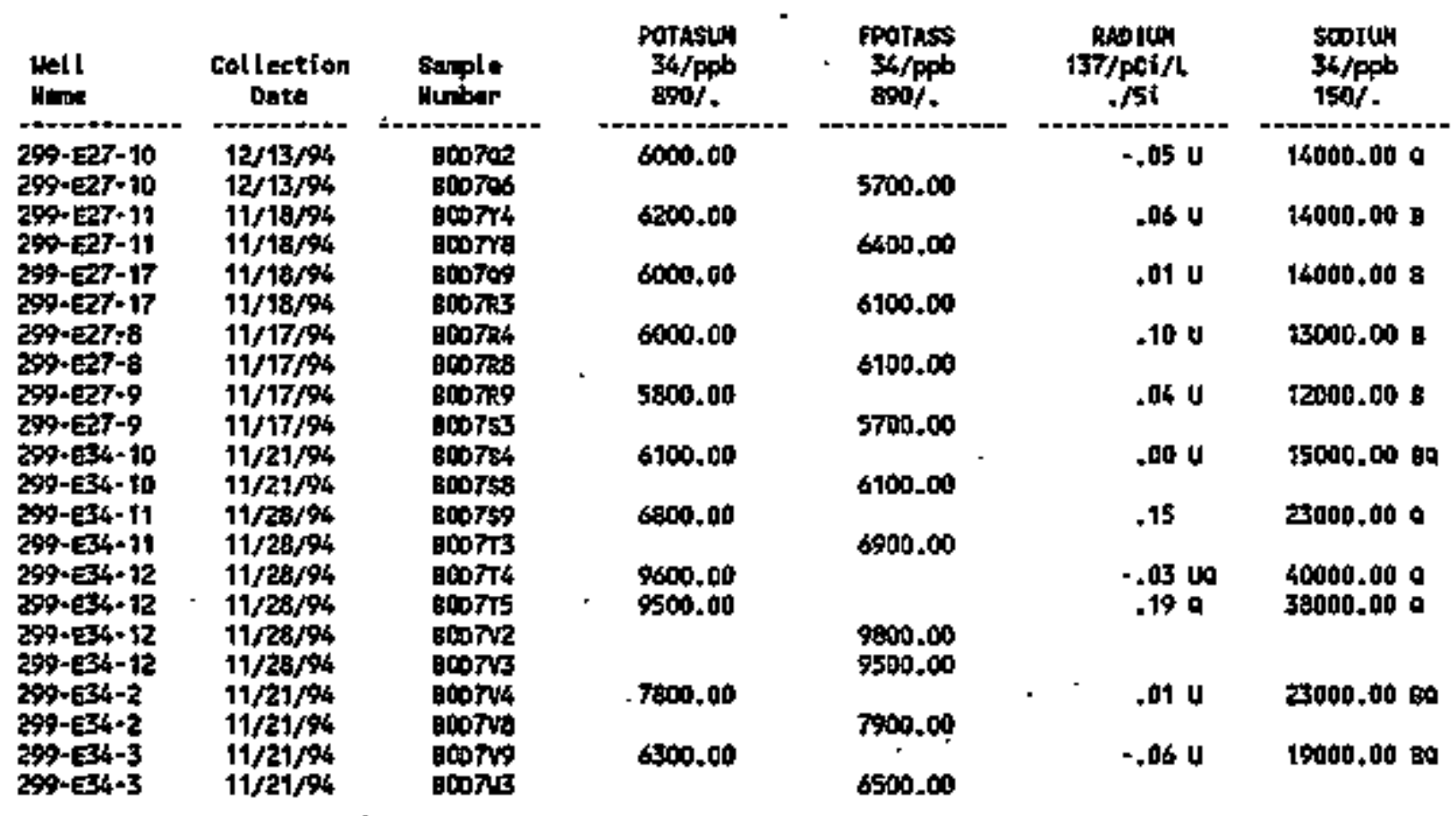


Table 14-16. Constituents with at Least One Detected Value for the Low-Level Waste Management Area 2 Data for Reporting Period October 1 through December 31, 1994. (sheet 7 of 8)

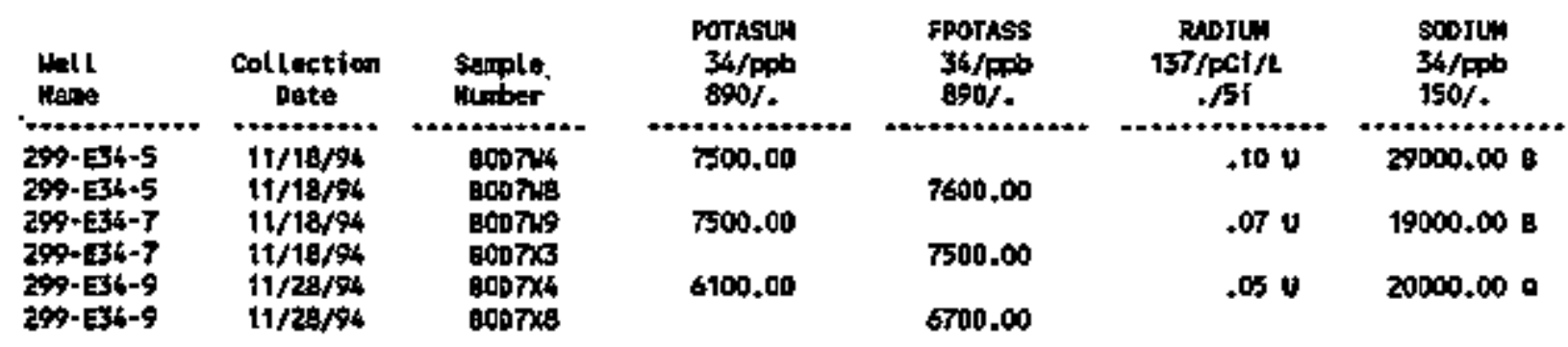

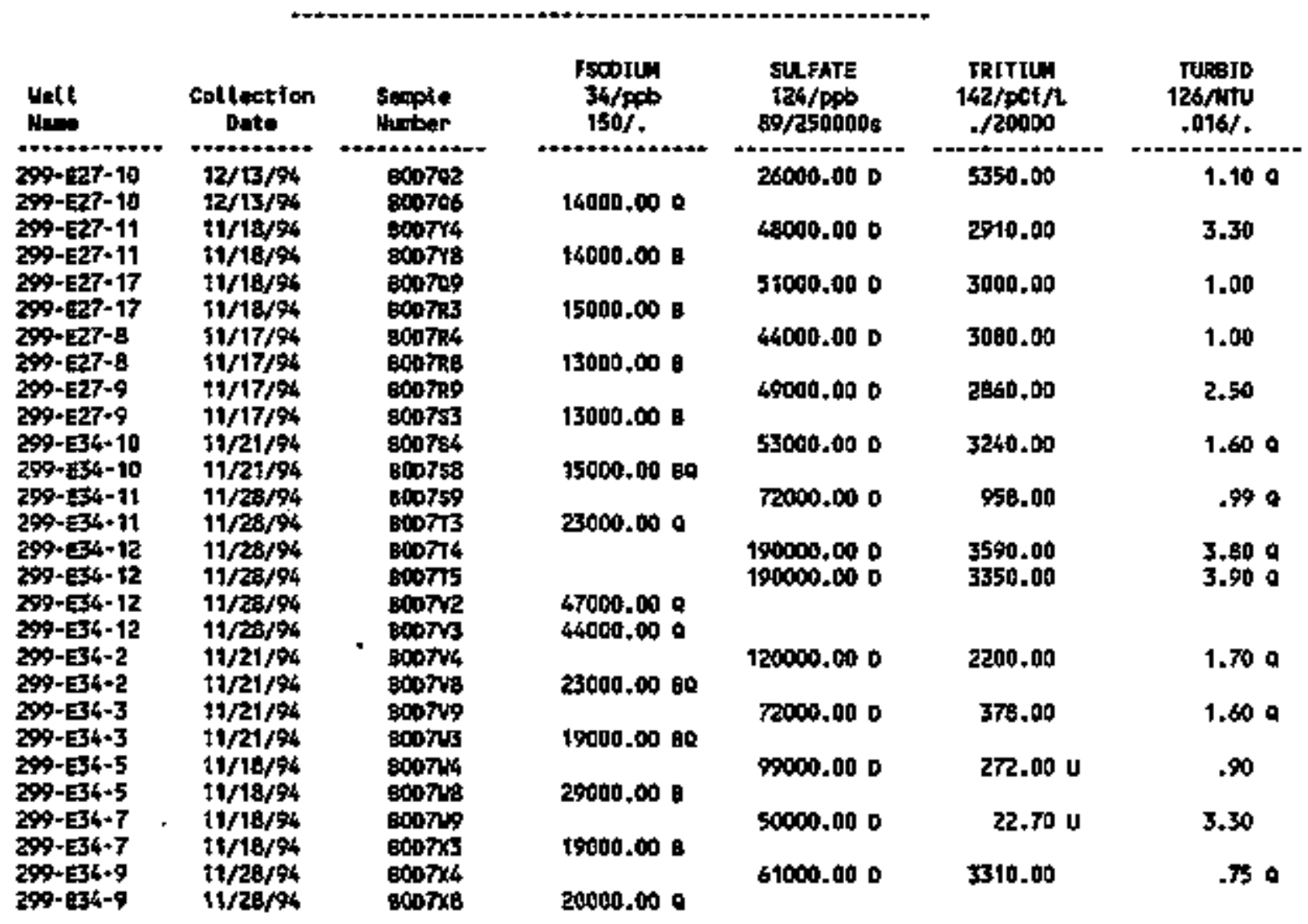


Table 14-16. Constituents with at Least One Detected Value for the Low-Level Waste Management Area 2 Data for Reporting Period October 1 through December 31, 1994.

(sheet 8 of 8 )

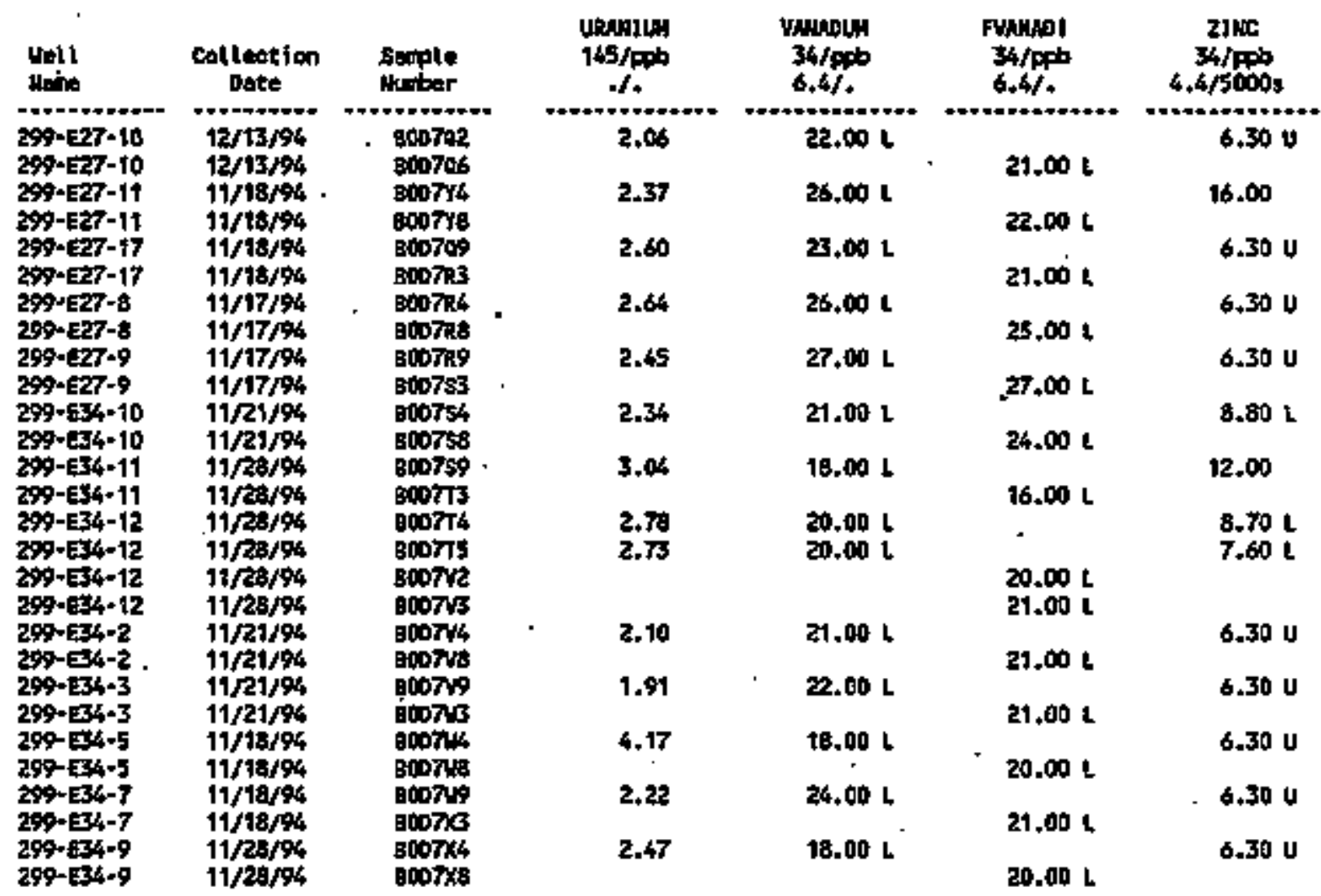

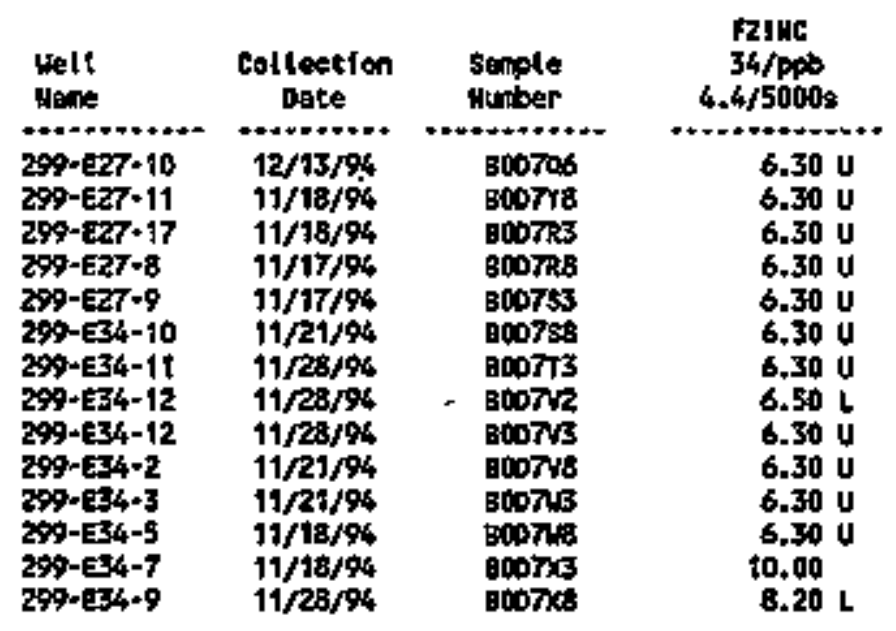

For wptanation of this trble, gee section 1.4 of report. 
Table 14-17. Contamination Indicator Parameters for the Low-Leve

Naste Management Area 2 Data for Reporting Perfod

October 1 through Decenber 31, 1994.

(sheet 1 of 2)

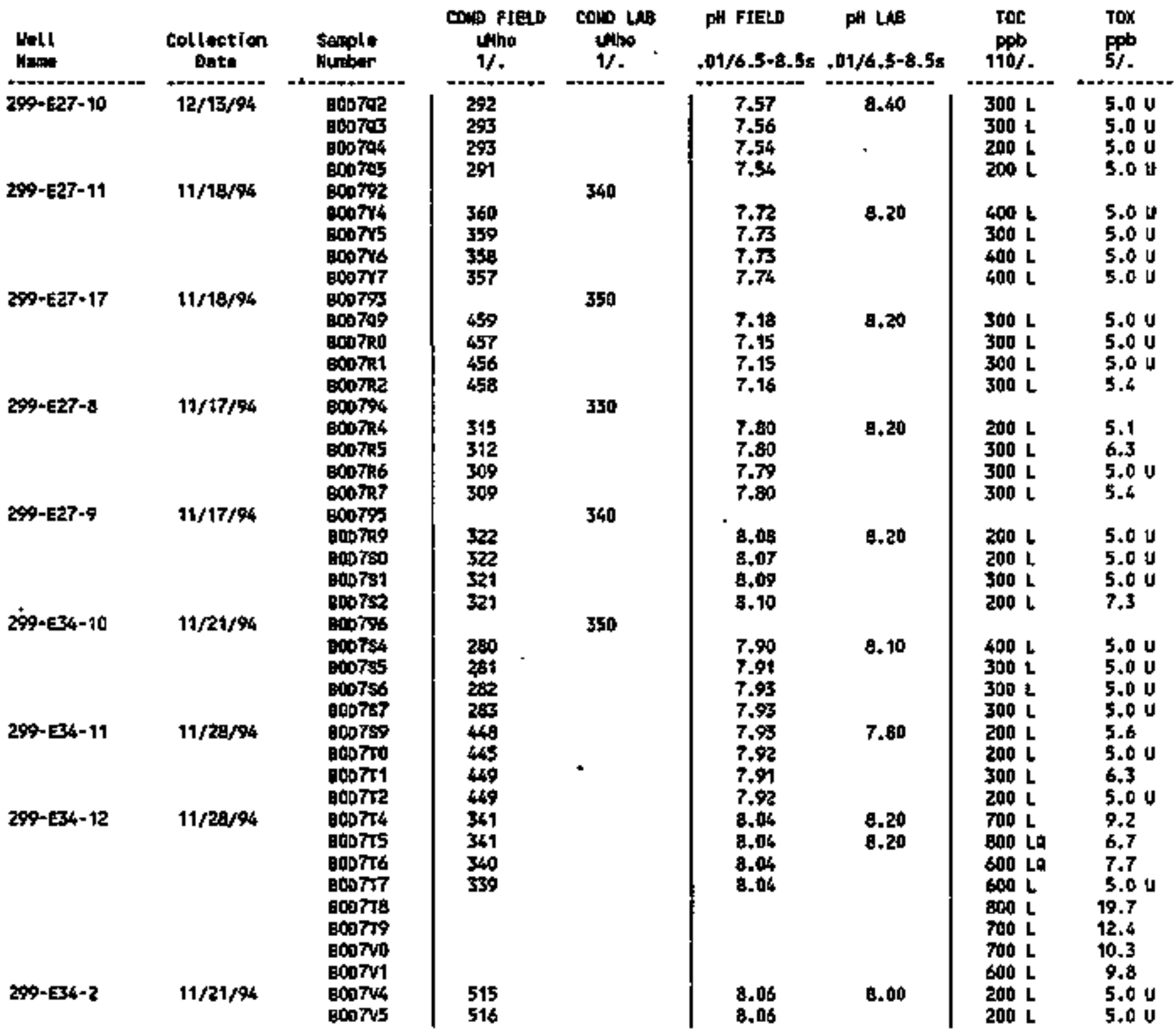




\section{DOE/RL-94-36-4}

Table 14-17. Contamination Indicator Parameters for the Low-Level Waste Management Area 2 Oata for Reporting Perjod October 1 through Decenber 31, 1994. (sheet 2 of 2)

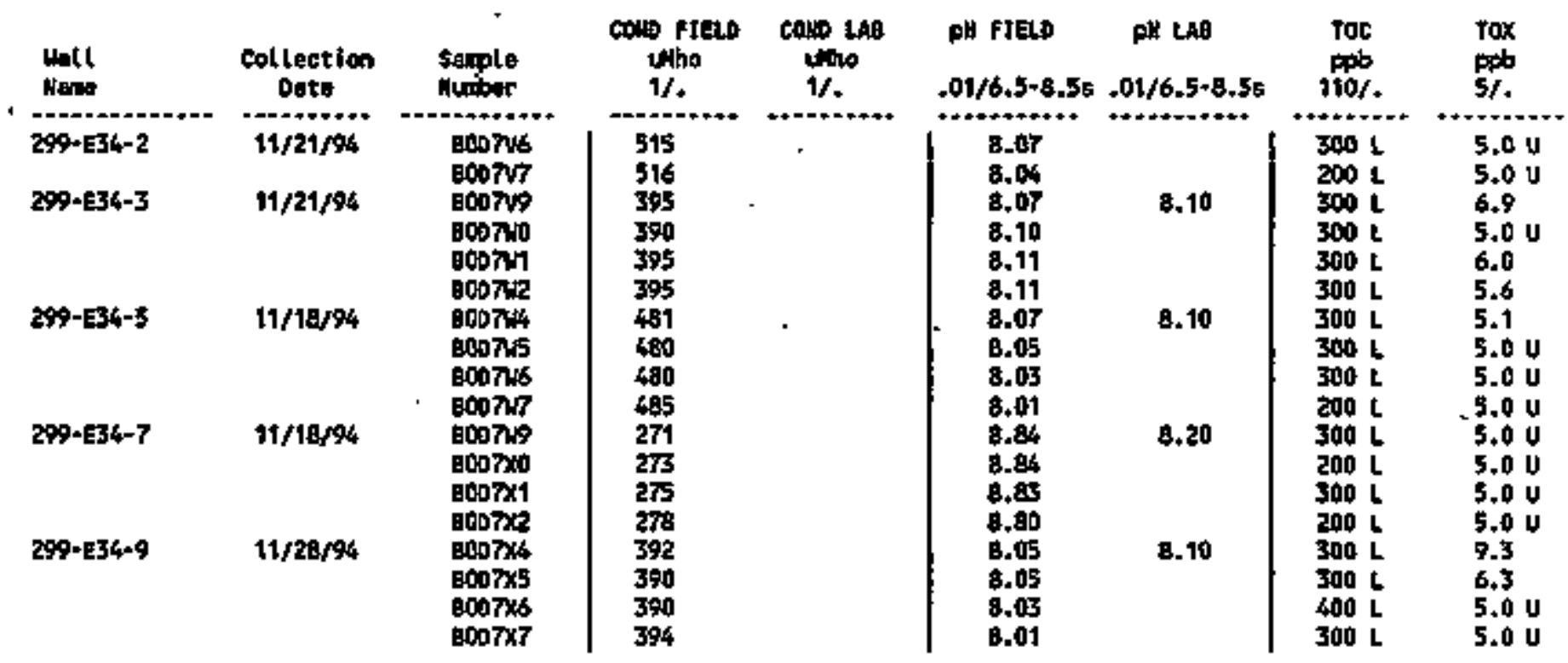

For explenation of this table, ses section 1.4 of report. 
Tabie 14-18. Constituent List and Summary of Results for the Low-Level Waste Management Area 3 Data for Reporting Period

October 1 through December 3I, 1994.

(sheet 1 of 3 )

\section{COHTMLMATIOH SLOLCATOR PARANETERS}

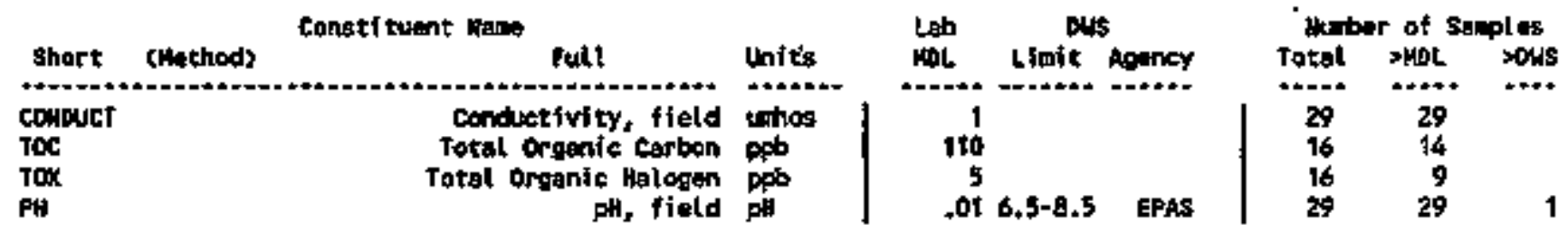

DRIHKTNG MRTER PARHETERS

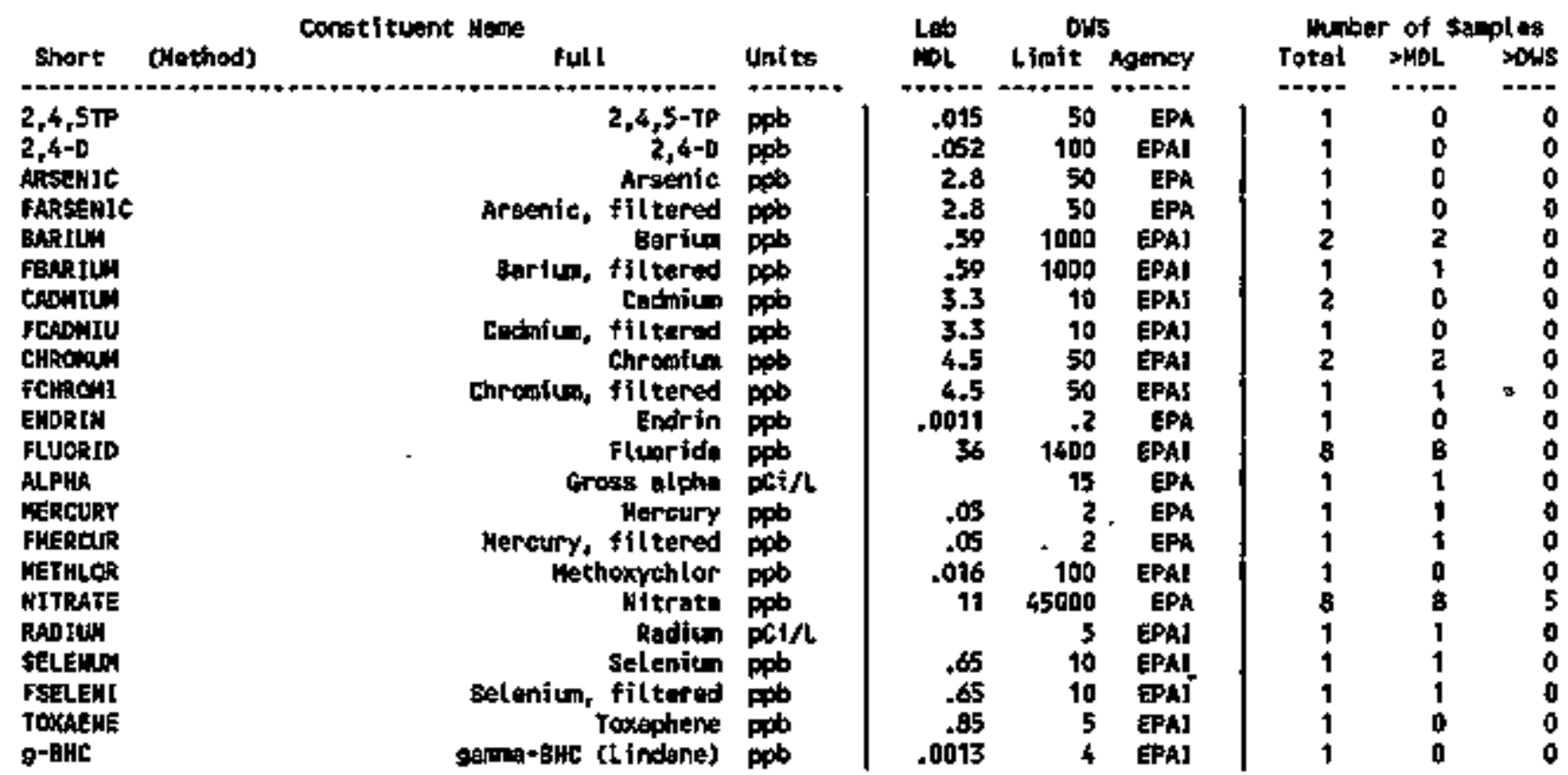

GROUNOWTER CULLJTY PARNETERS

\begin{tabular}{|c|c|c|c|c|c|c|c|c|c|}
\hline & & Conet'stuent Nane & & Lab & DHE & & 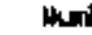 & $r$ of $s$ & ples \\
\hline short & (Hethod) & Full & Units & MDL & Linit & Agency & Totak & >WDL & sous \\
\hline & & $x+2+2=0$ & $+\infty+++\infty$ & $+2+2+4$ & $+2+x+4$ & $=0+\infty$ & $=0+$ & $\cdots+$ & $\cdots+$ \\
\hline ChLORto & & thitaride & pyb & 14 & 260000 & EPAS & $\mathbf{B}$ & 8 & 0 \\
\hline Inow & & Jron & pph & 5.24 & 300 & EFAS & 2 & 2 & 2 \\
\hline FIROM & & Iron, ffltered & pob & 5.24 & 300 & EPAS & 1 & 1 & 0 \\
\hline NANGESE & & Mangentse & pph & .72 & 50 & Pas & 2 & 2 & $\mathbf{0}$ \\
\hline Futhen & & Hongunest, fittered & pot & $-\pi$ & 50 & EPAS & 1 & 1 & 0 \\
\hline $\begin{array}{l}\text { LPtingl } \\
\text { soDIun }\end{array}$ & & Phenol & patb & -31 & & & 1 & 0 & \\
\hline Foolun & & sodiun, ffitrered & pos & 61 & & & $\begin{array}{l}2 \\
1\end{array}$ & $\frac{2}{1}$ & \\
\hline SUL.FATE & & Stufete & ppb & 37 & 250000 & EPAS & B & 8 & $\mathbf{0}$ \\
\hline
\end{tabular}


Tabie 14-18. Const1tuent L1st and Summary of Results for the Law-Leve1 Waste Management Area 3 Data for Reporting Period

october 1 through December 31, 1994.

(sheet 2 of 3)

SITE SPECIFIC AND OTंHER COHSTIJUEHTS

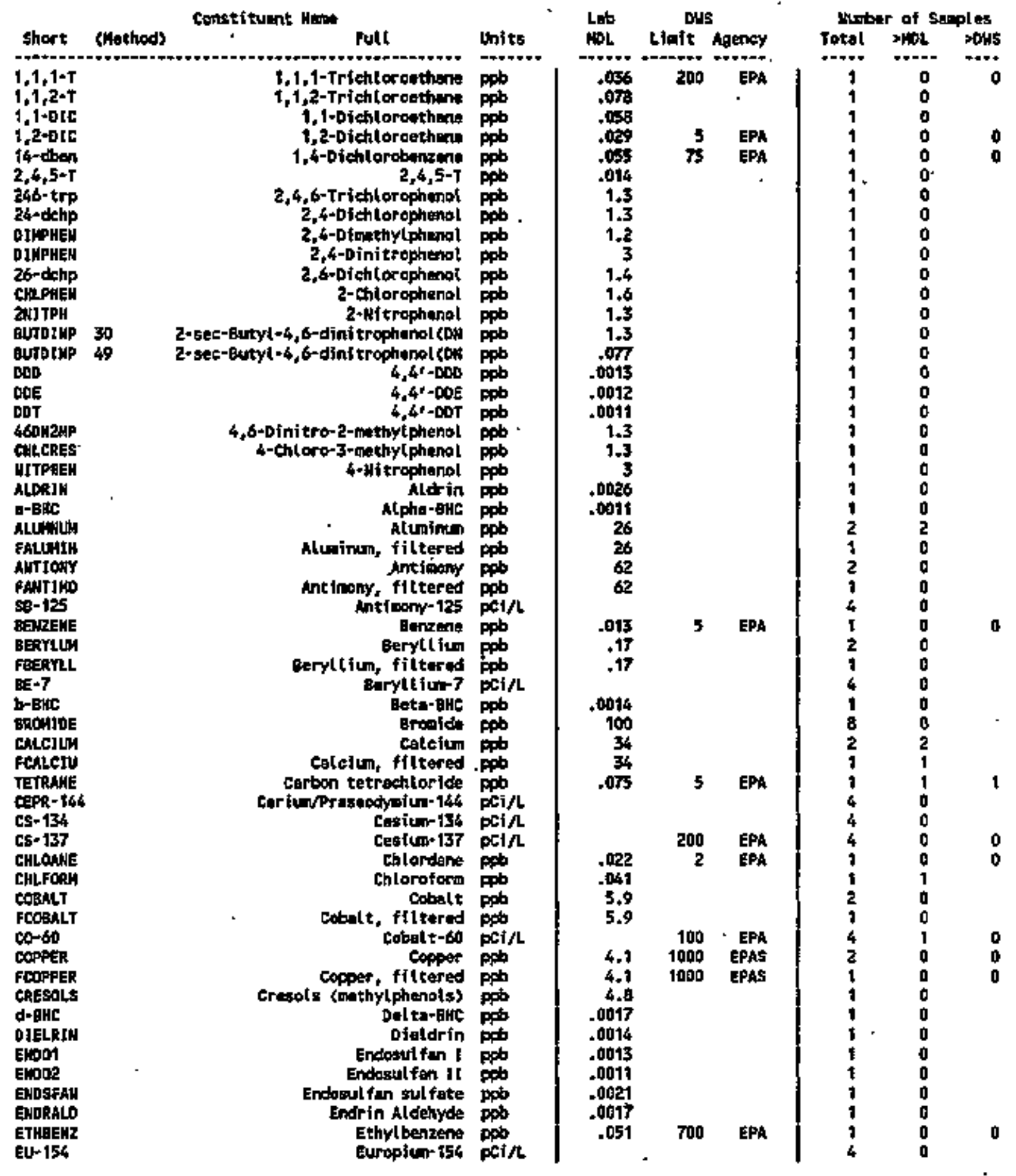


Table 14-18. Constituent List and Summary of Results for the Low-Level Waste Management Area 3 Data for Reporting Perjod

October 1 through Decenber 31, 1994.

(sheet 3 of 3 )

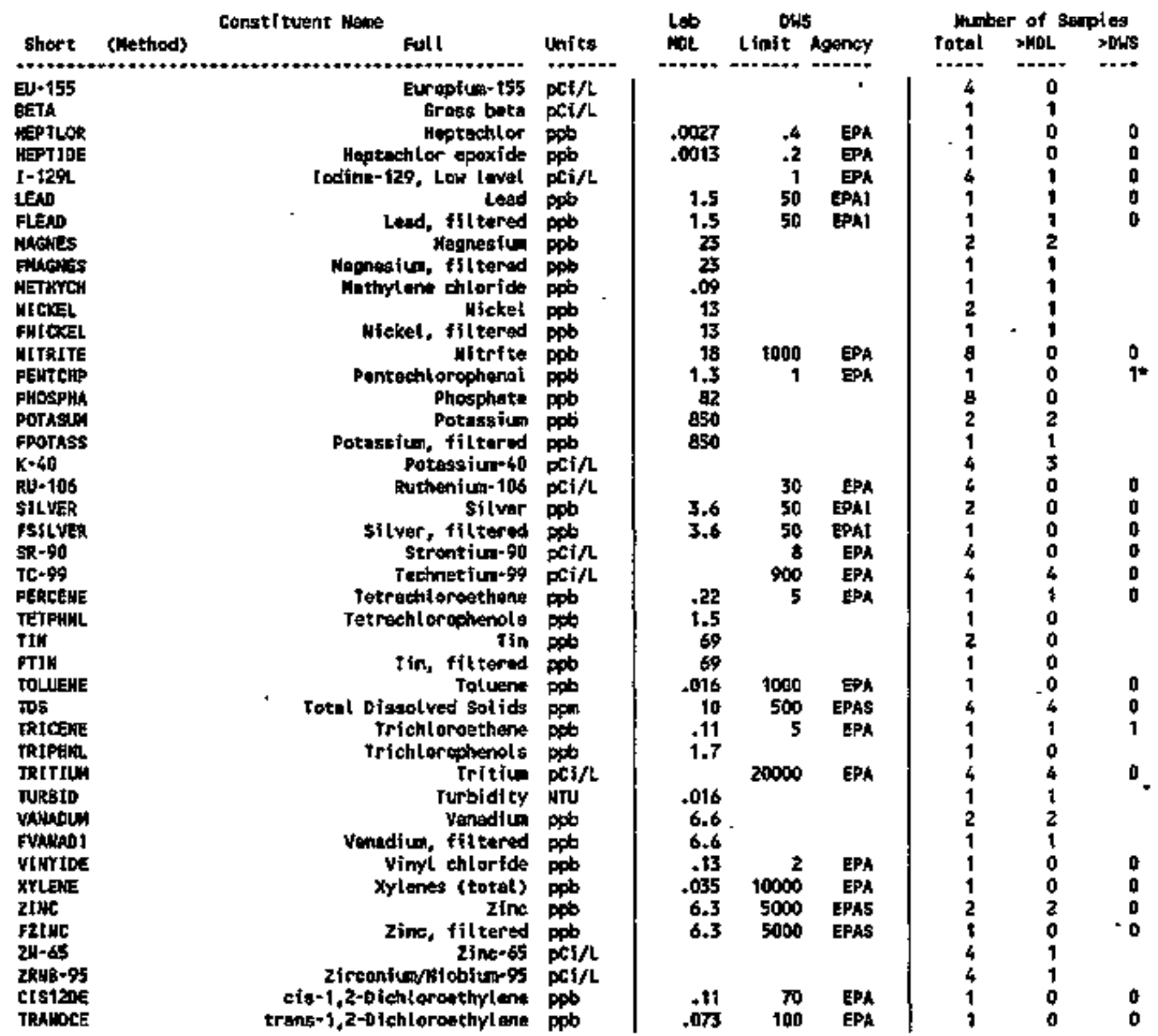

For explenetion of this table, see section 1.4 of report. 
Tab]e 14-19. Const $f$ tuents with at Least One Detected Value for the Low-Leve] Waste Management Area 3 Data for Reporting Period October 1 through December 31, 1994. (sheet 1 of 3 )

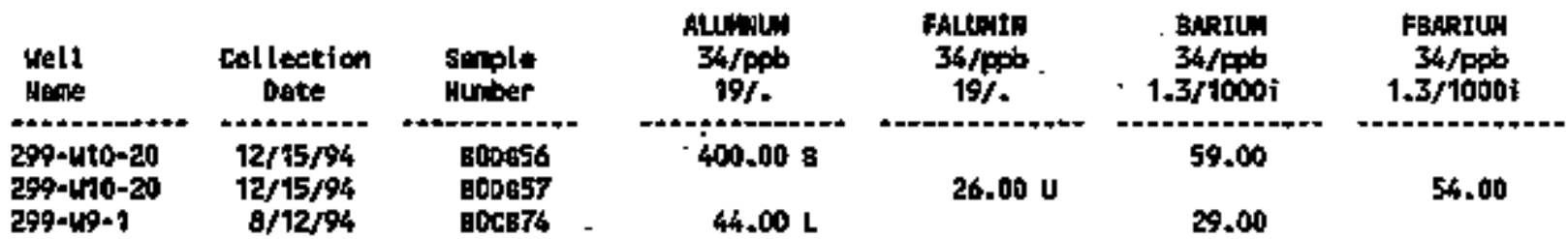

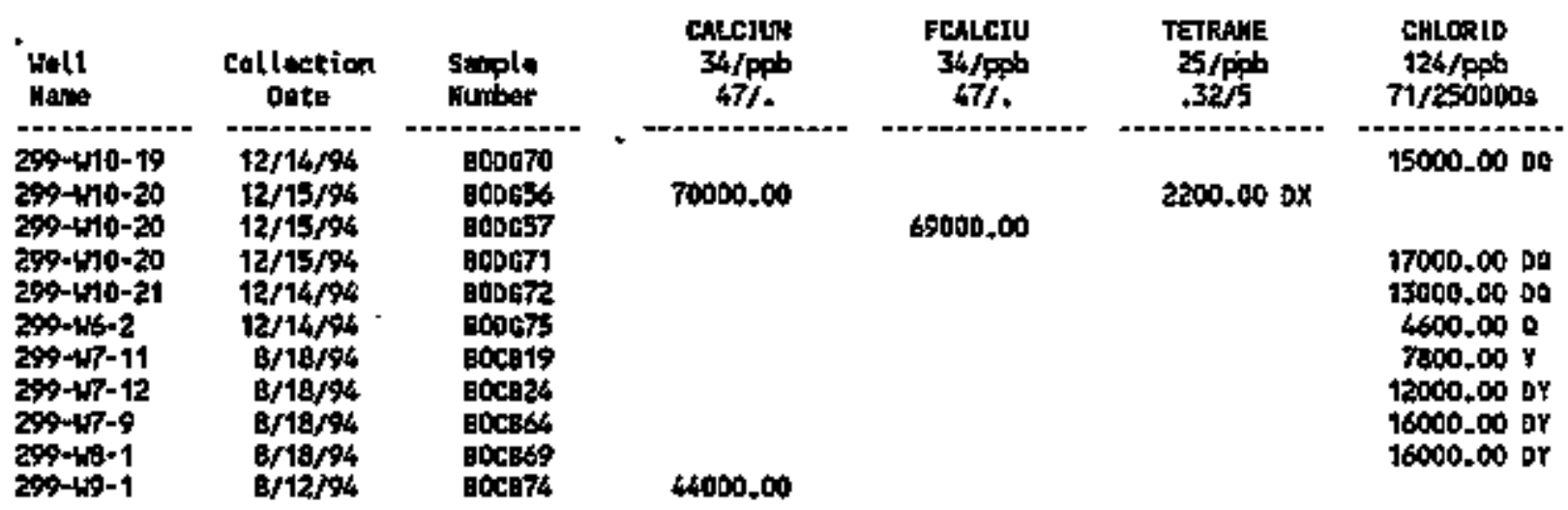

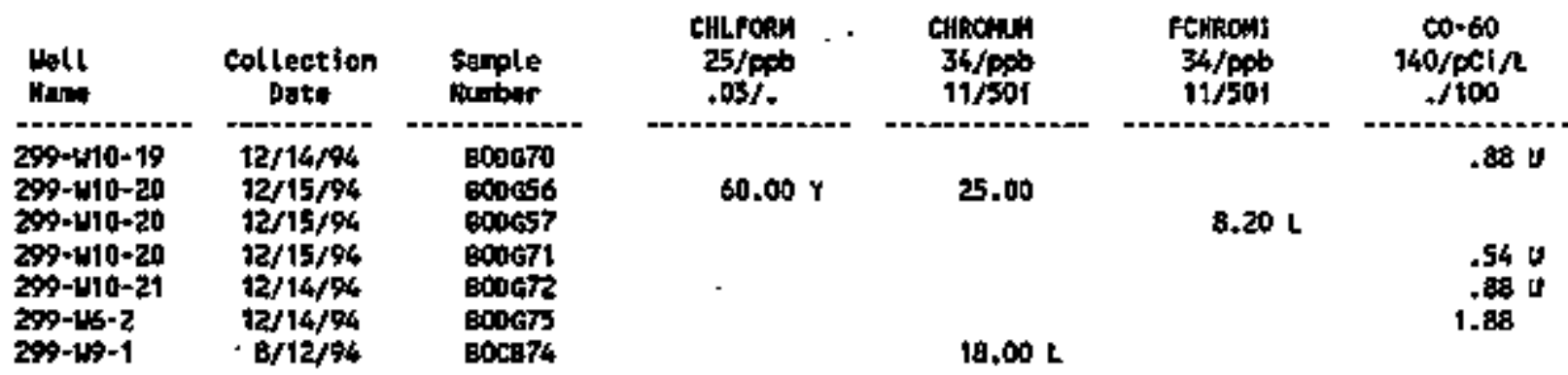

\begin{tabular}{|c|c|c|c|c|c|c|}
\hline Wett & $\begin{array}{c}\text { collection } \\
\text { trate }\end{array}$ & $\begin{array}{l}\text { semple } \\
\text { Hinitor }\end{array}$ & $\begin{array}{l}\text { Fttjorto } \\
124 / p+b \\
51 / 14001\end{array}$ & $\begin{array}{c}\text { ALPHA } \\
135 / \mathrm{pCD} / \mathrm{A} \\
. / 15\end{array}$ & $\begin{array}{c}\text { 日ETA } \\
136 / \mathrm{pC} 1 / \mathrm{L} \\
. / .\end{array}$ & $\begin{array}{c}\text { 1.129 } \\
\mathrm{t39} / \mathrm{pCi} / \mathrm{L} \\
. / 1\end{array}$ \\
\hline $\begin{array}{l}299-410-19 \\
299-410-20 \\
299-410-20 \\
299-410-21 \\
299-166-2 \\
299-47-11 \\
299-47-12 \\
299-177-9 \\
899-149+9\end{array}$ & $\begin{array}{l}12 / 14 / 94 \\
12 / 15 / 94 \\
12 / 15 / 94 \\
12 / 14 / 94 \\
12 / 14 / 94 \\
8 / 18 / 94 \\
8 / 48 / 94 \\
8 / 18 / 94 \\
8 / 18 / 94\end{array}$ & 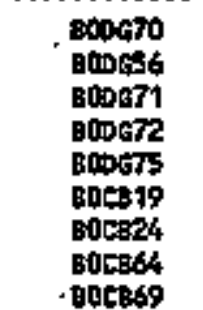 & $\begin{array}{l}500.00 \\
400.00 \\
400.00 \\
500.00 \\
800.00 Y \\
700.00 Y \\
700.00 Y \\
700.00 Y\end{array}$ & 1.57 & 24.60 & $\begin{array}{c}.01 \mathrm{U} \\
-.05 \mathrm{U} \\
.45 \\
.09 \mathrm{f}\end{array}$ \\
\hline
\end{tabular}


Table 14-19. Constituents with at Least One Detected Value for the Low-Leve] Waste Managerent Area 3 Data for Reporting Perjod

October 1 through December 31, 1994. (sheet 2 of 3 )

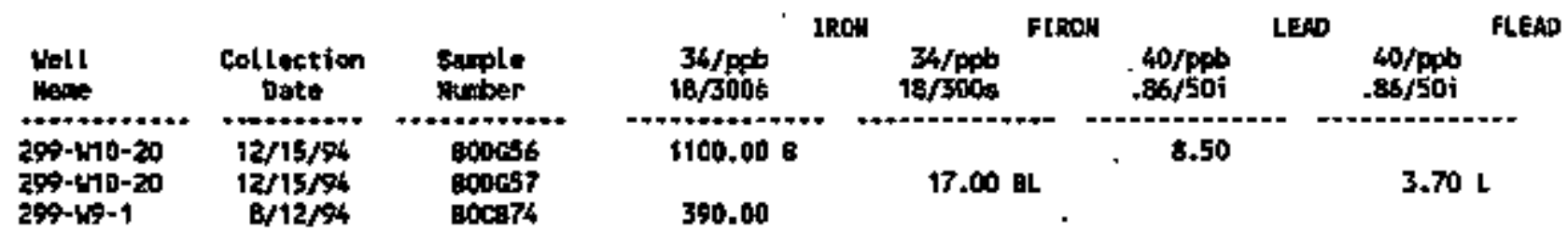

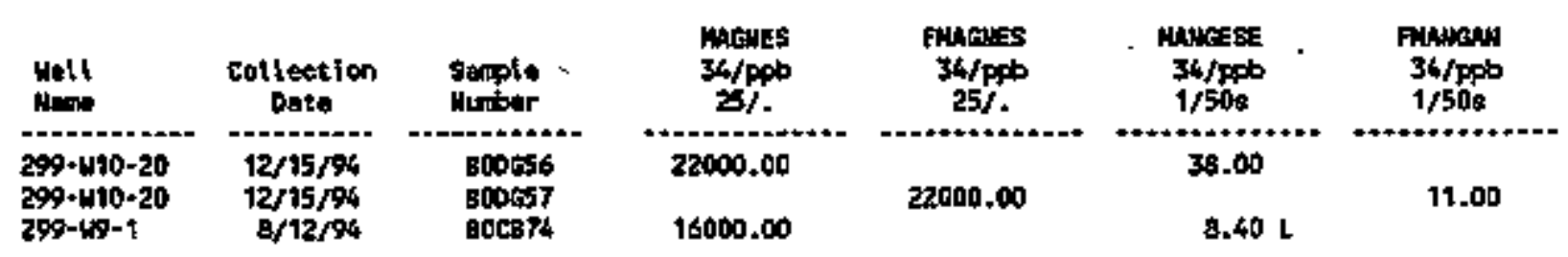

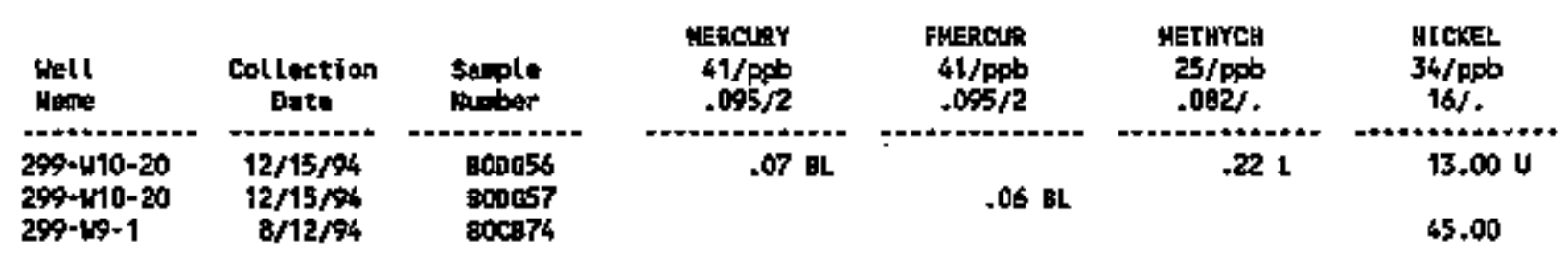

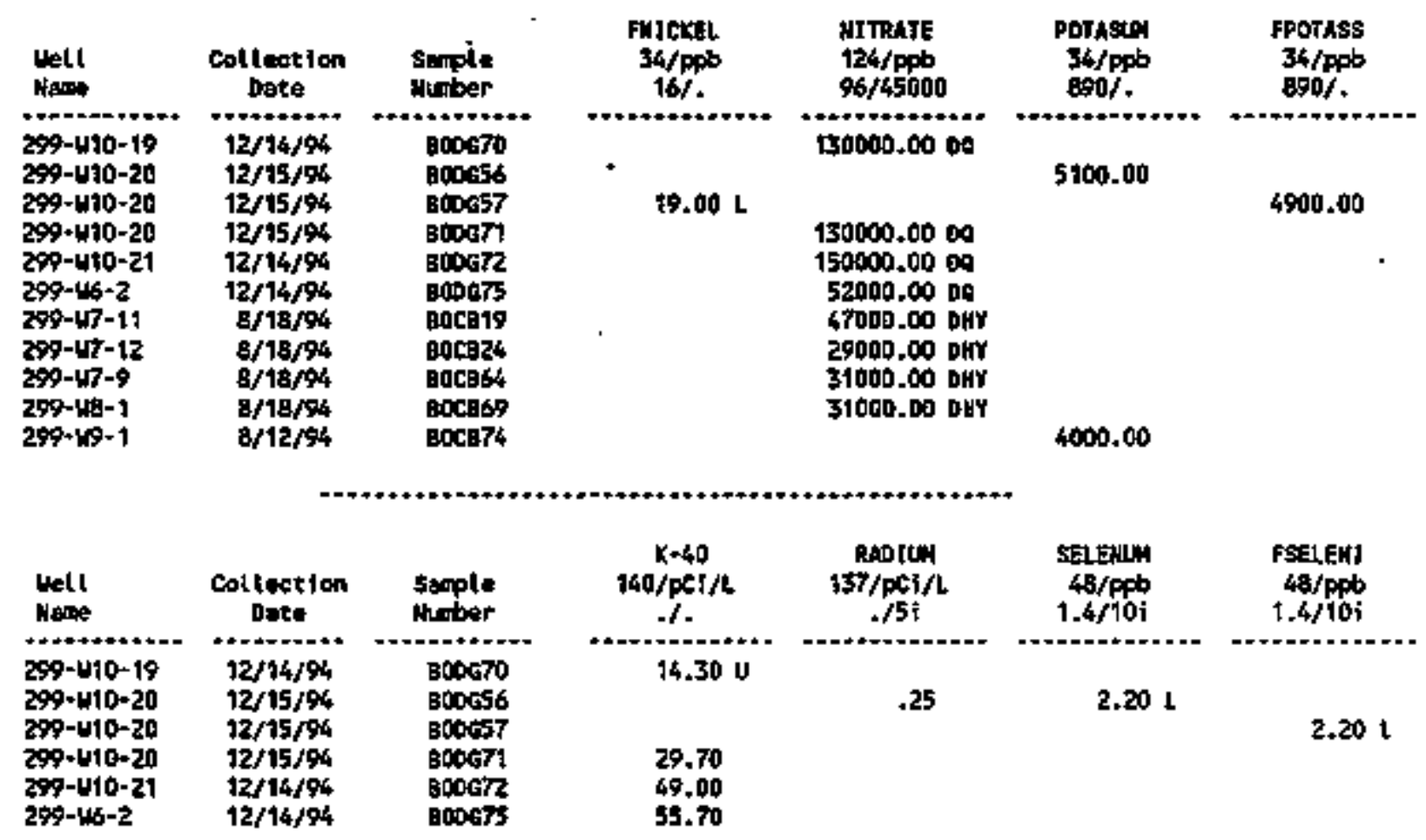


Table 14-19. Constituents with at Least One Detected Value for the Low-Level Waste Management Area 3 Data for Reporting Period October 1 through Decexber 31, 1994.

(sheet 3 of 3 )

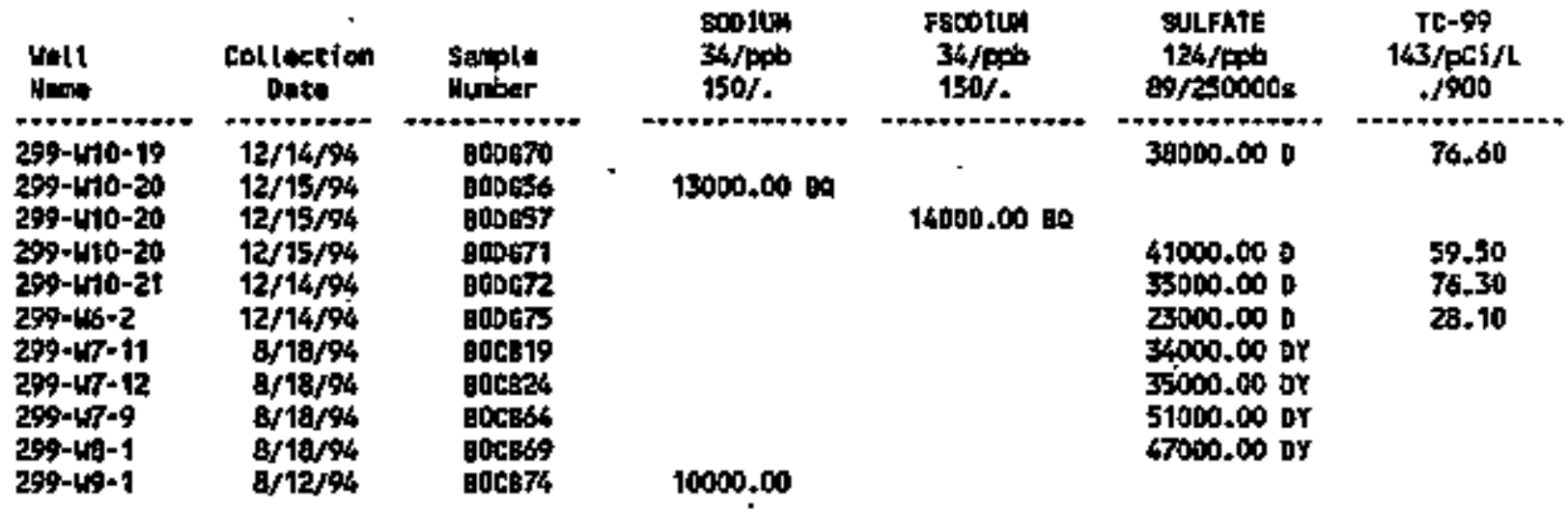

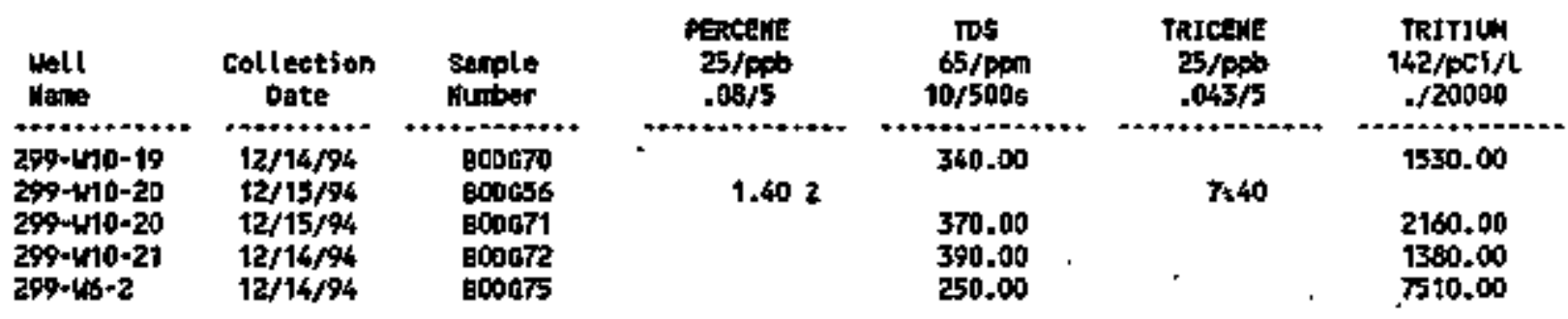

\begin{tabular}{|c|c|c|c|c|c|c|}
\hline + Uelt & $\begin{array}{c}\text { Coltection } \\
\text { Date }\end{array}$ & $\begin{array}{l}\text { Spipte } \\
\text { Heriber }\end{array}$ & $\begin{array}{c}\text { Tusiso } \\
\text { 126/uTu } \\
.016 / .\end{array}$ & $\begin{array}{l}\text { valanua } \\
\text { 34/pos } \\
6.4 / .\end{array}$ & $\begin{array}{c}\text { FWhips! } \\
34 / \mathrm{ppb} \\
6.4 / 6\end{array}$ & $\begin{array}{c}\text { 21uc } \\
34 / \mathrm{ppb} \\
4.4 / 5000 \mathrm{~s}\end{array}$ \\
\hline $\begin{array}{l}299-110-20 \\
299-10-20 \\
299-199-1\end{array}$ & $\begin{array}{l}12 / 15 / 94 \\
12 / 15 / 94 \\
8 / 12 / 94\end{array}$ & $\begin{array}{l}000056 \\
800557 \\
600374\end{array}$ & $24,00 \mathrm{n}$ & $\begin{array}{l}17.00 \mathrm{t} \\
21.00 \mathrm{~L}\end{array}$ & $13.00 \mathrm{~L}$ & $\begin{array}{l}13.00 \\
17.00 \mathrm{0}\end{array}$ \\
\hline
\end{tabular}

\begin{tabular}{|c|c|c|c|c|c|}
\hline Whetl & $\begin{array}{c}\text { Colliestion } \\
\text { Date }\end{array}$ & $\begin{array}{l}\text { Sepple } \\
\text { Hutiser. }\end{array}$ & $\begin{array}{c}\text { Ferwe } \\
34 / \mathrm{pob} \\
4.4 / 5600 \mathrm{~b}\end{array}$ & $\begin{array}{c}24-63 \\
940 / \mathrm{pc} i \mathrm{t} \\
. /\end{array}$ & 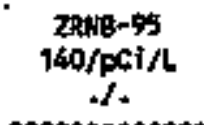 \\
\hline $\begin{array}{l}299-410-19 \\
299-410-20 \\
299-410-20 \\
299-410-21 \\
299-45-2\end{array}$ & $\begin{array}{l}12 / 14 / 94 \\
12 / 75 / 94 \\
12 / 15 / 94 \\
12 / 14 / 94 \\
12 / 14 / 94\end{array}$ & 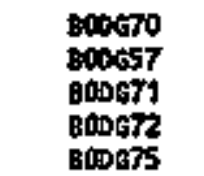 & $6.30 \mathrm{U}$ & $\begin{array}{r}2.11 \\
.72 \mathrm{U} \\
.75 \mathrm{U} \\
1.20 \mathrm{U}\end{array}$ & $\begin{array}{r}.2 .43 \mathrm{v} \\
2.45 \\
-1.13 \mathrm{U} \\
.52 \mathrm{U}\end{array}$ \\
\hline
\end{tabular}

For explanation of this toble, see Section 1.4 of report. 
Table 14-20. Contamination Indicator Parameters for the Low-Level Waste Management Area 3 Data for Reporting Period october 1 through December 31, 1994.

\begin{tabular}{|c|c|c|c|c|c|c|c|c|}
\hline Hell & $\begin{array}{l}\text { Collection } \\
\text { Date }\end{array}$ & $\begin{array}{l}\text { semple } \\
\text { nuber }\end{array}$ & $\begin{array}{l}\text { Contp fiElo } \\
\text { whe } \\
1 / .\end{array}$ & $\begin{array}{l}\text { COND LAB } \\
\text { ullho } \\
1 / .\end{array}$ & $\begin{array}{l}\text { PH FIELD } \\
.01 / 6.5-8.58\end{array}$ & $\begin{array}{l}\text { PAt LAB } \\
.01 / 6.5-8.58\end{array}$ & $\begin{array}{c}\text { Toc } \\
\text { ppb } \\
110 \%\end{array}$ & $\begin{array}{l}\mathrm{Tpx} \\
\mathrm{ppb} \\
5 \%\end{array}$ \\
\hline $299-410-13$ & $12 / 14 / 94$ & $\begin{array}{l}800058 \\
800659 \\
800060 \\
800651 \\
8000570\end{array}$ & $\begin{array}{l}452 \\
453 \\
450 \\
451 \\
558\end{array}$ & & $\begin{array}{l}8.51 \\
8.59 \\
8.61 \\
8.61 \\
8.77\end{array}$ & & $\begin{array}{l}110 L \\
200 L \\
140 L \\
120 L \\
300 L\end{array}$ & $\begin{array}{r}14.0 \\
16.4 \\
11.1 \\
11.1 \\
1000.0\end{array}$ \\
\hline $299-110+20$ & $12 / 15 / 94$ & $\begin{array}{l}\text { 800056 } \\
\text { 8000671 }\end{array}$ & & & & & & \\
\hline $\begin{array}{l}209+410-21 \\
207-46-2\end{array}$ & $\begin{array}{l}12 / 16 / 94 \\
11 / 50 / 94\end{array}$ & 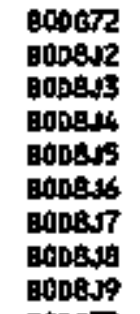 & $\begin{array}{l}598 \\
395 \\
395 \\
395 \\
398 \\
396 \\
395 \\
395 \\
397\end{array}$ & & $\begin{array}{l}8.20 \\
8.08 \\
8.08 \\
8.08 \\
8.08 \\
8.08 \\
8.08 \\
8.08 \\
8.07\end{array}$ & & 4001 & 444.0 \\
\hline $299-48-1$ & $\begin{array}{l}12 / 14 / 94 \\
11 / 30 / 94\end{array}$ & 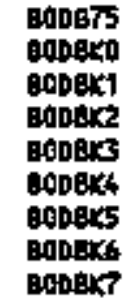 & $\begin{array}{l}370 \\
396 \\
396 \\
394 \\
393 \\
393 \\
393 \\
393 \\
393\end{array}$ & . & $\begin{array}{l}6.4 t \\
8.31 \\
8.29 \\
8.28 \\
8.28 \\
8.28 \\
8.30 \\
8.29 \\
8.28\end{array}$ & & $200 \mathrm{~L}$ & 119.0 \\
\hline $299-49+1$ & $12 / 14 / 94$ & $\begin{array}{l}800062 \\
800063 \\
000064 \\
800065 \\
000064 \\
000067 \\
600068 \\
000069\end{array}$ & $\begin{array}{l}450 \\
454 \\
458 \\
455\end{array}$ & & $\begin{array}{l}8.76 \\
8.75 \\
8.74 \\
8.74\end{array}$ & - & $\begin{array}{l}110 \mathrm{U} \\
110 \mathrm{U} \\
160 \mathrm{~L} \\
300 \mathrm{~L} \\
150 \mathrm{~L} \\
300 \mathrm{~L} \\
200 \mathrm{~L} \\
200 \mathrm{~L}\end{array}$ & $\begin{array}{l}5.0 \mathrm{U} \\
5.0 \mathrm{U} \\
5.0 \mathrm{U} \\
5.0 \mathrm{U} \\
5.0 \mathrm{U} \\
5.0 \mathrm{U} \\
5.3 \\
5.0 \mathrm{U}\end{array}$ \\
\hline
\end{tabular}

For explunation of this table, sen suction 1.4 of report. 
Table 14-21. Constituent List and:5ummary of Results for the Low-Level - Waste Management Area 4 Data for Reporting Period

October 1 through December 31, 1994.

(sheet 1 of 3)

Contantakt3 On trotenTOA PANNETERS

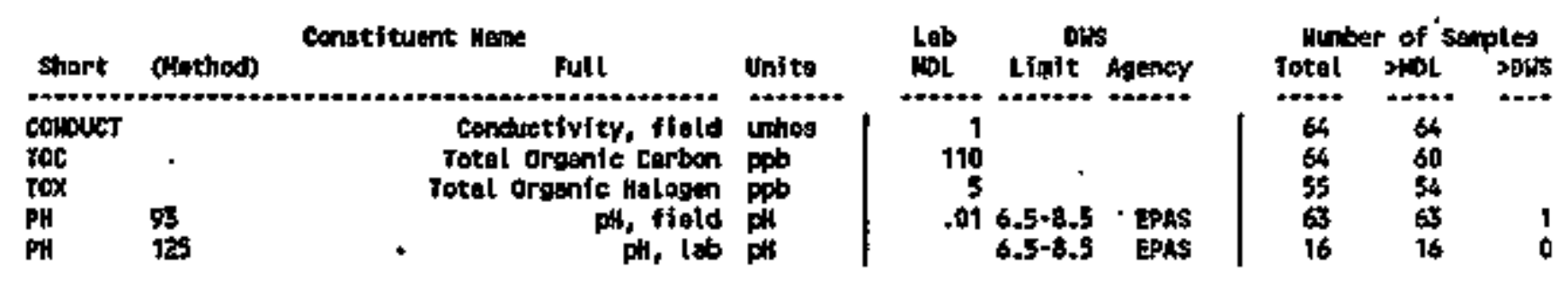

DRTMKIHG toter PARANLtERS

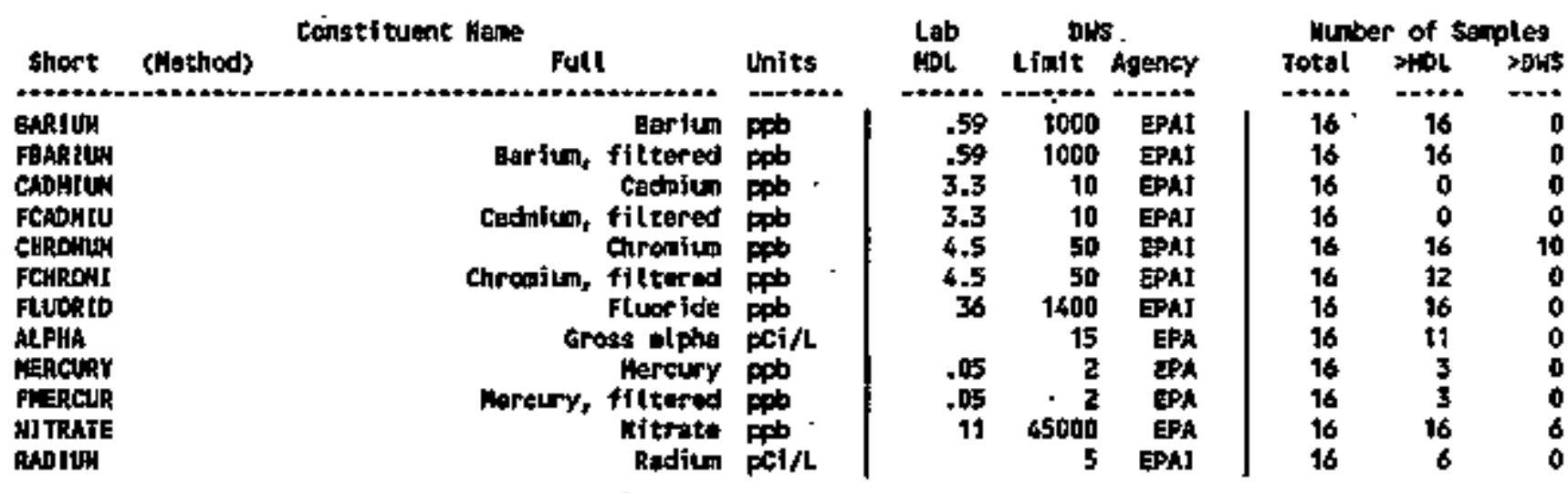

CROULOURTER GLALITY PARUI:TERS

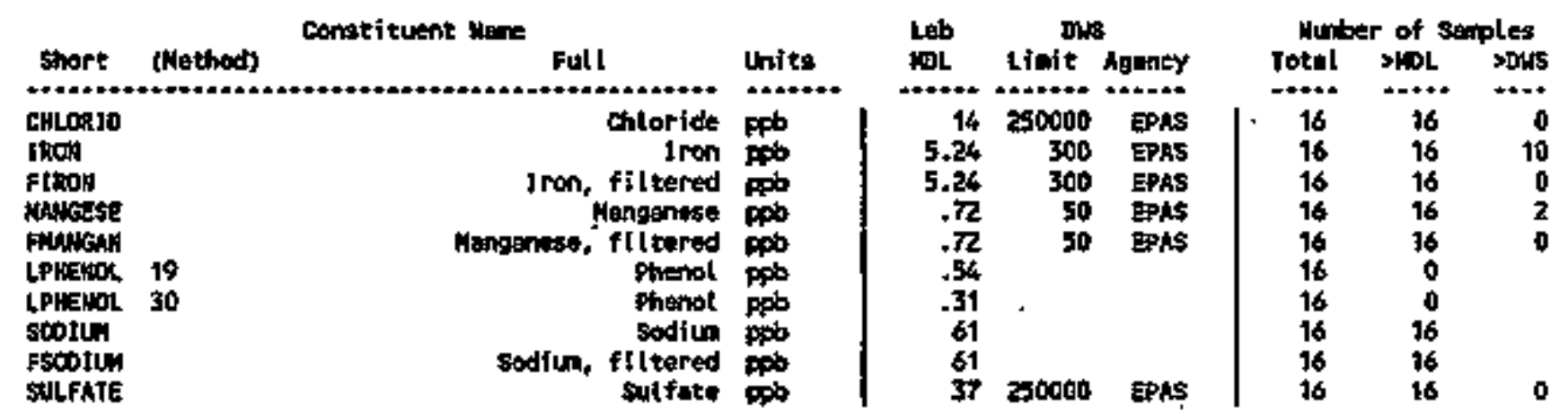


Table 14-21. Constituent List and Summary of Results for the Low-Level Waste Managenont Area 4 Data for Reporting Period

october 1 through December 31, 1994.

(sheet 2 of 3 )

SITE SPECIFIC AND OTHER COMSTITUENTS

\begin{tabular}{|c|c|c|c|c|c|c|c|c|c|}
\hline & & Consti buent kales & & Lab & tats & & Het & r of $s$ & ples \\
\hline Short & (Hethod) & Full & Unt to & $\mathrm{NOH}$ & Linit & hatency & Total & YHVL & SDN5 \\
\hline 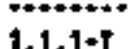 & 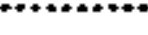 & 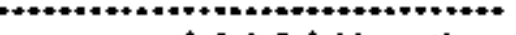 & $\cdots$ & $\cdots+\cdots$ & $+\infty$ & +-- & ---- & 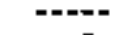 & $\cdots$ \\
\hline $\begin{array}{l}1,1,1-t \\
5,1,2-\tau\end{array}$ & & 1, 1,t-Jrichlaranthane & "מan & -036 & 200 & EPA & 16 & D & 0 \\
\hline $\begin{array}{l}1,1,2-1 \\
1,1-0, c\end{array}$ & & 1,1,2-7richloras fhane & מלp & .078 & & & 16 & D & \\
\hline $\begin{array}{l}1,1-016 \\
1,2-010\end{array}$ & & Pichlorosthane & ppt & .058 & & I- & 6 & 0 & \\
\hline $\begin{array}{l}1,2-0116 \\
14 \text {-dben }\end{array}$ & & $\begin{array}{l}\text { 1,2-bichlorouthane } \\
\text { 1, -biehlorobenzene }\end{array}$ & $\underset{0}{p}$ & 005 & $\frac{5}{5}$ & EPA & 14 & $\begin{array}{l}0 \\
0\end{array}$ & 0 \\
\hline 246-trp & & $2,4,6$-Trichlorophernol & $\operatorname{pob}$ & 1.3 & & & 16 & 0 & \\
\hline 24-dahp & 19 & 2,4-bichl orephenal & ppb & 4 & & & 16 & $\mathbf{0}$ & \\
\hline 24-dehp & 30 & 2,4-61chloreptingl & ppos & 1,3 & & & 16 & $\boldsymbol{0}$ & \\
\hline DINPKEN & & 2,4-Diusthyl phenol & ppos & 1.2 & & & 16 & $\mathbf{0}$ & \\
\hline OLMPREM & & 2,4-Dinitrophetol & ppb & 3 & & & 16 & 0 & \\
\hline 26-detpp & & 2,6-blehlor opbenal & ppb & 1.4 & & & 16 & 0 & \\
\hline CHLPREM & & 2-Chloroshenol & Fpb & 1.6 & & & 16 & 0 & \\
\hline ZWETHPB & & z-Nathylphenal & ppb & 5.1 & & & 16 & 0 & \\
\hline 2NITPA & 19 & 2-kttraphenol & ppb & 1.9 & & & 16 & $\mathbf{0}$ & \\
\hline 2NITPG & 30 & 2-N1trophimal & ppb & 1.3 & & & 16 & $\mathbf{0}$ & \\
\hline BVTOLn: & & 2-5et-Eutyl-4,6-dini trophenol (O) & ppb & 1.3 & & & 16 & 0 & \\
\hline ASDUZUP & & 4,6-binitro-7-methylphenol & ppb & 1,3 & & & 16 & 0 & \\
\hline CHLCAES & & 4-chloro-3+methylphenol & $\mathrm{ppb}$ & 1.3 & & & 16 & 0 & \\
\hline AMETKPH & & 4-Aathylphenol & pqb & 7 & & & 96 & (1) & \\
\hline MITPKEN & & 4-yft trophand & ppb & 3 & & & 16 & 0 & \\
\hline ALIDIMAM & & Mtunisum & ppb & 26 & & & It & 10 & \\
\hline FALUNTN & & Aluning, filtared & ppb & 26 & & & 16 & 1 & \\
\hline AKT IONY & & entimory & pho & 62 & & & 16 & 0 & \\
\hline FanF BNO & & Ant inony, filtered & ppos & 62 & & & 16 & 0 & \\
\hline GEWZEํㅔㄹㅡ & & Benzaris & pob & .013 & 5 & $E P A$ & 14 & $t$ & n \\
\hline GEMzOTH & & Benzothiarole & geb & 1.3 & & & 16 & 0 & \\
\hline ECRYLUN & & Berylliun & pob & .17 & & & 18 & 1 & \\
\hline PEERTLL & & Eerylljur, filtered & ppb & .17 & & & 16 & 0 & \\
\hline BISAEPH & . & Bistz-ethylhexyls phthalote & $\mathbf{P}$ & 5.3 & & & 16 & 0 & \\
\hline enontDE & & Bromide & $p+b$ & teo & & & 16 & 1 & \\
\hline chlc11y & & colcion & pep & 34 & - & & 14 & 16 & \\
\hline fatuclt & & Colciun, filtered & path & 34 & & & 16 & 16 & \\
\hline TETRAHE & & Carban tetrachloride & pats & .075 & 5 & EPA & 16 & 15 & 13 \\
\hline CHLFORH & & -Chlorafora & pos & .041 & & & 16 & 15 & \\
\hline costht & & Foble & pot & 5.9 & & & 16 & $\mathbf{0}$ & \\
\hline FCOBALT & & Cobale, filtered & ppb & 5.9 & & & 1 & 0 & \\
\hline COPPER & & Copper & pab & 4.1 & 1000 & EPAS & 1 & 9 & 0 \\
\hline FCOPFER & & Copper, ti l tered & ppb & 4.1 & 1000 & EPAS & is & 2 & D \\
\hline CRESOLS & & Crogola (nothylphonols) & pab & 4.8 & & & 1 & 0 & \\
\hline CraHTOE & & Cranide & ppb & & & & $t$ & 3 & \\
\hline DECHe & & Decane & ppb & 1.9 & & & 1 & a & \\
\hline ONOECAN & & Dodacmente & $\mathbf{p p b}$ & 1.1 & & & & 0 & \\
\hline ETHBEAZ & & Ethyibe & pob & .051 & 700 & EPA & 16 & 0 & 0 \\
\hline
\end{tabular}


Table 14-21. Constituent list and Summary of Results for the Low-Level Waste Management Area 4 Data for Reporting Period

October 1 through Decenber 31, 1994.

(sheet 3 of 3 )

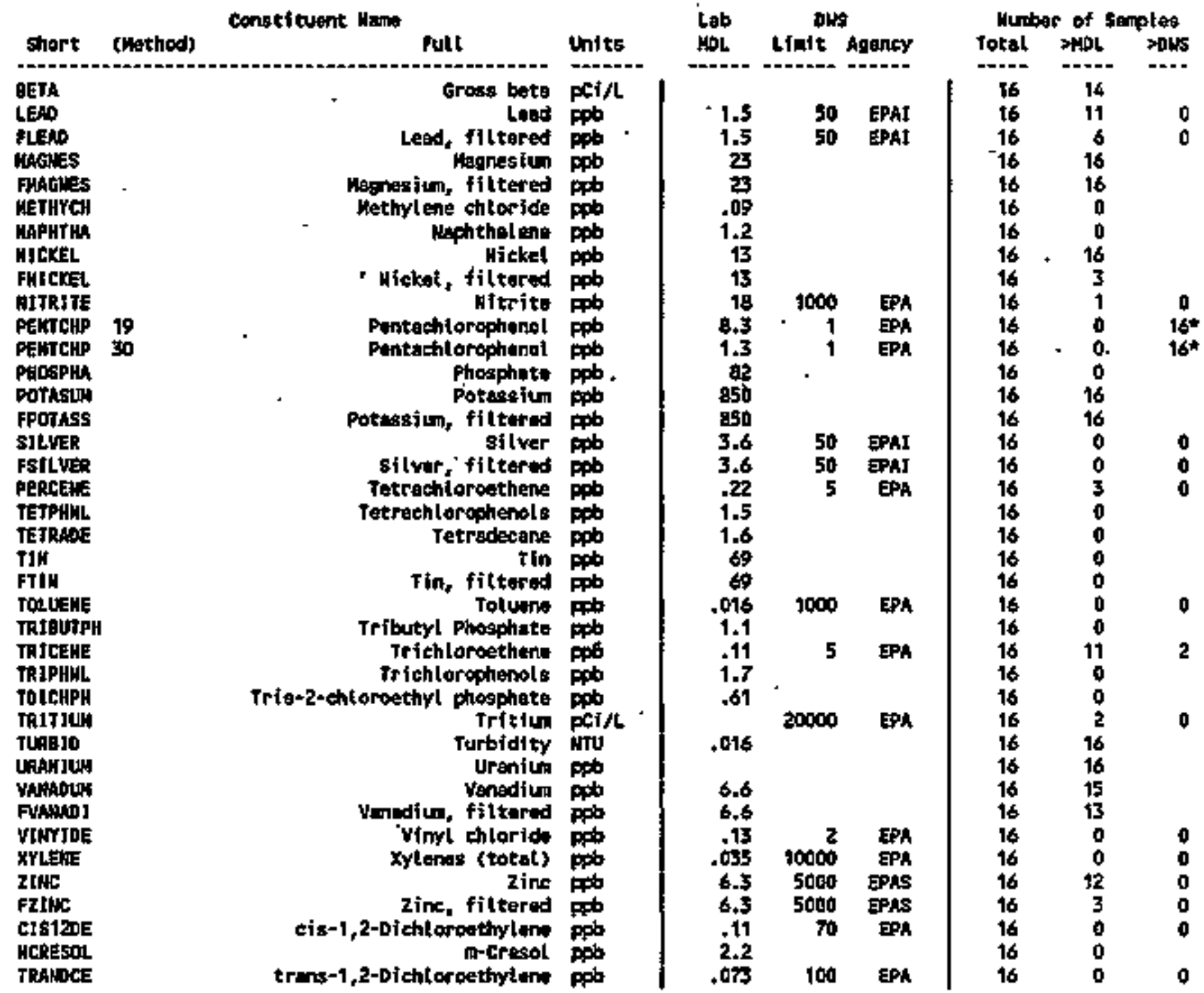

For explanation of this teble, see section 1.4 of report. 
Table 14-22. Constituents with at Least One Detected Value for the Low-Leve1 Waste Management Area 4 Data for Reporting Period

October 1 through December 31, 1994.

(sheet 1 of 9)

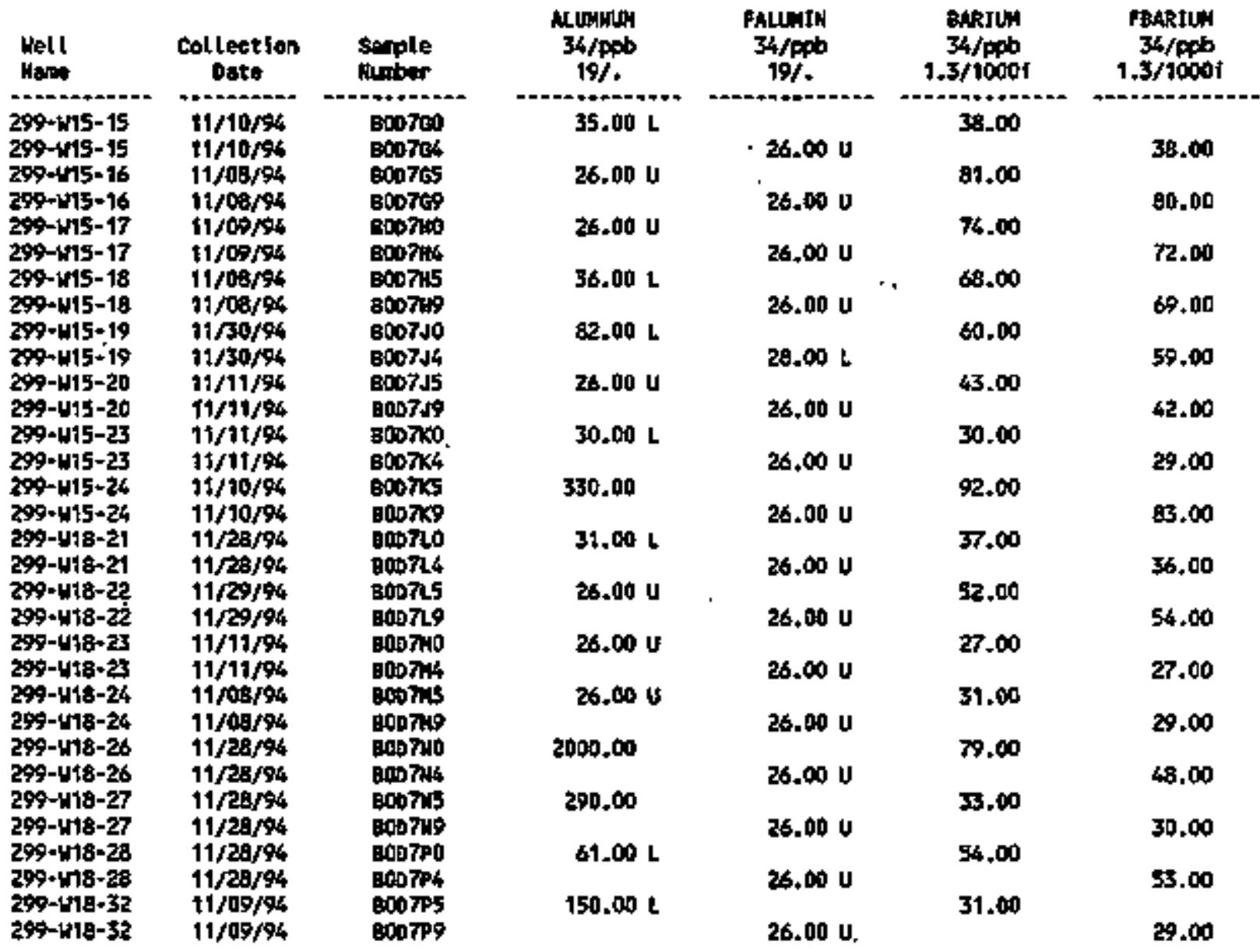

\begin{tabular}{|c|c|c|c|c|c|c|}
\hline Hell & $\begin{array}{c}\text { Collection } \\
\text { Dete }\end{array}$ & 5epple & $\begin{array}{l}\text { DENRENE } \\
25 / p \text { pb } \\
.24 / 5\end{array}$ & $\begin{array}{c}\text { CERYLUIM } \\
34 / \mathrm{ppb} \\
1.5 /\end{array}$ & $\begin{array}{c}\text { TBEEYLLL } \\
36 / p 96 \\
1.5 \%\end{array}$ & $\begin{array}{l}\text { BROWIOE } \\
124 / \mathrm{pPb} \\
110 \mathrm{H}\end{array}$ \\
\hline $9-415+15$ & $11 / 10 / 94$ & B00700 & $.01 \mathrm{u}$ & $.17 \mathrm{U}$ & & $100.00 \mathrm{U}$ \\
\hline $\begin{array}{l}299-W 15-15 \\
299-w 15-16\end{array}$ & $\begin{array}{l}11 / 10 / 96 \\
11 / 08 / 94\end{array}$ & $\begin{array}{l}600704 \\
800705\end{array}$ & .06 LH & $.17 \mathrm{U}$ & $.17 \mathrm{U}$ & $300.00 \mathrm{LY}$ \\
\hline $299-$-415-16 & $11 / 08 / 94$ & 600769 & & & $.17 \mathrm{U}$ & \\
\hline $\begin{array}{l}299-415-17 \\
299-U 15-17\end{array}$ & $\begin{array}{l}11 / 09 / 94 \\
11 / 00 / 94\end{array}$ & $\begin{array}{l}\text { Baprio } \\
\text { acorta4 }\end{array}$ & U & $.17 \mathrm{U}$ & ע 7. & $100.00 \mathrm{U}$ \\
\hline $\begin{array}{l}299-415-18 \\
299-415-18\end{array}$ & $\begin{array}{l}11 / 08 / 94 \\
11 / 08 / 94\end{array}$ & $\begin{array}{l}\text { 8007nis } \\
\text { Bop } 7199\end{array}$ & .01 Ua & $.17 \mathrm{~V}$ & $17 \mathrm{U}$ & $100.00 \mathrm{UT}$ \\
\hline $\begin{array}{l}299-415-19 \\
299 \cdot-15-19\end{array}$ & $\begin{array}{l}11 / 30 / 94 \\
11 / 30 / 94\end{array}$ & $\begin{array}{l}0007+10 \\
8007 \sqrt{14}\end{array}$ & .01 U & $.17 U$ & $.17 \mathrm{U}$ & $20.00 \mathrm{~L}$ \\
\hline $\begin{array}{l}299-415-20 \\
209-415-20\end{array}$ & $\begin{array}{l}11 / 11 / 94 \\
11 / 11 / 94\end{array}$ & $\begin{array}{l}\text { 80007 J5 } \\
\text { 8000799 }\end{array}$ & $.01 U$ & .174 & $17 \mathrm{U}$ & $109.00 \mathrm{v}$ \\
\hline $\begin{array}{l}299+415-23 \\
299-415-\frac{2}{2}\end{array}$ & $\begin{array}{l}11 / 11 / 94 \\
11 / 11 / 94\end{array}$ & $\begin{array}{l}8007 \times 0 \\
8007 \times 4\end{array}$ & $.01 \mathrm{U}$ & .17 U & $17 \mathrm{u}$ & $100.00 \mathrm{U}$ \\
\hline $\begin{array}{l}259-115+24 \\
299-115-24\end{array}$ & $\begin{array}{l}11 / 10 / 94 \\
11 / 10 / 94\end{array}$ & $\begin{array}{l}0007 \times 5 \\
0007 \times 9\end{array}$ & $.01 \mathrm{v}$ & $.37 \mathrm{~L}$ & $.17 \mathrm{u}$ & 100.000 \\
\hline
\end{tabular}


Tab]e 14-22. Constituents with at Least One Detected Value for the Low-Level Waste Managenent Area 4 Data for Reporting Period October 1 through Decemiber 31, 1994. (sheet 2 of 9)

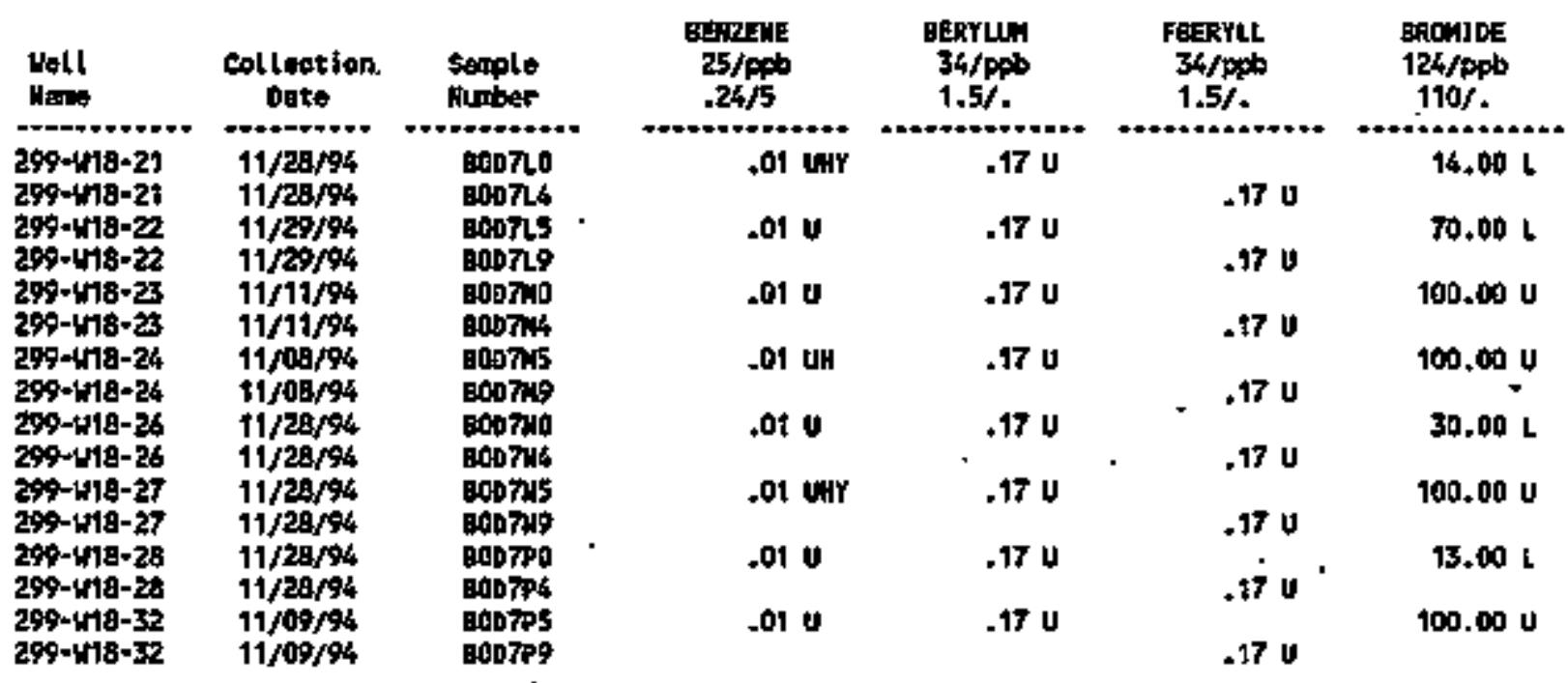

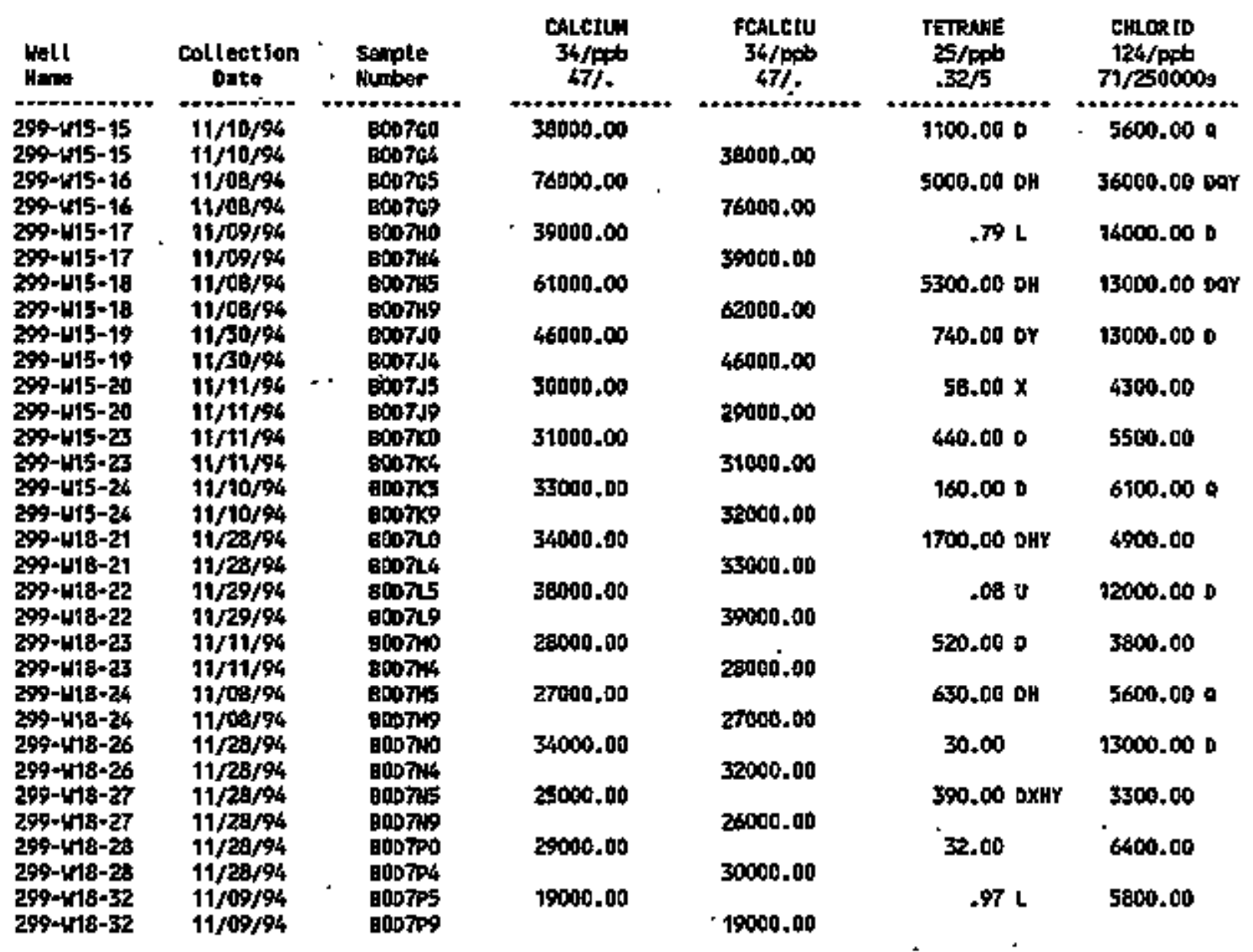


DOE/RL-94-36-4 
Table 14-22. Constituents with at Least one Detected Value for the Low-Leve] Waste Management Area 4 Data for Reporting Period October 1 through Decenber 31, 1994. (sheet 3 of 9)

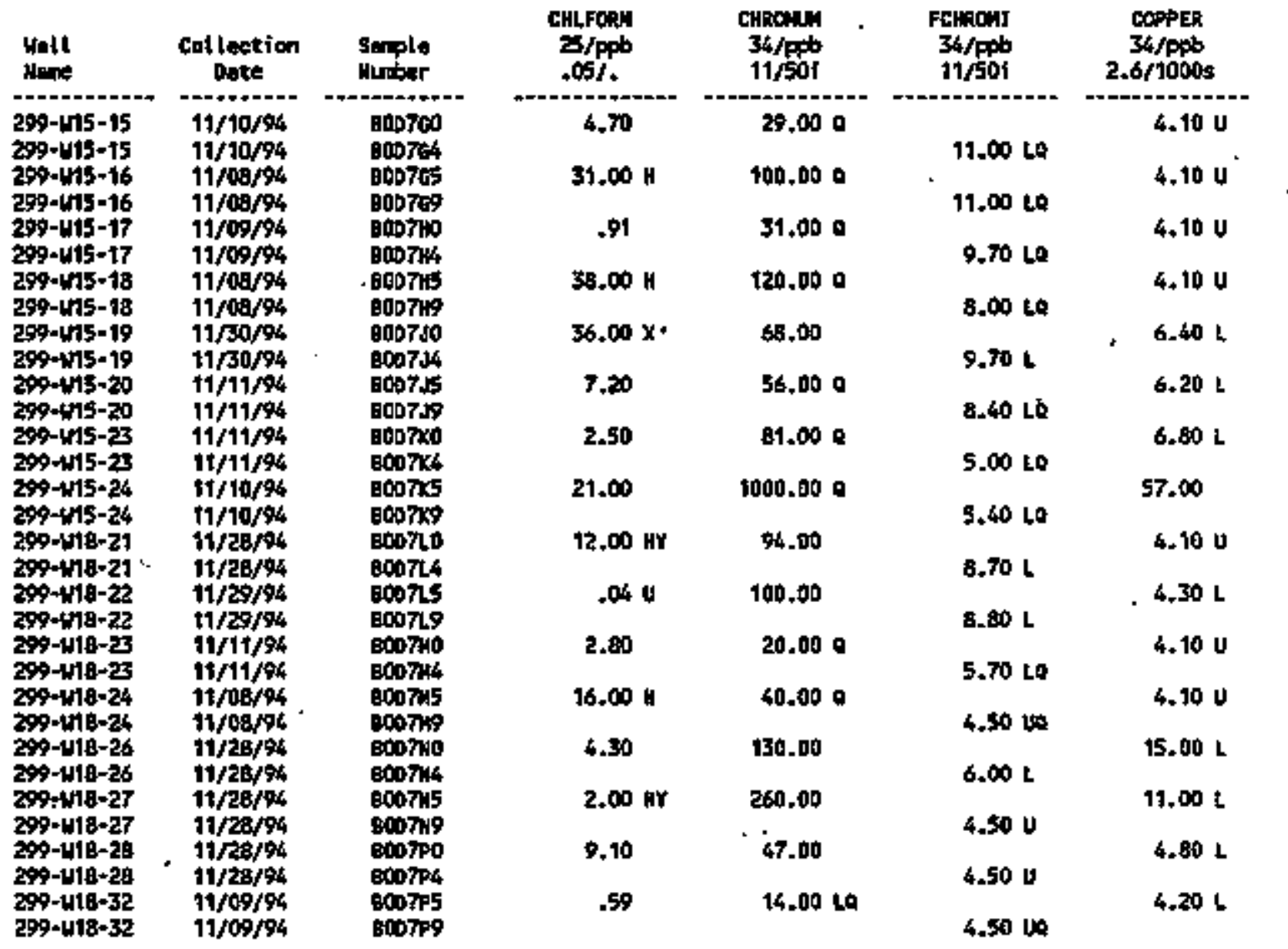

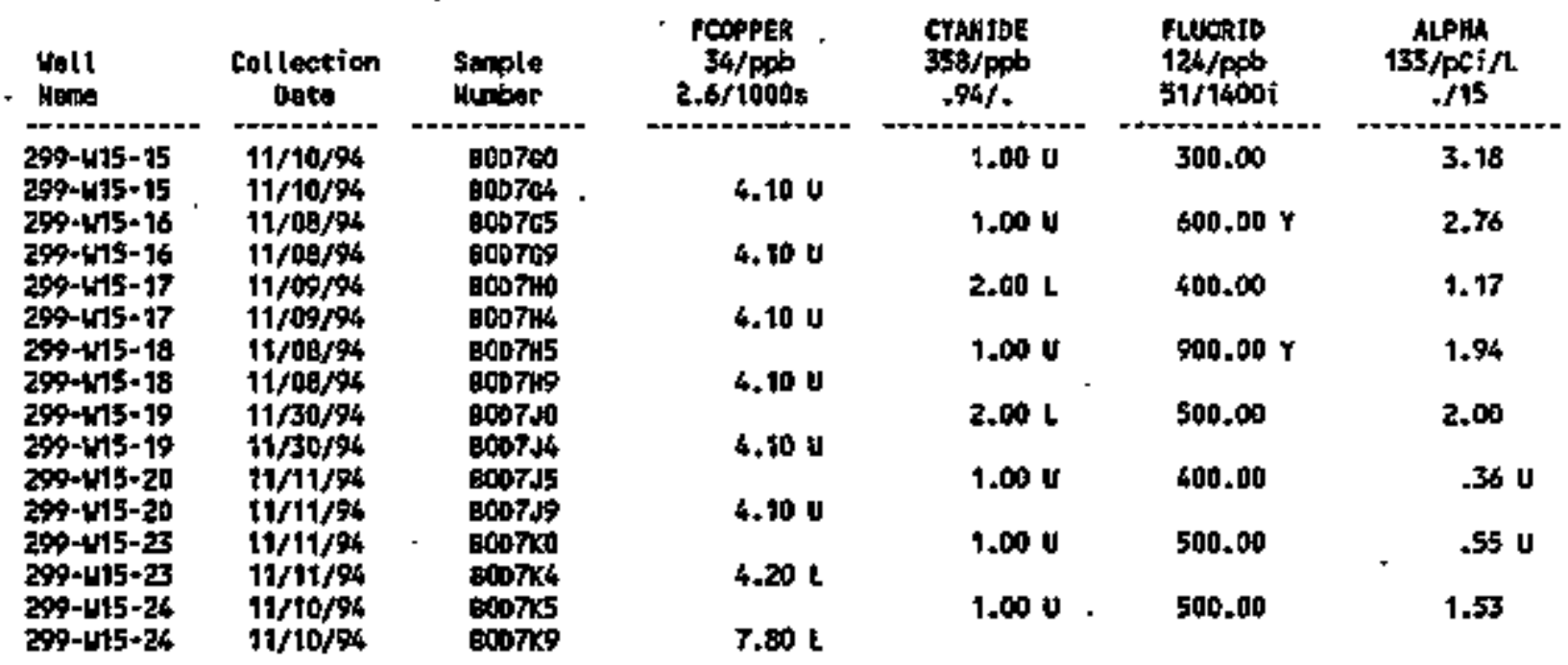


Table 14-22. Constituents with at Least One Detected Value for the Low-Leve1 Waste Managewent Area 4 Data for Reporting Period October 1 through December $31,1994$.

(sheet 4 of 9 )

\begin{tabular}{|c|c|c|c|c|c|c|}
\hline Holl & $\begin{array}{l}\text { Collestion } \\
\text { Dote }\end{array}$ & $\begin{array}{l}\text { sample } \\
\text { Munber }\end{array}$ & $\begin{array}{c}\text { Ftopele } \\
34 / \mathrm{ppb} \\
2.6 / 1000 \mathrm{~s}\end{array}$ & $\begin{array}{c}\text { CYAMH10E } \\
\text { 358/ppb } \\
. \quad .96 / .\end{array}$ & $\begin{array}{l}\text { Ftuse ID } \\
124 / \mathrm{ppb} \\
51 / 1400 \mathrm{t}\end{array}$ & $\begin{array}{c}\text { ALPHA } \\
\text { t35/pCi/A } \\
. / 15\end{array}$ \\
\hline $299-478-2 t$ & $11 / 28 /{ }_{4}$ & Bopro & & $1.00 \mathrm{U}$ & 500,00 & 8.96 \\
\hline $259-418-22$ & $11 / 29 / 44$ & e0075 & 100 & 1.000 & 400.00 & .79 u \\
\hline $299-410-23$ & $11 / 11 / 94$ & Boorno & $\begin{array}{l}4.100 \\
4.10 \mathrm{U}\end{array}$ & 1,000 & 500.00 & 1.69 \\
\hline $\begin{array}{l}299-118-34 \\
299-118-24\end{array}$ & $\begin{array}{l}11 / 0 \mathrm{~g} / 94 \\
11 / 0 \mathrm{~d} / \mathrm{s4}\end{array}$ & 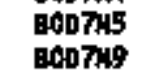 & $\$, 10 \mathrm{U}$ & 1.00 & 900,00 & $.65 \mathrm{~V}$ \\
\hline $\begin{array}{l}299-118-26 \\
299-418-26\end{array}$ & $\begin{array}{l}11 / 28 / 94 \\
11 / 20 / 94\end{array}$ & $\begin{array}{l}600710 \\
0007 \times 14\end{array}$ & 4.100 & $1.00 \mathrm{U}$ & 600.00 & 4.29 \\
\hline $\begin{array}{l}259-418-27 \\
299-418-27\end{array}$ & $\begin{array}{l}11 / 28 / 94 \\
11 / 28 / 94\end{array}$ & $\begin{array}{l}\text { Bephis } \\
\text { Boonis }\end{array}$ & $4.10 \mathrm{U}$ & 1.00 & 400.00 & .80 \\
\hline $\begin{array}{l}299-118-28 \\
299-118-28\end{array}$ & $\begin{array}{l}11 / 28 / 94^{\circ} \\
11 / 28 / 94\end{array}$ & $\begin{array}{l}\text { E007P0 } \\
\text { g007P4 }\end{array}$ & $4.10 \mathrm{U}$ & $1.00 \mathrm{~V}$ & 400.00 & 1.26 \\
\hline $\begin{array}{l}299-418-32 \\
299-118-32\end{array}$ & $\begin{array}{l}11 / 00 / 94 \\
11 / 09 / 94\end{array}$ & $\begin{array}{l}300775 \\
3007 \% 9\end{array}$ & $4.10 \mathrm{U}$ & $1.00 \mathrm{~L}$ & 600.00 & 2.56 \\
\hline
\end{tabular}

\begin{tabular}{|c|c|c|c|c|c|c|}
\hline $\begin{array}{l}\text { Mell } \\
\text { Mant }\end{array}$ & $\begin{array}{l}\text { Cotlection } \\
\text { Dete }\end{array}$ & $\begin{array}{l}\text { sonple } \\
\text { Himber }\end{array}$ & $\begin{array}{c}\text { 日EFA } \\
\text { 136/pGi/L } \\
.7 .\end{array}$ & $\begin{array}{c}\text { IfOOM } \\
34 / \mathrm{pqb} \\
18 / 3000\end{array}$ & $\begin{array}{c}\text { FJROW } \\
\text { 34/ppb } \\
\{8 / 300 \mathrm{~s}\end{array}$ & $\begin{array}{l}\text { LEAD } \\
\text { 40/ppt } \\
.86 / 501\end{array}$ \\
\hline $299-115-15$ & $11 / 10 / 94$ & 900700 & 7.05 & $170.00 \mathrm{a}$ & & 7.10 \\
\hline $\begin{array}{l}299-w 15-15 \\
299-\text { w15-16 }\end{array}$ & $\begin{array}{l}t 1 / 10 / 94 \\
t 1 / 08 / 94\end{array}$ & $\begin{array}{l}B 00704 \\
800765\end{array}$ & 15.10 & 420.009 & 45.000 & $1.80 \mathrm{~L}$ \\
\hline $299 \cdot w 15 \cdot 16$ & $11 / 08 / 94$ & 800769 & & & 34.000 & \\
\hline 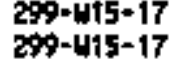 & $\begin{array}{l}11 / 09 / 94 \\
11 / 09 / 94\end{array}$ & $\begin{array}{l}\text { 80007Ho } \\
\text { 60077H4 }\end{array}$ & 7.58 & 150.000 & 46.000 & $1.50 \mathrm{~V}$ \\
\hline $\begin{array}{l}299-415-18 \\
299-4 t 5-18\end{array}$ & $\begin{array}{l}11 / 08 / 94 \\
11 / 03 / 94\end{array}$ & 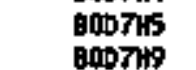 & 6.51 & 530.000 & 30.009 & $1.50 \overline{\mathrm{U}}$ \\
\hline $\begin{array}{l}z \$ 9-4 t 5-19 \\
z \$ 9-4 t 5-19\end{array}$ & $\begin{array}{l}11 / 30 / 94 \\
11 / 50 / 94\end{array}$ & $\begin{array}{l}\text { DOD7\$0 } \\
\text { BDD7 } \$ 4\end{array}$ & $\$ 1.90$ & 320.00 日9 & $26.00 \mathrm{BO}$ & $1.60 \mathrm{BL}$ \\
\hline $\begin{array}{l}2 x=-415-20 \\
299-455-20\end{array}$ & $\begin{array}{l}11 / 11 / 94 \\
11 / 11 / 94\end{array}$ & $\begin{array}{l}0007 / 5 \\
000759\end{array}$ & $1.68 \mathrm{~d}$ & $250.00 \mathrm{a}$ & & $1,50 \mathrm{~W}$ \\
\hline $\begin{array}{l}269-4 t 5-23 \\
299-4 t 5-23\end{array}$ & $\begin{array}{l}11 / 11 / 94 \\
11 / 11 / 94\end{array}$ & $\begin{array}{l}8007 \times 0 \\
8007 \times 4\end{array}$ & 2.95 & $\$ 10.000$ & $16.00 \mathrm{LQ}$ & $2.10 \mathrm{~L}$ \\
\hline $\begin{array}{l}249-475-24 \\
299-415-24\end{array}$ & $\begin{array}{l}11 / 10 / 94 \\
11 / 10 / 94\end{array}$ & $\begin{array}{l}\text { Bop 7ks } \\
\text { Bopros }\end{array}$ & 7.66 & 4900.00 & $64.00 \mathrm{~B}$ & $1.60 \mathrm{~L}$ \\
\hline $\begin{array}{l}299-418-21 \\
299-418-21\end{array}$ & $\begin{array}{l}11 / 29 / 94 \\
11 / 29 / 44\end{array}$ & $\begin{array}{l}\text { Botho } \\
\text { Bep7L6 }\end{array}$ & 9.53 & 420.000 & 52.000 & 11.00 \\
\hline $\begin{array}{l}299-418-22 \\
299-418-22\end{array}$ & $\begin{array}{l}11 / 29 / 94 \\
11 / 29 / 94\end{array}$ & $\begin{array}{l}\text { 800725 } \\
\text { 8007L5 }\end{array}$ & $\$, \infty 0$ & 420,000 & $30.00 \mathrm{BO}$ & $1.90 \mathrm{t}$ \\
\hline $\begin{array}{l}297-418-23 \\
299-418-23\end{array}$ & $\begin{array}{l}11 / 11 / 94 \\
11 / 11 / 94\end{array}$ & $\begin{array}{l}8007 \times 10 \\
\text { BODOHNG }\end{array}$ & .540 & $95.00 \mathrm{e}$ & $25.00 \mathrm{a}$ & $1.50 \mathrm{~V}$ \\
\hline $\begin{array}{l}299-418-24 \\
296-w 18-24\end{array}$ & $\begin{array}{l}11 / 08 / 84 \\
11 / 00 / \% 4\end{array}$ & $\begin{array}{l}\text { 6007ns } \\
\text { B0074\% }\end{array}$ & 5.76 & 220,000 & 29,000 & $1.50 \mathrm{U}$ \\
\hline $\begin{array}{l}299-418-26 \\
299-418+26\end{array}$ & $\begin{array}{l}11 / 28 / \% 4 \\
11 / 28 / \% 4\end{array}$ & $\begin{array}{l}8007100 \\
8007144\end{array}$ & 9.02 & 4100,000 & 21,000 & $5.00 \mathrm{~L}$ \\
\hline $\begin{array}{l}297-418-27 \\
259-478-27\end{array}$ & $11 / 28 / 94$ & $\begin{array}{l}800745 \\
600749\end{array}$ & 3.46 & 1600.000 & 29,000 & $1.90 \mathrm{l}$ \\
\hline $\begin{array}{l}299-w_{1 B}-28 \\
299-v_{18-28}\end{array}$ & $\begin{array}{l}11 / 28 / 94 \\
11 / 28 / 94\end{array}$ & $\begin{array}{l}800700 \\
800704\end{array}$ & 5.01 & 250.000 & 12,00 Lo & $5.00 \mathrm{t}$ \\
\hline $\begin{array}{l}299-w 18-32 \\
299-w 18-32\end{array}$ & $\begin{array}{l}11 / 09 / 94 \\
1 \$ / 00 / 44\end{array}$ & $\begin{array}{l}8007 \mathrm{ps} \\
\text { 8007ps }\end{array}$ & 3.8 & 900.000 & $26,00 \mathrm{a}$ & $2.00 \mathrm{t}$ \\
\hline
\end{tabular}


Table 14-22. Constituents with at Least One Betected Value for the Low-Level Waste Maragement Area 4 Data for Reporting Perjod October 1 through December 31, 1994. (sheet 5 of 9)

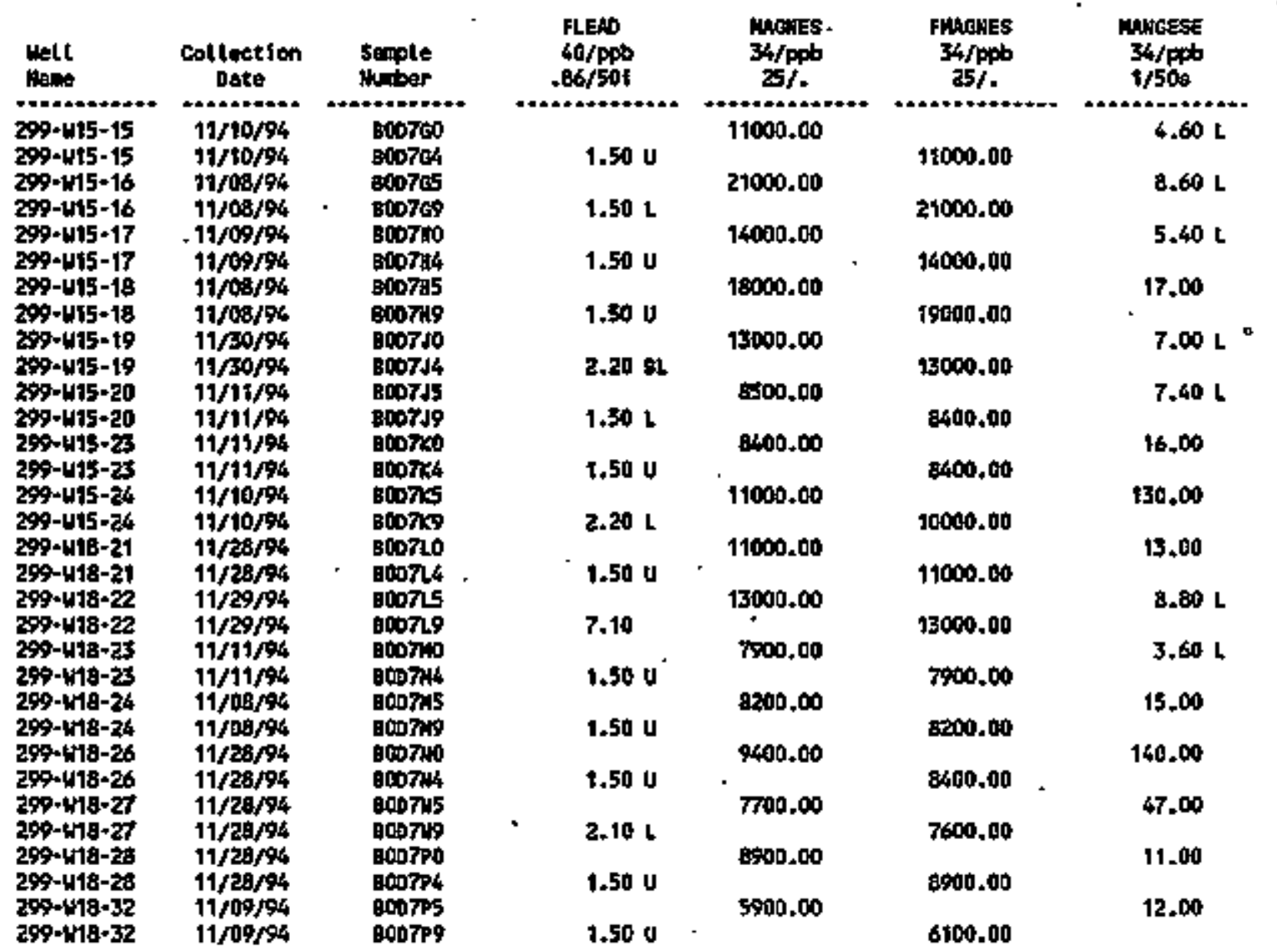

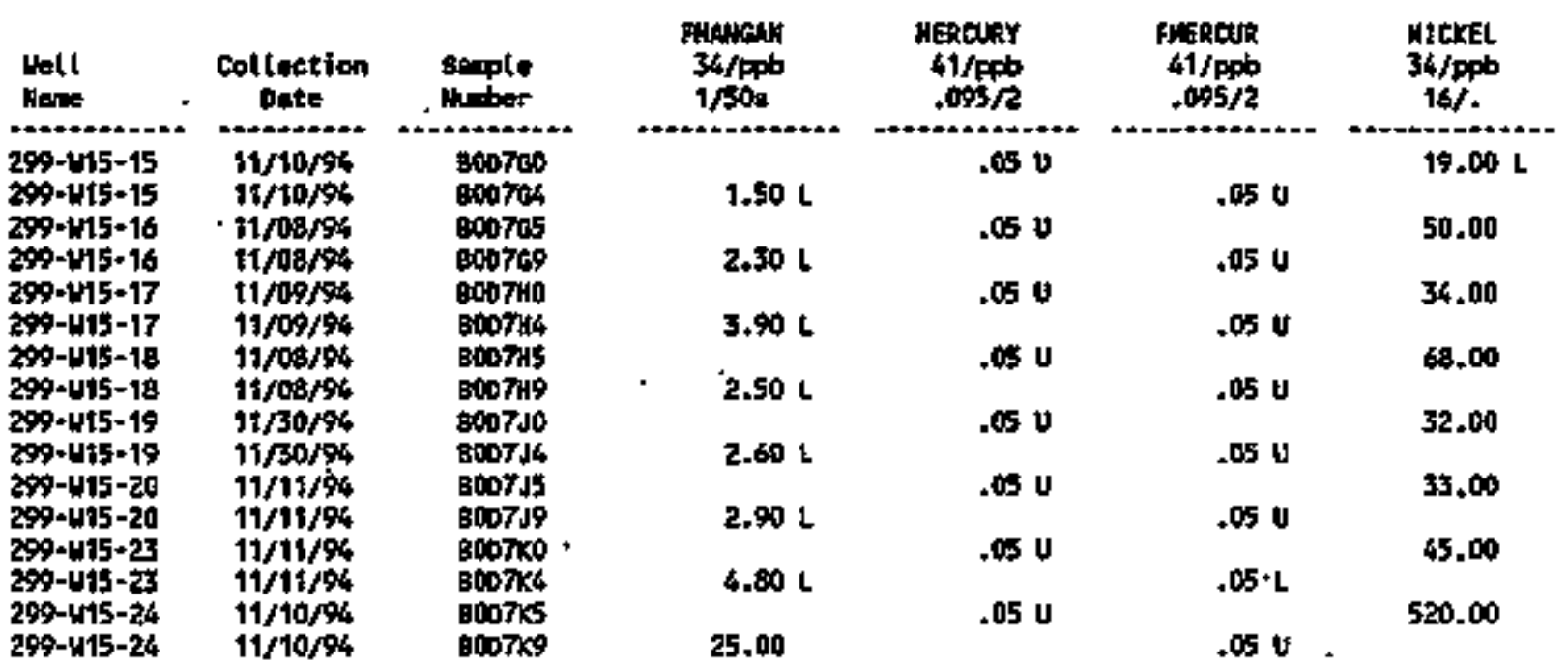


Table 14-22. Constituents with at Least One Detected Value for the Low-Level Waste Management Area 4 Data for Reporting Period

October I through December 31, 1994.

(sheet 6 of 9)

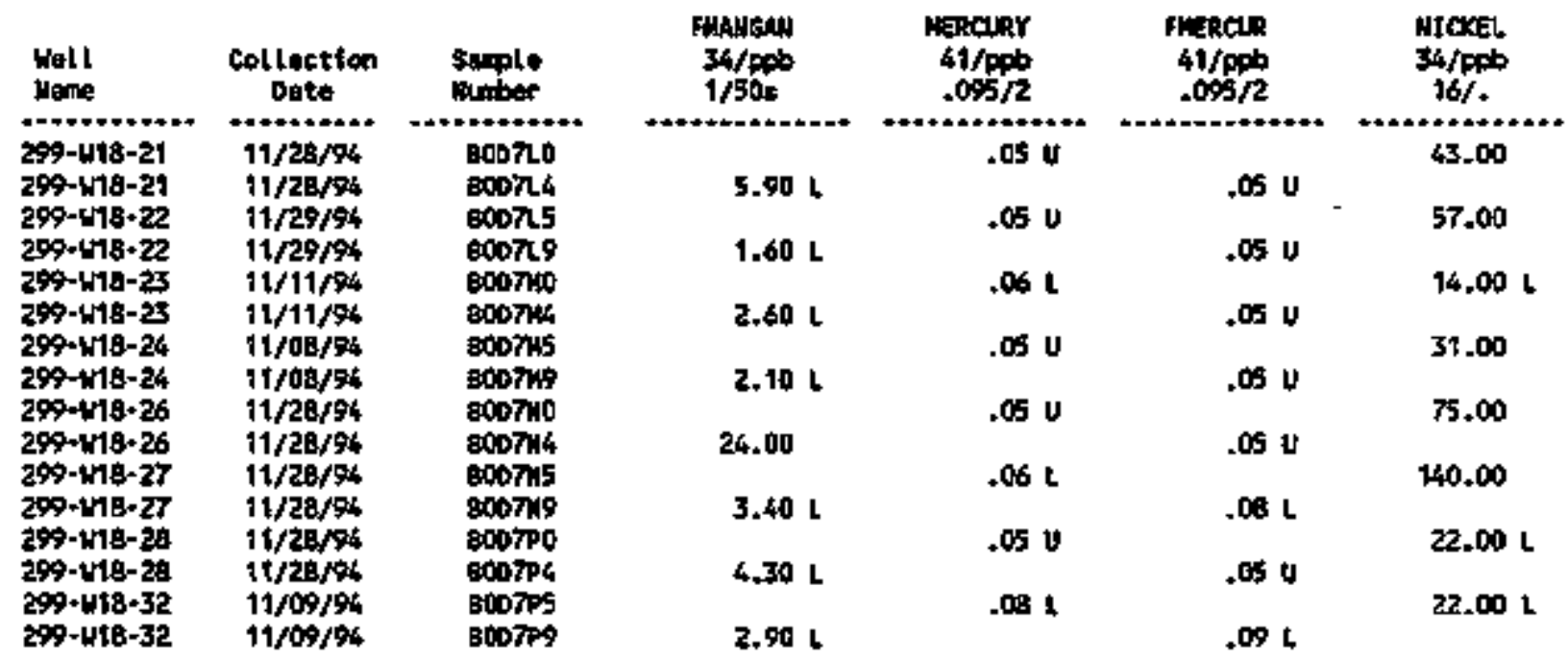

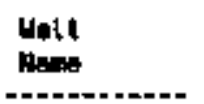

29-U15-15

$29 \cdot 415+15$

299-15-16

$299 \cdot 415 \cdot 16$

299-415-17

$2 \%-415-17$

$259-415+18$

29-415-18

299-4t5-10

$299 \cdot$ - $15 \cdot 19$

299-415-20

299-415-20

2\%8-415-23

299-415-23

299+W75-24

299-415-24

$29 \%-w 18-21$

299-418-21

299.+118-22

299-418-22

299-118-23

295-418-25

299-118-24

299-W18-24

299-พ18-26

299+w18-26

299-418-27

295-418-27

290-418-28

299-U1B-2B

29-Ut8-32

$299 \cdot 418+32$

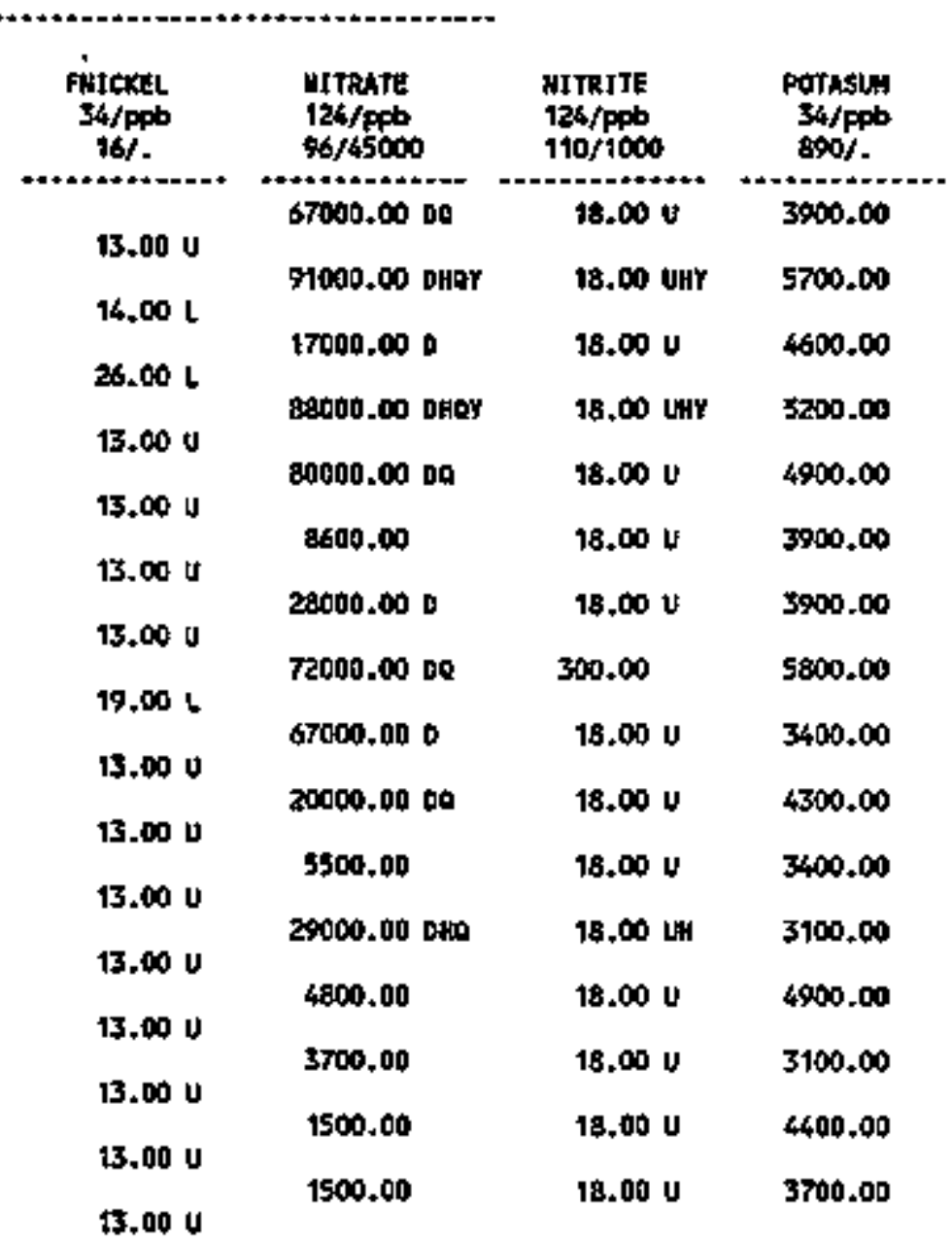


Table 14-22. Constituents with at Least One Detected Va7ue for the Low-Level Waste Managenent Area 4 Data for Reporting Period October 1 through December 31, 1994. (sheet 7 of 9)

\begin{tabular}{|c|c|c|c|c|c|c|}
\hline Noll & $\underset{\text { Dotito }}{\text { Colloction }}$ & $\begin{array}{l}\text { Somple } \\
\text { Nuliber }\end{array}$ & $\begin{array}{l}\text { lpotass } \\
34 / \mathrm{pph} \\
890 \% .\end{array}$ & 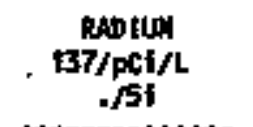 & $\begin{array}{l}\text { scotun } \\
34 / F{ }^{2} \\
150 \%\end{array}$ & $\begin{array}{l}\text { P5001up } \\
34 / p \% 6 \\
150 \% .\end{array}$ \\
\hline 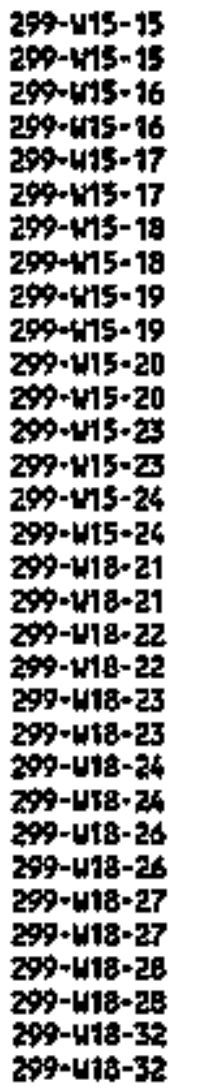 & $\begin{array}{l}11 / 10 / 94 \\
11 / 10 / 94 \\
11 / 09 / 94 \\
11 / 09 / 94 \\
11 / 09 / 94 \\
11 / 09 / 94 \\
11 / 09 / 94 \\
11 / 08 / 94 \\
11 / 30 / 94 \\
11 / 30 / 94 \\
11 / 11 / 94 \\
11 / 11 / 94 \\
11 / 11 / 94 \\
11 / 11 / 94 \\
11 / 10 / 94 \\
11 / 10 / 94 \\
11 / 28 / 94 \\
11 / 28 / 94 \\
11 / 29 / 94 \\
11 / 29 / 94 \\
11 / 11 / 94 \\
11 / 11 / 94 \\
11 / 68 / 94 \\
11 / 08 / 94 \\
11 / 28 / 94 \\
11 / 28 / 94 \\
11 / 28 / 94 \\
111 / 28 / 94 \\
111 / 28 / 94 \\
111 / 28 / 94 \\
11199 / 94 \\
111 / 09 / 94\end{array}$ & 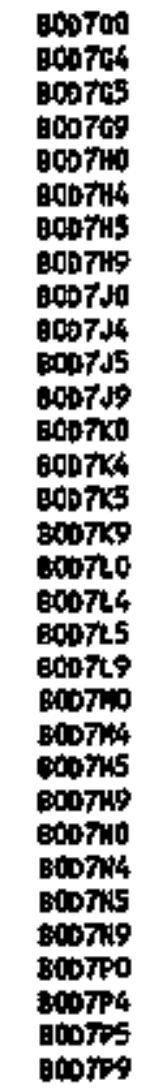 & $\begin{array}{l}3900.00 \\
5600.00 \\
4700.00 \\
5300.00 \\
4500.00 \\
4000.00 \\
3400.00 \\
5700.00 \\
3200.00 \\
4100.00 \\
3400.00 \\
3500.00 \\
4200.00 \\
3800.00 \\
4500.00 \\
3300.00\end{array}$ & $\begin{array}{l}.18 \\
.11 \mathrm{U} \\
.11 \mathrm{U} \\
.32 \\
.25 \\
.11 \mathrm{U} \\
.30 \\
.06 \mathrm{U} \\
. .02 \mathrm{U} \\
.13 \\
.09 \mathrm{U} \\
.12 \mathrm{U} \\
.67 \\
.03 \mathrm{U} \\
.13 \mathrm{U} \\
.03 \mathrm{U}\end{array}$ & $\begin{array}{l}21000.00 \\
42000.00 \\
16000.00 \\
27000.00 \\
23000.000 \\
12000.00 \\
20000.00 \\
20000.00 \\
25000.000 \\
14000.0080 \\
14000.00 \\
17000.00 \\
20000.000 \\
12000.000 \\
14000.000 \\
14000.00\end{array}$ & $\begin{array}{l}21000.00 \\
42000.00 \\
16000.00 \\
28000.00 \\
24000.000 \\
14000.00 \\
20000.00 \\
20000.00 \\
24000.000 \\
15000.0000 \\
14000.00 \\
17000.00 \\
20000.000 \\
13000.000 \\
14000.000 \\
14000.00\end{array}$ \\
\hline $\begin{array}{l}\text { Well } \\
\text { Hente }\end{array}$ & $\begin{array}{l}\text { Collection, } \\
\text { Dote }\end{array}$ & $\begin{array}{l}\text { sample } \\
\text { Nuber }\end{array}$ & $\begin{array}{l}\text { SULFKTE } \\
\text { 124/ppb } \\
89 / 250000 \mathrm{~s}\end{array}$ & $\begin{array}{l}\text { PESCENE } \\
\text { Z S/Pph } \\
\text {.08/5 }\end{array}$ & $\begin{array}{l}\text { TRLCEXE } \\
25 / \text { pक } \\
.063 / 5\end{array}$ & $\begin{array}{l}\text { TRITIUN } \\
\text { 142/pCI/LL } \\
. / 20000\end{array}$ \\
\hline 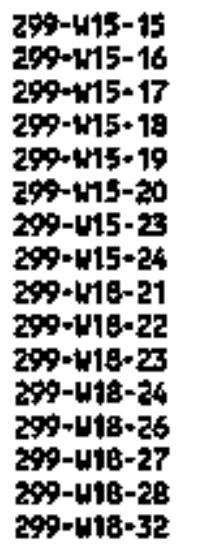 & $\begin{array}{l}11 / 10 / 94 \\
11 / 00 / 94 \\
11 / 09 / 94 \\
11 / 00 / 94 \\
11 / 30 / 94 \\
11 / 11 / 94 \\
11 / 11 / 94 \\
11 / 10 / 94 \\
11 / 28 / 94 \\
11 / 29 / 94 \\
11 / 11 / 94 \\
11 / 08 / 94 \\
11 / 28 / 94 \\
11 / 20 / 94 \\
11 / 289 / 94 \\
11 / 09 / 94\end{array}$ & 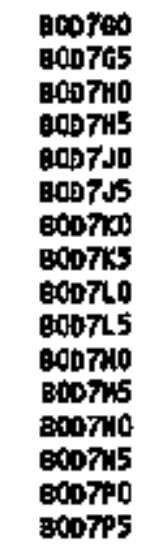 & $\begin{array}{l}18000.00 \mathrm{D} \\
91000.00 \mathrm{or} \\
28000.00 \mathrm{D} \\
46000.00 \mathrm{or} \\
28000.00 \mathrm{D} \\
15000.00 \mathrm{D} \\
16000.00 \mathrm{D} \\
17000.00 \mathrm{D} \\
20000.00 \mathrm{D} \\
19000.00 \mathrm{D} \\
16000.00 \mathrm{0} \\
17000.00 \mathrm{D} \\
21000.00 \mathrm{D} \\
16000.00 \mathrm{D} \\
18000.00 \mathrm{D} \\
12000.00 \mathrm{D}\end{array}$ & $\begin{array}{l}.22 \mathrm{U} \\
.42 \mathrm{UH} \\
.22 \mathrm{U} \\
1.50 \mathrm{H} \\
.22 \mathrm{U} \\
.22 \mathrm{U} \\
.22 \mathrm{U} \\
.22 \mathrm{U} \\
.22 \mathrm{UHY} \\
.22 \mathrm{U} \\
.22 \mathrm{U} \\
.22 \mathrm{UH} \\
.22 \mathrm{U} \\
.30 \mathrm{HY} \\
.22 \mathrm{~V} \\
.22 \mathrm{~V}\end{array}$ & 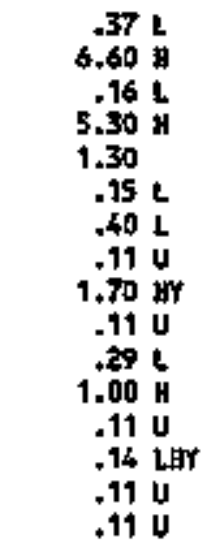 & $\begin{array}{r}198.00 \mathrm{U} \\
83.50 \mathrm{U} \\
7.68 \mathrm{U} \\
166.00 \mathrm{U} \\
167.00 \mathrm{U} \\
213.09 \mathrm{U} \\
265.00 \mathrm{U} \\
185.00 \mathrm{U} \\
150.00 \mathrm{U} \\
132.00 \mathrm{U} \\
140.00 \mathrm{U} \\
76.90 \mathrm{U} \\
372.00 \\
22.40 \mathrm{U} \\
107.00 \mathrm{U} \\
206.00 \mathrm{U}\end{array}$ \\
\hline
\end{tabular}


Table 14-22. Constituents with at Least One Detected Value for the Low-Level Haste Management Area 4 Data for Reporting Period October 1 through December 31, 1994.

(sheet. 8 of 9)

\begin{tabular}{|c|c|c|c|c|c|c|}
\hline $\begin{array}{l}\text { Sall } \\
\text { Mange }\end{array}$ & $\begin{array}{c}\text { col lection } \\
\text { pate }\end{array}$ & $\begin{array}{l}\text { Sapple } \\
\text { Huber }\end{array}$ & $\begin{array}{l}\text { TURBIO } \\
126 / \mathrm{MTU} \\
.016 / .\end{array}$ & $\begin{array}{l}\text { URANulus } \\
145 / \mathrm{ppb} \\
\times /\end{array}$ & $\begin{array}{l}\text { VAYADLM } \\
\text { 34/ppb } \\
6+4 \%\end{array}$ & $\begin{array}{c}\text { FWhHADI } \\
34 / \mathrm{ppb} \\
6.4 \%\end{array}$ \\
\hline 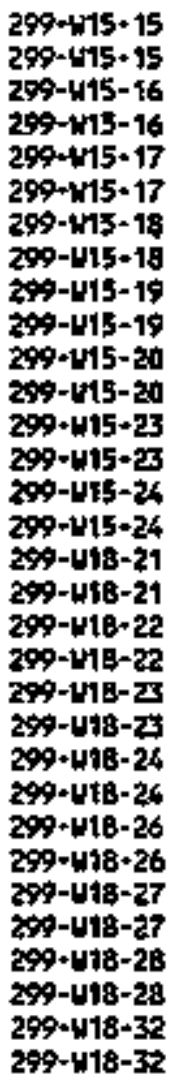 & $\begin{array}{l}11 / 10 / 94 \\
11 / 10 / 94 \\
11 / 08 / 94 \\
11 / 08 / 94 \\
11 / 09 / 94 \\
11 / 09 / 94 \\
11 / 08 / 94 \\
11 / 08 / 96 \\
11 / 50 / 96 \\
11 / 50 / 94 \\
11 / 11 / 96 \\
11 / 11 / 44 \\
11 / 11 / 94 \\
11 / 11 / 94 \\
11 / 10 / 94 \\
11 / 10 / 94 \\
11 / 28 / 94 \\
11 / 28 / 94 \\
11 / 29 / 94 \\
11 / 29 / 94 \\
11 / 11 / 94 \\
11 / 11 / 94 \\
11 / 08 / 94 \\
11 / 08 / 94 \\
11 / 28 / 94 \\
11 / 29 / 94 \\
11 / 28 / 94 \\
11 / 28 / 94 \\
11 / 28 / 94 \\
11 / 28 / 94 \\
11 / 09 / 94 \\
11 / 09 / 94\end{array}$ & 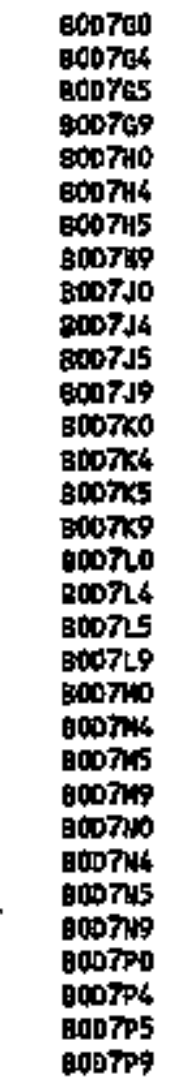 & $\begin{array}{l}2.40 \\
1.700 \\
1.300 \\
2.300 \\
1.700 \\
2.40 \\
1.80 \\
19.00 \\
1.500 \\
1.500 \\
.95 \\
1.700 \\
150.000 \\
9.909 \\
1.409 \\
18.000\end{array}$ & $\begin{array}{l}5.04 \\
2.43 \\
1.09 \\
1.81 \\
2.61 \\
.79 \\
.93 \\
.85 \\
20.20 \\
.82 \\
1.31 \\
.96 \\
1.75 \\
.99 \\
.91\end{array}$ & $\begin{array}{l}25.00 \mathrm{~L} \\
24.00 \mathrm{~L} \\
30.00 \mathrm{~L} \\
24.00 \mathrm{~L} \\
16.00 \mathrm{~L} \\
15.00 \mathrm{~L} \\
29.00 \mathrm{~L} \\
13.00 \mathrm{~L} \\
23.00 \mathrm{~L} \\
25.00 \mathrm{~L} \\
26.00 \mathrm{~L} \\
35.00 \\
11.00 \mathrm{~L} \\
29.00 \mathrm{~L} \\
6.60 \mathrm{U} \\
51.00\end{array}$ & $\begin{array}{l}24.00 \mathrm{~L} \\
23.00 \mathrm{~L} \\
28.00 \mathrm{~L} \\
24.00 \mathrm{~L} \\
13.00 \mathrm{~L} \\
16.00 \mathrm{~L} \\
28.00 \mathrm{~L} \\
6.60 \mathrm{U} \\
28.00 \mathrm{~L} \\
25.00 \mathrm{~L} \\
29.00 \mathrm{~L} \\
34.00 \\
6.00 \mathrm{U} \\
26.00 \mathrm{I} \\
6.60 \mathrm{~L} \\
47.00\end{array}$ \\
\hline
\end{tabular}


Table 14-22. Constituents with at Least One Detected Value for the Low-Leve]

- Waste Management Area 4 Data for Reporting Period

October 1 through December 31, 1994. (sheet 9 of 9 )

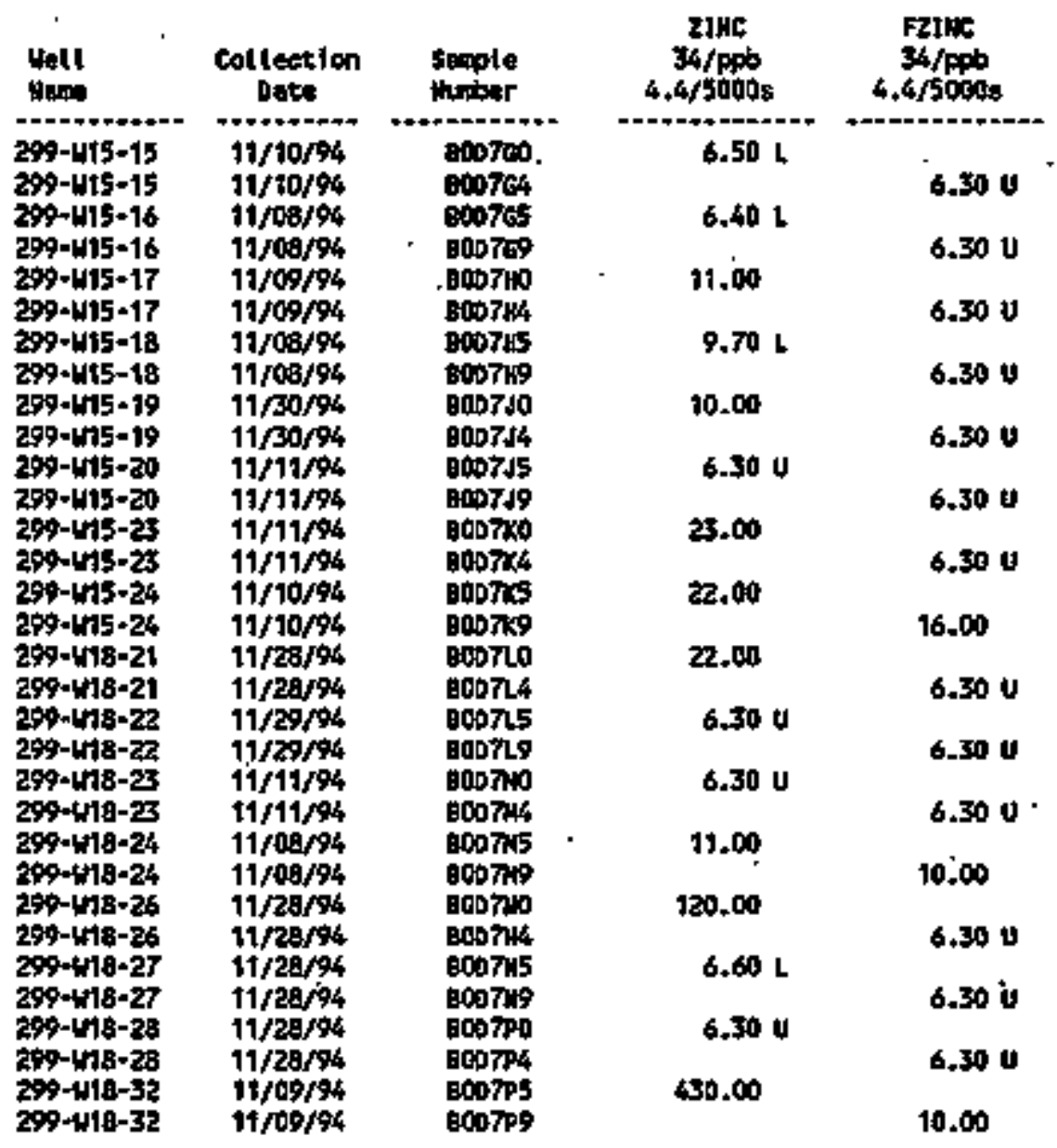

For explanatian of this table, see section 1.4 of report. 
Table 14-23. Contamination Indicator Parameters for the Low-Leve] Waste Management Area 4 Data for Reporting Perfod October 1 through December 31, 1994.

(sheet 1 of 2)

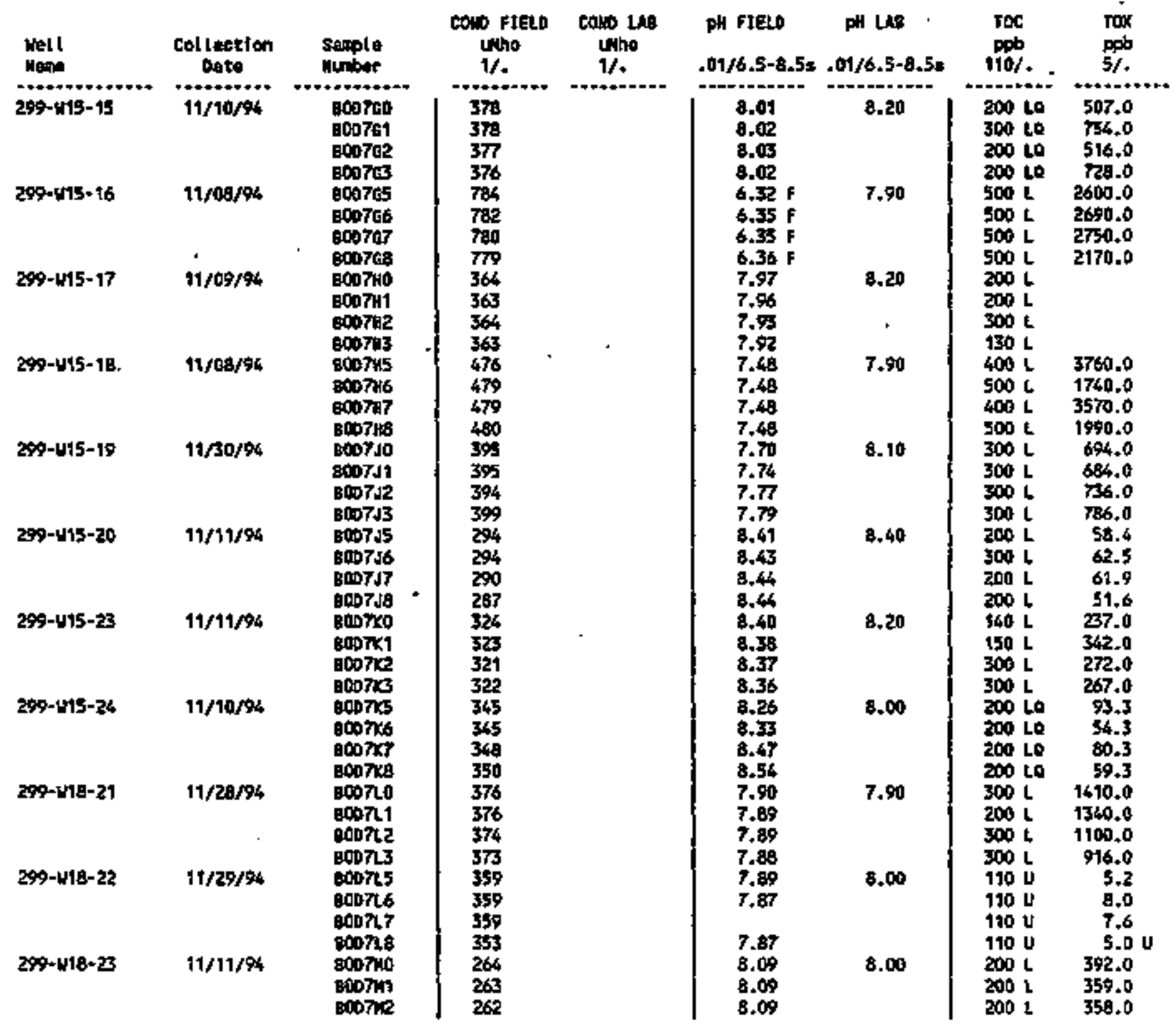


Table 14-23. Contamination Indicator Parameters for the Low-Leve1 Waste Hanagenent Area 4 Data for Reporting Period October 1 through Decenber 31, 1994. (sheet 2 of 2)

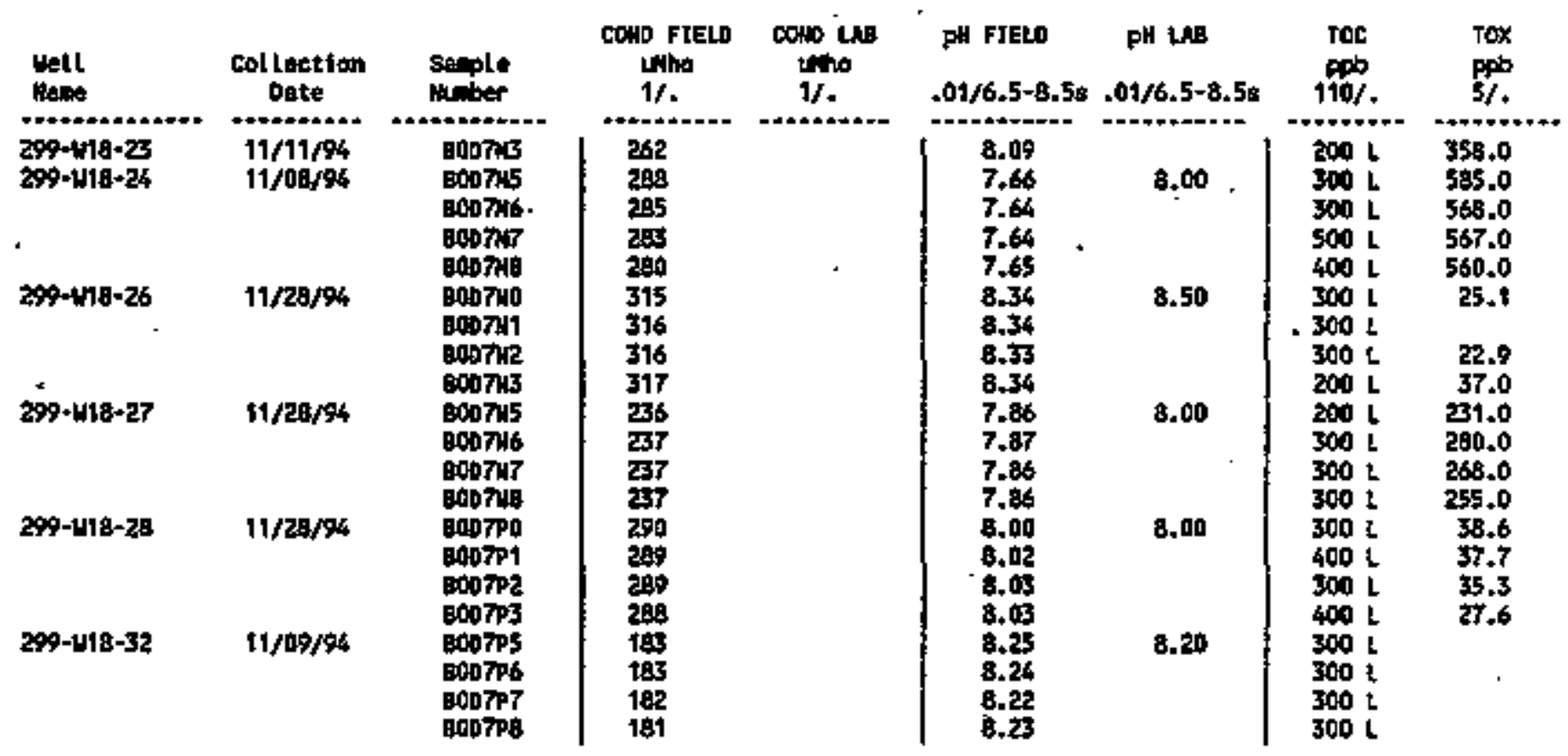

For explanation of this table, ses Section 1.4 of raport. 
Tab7e 14-24. Constituent L1st and Summary of Results for the Low-Level Waste Management Area 5 Data for Reporting Period

October 1 through Decenber 31, 1994.

COITHAIMATION IMDJCATOR PARANETER:

\begin{tabular}{|c|c|c|c|c|c|c|c|c|}
\hline & & Constituent thane & & tab & & Aur & $r$ of $s$ & aples \\
\hline short & (Methed) & Full & Inits & Linte & Agancy & Totel & $\$ O L$ & $>048$ \\
\hline $\begin{array}{l}\text { Conduct } \\
\text { Tac } \\
\text { Tax } \\
\text { PH. }\end{array}$ & & $\begin{array}{l}\text { Conductivity, field } \\
\text { Total organic Carban } \\
\text { Total orpanic Hslogen } \\
\text { pH, field }\end{array}$ & $\begin{array}{l}\text { uhos } \\
\text { ppb } \\
\text { ppb } \\
\text { ph }\end{array}$ & $\begin{array}{l}1 \\
110 \\
5 \\
.01\end{array} 6.5 \cdot 8.5$ & EPAS & $\begin{array}{r}12 \\
5 \\
5 \\
12\end{array}$ & $\begin{array}{r}12 \\
5 \\
5 \\
12\end{array}$ & 1 \\
\hline
\end{tabular}

\section{DRIAKIHG WATER PLRAMETERS}

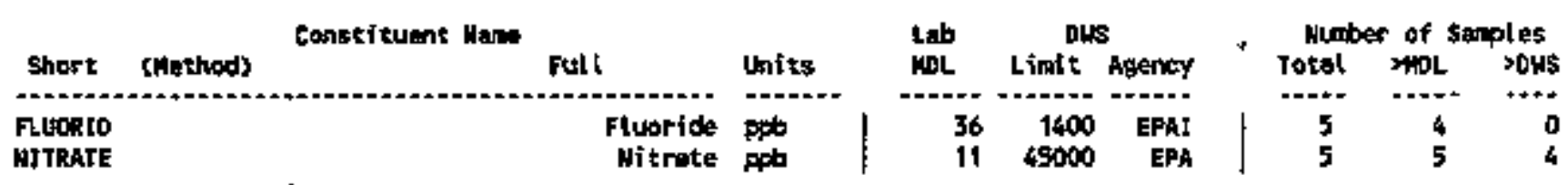

\section{GROUIDUATER OUALITY PNRAMETERS}

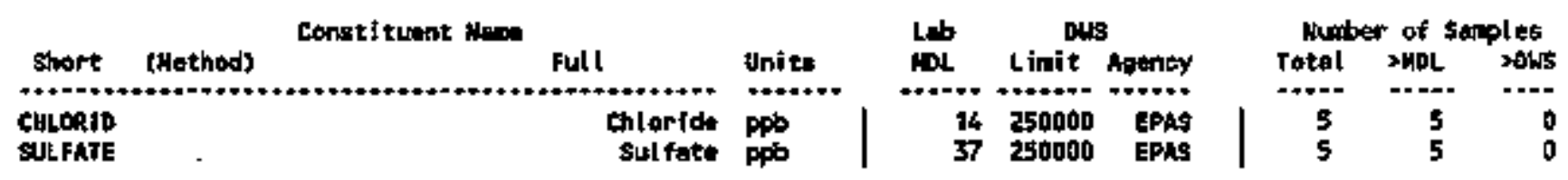

SITE SPECJFIC MND OTHER CONSTITUENTS

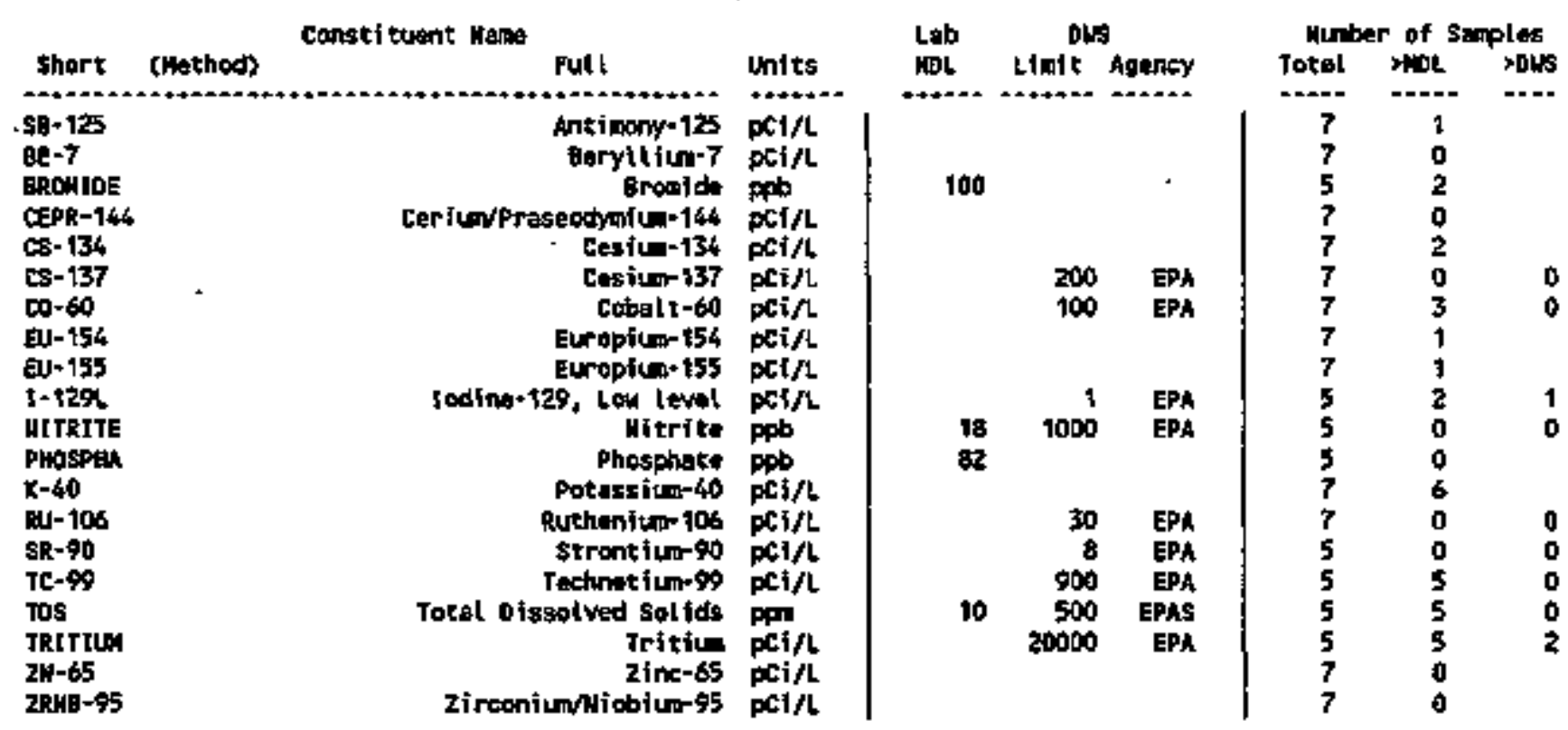

For explanation of this table, sea section 1.4 of report, 
Tab7e 14-25. Constituents with at Least One Detected Value for the Low-Leve] Waste Management Area 5 Data for Reporting Period . October 1 through December 31, 1994.

\begin{tabular}{|c|c|c|c|c|c|c|}
\hline Well & $\begin{array}{l}\text { Colluction } \\
\text { Date }\end{array}$ & $\begin{array}{l}\text { Sample } \\
\text { Nunber }\end{array}$ & $\begin{array}{c}88-125 \\
140 / \mathrm{pci} / \mathrm{L} \\
.7\end{array}$ & $\begin{array}{l}\text { ARowtDE } \\
\text { 226/pos } \\
110 \% .\end{array}$ & $\begin{array}{c}\mathrm{c5}+134 \\
140 / p \mathrm{i} / \Omega \\
-f .\end{array}$ & $\begin{array}{c}\text { CHLORID } \\
124 / 006 \\
71 / 250000\end{array}$ \\
\hline $\begin{array}{l}299-411-31 \\
299-411-31 \\
299-146-10 \\
299-146-2 \\
299-146-4 \\
299-18-9 \\
299-14-9\end{array}$ & $\begin{array}{r}8 / 22 / 94 \\
12 / 14 / 94 \\
8 / 23 / 94 \\
12 / 14 / 94 \\
12 / 14 / 94 \\
12 / 13 / 94 \\
12 / 13 / 94 .\end{array}$ & 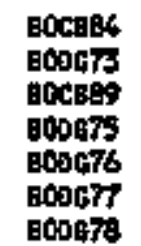 & $\begin{array}{r}2.53 \mathrm{v} \\
3.40 \\
-1.67 \mathrm{v} \\
1.68 \mathrm{v} \\
1.90 \mathrm{v} \\
-2.36 \mathrm{u} \\
2.32 \mathrm{u}\end{array}$ & 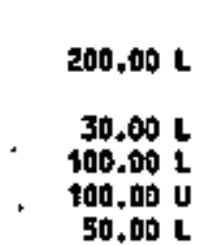 & $\begin{array}{l}.10 \mathrm{U} \\
.27 \mathrm{v} \\
.73 \mathrm{U} \\
.28 \mathrm{v} \\
. .08 \\
1.17 \\
.08 \mathrm{v}\end{array}$ & $\begin{array}{l}39000.0000 \\
-\$ 600.000 \\
9200,000 \\
\$ 0,000 \\
8100.000\end{array}$ \\
\hline
\end{tabular}

\begin{tabular}{|c|c|c|c|c|c|c|}
\hline Matl & $\begin{array}{l}\text { Collectian } \\
\text { Date }\end{array}$ & $\begin{array}{l}\text { Suple } \\
\text { Iunber }\end{array}$ & $\begin{array}{c}00-60 \\
140 / p c 1 / L \\
. / 100\end{array}$ & $\begin{array}{c}\text { EU-154 } \\
\text { t40/pct/lt } \\
\text {. } / .\end{array}$ & $\begin{array}{c}\text { EU-155 } \\
140 / \mathrm{pCT} / \mathrm{C} \\
. /\end{array}$ & $\begin{array}{l}\text { FLudRto } \\
124 / \mathrm{p} \times \mathrm{b} \\
51 / 14001\end{array}$ \\
\hline $\begin{array}{l}299-11-31 \\
299-11-31 \\
299-14-10 \\
299-14-2 \\
299-14-4 \\
299-45-9 \\
299-16-9\end{array}$ & $\begin{array}{r}1 / 22 / 94 \\
12 / 14 / 94 \\
1 / 23 / 94 \\
12 / 14 / 94 \\
12 / 14 / 94 \\
12 / 13 / 94 \\
12 / 13 / 94\end{array}$ & $\begin{array}{l}\text { E0ca84 } \\
\text { E00073 } \\
\text { E0cas } \\
\text { E00475 } \\
\text { E00076 } \\
\text { E00077 } \\
\text { e00678 }\end{array}$ & $\begin{array}{c}-.06 \mathrm{u} \\
.69 \mathrm{v} \\
1.32 \\
1.88 \\
.81 \mathrm{u} \\
.89 \\
.51 \mathrm{v}\end{array}$ & $\begin{array}{r}-29 \mathrm{U} \\
-2.79 \mathrm{U} \\
2.51 \mathrm{U} \\
-4.16 \mathrm{U} \\
4.05 \\
=.80 \mathrm{U} \\
2.19 \mathrm{U}\end{array}$ & $\begin{array}{r}-1.03 \mathrm{U} \\
2.71 \\
.11 \mathrm{U} \\
.29 \mathrm{u} \\
-.64 \mathrm{U} \\
1.05 \mathrm{v} \\
-1.11 \mathrm{U}\end{array}$ & $\begin{array}{l}1200.00 \\
500.00 \\
500.00 \\
30.00 \text { UF } \\
800.00\end{array}$ \\
\hline
\end{tabular}

\begin{tabular}{|c|c|c|c|c|c|c|}
\hline Well & $\begin{array}{l}\text { Collection } \\
\text { det: }\end{array}$ & $\begin{array}{l}\text { serpte } \\
\text { striber }\end{array}$ & $\begin{array}{c}1-129 \\
13 \% / p c t / c \\
. / 1\end{array}$ & $\begin{array}{l}\text { NIJRATE } \\
126 / \mathrm{ppb} \\
96 / 45000\end{array}$ & $\begin{array}{c}K \times 40 \\
140 / p 6 i / L \\
. /\end{array}$ & $\begin{array}{c}\text { SULFRTE } \\
\text { 124/ppb } \\
89 / 250000 \mathrm{~s}\end{array}$ \\
\hline $\begin{array}{l}299-111-31 \\
299-1111-31 \\
299-116-10 \\
299-16-2 \\
299-116-4 \\
299-116-9 \\
299-116-9\end{array}$ & $\begin{array}{r}8 / 22 / 94 \\
12 / 14 / 94 \\
8 / 2 / 3 / 4 \\
12 / 14 / 94 \\
12 / 14 / 94 \\
12 / 13 / 94 \\
12 / 13 / 94\end{array}$ & 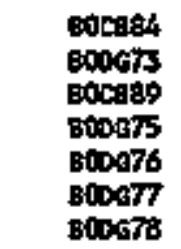 & $\begin{array}{l}1.85 \\
.09 \mathrm{u} \\
.57 \\
.16 \mathrm{u} \\
.01 \mathrm{u}\end{array}$ & $\begin{array}{c}120000.00 \mathrm{De} \\
52000.00 \mathrm{DO} \\
97000.00 \mathrm{DO} \\
800.00 \mathrm{D} \\
69000.00 \mathrm{DO}\end{array}$ & $\begin{array}{l}51.00 \\
25.90 \\
6.22 \mathrm{u} \\
55.70 \\
52.50 \\
45.40 \\
60.90\end{array}$ & $\begin{array}{r}63000.00 \mathrm{D} \\
23000.00 \mathrm{D} \\
38000.00 \mathrm{D} \\
200.00 \mathrm{LF} \\
25000.00 \mathrm{D}\end{array}$ \\
\hline
\end{tabular}

\begin{tabular}{|c|c|c|c|c|c|}
\hline Uela & $\begin{array}{c}\text { Collection } \\
\text { Date }\end{array}$ & $\begin{array}{l}\text { Strple } \\
\text { Huniber }\end{array}$ & $\begin{array}{c}\mathrm{TC}-99 \\
143 / \mathrm{pCi} / \mathrm{L} \\
/ 900\end{array}$ & $\begin{array}{c}\text { Tos } \\
65 / \mathrm{pom} \\
10 / 5000\end{array}$ & $\begin{array}{c}\text { TRITIU } \\
142 / \mathrm{Fi} / \mathrm{L} \\
. / 20000\end{array}$ \\
\hline $\begin{array}{l}279-111-3 t \\
294-146-2 \\
299-16-4 \\
299-116-9 \\
299-116-9\end{array}$ & $\begin{array}{l}12 / 14 / 94 \\
12 / 14 / 94 \\
12 / 14 / 94 \\
12 / 13 / 94 \\
12 / 13 / 94\end{array}$ & $\begin{array}{l}\text { gabs7s } \\
\text { Baps75 } \\
\text { Babe7t } \\
\text { Bober7 } \\
\text { adog78 }\end{array}$ & $\begin{array}{r}317.00 \\
28.10 \\
55.00 \\
45.70 \\
43.30\end{array}$ & $\begin{array}{l}480.00 \\
250.00 \\
280.00 \\
270.00 \\
270.00\end{array}$ & $\begin{array}{r}74000.00 \\
7510.00 \\
32900.00 \\
10700.00 \\
10300.00\end{array}$ \\
\hline
\end{tabular}

For explenterton of this tabli, see section 1.4 of report. 
Table 14-26. Contamination Indicator Parameters for the Low-Level . Waste Management Area 5 Data for Reporting Period October 1 through December 31, 1994.

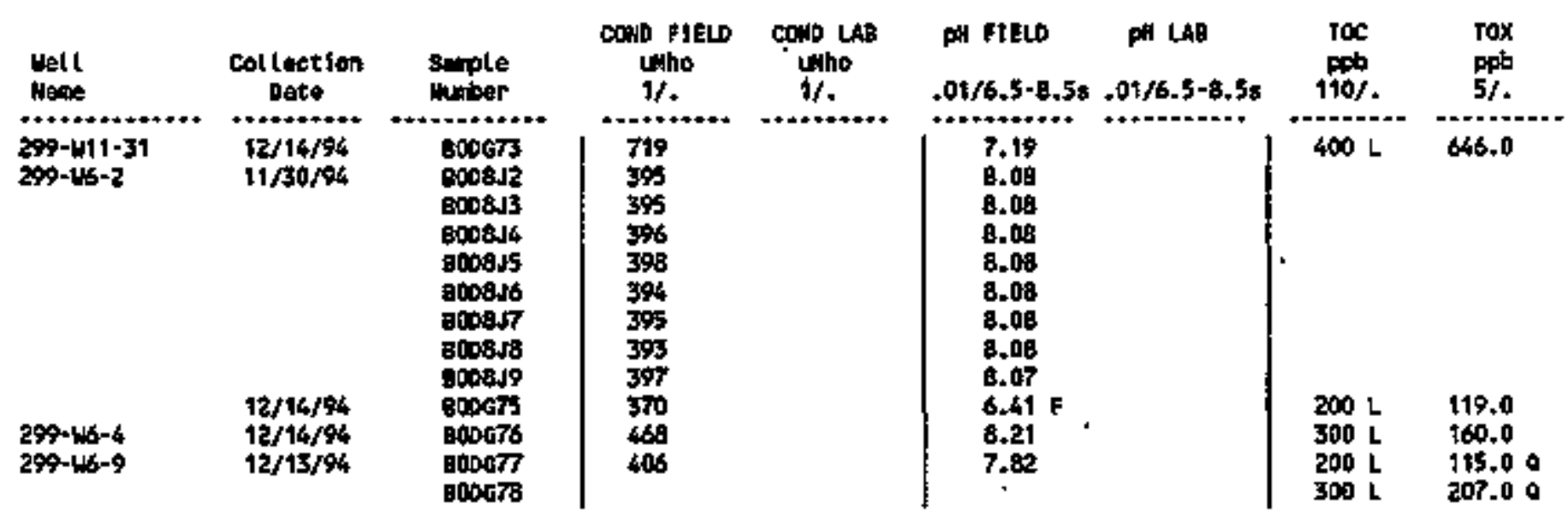

For explenution of this tuble, sere suction 1.4 of report.

Tab7e 14-27. Water Quality Standards Exceeded at Low-Level Waste Management Area 1.

\begin{tabular}{|c|c|c|c|}
\hline $\begin{array}{l}\text { Field } \mathrm{pH}^{\mathrm{pH}} \\
(6.5-8.5)\end{array}$ & $299-E 32-8$ & & \\
\hline $\begin{array}{l}\text { Chromfuin, unftltered } \\
(50 \mathrm{ppb})\end{array}$ & $\begin{array}{l}299-E 28-26 \\
299-E 32-10\end{array}$ & $\begin{array}{l}\text { 299-E32-2 } \\
299-\mathrm{E} 33-30\end{array}$ & 299-E32-9 \\
\hline $\begin{array}{l}\text { Iron, unfiltered } \\
(300 \mathrm{ppb})\end{array}$ & $\begin{array}{l}\text { 299-E28-26* } \\
299-E 32-9^{*}\end{array}$ & $\begin{array}{l}299-E 32-4 * \\
299-E 32-10^{*}\end{array}$ & $299-E 32-8^{*}$ \\
\hline $\begin{array}{l}\text { Tritium } \\
(20,000 \mathrm{pC}(1 / 1)\end{array}$ & $\begin{array}{l}299-E 28-27 \\
299-E 32-6\end{array}$ & $299-E 32-2$ & $299-E 32-3$ \\
\hline
\end{tabular}

- QC data associated with this sample was outside of established limits. 
Tab]e 14-30. Water Qual jty Standards Exceeded at Low-Level Waste Management Areà 4.

\begin{tabular}{|c|c|c|c|}
\hline Field pH & $299-W 15-16$ & & \\
\hline $\begin{array}{l}\text { Chromium, unfil tered } \\
\text { (50 pgb) }\end{array}$ & $\begin{array}{l}299-w 15-16^{*} \\
299-W 15-20^{*} \\
299-W 18-21 \\
299-W 18-27\end{array}$ & $\begin{array}{l}299-W 15-18 * \\
299-W 15-23 \\
299-W 18-22\end{array}$ & $\begin{array}{l}299-415-19 \\
299-415-24 \\
299-418-26\end{array}$ \\
\hline $\begin{array}{l}\text { Iron, unftitered } \\
(300 \mathrm{ppb})\end{array}$ & $\begin{array}{l}299-415-16^{*} \\
299-415-23^{*} \\
299-N 18-22^{*} \\
299-418-32^{*}\end{array}$ & $\begin{array}{c}299-W 15-18^{*} \\
299-W 15-24^{*} \\
299-W 18-26^{*}\end{array}$ & $\begin{array}{l}299-W 15-19^{*} \\
299-418-21^{*} \\
299-418-27^{*}\end{array}$ \\
\hline $\begin{array}{l}\text { Manganese, unfij7tered } \\
(50 \mathrm{ppb})\end{array}$ & $299-W 15-24$ & $299-W 18-26$ & \\
\hline $\begin{array}{l}\text { Carbon Tetrachloride } \\
(5 \mathrm{ppb})\end{array}$ & $\begin{array}{l}299-W 15-15 \\
299-W 15-19 \\
299-k 15-24 \\
299-w 18-24 \\
299-k 118-28\end{array}$ & $\begin{array}{l}299-W 15-16^{*} \\
299-W 15-20 \\
299-W 18-21^{*} \\
299-418-26\end{array}$ & $\begin{array}{l}299-W 15-18 \\
299-415-23 \\
299-W 18-23 \\
299-W 18-27^{*}\end{array}$ \\
\hline $\begin{array}{l}\text { Trichloroethene } \\
\text { (5 ppb) }\end{array}$ & $299-W 15-16^{*}$ & $299-W 15-18^{*}$ & . \\
\hline
\end{tabular}

- QC data associated with this sample was outside of estab]ished limits.

Table 14-31. Water Qual ity Standards Exceeded at Low-Level Waste Management Area 5.

\begin{tabular}{|c|c|c|c|}
\hline $\begin{array}{l}\text { Field pH } \\
(6.5-8.5)\end{array}$ & $299-w 6-2$ & & \\
\hline $\begin{array}{l}\text { Nitrate } \\
(45,000 \mathrm{ppb})\end{array}$ & $\begin{array}{l}299-W 6-2^{*} \\
299-W 11-31^{*}\end{array}$ & $299-116-4^{*}$ & $299-46-9^{*}$ \\
\hline $\begin{array}{l}\text { Iodine-129 } \\
\text { (1 } \mathrm{pC} i / \mathrm{L})\end{array}$ & $299-411-31$ & & \\
\hline $\begin{array}{l}\text { Tritium } \\
(20,000 \mathrm{pCi} / 1)\end{array}$ & $\begin{array}{l}299-w 6-4 \\
299-W 11-31\end{array}$ & $299-1 / 6-5$ & $299-\$ 6-10$ \\
\hline
\end{tabular}

* QC data assoctated with this sample was outside of established limits. 
DOE/RL-94-36-4

This page intentionally left blank.

14-76 


\section{CONTENTS}

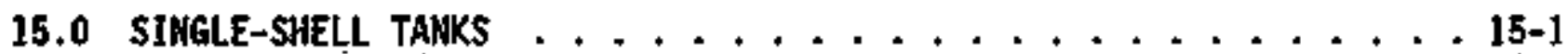

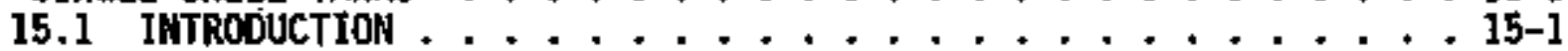

15.2 WATER LEVEL MEASUREMENTS . . . . . . . . . . - 15-2

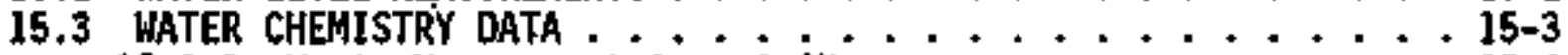

15.3.1 Waste Management Area $A-A X \ldots \ldots . . . . . .15-4$

15.3.2 Waste Management Area B-BX-BY . .............. 15-5

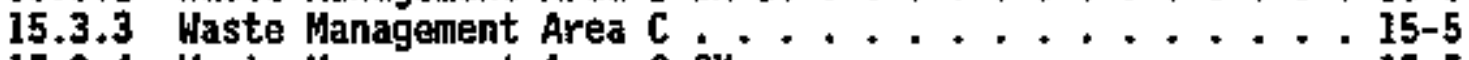

15.3.4 Waste Management Area $\mathrm{S}-\mathrm{SX} \ldots \ldots \ldots \ldots . . . . . .15-5$

15.3.5 Waste Management Area $\mathbf{T} \ldots \ldots \ldots$. . . . . . . . $\ldots$

15.3.6 Waste Management Area TX-TY . . . . . . . 15-6

15.3.7 Waste Management Area $0 \ldots \ldots$ 15-7 
BOE/RL-94-36-4

\section{LIST OF FIEURES}

15-1 Single-She11 Tank Waste Management Areas in the 200 East Area . . 15-8

15-2 Single-Shell Tank Waste Management Areas in the 200 West Area. . . 15-9

15-3 Well Location Map for the Single-Shell Tank Maste

Management Areas in the 200 East Area. ......... 15-10

15-4 Weli Location Hap for the Single-Sheli Tank

Waste Management Areas in the 200 West Area. . . . . . . . 15-11 


\section{LIST OF TABLES}

15-I Groundwater Honitoring We]ls for the Single-She11 Tanks . . . 15-12

15-2 RCRA Water Level Measurement Report Single-Shel] Tanks,

Fourth Quarter $1994 \ldots . . . . . . . . . . .15-15$.

15-3 Constituent List and Suramary of Results for the Single-Sheli Tank Haste Management Area A-AX Data for Reporting Period

October 1 through Decenber $31,1994 \ldots . . . . . .15-22$

15-4 Constituents with at.Least One Detected Value for the

Single-Shell Tank Waste Management Area A-AX Data for

Reporting Period October 1 through Decenber 31, 1994 . . . . 15-22

15-5 Contamination Indjcator Parameters for the

sing Te-She11 Tank Waste Management Area A-AX Data for

Reporting Period October 1 through December 31, 1994 . . . . . 15-22

15-6. Constytuent List and Summary of Results for the

Single-She11 Tank Waste Management Area B-BY-BX Data for

Reporting Period October 1 through Decenber 31, $1994 \ldots . .$. 15-23

15-7 Constituents with at Least One Detected Value for

the Single-Shell Tank Waste Management Area B-BY-BX Data

for Reporting Period october I through December 31, 1994 . . . 15-25

15-8 Contamination Indjcator Parameters for the

Single-Shell Tank Waste Management Area B-BY-BX Data

for Reporting Reriod 0ctober 1 through December 31, 1994 . . . . 15-27

15-9 Constituent List and Summary of Results for the

Single-Shell Tank Waste Management Area $C$ Data for

Reporting Period October I through December 31, 1994 . . . . 15-27

15-10 Constituents with at Least One Detected Value for the

Single-Shel1 Tank Haste Management Area C Data for

Reporting Period October 1 through December 31, $1994 \ldots$. . . . 15-28

15-11 Contamtnation Indicator Parameters for the

Single-5he11 Tank Waste Management Area C Data for

Reporting Period October 1 through Decenber 31, 1994 . . . . 15-28

15-12 Const 1tuent 1ist and Summary of Results for the .

Single-Shell Tank Waste Management Area S-SX Data for

Reporting Perlod October I through December 31, 1994 . . . . . 15-29

15-13 Constituents with at Least One Detected Value for the

Single-Shelf Tank Waste Management Area S-SX Data for

Reporting Perlod October 1 through December $31,1994 \ldots \ldots$. . . 15-30

15-14 Contamtnation Indicator Parameters for the

Singlo-Sheil Tank Waste Management Area S-SX Data for

Reporting Period October 1 through December 31, $1994 \ldots \ldots \ldots$. 15-31

15-15 Const turent List and Summary of Results for the

Single-She 17 Tank Waste Management Area T Data for

Reporting Period October 1 through December 31, 1994 . . . . 15-32

15-16 Constituents with at Least One Detected Value for the

Singlo-She11 Tank Waste Management Area T Data for

Reporting Period October 1 through December 31, $1994 \ldots \ldots$. . . 15-35

15-17 Contamination Indicator Parameters for the

Single-Shell Tank Waste Managenent Area T Data for

Reporting Period October 1 through December 31, 1994 . . . . 15-44

15-18 Constituent List and Summary of Results for the

Single-She11 Tank Waste Management Area TX-TY Data for

Reporting Period October 1 through December $31,1994 \ldots$. . . 15-45 


\section{LIST OF TABLES (cont)}

15-19 Constjtuents with at Least one Detected Value for the Single-She]1 Tank Waste Hanagenent Area TX-TY Data for Reporting Period October I through December 31, 1994 . . . . . . 15-48

15-20 Contastnation Indicator Parameters for the Single-Shejl Tank Waste Wanagement Area TX-TY Data for Reporting Period October 1 through December 31, 1994 . . . . . , 15-53

15-21 Constituent List and Summary of Results for the Single-Shell Tank Waste Management Area U Data for Reporting Period October 1 through December 31, 1994 . . . . . I5-53

15-22 Constituents with at Least one Detected Value for the Single-She11 Tank Waste Management Area U Data for Reporting Period October 1 through December 31, $1994 \ldots$. . . . 15-54

15-23 Contamination Indtcator Parameters for the Single-Shel] Tank Waste Management Area U Data for Reporting Period October 1 through Decenber 31, $1994 \ldots \ldots$. . . 15-54 


$$
\text { DOE/RL-94-36-4 }
$$

\title{
15.0 SIHGLE-SHELL TANKS
}

\author{
J. A. Caggiano \\ Westinghouse Häford Company
}

\subsection{IMTRODUCTION}

A] though decommissioned in 1980, the $55 T$ s are considered to be actively storing hazardous and radioactive waste and have been designated as RCRA facilittes. The SSTs received-metal and first-cycle waste from the chemical processing of uraniua-bearing spent fuel rods. Spent fuel rods from reactors in the 100 Areas were processed using the bismuth phosphate, REDOX, or PUREX process. Hetal waste is that which was produced in the first step of the process of dissolving spent fuels, and contains about $90 \%$ of fission product radionuclides as well as uranium and some plutonium. Other waste streams (e.g., coating, noncomplexed, and deionjzing waste) also were sent to SSTs at some time in their history (Anderson 1990 discusses types of waste sent to various SSTs from startup until the tank was no longer used). Chemical and radiological contents of the various 5STs were sumarized in WHC (1993C).

The 149 SSTs are located in 7 waste management areas (MMA) each containing 1 or more tank farms (Figures 15-1 and 15-2). Three WMAs are in the 200 East Area (A-AX, B-BX-BY, and $C$ ) and four are in the 200 West Area (S-SX, T, TX-TY, and U). Each tank farm contains from 4 (AX Tank Farm) to 18 (TX Tank Farn) tanks, each of which is an underground, reinforced-concrete tank with a single 1 iner of carbon steel. The jarger tanks have a diameter of $23 \mathrm{~m}$ (75 ft), vary in height, and are burfed at least $2 \mathrm{~m}(6 \mathrm{ft})$.below the ground surface. The capacity of these tanks varies from 2,000,000 to $3,800,000 \mathrm{~L}(530,000$ to $1,000,000 \mathrm{ga})$. The sixteen 200-serjes tanks hald $208,000 \mathrm{~L}(55,000 \mathrm{gat})$ each. Four 200 -series tanks are located in each of the orlginal four tank farms: 241-B, 24I-C, 24I-T, and 24I-U. The ortginal four tank farms were built in 1944; SSTs were bujit from 1944 to 1964.

Interin-status, detection-level groundwater monitoring was initiated at the SSTs in 1989 with the construction of 12 RCRA-standard wel]s. Sampling was initiated in February 1990 at WMAs A-AX, B-BX-BY, C, and T, but was suspended until July 1991 because of the absence of analytical laboratory support. Sampling resumed in 1991 and cont fnued quarterly through early 1993. Host wells have been sampled for more than four quarters. All WMAs, except T and $T X-T Y$, are now in detection-7evel indicator parameter evaluation status and are sampled semiannually. MHAs $T$ and TX-TY continue to be sampled quarterly because these sites are now in assessment-level monitoring status because of elevated specific conductance. A groundwater quality assessment monjtoring plan (Cagglano and Chou 1993) for WHAs $T$ and TX-TY was submitted to Ecology in July 1993. The \$ST WHA well locations are shown in Figures 15-3 and 15-4. Wells in the SST monitoring networks are 1isted in Table 15-1. Groundwater monitoring at the SSTs follows the groundwater monitoring plan (Jensen et al. 1989) as revised (Cagglano and Goodwin 1991).

Hunerous older wells in the vicinity of the tank farms are constructed of carbon steel casing that is perforated and open to different levels in the unconfined aquifer. These wells were part of an operational groundwater 


$$
\text { DOE/RL-94-35-4 }
$$

monitoring system at some point in their history and currently are used only for water level measurements or to collect samples for operational monitoring. Some are used for environmental surveillance onitoring to check for confortance to DOE orders (e.g., Schmidt et al. 1992). These older wells do not comply with the construction specifications of MAC 173-160 and are not used for collection of RCRA samples. One exception is well 2-E27-7, wh1ch was constructed in 1982 and has a carbon steel casing, an annular seat, and a stainless steel screen belor the carbon steel casing. No carbon steel is in contact with groundwater in weI] 2-E27-7. Samples are collected from this woll, which is upgradient of bWt $C$, but data from this well are not used in statistical evaluation.

\subsection{WATER LEVEL MEASUREMENTS}

Water levels measured in 61 wells for this quarter are given in Table 15-2. These data include water level weasurements taken when samp]ing, as we]1 as monthly measurements made by Well Services personnel. Suspected errors in water level measurenents this quarter are flagged in Table 15-2. A11 water levels in well 299-W23-15 depart significantly from the historical trend of water levels in thts well and are flagged.

The water table in the 200 East Area is nearly flat because of declining water levels and the high transmissivity of the Hanford formation. The water table map for June 1994 (DOE-RL 1995, Fig 4.11-5) illustrates the very low 5lope of the water table. Water levels have been declining steadily over the past several years at all SST sites (see hydrographs of ali WMAs in DOE-RL [1995]). However, data for 1994 in the 200 East area exhibtted an increase in water level from March through June that was followed by a decline of similar magnitude. Data from the third quarter indicated very little change in water leve]. Hater levels rose in almost at SST mells in the 200 East area in November by up to $6 \mathrm{~cm}(0.20 \mathrm{ft})$. Nater levels declined again in December, resulting in no appreciable change of water level in SST wells in the 200 East area for the quarter as well. as for the calendar year.

The change from a pattern of continuing steady decline in the water table in the previous few years in the 200 East area appears unrelated to any increased precipitation or natura] recharge. It may be accounted for by 1 nereased liquid effluent discharges to the 216-B-3 Pond and elimination of open ditches carrying water to B Pond. With the startup of the 242-A Evaporator, discharges to $B$ Pond have doubled. Discharges to the $B$ Pond now flow directly to the 216-B-3C lobe of the pond via an underground pipeline, thus eliminating previous losses of liquid in transit via inftitration and evapotranspiration. Several brief campaigns of the 242-A Evaporator were run in calendar year 1994.

A barrfer may be constructed over the 216-BY cribs in operable unit 200-BP-1 located north of WHA B-BX-BY. A modified RCRA cover was the preferred alternative in the Record of Decistion that is going out for public comment. After comments are addressed, the definitive design of the cover will commence. However, it is anticipated that all wells in the area of the 216-BY cribs will be decomissioned to ease construction and to eiminate potential pathways through the cover. New groundwater monitoring wells will be constructed beyond the new cover. Well 2-E33-38, along with nine older 
carbon steel wells formerly used by the Operational Honitoring program to monitor the 216-BY cribs, will be decommissioned. We]ls 2-E33-1 and 2-E33-5, along with well 2-E33-38, have been used to nonitor water levels for the SSTs in this area of very low water table gradient in 200 East Area. Contingency planning for the location and design of replacentent wells has been-initiated, but the project is on hold pending review of the Record of Decision by the Yakama Nation.

Water levels in 200 West area SST wells continued to decline as they have since the inception of water level monitoring in. RCRA wells in 1990. Hater levels in wel1s 2-W18-25 and 2-418-31 continue to be lower than in wells 2-W19-31 and 2-W19-32 at Min $U$. Five wells have been added to the water level monitoring network in the vicinity of the 241-U Tank Farm to deterwine . the extent of this change (see Table 15-2). Water level data for this quarter continue to confirm the existence of a local high point in the water tabie slightly south and east of WMA $U$. However, the highest point of the water table in the 200 West area continues to be to the' north of the 241-U Tank Farm. Groundwater flow now appears to be to the northwest (at least locally) beneath WHA $U$; an addittonal well is being considered noar the northwest corner of the 24I-U Tank Farm. PTans are being made to run the borehole velocity flowmeter in wells 299-W19-31 and 299-W19-32 to get a more accurate measure (compared with water level measurements in scattered wells) of the direction of groundwater flow beneath HMA $U$. An engineering change notice may be prepared to revise the groundwater monitoring plan when these measurements of groundwater flow direction have been completed. The original monitoring network was estabitshed in 1989 when grounchater was flowing to the northeast. because of the water table mound that originated beneath the now-

deconissioned 216- $4-10$ Pond in the southwest corner of the 200 West Area.

\subsection{WATER CHEMISTRY DATA}

Data recefved from analyses of samples collected during the fourth quarter of 1994 are provided in tables 15-3 through 15-23. Most S5T wells were not sampled this quarter; however, some data from samples collected in September 1994 are reported because they were recesved after the cutoff date for the July-to-September-1994 quarterly report. Quarterly sampling is presently conducted in WAS $T$ and $T X-T Y$ because these sites are in groundwater assessment monttoring under interim status (40 CFR 265). Al] other WMAs are sampled seniannually.

Wuch of the data for nitrate and some of the data for ToX and other constituents (e.g., metals analyzed by induct fvely coupled plasma) are flagged with a " $Q$ " indicating some qual'jty control problems during laboratory analyses of the samples. Data flags are explajned tn Chapter 1. Data for potassium-40 are reported as part of ganma scan analyses; however, potassfun-40 is a naturaily occurring isotope and is the biggest contributor to natural background radiation.

Several regulatory drinking water 1 imits for constituent concentration have been added to the data tables, resulting in sone constituents that had not previously been jdentified in quarterly reports exceeding concentration 1imits. For exampie, carbon tetrachloride exceeds the 5-ppb 1 imit in a number of wells in the 200 West Area because the groundwater contains a widespread 
plume of this constituent that originated from discharges to PFP cribs (BOE-RL 1994, Ftgure 4.1-19). Similar7y, trichToroethene has exceeded the 1 imit in some 200 West Area wells.

Regulatory limits for fluoride are not consistent. The primary DWS for fluoride is 4,000 ppb (40 CFR 141); the secondary standard (40 CFR 143.3) is $2,000 \mathrm{ppb}$. Washington State standards in Revised Code of Hashington (RCW) 248 and WAC 173-200 are 4,000 ppb. However, Appendix III of 40 CFR 265 (EPA Interim Primary DWS) 1ists a range of 1.4 to $2.4 \mathrm{ppm}(1,400$ to $2,400 \mathrm{ppb}$ ) for fluoride. The preponderance of promulgated standards is 4,000 ppb. Groundwater qual ity beneath WHA $\mathbf{T}$ is degraded because of fluoride, which has exceeded the 4,000-ppb standard in the past and continues to do so in some wells. Fluorine-bearing compounds were used in the bismuth-phosphate process in 221-T (T P1 ant) and were discharged to SSTs as well as liquid effluent factlities in the northern part of 200 Vest Area. Tables 15-12 and 15-13 use the EPA Interim Primary DAS (1.e., 1,400-ppb standard) for fluoride.

Four quarters of background data have been. obtained from wells in a11 WAs and the data have been statistically evaluated for indications of contanination through analyses of TOC, pH, and specific conductance for each site. Replicate average values from downgradient wells are compared to the critical means for indicator parameters to determine whether the facilities in any SST HAs may be affecting groundwater quality. Values for TOX were not used to calculate a critica] mean for each WMA because of QC problens in the laboratory. ToX samples, previously analyzed by Datachem Laboratory, are now being analyzed by Heston. If TOX values from background wells are historlcally non-detects, a likit of quantitation will be used as a surrogate. upgradient/downgradient comparison value. TOX data frow downgradient wel is will be compared accordingly. The change in groundwater flow direction beneath WMA $U$ wili necessitate a new calculation of background for this site.

Several LLBG RCRA standard wells have been added to the expanded network for groundwater quality assessment monjtoring at WMAs $T$ and TX-TY.

We1ts 299-46-2, 299-46-4, 299-W6-6, 299-W6-9, 299-W10-19, 299-W10-20, 299-W10-21 and 299-W11-31 may be found on Figures 14-3 (for LLBG WMA 3) or Figure 14-5 (for LLBG WMA 5). Several radionucl ides from the 11 st of site-spectfic constituents for the \$STs have been added to the sampling list for these wells, all of wich are located north or west of wMA T. Some data on radionuclides from these wells were received this quarter and are included in Tables 15-15 through 15-17. Additional wells to supplement the groundwater quality assessment program south of MMAs $T$ and $T X-T Y$ are being identified and checked before having any remedia] work performed and adding them to the SST network. These two intas are in groundwater quality assessment status because of elevated specific conductance. Wells are being added to these networks to provide data for determining the possible source and extent of the high conductivity plume in groundwater beneath these two WMAs.

Several TOX samples were not collected and analyzed in quadruplicate as requested. This oversight has been corrected for future sampling. Single results for Tox will not be used in calculating the critical mean and other statistical parameters. 


\subsubsection{Waste Managenent Area A-AX}

None of the wells in WMA A-AX were sampled this quarter. However, some data not received in time for the last quarterly report are included in Tables $15-3,15-4$, and $15-5$.

\subsubsection{Waste' Management Area B-BX-BY}

Two wells (299-E33-33 and 299-E33-36) were sampled this quarter. These. wells are cosampled for the 216-B-63 trench project for which sampling was performed this quarter. Data for other wells in the B-BX-BY networkthat did not arrive in time for the 1ast quarterly report are included in Tables 15-6 through 15-8. Iodine-129 results for these two wells this quarter were 2.76 and $5.93 \mathrm{pCi} / \mathrm{L}$, respectively.

Ho exceedances of the critical mean (or critical range) for the reported indicator parameters for we11s 299-E33-33 and 299-E33-36 occurred this guarter. However, the four values for $\mathrm{pH}$ for well 299-E33-36 (6.42, 6.46, $6.46,6.48$ ) depart significantly fron the historical trend for $\mathrm{pH}$ in this ve11, are very unusual for Hanford S1te groundwater, and are likely in error. A RAOE has been stbmitted to check the validity of these data. We1l 299-E3336 is an upgradtent we]1 for SST LNMA B-BX-BY.

Groundwater contaminant plumes north and south of WHA B-BX-BY arising from the 216-BY cribs (Operable Un't 200-BP-1) and 216-B-5 reverse welT (Operable Unit 200-BP-5) are currently being treated. At both sites, groundwater is being extracted, run through a charcoal and zeolite treatment system, and the resulting effluent injected into the ground. No effect has been observed on water levels or water quality to date at MMA B-BX-BY during the operation of these pump-and-treat systems. The operations are scheduled to conclude in April 1995.

\subsubsection{Maste Management Area $\mathrm{C}$}

Mo samples were collected this quarter from wel]s in WMA C. Data from gamoa scan analyses that were not reported in the previous quarterly report are included in Tables 15-9 and 15-10.

\subsubsection{Waste Management Area 5-SX}

He11 299-W22-44 was sampled early this quarter; sampling at other+we11s in the network was completed in late September and most of the data from that sampling were included in the previous quarterly report. Mo exceedances of the critical mean for field specific conductance, TOX, TOC or pH occurred for downgradient we11 299-W22-44.

\subsubsection{Naste Nanagement Area $T$}

A11 wells in WMA $T$ and the expanded network were sampled this quarter. Data in Tables 15-15 through 15-17 include some from wells added to the 
expanded network for assessment monitoring. Addttional data are from weils that are part of the RCRA monitoring network for LLBG WMAs 3 and 5. Analytical data from unfiltered samples for iron and chromium al1 exceed limits, but all data fron filtered sample analyses are below liaits except. for flltered chromilm. Fittered chromium exceeds the 50-ppb 1imit in we11s 299-410-15 and 299-k10-16. Nitrate and fluoride continue to exceed 1 imits in many wells. Gross beta values of $106.00,92.7$, and $86.3 \mathrm{pCj} / \mathrm{L}$ in welis 299-d10-15, 299-w11-27 and 299-w11-28 (respectively) correspond with values of $467,11.70$, and $455 \mathrm{pC} / \mathrm{L}$. Tritium exceeds the 20,000-pC1/L 13mit in a]1 WHA T network wells except 299-W11-27 and also exceeds the limtt in two LLBG wells in the expanded network.

Turbtdity ranged between 1.9 and 24.00 MTUs this quarter, with four of five samples below 6.2 NTUs.

Field specific conductance continues to be elevated in downgradient we11 299-W10-15 and is the paraneter that triggered this site into groundwater quality assessment monitoring under interim-status regulations.

We11. 2-W11-28 at WMA T was remediated in late 1993 and has been sampled on several occastions during 1994. After remedjation, the well was redeveloped until turbidity reached 2.5 NTUs. Carbon steel casing rollers used in the remediation of this well broke from the dritil string during remediation and now rest at the bottom of the screen. Data continue to be flagged (a $P$ flag follows values for this we11 in Tables 15-15 through 15-17) until no effects of the steel on groundwater quality are observed. After remediation, an NCR was prepared because the vell departs from conditions in the generic specification for well construction and because of elevated turbldity (WHC 3992a). The rell was redeveloped on October 26 and a sample was collected. The sample collected in October is the first to have acceptable levels of turbidity. A Tengthy purge at a low fion rate $(5.7 \mathrm{~L} / \mathrm{i}[1.5 \mathrm{gpm}]$ ) succeeded in obtaining a sampie with a turbidtty of $\mathbf{5 . 9}$ MTU. Analyses of unfiltered samples from this well yielded values for iron and chromium that exceeded 1 inits, but values for analyses of filtered samples were below limits.

\subsubsection{Waste Management Area TX-TY}

A11 wells in WMA TX-TY were sampled this quarter. Nitrate exceeded the DUS in a]l wells this quarter, with a maximum of $300,000 \mathrm{ppb}$ in well 299-W14-12. Gross beta is elevated in wells 299-W14-12 and 299-W15-22 (see Table 15-19). Technet lum-99 is elevated in the same wells and may account for elevated gross beta. Samples from wells 299-W10-17 and 299-W10-18 exceed the $1,400-p p b 1$ imit for fluoride, but not the 4,000-ppb primary DWS (40 CFR 14i). Unfiltered chromitu and iron exceed the 1 imit in a 71 wells, while unfiltered aanganese exceeds the $1 \mathrm{imit}$ in all but one sampla. AnaTyses of fi]tered samples for these constttuents are below the litit except for one 5 ample for filtered analyses for chronium. Tritium exceeds the $]$ imit of $20,000 \mathrm{pCi} / \mathrm{L}$ in all wells except 299-W10-18. Two values for ${ }^{129}$ I in well 299-W14-12 are 34.80 and $29.00 \mathrm{pCi} / \mathrm{L}$. Turbidjty ranges from $5.3 \mathrm{NTU}$ to $82 \mathrm{NTUS}$, with ait but one value for turbidity equaliting or exceeding 43 NTUs. Total dissolved solid exceeds the WAC limjt of 500 ppm in well 299-Wl4-12. 
DOE/RL-94-36-4

Field specific conductance continues to exceed the critical mean in dormgradient we lIs 299-W14-12 and 299-W10-17 and is the reason WMA TX-TY has been triggered into groundwater quality assessment monitoring under interimstatus regulations.

15.3.7 Waste Management Area U

No samples were collected this quarter at WMA U. Data from gamma scan analyses that did not arrive in time for inclusion in the previous quarterly report are presented in Tables 15-21 and 15-22.

15-7 
Figure 15-1. Single-Shell Tank Waste Managenent Areas in the 200 East Area.

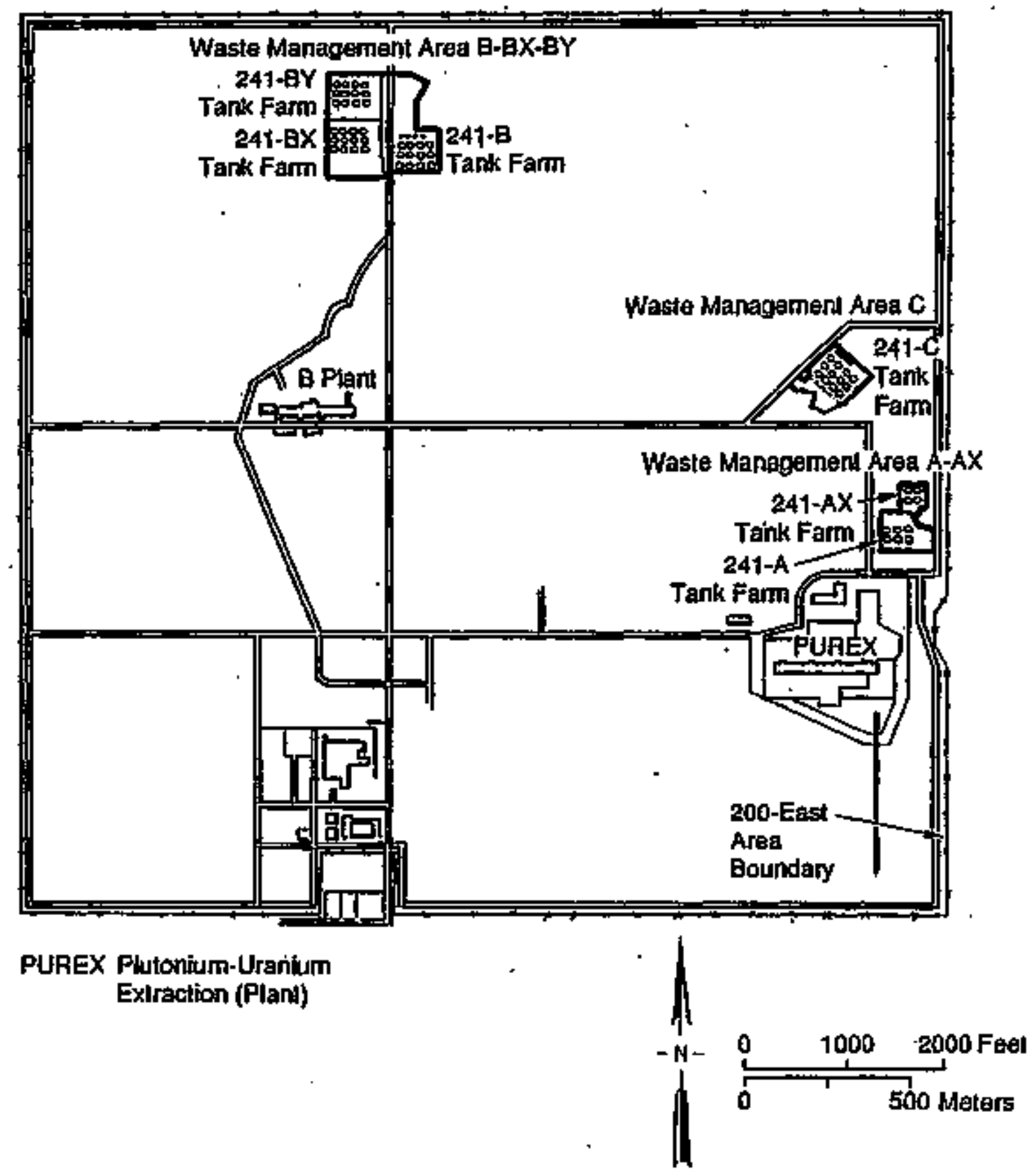

P5-10-71 
Figure 15-2. Single-Shell Tank Waste Management Areas in the 200 West Area.

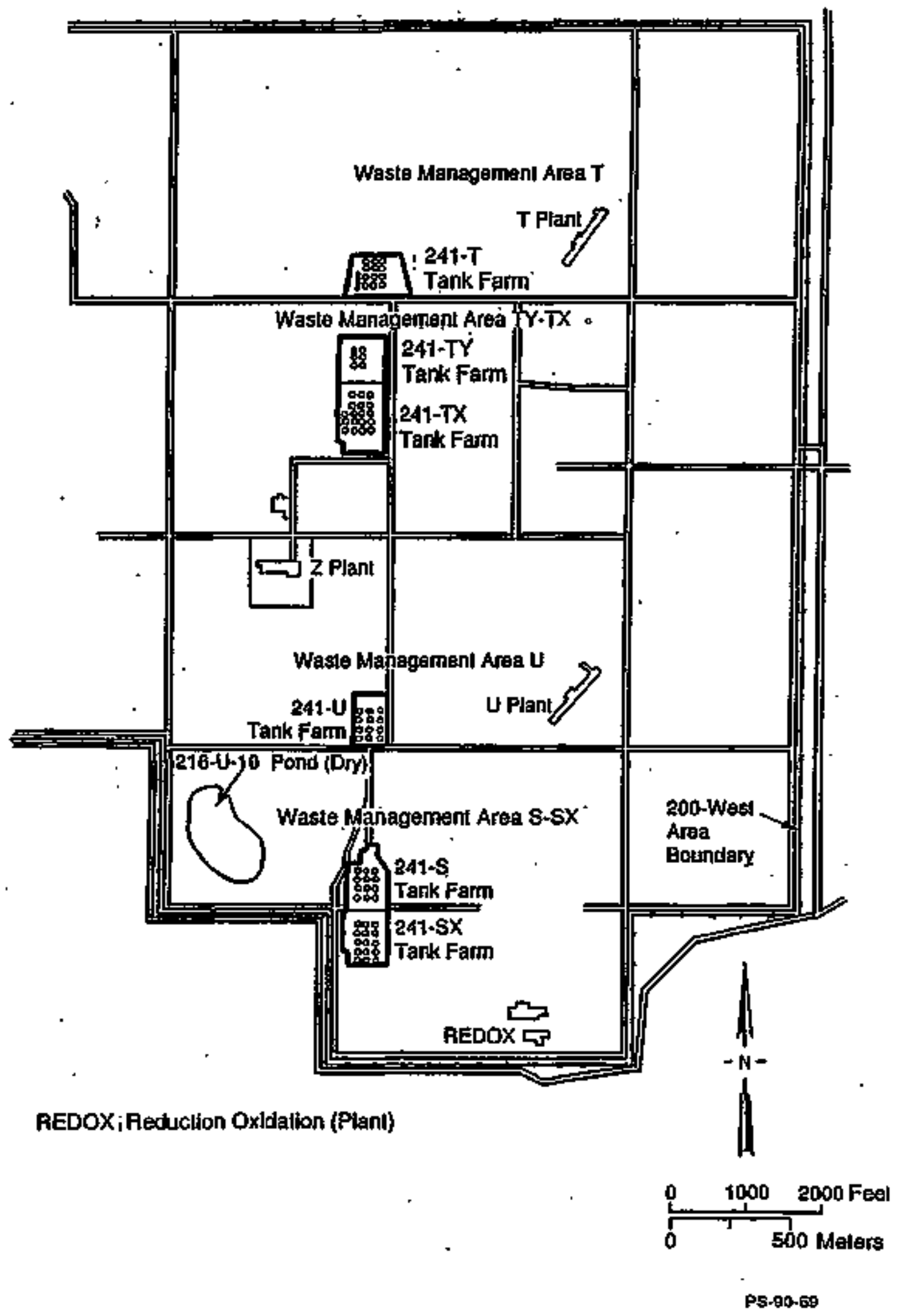




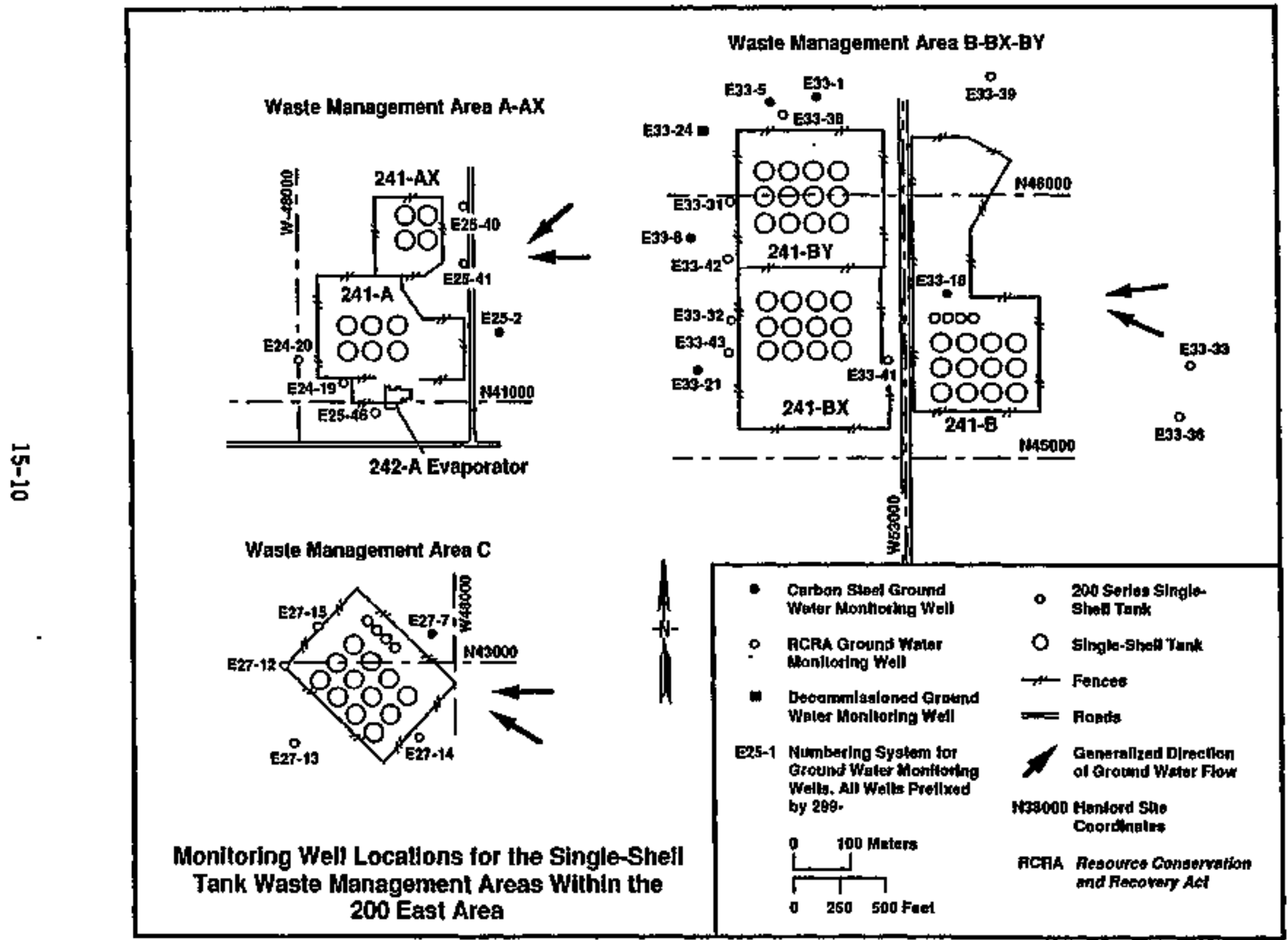

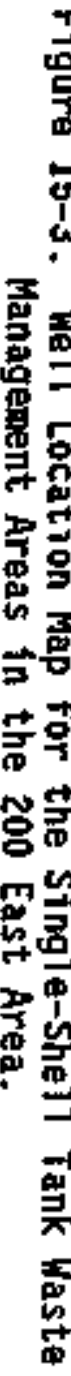




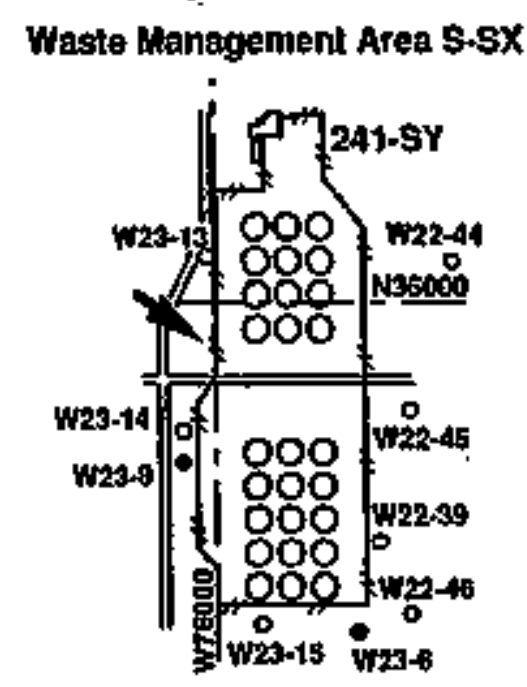

Waste Mangemenl Area T

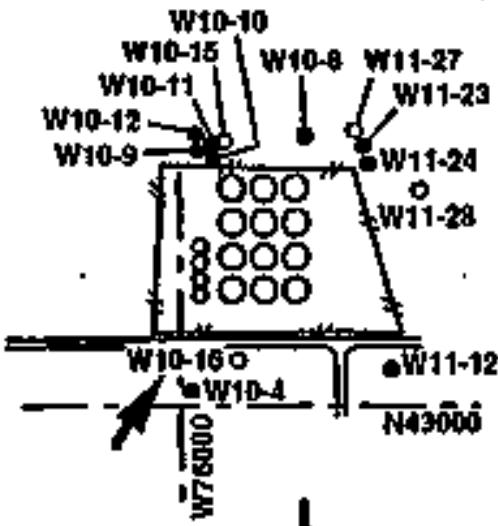

少

Waste Management Area TX-TY

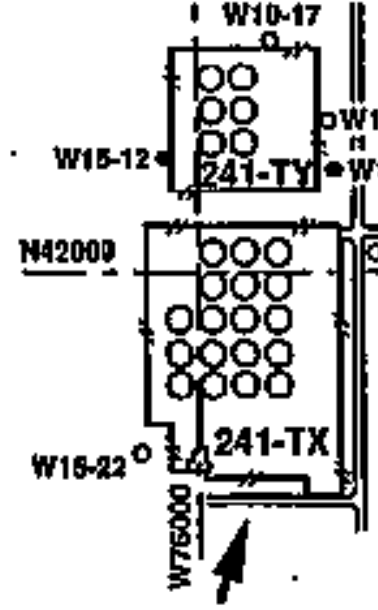

Waste Management Area U

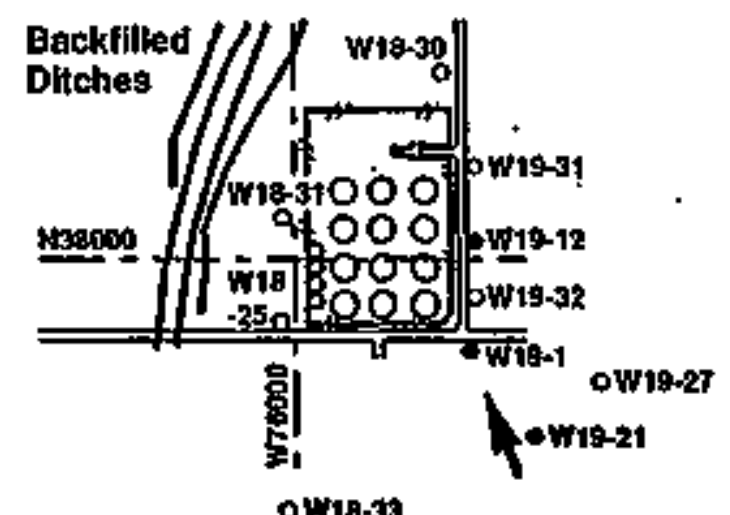

Wiget

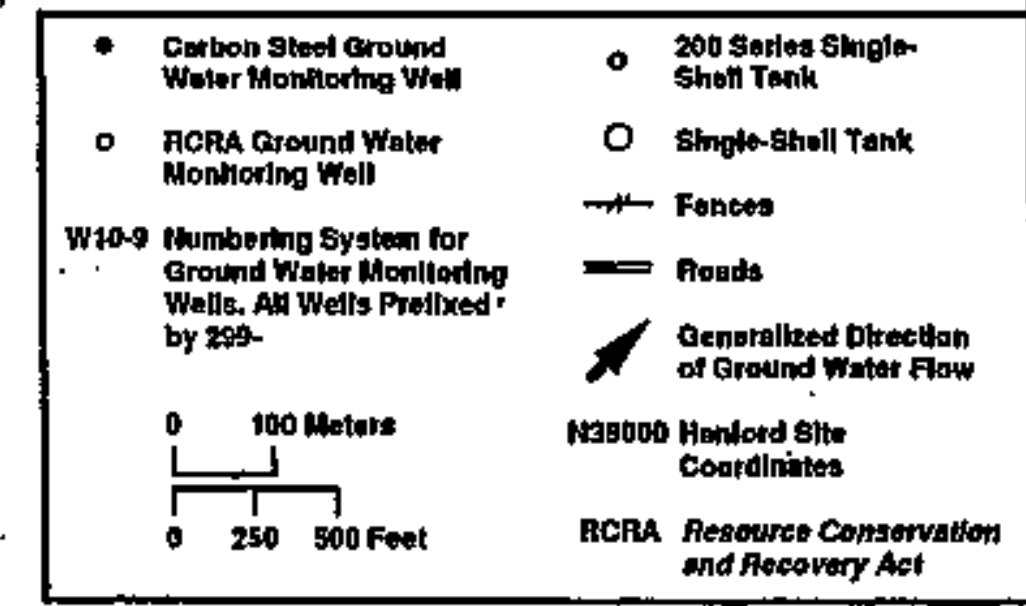

Monjtoring Well Locations tor the Single-Shell Tank Waste Management Areas Within the 200 West Area 
Table 15-1. Groundwater Monftorting Wells for the Single-Shell Tanks. (3 sheets)

\begin{tabular}{|c|c|c|c|}
\hline $\begin{array}{l}\text { Well no. } \\
\text { (299-) }\end{array}$ & Aquifer & $\begin{array}{l}\text { Sample } \\
\text { frequency }\end{array}$ & $\begin{array}{l}\text { Sample date, } \\
\text { 4th otr } 1994\end{array}$ \\
\hline \multicolumn{4}{|c|}{ Single-She11 Tanks Waste Management Area A-AX } \\
\hline$E 24-19^{95}$ & Ringold: Water Table & Seniannually & Not Sampied \\
\hline$E 24-20^{91}$ & Ringold: Water Table & Semtannuatiy & Not Sampled \\
\hline $625-40^{89}$ & Ringold: Water Table & Semiamtial1y & Hot Sampied \\
\hline $\mathrm{E} 25-41^{83}$ & Ringold: Water Table & SemiannщaT7y & Not Satepled \\
\hline$E 25-46^{92}$ & Ringoid: Water Table & Semiannually & Not Sampled \\
\hline \multicolumn{4}{|c|}{ Single-Shell Tanks Waste Management Area B-BX-BY } \\
\hline $\mathrm{E} 33-31^{69}$ & Hanford: Water Table & Semiannually & Hot Sampied \\
\hline$E 33-32^{89}$ & Hanford: Water Table & Semiannual1y & Not Sampled \\
\hline$E 33-33^{9}$ & Hanford: Water Table & SemiamnuaTyy & $11 / 21 / 94^{d}$ \\
\hline$E 33-36^{93}$ & Hanford: Water Tab]e & SemjannuaTly & $11 / 21 / 94^{\circ}$ \\
\hline$E 33-41^{916}$ & Hanford: Water Table & Semiannually & Not Sampled \\
\hline$E 33-38^{91 c}$ & Hanford: Water Table & Selniannua]1y & Not sampled \\
\hline E33-3991c & Hanford: Water Table & Semiannua]1y & Not samp]ed \\
\hline$E 33-42^{\text {I1 }}$ & Hanford: Nater Table & Semiannual1y & Not Sampled \\
\hline$E 33-43^{\text {g1 }}$ & Hanford: Water Table & Semf annua $17 y$ & Not Sampled \\
\hline \multicolumn{4}{|c|}{ Single-Shell Tank Waste Management Area C } \\
\hline$E 27-12^{89}$ & Ringold: Hater Tabie & Semiannually & Hot Sampled \\
\hline$E 27-13^{89}$ & Ringold: Water Table & Sen1annua11y & Not Sampled \\
\hline$E 27-14^{89}$ & Ringold: Water Table & Semiannua17y & Not Sampled \\
\hline$E 27-15^{89}$ & Ringold: Water Table & Semiannually & Not Sampled \\
\hline $\mathrm{E} 27-7^{82}$ & Ringold: Water Table & Semiannua17y & Hot Sampled \\
\hline \multicolumn{4}{|c|}{ Single-Shel] Tanks Waste Management Area S-5X } \\
\hline $122-39^{90}$ & Ringold: Water Table & Semíanntă $11 \mathrm{y}$ & Not. Sampled \\
\hline$W 22-44^{91}$ & Ringold: Water Table & Semt amnua $1 \mathrm{y}$ & $10 / 04 / 94$ \\
\hline W22-45 & Ringold: Water Table & Semiannual Iy & Not Sampled \\
\hline W22-46 & Ringold: Water Table & Semiannual ly & Not SampTed \\
\hline $123-13^{90}$ & Ringoid: Water Table & Semiannuatiy & Not Sampled \\
\hline $123-14^{90}$ & Ringold: Nater TabTe & Semiannual $7 y$ & Not Sampied \\
\hline
\end{tabular}


Table 15-1. Groundwater Monitoring We11s for the Single-Shell Tanks. (3 sheets)

\begin{tabular}{|c|c|c|c|}
\hline $\begin{array}{c}\text { Wel1 no. } \\
(299-)\end{array}$ & Aquifer" & $\begin{array}{l}\text { Sample } \\
\text { frequency }\end{array}$ & $\begin{array}{l}\text { Sample date, } \\
\text { 4th Qtr } 1994\end{array}$ \\
\hline $423-15^{91}$ & Ringold: Water Tabie & Semiannually & Not Sampled \\
\hline \multicolumn{4}{|c|}{ Single-Shell Tanks Waste Management Area T } \\
\hline $410-15^{59}$ & Ringold: Hater Table & Quarterly & $11 / 30 / 94$ \\
\hline $110-16^{69}$ & Ringold: Water, Table & Quarteriy & $11 / 29 / 94$ \\
\hline $410-19^{92}$ & Ringold: Water Table & Quarterly & $12 / 14 / 94$ \\
\hline$\$ 10-20^{93}$ & Ringold: Water Table & Quarteriy & $12 / 15 / 94$ \\
\hline$\$ 10-21^{\$ 3}$ & Ringold: Water Table & Quarterly & $12 / 14 / 94$ \\
\hline $111-27^{91}$ & Ringold: Water Table & Quarteriy & $11 / 30 / 94$ \\
\hline N11-28 & Ringold: Hater Table & Quarterly & $10 / 26 / 94$ \\
\hline $411-31^{92}$ & Ringold: Water Table & Quarterìy & $12 / 14 / 94$ \\
\hline \multicolumn{4}{|c|}{ Single-Shell Tanks Waste Management Area TX-TY } \\
\hline $110-17^{90}$ & Ringold: Hater Table & Quarterly & $11 / 30 / 94$ \\
\hline$W 10-18^{90}$ & Ringold: Water-Table & QuarterIy & $11 / 30 / 94$ \\
\hline$W 14-12^{91}$ & Ringold: Water Table & Quarterly & $11 / 30 / 94$ \\
\hline $415-22^{90}$ & Ringoid: Water Table & Quarterly & $11 / 30 / 94$ \\
\hline \multicolumn{4}{|c|}{ Single-Shel] Tanks Waste Management Area U } \\
\hline$W 18-25^{90}$ & Ríngold: Water Table & Semiannually & Not Sampled \\
\hline W18-3091 & Ringold: Water Table & Semiannttal 1y & Not Sampled \\
\hline W18-3191 & Ringold: Water Table & Semiannually & Not Sampled \\
\hline N19-3194 & Ringold: Water Table & Semiannuat1y & Not Sampled \\
\hline
\end{tabular}


Table 15-1. Groundwater Monitoring Wells for the Single-Shell Tanks. (3 sheets)

\begin{tabular}{|c|c|c|c|}
\hline $\begin{array}{l}\text { Well no. } \\
(299-)\end{array}$ & Aquifer ${ }^{a}$ & $\begin{array}{l}\text { Sample } \\
\text { frequency. }\end{array}$ & $\begin{array}{l}\text { Sample date, } \\
\text { 4th Qtr } 1994\end{array}$ \\
\hline Writ $32 \%$ & 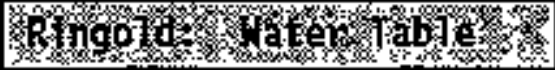 & 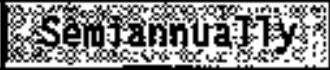 & Yot sampled \\
\hline Hog 98 & 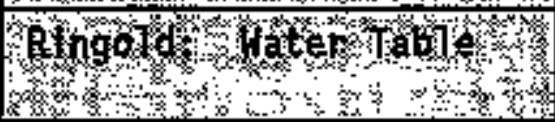 & S & Hot $=5$ \\
\hline
\end{tabular}

Motes: Shading denotes upgradient wells. Superscript following we11 number denotes the year of well conpletion.

"Wells are screened either in sand and grave? of the Hanford formation or in matrix-supported silty sandy gravel of the Ringold Formation.

Well in middle of WMA B-BX-BY and not clearly upgradjent or downgradient. Therefore, data from sampling/analyses in this well are not used in statistical evaluations.

"Part of 200-BP-1 operable unjt network. Mot sampled regularly as RCRA wells.

"Part of network for 216-B-63 that was sampled this quarter. 
Table 15-2. RCRA Water Level Measurement Report.

Single-Shell Tanks, Fourth Quarter 1994.

(sheet 1 of 7)

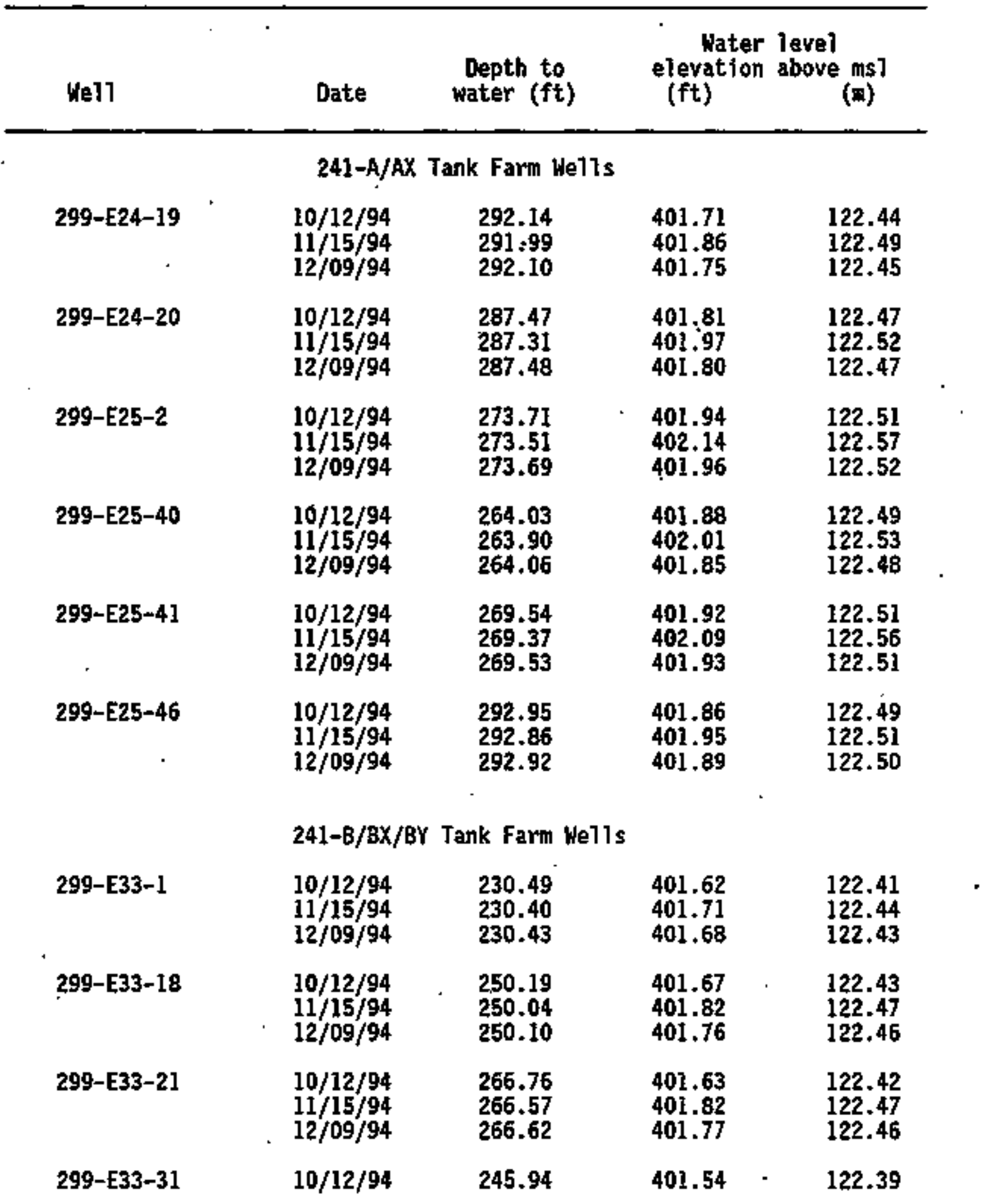


Table 15-2. RCRA Water Leve] Measurement Report Single-She11 Tanks, Fourth Quarter 1994.

(sheet 2 of 7)

\begin{tabular}{|c|c|c|c|c|}
\hline Hel1 & Date & $\begin{array}{l}\text { Depth to } \\
\text { water (ft) }\end{array}$ & $\begin{array}{l}\text { Water } \\
\text { elevation } \\
\text { (ft) }\end{array}$ & $\begin{array}{l}\text { level } \\
\text { above ms] } \\
\text { (m) }\end{array}$ \\
\hline & \multicolumn{3}{|c|}{ 241-B/BX/BY Tank Fari Wel1s } & \\
\hline 299-E33-31 & $\begin{array}{l}11 / 15 / 94 \\
12 / 09 / 94\end{array}$ & $\begin{array}{l}245.80 \\
245.82\end{array}$ & $\begin{array}{l}401.68 \\
401.66\end{array}$ & $\begin{array}{l}122.43 \\
122.43\end{array}$ \\
\hline $299-E 33-32$ & $\begin{array}{l}10 / 12 / 94 \\
11 / 15 / 94 \\
12 / 09 / 94\end{array}$ & $\begin{array}{l}258.58 \\
258.47 \\
258.47\end{array}$ & $\begin{array}{l}401.44 \\
401.55 \\
401.55\end{array}$ & $\begin{array}{l}122.36 \\
122.39 \\
122.39\end{array}$ \\
\hline $299-E 33-33$ & $\begin{array}{l}10 / 12 / 94 \\
11 / 15 / 94 \\
11 / 21 / 94 \\
12 / 09 / 94\end{array}$ & $\begin{array}{l}238.81 \\
238.65 \\
238.94 \\
238.74\end{array}$ & $\begin{array}{l}401.58 \\
401.74 \\
40 \mathrm{I} .45^{\star} \\
401.65\end{array}$ & $\begin{array}{l}122.40 \\
122.45 \\
122.36 \\
122.42\end{array}$ \\
\hline $299-E 33-36$ & $\begin{array}{l}11 / 21 / 94 \\
12 / 21 / 94\end{array}$ & $\begin{array}{l}248.25 \\
246.76\end{array}$ & $\begin{array}{l}398.42^{\star} \\
399.91\end{array}$ & $\begin{array}{l}121.44 \\
121.89\end{array}$ \\
\hline 299-E33-38 & $12 / 13 / 94$ & 230.48 & $40 I .47$ & 122.37 \\
\hline 299-E33-39 & $12 / 13 / 94$ & 222.04 & 401.25 & 122.30 \\
\hline 299-E33-41 & $\begin{array}{l}10 / 12 / 94 \\
11 / 15 / 94 \\
12 / 09 / 94\end{array}$ & $\begin{array}{l}253.32 \\
253.33 \\
253.63\end{array}$ & $\begin{array}{l}401.63 \\
401.62 \\
401.32\end{array}$ & $\begin{array}{l}122.42 \\
122.41 \\
122.32\end{array}$ \\
\hline $299-E 33-42$ & $\begin{array}{l}10 / 12 / 94 \\
11 / 15 / 94 \\
12 / 09 / 94\end{array}$ & $\begin{array}{l}252.73 \\
252.62 \\
252.64\end{array}$ & $\begin{array}{l}401.57 \\
401.68 \\
401.66\end{array}$ & $\begin{array}{l}122.40 \\
122.43 \\
122.43\end{array}$ \\
\hline $299-E 33-43$ & $11 / 15 / 94$ & 261.15 & 401.53 & 122.39 \\
\hline 299-E33-5 & $\begin{array}{l}10 / 12 / 94 \\
11 / 15 / 94 \\
12 / 09 / 94\end{array}$ & $\begin{array}{l}233.05 \\
232.95 \\
232.99\end{array}$ & $\begin{array}{l}401.67 \\
401.77 \\
401.73\end{array}$ & $\begin{array}{l}122.43 \\
122.46 \\
122.45\end{array}$ \\
\hline $299-E 33-8$ & $\begin{array}{l}10 / 12 / 94 \\
11 / 15 / 94 \\
12 / 09 / 94\end{array}$ & $\begin{array}{l}249.31 \\
249.18 \\
249.23\end{array}$ & $\begin{array}{l}401.68 \\
401.81 \\
401.76\end{array}$ & $\begin{array}{l}122.43 \\
122.47 \\
122.46\end{array}$ \\
\hline
\end{tabular}


Table 15-2. RCRA Water Leve] Measurement Report Single-Shell Tanks, Fourth Quarter 1994.

(sheet 3 of 7 )

\begin{tabular}{|c|c|c|c|c|}
\hline Well & Date & $\begin{array}{l}\text { Depth to } \\
\text { water (ft) }\end{array}$ & $\begin{array}{l}\text { Water } \\
\text { elevation } \\
\text { (ft) }\end{array}$ & $\begin{array}{l}\text { levet } \\
\text { n above msi } \\
\text { (m) }\end{array}$ \\
\hline \multicolumn{5}{|c|}{ 241-C Tank Farm We]Is } \\
\hline 299-E27-12., & $\begin{array}{l}10 / 12 / 94 \\
11 / 15 / 94 \\
12 / 09 / 94\end{array}$ & $\begin{array}{l}259.47 \\
259.34 \\
259.44\end{array}$ & $\begin{array}{l}401.68 \\
401.81 \\
401.71\end{array}$ & $\begin{array}{l}122.43 \\
122.47 \\
122.44\end{array}$ \\
\hline $299-E 27-13$ & $\begin{array}{l}10 / 12 / 94 \\
11 / 15 / 94 \\
12 / 09 / 94\end{array}$ & $\begin{array}{l}267.30 \\
267.20 \\
267.34\end{array}$ & $\begin{array}{l}401.89 \\
401.99 \\
401.85\end{array}$ & $\begin{array}{r}122.50 \\
122.53 \\
122.48\end{array}$ \\
\hline $299-E 27-14$ & $\begin{array}{l}10 / 12 / 94 \\
11 / 15 / 94 \\
12 / 09 / 94\end{array}$ & $\begin{array}{l}256.85 \\
256.70 \\
256.77\end{array}$ & $\begin{array}{l}401.69 \\
401.84 \\
401.77\end{array}$ & $\begin{array}{l}122.44 \\
122.48 \\
122.46\end{array}$ \\
\hline 299-E27-15 & $\begin{array}{l}10 / 12 / 94 \\
11 / 15 / 94 \\
12 / 09 / 94\end{array}$ & $\begin{array}{l}251.54 \\
251.42 \\
251.48\end{array}$ & $\begin{array}{l}401.33 \\
401.45 \\
401.39\end{array}$ & $\begin{array}{r}122.33 \\
122.36 \\
122.34\end{array}$ \\
\hline 299-E27-7 & $\begin{array}{l}10 / 12 / 94 \\
12 / 09 / 94\end{array}$ & $\begin{array}{l}232.99 \\
232.96\end{array}$ & $\begin{array}{l}401.88 \\
401.91\end{array}$ & $\begin{array}{l}122.49 \\
122.50\end{array}$ \\
\hline \multicolumn{5}{|c|}{ 241-S/SX Tank Farm Wells } \\
\hline 299-W22-39 & $\begin{array}{l}10 / 13 / 94 \\
11 / 14 / 94 \\
12 / 09 / 94\end{array}$ & $\begin{array}{l}211.25 \\
211.62 \\
211.79\end{array}$ & $\begin{array}{l}457.01 \\
456.64 \\
456.47\end{array}$ & $\begin{array}{l}139.30 \\
139.18 \\
139.13\end{array}$ \\
\hline $299-W 22-44$ & $\begin{array}{l}10 / 04 / 94 \\
10 / 13 / 94 \\
11 / 14 / 94 \\
12 / 09 / 94\end{array}$ & $\begin{array}{l}219.11 \\
219.27 \\
219.67 \\
219.86\end{array}$ & $\begin{array}{l}459.02^{*} \\
458.86 \\
458.46 \\
458.27\end{array}$ & $\begin{array}{l}139.91 \\
139.86 \\
139.74 \\
139.68\end{array}$ \\
\hline 299-W22-45 & $\begin{array}{l}10 / 13 / 94 \\
11 / 14 / 94 \\
12 / 09 / 94\end{array}$ & $\begin{array}{l}208.64 \\
209.02 \\
209.23\end{array}$ & $\begin{array}{l}457.57 \\
457.19 \\
456.98\end{array}$ & $\begin{array}{l}139.47 \\
139.35 \\
139.29\end{array}$ \\
\hline $299-122-46$ & $\begin{array}{l}10 / 13 / 94 \\
11 / 14 / 94 \\
12 / 09 / 94\end{array}$ & $\begin{array}{l}214.44 \\
214.80 \\
214.93\end{array}$ & $\begin{array}{l}456.74 \\
456.38 \\
456.25\end{array}$ & $\begin{array}{l}139.21 \\
139.10 \\
139.07\end{array}$ \\
\hline $299-123-13$ & $10 / 13 / 94$ & 206.95 & 459.38 & 140.02 \\
\hline
\end{tabular}


Table 15-2. RCRA Water Level Measurement Report Single-She11 Tanks, Fourth Quarter 1994.

(sheet 4 of 7 )

\begin{tabular}{|c|c|c|c|c|}
\hline WeT] & Date & $\begin{array}{l}\text { Depth to } \\
\text { water (ft) }\end{array}$ & $\begin{array}{l}\text { Water } \\
\text { elevation } \\
\text { (ft) }\end{array}$ & $\begin{array}{l}\text { leve] } \\
\text { above nts1 } \\
\text { (n) }\end{array}$ \\
\hline \multicolumn{5}{|c|}{ 241-S/SX Tank Farw Wells } \\
\hline $299-423-13$ & $\begin{array}{l}11 / 14 / 94 \\
12 / 09 / 94\end{array}$ & $\begin{array}{l}207.38 \\
207.50\end{array}$ & $\begin{array}{l}458.95 \\
458.83\end{array}$ & $\begin{array}{l}139.89 \\
139.85\end{array}$ \\
\hline $299-423-14$ & $\begin{array}{l}10 / 13 / 94 \\
11 / 14 / 94 \\
12 / 09 / 94\end{array}$ & $\begin{array}{l}205.11 \\
205.53 \\
205.67\end{array}$ & $\begin{array}{l}458.89 \\
458.47 \\
458.33\end{array}$ & $\begin{array}{l}139.87 \\
139.74 \\
139.70\end{array}$ \\
\hline $299-1423-15$ & $\begin{array}{l}10 / 13 / 94 \\
11 / 14 / 94 \\
12 / 09 / 94\end{array}$ & $\begin{array}{l}197.84 \\
198.19 \\
198.29\end{array}$ & $\begin{array}{l}467.60+ \\
467.25+ \\
467.15+\end{array}$ & $\begin{array}{l}142.52 \\
142.42 \\
142.39\end{array}$ \\
\hline $299-123-6$ & $\begin{array}{l}10 / 13 / 94 \\
11 / 14 / 94 \\
12 / 09 / 94\end{array}$ & $\begin{array}{l}209.92 \\
210.25 \\
210.40\end{array}$ & $\begin{array}{l}457.06 \\
456.73 \\
456.58\end{array}$ & $\begin{array}{l}139.31 \\
139.21 \\
139.17\end{array}$ \\
\hline $299-W 23-8$ & $\begin{array}{l}10 / 13 / 94 \\
11 / 14 / 94 \\
12 / 09 / 94\end{array}$ & $\begin{array}{l}204.88 \\
205.33 \\
205.47\end{array}$ & $\begin{array}{r}459.07 \\
458.62 \\
458.48\end{array}$ & $\begin{array}{l}139.92 \\
139.79 \\
139.74\end{array}$ \\
\hline \multicolumn{5}{|c|}{ 241-T Tank Farm Hells } \\
\hline $299-410-10$ & $\begin{array}{l}10 / 13 / 94 \\
11 / 14 / 94 \\
12 / 09 / 94\end{array}$ & $\begin{array}{l}214.58 \\
.214 .94 \\
214.99\end{array}$ & $\begin{array}{l}460.77 \\
460.41 \\
460.36\end{array}$ & $\begin{array}{l}140.44 \\
140.33 \\
140.32\end{array}$ \\
\hline $299-410-11$ & $\begin{array}{l}10 / 13 / 94 \\
11 / 14 / 94 \\
12 / 09 / 94\end{array}$ & $\begin{array}{l}214.58 \\
214.78 \\
214.93\end{array}$ & $\begin{array}{l}460.94 \\
460.74 \\
460.59\end{array}$ & $\begin{array}{l}140.49 \\
140.43 \\
140.39\end{array}$ \\
\hline $299-410-12$ & $\begin{array}{l}10 / 13 / 94 \\
11 / 14 / 94 \\
12 / 09 / 94\end{array}$ & $\begin{array}{l}214.32 \\
214.53 \\
214.67\end{array}$ & $\begin{array}{l}460.69 \\
460.48 \\
460.34\end{array}$ & $\begin{array}{l}140.42 \\
140.35 \\
140.31\end{array}$ \\
\hline 299-W10-15 & $\begin{array}{l}10 / 13 / 94 \\
11 / 14 / 94 \\
12 / 09 / 94\end{array}$ & $\begin{array}{l}215.26 \\
215.48 \\
215.51\end{array}$ & $\begin{array}{l}460.64 \\
460.42 \\
460.29\end{array}$ & $\begin{array}{l}140.40 \\
140.34 \\
140.30\end{array}$ \\
\hline 299-W10-16 & $\begin{array}{l}10 / 13 / 94 \\
11 / 14 / 94\end{array}$ & $\begin{array}{l}211.98 \\
212.23\end{array}$ & $\begin{array}{l}460.78 \\
460.53\end{array}$ & $\begin{array}{l}140.45 \\
140.37\end{array}$ \\
\hline
\end{tabular}


Table 15-2. RCRA Water Level Measurement Report single-Shelt Tanks, fourth Qtarter 1994.

(sheet 5 of 7)

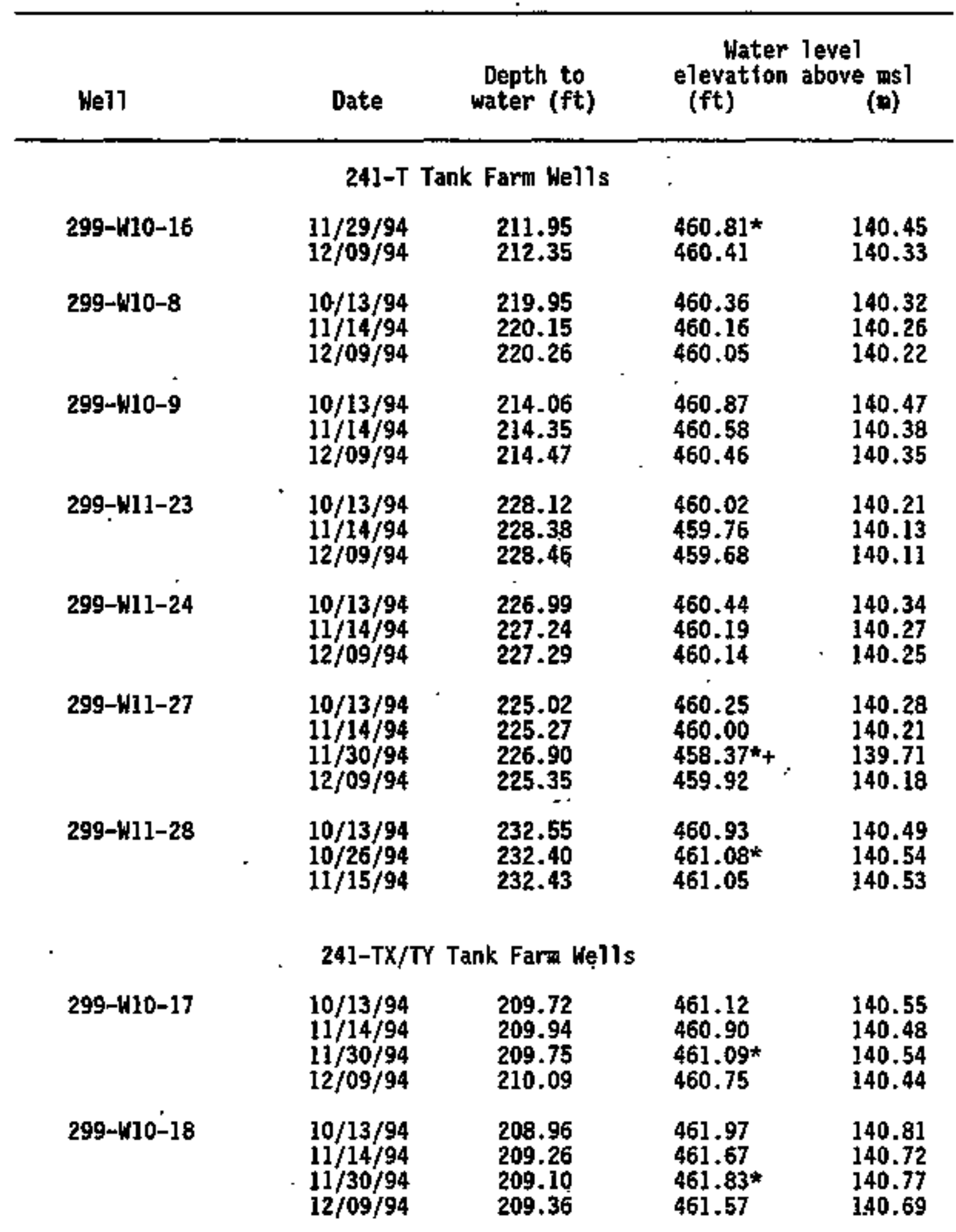


- Table 15-2. RCRA Water Level Measurement Report Single-She11 Tanks, Fourth Quarter 1994.

(sheet 6 of 7 )

\begin{tabular}{|c|c|c|c|c|}
\hline Well & Date & $\begin{array}{l}\text { Depth to } \\
\text { water (ft) }\end{array}$ & $\begin{array}{l}\text { Hater } \\
\text { elevation } \\
\text { (ft) }\end{array}$ & $\begin{array}{l}\text { level } \\
\text { above गs 1 } \\
\text { (m) }\end{array}$ \\
\hline \multicolumn{5}{|c|}{ 241-TX/TY Tank Fare Wells } \\
\hline 299-W14-12 & $\begin{array}{l}10 / 13 / 94 \\
11 / 30 / 94 \\
12 / 09 / 94\end{array}$ & $\begin{array}{l}207.76 \\
208.05 \\
208.16\end{array}$ & $\begin{array}{l}462.76 \\
462.47^{*} \\
462.36\end{array}$ & $\begin{array}{l}141.05 \\
140.96 \\
140.93\end{array}$ \\
\hline 299-비 5-12 & $\begin{array}{l}10 / 13 / 94 \\
11 / 14 / 94 \\
12 / 09 / 94\end{array}$ & $\begin{array}{l}208.04 \\
208.33 \\
208.45\end{array}$ & $\begin{array}{l}462.03 \\
461.74 \\
461.62\end{array}$ & $\begin{array}{l}140.83 \\
140.74 \\
140.70\end{array}$ \\
\hline $299-415-13$ & $\begin{array}{l}10 / 13 / 94 \\
11 / 14 / 94 \\
12 / 09 / 94\end{array}$ & $\begin{array}{l}207.99 \\
208.28 \\
208.36\end{array}$ & $\begin{array}{l}462.13 \\
461.84 \\
461.76\end{array}$ & $\begin{array}{l}140.86 \\
140.77 \\
140.74\end{array}$ \\
\hline \multirow[t]{2}{*}{$299-615-22$} & $\begin{array}{l}10 / 13 / 94 \\
11 / 14 / 94 \\
11 / 30 / 94 \\
12 / 09 / 94\end{array}$ & $\begin{array}{l}207.18 \\
207.53 \\
208.35 \\
207.54\end{array}$ & $\begin{array}{l}463.59 \\
463.24 \\
462.42^{*} \\
463.23\end{array}$ & $\begin{array}{l}141.30 \\
141.20 \\
140.95 \\
141.19\end{array}$ \\
\hline & \multicolumn{2}{|c|}{ 241-U Tank Farmu Wells } & . & \\
\hline $299-418-25$ & $\begin{array}{l}10 / 13 / 94 \\
11 / 14 / 94 \\
12 / 09 / 94\end{array}$ & $\begin{array}{l}203.63 \\
204.07 \\
204.28\end{array}$ & $\begin{array}{l}462.41 \\
461.97 \\
.461 .76\end{array}$ & $\begin{array}{l}140.94 \\
140.81 \\
140.74\end{array}$ \\
\hline $299-W 18-30$ & $\begin{array}{l}10 / 13 / 94 \\
11 / 14 / 94 \\
12 / 09 / 94\end{array}$ & $\begin{array}{l}210.03 \\
210.43 \\
210.63\end{array}$ & $\begin{array}{l}462.81 \\
462.41 \\
462.21\end{array}$ & $\begin{array}{l}141.06 \\
140.94 \\
140.88\end{array}$ \\
\hline $299-W 18-31$ & $\begin{array}{l}10 / 13 / 94 \\
11 / 14 / 94 \\
12 / 09 / 94\end{array}$ & $\begin{array}{l}201.65 \\
202.09 \\
202.29\end{array}$ & $\begin{array}{l}462.51 \\
462.07 \\
461.87\end{array}$ & $\begin{array}{l}140.97 \\
140.84 \\
140.78\end{array}$ \\
\hline $299-118-33$ & $\begin{array}{l}10 / 13 / 94 \\
11 / 14 / 94 \\
12 / 09 / 94\end{array}$ & $\begin{array}{l}206.99 \\
207.53 \\
207.71\end{array}$ & $\begin{array}{l}461.92 \\
461.38 \\
461.20\end{array}$ & $\begin{array}{l}140.79 \\
140.63 \\
140.57\end{array}$ \\
\hline 299-W19-12 & $\begin{array}{l}10 / 13 / 94 \\
11 / 14 / 94 \\
12 / 09 / 94\end{array}$ & $\begin{array}{l}210.42 \\
210.84 \\
211.04\end{array}$ & $\begin{array}{l}462.83 \\
462.41 \\
462.21\end{array}$ & $\begin{array}{l}141.07 \\
140.94 \\
140.88\end{array}$ \\
\hline
\end{tabular}


Table 15-2. RCRA Water Leve] Measurement Report Single-She11 Tanks, Fourth quarter 1994.

(sheet 7 of 7)

\begin{tabular}{|c|c|c|c|c|}
\hline Mell & Date & $\begin{array}{l}\text { Depth to } \\
\text { water (ft) }\end{array}$ & $\begin{array}{l}\text { Water } \\
\text { elevation } \\
\text { (ft) }\end{array}$ & $\begin{array}{l}\text { Teve1 } \\
\text { above ms1 } \\
\text { (m) }\end{array}$ \\
\hline . & \multicolumn{2}{|c|}{ 241-U Tank Farm We]ls } & & \\
\hline $299-419-21$ & $\begin{array}{l}10 / 13 / 94 \\
11 / 14 / 94 \\
12 / 09 / 94\end{array}$ & $\begin{array}{l}216.26 \\
216.85 \\
217.10\end{array}$ & $\begin{array}{r}462.53 \\
461.94 \\
461.69\end{array}$ & $\begin{array}{r}140.98 \\
\cdot \quad 140.80 \\
140.72\end{array}$ \\
\hline 299-W19-27 & $\begin{array}{l}10 / 13 / 94 \\
11 / 14 / 94 \\
12 / 09 / 94\end{array}$ & $\begin{array}{l}221.36 \\
221.93 \\
220.14\end{array}$ & $\begin{array}{l}462.55 \\
461.98 \\
463.77+\end{array}$ & $\begin{array}{l}140.99 \\
140.81 \\
141.36\end{array}$ \\
\hline 299-419-31 & $\begin{array}{l}10 / 13 / 94 \\
11 / 14 / 94 \\
12 / 09 / 94\end{array}$ & $\begin{array}{l}211.37 \\
211.77 \\
211.94\end{array}$ & $\begin{array}{l}462.82 \\
462.42 \\
462.25\end{array}$ & $\begin{array}{l}140.07 \\
140.95 \\
140.89\end{array}$ \\
\hline 299-419-32 & $\begin{array}{l}10 / 13 / 94 \\
11 / 14 / 94 \\
12 / 09 / 94\end{array}$ & $\begin{array}{l}212.05 \\
212.54 \\
212.69\end{array}$ & $\begin{array}{l}462.85 \\
462.36 \\
462.21\end{array}$ & $\begin{array}{l}141.08 \\
140.93 \\
140.88\end{array}$ \\
\hline $299-W 19-6$ & $\begin{array}{l}10 / 13 / 94 \\
11 / 14 / 94 \\
12 / 09 / 94\end{array}$ & $\begin{array}{r}227.88 \\
-\quad 228.35 \\
\quad 228.51\end{array}$ & $\begin{array}{l}458.72 \\
458.25 \\
458.09\end{array}$ & $\begin{array}{l}139.82 \\
139.67 \\
139.63\end{array}$ \\
\hline & & & . & \\
\hline
\end{tabular}

HOTES: 1. Hater level elevations are calculated by subtracting the measured depth-to-water from the surveyed elevation for the well.

2. Depth-to-water values are transcribed from field records.

3. Elevations marked with an $1 * 1$ were measured at the time of sampling.

4. ETevations marked with a ' + ' are outside of the expected range, and are suspectad of error. 
Table 15-3. Constjtuent List and Sunmary of Results for the Single-Shell Tank Waste Management Area A-AX Data for Reporting Period October 1 through December 31, 1994.

SITE SPECIFIC AD OTHER COHSTIJUEMTS

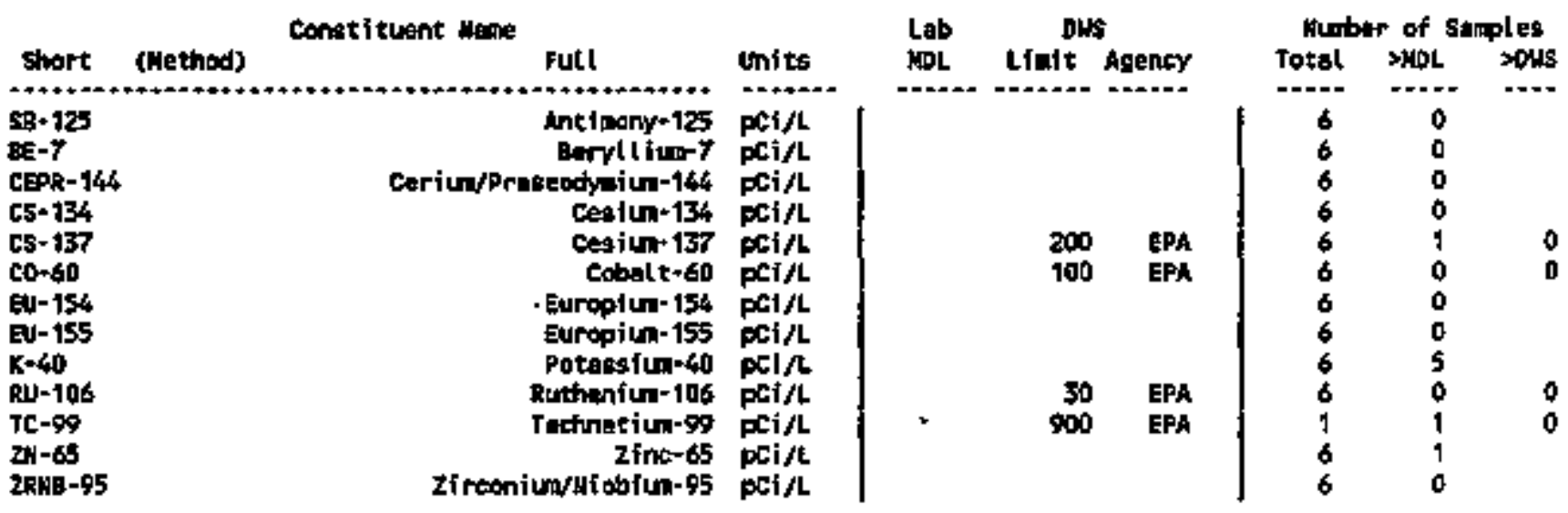

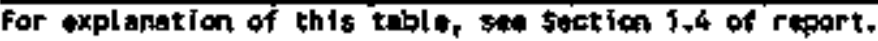

TabTe 15-4. Constftuents with at Least One Detected Value for the Single-She]1 Tank Waste Hanagewent Area A-AX Data for Reporting Period October 1 through December 31, 1994.

\begin{tabular}{|c|c|c|c|c|c|c|}
\hline $\begin{array}{l}\text { Welt } \\
\text { Hene }\end{array}$ & $\begin{array}{c}\text { Col lection } \\
\text { Date }\end{array}$ & $\begin{array}{l}\text { Sample } \\
\text { Hunbar }\end{array}$ & $\begin{array}{c}65-137 \\
140 / 061 / 1 \\
-/ 200\end{array}$ & $\begin{array}{c}x-40 \\
140 / p C 1 / 1 \\
. /\end{array}$ & $\begin{array}{c}16-99 \\
143 / p \times 1 / t \\
-/ 900\end{array}$ & $\begin{array}{c}24-65 \\
140 / \mathrm{pCl}^{-1 / 2} \\
.1\end{array}$ \\
\hline $\begin{array}{l}299-E 24-19 \\
299-624-20 \\
299-E 25-40 \\
299-E 25+41 \\
299-E 25-46 \\
299-64+46\end{array}$ & $\begin{array}{l}9 / 16 / 94 \\
9 / 14 / 94 \\
\$ / 14 / 94 \\
9 / 14 / 94 \\
\$ / 14 / 94 \\
9 / 14 / 94\end{array}$ & 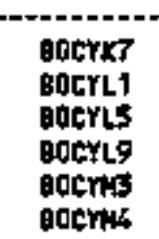 & $\begin{array}{r}.75 v \\
+2.00 v \\
2.45 \\
.62 v \\
-.81 \mathrm{v} \\
.54 \mathrm{v}\end{array}$ & $\begin{array}{r}101.00 \\
51.00 \\
22.40 \\
71.00 \\
2.2600 \\
59.900\end{array}$ & 47,60 & $\begin{array}{r}3.58 \mathrm{U} \\
1.85 \\
-5.93 \mathrm{U} \\
.75 \mathrm{U} \\
-2.45 \mathrm{U} \\
-1.16 \mathrm{U}\end{array}$ \\
\hline
\end{tabular}

For explanation of this table, see section $\$ .4$ of report.

Table 15-5. Contamination Indicator Parameters for the Single-Shell Tank Waste Management Area A-AX Data for Reporting Period October 1 through December 31, 1994.

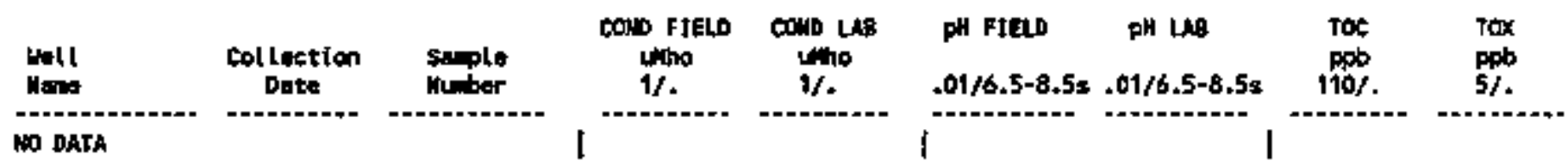

Far explanditan of this table, sect section 1.4 of ceport. 
TabTe 15-6. Constituent List and Summary of Results for the Single-Shel] Tank Waste Management Area B-BY-BX Data for Reporting Period October I through December 31, 1994.

(sheet 1 of 2)

COATANLWhTON ILOICATOR PARAHETERS

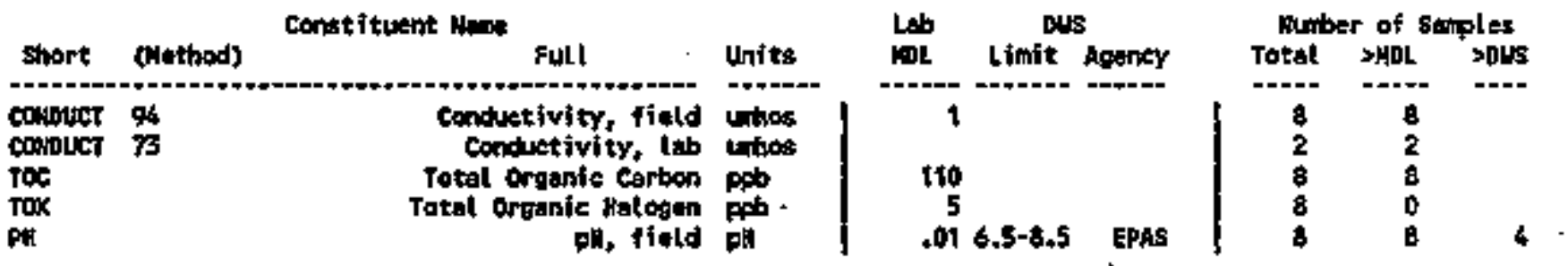

DRLMXIAN HATER PAGNIETERS

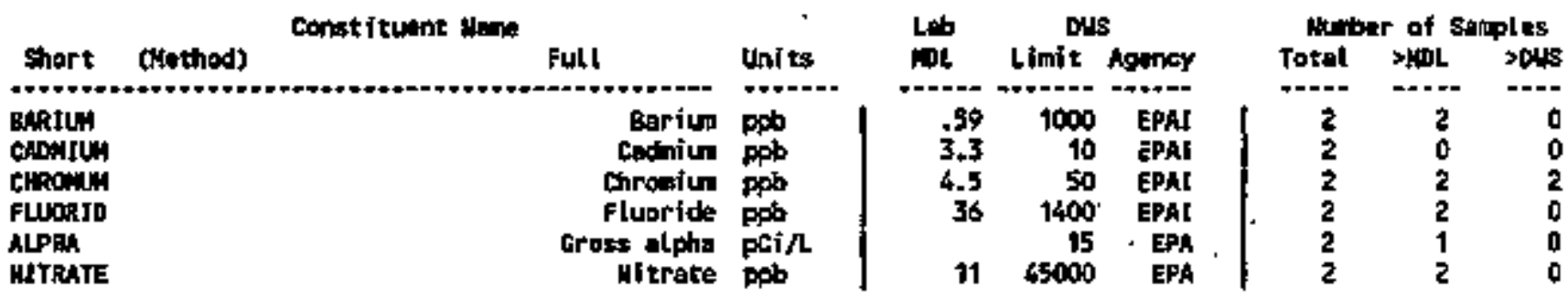

GROLWOLKTER AHALITY PARANETERS

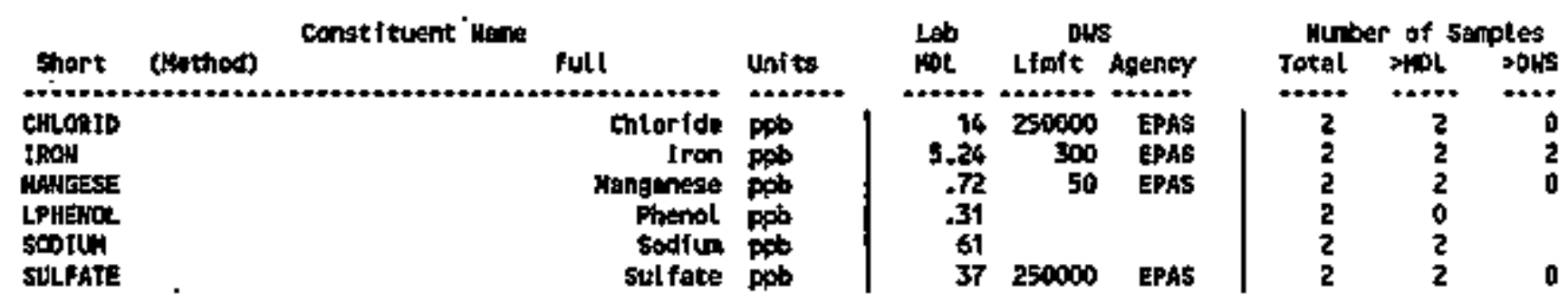

SITE SPECIFtC AHD OTHER COUSTITUEMTS

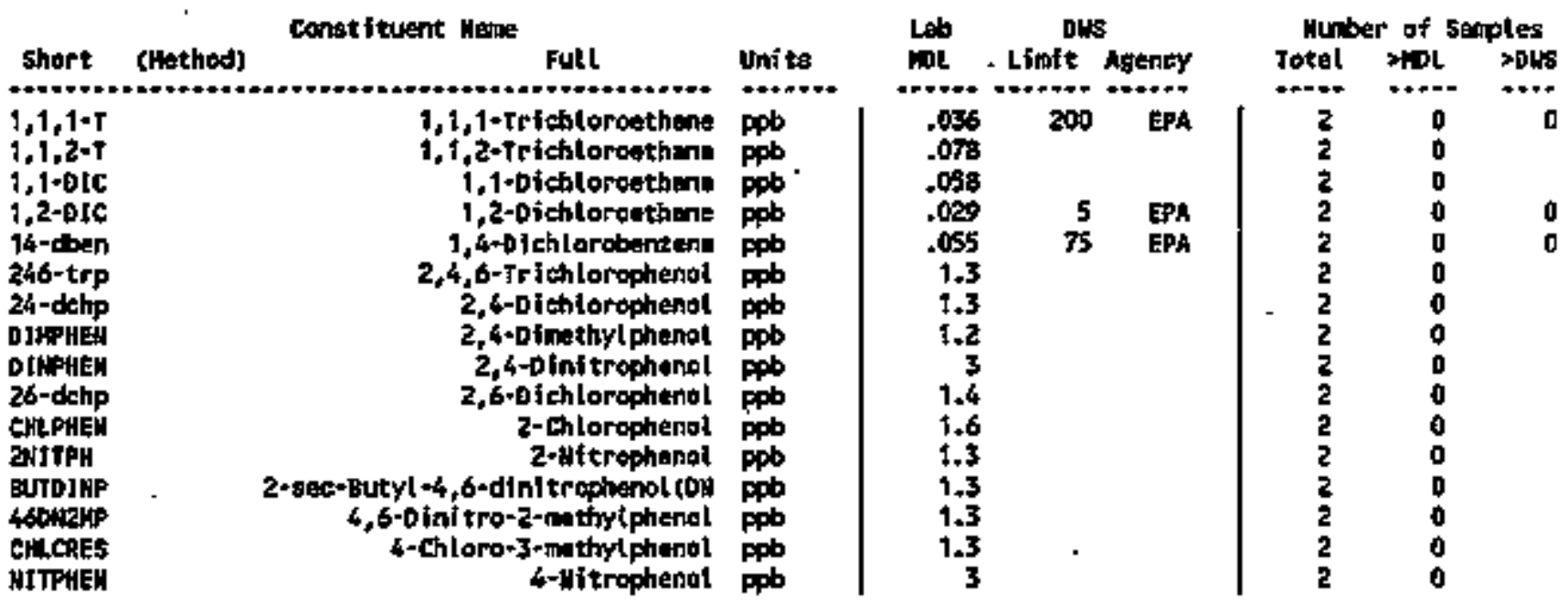


Table 15-6. Constituent List and Summary of Results for the Single-Shell Tank Waste Management Area B-BY-BX Data for

Reporting Period October I through December 31, 1994. (sheet 2 of 2)

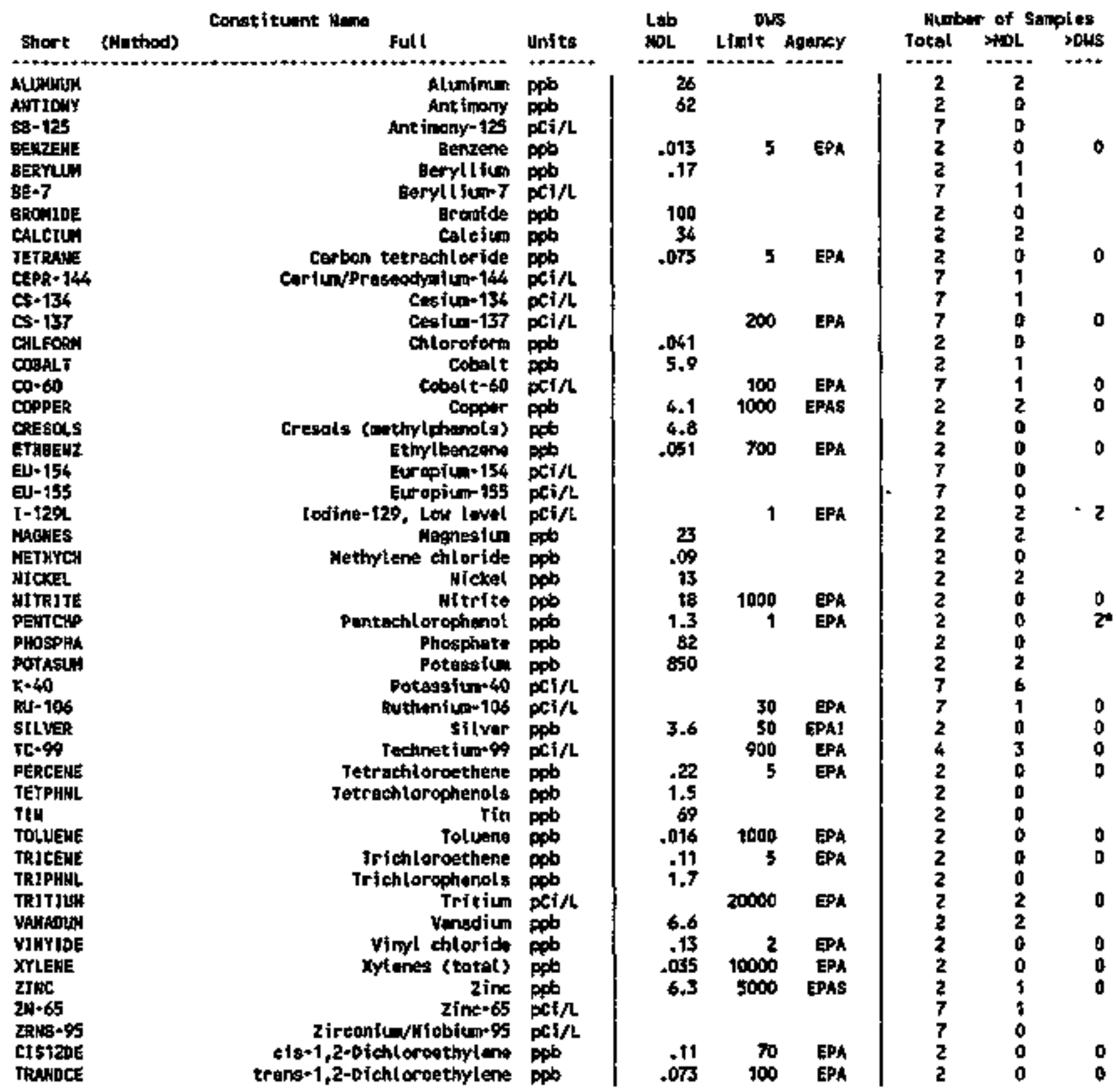

For axplanstion of this table, ate section 1.4 of report. 
Table 15-7. Constituents with at Least One Detected Value for the Single-She11 Tank Waste Management Area B-BY-BX Data for Reporting Period October 1 through December 31, 1994.

(sheet 1 of 2)

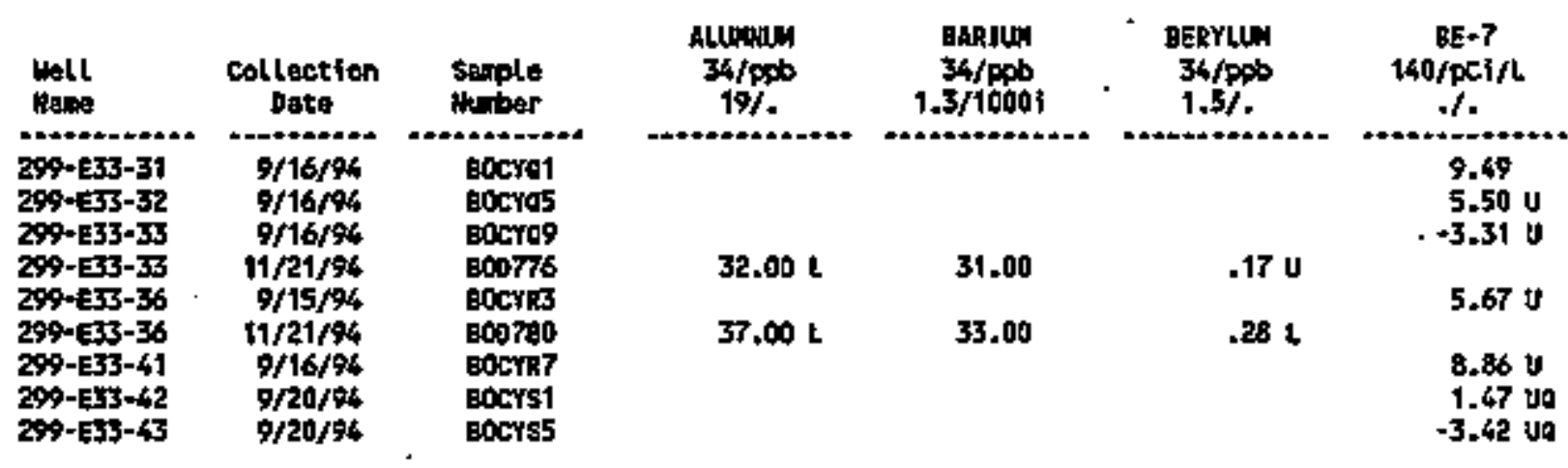

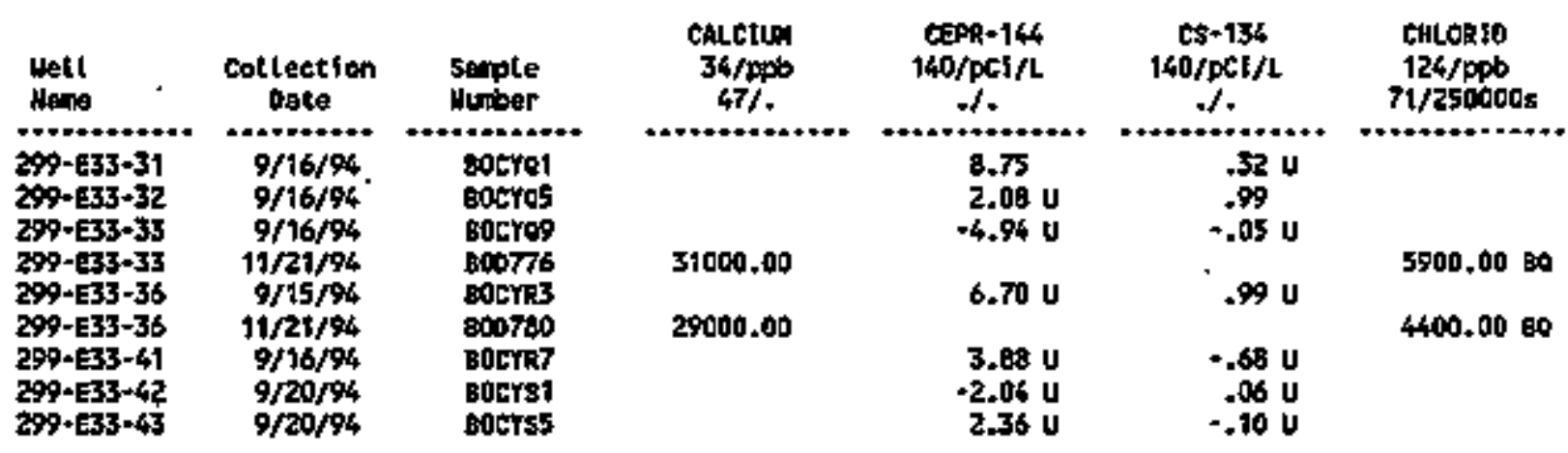

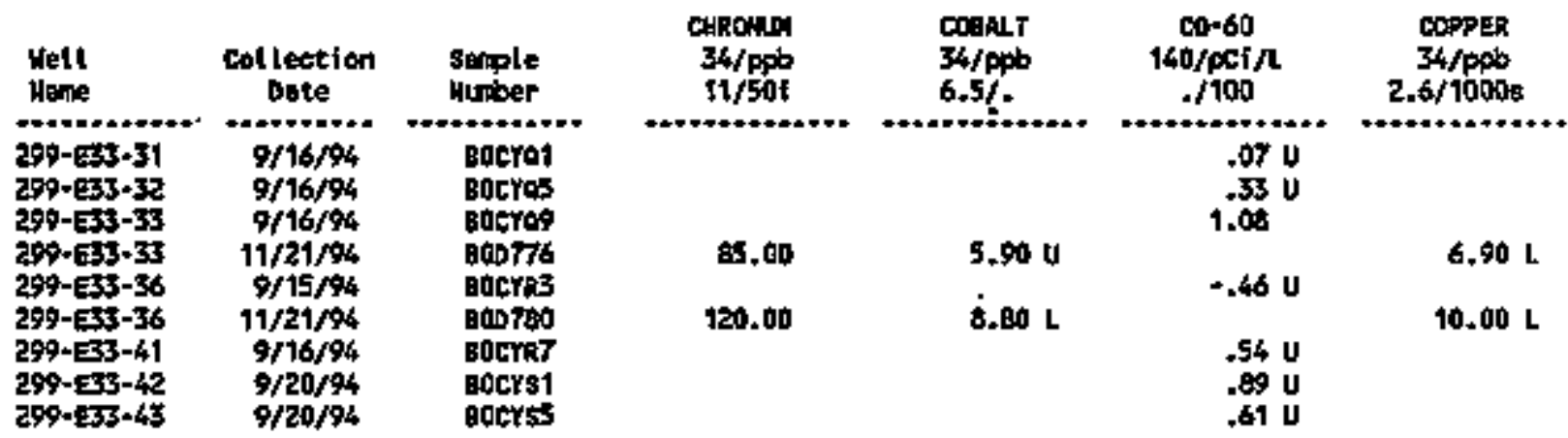

\begin{tabular}{|c|c|c|c|c|c|c|}
\hline $\begin{array}{l}\text { Holl } \\
\text { Hane }\end{array}$ & $\begin{array}{l}\text { Collection } \\
\text { Date }\end{array}$ & $\begin{array}{l}\text { Supplo" } \\
\text { Number }\end{array}$ & $\begin{array}{l}\text { Fuprug } \\
124 / \mathrm{ppb} \\
51 / 14001\end{array}$ & $\begin{array}{c}\text { ALPHA } \\
135 / \mathrm{pCI} / \mathrm{L} \\
. / 15\end{array}$ & $\begin{array}{c}l-129 \\
139 / \mathrm{pCi} / \mathrm{L} \\
. / 1\end{array}$ & $\begin{array}{c}\text { tron } \\
34 / \mathrm{ppb} \\
18 / 300 \mathrm{~s}\end{array}$ \\
\hline $9-\cos -33$ & $\begin{array}{l}11 / 21 / 94 \\
11 / 21 / 44\end{array}$ & $\begin{array}{l}\text { Bont76 } \\
\text { Bog7to }\end{array}$ & $\begin{array}{l}400.00 \\
400.00\end{array}$ & $\begin{array}{r}.56 \mathrm{U} \\
t .11\end{array}$ & $\begin{array}{l}2.76 \\
5.93\end{array}$ & $\begin{array}{l}360 \\
510\end{array}$ \\
\hline
\end{tabular}


Table 15-7. Constituents with at Least One Detected Vaitue for the Single-She11 Tank Waste Management Area B-BY-BX Data for Reporting Period October 1 through Decenber 31, 1994. (sheet 2 of 2)

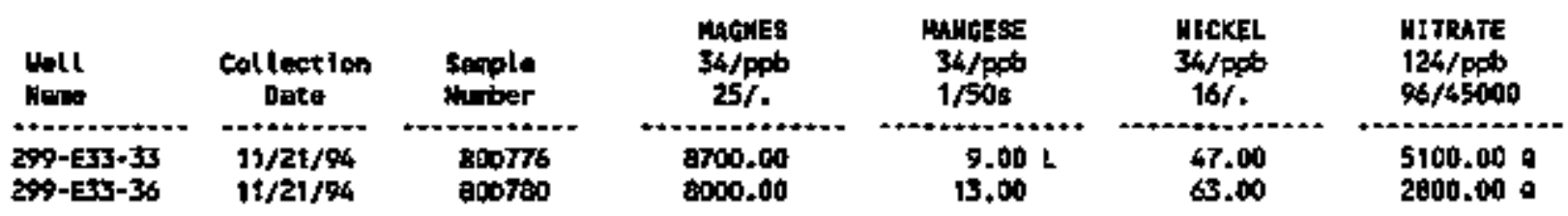

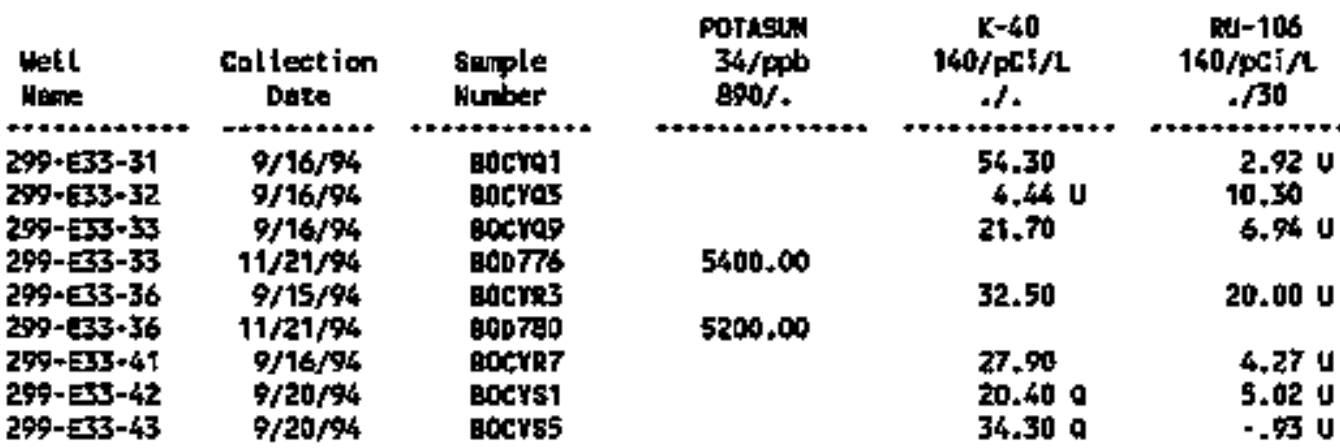

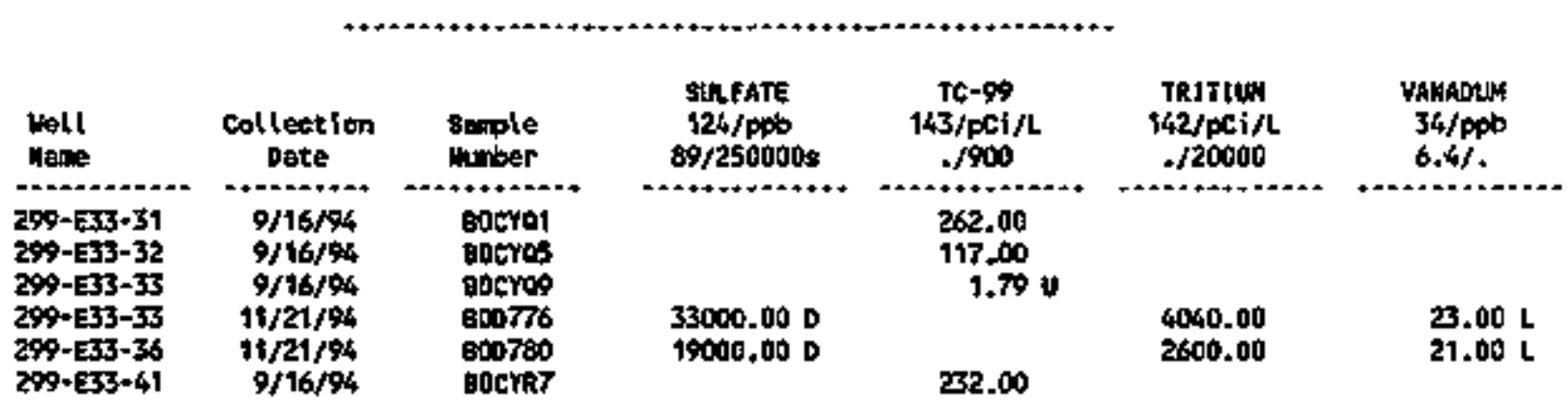

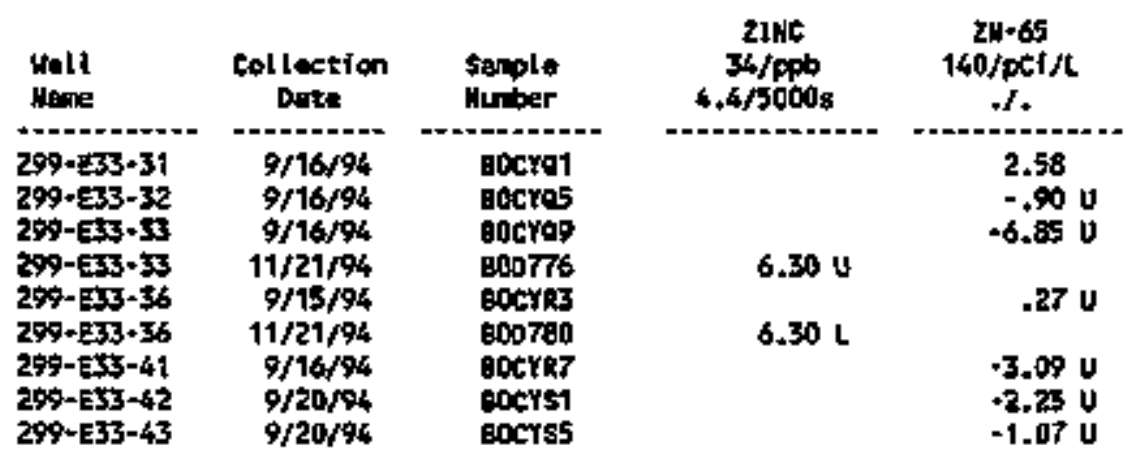


Table 15-8. Contamination Indicator Parameters for the

Single-Shell Tank Waste Management Area B-BY-BX Data for Reporting Period October 1

through December 31, 1994.

\begin{tabular}{|c|c|c|c|c|c|c|c|c|}
\hline Noll & $\underset{\text { Dat: }}{\text { collection }}$ & $\begin{array}{l}\text { sanple } \\
\text { inipur }\end{array}$ & $\begin{array}{l}\text { cond Fieno } \\
\text { 1wino } \\
1 / .\end{array}$ & $\begin{array}{c}\text { COND LAB } \\
\text { Who } \\
1 / .\end{array}$ & $\begin{array}{l}\text { ph fteto } \\
.01 / 6.5-8.55\end{array}$ & $\begin{array}{l}\text { pH LAB } \\
.01 / 6.5-8.58\end{array}$ & $\begin{array}{c}T 00 \\
110 \%\end{array}$ & $\begin{array}{l}10 x \\
p p o b \\
5 \%\end{array}$ \\
\hline $299-153-33$ & $11 / 21 / 44$ & $\begin{array}{l}800776 \\
000777 \\
800778 \\
000779 \\
800790 \\
600781 \\
800782 \\
000783\end{array}$ & $\begin{array}{l}240 \\
240 \\
237 \\
236 \\
255 \\
255 \\
253 \\
255\end{array}$ & 300 & $\begin{array}{l}8.04 \\
8.04 \\
8.02 \\
8.02 \\
6.42 \mathrm{~F} \\
6.46 \mathrm{~F} \\
6.46 \mathrm{~F} \\
6.48 \mathrm{~F}\end{array}$ & $\checkmark$ & $\begin{array}{l}200 \mathrm{~L} \\
300 \mathrm{~L} \\
300 \mathrm{~L} \\
200 \mathrm{~L} \\
300 \mathrm{~L} \\
300 \mathrm{~L} \\
300 \mathrm{~L} \\
501 \mathrm{l}\end{array}$ & $\begin{array}{l}5.0 \mathrm{U} \\
5.0 \mathrm{U} \\
5.0 \mathrm{U} \\
5.0 \mathrm{U} \\
5.0 \mathrm{U} \\
5.0 \mathrm{U} \\
5.0 \mathrm{U} \\
5.0 \mathrm{U}\end{array}$ \\
\hline
\end{tabular}

For explanation of this table, sec section 1.4 of report.

Table 15-9. Constituent List and Summary of Resuits for the Single-She1I Tank Waste Managenent Area C Data for Reporting Period October 1 through December $31,1994$.

SITE EPECIFIC NA OTYER CONST1TUENTS

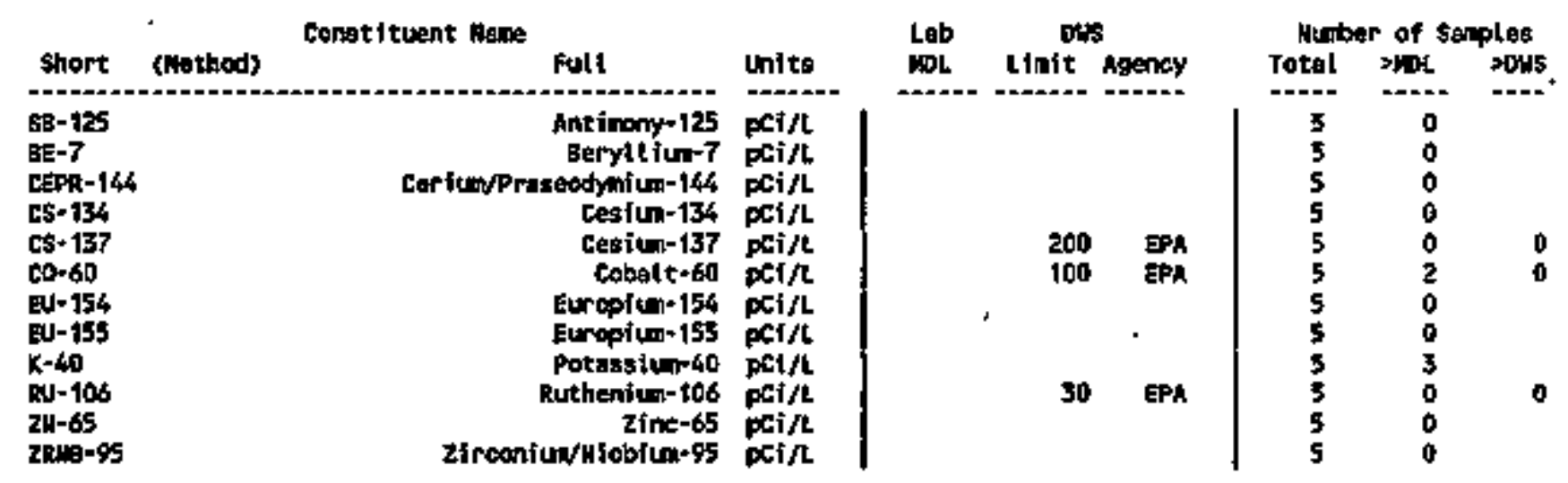

For explanstion of this trble, sut section 1.4 of tuport. 


\section{DOE/RL-94-36-4}

Table 15-10. Constituents with at Least one Detected Value for the Single-Shell Tank Waste Managenent Area C Data for Reporting Period October I through December 31, 1994.

\begin{tabular}{|c|c|c|c|c|}
\hline Lall & $\begin{array}{c}\text { Collection } \\
\text { bate }\end{array}$ & $\begin{array}{l}\text { temple } \\
\text { Humber }\end{array}$ & $\begin{array}{c}c 0-60 \\
140 / p c i / L \\
. / 100\end{array}$ & $\begin{array}{c}K=40 \\
140 / p 6 i / L \\
-/ .\end{array}$ \\
\hline $\begin{array}{l}299-E 27-12 \\
299-E 27-13 \\
299-677-14 \\
299-E 27-15 \\
299-E 27-7\end{array}$ & $\begin{array}{l}9 / 15 / 94 \\
9 / 21 / 94 \\
9 / 21 / 94 \\
9 / 15 / 44 \\
9 / 15 / 94\end{array}$ & $\begin{array}{l}\text { Bocrat } \\
\text { gotras } \\
\text { gocris? } \\
\text { gocres } \\
\text { gocre? }\end{array}$ & $\begin{array}{r}.75 \\
1.47 \\
-2.75 \mathrm{v} \\
. .62 \mathrm{v} \\
.42 \mathrm{v}\end{array}$ & $\begin{array}{l}18.60 \mathrm{v} \\
45.40 \\
4 t .50 \\
28.60 \\
7.75 \mathrm{v}\end{array}$ \\
\hline
\end{tabular}

For explanation of this tibla, see Section 1.4 of report.

Table 15-11. Contamination Indicator Parameters for the Single-Shel] Tank Yaste Management Area C Data for Reporting Period October 1 through Decenber 31, 1994.

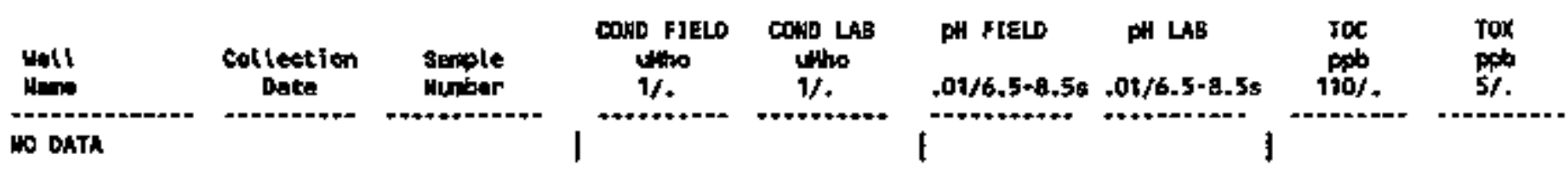

For explanation of this table, ses Section 1.6 of report. 
Table 15-12. Constituent L1st and Summary of Results for the Single-Shell Tank Waste Management Area S-SX Data for Reporting Period October 1 through December 31, 1994.

COATANIMATIOK fMICATOB PARAMETERS

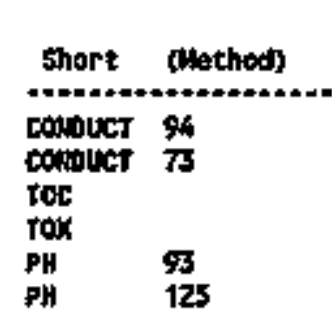

Constituent Mere

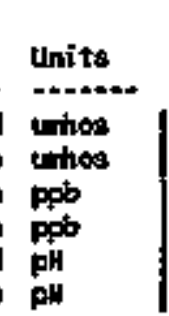

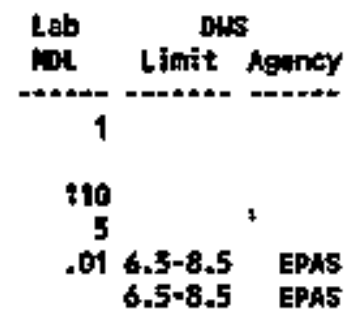

\begin{tabular}{ccc} 
Muribe of somples \\
Total & $>$ >ot & >ous \\
\hline 4 & 4 & \\
1 & 1 & \\
4 & 4 & \\
4 & 3 & \\
4 & 4 & 0 \\
1 & 1 & 0
\end{tabular}

DRIMKING WATER PARANETERS

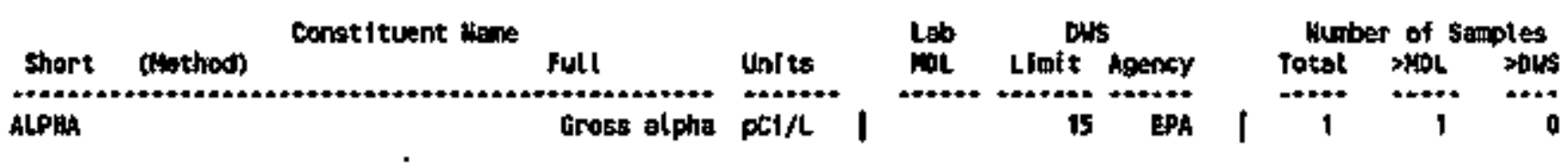

SITE SPECLFIC AHD OTHER CONSTITUENTS

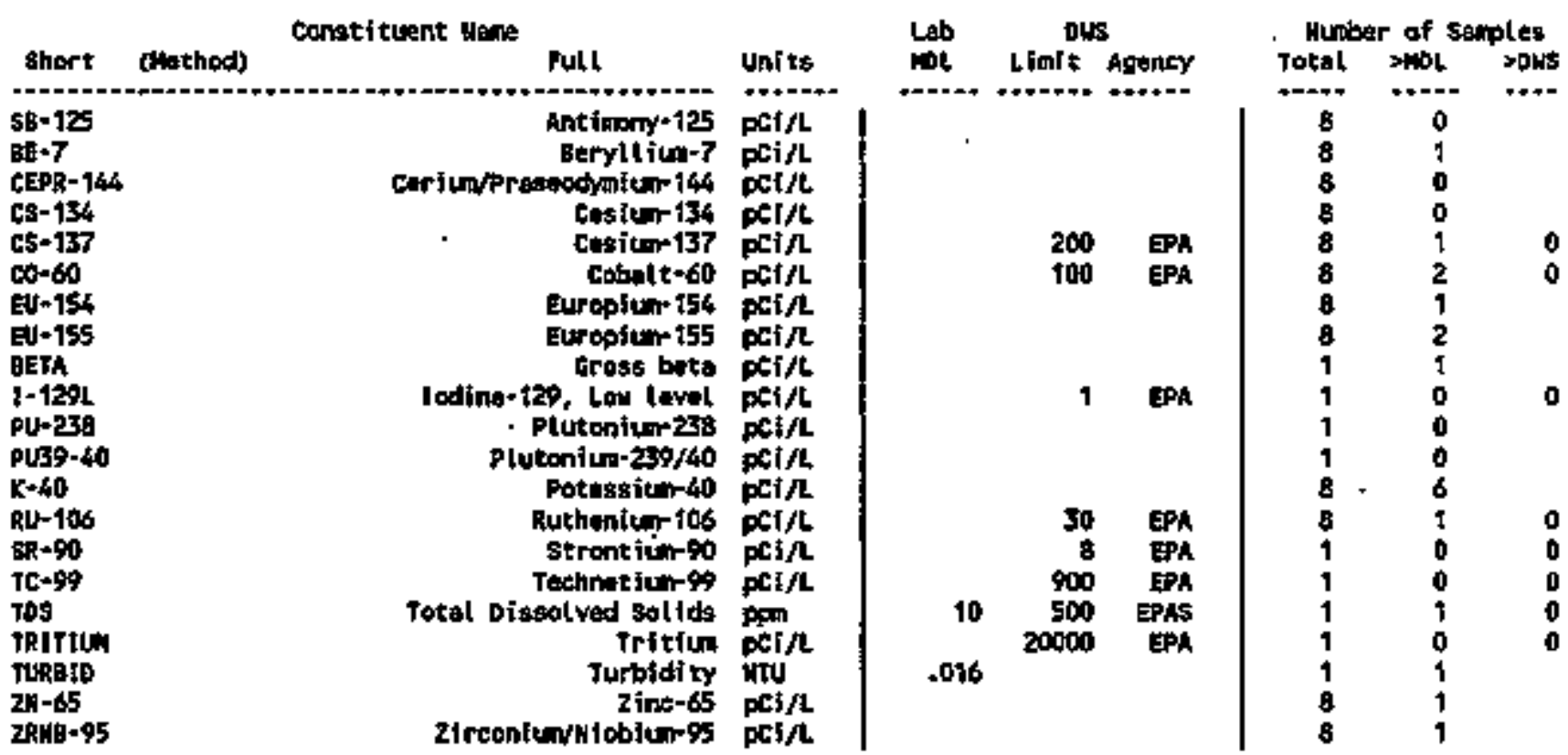

For explantion of this tbibles section 1.4 of report. 
Table 15-13. Const1tuents with at Least One Detected Value for the Single-Shell Tank Waste Management Area S-SX Data for Reporting Period October 1 through December 31, 1994.

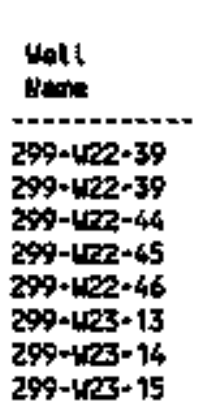

\begin{tabular}{|c|c|}
\hline $\begin{array}{c}\text { Cal leetion } \\
\text { Date }\end{array}$ & $\begin{array}{l}\text { Sample } \\
\text { Hutitar }\end{array}$ \\
\hline $\begin{array}{c}9 / 20 / 94 \\
9 / 20 / 94 \\
10 / 04 / 94 \\
9 / 20 / 94 \\
9 / 20 / 94 \\
9 / 20 / 94 \\
9 / 20 / 94 \\
9 / 20 / 94\end{array}$ & 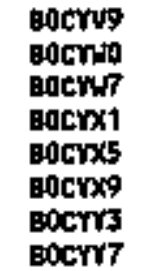 \\
\hline
\end{tabular}

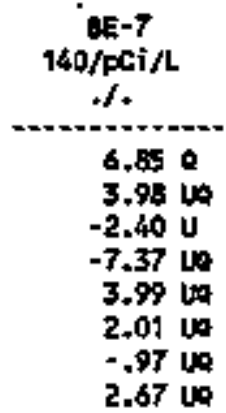
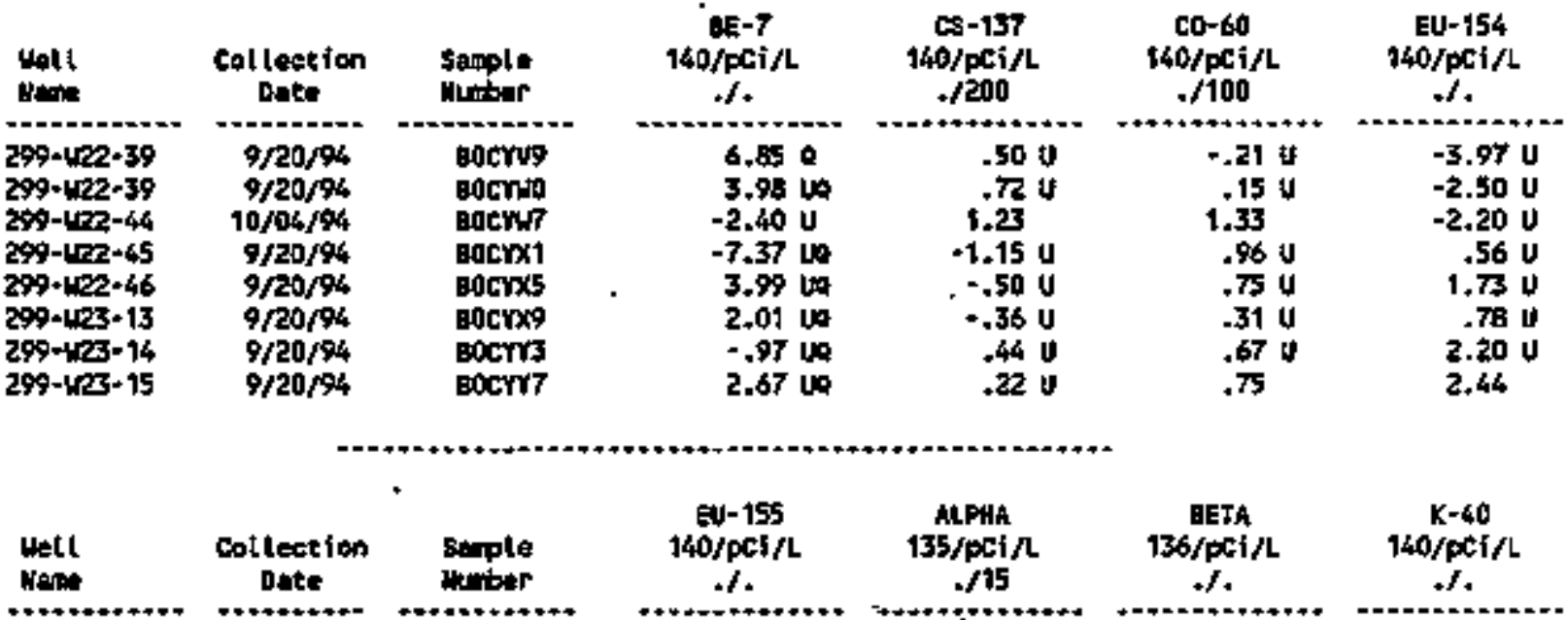

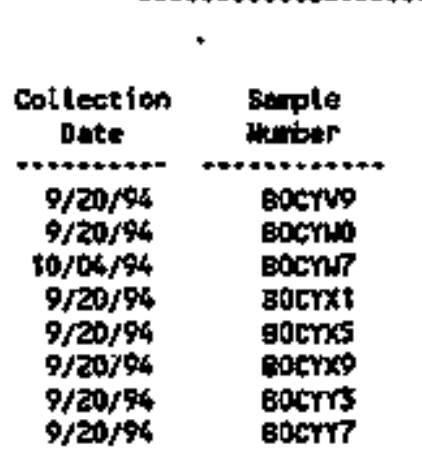
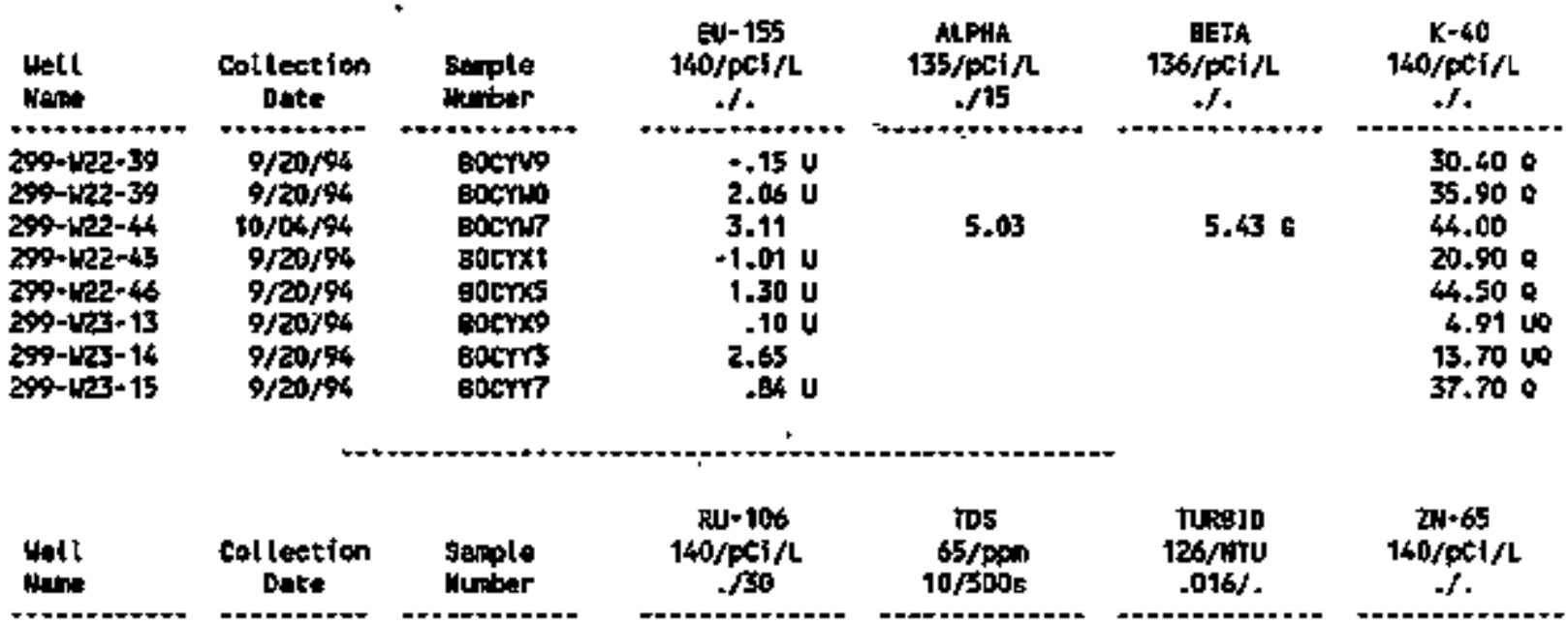

\begin{tabular}{|c|c|}
\hline $\begin{array}{c}\text { Col leetion } \\
\text { Date }\end{array}$ & $\begin{array}{l}\text { Serple } \\
\text { Murber }\end{array}$ \\
\hline $\begin{array}{r}9 / 20 / 94 \\
9 / 20 / 94 \\
10 / 04 / 94 \\
9 / 20 / 94 \\
9 / 20 / 94 \\
9 / 20 / 94 \\
9 / 20 / 94 \\
9 / 20 / 94\end{array}$ & 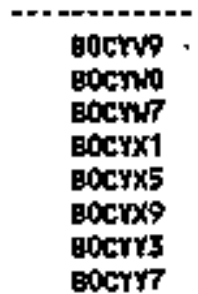 \\
\hline
\end{tabular}
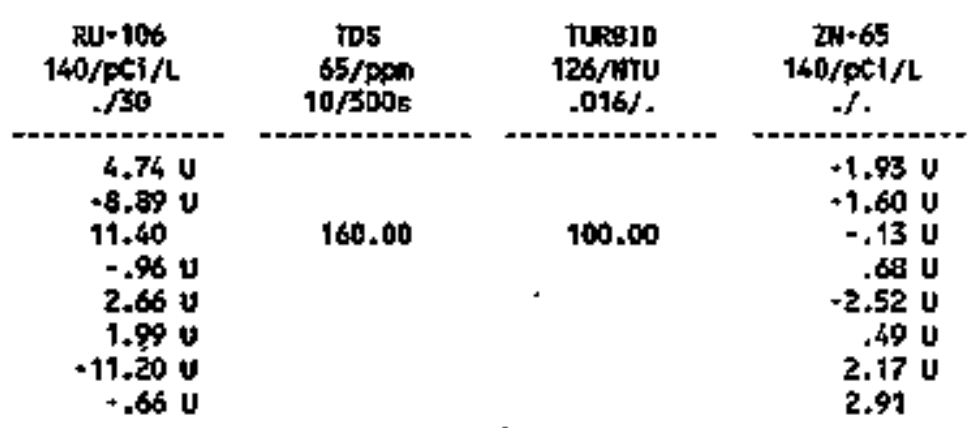

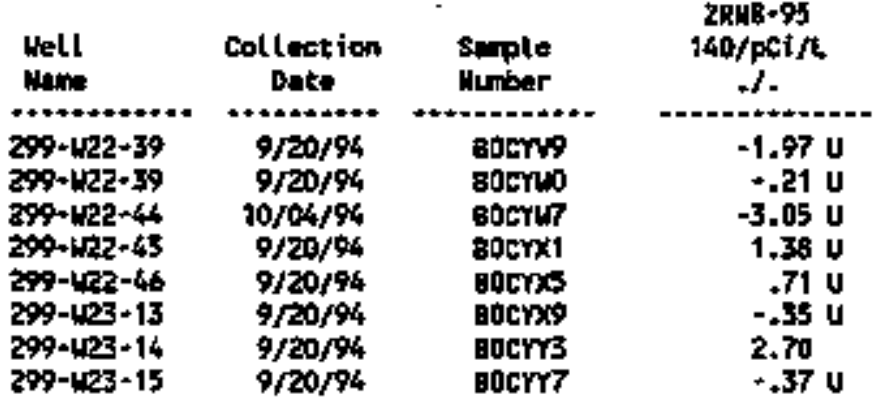


Table 15-14. Contamination Indicator Parameters for the Single-Shell Tank Waste Managenent Area 5-5X Data for Reporting Period October 1 through December 31, 1994.

\begin{tabular}{|c|c|c|c|c|c|c|c|c|}
\hline $\begin{array}{l}\text { Nell } \\
\text { Mane }\end{array}$ & $\begin{array}{l}\text { Collection } \\
\text { Oate }\end{array}$ & $\begin{array}{l}\text { Semple } \\
\text { Munber }\end{array}$ & $\begin{array}{c}\text { Conth FIELD } \\
\text { who } \\
1 \%\end{array}$ & $\begin{array}{c}\text { Cowd LAB } \\
\text { who } \\
1 / .\end{array}$ & $\begin{array}{l}\text { PH FIELD } \\
.01 / 6.5-8.5 \$\end{array}$ & $\begin{array}{l}\text { PH LAB } \\
.01 / 6.5-8.58\end{array}$ & $\begin{array}{c}\mathrm{JOC} \\
\mathrm{ppb} \\
110 / .\end{array}$ & $\begin{array}{l}\text { Tox } \\
\text { ppb } \\
5 / .\end{array}$ \\
\hline $0-1222-44$ & $10 / 04 / 94$ & $\begin{array}{l}\text { Boctrus } \\
\text { Bocitus } \\
\text { Botrus } \\
\text { Bostrxo }\end{array}$ & $\begin{array}{r}196 \\
.196 \\
195 \\
195\end{array}$ & 200 & $\begin{array}{l}7.80 \\
7.82 \\
7.80 \\
7.80\end{array}$ & 8.00 & $\begin{array}{l}3001 \\
3001 \\
3001 \\
3001\end{array}$ & $\begin{array}{l}6.5 \\
5.5 \\
6.1 \\
5.00\end{array}$ \\
\hline
\end{tabular}

Far explanation of this table, sta section 1.4 of raport. 
Table 15-15. Constituent List and Summary of Results for the Single-SheTl Tank Haste Managenent Area T Data for

Reporting Period October 1 through

December 31, 1994.

(sheet 1 of 3 )

CONTAMIMATTOOS TMOICATOR PARAMETERS

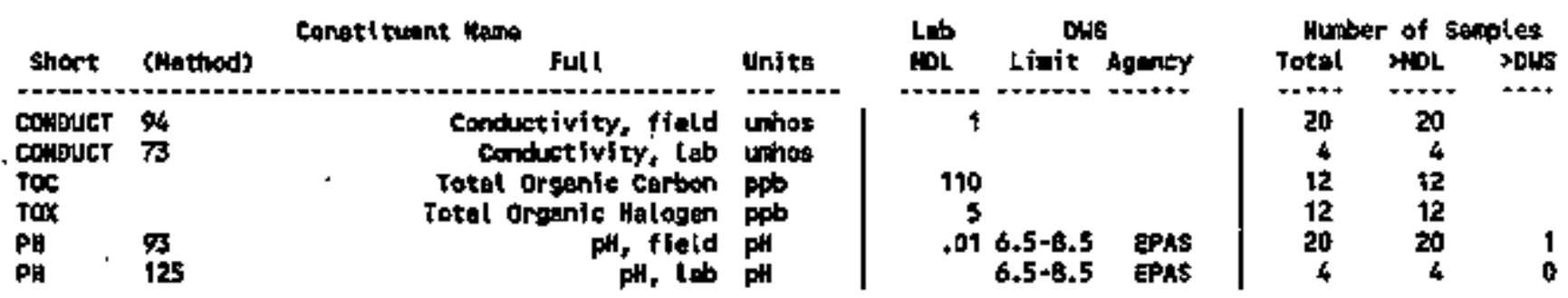

DRINXIHC WATER PARANETERS

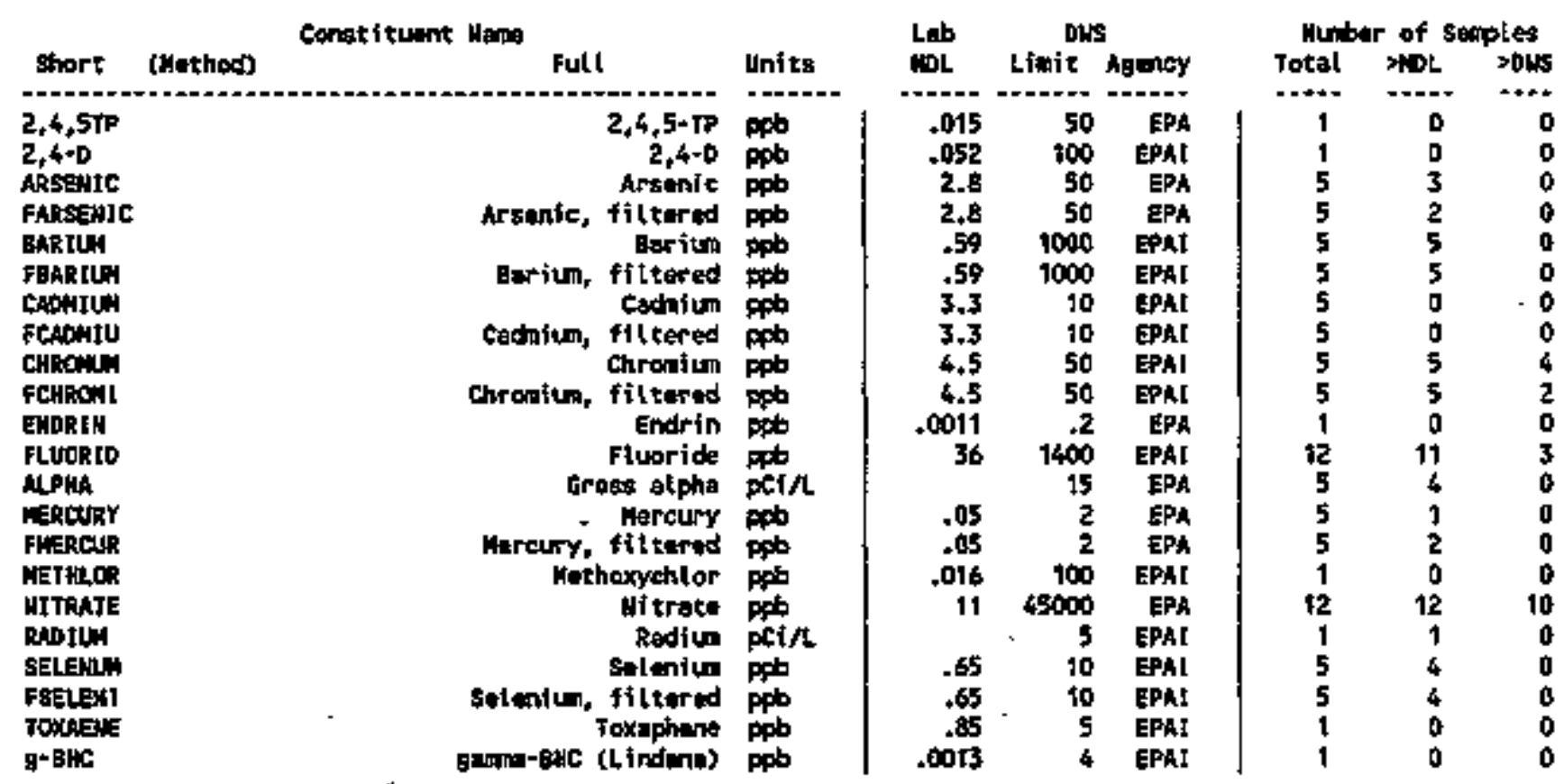

GROABDUSER CUALITY PARANETERS

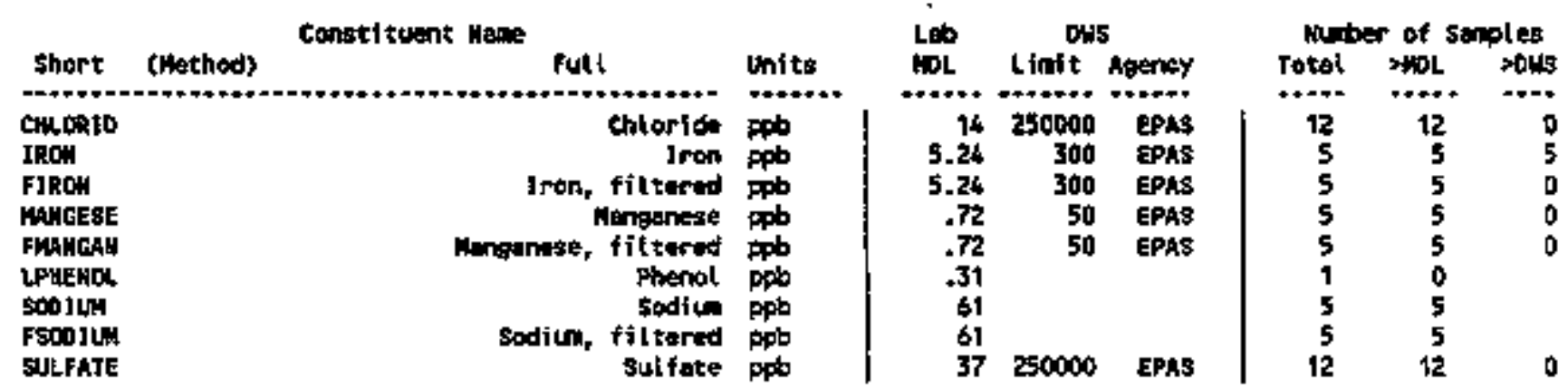


Table 15-15. Constituent List and Sumpary of Results for the Single-She11 Tank Waste Maragement Area T Data for Reporting Period October 1 through December 31, 1994.

(sheet 2 of 3 )

SITE SPECIFIC AND OTHER ConsTITUENTS

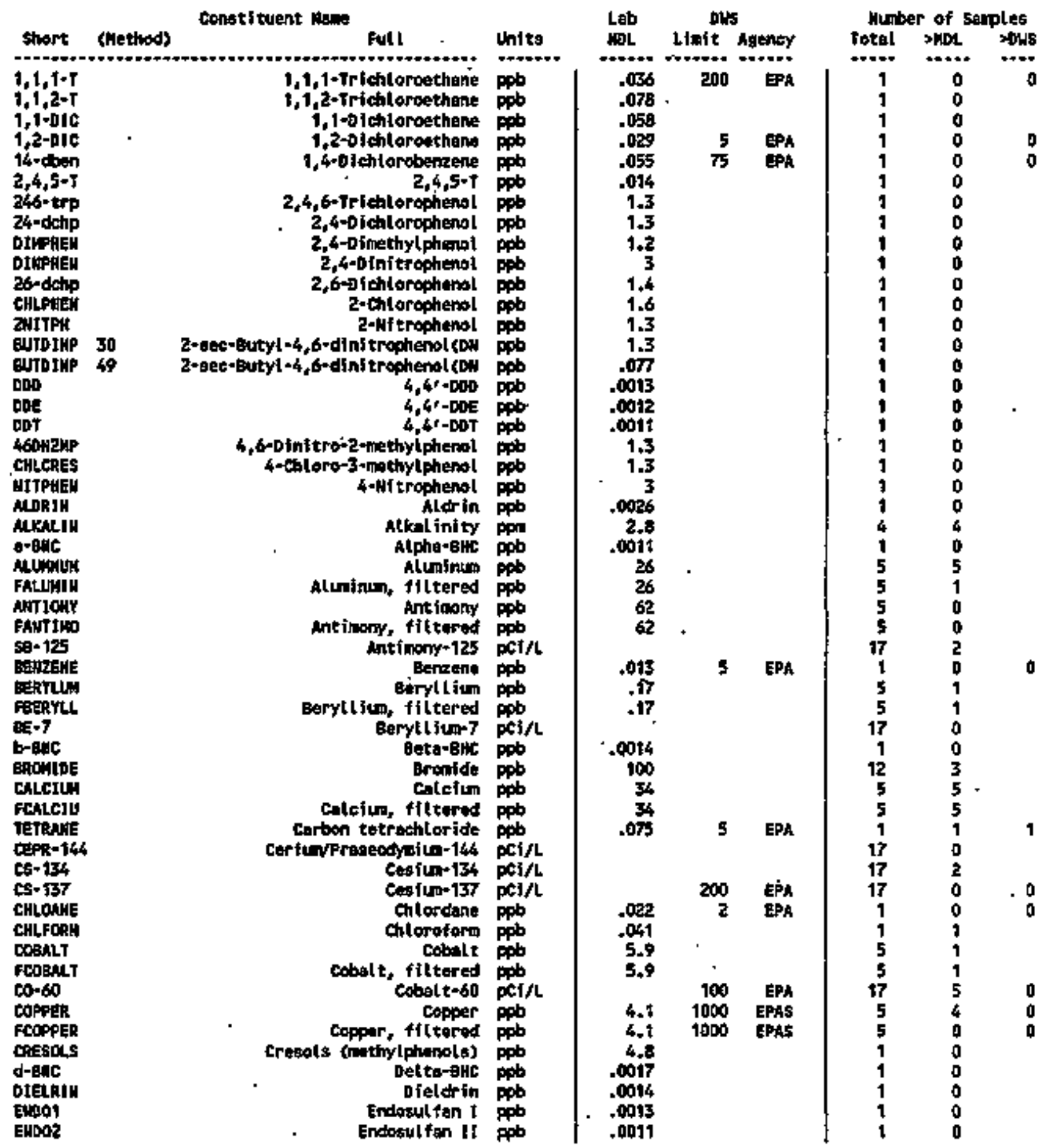


Table 15-15. Constftuent List and Summary of Results for the Single-Shell Tank Waste Hanagement Area $T$ Data for

Reporting Perjod October 1 through

December 31, 1994 .

(shest 3 of 3 ).

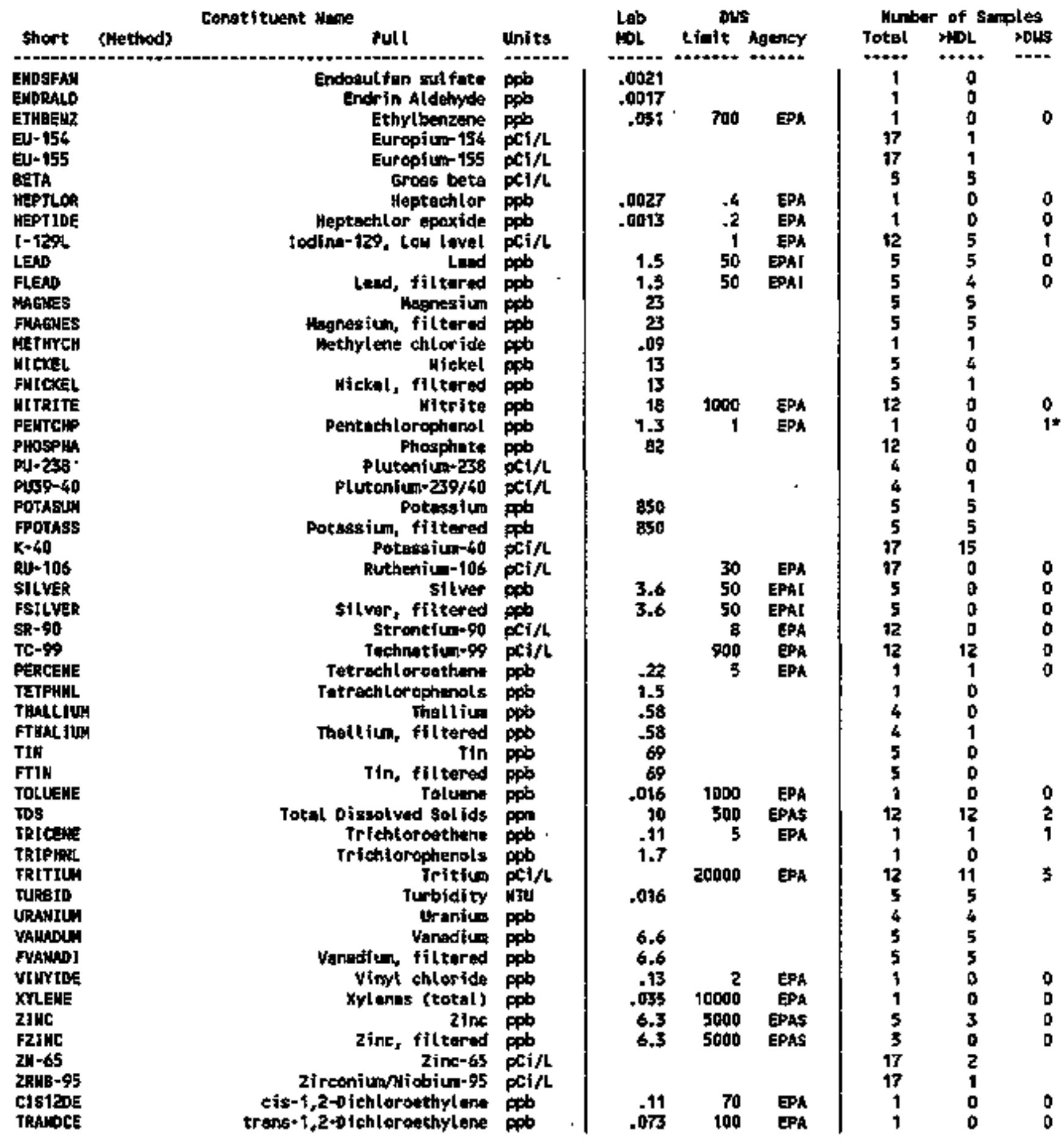

Far explanation of this table, cot section 1.4 of repart. 
Tab7e 15-16. Constituents with at Least One Detected Value for the Single-She11 Tank Waste Management Area T Data for Reporting Perjod October 1 through

December 31, 1994.

(sheet 1 of 9 )

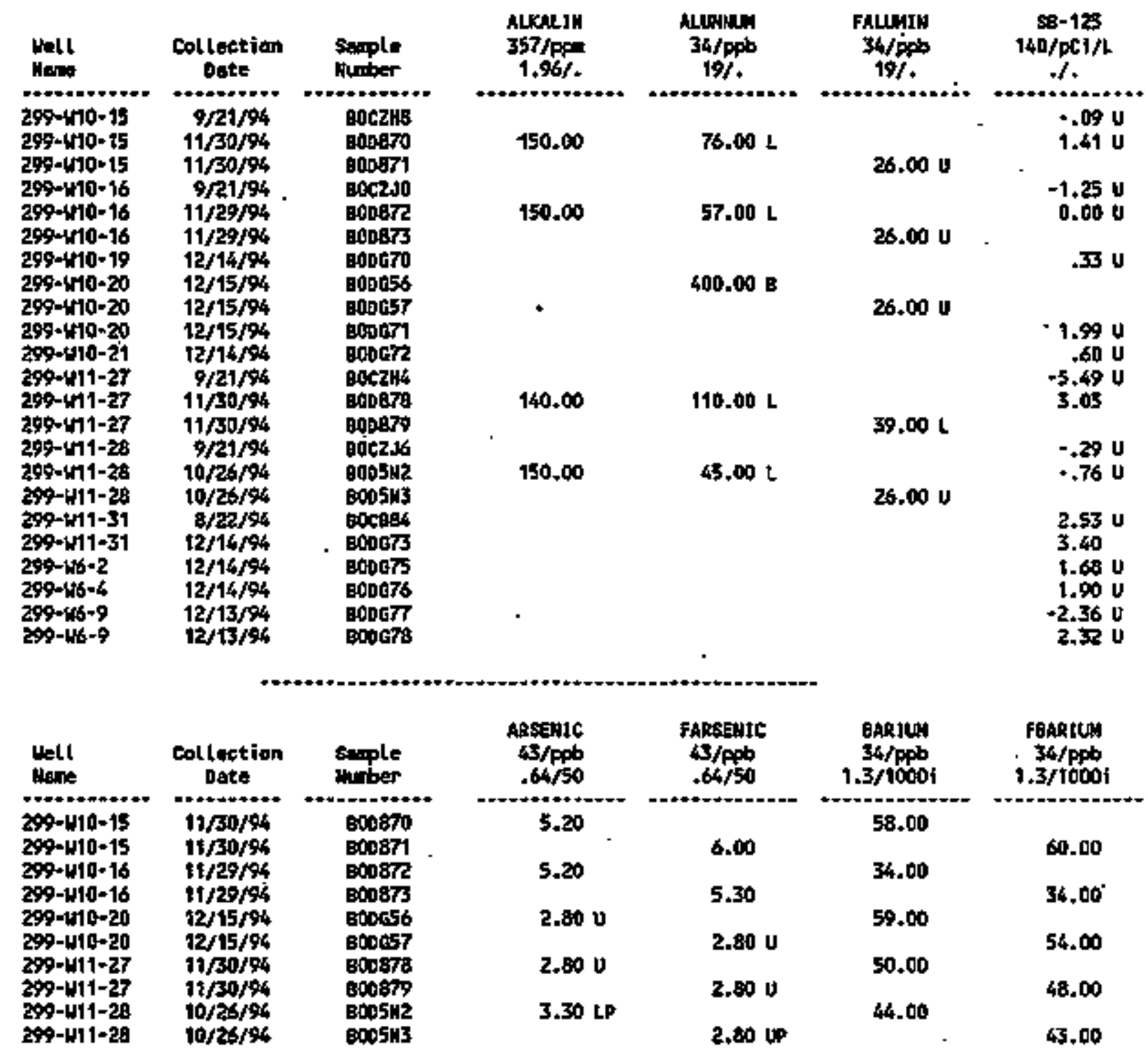


Table 15-16. Constituents with at Least One Detected Value for the Single-Shel1 Tank Maste Management Area T Data for Reporting Period October 1 through

December 31, 1994.

(sheet 2 of 9 )

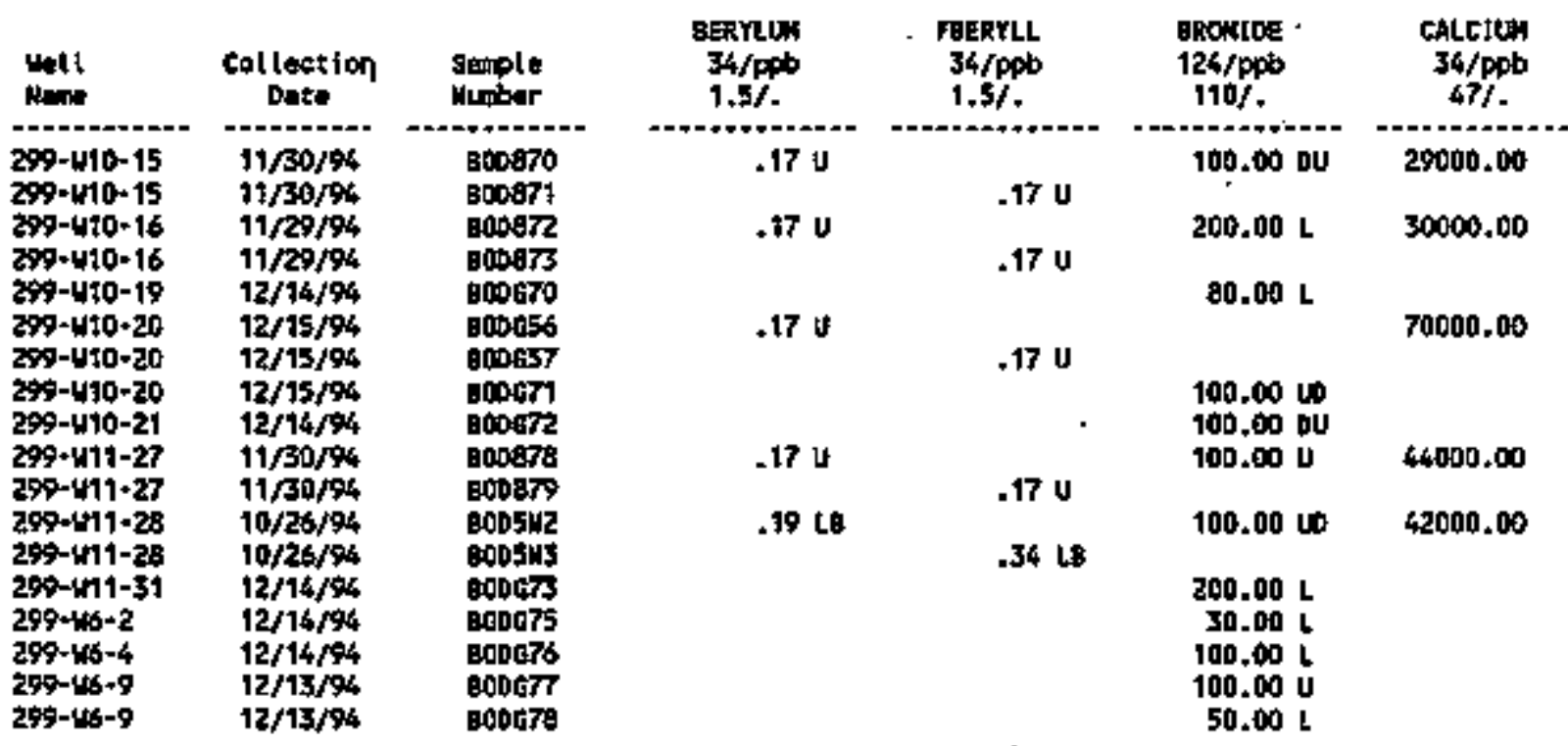

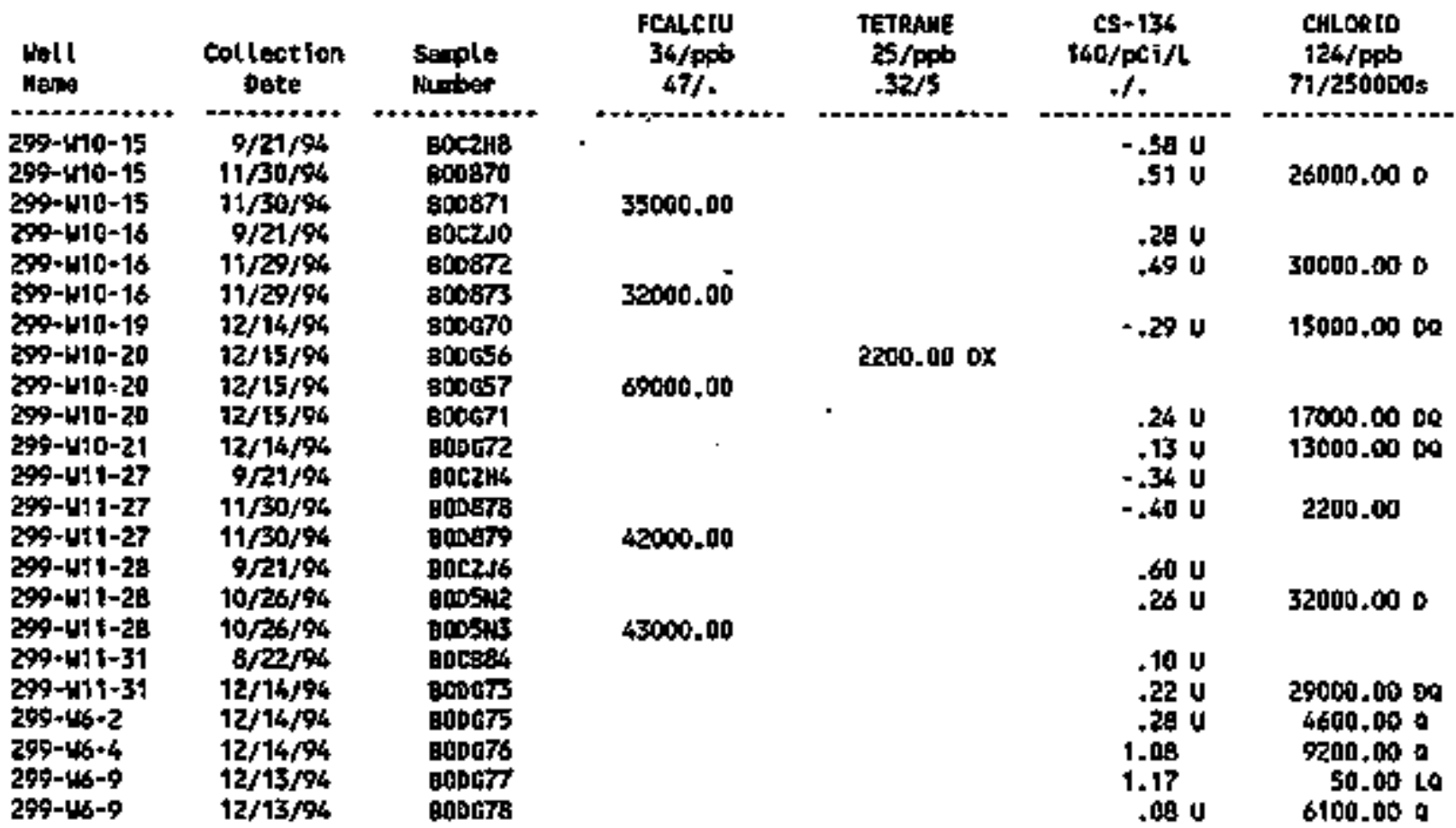


Table 15-16. Constituents with at Least One Betected Value for the Single-Shel1 Tank . Waste Management Area T Data for

Reporting Period October 1 through

December 31, 1994.

(sheet 3 of 9)

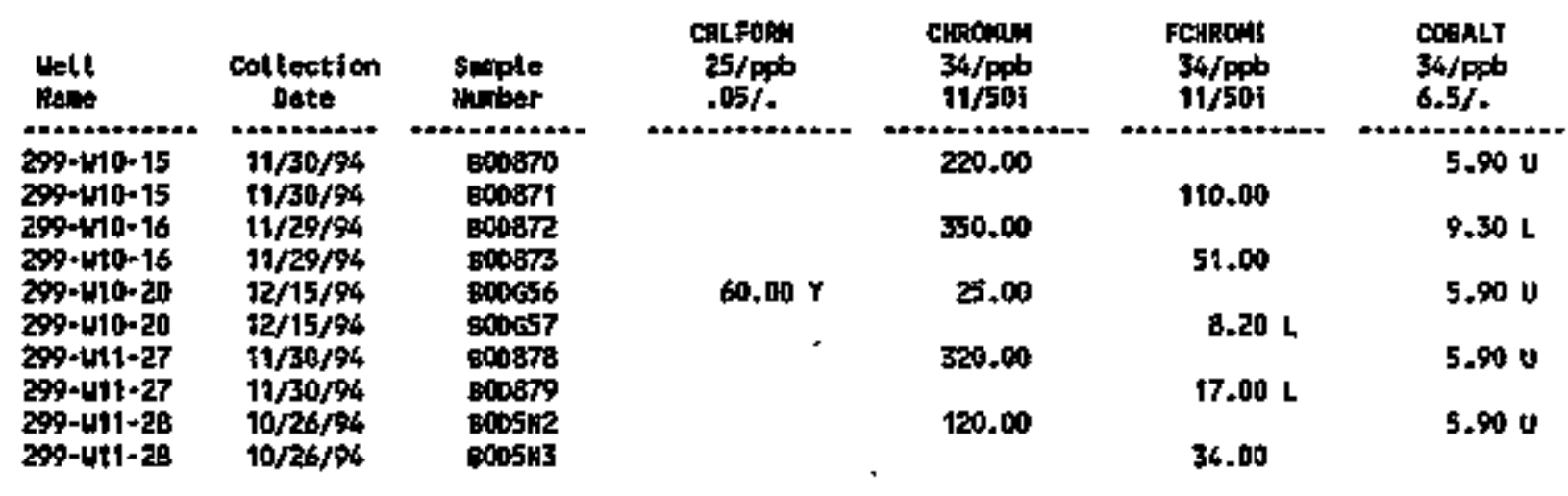

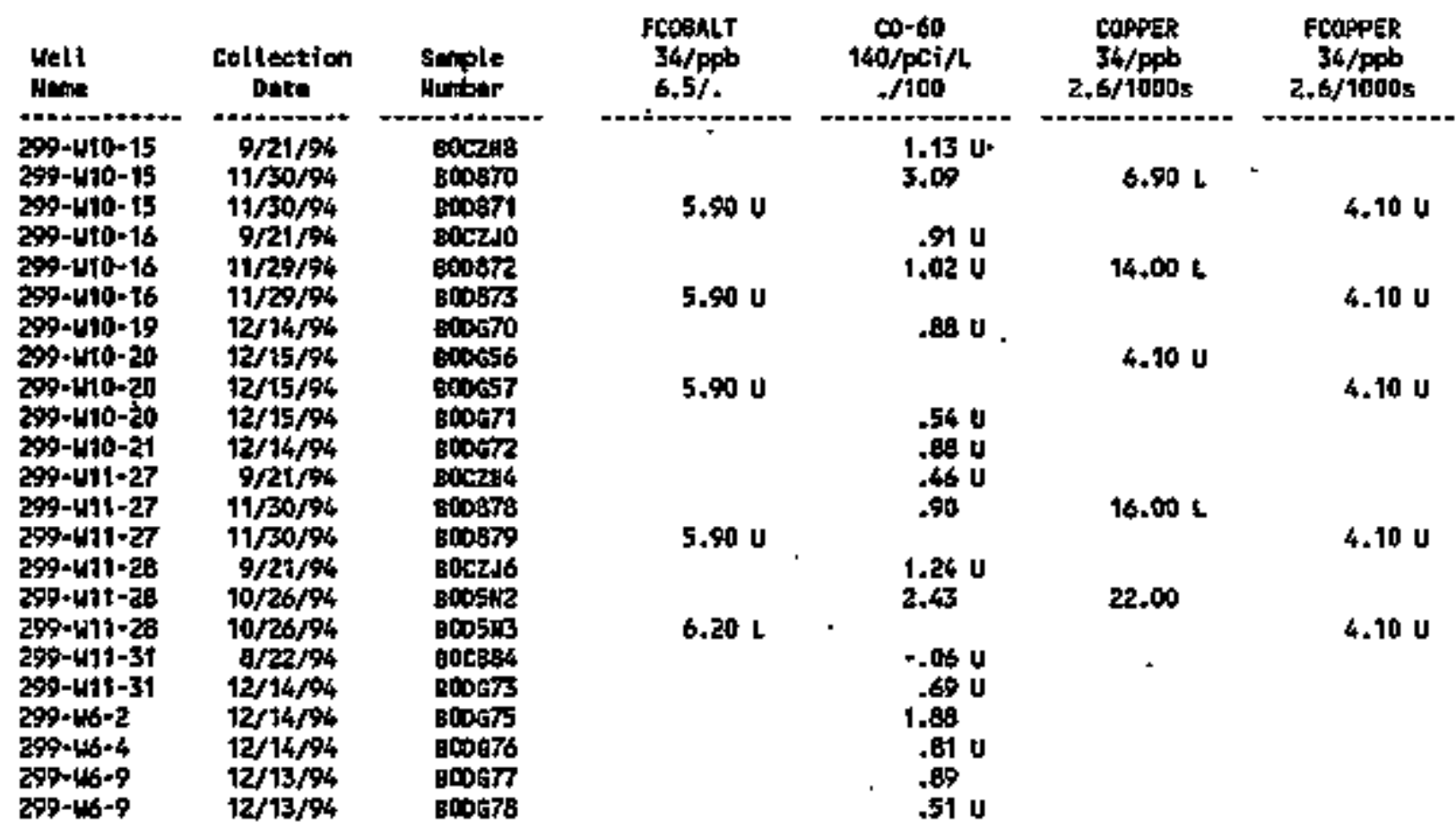


Table 15-16. Constituents wtth at Least One Detected Value for the Single-Shel] Tank Waste Management Area T Data for Reporting Period October 1 through

December 31, 1994.

(sheet 4 of 9 )

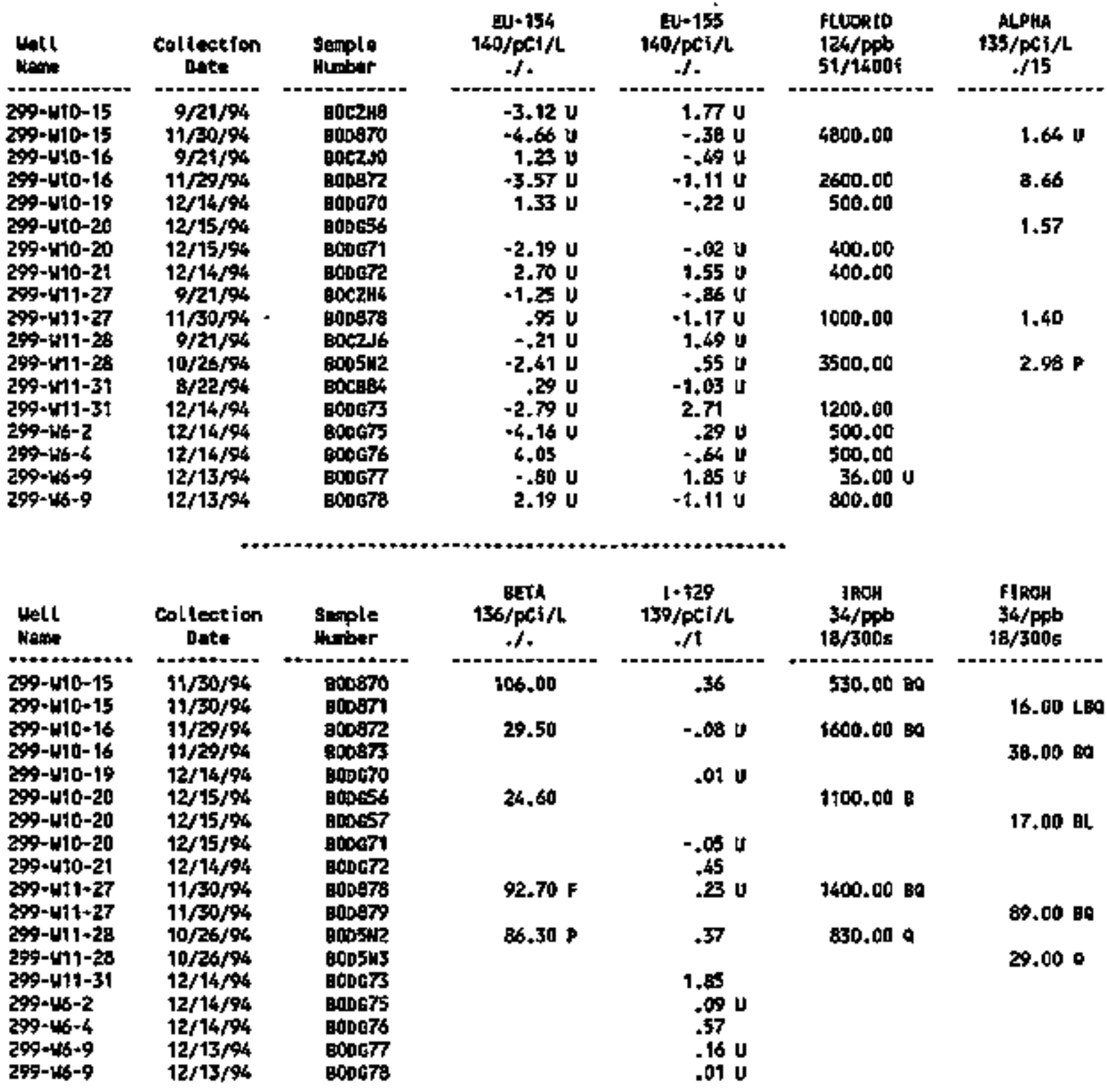


Table 15-16. Constituents with at Least One Detected Value for the Single-Shell Tank Haste Management Area T Data for Reporting Period October 1 through Decenber 31, 1994. . (sheet 5 of 9 )

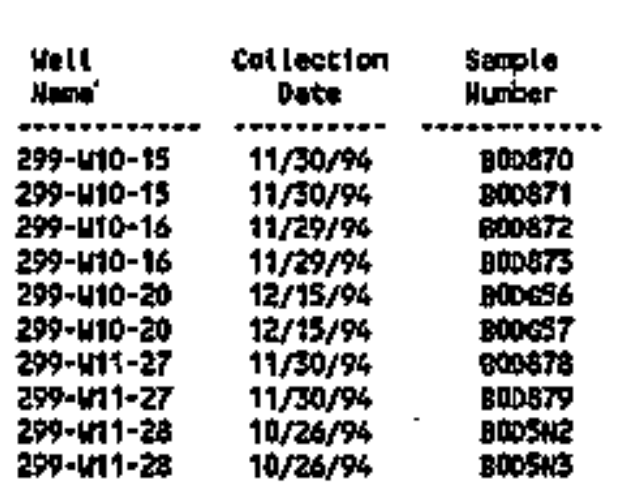

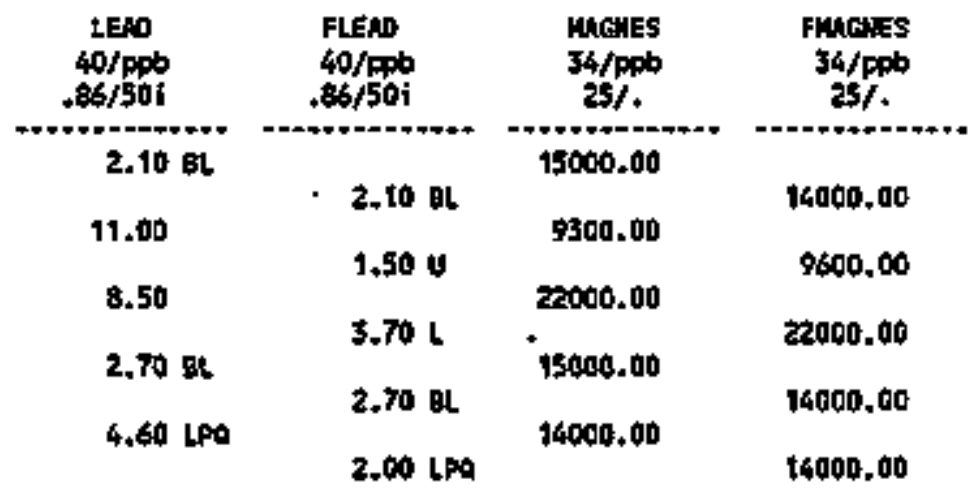

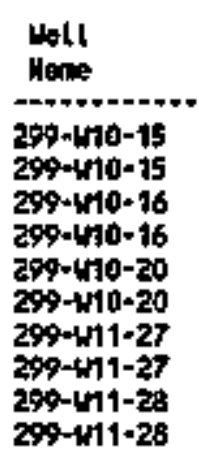

\begin{tabular}{|c|c|}
\hline $\begin{array}{l}\text { Coll lagtion } \\
\text { Date }\end{array}$ & $\begin{array}{l}\text { Sappla } \\
\text { Hunber }\end{array}$ \\
\hline $\begin{array}{l}11 / 30 / 94 \\
11 / 30 / 94 \\
11 / 29 / 94 \\
11 / 29 / 94 \\
12 / 15 / 94 \\
12 / 15 / 94 \\
11 / 30 / 94 \\
11 / 30 / 94 \\
10 / 26 / 94 \\
10 / 26 / 94\end{array}$ & 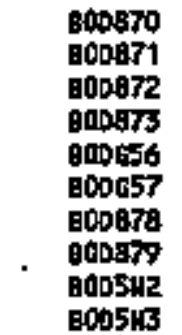 \\
\hline
\end{tabular}

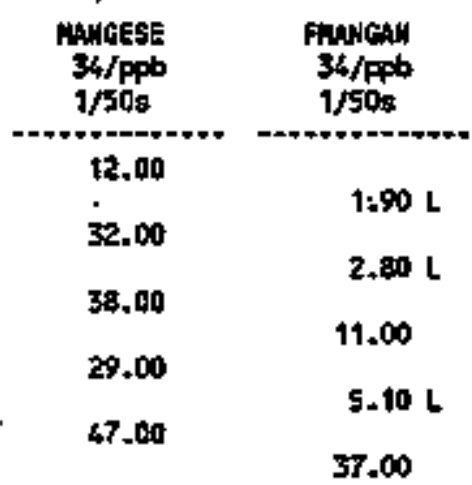

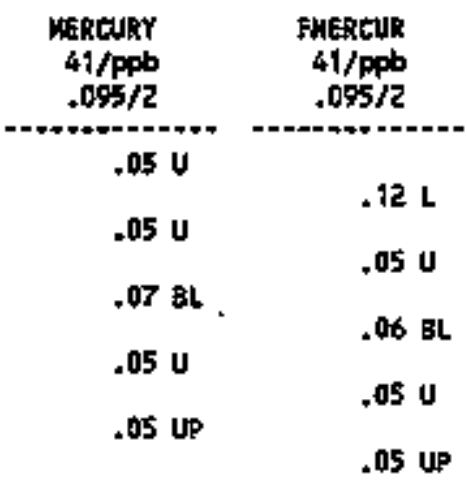

Whan

299-110-15

290-110-15

$2 \%+10-16$

299-4to-16

$299-10-19$

299-w10-20

$290-110-20$

$209-410-20$

299-410-21

299-411-27

299-U11-27

259-41f-28

298-411 -28

299-U1 1-31

299-145-2

258-16-4

299-116-9

259-115-9

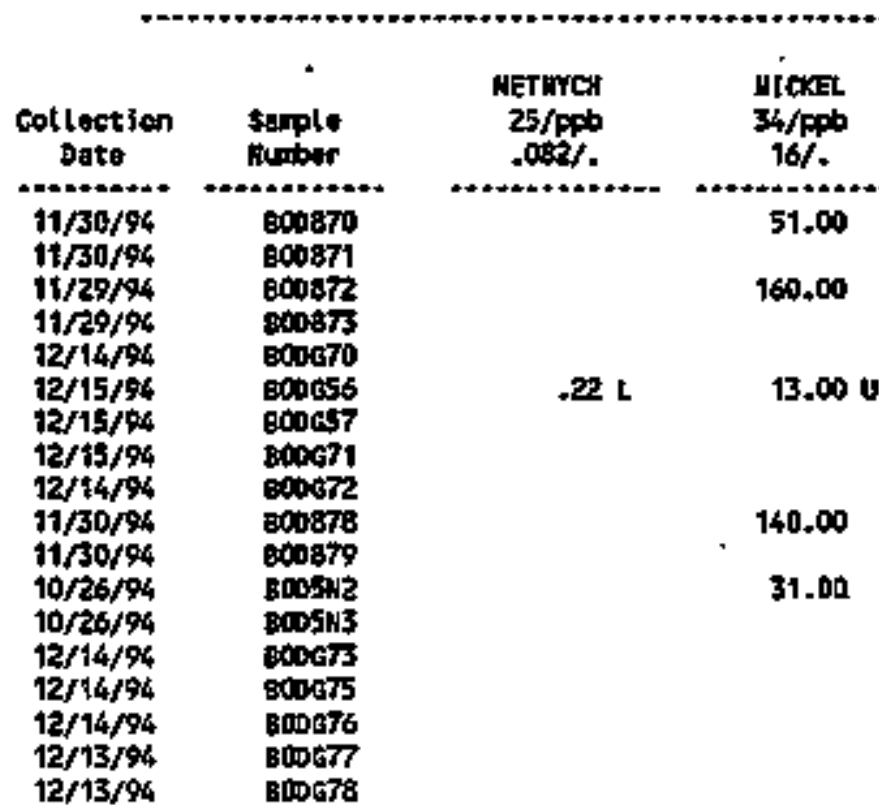

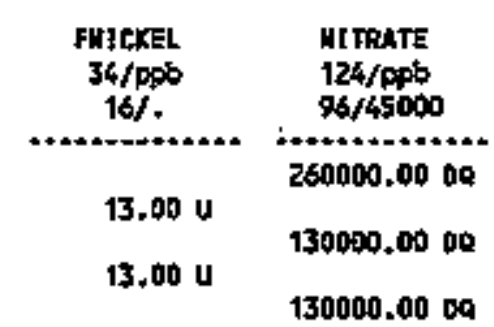

19,001

130000,0000 150000.0000

6300,000

210000.000

120000,0000 52000.00 DO 97000.0000 600.000 69000.00 09 
Table 15-16. Constituents with at Least One Detected Value for the Single-Shell Tank Waste Managenent Area T Data for Reporting Period October I through

December $31,1994$.

(sheet 6 of 9)

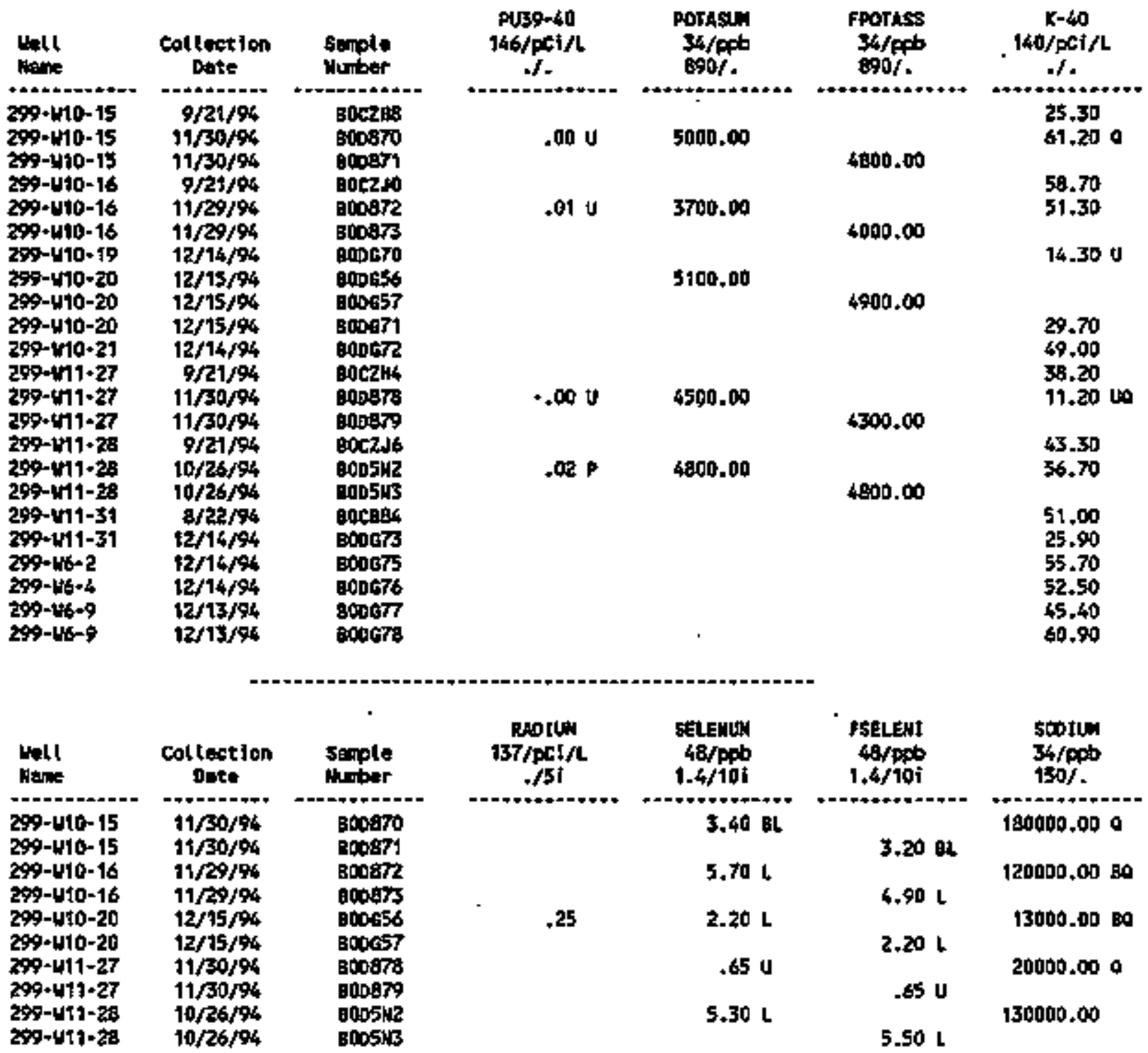




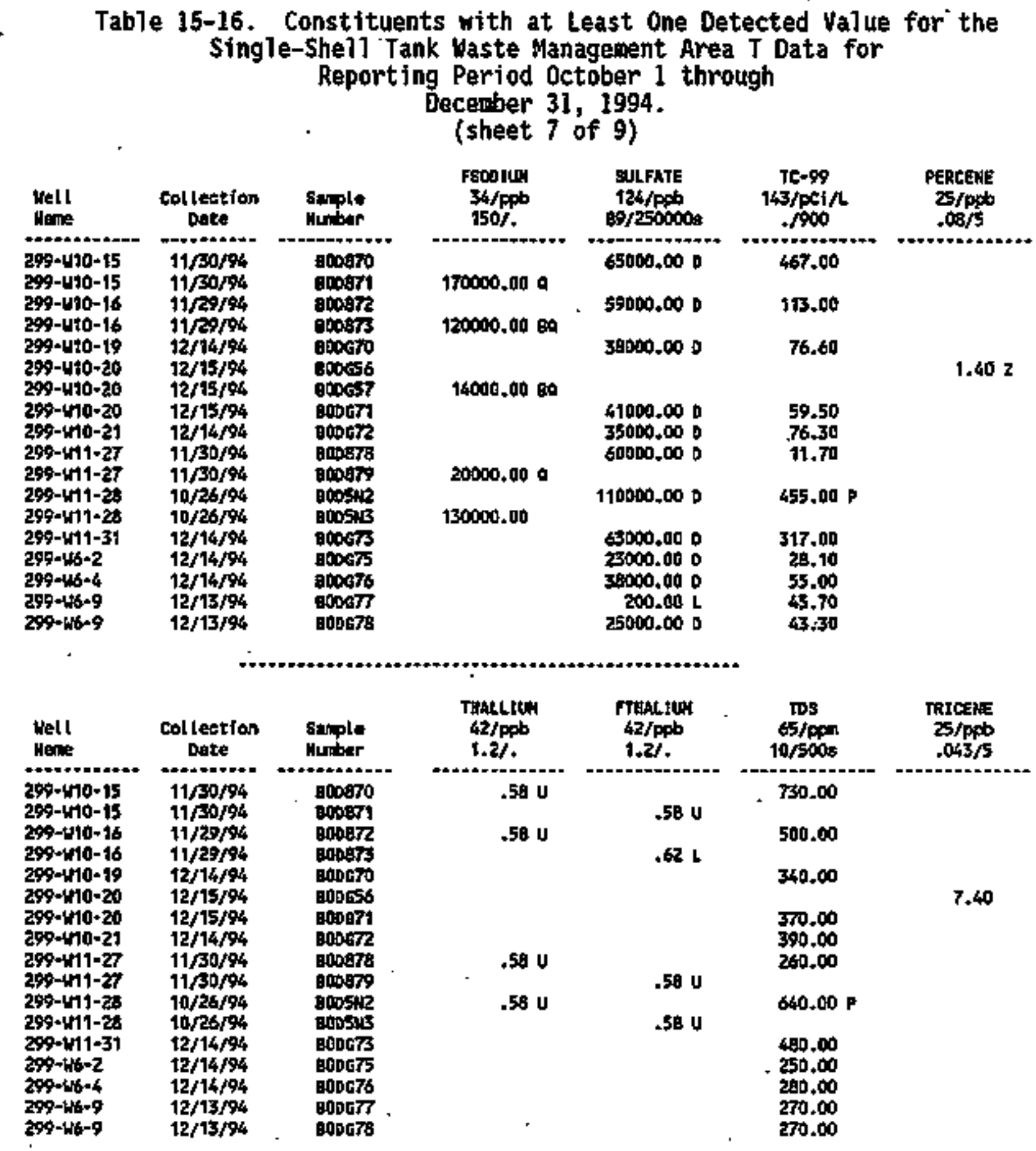


Table 15-16. Constituents with at Least One Detected Value for the Single-Shell Tank Waste Management Area T Data for Reporting Period October 1 through December 31, 1994.

(sheet 8 of 9)

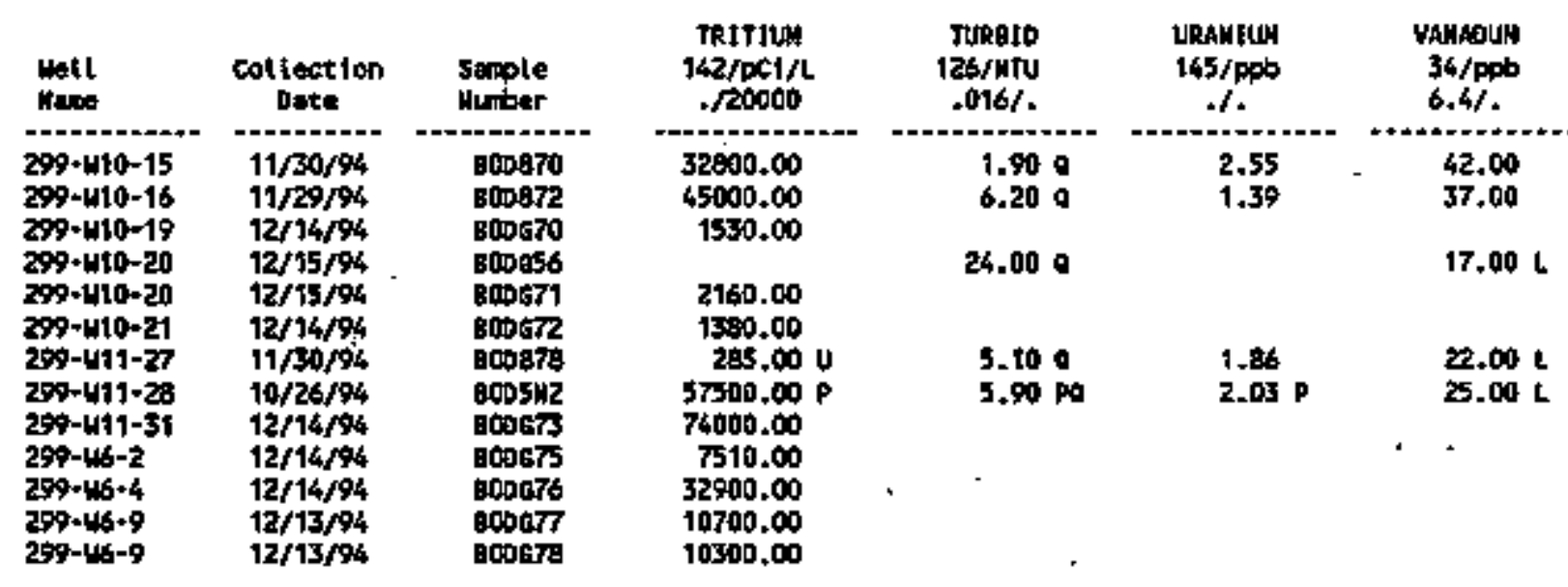

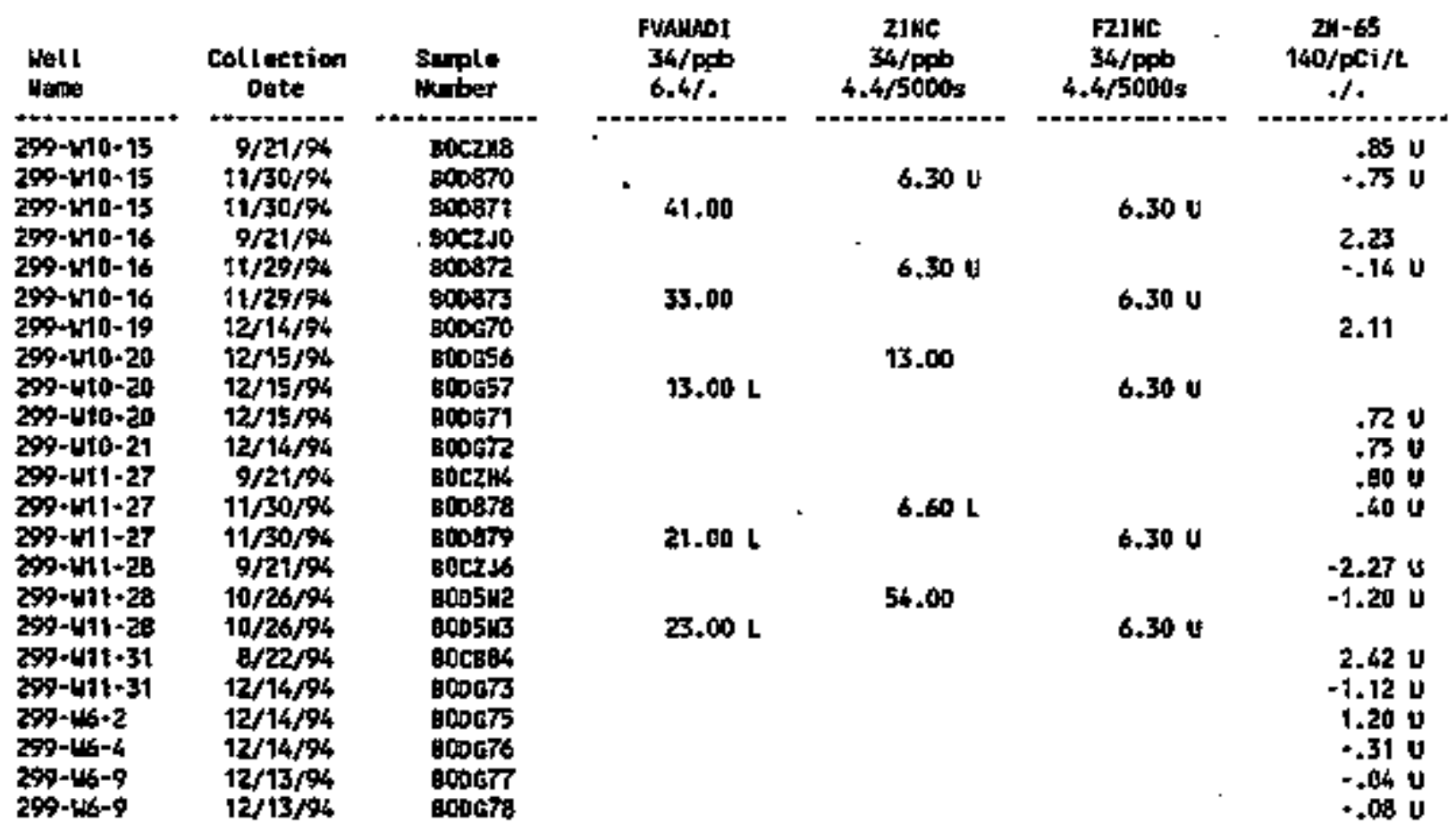


Table 15-16. Constituents with at Least One Detected Value for the Single-Shell Tank Waste Managenent Area T Data for Reporting Period October 1 through December 31, 1994. (sheet 9 of 9 )

\begin{tabular}{|c|c|c|c|}
\hline 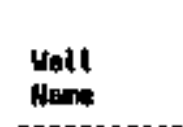 & $\begin{array}{c}\text { Collection } \\
\text { date }\end{array}$ & $\begin{array}{l}\text { sample } \\
\text { luxptar }\end{array}$ & $\begin{array}{c}2 \mathrm{RHQ}, \mathrm{BS} \\
140 / \mathrm{pCH} / \mathrm{L} \\
.\end{array}$ \\
\hline 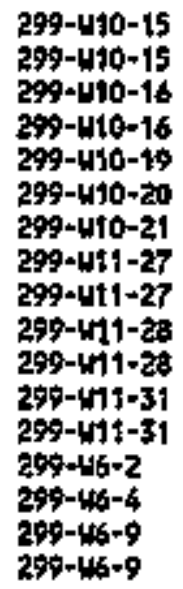 & $\begin{array}{r}9 / 21 / 94 \\
11 / 90 / 94 \\
9 / 21 / 94 \\
11 / 29 / 94 \\
12 / 14 / 94 \\
12 / 15 / 94 \\
12 / 14 / 94 \\
9 / 21 / 94 \\
11 / 30 / 94 \\
9 / 21 / 94 \\
10 / 26 / 94 \\
8 / 22 / 94 \\
12 / 14 / 94 \\
12 / 14 / 94 \\
12 / 14 / 94 \\
12 / 13 / 94 \\
12 / 13 / 94\end{array}$ & 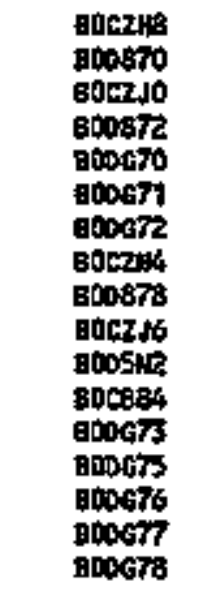 & $\begin{array}{r}-.90 \mathrm{U} \\
1.70 \mathrm{U} \\
1.75 \mathrm{U} \\
-1.15 \mathrm{U} \\
-2.43 \mathrm{U} \\
2.45 \mathrm{U} \\
-1.13 \mathrm{U} \\
-1.02 \mathrm{U} \\
.49 \mathrm{U} \\
.07 \mathrm{U} \\
2.11 \mathrm{U} \\
1.51 \mathrm{U} \\
.26 \mathrm{U} \\
.32 \mathrm{U} \\
-2.00 \mathrm{U} \\
-.47 \mathrm{U} \\
-.10 \mathrm{U}\end{array}$ \\
\hline
\end{tabular}

For explanstion of this teble, 394 section 1.4 of report. 
Table 15-17. Contamination Indicator Parameters for the S1ngle-Shell Tank Waste Management Area T Data for Reporting Period October 1 through December 31, 1994.

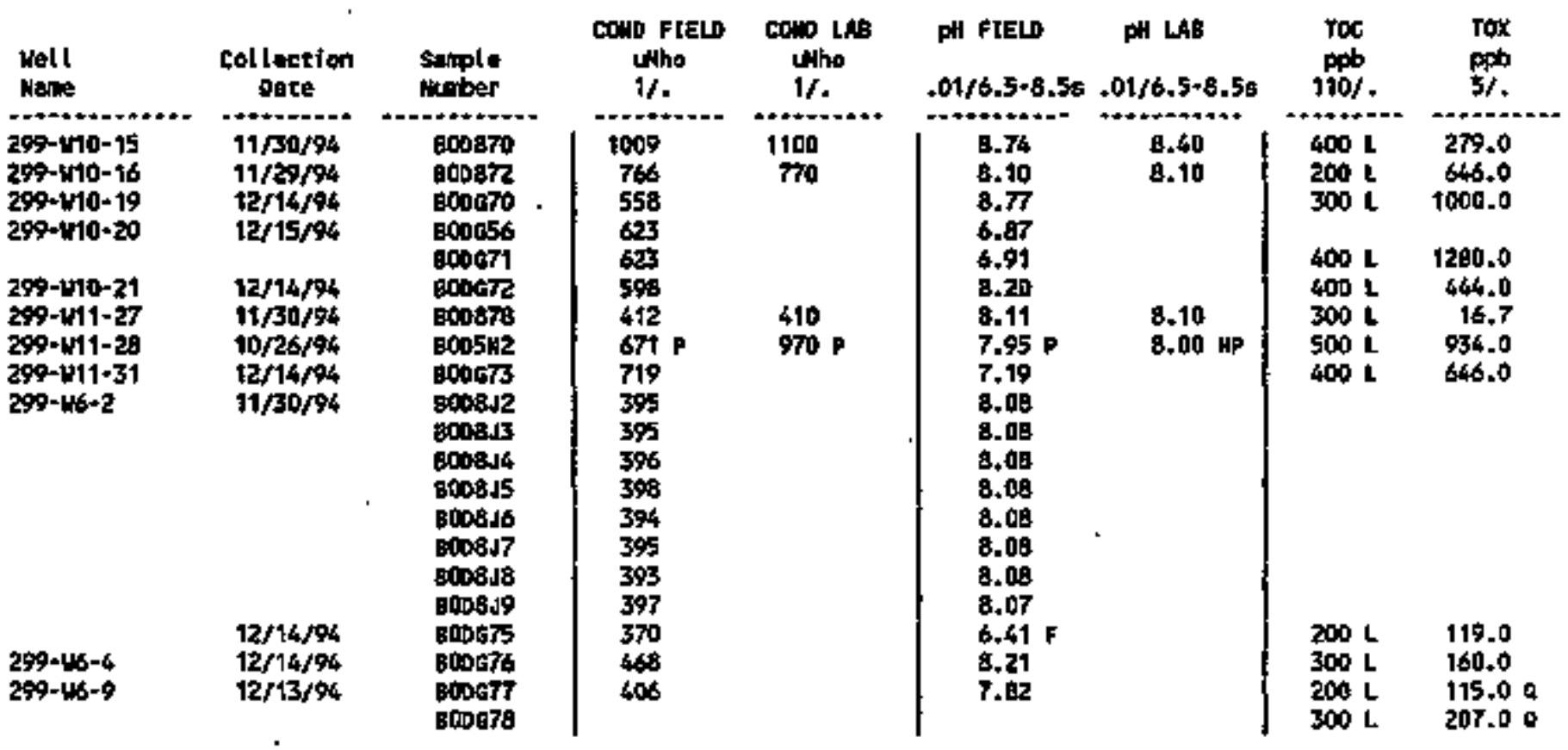

for axplenstion of this table, ses section 1.4 of report. 
Table 15-18. Constituent List and Summary of Resuits for the Single-Sheli Tank haste Managensent Area TX-TY Gata for Reporting Period October 1 through December 31, 1994. (sheet 1 of 3 )

CONTAUIBATIOH IMDTCATOR PARAHETERS

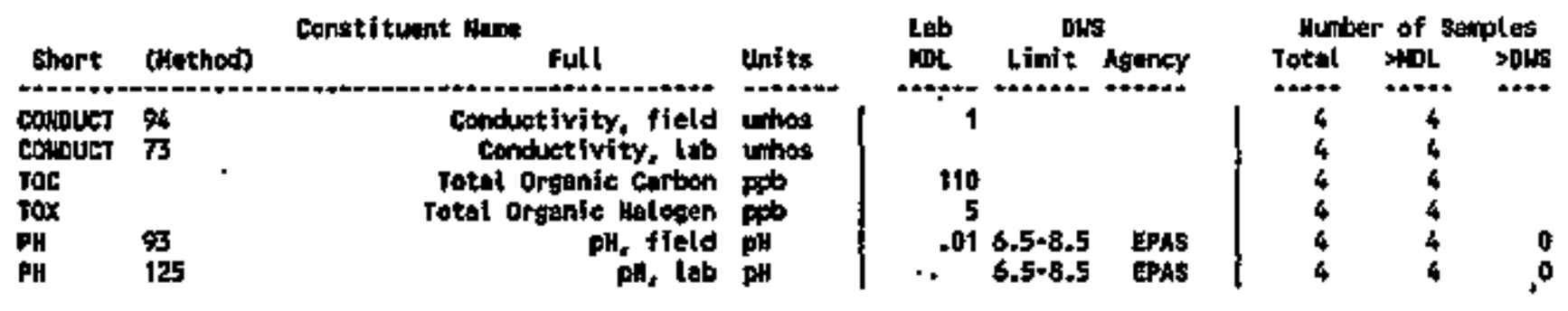

DR1HKTUS WHER PMANETERS

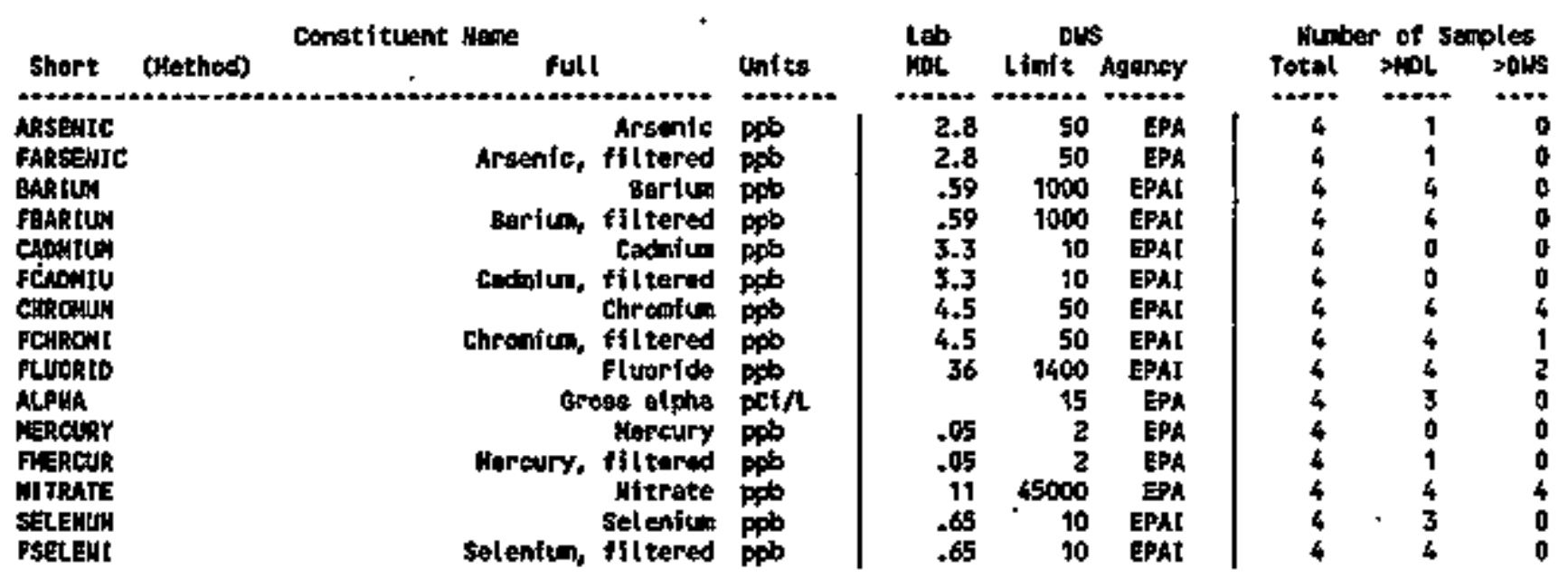

CROAHOWATER QUALITY PARALETERS

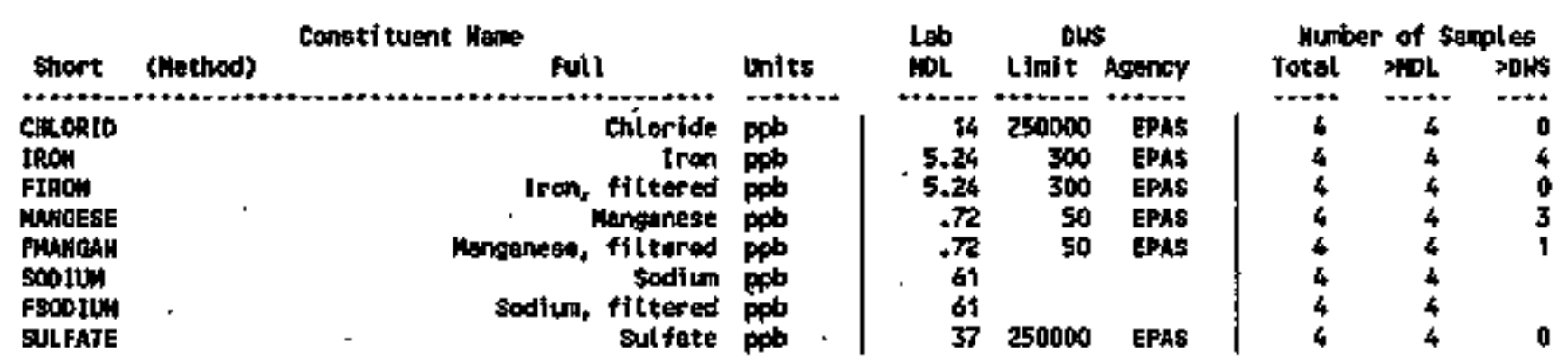


Table 15-1B. Constituent List and Summary of Results for the Single-She11 Tank Waste Management Area TX-TY Data for Reporting Period October 1 through December 31, 1994. (sheet 2 of 3 )

SITE SPECIFIC AHO OTHER COASTITUENTS

\begin{tabular}{|c|c|c|c|c|c|c|c|c|c|}
\hline Short & (Mothod) & tonstit tupht hame Full & Units & Lot & $\begin{array}{l}\text { ONS } \\
\text { Linit }\end{array}$ & 3 Apency & $\begin{array}{c}\text { Nunbe } \\
\text { Totel }\end{array}$ & $\begin{array}{l}\text { er of } 5 \\
\text { sWoL" }\end{array}$ & ancles \\
\hline & & & & $\cdots$ & & & $\cdots$ & +- & \\
\hline ALKALIN & & $\begin{array}{l}\text { Alkalinity } \\
\text { Mluminum }\end{array}$ & $\operatorname{lom}_{0 \times \infty}$ & $\begin{array}{r}2.8 \\
26\end{array}$ & & & 4 & $\begin{array}{l}4 \\
4\end{array}$ & \\
\hline PALUYII & $\cdot$ & Aluninum, fitteres & $p \infty$ & 36 & & - & 4 & 3 & \\
\hline AKT JONY & & Antinony & ppt & 62 & & & 4 & a & \\
\hline $\begin{array}{l}\text { Fantino } \\
\text { sg- } 125\end{array}$ & & $\begin{array}{l}\text { Ant inony, fti tered } \\
\text { Ant Inomy-125 }\end{array}$ & peis & 62 & & & $\begin{array}{l}4 \\
8\end{array}$ & $\begin{array}{l}0 \\
0\end{array}$ & \\
\hline BERYLLIH & & Beryltio & $\mathrm{ppb}$ & .17 & & & 4 & 0 & \\
\hline $\begin{array}{l}\text { PBERYLL } \\
\text { BE-7 }\end{array}$ & & $\begin{array}{r}\text { Beryttiun, filtered } \\
\text { eeryllium-7 }\end{array}$ & $\operatorname{ppt}_{\mathrm{C} / \mathrm{i} / \mathrm{s}}$ & .17 & & & 4 & $\begin{array}{l}0 \\
0\end{array}$ & \\
\hline gRONIDE & & Sromido & PA & 100 & & . & 4 & 3 & \\
\hline GRLCIUH & & Goleitn & $\mathbf{p p b}$ & 34 & & & 4 & 4 & \\
\hline renciv & & Calciun, filtered & ppos & 34 & & & 4 & 4 & \\
\hline $\begin{array}{l}\text { cteph-144 } \\
\text { cs-134 }\end{array}$ & & 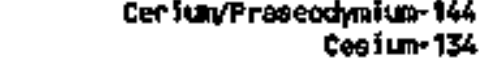 & $\begin{array}{l}\mathrm{PCF} / \mathrm{L} \\
\mathrm{PC} \mathbf{C} \mathrm{L}\end{array}$ & & & & 8 & $i$ & \\
\hline$c \$-137$ & & Cesium 137 & $\mathrm{DC} B / \mathrm{L}$ & & 200 & EPA & 8 & 1 & 0 \\
\hline $\begin{array}{l}\text { Cosint } \\
\text { FCOBALT }\end{array}$ & & Cobalt, filtered & $\begin{array}{l}\text { ppb } \\
\text { ppb }\end{array}$ & $\begin{array}{l}5.9 \\
3.9\end{array}$ & & & 4 & $\begin{array}{l}1 \\
0\end{array}$ & \\
\hline $10-60$ & & cobelt $t-\infty 0$ & $\mathrm{PCj} / \mathrm{L}$ & & 100 & EPh & a & 2 & - 10 \\
\hline $\begin{array}{l}\text { COPPER } \\
\text { FCOPPER }\end{array}$ & & Coppar & ppb & 4.1 & 1000 & $\begin{array}{l}\text { EPAS } \\
\text { gPAS }\end{array}$ & 4 & 4 & a \\
\hline EN-154 & & $\begin{array}{l}\text { Copper, filtered } \\
\text { Europiun-154 }\end{array}$ & $\begin{array}{l}\mathrm{Ppo} \\
\mathrm{PC} i / L\end{array}$ & & 1000 & EPAS & 8 & 0 & a \\
\hline EJ-15S & & Europium-155 & $\mathrm{PC} 1 / \mathrm{L}$ & & & & $\vec{B}$ & $i$ & \\
\hline $\begin{array}{l}\text { BETh } \\
j=129 L\end{array}$ & & Jodine-129, Low level bel & $\mathrm{PC} ; \mathrm{LL}$ & & 1 & & 4 & 4 & \\
\hline LEND & & 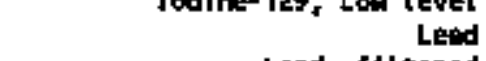 & ppb & 1.5 & 50 & EPA] & 4 & 4 & c \\
\hline FLEAD & & Leed, fil tered & ppb & 1.5 & 50 & EPA] & 4 & 1 & 0 \\
\hline $\begin{array}{l}\text { YGAGNES } \\
\text { FHALYUES }\end{array}$ & & $\begin{array}{l}\text { Mognisium } \\
\text { hegnesiung filtered }\end{array}$ & ppb & $\mathbf{2 3}$ & & & 4 & 4 & \\
\hline MTEKEL & & nognestivin, Thited & ppb & 13 & & & 4 & 4 & \\
\hline FHI CKEL & & Hicket, filtered & pob & 13 & & & 4 & 2 & \\
\hline AITR!TE & & Hitrite & b & 18 & 1000 & EPA & 4 & 0 & 0 \\
\hline FHOSPHA & & Phosphate & ppo & 82 & & & 4 & 0 & \\
\hline 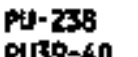 & & Plutoniun-238 & Cill & & & & 4 & 0 & \\
\hline $\begin{array}{l}\text { PUBS-40 } \\
\text { POTASTOA }\end{array}$ & & $\begin{array}{l}\text { Plutonitur-239/40 } \\
\text { Potossjum }\end{array}$ & $\underset{m b}{\infty i / L}$ & 850 & & & 4 & $\begin{array}{l}0 \\
4\end{array}$ & \\
\hline fpotasts & & Patossium, fittered & $\underset{\infty \infty b}{p p o b}$ & 850 & & & 4 & 4 & \\
\hline$k-60$ & & Potésoive 40 & $\bar{x} \boldsymbol{T} / \mathrm{L}$ & & & & 8 & 7 & \\
\hline RU-10S & & Ruthentich-106 & $\mathrm{plih}$ & & 30 & EPA & 8 & 0 & 0 \\
\hline BILVER & & silver & peb & 3.6 & 50 & Eend & 4 & 0 & 0 \\
\hline FSILVER & & . sitver, filtered & ppo & 3.6 & so & EPAI & 6 & 0 & 0 \\
\hline $\begin{array}{l}\mathbf{s h} \cdot 90 \\
\mathrm{TC}-99\end{array}$ & & Strantion-90 & pci/l & ‘ & $900^{\circ}$ & EPA & 8 & 8 & $\begin{array}{l}0 \\
1\end{array}$ \\
\hline TEAtLION & & Thartiun & $x_{\infty \rightarrow \infty}^{\infty 112}$ & .58 & & $E_{P h}$ & 4 & 0 & \\
\hline FTakesuth & & Thaldfur, filtered & 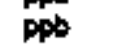 & .58 & & & 4 & 0 & \\
\hline TIR & & & ppo & 69 & & & 4 & 0 & \\
\hline FTIF & & Tin, filtered & 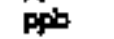 & 69 & & & 4 & 0 & \\
\hline TDS & & Total Dissolved Solidg & ppn & 10 & 500 & EPAS & 4 & 4 & 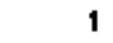 \\
\hline
\end{tabular}


Table 15-18. Const1tuent List and Sumsary of Results for the Single-Shell Tank Waste Management Area TX-TY Data for Reporting Period 0ctober 1 through December 31, 1994.

(sheat 3 of 3 )

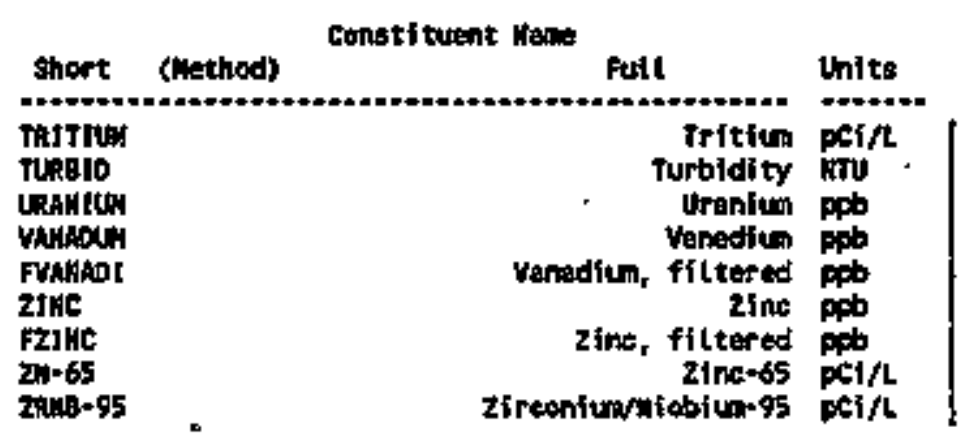

\begin{tabular}{|c|c|c|c|c|c|}
\hline Leb & ous & & Hum & $r$ of $s$ & plos \\
\hline $\mathrm{COL}$ & Lftett & Ageroy & fotel & $3 \mathrm{KDL}$ & riss \\
\hline & (20000 & mis & $+\infty$ & $\cdots$ & $\cdots$ \\
\hline - ,016 & 2000 & EAPA & 4 & 4 & 3 \\
\hline 6.6 & & & 4 & 4 & \\
\hline 6.6 & & & 4 & 4 & \\
\hline 6.3 & 5000 & EPAS & 4 & 2 & 0 \\
\hline & & - & $v$ & Q & \\
\hline
\end{tabular}

For explanation of this tabie, see section 1.4 of report. 
Table 15-19. Constituents with at Least One Detected Value for the Single-She1] Tank Yaste Management Area TX-TY Data for Reporting Period October 1 through December 31, 1994. (sheet 1 of 5 )

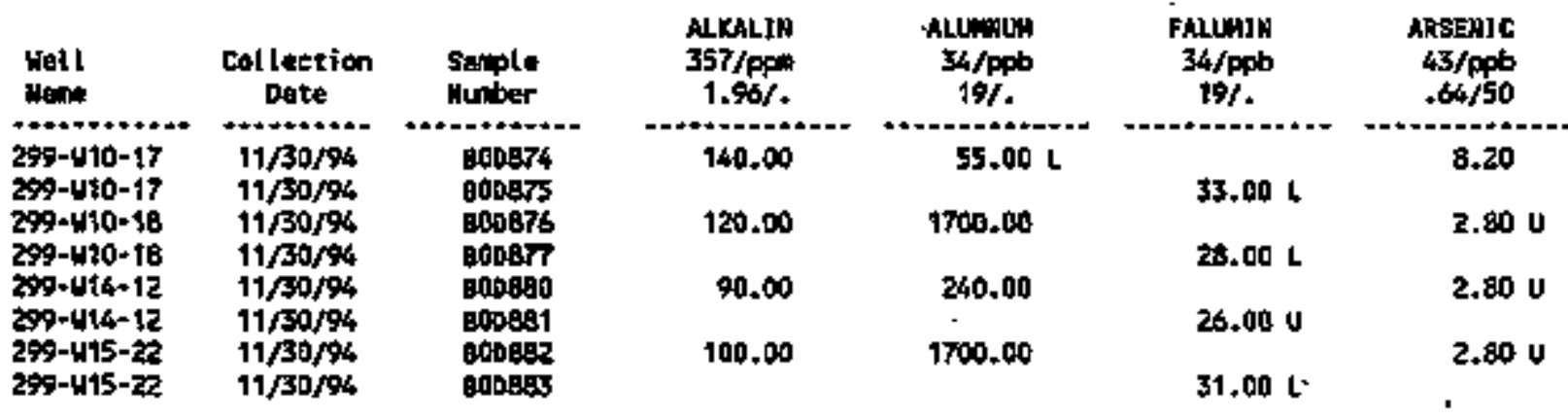

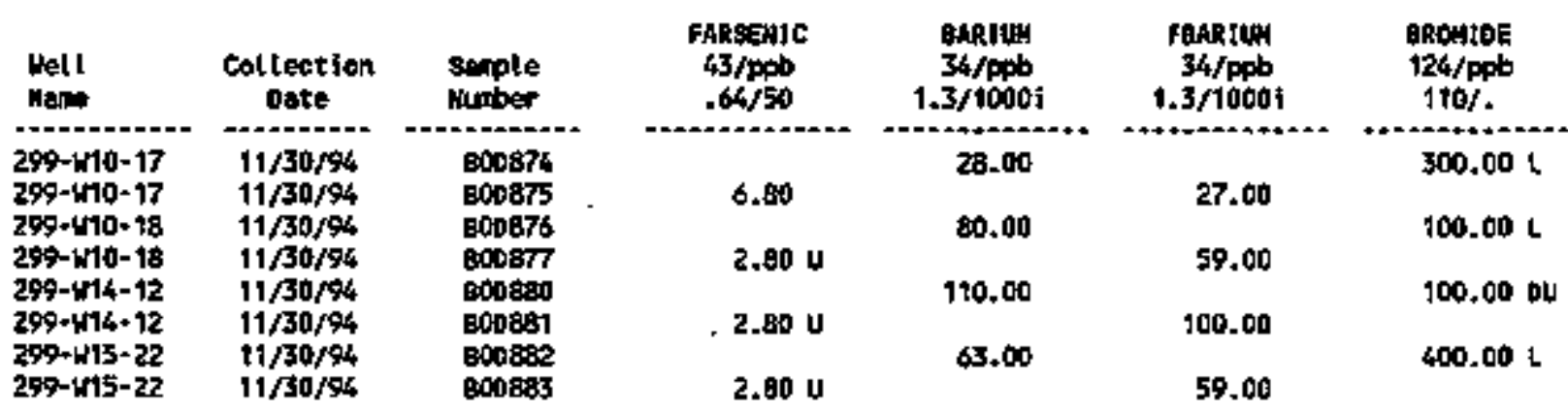

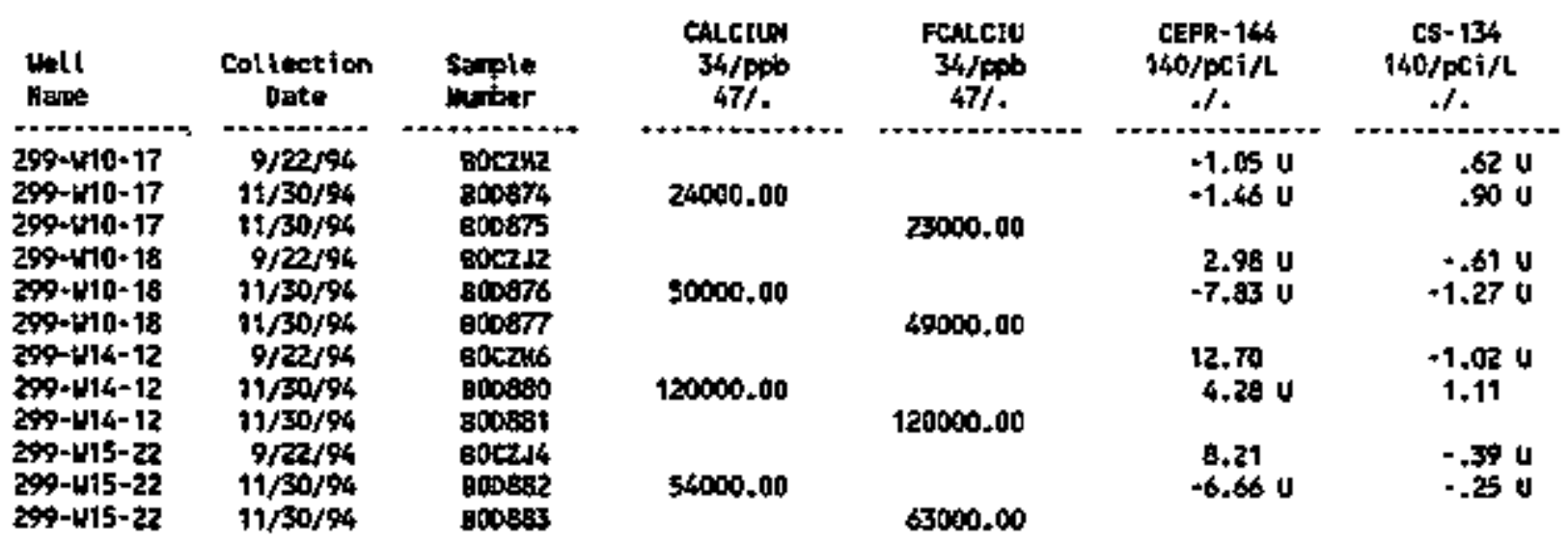


Table 15-19. Constituents with at Least One Detected Value for the Single-Shell Tank Waste Management Area TX-TY Data for Reporting Period October 1 through December 31, 1994. (sheet 2 of 5 ).

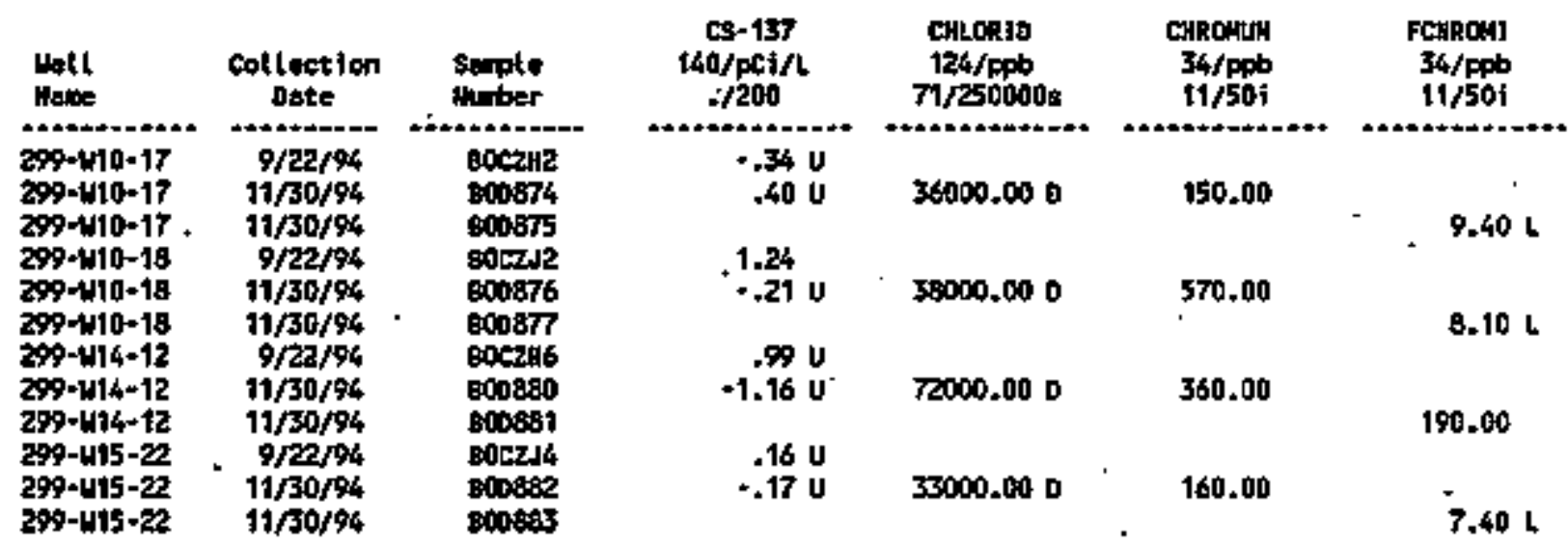

\begin{tabular}{|c|c|c|c|c|c|c|}
\hline $\begin{array}{l}\text { Well } \\
\text { Nome }\end{array}$ & $\begin{array}{l}\text { Collection } \\
\text { Duto }\end{array}$ & $\begin{array}{l}\text { 3erple } \\
\text { Huber }\end{array}$ & $\begin{array}{l}\text { COAALT } \\
34 / p p b \\
6.5 \%\end{array}$ & $\begin{array}{l}\text { FCOAALI } \\
34 / \mathrm{ppb} \\
6.5 \% .\end{array}$ & $\begin{array}{c}\mathrm{CD}-60 \\
140 / \mathrm{pCD} / \mathrm{L} \\
-/ 100\end{array}$ & $\begin{array}{c}\text { COPPER } \\
34 / \mathrm{ppb} \\
2.6 / 1000 \mathrm{~s}\end{array}$ \\
\hline $\begin{array}{l}299-\mathrm{ulo-17} \\
299-\mathrm{u10}-17 \\
299-\mathrm{u10}-17\end{array}$ & $\begin{array}{r}9 / 22 / 94 \\
11 / / 30 / 44 \\
11 / 30 / 94\end{array}$ & $\begin{array}{l}8002112 \\
800874 \\
600875\end{array}$ & $5.90 \mathrm{U}$ & $5.90 \mathrm{U}$ & $.22 \mathrm{U}$ & $7.90 \mathrm{~L}$ \\
\hline $\begin{array}{l}299-410-18 \\
299-410-18 \\
299-410-18\end{array}$ & $\begin{array}{r}9 / 22 / 94 \\
11 / 30 / 94 \\
11 / 30 / 94\end{array}$ & $\begin{array}{l}\text { B0c2.12 } \\
\text { B000876 } \\
1000877\end{array}$ & 26.00 & 5.900 & $\begin{aligned} .61 \mathrm{U} \\
.30 \mathrm{U}\end{aligned}$ & $37.00^{\circ}$ \\
\hline $\begin{array}{l}299-414-12 \\
299-414-12 \\
299-4 t 4-12\end{array}$ & $\begin{array}{r}9 / 20 / 94 \\
11 / 30 / 94 \\
11 / 30 / 94\end{array}$ & 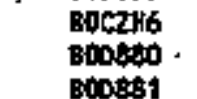 & $5.90 \mathrm{D}$ & $5.50 \mathrm{U}$ & $\begin{array}{r}13.20 \\
7.80\end{array}$ & $6.40 \mathrm{~L}$ \\
\hline $\begin{array}{l}299-415-22 \\
299-415-22 \\
299-(1515-22\end{array}$ & $\begin{array}{r}9 / 20 / 94 \\
11 / 30 / 94 \\
11 / 30 / 94\end{array}$ & 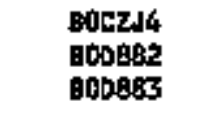 & $5.90 \mathrm{U}$ & $5.90 \mathrm{U}$ & $\begin{array}{l}-.21 \\
.22 \\
.23\end{array}$ & $12.00 \mathrm{~L}$ \\
\hline $\begin{array}{l}\text { Hell } \\
\text { Herou }\end{array}$ & $\begin{array}{c}\text { Collection } \\
\text { Date }\end{array}$ & $\begin{array}{l}\text { 3aple } \\
\text { Mubar }\end{array}$ & $\begin{array}{c}\text { FCDPPER } \\
34 / \mathrm{PPD} \\
2.6 / 1000 \mathrm{DS}\end{array}$ & $\begin{array}{c}\mathrm{Ej} 155 \\
140 / \mathrm{pci} / \mathrm{h} \\
. / .\end{array}$ & $\begin{array}{l}\text { FLEGRID } \\
124 / \text { pph } \\
51 / 14001\end{array}$ & $\begin{array}{c}\text { ALPAR } \\
135 / \mathrm{PSI}^{\prime} / \mathrm{A} \\
+/ 15\end{array}$ \\
\hline $\begin{array}{l}299-w 10-17 \\
299-410-17 \\
299-w 10-17\end{array}$ & $\begin{array}{r}9 / 22 / 94 \\
11 / 30 / 94 \\
11 / 30 / 94\end{array}$ & $\begin{array}{l}\text { 日0C2H2 } \\
\text { B00874 } \\
\text { 800875 }\end{array}$ & $4.20 \mathrm{~L}$ & $\begin{array}{l}57 \mathrm{U} \\
.64 \mathrm{U}\end{array}$ & 2200.00 & 1.97 \\
\hline $\begin{array}{l}299-410-18 \\
299-410-18 \\
299-410-18\end{array}$ & $\begin{array}{r}9 / 22 / 94 \\
11 / 30 / 94 \\
11 / 30 / 94\end{array}$ & $\begin{array}{l}8002.52 \\
800876 \\
800 \$ 77\end{array}$ & $4.10 \mathrm{U}$ & $\begin{array}{r}-1.50 \mathrm{U} \\
.76 \mathrm{U}\end{array}$ & 1800.00 & 2.77 \\
\hline $\begin{array}{l}299-144-12 \\
299-114-12 \\
299-w_{14-12}\end{array}$ & $\begin{array}{r}9 / 22 / 94 \\
11 / 30 / 46 \\
11 / 30 / 94\end{array}$ & $\begin{array}{l}\text { Bocahs } \\
\text { Bopsad } \\
\text { BOpgas1 }\end{array}$ & $4.10 \mathrm{U}$ & $-1.48 \mathrm{U}$ & 9000.00 & $3.56 \mathrm{U}$ \\
\hline $\begin{array}{l}299-115-22 \\
299-w 15-22 \\
299-115-22\end{array}$ & $\begin{array}{r}9 / 22 / 94 \\
11 / 30 / 94 \\
11 / 30 / 94\end{array}$ & $\begin{array}{l}\text { E0c7J4 } \\
800802 \\
800883\end{array}$ & $4.10 \mathrm{v}$ & $\begin{array}{l}-.03 \\
2.20\end{array}$ & 500.00 & 2.17 \\
\hline
\end{tabular}


Table 15-19. Constituents with at Least One Betected Value for the Single-She]1 Tank Haste Management Area TX-TY Data for Reporting Period October 1 through December 31, 1994. (sheet 3 of 5 )

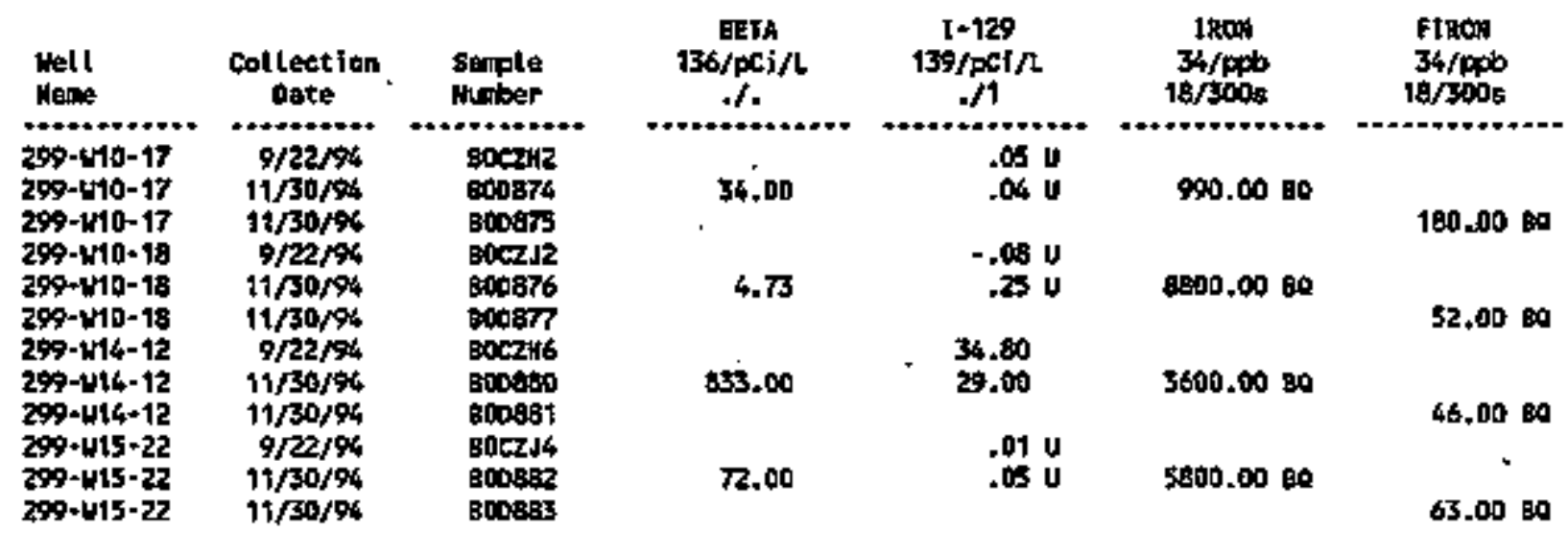

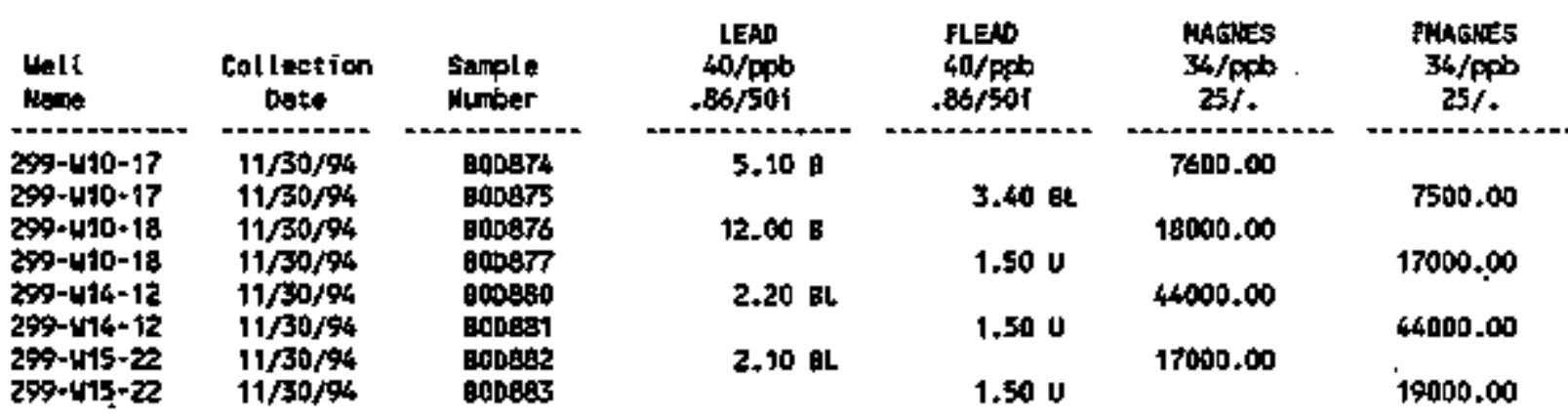

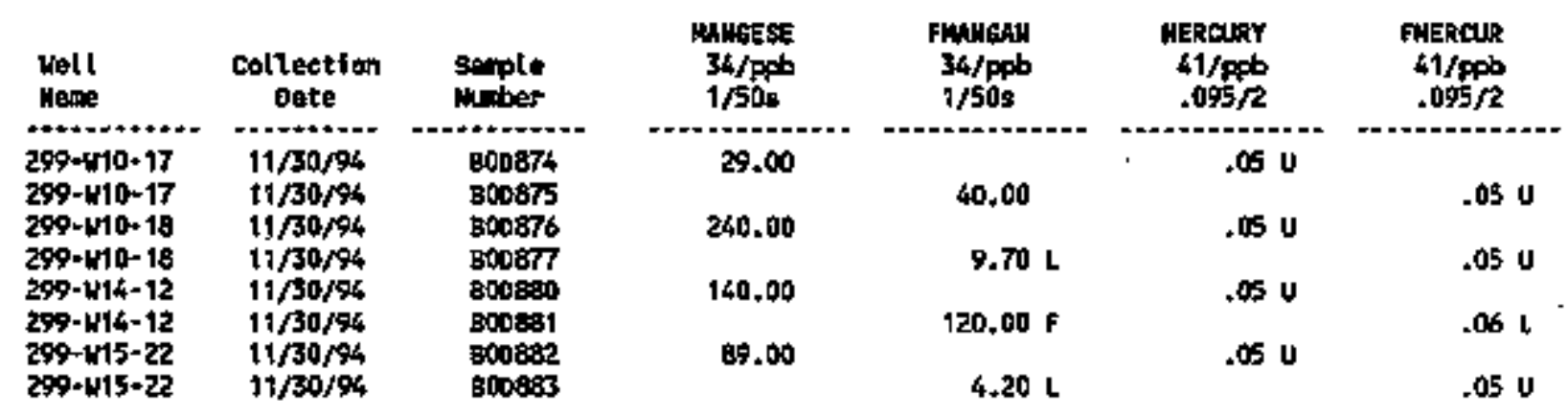


Table 15-19. Constituents with at Least One Detected Value for the Single-Shell Tank Waste Management Area TX-TY Data for Reporting Period October I through December 31, 1994. (sheet 4 of 5 )

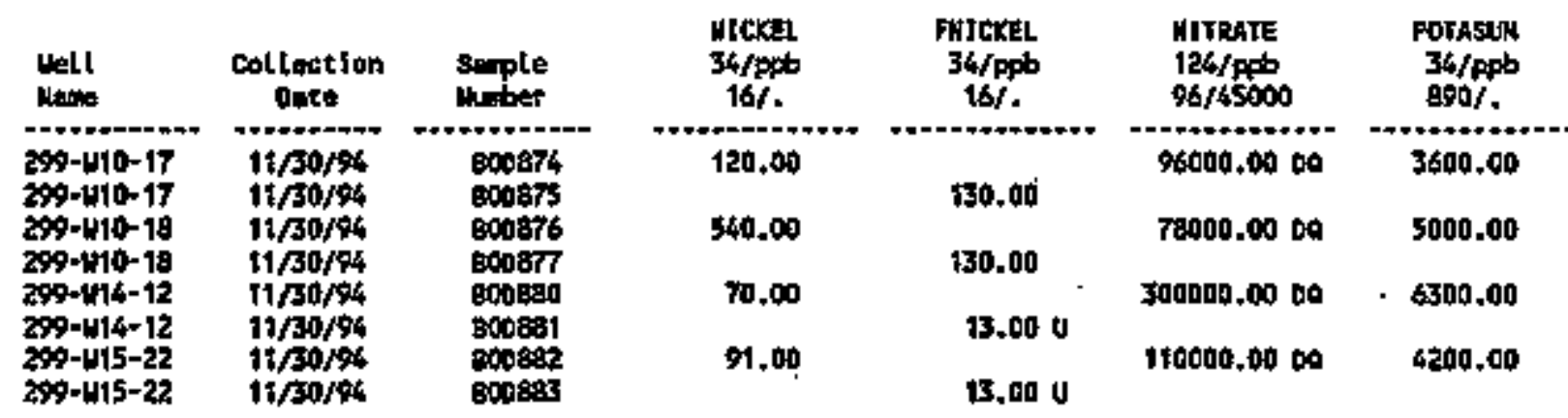

\begin{tabular}{|c|c|c|c|c|c|c|}
\hline $\begin{array}{l}\text { Wet } 1 \\
\text { Hente }\end{array}$ & $\begin{array}{l}\text { Colleat fon } \\
\text { Date }\end{array}$ & $\begin{array}{l}\text { semple } \\
\text { Ntmber }\end{array}$ & $\begin{array}{l}\text { Fporiss } \\
34 / 0+b \\
690 / .\end{array}$ & $\begin{array}{c}\mathrm{K}-40 \\
140 / \mathrm{pci} / \mathrm{L} \\
. /\end{array}$ & $\begin{array}{l}\text { SELEM:A } \\
48 / \mathrm{pps} \\
1.4 / 10 \mathrm{i}\end{array}$ & $\begin{array}{l}\text { FSELEHI } \\
\text { 48/pp' } \\
1.6 / 101\end{array}$ \\
\hline 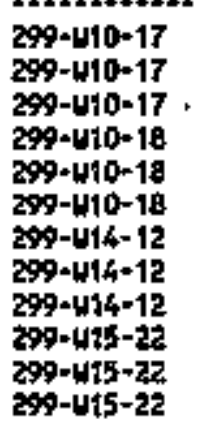 & $\begin{array}{r}9 / 22 / 94 \\
11 / 30 / 44 \\
11 / 30 / 94 \\
9 / 22 / 94 \\
11 / 30 / 94 \\
11 / 50 / 94 \\
9 / 20 / 96 \\
11 / 30 / 94 \\
11 / 30 / 94 \\
9 / 22 / 94 \\
11 / 30 / 94 \\
11 / 50 / 94\end{array}$ & 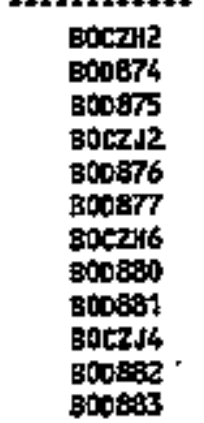 & $\begin{array}{l}3700.00 \\
4800.00 \\
6400.00 \\
4800.00\end{array}$ & $\begin{array}{l}30.60 \\
8.77 \text { uo } \\
33.30 \\
46.00 \mathrm{a} \\
61.70 \\
49.200 \\
42.10 \\
46.20 \mathrm{a}\end{array}$ & $\begin{array}{l}2.00 \mathrm{EL} \\
.65 \mathrm{U} \\
1.50 \mathrm{BL} \\
2.00 \mathrm{EL}\end{array}$ & $\begin{array}{l}3.10 \mathrm{BL} \\
1.50 \mathrm{8L} \\
1.70 \mathrm{gL} \\
3.00 \mathrm{EL}\end{array}$ \\
\hline
\end{tabular}

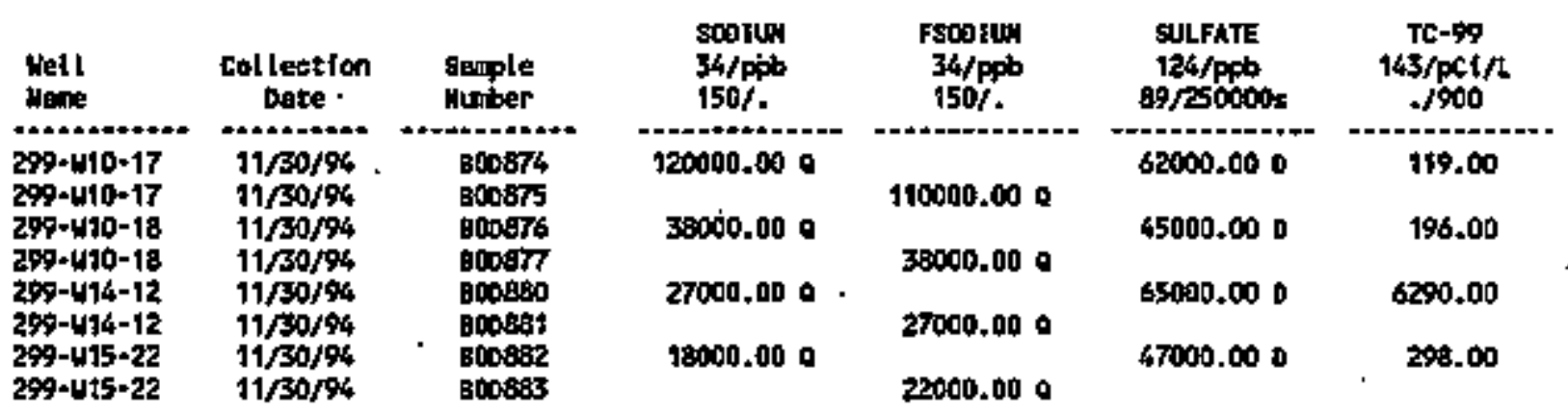




\section{DOE/RL-94-36-4}

Table 15-19. Constituents with at Least One Detected Value for the Single-Shell Tank Waste Management Area TX-TY Data for Reporting Period October 1 through Decenter 31, 1994. (sheet 5 of 5 )

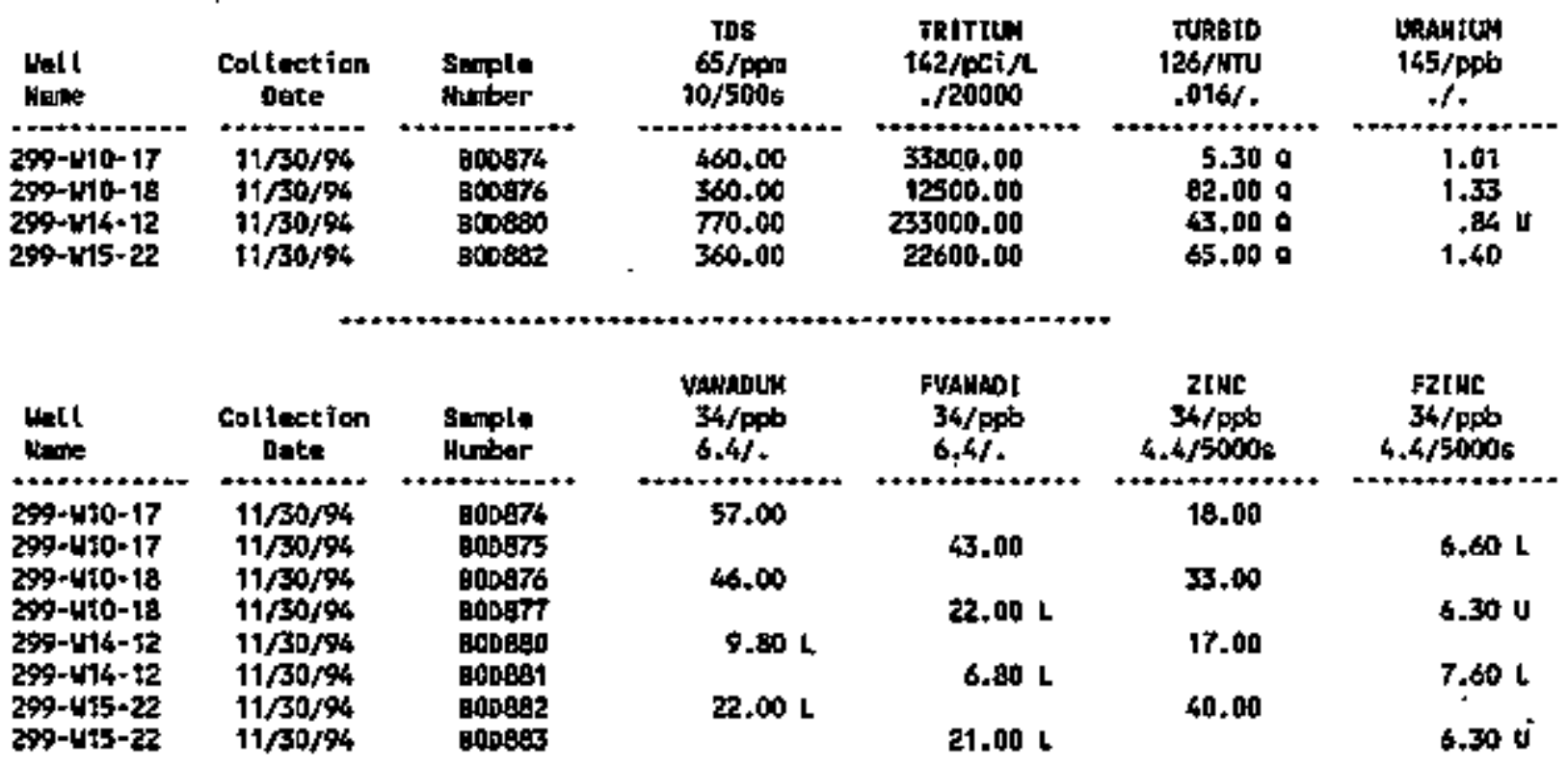

For explanetion of this table, aee section 1.4 of report. 
Table 15-20. Contamination Indicator Parameters for the

Single-She11 Tank Haste Management Area TX-TY Data for

Reporting Period Dctober 1 through December 31, 1994.

\begin{tabular}{|c|c|c|c|c|c|c|c|c|}
\hline Letl & $\begin{array}{c}\text { cotlection } \\
\text { Date }\end{array}$ & $\begin{array}{l}\text { senple } \\
\text { luniber }\end{array}$ & $\begin{array}{c}\text { COAdo FIELP } \\
\text { utho } \\
4 /\end{array}$ & $\begin{array}{l}\text { conto LAB } \\
\text { Wha } \\
\text { W/. }\end{array}$ & $\begin{array}{l}\text { PH FIELD } \\
.01 / 6.5-8.588\end{array}$ & $\begin{array}{c}\text { PH LAB } \\
.01 / 6.5-8.58\end{array}$ & $\begin{array}{c}\text { Toc } \\
\text { ppb } \\
\text { t10/ }\end{array}$ & $\begin{array}{r}\text { Jox } \\
\mathrm{Ppb} \\
5 / .\end{array}$ \\
\hline
\end{tabular}

For explanstion of thie toble, see section 1.4 of report.

Table 15-21. Constituent List and Sumary of Results for the Single-Shell Tank Maste Management Area U Data for Reporting Period October 1 through December 31, 1994.

DRJMKL WS WATER PARNATERS

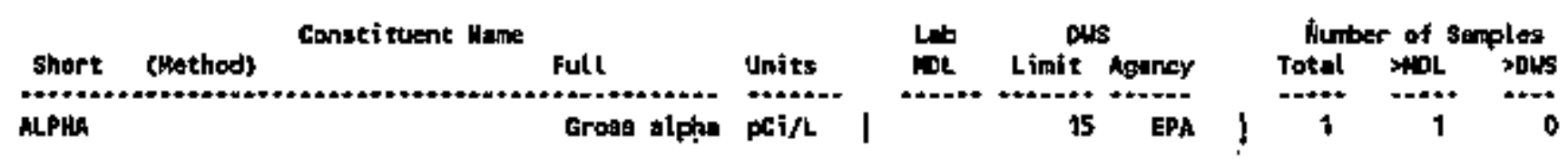

SITE SFECIFtC AMD OTHER constrtrugts

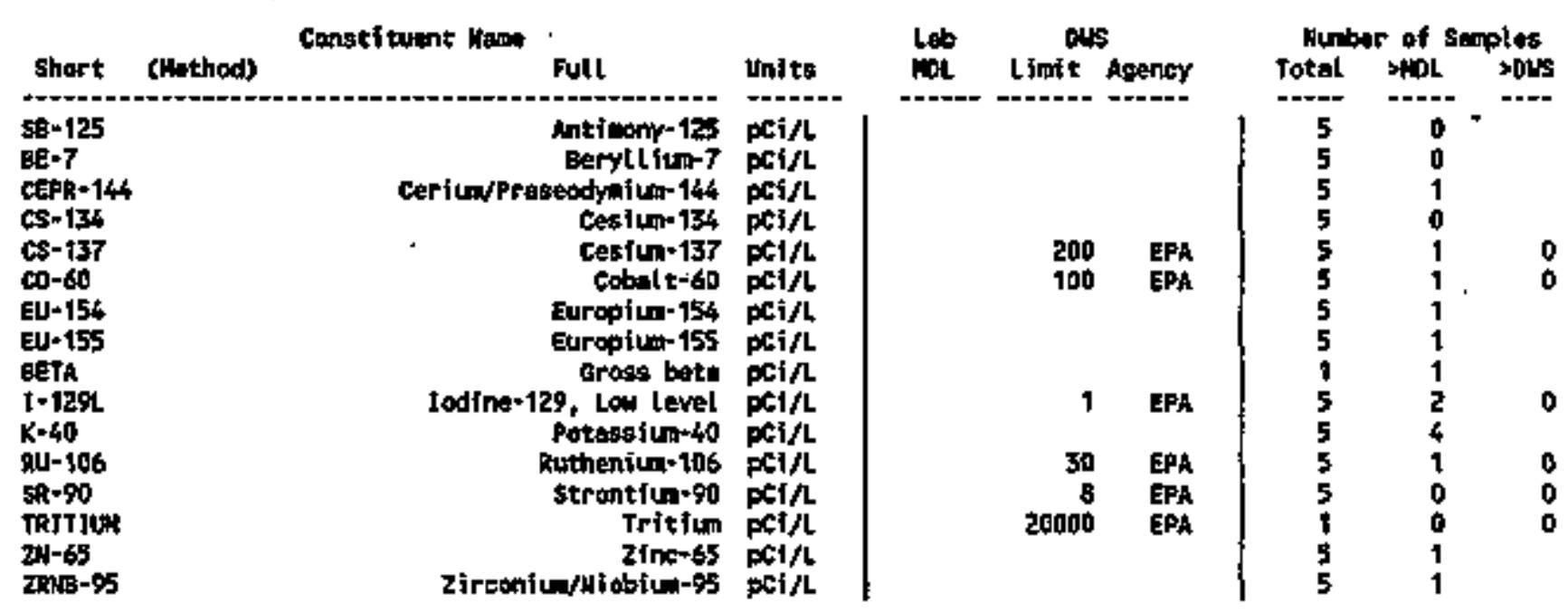

for explangtion af this table, sate Section 1.4 of report. 
Table 15-22. Constituents with at Least One Detected Value for the Single-She]l Tank Waste Management Area U Data for Reporting Perjod October 1 through December 31, 1994.

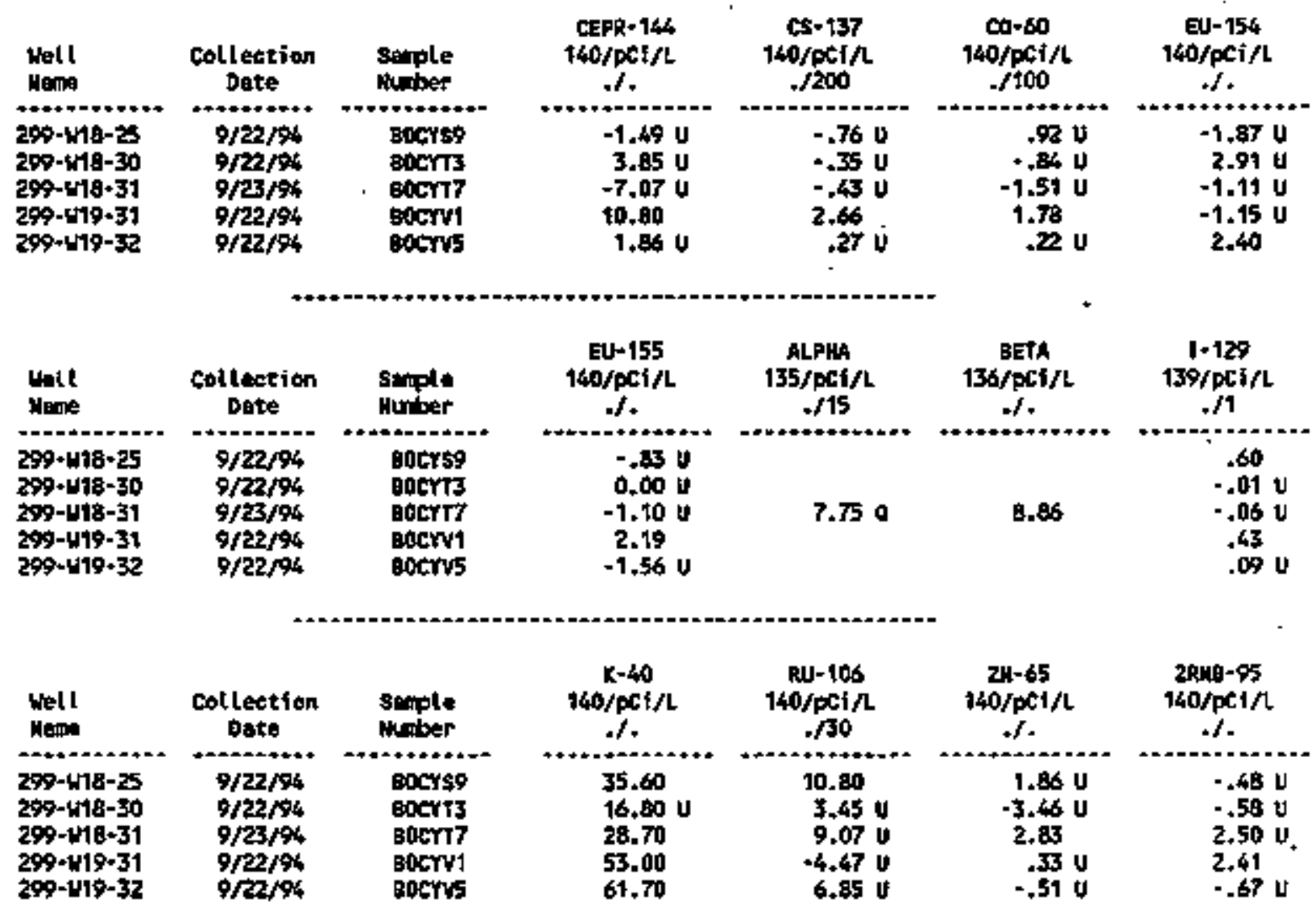

For explantion of this table, set section 1.4 of raport.

Table 15-23. Contamination Indicator Parameters for the Single-Shell Tank Waste Management Area U Data for Reporting Period October 1 through December 31, 1994.

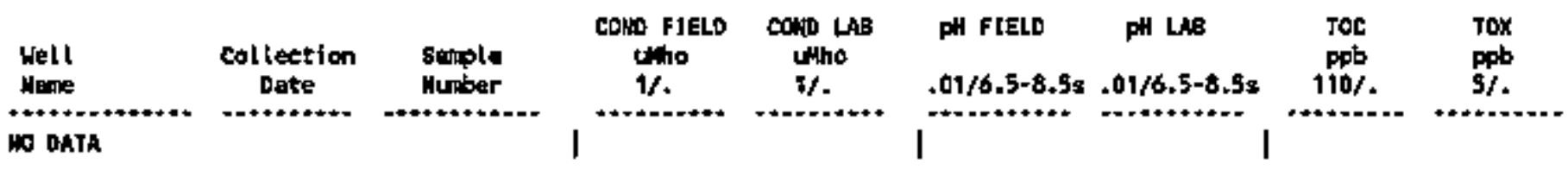

For explernation of this table, see sextidi 1.4 of report. 
DOE/RL-94-36-4

\section{CONTENTS}

16.0300 AREA PROCESS TRENCHES .................. 16-1

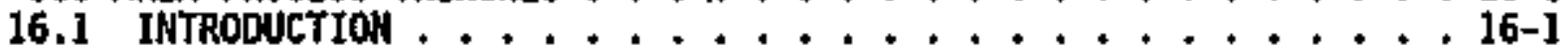

16.2 hater level MEASUREMENTS ................ 16-1

16.3 HATER CHEMISTRY DATA . .................. 16-1 


\section{DOE/RL-94-36-4}

\section{LIST OF FIEURES}

16-1 Well Location Map for the 300 Area Process Trenches . . . . . 16-3

\section{LIST OF TABLES}

16-1 Monitoring Well Purpose and Sampling Schedule for the 300 Area Process Trenches Metwork .............. 16-4

16-2 RCRA Water Lieve1 Measurenent Report 300 Area Process Trenches, Fourth Quarter $1994 \ldots . . . .16-5$

16-3 Constituent List and Sumemary of Results for the, 300 Area Process Trenches Data for Reporting Period October 1 through December $31,1994 \ldots \ldots$ 16-10

16-4 Constituents with at Least One Detected Vajue for the 300 Area - Process Trenches Data for Reporting Period October 1 through December 31, $1994 \ldots \ldots$ 16-13

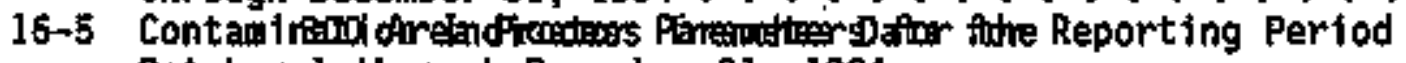
october 1 through Decerber $31,1994 \ldots 16-21$ 
DOE/RL-94-36-4

\title{
16.0 300 AREA PROCESS TREICHES
}

\author{
J. W. Lindberg \\ Hestinghouse hanford Company
}

\section{I INTRODUCTION}

The groundwater near the 300 Area Process Trenches (APT) (316-5) has been monitored by a RCRA wel1 network since June 1985. The groundwater mont toring is being conducted under interin-status regulations (40 CFR 265) and is an assessment-level program (Schalla 1988). Monitoring wells were constructed in response to a Consent Agreement and Compliance Order (Ecology and EPA 1986). Locations of groundwater wells currently used to monitor the facj7ity are shown in Figure 16-1. Table 16-1 lists the wells of the monjtoring network and provides information about their relative Tocation, the portion of the aquffer sampled, sampling frequency, and last sampling date. Mine of the 11 wells in the monitoring network are RCRA standard wel's while two are older, noncoawl fant wells.

The 300 APT are located in the northern portion of the 300 Area and were used for the disposal of wastewater from various 300 Area operations. Adninistrative isolation of the trenches was completed and all discharges to the trenches were terminated. Complete physical isolation of the trenches will occur by the enid of January 1995. Just before the trenches were taken out of use, the wastewater primarily consisted of cooling water, with small quantities of nonhazardous maintenance and process waste.

\subsection{WATER LEVEL MEASUREMEKTS}

Water levels are measured monthly and at the time of sampling in selected 300 Area wells. Water level measurenents obtained during the fourth quarter of 1994 are presented in Table 16-2.

\subsection{WATER CHEMISTRY DATA}

Water chemistry samples are collected semiannually from the 300 APT monitoring wells. In addition to semiannual samples, samples are collected quarterly fro: we]T 399-I-17A to provide near-trench aonitoring of contawinants. Sampling dates are 1isted in Table 16-1. Analytical results received during the fourth quarter are presented in Tables 16-3 through 16-5.

Sampling and analysis during the fourth quarter showed that one drinking water parameter, two groundwater quality parameters, and two site-specific and other constituents exceed drinking water standards (Tables 16-3 and 16-4). Iron and manganese are assumed to be the remnants of well construction or caused by the use of stainless steel screens. The exceedance of gross alpha is probably related to uranium that is detected in downgradient wells. If the uranium component is subtracted from the gross alpha, the remainder will probably not exceed the drinking water standards. We11 399-1-16B continues to 
show elevated levels of trichloroethene and e1s-dicloroethere with a corresponding elevated level in the indicator parameter ToX (Table 16-5).

Field-measured pH collected from well 399-1-17A on 0ctober 7, 1994, is probably in error. The four replicates (ranging from 6.85 to 6.87) are inconsistent with historical trends, the laboratory value from a sample collected at the same time $(7.30)$, and replicates collected December 15, 1994 (7.34-7.50). A RADE has been submitted for these data. 
Figure 16-1. Hel] Location Map for the 300 Area Process Trenches.

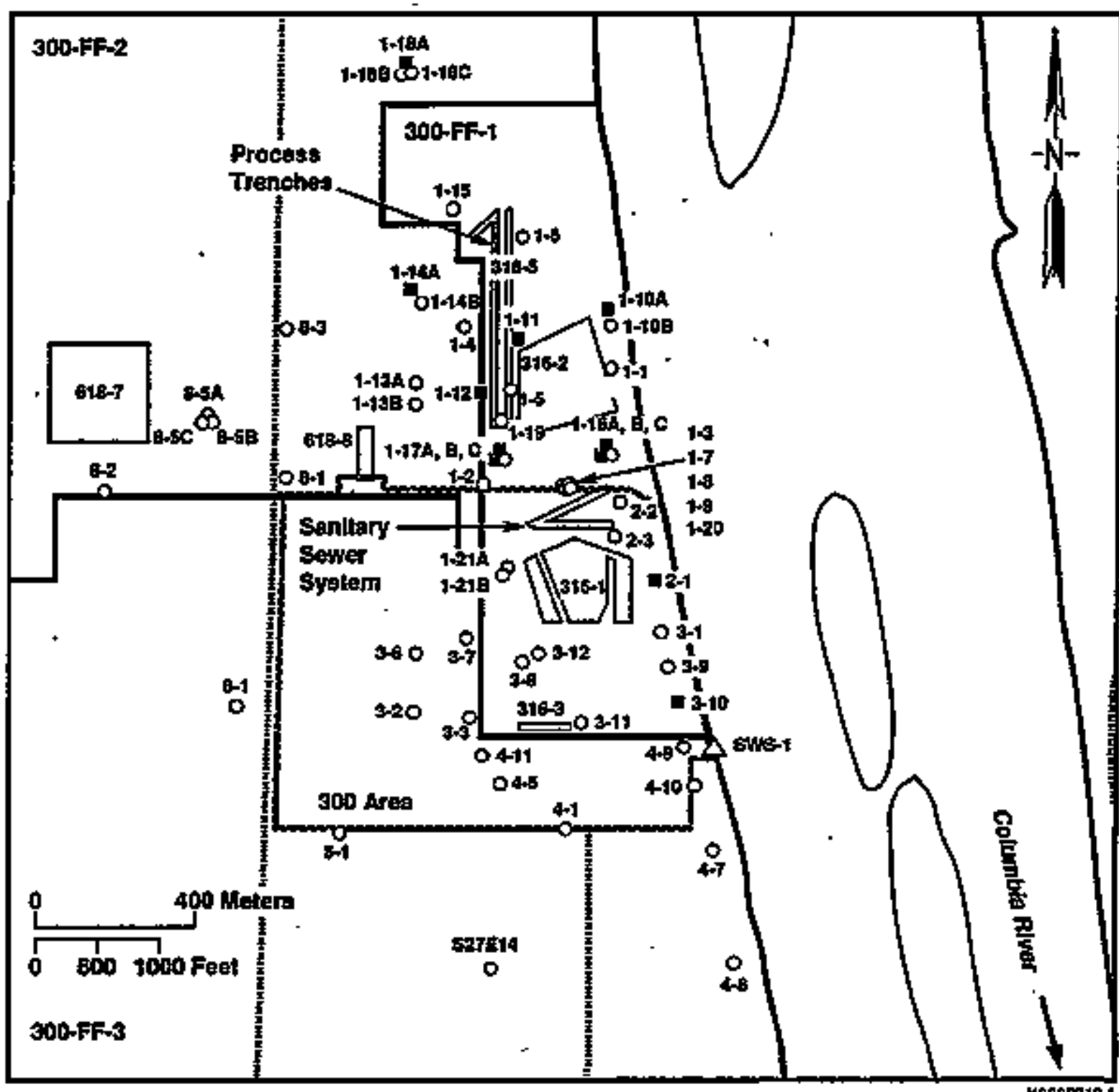

- 1-12 Well Lotatlan and Mumber (Welle Prolixad by 399. Except Tho: Beglnimg with S are Prellxed with 6g日-)

- 47 Monftoring Nehirork Wod

A sWs-1 Surface-Water Monltoring Station

mrmmanimin, Roget. 
Table 16-1. Monitoring We11 Purpose and Sampling Schedule for the 300 Area Process Trenches Network.

\begin{tabular}{|c|c|c|c|c|}
\hline $\begin{array}{l}\text { We]1 no. } \\
\text { (399-) }\end{array}$ & $\begin{array}{l}\text { Relative } \\
\text { position }\end{array}$ & Hydrogeologic unit & $\begin{array}{l}\text { Sample } \\
\text { frequtency }\end{array}$ & $\begin{array}{c}\text { Last sampling } \\
\text { date }\end{array}$ \\
\hline $1-10 A$ & Downgradient & $\begin{array}{l}\text { Hanford/Ringold: } \\
\text { Hater Table }\end{array}$ & Semiannual1y & $10 / 05 / 94$ \\
\hline $1-I 1$ & Adjacent & $\begin{array}{l}\text { Hanford/Ringold: } \\
\text { Water Table }\end{array}$ & Semiannually & $10 / 05 / 94$ \\
\hline $1-12$ & Downgradtent & $\begin{array}{l}\text { Hanford/Ringold: } \\
\text { Nater Table }\end{array}$ & Semiannua 11y & $\begin{array}{l}10 / 05 / 94 \\
10 / 06 / 94\end{array}$ \\
\hline $1-14 \mathrm{~A}$ & Adjacent & $\begin{array}{l}\text { Hanford: Water } \\
\text { Table }\end{array}$ & Semfannuajly & $10 / 05 / 94$ \\
\hline $1-16 A$ & Downgradtent & $\begin{array}{l}\text { Ringold: Water } \\
\text { Table }\end{array}$ & Semiannuat1y & $10 / 07 / 94$ \\
\hline $1-168$ & Downgradient & $\begin{array}{l}\text { Ringold: Bottom of } \\
\text { Unconfined Aquifer }\end{array}$ & Semiannually & $10 / 10 / 94$ \\
\hline $1-17 \mathrm{~A}$ & Downgradient & $\begin{array}{l}\text { Ringold: Water } \\
\text { Table }\end{array}$ & Quarterly & $\begin{array}{l}10 / 07 / 94 \\
12 / 15 / 94\end{array}$ \\
\hline $1-17 B$ & Downgradient & $\begin{array}{l}\text { Ringold: Bottom of } \\
\text { Unconf ined Aquifer }\end{array}$ & Semiannua11y & $10 / 07 / 94$ \\
\hline$I-18 A$ & Upgradient & $\begin{array}{l}\text { Ringold: Water } \\
\text { Table }\end{array}$ & Semiannually & $10 / 05 / 94$ \\
\hline $2-1^{8}$ & Downgradient & $\begin{array}{l}\text { Hanford/Ringold: } \\
\text { Water Tabie }\end{array}$ & Semiannually & $10 / 05 / 94$ \\
\hline $3-10^{8}$ & Downgradient & $\begin{array}{l}\text { Hanford: Water } \\
\text { Table }\end{array}$ & Semiannually & $10 / 10 / 94$ \\
\hline
\end{tabular}

Note: Hydrogeologic units include the sandy gravels of the Hanford formation and silty sands of the Ringold Formation. Water levels are measured in all wells monthly.

"Wells 399-2-1 and 399-3-10 are not RCRA standard wells. The results are used as a supplemental source of data only. 
Table 16-2. RCRA Water Leve] Measurement Report 300 Area Process Trenches, Fourth Quarter 1994. (sheet 1 of 5)

Hel1 . Date $\quad \begin{gathered}\text { Depth to } \\
\text { water (ft) }\end{gathered}$\begin{tabular}{c}
$\begin{array}{c}\text { Water level } \\
\text { elevation above ms } \\
\text { (ft) }\end{array}$ \\
\hline
\end{tabular}

Wells Monitoring the Top of the Unconfined Aquifer

399-1-1

$399-1-11$

$399-1-12$

399-1-14A

$399-1-15$

$399-1-16 A$

399-1-17A

$399-1-18 A$
$10 / 25 / 94$

$11 / 22 / 94$

$12 / 19 / 94$

$10 / 05 / 94$

$10 / 25 / 94$

$11 / 22 / 94$

$12 / 19 / 94$

$10 / 05 / 94$

$11 / 22 / 94$

$12 / 19 / 94$

$10 / 06 / 94$

$10 / 25 / 94$

$11 / 22 / 94$

$12 / 19 / 94$

10/05/94

$10 / 25 / 94$

$11 / 22 / 94$

$12 / 20 / 94$

$10 / 25 / 94$

$11 / 22 / 94$

$12 / 19 / 94$

10/07/94

$10 / 25 / 94$

$11 / 22 / 94$

$12 / 19 / 94$

10/07/94

10/25/94

$11 / 22 / 94$

$12 / 15 / 94$

$12 / 19 / 94$

$10 / 05 / 94$

$10 / 25 / 94$
35.49

34.61

35.15

32.94

32.35

31.55

32.15

36.77

35.61

35.49

43.88

43.43

42.58

42.48

41.02

41.72

41.03

40.87

37.96

37.25

37.02

41.16

40.60

39.69

40.07

35.86

36.54

35.66

34.72

35.69

49.06

48.77
341.17

342.05

341.51

$340.63^{*}$

341.22

342.02

341.42

340.95*

342.11

342.23

$340.53 *$

340.98

341.83

341.93

342.21 *

341.51

342.20

342.36

341.57

342.28

342.51

340.31 *

340.87

341.78

341.40

$341.58 *$

340.90

341.78

$342.72^{\star}$

341.75

$341.72 *$

342.01
103.99

104.26

104.09

103.82

104.00

104.25

104.06

103.92

104.28

104.31

103.79

103.93

104.19

104.22

104.31

104.09

104.30

104.35

104.11

104.33

104.40

103.73

103.90

104.17

104.06

104.11

103.91

104.17

104.46

104.17

104.16

104.24 
Table 16-2. RCRA Nater Level Heasurement Report 300 Area Process Trenches, Fourth Quarter 1994. (sheet 2 of 5 )

\begin{tabular}{|c|c|c|c|c|}
\hline We]l & Date & $\begin{array}{l}\text { Depth to } \\
\text { water (ft) }\end{array}$ & $\begin{array}{l}\text { Water } \\
\text { elevation } \\
\text { (ft) }\end{array}$ & $\begin{array}{l}\text { leve] } \\
\text { above asl } \\
\text { (in) }\end{array}$ \\
\hline \multicolumn{5}{|c|}{ Wells Monitoring the Top of the Unconfined Aquifer } \\
\hline 399-1-18A & $\begin{array}{l}11 / 22 / 94 \\
12 / 20 / 94\end{array}$ & $\begin{array}{l}48.28 \\
47.96\end{array}$ & $\begin{array}{l}342.50 \\
342.82\end{array}$ & $\begin{array}{l}104.39 \\
104.49\end{array}$ \\
\hline $399-1-19$ & $\begin{array}{l}10 / 25 / 94 \\
11 / 22 / 94 \\
12 / 19 / 94\end{array}$ & $\begin{array}{l}33.59 \\
32.75 \\
32.65\end{array}$ & $\begin{array}{l}341.05 \\
341.89 \\
341.99\end{array}$ & $\begin{array}{l}103.95 \\
104.21 \\
104.24\end{array}$ \\
\hline 399-1-3 & $\begin{array}{l}10 / 25 / 94 \\
11 / 22 / 94 \\
12 / 19 / 94\end{array}$ & $\begin{array}{l}43.80 \\
42.90 \\
43.18\end{array}$ & $\begin{array}{l}340.91 \\
341.81 \\
341.53\end{array}$ & $\begin{array}{l}103.91 \\
104.18 \\
104.10\end{array}$ \\
\hline $399-1-4$ & $\begin{array}{l}10 / 25 / 94 \\
11 / 22 / 94 \\
12 / 20 / 94\end{array}$ & $\begin{array}{l}39.20 \\
38.42 \\
38.37\end{array}$ & $\begin{array}{l}341.38 \\
342.16 \\
342.21\end{array}$ & $\begin{array}{l}104.05 \\
104.29 \\
104.31\end{array}$ \\
\hline $399-1-5$ & $\begin{array}{l}10 / 25 / 94 \\
11 / 22 / 94 \\
12 / 19 / 94\end{array}$ & $\begin{array}{l}38.79 \\
37.93 \\
37.86\end{array}$ & $\begin{array}{l}340.98 \\
341.84 \\
341.91\end{array}$ & $\begin{array}{l}103.93 \\
104.19 \\
104.21\end{array}$ \\
\hline $399-1-7$ & $\begin{array}{l}10 / 25 / 94 \\
11 / 22 / 94 \\
12 / 19 / 94\end{array}$ & $\begin{array}{l}44.70 \\
43.79 \\
44.19\end{array}$ & $\begin{array}{l}340.90 \\
341.81 \\
341.41\end{array}$ & $\begin{array}{l}103.91 \\
104.18 \\
104.06\end{array}$ \\
\hline $399-1-8$ & $\begin{array}{l}10 / 25 / 94 \\
11 / 22 / 94 \\
12 / 19 / 94\end{array}$ & $\begin{array}{l}43.98 \\
43.08 \\
43.28\end{array}$ & $\begin{array}{l}340.90 \\
341.80 \\
341.60\end{array}$ & $\begin{array}{l}103.91 \\
104.18 \\
104.12\end{array}$ \\
\hline $399-2-1$ & $\begin{array}{l}10 / 05 / 94 \\
10 / 25 / 94 \\
11 / 22 / 94 \\
12 / 19 / 94\end{array}$ & $\begin{array}{l}33.81 \\
34.34 \\
33.56 \\
34.05\end{array}$ & $\begin{array}{l}341.42^{\star} \\
340.89 \\
341.67 \\
341.18\end{array}$ & $\begin{array}{l}104.06 \\
103.90 \\
104.14 \\
103.99\end{array}$ \\
\hline $399-2-2$ & $\begin{array}{l}10 / 25 / 94 \\
11 / 22 / 94 \\
12 / 19 / 94\end{array}$ & $\begin{array}{l}36.61 \\
35.75 \\
36.18\end{array}$ & $\begin{array}{l}340.91 \\
341.77 \\
341.34\end{array}$ & $\begin{array}{l}103.91 \\
104.17 \\
104.04\end{array}$ \\
\hline $399-2-3$ & $\begin{array}{l}10 / 25 / 94 \\
11 / 22 / 94 \\
12 / 19 / 94\end{array}$ & $\begin{array}{l}34.50 \\
33.64 \\
34.13\end{array}$ & $\begin{array}{l}340.92 \\
341.78 \\
341.29\end{array}$ & $\begin{array}{l}103.91 \\
104.17 \\
104.03\end{array}$ \\
\hline
\end{tabular}


Table 16-2. RCRA Water Level Measurement Report 300 Area Process Trenches, Fourth Quarter 1994. (sheet 3 of 5)

\begin{tabular}{|c|c|c|c|c|}
\hline Wel1 & Date & $\begin{array}{l}\text { Depth to } \\
\text { water (ft) }\end{array}$ & $\begin{array}{l}\text { Water } \\
\text { elevation } \\
\text { (ft) }\end{array}$ & $\begin{array}{l}\text { level } \\
\text { above ms1 } \\
\text { (m) }\end{array}$ \\
\hline \multicolumn{5}{|c|}{ Heils Monitoring the Top of the Unconfined Aquifer } \\
\hline $\begin{array}{c}\text { 399-3-1 } \\
\end{array}$ & $\begin{array}{l}10 / 25 / 94 \\
11 / 22 / 94 \\
12 / 20 / 94\end{array}$ & $\begin{array}{r}43.62 \\
42.78 \\
43.08\end{array}$ & $\begin{array}{l}340.77 \\
341.61 \\
341.31\end{array}$ & $\begin{array}{l}103.87 \\
104.12 \\
104.03\end{array}$ \\
\hline $399-3-10$ & $\begin{array}{l}10 / 10 / 94 \\
10 / 25 / 94 \\
11 / 21 / 94 \\
12 / 20 / 94\end{array}$ & $\begin{array}{r}145.03 \\
44.67 \\
44.16 \\
44.16\end{array}$ & $\begin{array}{l}240.32 *+ \\
340.68 \\
341.19 \\
341.19\end{array}$ & $\begin{array}{r}73.25 \\
103.84 \\
103.99 \\
103.99\end{array}$ \\
\hline $399-3-6$ & $\begin{array}{l}10 / 25 / 94 \\
11 / 21 / 94 \\
12 / 20 / 94\end{array}$ & $\begin{array}{l}51.98 \\
51.23 \\
51.18\end{array}$ & $\begin{array}{l}340.82 \\
341.57 \\
341.62\end{array}$ & $\begin{array}{l}103.88 \\
104.11 \\
104.13\end{array}$ \\
\hline $399-3-9$ & $\begin{array}{l}10 / 25 / 94 \\
11 / 21 / 94 \\
12 / 20 / 94\end{array}$ & $\begin{array}{l}47.38 \\
46.80 \\
46.78\end{array}$ & $\begin{array}{l}340.70 \\
341.28 \\
341.30\end{array}$ & $\begin{array}{l}103.85 \\
104.02 \\
104.03\end{array}$ \\
\hline $399-4-1$ & $\begin{array}{l}10 / 25 / 94 \\
11 / 21 / 94 \\
12 / 20 / 94\end{array}$ & $\begin{array}{l}55.02 \\
54.38 \\
54.44\end{array}$ & $\begin{array}{l}340.58 \\
341.22 \\
341.16\end{array}$ & $\begin{array}{l}103.81 \\
104.00 \\
103.99\end{array}$ \\
\hline $399-4-10$ & $\begin{array}{l}10 / 25 / 94 \\
11 / 21 / 94 \\
12 / 20 / 94\end{array}$ & $\begin{array}{l}37.71 \\
37.40 \\
37.41\end{array}$ & $\begin{array}{l}340.83 \\
341.14 \\
341.13\end{array}$ & $\begin{array}{l}103.88 \\
103.98 \\
103.98\end{array}$ \\
\hline $399-4-11$ & $\begin{array}{l}10 / 25 / 94 \\
11 / 21 / 94 \\
12 / 20 / 94\end{array}$ & $\begin{array}{l}63.84 \\
63.11 \\
63.16\end{array}$ & $\begin{array}{l}340 \cdot 61 \\
341.34 \\
341.29\end{array}$ & $\begin{array}{l}103.82 \\
104.04 \\
104.03\end{array}$ \\
\hline $399-4-7$ & $\begin{array}{l}10 / 25 / 94 \\
11 / 21 / 94 \\
12 / 20 / 94\end{array}$ & $\begin{array}{l}37.89 \\
37.46 \\
37.49\end{array}$ & $\begin{array}{l}340.67 \\
341.10 \\
341.07\end{array}$ & $\begin{array}{l}103.84 \\
103.97 \\
103.96\end{array}$ \\
\hline $399-5-1$ & $\begin{array}{l}10 / 25 / 94 \\
11 / 21 / 94 \\
12 / 20 / 94\end{array}$ & $\begin{array}{l}54.52 \\
53.83 \\
53.65\end{array}$ & $\begin{array}{l}341.01 \\
341.70 \\
341.88\end{array}$ & $\begin{array}{l}103.94 \\
104.15 \\
104.21\end{array}$ \\
\hline $399-6-1$ & $\begin{array}{r}10 / 26 / 94 \\
11 / 21 / 94 \\
12 / 20 / 94\end{array}$ & $\begin{array}{r}47.49 \\
46.91 \\
46.97\end{array}$ & $\begin{array}{l}341.30 \\
341.88 \\
341.82\end{array}$ & $\begin{array}{l}104.03 \\
104.21 \\
104.19\end{array}$ \\
\hline
\end{tabular}


Table 16-2. RCRA Water Level Measurement Report 300 Area Process Trenches, Fourth Quarter 1994. (sheet 4 of 5)

\begin{tabular}{|c|c|c|c|}
\hline Well & Date & $\begin{array}{l}\text { Depth to } \\
\text { water (ft) }\end{array}$ & $\begin{array}{l}\text { Water level } \\
\text { elevatjon above msl } \\
\text { (ft) }\end{array}$ \\
\hline
\end{tabular}

Wells Monitoring the Top of the Unconfined Aquifer.

$\begin{array}{ccccc}399-8-1 & 10 / 25 / 94 & 54.92 & 341.21 & 104.00 \\ & 11 / 21 / 94 & 54.29 & 341.84 & 104.19 \\ 12 / 19 / 94 & 53.81 & 342.32 & 104.34 \\ 399-8-2 & 10 / 25 / 94 & 56.06 & 341.96 & 104.23 \\ & 11 / 21 / 94 & 55.56 & 342.46 & 104.38 \\ & 12 / 20 / 94 & 55.08 & 342.94 & 104.53 \\ 399-8-3 & 10 / 25 / 94 & 53.25 & 341.50 & 104.09 \\ & 11 / 22 / 94 & 52.71 & 342.04 & 104.25 \\ & 12 / 19 / 94 & 52.12 & 342.53 & 104.43 \\ 699-527-E 14 & 10 / 25 / 94 & 59.16 & 340.52 & 103.79 \\ & 11 / 21 / 94 & 58.48 & 341.20 & 1.04 .00 \\ & 12 / 20 / 94 & 58.59 & 341.09 & 103.96\end{array}$

We1ls Monitoring the Bottom of the Unconfined Aquifer

\begin{tabular}{|c|c|c|c|c|}
\hline $399-1-16 \mathrm{~B}$ & $\begin{array}{r}10 / 10 / 94 \\
-10 / 25 / 94 \\
11 / 22 / 94 \\
12 / 19 / 94\end{array}$ & $\begin{array}{l}39.54 \\
40.09 \\
39.19 \\
39.60\end{array}$ & $\begin{array}{l}341.54 \\
340.99 \\
341.89 \\
341.48\end{array}$ & $\begin{array}{l}104.10 \\
103.93 \\
104.21 \\
104.08\end{array}$ \\
\hline $399-1-17 \mathrm{~B}$ & $\begin{array}{l}10 / 07 / 94 \\
10 / 25 / 94 \\
11 / 22 / 94 \\
12 / 19 / 94\end{array}$ & $\begin{array}{l}37.34 \\
36.80 \\
35.91 \\
35.97\end{array}$ & $\begin{array}{l}340.46^{\star} \\
341.00 \\
341.89 \\
341.83\end{array}$ & $\begin{array}{l}103.77 \\
103.94 \\
104.21 \\
104.19\end{array}$ \\
\hline $399-1-18 B$ & $\begin{array}{l}10 / 25 / 94 \\
11 / 22 / 94 \\
12 / 20 / 94\end{array}$ & $\begin{array}{l}47.55 \\
46.89 \\
46.81\end{array}$ & $\begin{array}{l}342.34 \\
343.00 \\
343.08\end{array}$ & $\begin{array}{l}104.35 \\
104.55 \\
104.57\end{array}$ \\
\hline
\end{tabular}

Wells Monjtoring the Confined Aquifer

$399-1-17 C$

$$
\begin{aligned}
& 10 / 25 / 94 \\
& 11 / 22 / 94 \\
& 12 / 19 / 94
\end{aligned}
$$

2.55

2.92

3.22

375.51

375.14

374.84

114.46

114.34

114.25 
Table 16-2. RCRA Water Level Measurement Report

300 Area Process Trenches, Fourth Quarter 1994. (sheet 5 of 5 )

\begin{tabular}{|c|c|c|c|c|}
\hline Well & Date & $\begin{array}{l}\text { Depth to } \\
\text { water (ft) }\end{array}$ & $\begin{array}{l}\text { Hater } \\
\text { eTevation } \\
\text { (ft) }\end{array}$ & $\begin{array}{l}\text { level } \\
\text { above ms } 1 \\
\qquad(m)\end{array}$ \\
\hline & \multicolumn{3}{|c|}{ Wells Monitoring the Confined Aquifer } & . \\
\hline 399-1-18C & $\begin{array}{l}10 / 25 / 94 \\
11 / 22 / 94 \\
12 / 20 / 94\end{array}$ & $\begin{array}{l}45.11 \\
44.47 \\
44.32\end{array}$ & $\begin{array}{l}342.90 \\
343.54 \\
343.69\end{array}$ & $\begin{array}{l}104.52 \\
104.71 \\
104.76\end{array}$ \\
\hline $399-1-9$ & $\begin{array}{l}10 / 25 / 94 \\
11 / 22 / 94\end{array}$ & $\begin{array}{l}27.21 \\
26.83\end{array}$ & $\begin{array}{l}357.55 \\
357.93\end{array}$ & $\begin{array}{l}108.98 \\
109.10\end{array}$ \\
\hline
\end{tabular}

MOTES: 1. Water level elevations are calculated by subtracting the measured depth-to-water from the surveyed elevation for the well.

2. Depth-to-water values are transcribed from field records.

3. Elevations marked with an $* 1$ were measured at the time of sampling.

4. Elevations marked with a ' + ' are outside of the expected range, and are suspected of error. 
Table 16-3. Constituent List and Summary of Results for the 300 Area Process Trenches Data for Reporting Pertod October 1 through December $31,1994$.

(sheet 1 of 3)

COMTANLHATIOO IUDICATOR PARAMETERS

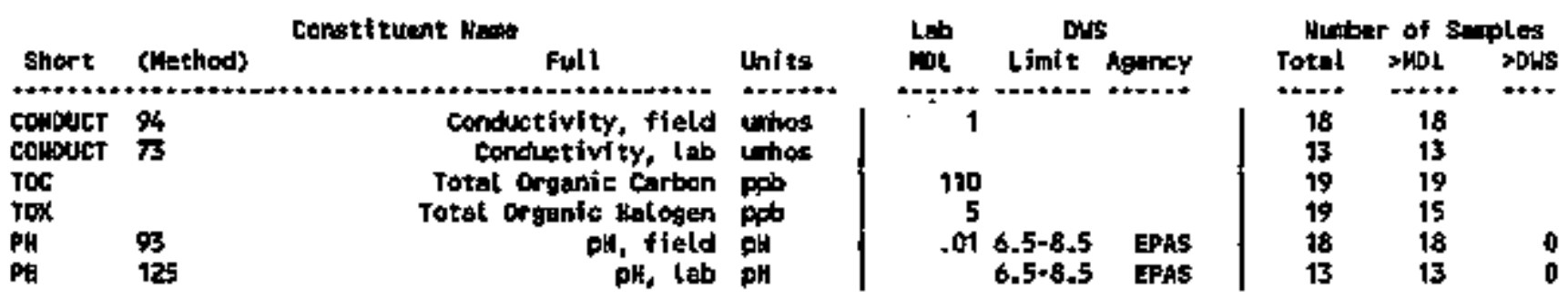

DRInKTHo WAtER PARAMRTERS

\begin{tabular}{|c|c|c|c|c|c|c|c|c|c|}
\hline & & Constituant Nape & & Let & on & & mith & r of $s$ & ples \\
\hline short & (Nethod) & Ful l & Units: & DLt. & Limit & Agancy & Tot:el & HDL & rows \\
\hline ARstujC & & Arsenic & & 2 & n & & & & \\
\hline Farstenc & & Arkenic, filtered & pos & $\begin{array}{l}2.8 \\
2.8\end{array}$ & $\begin{array}{l}30 \\
50\end{array}$ & $\begin{array}{l}\text { EPA } \\
\text { EPA }\end{array}$ & $\begin{array}{l}12 \\
12\end{array}$ & 2 & 0 \\
\hline BARJuK & & Bariun & pot & 59 & 1000 & EPAJ & 12 & $1 \overline{2}$ & 0 \\
\hline FEARIUN & & Bariun, fittered & pow & 59 & 1000 & EPA] & 12 & 12 & 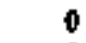 \\
\hline САРнाОМ & & cachiu & pab & 3.3 & 10 & EPAI & 12 & -0 & 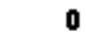 \\
\hline FCApHIJ & & Cudpiun, f f ltered & pob & 3.5 & 10 & EPAA & 12 & $\mathbf{0}$ & $=$ \\
\hline CHRONuts & & Throaliu & $\mathrm{pob}$ & 4.5 & 50 & EPAI & 12 & 12 & 3 \\
\hline FCHow1 & & Chrogiun, filtered & $\mathbf{p} p b$ & 4.5 & 50 & EPA] & 12 & 8 & 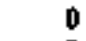 \\
\hline Funkio & & fluoride & pob & 36 & 1400 & EPAT & $\uparrow$ & 1 & - \\
\hline ALPH & & Gross alphe & $\mathrm{pc} \$ / \mathrm{L}$ & & 13 & EPA & 12 & 12 & 8 \\
\hline HERCLRY & & Nercimy & $p$ & .05 & 2 & EPA & $1 \mathbf{z}$ & 5 & 0 \\
\hline FMEREUR & & Mereury, ffltered & apple & .05 & & EPA & 12 & 4 & Q \\
\hline NITRATE & & If trate & pos & 11 & 65000 & EPA & 1 & 1 & 0 \\
\hline adbutu & & Radiun & pcis & & & EPAI & 12 & 0 & 0 \\
\hline SELEMUA & & sęlenium & ppos & 65 & 10 & EFAI & 12 & 3 & 0 \\
\hline FSELEN] & & selenilu, filtered & Dots & . 65 & 10 & EPAI & 12 & 4 & 0 \\
\hline
\end{tabular}

GROUMDHATEA OUALITT PARANETERS

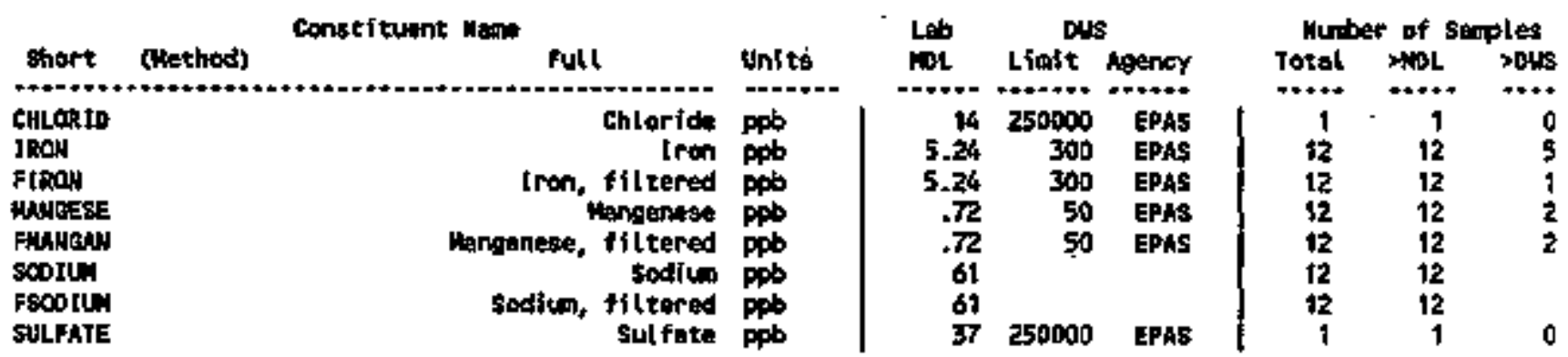


Table 16-3. Constituent List and Summary of Results for the 300 Area Process Trenches Data for Reporting Period October 1 through December 31, 1994.

(sheet 2 of 3)

8ITE SPECIFIC AND OTHER const!TUENTS

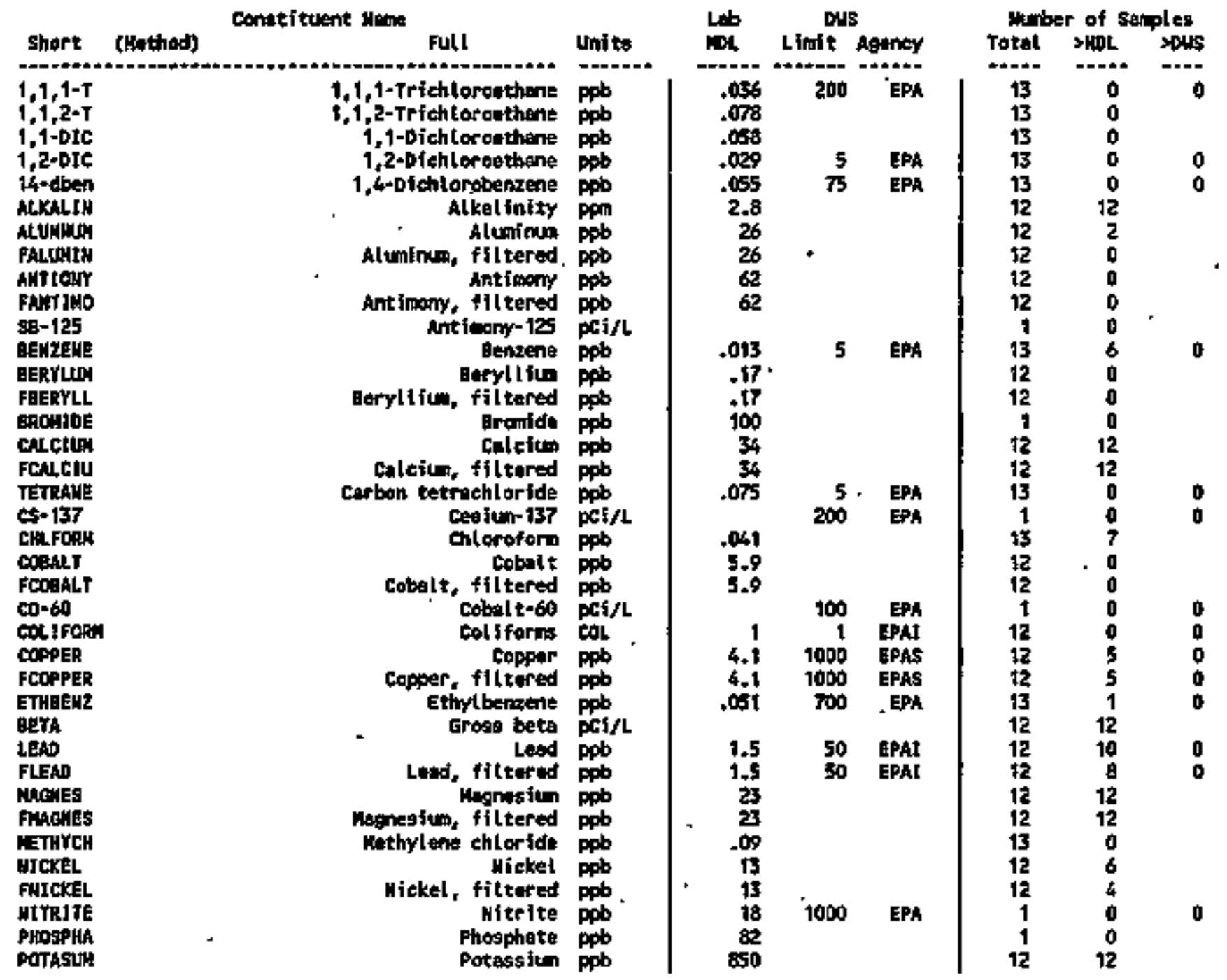


Table 16-3. Constituent List and Sumnary of Results for the 300 Area Process Trenches Data for Reporting Period October 1 through December 31, 1994.

(sheet 3 of 3 )

\begin{tabular}{|c|c|c|c|c|c|c|c|c|c|}
\hline & & Gorstitituart hast & & Leb & DU & & $m$ & $r$ of $s$ & \\
\hline Shapt & (Wethod) & Fuld & Unito & $\operatorname{mot}$ & tinfit & Asenty & Totel & $>$ Hot & rows \\
\hline fPoriat: & & Potessing, filtered & $\infty$ & 850 & & & 12 & 12 & \\
\hline Rit-10s & & Ruthcion-tos & Ei & & 30 & EPA & & 0 & D \\
\hline SILVER & & S1lves & Doph & 3.6 & $\$ 0$ & EPal & & 0 & 0 \\
\hline F\$ILVER & & stlver, fittered & Ppos & 3.6 & 50 & EPA] & 12 & 0 & 0 \\
\hline SR-90 & & struntiu=90 & pсiA & & 8 & EPA & 1 & 1 & e \\
\hline PEREEAE & & Tetrachloroethene & pot & .22 & 5 & EPA & 13 & 9 & 0 \\
\hline FTנH & & $\pi$ in, filtered & pob & 69 & & & 12 & 0 & \\
\hline TQLUENE & & Taluent & ppos & .018 & 1000 & EPh & 13 & 7 & 0 \\
\hline TRJCENE & & Trichlorothene & pos & $.1 \mathrm{t}$ & & EPA & 13 & 8 & 1 \\
\hline TRITIUA & & $\begin{array}{l}\text { Tritiun } \\
\text { tureniug }\end{array}$ & $\underset{p \rightarrow b}{\mathrm{pCi} / \mathrm{L}}$ & 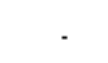 & 20000 & EPh & 12 & 11 & 0 \\
\hline$U-734$ & & Iranitur 234 & $\min$ & & & & 1 & 1 & \\
\hline v-235 & & Uranium-235 & $\mathrm{pCi} / \mathrm{L}$ & & & & 1 & 1 & \\
\hline $\mathbf{v}+238$ & & Uraniuni-2z8 & $\mathrm{PCi} / \mathrm{L}$ & 64 & & & 1 & 1 & \\
\hline FWAFADS & & Venediug, ffltered & $P p D$ & $\begin{array}{l}0.0 \\
6.6\end{array}$ & & & $\begin{array}{l}16 \\
12\end{array}$ & 3 & \\
\hline VIATIDE. & & Vtryt chloride & Dob & .13 & & $E P A$ & 13 & 0 & 0 \\
\hline XYLENE & & Xylanes (tatol) & Ppb & .035 & 10000 & EPA & 13 & 3 & n \\
\hline Fint & & zine, filtered & ppb & 4.3 & 5000 & $\begin{array}{l}\text { EPhS } \\
\text { EPAs }\end{array}$ & 12 & $\begin{array}{r}17 \\
8\end{array}$ & o \\
\hline $\begin{array}{l}\text { CIF120E } \\
\text { TRALDEE }\end{array}$ & - & $\begin{array}{l}\text { efis-1,2-0ithloroethylent } \\
\text { trans-1,2-Dithlorosthylene }\end{array}$ & ppb & .11 & $\begin{array}{r}70 \\
100\end{array}$ & $\begin{array}{l}\text { EPA } \\
\text { EPA }\end{array}$ & $\begin{array}{l}13 \\
13\end{array}$ & 0 & 0 \\
\hline
\end{tabular}

For explention of this table, ses Spation 1,6 of report. 
Table. 16-4. Constituents with at Least One Detected Value for the 300 Area Process Trenches Data for Reporting Period October 1 through Decenber 31, 1994.

(sheet 1 of 8 )

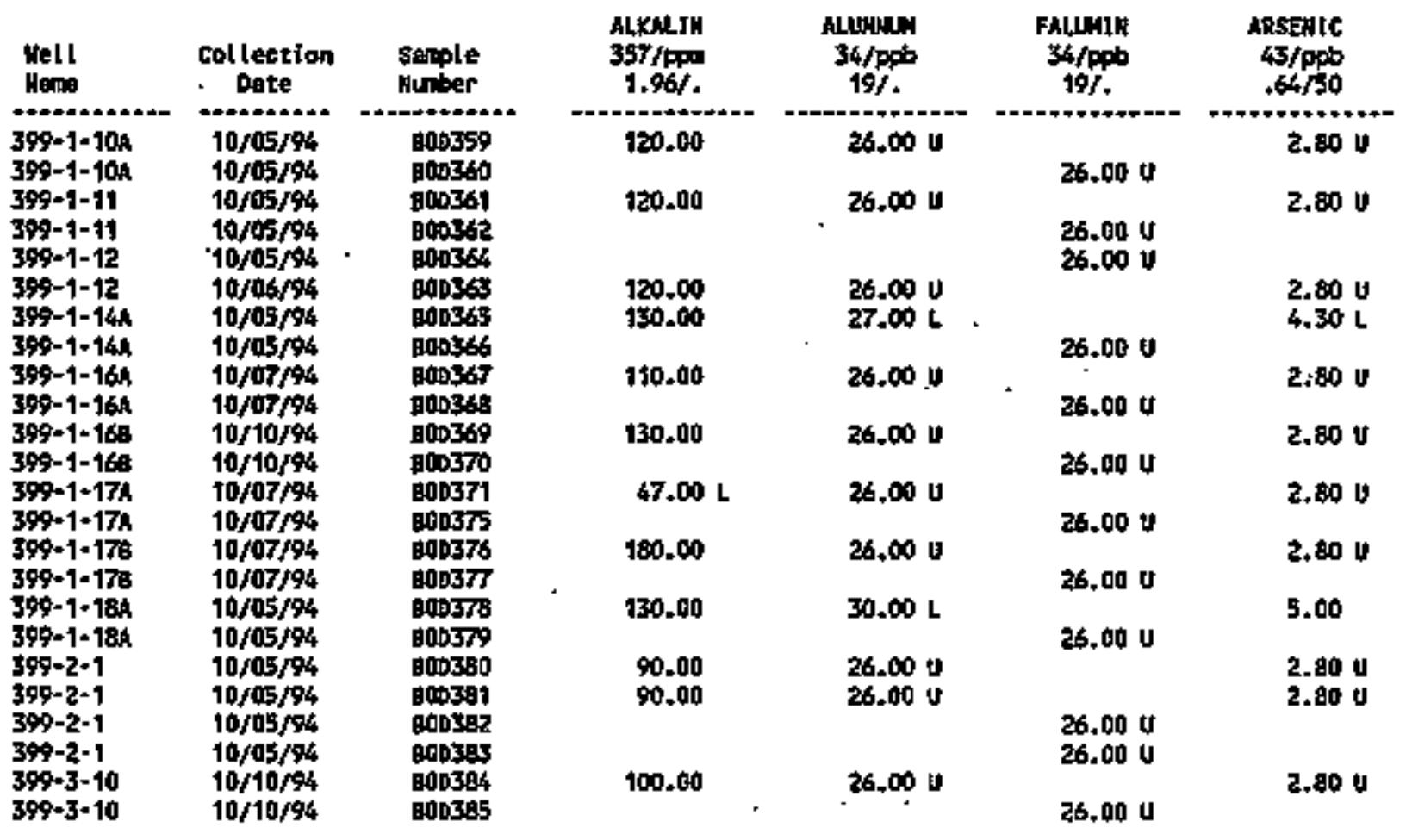

\begin{tabular}{|c|c|c|c|c|c|c|}
\hline Moll & $\begin{array}{c}\text { Collectson } \\
\text { Dete }\end{array}$ & $\begin{array}{l}\text { sample } \\
\text { Hunber }\end{array}$ & $\begin{array}{l}\text { FARSENIC } \\
43 / \mathrm{ppb} \\
.64 / 50\end{array}$ & 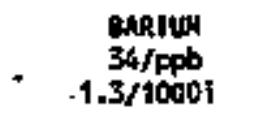 & $\begin{array}{l}\text { FEARIUW } \\
\text { 34/PF' } \\
\text { t.3/100Dit }\end{array}$ & $\begin{array}{l}\text { BENZELE } \\
\text { 25fRpb } \\
.24 / 5\end{array}$ \\
\hline 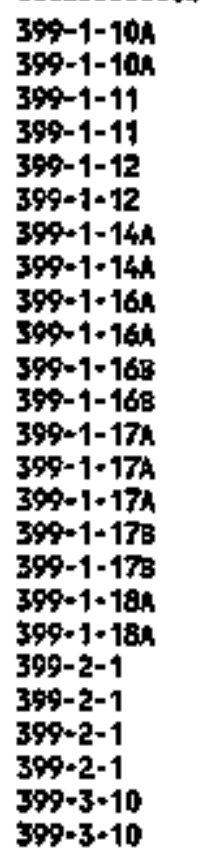 & $\begin{array}{l}10 / 05 / 94 \\
10 / 05 / 94 \\
10 / 05 / 94 \\
10 / 05 / 94 \\
10 / 05 / 94 \\
10 / 05 / 94 \\
10 / 05 / 94 \\
10 / 05 / 94 \\
10 / 07 / 94 \\
10 / 07 / 94 \\
10 / 10 / 94 \\
10 / 10 / 94 \\
10 / 07 / 94 \\
10 / 07 / 94 \\
12 / 15 / 44 \\
10 / 07 / 94 \\
10 / 07 / 94 \\
10 / 05 / 94 \\
10 / 05 / 94 \\
10 / 05 / 94 \\
10 / 05 / 94 \\
10 / 05 / 94 \\
10 / 05 / 94 \\
10 / 10 / 94 \\
10 / 10 / 94\end{array}$ & 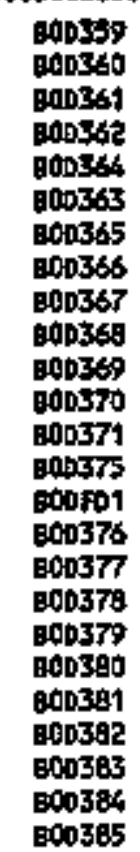 & $\begin{array}{l}2.80 \mathrm{U} \\
2.80 \mathrm{U} \\
2.90 \mathrm{U} \\
3.60 \mathrm{~L} \\
2.60 \mathrm{U} . \\
2.80 \mathrm{U} \\
2.80 \mathrm{U} \\
2.80 \mathrm{U} \\
6.10 \\
2.80 \mathrm{U} \\
2.80 \mathrm{U} \\
2.80 \mathrm{U}\end{array}$ & $\begin{array}{l}53.00 \\
42.00 \\
40.00 \\
41.00 \\
52.00 \\
47.00 \\
28.00 \\
63.00 \\
44.00 \\
63.00 \\
64.00\end{array}$ & $\begin{array}{l}51.00 \\
41.00 \\
40.00 \\
41.00 \\
52.00 \\
47.00 \\
29.00\end{array}$ & 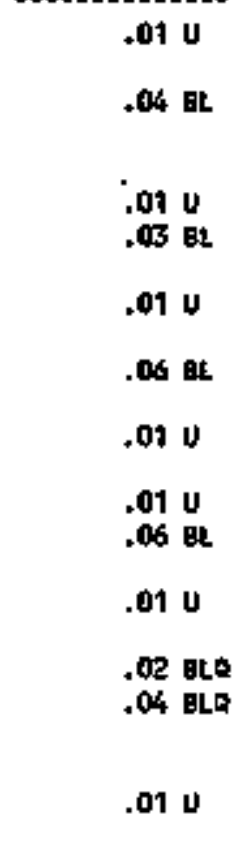 \\
\hline
\end{tabular}


Table 16-4. Constftuents with at Least One Detected VaTue for the 300 Area Process Trenches Data for Reporting Period

October 1 through December 31, 1994.

- (sheet 2 of 8 )

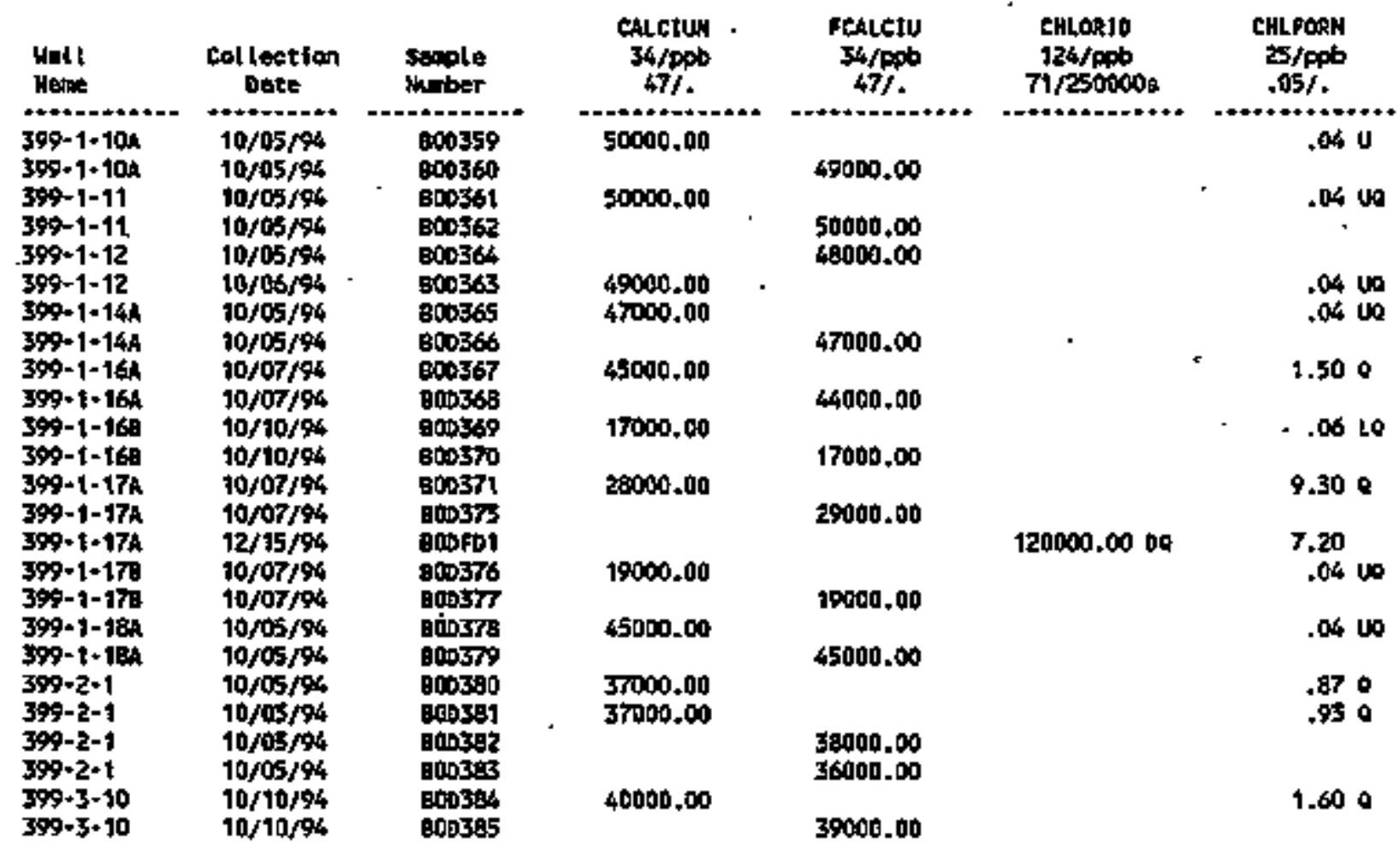


Table 16-4. Constituents with at Least One Detected value for the 300 Area Process Trenches Data for Reporting Period October I through December 31, 1994.

(sheet 3 of 8 )

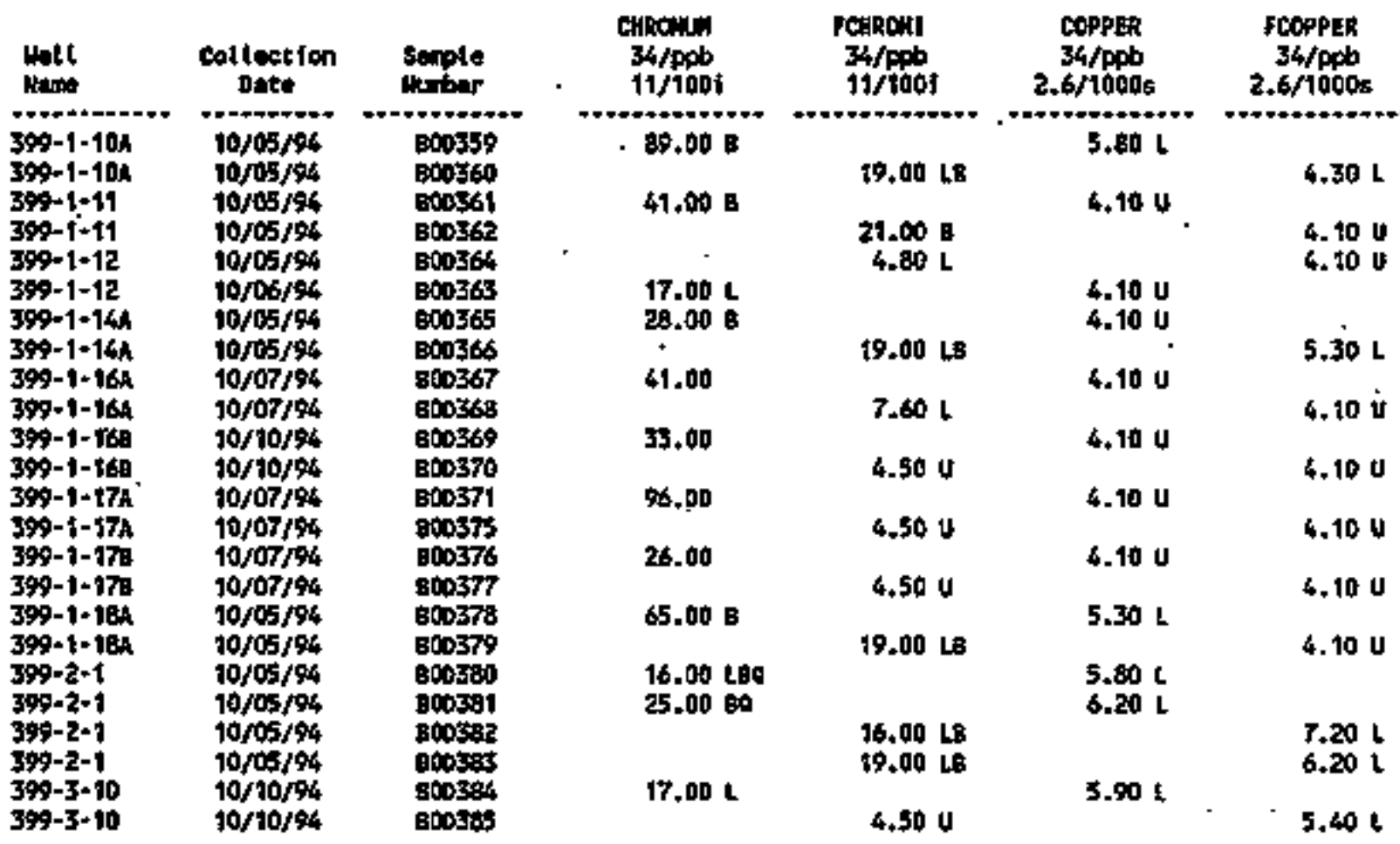

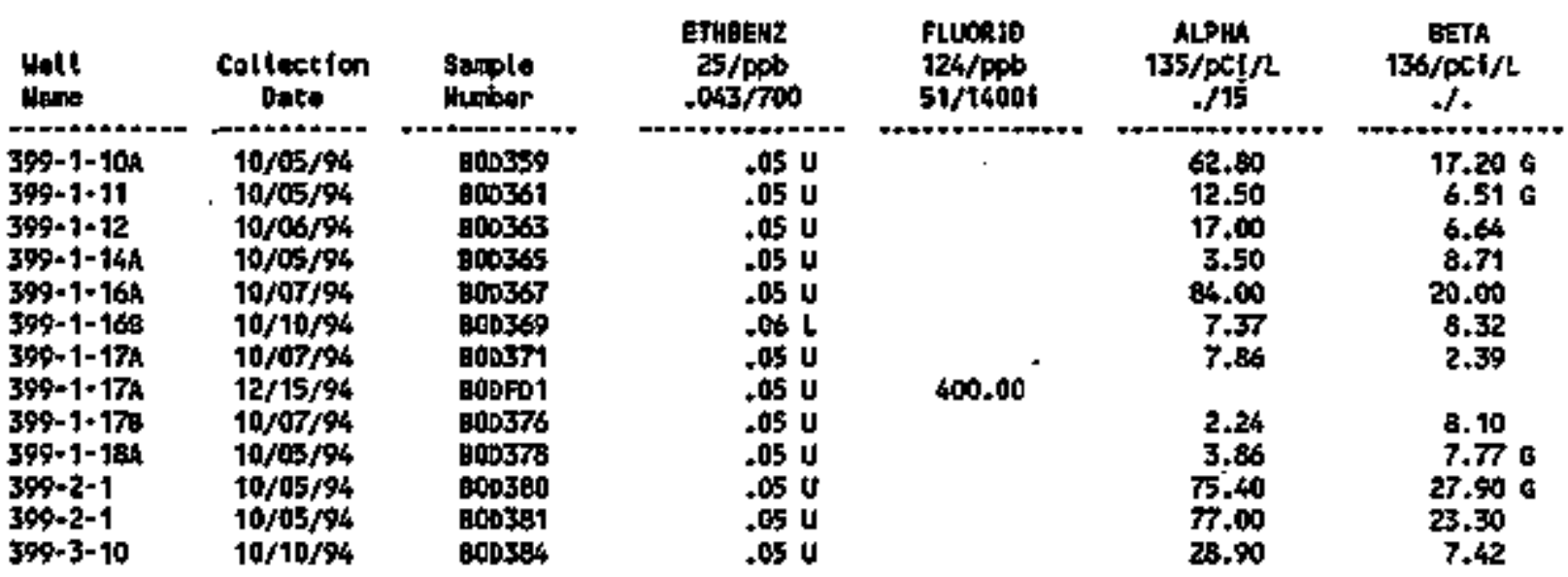


Table 16-4. Constituents with at Least One Detected Value for the 300 Area Process Trenches Data for Reporting Period October I through December 31, 1994.

(sheet 4 of 8 )

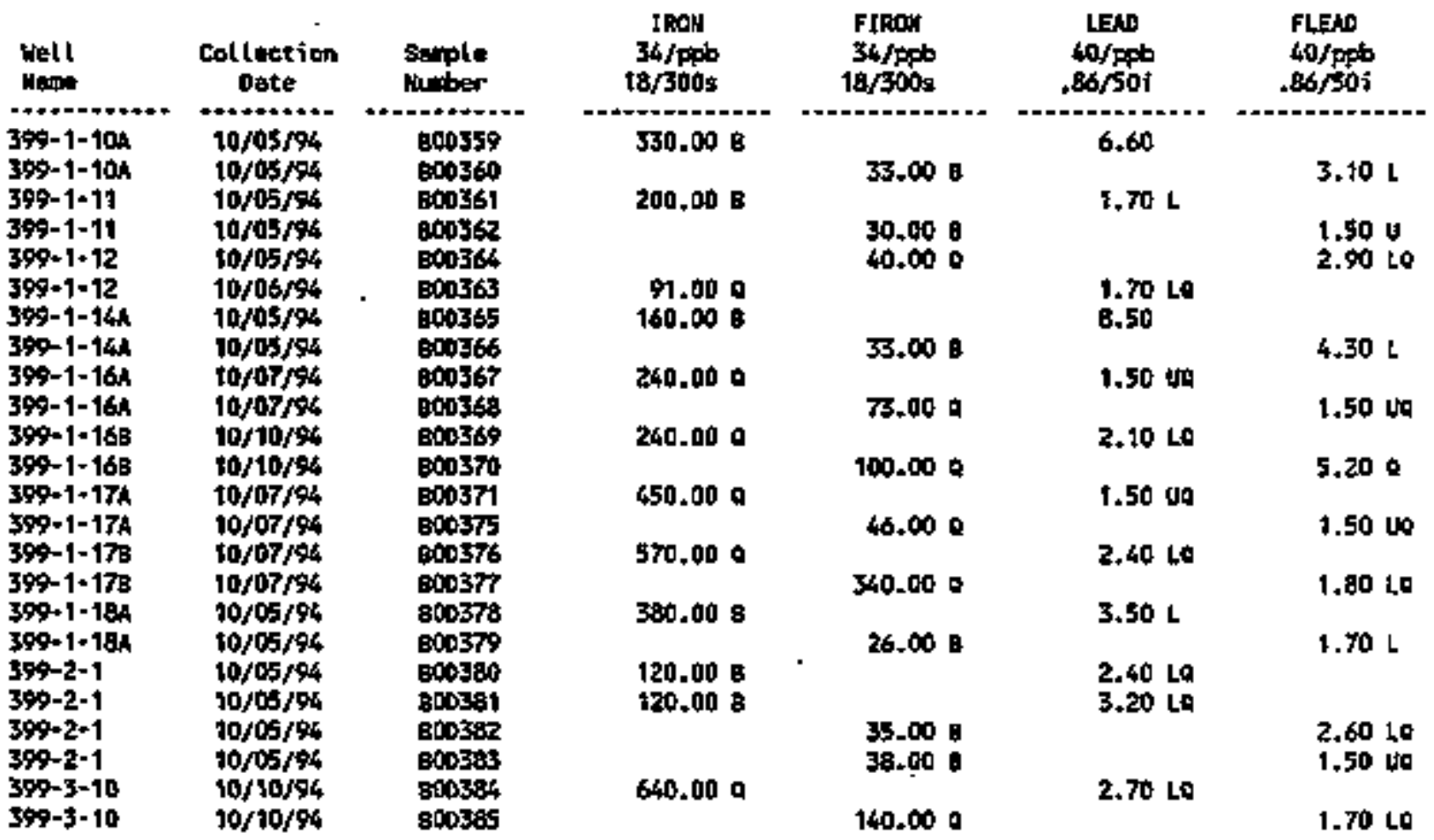

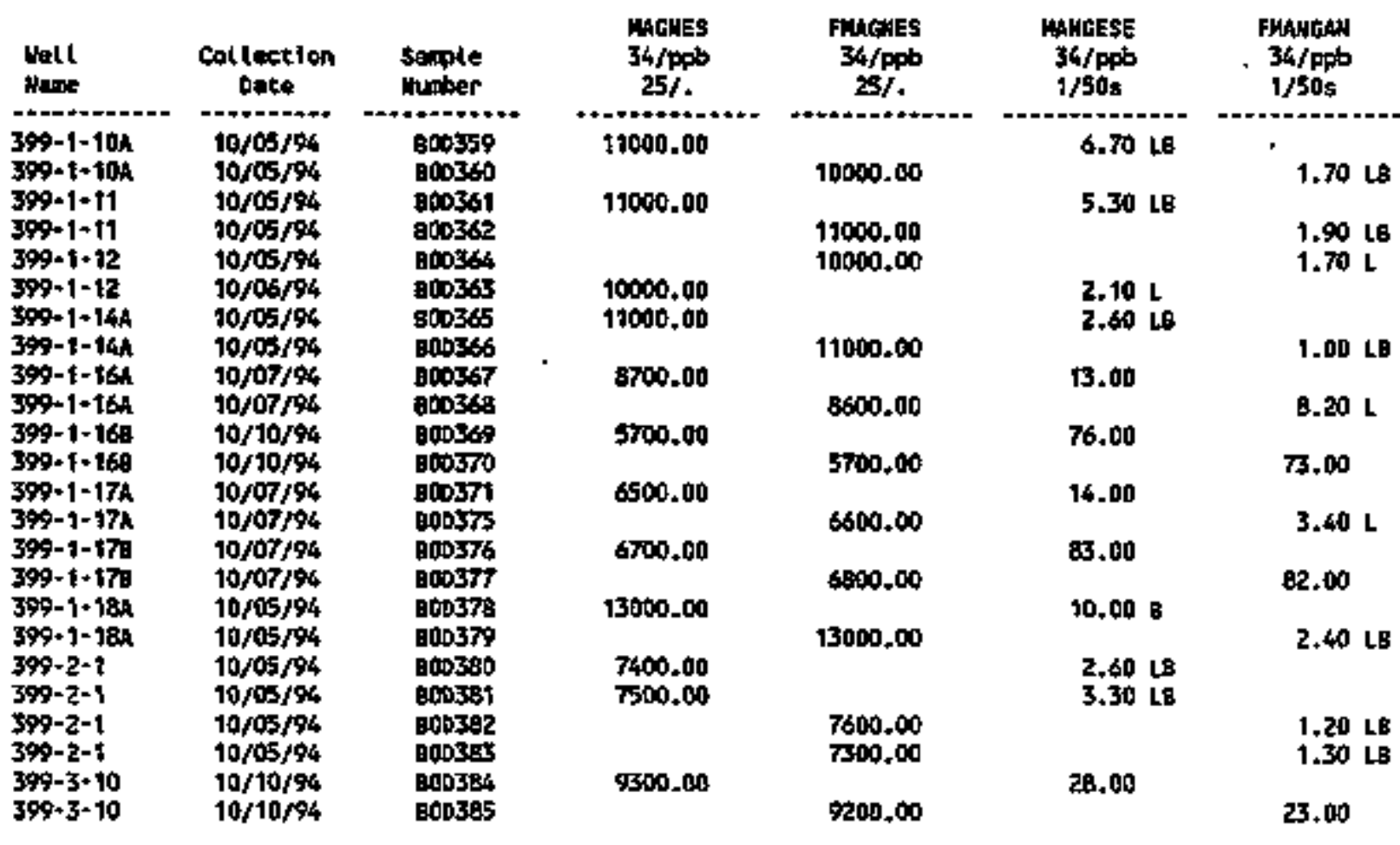


Table 16-4. Constituents with at Least One Detected Value for the 300 Area Process Trenches Data for Reporting Period October 1 through Decenber 31, 1994. (sheet. 5 of 8 )

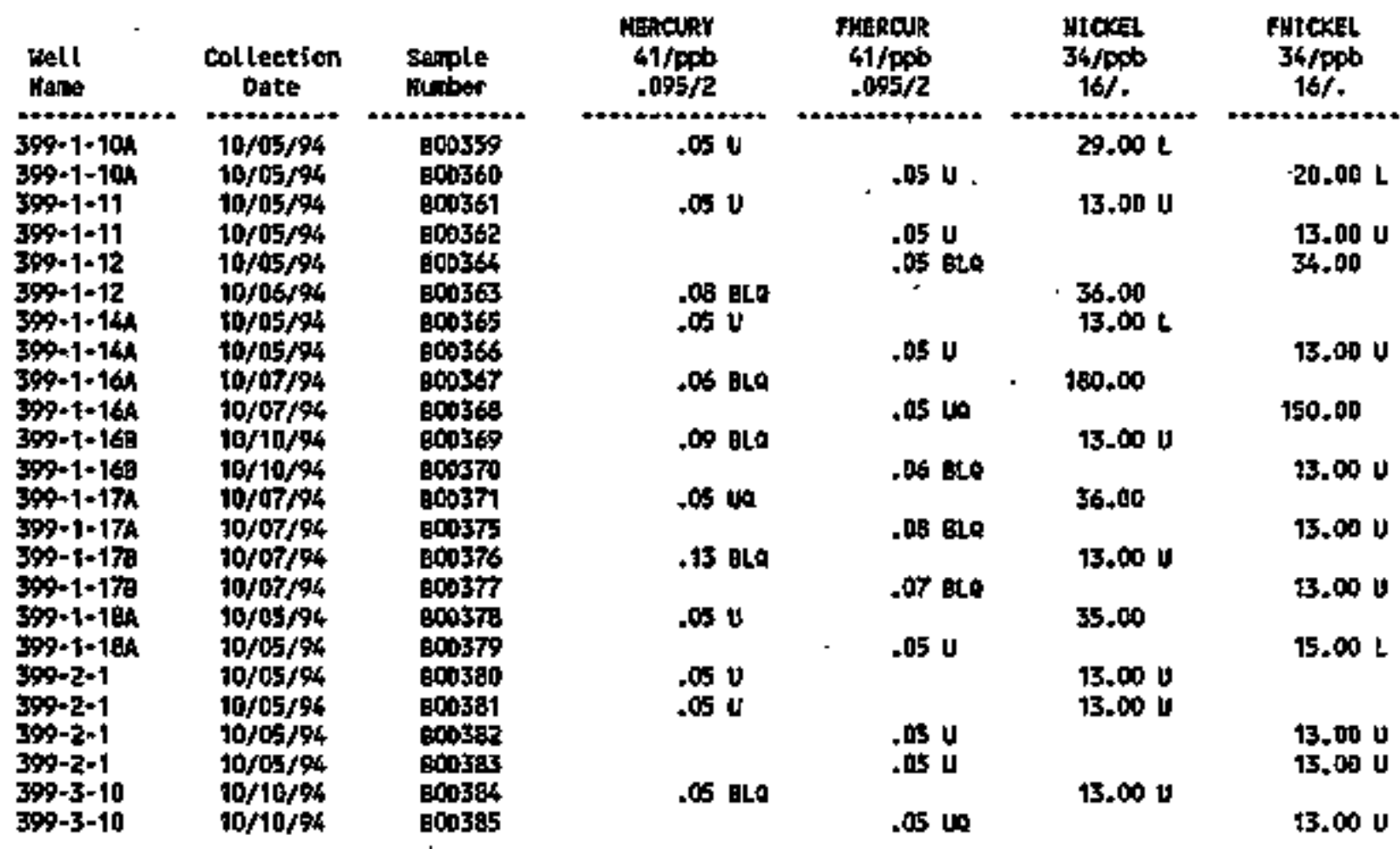

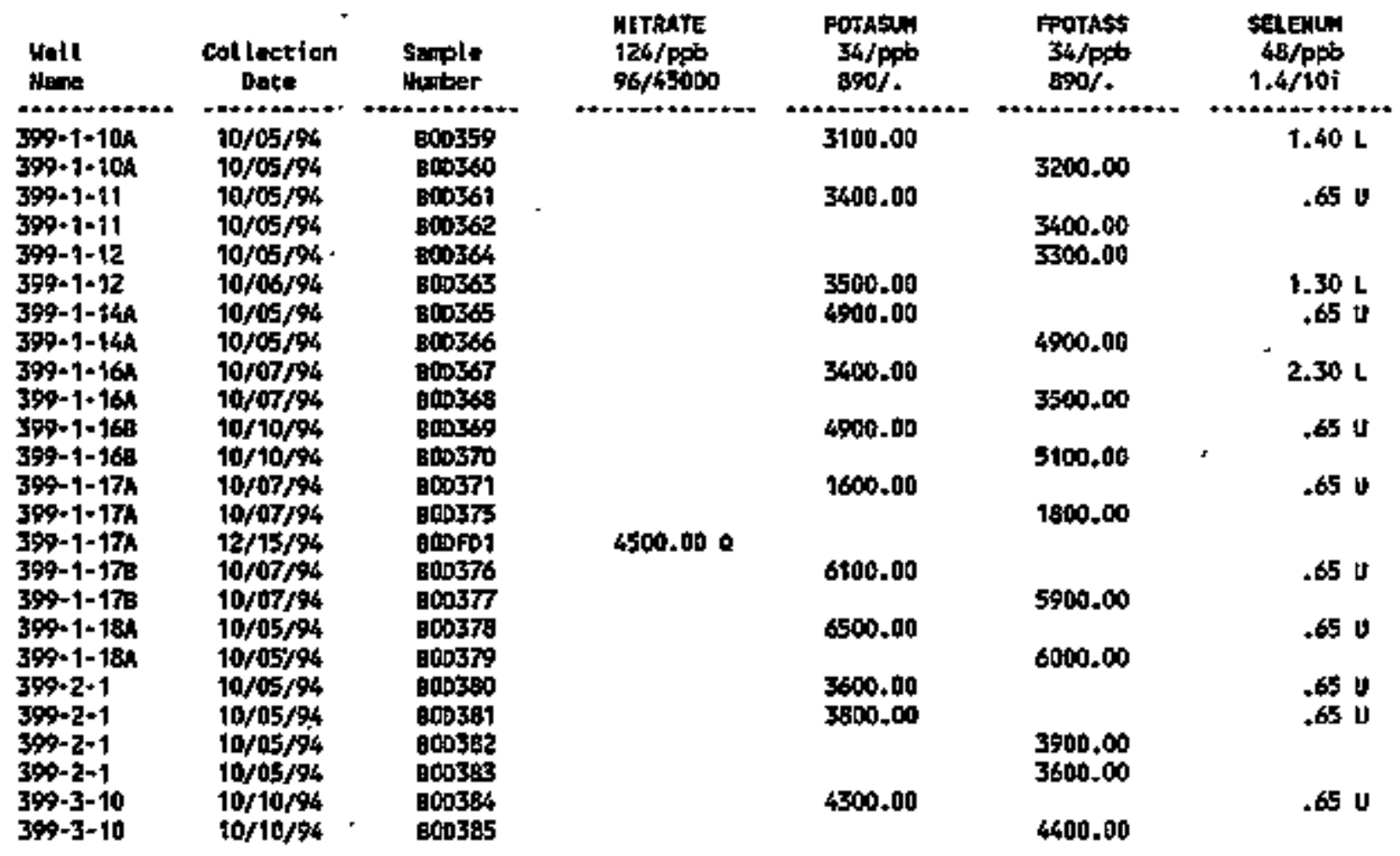


Table 16-4. Constituents with at Least One Detected Value for the 300 Area Process Trenches Data for Reporting Period October 1 through Decenber 31 , 1994.

(sheet 6 of 8 )

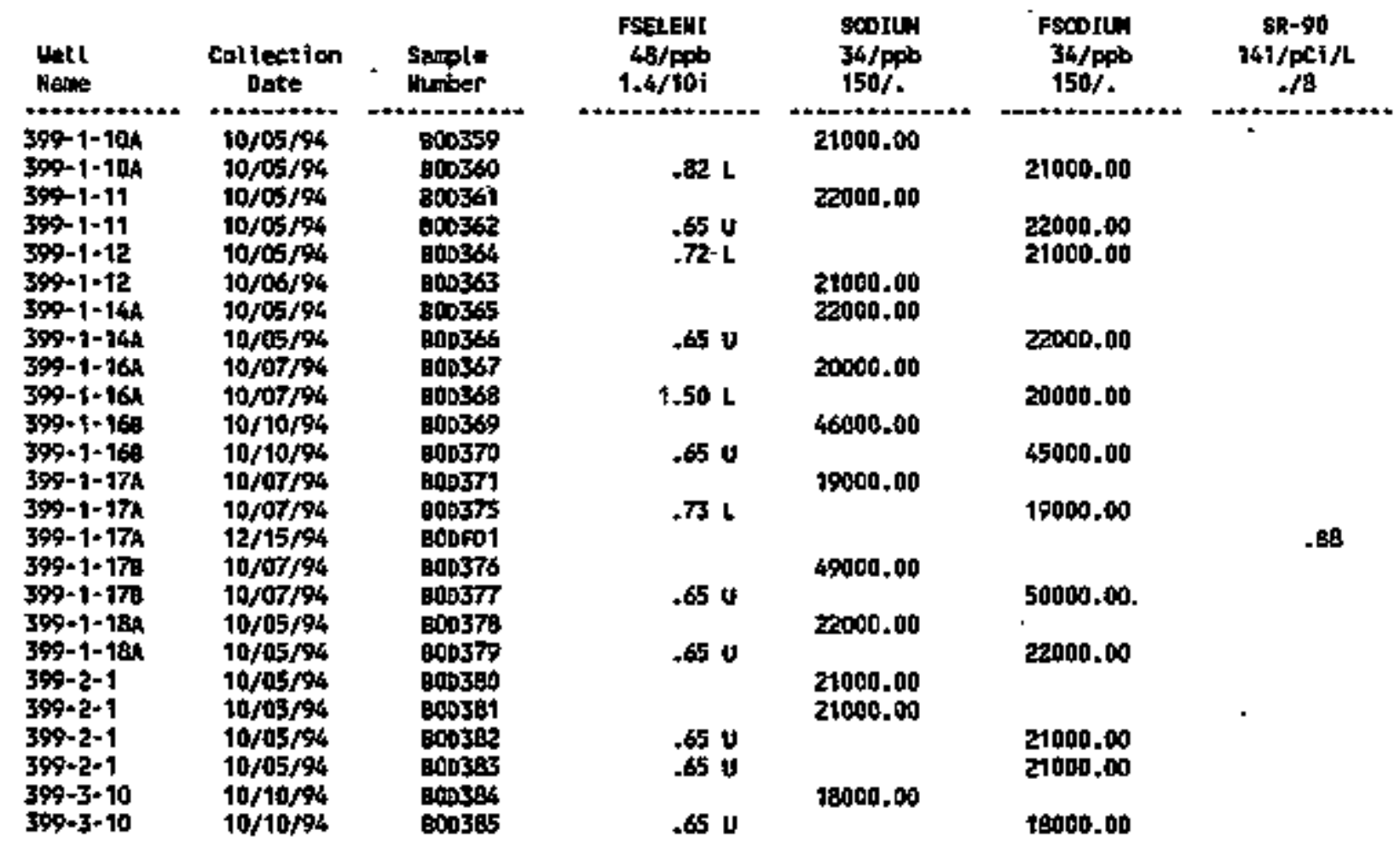

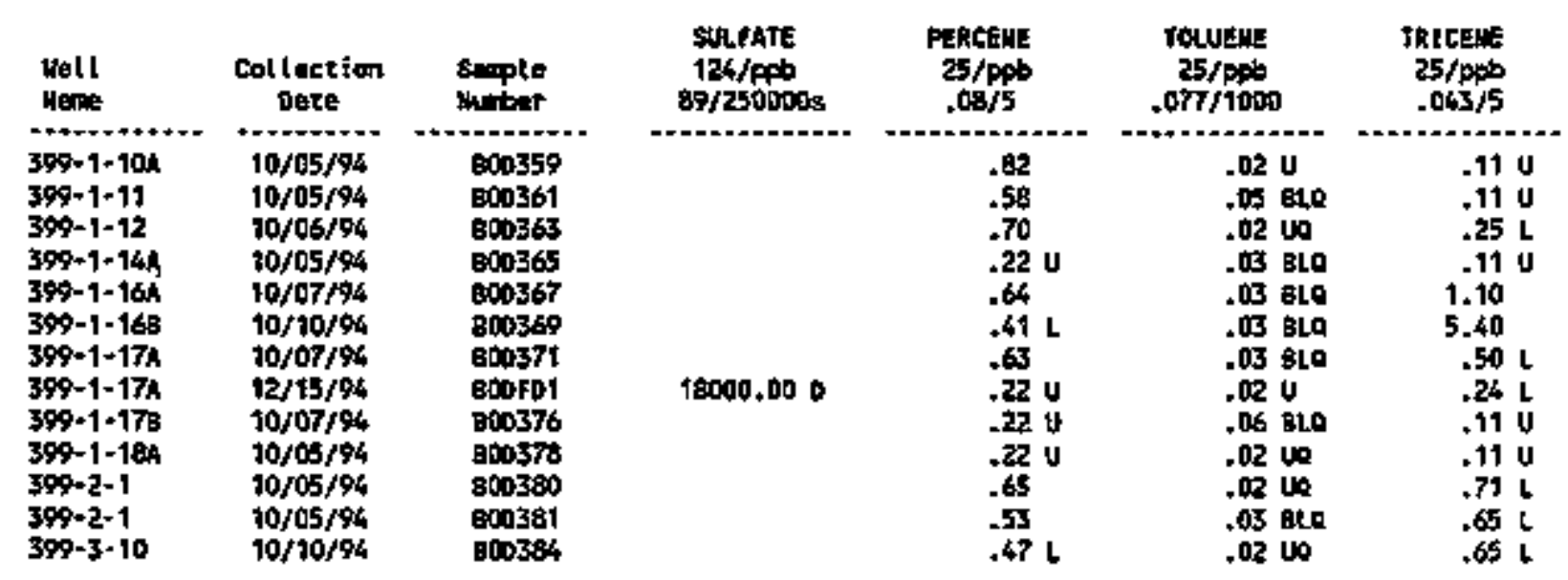


Table 16-4. Constituents with at least One Detected Value for the 300 Area Process Trenches Data for Reporting Period

October 1 through December 31, 1994.

(sheet 7 of 8 )

\begin{tabular}{|c|c|c|c|c|c|c|}
\hline "Wotl & $\begin{array}{c}\text { Collection } \\
\text { Dats }\end{array}$ & Sample & 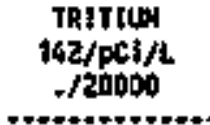 & $\begin{array}{l}\text { urtwiluy } \\
145 / \mathrm{ppb} \\
-1 /\end{array}$ & $\begin{array}{c}1-234 \\
148 / \mathrm{pe} / \mathrm{L} \\
. / 1\end{array}$ & $\begin{array}{c}11-235 \\
148 / \mathrm{pci} / \mathrm{L} \\
-/\end{array}$ \\
\hline $\begin{array}{l}399-1-10 \alpha \\
399-1-11 \\
399-1-12 \\
399-1-12 \\
399-1-16 A \\
399-1-16 A \\
399-1-16 \beta \\
399-1=17 A \\
399-i-17 A \\
399-i=171 \\
399-1-16 A \\
399-2-1 \\
399-2-1 \\
399-3-10\end{array}$ & $\begin{array}{l}10 / 05 / 94 \\
10 / 05 / 94 \\
10 / 05 / 94 \\
10 / 06 / 94 \\
10 / 05 / 94 \\
10 / 07 / 94 \\
10 / 10 / 94 \\
10 / 07 / 94 \\
12 / 15 / 94 \\
10 / 07 / 94 \\
10 / 05 / 94 \\
10 / 05 / 94 \\
10 / 05 / 94 \\
10 / 10 / 94\end{array}$ & $\begin{array}{l}800354 \\
80036 t \\
800363 \\
800363 \\
600365 \\
600367 \\
800369 \\
800371 \\
800 \mathrm{Ft} t \\
800576 \\
800378 \\
800380 \\
600581 \\
800384\end{array}$ & $\begin{array}{r}10900.00 \\
9530.00 \\
8900.00 \\
6900.00 \\
7610.00 \\
6450.00 \\
64.00 \\
226.00 \\
141.00 \mathrm{U} \\
11500.00 \\
4510.00 \\
4490.00 \\
3750.00\end{array}$ & $\begin{array}{r}25.80 \\
14.40 \\
19.90 \\
19.90 \\
3.68 \\
10.90 \\
5.52 \\
9.17 \\
44.60 \\
. .20 \\
3.97 \\
31.30 \\
\$ 3.40 \\
17.60\end{array}$ & 24.80 &. \\
\hline
\end{tabular}

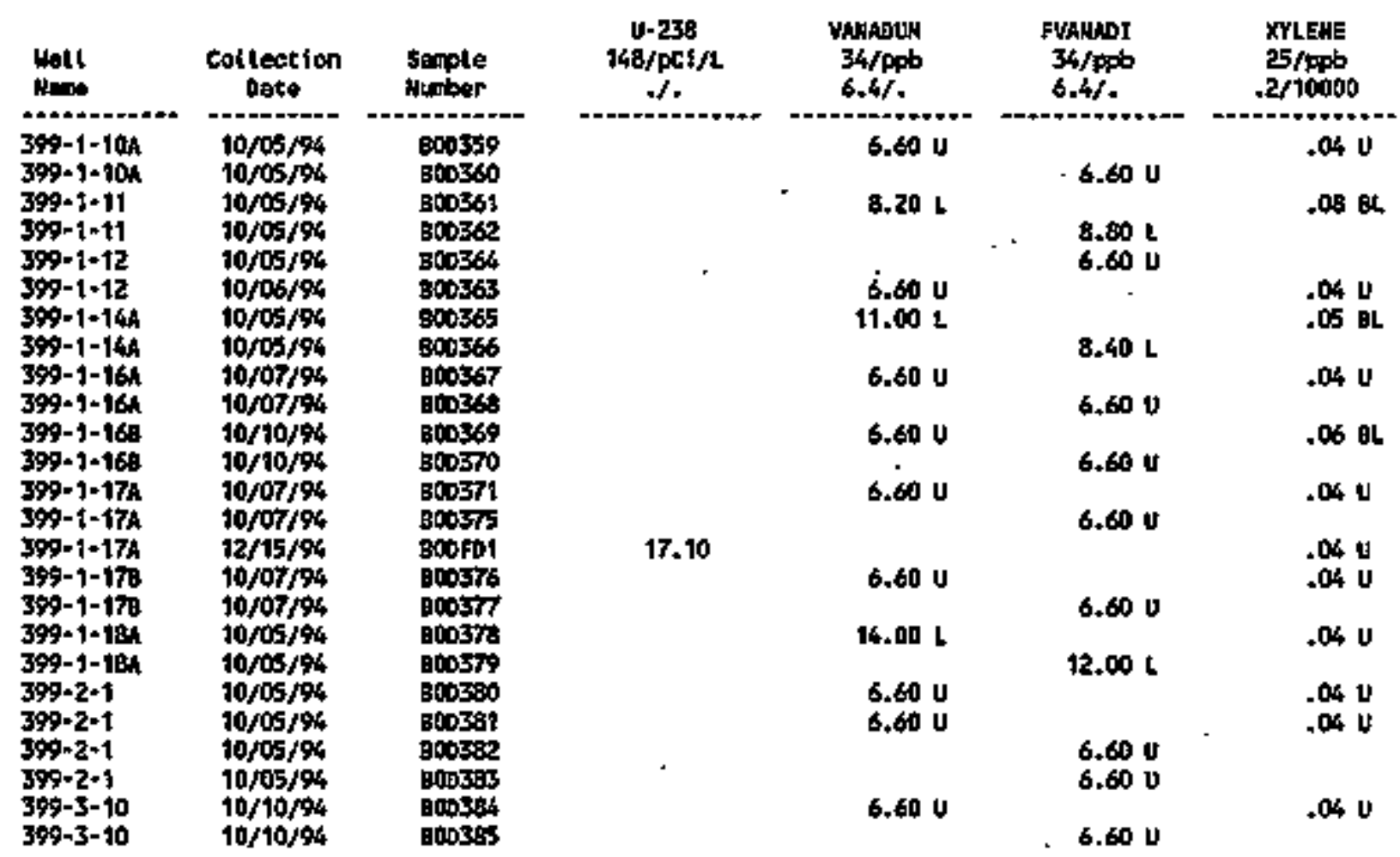


Table 16-4. Constituents with at Least One Betected Value for the 300 Area Process Trenches Data for Reporting Period October 1 through December 31, 1994. (sheet 8 of 8)

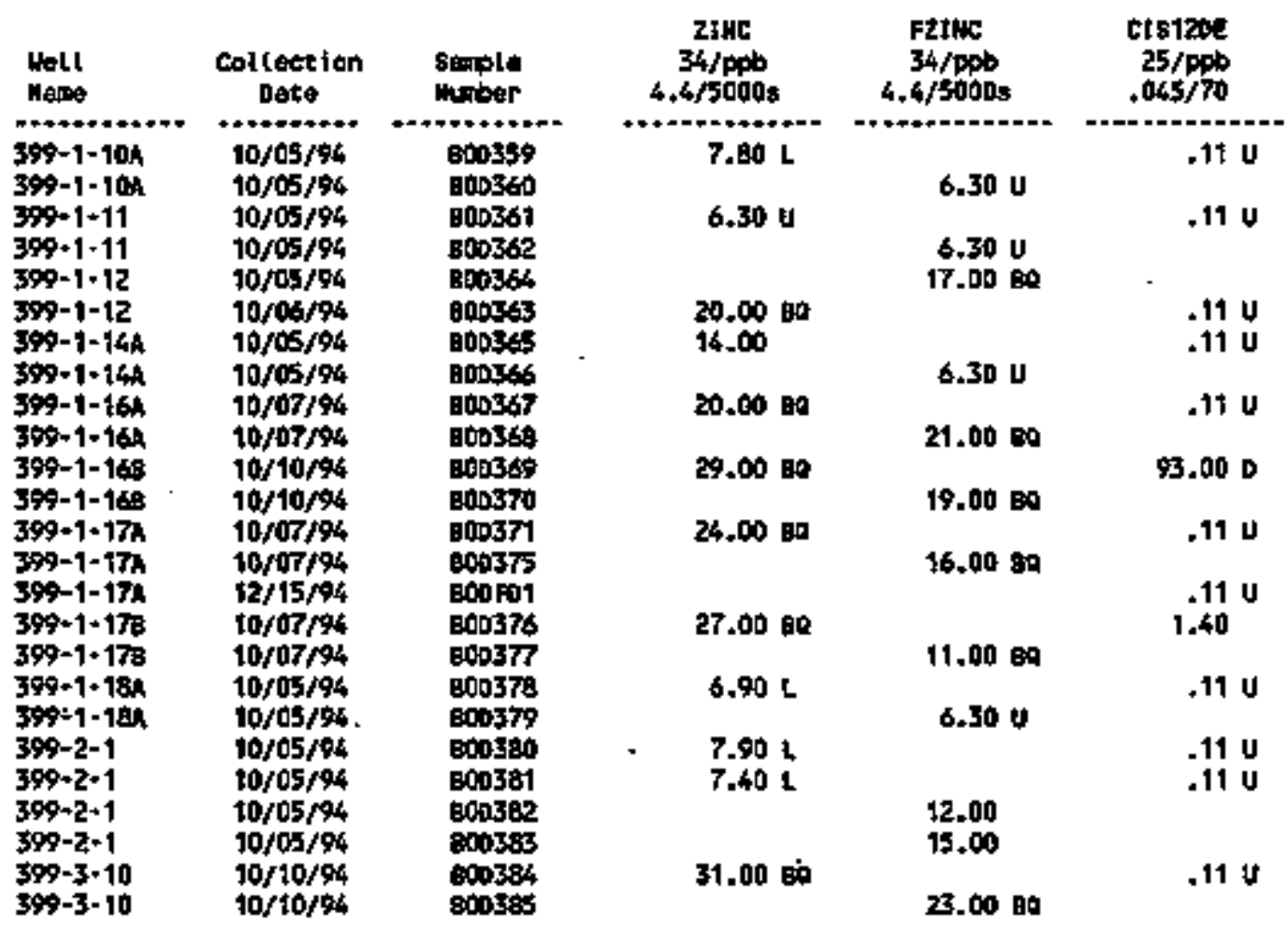

For explangtion of this tabe, sat Section 1,4 of raport: 
Table 16-5. Contamination Indicator Parameters for the 300 Area Process Trenches Data for Reporting Period October 1 through December 31, 1994.

\begin{tabular}{|c|c|c|c|c|c|c|c|c|}
\hline $\begin{array}{l}\text { Wett } \\
\text { Neme }\end{array}$ & $\begin{array}{c}\text { Coliection } \\
\text { Date } \\
-\end{array}$ & $\begin{array}{c}\text { scople } \\
\text { wimber }\end{array}$ & $\begin{array}{c}\text { Cowl f1ELD } \\
\text { who } \\
\text { I/ }\end{array}$ & 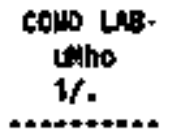 & $\begin{array}{l}\text { PH FIEAD } \\
\text {.01/6.5-8.5s }\end{array}$ & $\begin{array}{c}\text { PH LAB } \\
.01 / 6.5-8.53\end{array}$ & $\begin{array}{c}\text { TOC } \\
\text { Ppos } \\
110 / .\end{array}$ & $\begin{array}{c}\text { Tox } \\
\text { Fph } \\
\$ 1 / .\end{array}$ \\
\hline
\end{tabular}

For explantion of this table, aee section 1.4 of repoet. 
DOE/RL-94-36-4

This page tntentionally left blank.

16-22 
DOE/RL-94-36-4

\section{CONTENTS}

17.0 NONRADIOACTIVE DANGEROUS WASTE LANDFILL $\ldots \ldots \ldots \ldots$

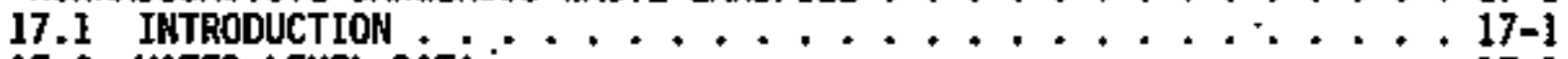

17.2 WATER LEVEL DATA $\ldots \ldots \ldots \ldots \ldots \ldots$ 17-1

17.3 HATER CHENISTRY DATA ........................ 


\section{LIST OF FIGURES}

17-1 Nell Location Map for the Monradioactive Dangerous Waste Landfill and Solid Waste Landftil .............. . 17-2

\section{LIST OF TABLES}

17-1 Monitoring Well Purpose and Saspling Schadule for the Nonradioact lve Dangerous Waste Landfill Metwork . . . . . . 17-3

17-2 RCRA Water Leve1 Heasurement Report NRDHL, Fourth quarter 1994 . . . . . . . . . . . . . 17-4 
DOE/RL-94-36-4

\title{
17.0 MONRADIOACTIVE DANGEROUS WASTE LANDFILL
}

\author{
F. H. Hodges \\ Westinghouse Hanford Company
}

\subsection{INTRODUCTION ,}

The Nonradioactive Dangerous Waste Landfil1 (NRONL) is part of the Hanford Central Landfill located approximately $5.6 \mathrm{~km}(3.5 \mathrm{mi})$ southeast of the 200 East Area. The NRDUL, covered under the Consent Agreement and Cosipli ance Order (Ecology and EPA 1986), is currently under an interim-status, detection level groundwater monitoring progran (WHC 1993b). The groundwater monitoring network consists of three upgradient and six downgradient wells (Table 17-1). Two upgradient and five downgradient wells are screened at the top of the water table. One upgradient and one downgradient well are screened at the top of a low-permeability untt approximately $21.3 \mathrm{~m}(70 \mathrm{ft})$ below the top of the water table. Figure 17-1 shows well locations. It should be noted that we11 699-26-34A was previously named 699-26-34.

\subsection{MATER LEYEL DATA}

Water level measurements were carried out on a monthly schedule for nine HRDWL monitoring wells, the six older monitoring wells of the adjacent Solid Naste Landfil1, and in severa] nearby non-RCRA wells. Results of the water level measurements are reported in Table 17-2.

\subsection{MATER CHENISTRY DATA}

The monitoring network for the HRDWh is on a semiannual sampiting schedule. The monitoring network was last sampled in August/September 1994 and was not sampled during this quartar. The next scheduled sampling for the MRDWL, network is in February 1995. There is no new data to report at this time. 
Figure 17-1. Well Location Map for the Nonradioactive Dangerous Waste Landfi11 and Sol io Waste Landfill.

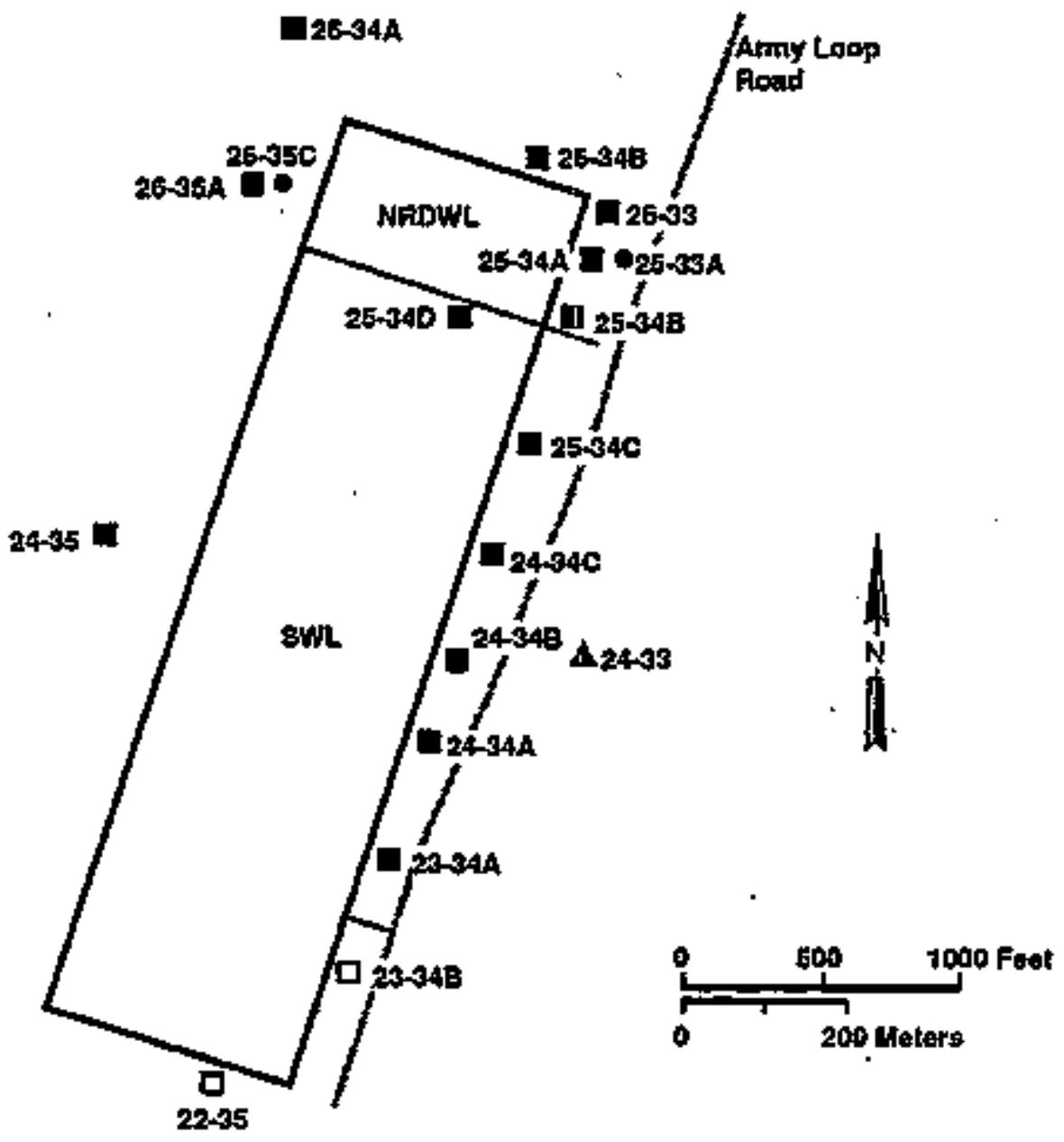

Weil completed at the top of the uncanitned equilter

Whil eornpletid in the upper Fingold Fommution

- WaH not cometrucled to RCRA spacitionatons

口 Now RCRA welle complated In 1992.

NADWL Nonradloactwe Dangerang Waste Lindfal

SWL Bolid Weste Lenciril

All wells preitixed by 698

Hidoptos.24 
Table 17-1. Monitoring Well Purpose and Sampling Schedule for the Honradioactive Dangerous Haste Landfi11 Network.

\begin{tabular}{|c|c|c|c|c|}
\hline $\begin{array}{c}\text { Well no. } \\
(699-)\end{array}$ & $\begin{array}{l}\text { Relative } \\
\text { position }\end{array}$ & Hydrogeologic unit & $\begin{array}{l}\text { Sample } \\
\text { frequency }\end{array}$ & $\begin{array}{l}\text { Sample date, } \\
\text { 4th Qtr } 1994\end{array}$ \\
\hline $26-34 A$ & Upgradtent & $\begin{array}{l}\text { Hanford: Water } \\
\text { Table }\end{array}$ & Semiannually & n.a. \\
\hline $26-35 A$ & Upgradient & $\begin{array}{c}\text { Hanford: Water } \\
\text { Table }\end{array}$ & Semiannually & n.a. \\
\hline $26-35 C$ & Upgradient & Top of Ringold & Semfiannualty & n.a. \\
\hline $25-33 A$ & Downgradient & Top of Ringold & Sent annually & n.a. \\
\hline $25-34 A$ & Downgradient & $\begin{array}{l}\text { Hanford: Hater } \\
\text { Table }\end{array}$ & Seni annually & n.a. \\
\hline $25-34 B$ & Downgradient & $\begin{array}{l}\text { Hanford: Water } \\
\text { Table }\end{array}$ & Sem tannually & n.a. \\
\hline $25-34 D$ & Downgradjent & $\begin{array}{c}\text { Hanford: Water } \\
\text { Table }\end{array}$ & Semíanntually & n.a. \\
\hline $26-33$ & Downgradient & $\begin{array}{l}\text { Hanford: Water } \\
\text { Table }\end{array}$ & Semi annually & n.a. \\
\hline $26-34 B$ & Downgradtent & $\begin{array}{l}\text { Hanford: Water } \\
\text { Table }\end{array}$ & Semi annual ly & n.a. \\
\hline
\end{tabular}

n.a. not appijcable

Hote: Hydrogeologic units include the sandy gravels of the Hanford formation and silty sands of the Rfingold Formation. Water levels are maasured monthly in all wells. 
Table 17-2. RCRA Hater LeveI Measurement Report NRONL, Fourth Quarter 1994.

(sheet 1 of 3)

\begin{tabular}{|c|c|c|c|c|c|}
\hline Wel] & Date & $\begin{array}{l}\text { Depth to } \\
\text { water (ft) }\end{array}$ & $\begin{array}{l}\text { Water } \\
\text { elevatjon } \\
\text { (ft) }\end{array}$ & $\begin{array}{c}\text { level } \\
\text { above ms } \\
\text { (m) }\end{array}$ & \\
\hline \multicolumn{6}{|c|}{ MRDHL Wells Monitoring the Top of the Unconfined Aquifer at the Water Table } \\
\hline $699-25-34 A$ & $\begin{array}{l}10 / 27 / 94 \\
11 / 28 / 94 \\
12 / 15 / 94\end{array}$ & $\begin{array}{l}129.70 \\
129.85 \\
129.89\end{array}$ & $\begin{array}{l}400.61 \\
400.46 \\
400.42\end{array}$ & $\begin{array}{l}122.11 \\
122.06 \\
122.05\end{array}$ & \\
\hline $699-25-34 B$ & $\begin{array}{l}10 / 27 / 94 \\
12 / 15 / 94\end{array}$ & $\begin{array}{l}129.70 \\
129.89\end{array}$ & $\begin{array}{l}399.70+ \\
399.51+\end{array}$ & $\begin{array}{l}121.83 \\
121.77\end{array}$ & • \\
\hline $699-25-340$ & $\begin{array}{l}10 / 27 / 94 \\
11 / 28 / 94 \\
12 / 16 / 94\end{array}$ & $\begin{array}{l}136.84 \\
136.83 \\
136.86\end{array}$ & $\begin{array}{l}401.07 \\
401.08 \\
401.05\end{array}$ & $\begin{array}{l}122.25 \\
122.25 \\
122.24\end{array}$ & \\
\hline $699-26-33$ & $\begin{array}{l}10 / 27 / 94 \\
11 / 28 / 94 \\
12 / 15 / 94\end{array}$ & $\begin{array}{l}135.57 \\
134.78 \\
134.90\end{array}$ & $\begin{array}{l}400.09 \\
400.88 \\
400.76\end{array}$ & $\begin{array}{l}121.95 \\
122.19 \\
122.15\end{array}$ & $\cdot$ \\
\hline $699-26-34 A$ & $\begin{array}{l}10 / 27 / 94 \\
11 / 01 / 94 \\
11 / 28 / 94 \\
12 / 15 / 94\end{array}$ & $\begin{array}{l}127.44 \\
132.76 \\
127.55 \\
127.71\end{array}$ & $\begin{array}{l}400.96 \\
395.64 *+ \\
400.85 \\
400.69\end{array}$ & $\begin{array}{l}122.21 \\
120.59 \\
122.18 \\
122.13\end{array}$ & \\
\hline $699-26-34 B$ & $\begin{array}{l}10 / 27 / 94 \\
11 / 28 / 94 \\
12 / 15 / 94\end{array}$ & $\begin{array}{l}129.17 \\
129.19 \\
129.21\end{array}$ & $\begin{array}{l}401.10 \\
401.08 \\
401.06\end{array}$ & $\begin{array}{l}122.26 \\
122.25 \\
122.24\end{array}$ & \\
\hline \multirow[t]{2}{*}{$699-26-35 A$} & $\begin{array}{l}10 / 27 / 94 \\
11 / 01 / 94 \\
11 / 28 / 94 \\
12 / 15 / 94\end{array}$ & $\begin{array}{l}131.93 \\
131.68 \\
131.59 \\
132.03\end{array}$ & $\begin{array}{l}400.73 \\
400.98^{*} \\
401.07 \\
400.63\end{array}$ & $\begin{array}{l}122.14 \\
122.22 \\
122.25 \\
122.11\end{array}$ & \\
\hline & \multicolumn{4}{|c|}{ HRDWL Wells Monitoring the Upper Ringold } & \\
\hline $699-25-33 A$ & $\begin{array}{l}10 / 27 / 94 \\
11 / 28 / 94 \\
12 / 15 / 94\end{array}$ & $\begin{array}{l}127.99 \\
128.08 \\
127.80\end{array}$ & $\begin{array}{l}400.98 \\
400.89 \\
401.17\end{array}$ & $\begin{array}{l}122.22 \\
122.19 \\
122.28\end{array}$ & \\
\hline $699-26-35 C$ & $\begin{array}{l}10 / 27 / 94 \\
11 / 28 / 94 \\
12 / 15 / 94\end{array}$ & $\begin{array}{l}131.58 \\
131.61 \\
131.62\end{array}$ & $\begin{array}{l}401.10 \\
401.07 \\
401.06\end{array}$ & $\begin{array}{l}122.26 \\
122.25 \\
122.24\end{array}$ & \\
\hline
\end{tabular}


Table 17-2. RCRA Hater Level Measurement Report NRDiL, Fourth Quarter 1994.

(sheet 2 of 3 )

We1] Date $\quad \begin{gathered}\text { Depth to } \\ \text { water (ft) }\end{gathered} \begin{gathered}\begin{array}{c}\text { Water level } \\ \text { elevation above ms1 } \\ \text { (ft) }\end{array} \\ \text { (m) }\end{gathered}$

MRDWL Wells Monitoring the Upper Ringold

Other Hel]s Associated with MRONL

$699-20-20$

$699-20-39$

$699-22-35$

699-23-34A

699-23-34B

$699-24-33$

$699-24-34 A$

$699-24-34 B$

$699-24-34 C$

699-24-35
$10 / 27 / 94$

$11 / 28 / 94$

$12 / 15 / 94$

$10 / 27 / 94$

$11 / 28 / 94$

$12 / 15 / 94$

11/02/94

$10 / 27 / 94$

$11 / 02 / 94$

$11 / 28 / 94$

$12 / 15 / 94$

$11 / 02 / 94$

$11 / 02 / 94$

$11 / 28 / 94$

$12 / 15 / 94$

$10 / 27 / 94$

$11 / 01 / 94$

$11 / 28 / 94$

$12 / 15 / 94$

$10 / 27 / 94$

$11 / 01 / 94$

$11 / 28 / 94$

$12 / 15 / 94$

$10 / 27 / 94$

$11 / 02 / 94$

$11 / 28 / 94$

$12 / 15 / 94$

$10 / 27 / 94$
106.04

106.17

106.12

138.61

138.77

138.65

132.97

131.82

131.80

131:83

I31.84

132.48

$123.40^{\circ}$

123.22

123.24

132.84

132.76

132.84

132.86

132.43

132.39

132.43

132.45

131.54

131.50

131.54

131.57

137.74
399.54

399.41

399.46

401.37

401.21

401.33

401.00*

401.04

$401.05^{*}$

401.03

401.02

401.02*

400:87*

401.05

401.03

401.04

$401.12 *$

401.04

401.02

401.07

$401.11 *$

401.07

401.05

401.05

$401.09 *$

401.05

401.02

401.07
121.78

121.74

121.76

122.34

122.29

122.33

122.22

122.24

122.24

122.23

$\$ 22.23$

122.23

122.19

122.24

122.23

122.24

I22. 26

122.24

122.23

122.25

I 22.26

122.25

122.24

$\$ 22.24$

122.25

I22. 24

122.23

122.25 
Table 17-2. RCRA Water Level Measurement Report NRONL, Fourth Quarter 1994. (sheet 3 of 3 )

\begin{tabular}{|c|c|c|c|c|}
\hline Hell & Date & $\begin{array}{l}\text { Depth to } \\
\text { water (ft) }\end{array}$ & $\begin{array}{l}\text { Water } \\
\text { elevation } \\
\text { (ft) }\end{array}$ & $\begin{array}{l}\text { level } \\
\text { above ms1 } \\
\text { (m) }\end{array}$ \\
\hline . & \multicolumn{4}{|c|}{ other Wells Associated with NRDUL } \\
\hline $699-24-35$ & $\begin{array}{l}11 / 01 / 94 \\
11 / 28 / 94 \\
12 / 15 / 94\end{array}$ & $\begin{array}{l}137.71 \\
137.75 \\
137.75\end{array}$ & $\begin{array}{ll}401.10^{*} \\
401.06 \\
401.06\end{array}$ & $\begin{array}{l}122.26 \\
122.24 \\
122.24\end{array}$ \\
\hline $\begin{array}{c}699-25-34 C \\
-\end{array}$ & $\begin{array}{l}10 / 27 / 94 \\
11 / 02 / 94 \\
11 / 28 / 94 \\
12 / 15 / 94\end{array}$ & $\begin{array}{l}134.41 \\
134.38 \\
134.42 \\
134.44\end{array}$ & $\begin{array}{l}401.05 \\
401.08^{\star} \\
401.04 \\
401.02\end{array}$ & $\begin{array}{l}122.24 \\
122.25 \\
122.24 \\
122.23\end{array}$ \\
\hline $699-28-40$ & $\begin{array}{l}10 / 27 / 94 \\
11 / 28 / 94 \\
12 / 15 / 94\end{array}$ & $\begin{array}{l}158.05 \\
158.12 \\
158.01\end{array}$ & $\begin{array}{l}401.39 \\
401.32 \\
401.43\end{array}$ & $\begin{array}{l}122.34 \\
122.32 \\
122.36\end{array}$ \\
\hline $699-31-31$ & $\begin{array}{l}10 / 27 / 94 \\
11 / 28 / 94 \\
12 / 15 / 94\end{array}$ & $\begin{array}{l}12 B \cdot 38 \\
128.37 \\
128.28\end{array}$ & $\begin{array}{l}400.94 \\
400.95 \\
401.04\end{array}$ & $\begin{array}{l}122.21 \\
122.21 \\
122.24\end{array}$ \\
\hline $699-34-39 A$ & $\begin{array}{l}10 / 27 / 94 \\
11 / 28 / 94 \\
12 / 15 / 94\end{array}$ & $\begin{array}{l}135.57 \\
135.61 \\
135.55\end{array}$ & $\begin{array}{l}401.50 \\
401.46 \\
401.52\end{array}$ & $\begin{array}{l}122.38 \\
122.37 \\
122.38\end{array}$ \\
\hline
\end{tabular}

NDTES: 1. Water level elevations are calculated by subtracting the measured depth-to-water from the surveyed elevation for the well.

2. Depth-to-water values are transcribed from field records.

3. Elevations marked with an $' * 1$ were measured at the time of sampling.

4. Elevations marked with a " + ' are outstde of the expected range, and are suspected of error. 


\subsection{REFEREAICES}

40 CFR 141, "Nationa] Primary Orinking Water Reguiations," Code of Federal Regutations, as amended.

40 CFR 143, "National Secondary Orinking Water Regulations," Cade of Federal Reguitions, as amended.

40 CFR 265, "Interim Status Standards for Owners and Operators of Hazardous Waste. Treatwent, Storage, and Disposal Facilities," Code of Federal Regulations, as amended.

Anderson, J. D., 1990, A History of the 200 Area Tank Farms: Westinghouse Hanford Company, WHC-MR-0132, Westinghouse Hanford Company, Richland, Hashington.

APHA, 1989, Standand Methods for Evajuation of Hater and Wastewater, 17th ed., American Public Health Association, Washington, D.C.

ASTH, 1991, Annual Book of ASTM Standards, Vol. 11.01, Water and Environmental Techrology, Phtladee]phia, Pennsylvania.

Caggiano, J. A., and C. J. Chou, 1993, Interim-Status Groundwater Quality Assessment Plan for the Single Shell Tank Waste Hanagement Areas $T$ and TX-TY: Richland, Washington, Westinghouse Hanford Company, WHC-SD-EN-AP-132, Rev. 0, Hestinghouse Hanford Company, Richland, Washington.

Caggiano, J. A., and S. M. Goodwin, 1991, Interim-Status Groundwater Monftoring Plan for the Single-Shelf Tanks, WHC-SD-EN-AP-012, Rev. 1, Westinghouse Hanford Company, Rich 1 and, Washington.

Chamness, M. A., S. P. Luttre11, and S. Dudziak, 1989, 40 CFR 265 Interin Status Ground-Water Nonftoring P1 an for the 2101-N Pond, PNL-6851, Pacific Northwest Laboratory, Rich1and, Washington.

Chou, C. J., G. L. Kasza, and R. B. Hercer, 1990, Interim-Status Groundwater Qualfty Assessment Plan for the 216-A-29 Ditch, WHC-SD-EN-AP-031, Rev. 0, Westinghouse Hanford Company, Richland, Mashington.

DOE-RL, 1994, Annua7 Report for RCRA Groundwater Monitoring Projects at Hanford Site factifties for 1993, DOE/RL-93-88, U.S. Department of Energy, Richl and Operations office, Richland, Washington.

DOE-RL, 1995, AnnuaI Report for RCRA Groundwater Monitoring Projects at Hanford Site Facilities for 1994, DOE/RL-94-136, U.S. Department of Energy, Richl and Operations, Office, Richland, Washington.

Ecology, 1994, Dangerous Waste Portion of the Resource Conservation and Recovery Act Perrit for the Treatment, Storage, and Disposal of Dangerous Waste, Permit No. WA7890008967, effective Septemer 28, Washington State Department of Ecology, 0lympia, Hashington.. 
Ecology and EPA, 1986, Consent Agraement. and Compliance Order, Ecology Ho. DE 86-133, Hashington State Department of Ecology and the U.S. Environmental Protection Agency, 0lympta, Washington.

Ecology, EPA, and DOE, 1992, Hanford Federal Factitty Agreement and Consent Order, 2 vols, Washtugton State Department of Ecology, U.S. Environienta] Protection Agency, and U.S. Department of Energy, olympia, Washington.

EPA, 1979, Hethods for Chemical Analysis of Water and Waste, EPA-600/4-79-020, U.S. Envtronmental Protection Agency, Environmental Monitioring and Support Laboratory, Cincinati, Ohto.

EPA, 1986a, RCRA Groundwater Honttoring Technical Enforcement Guidance Docunent, OSWER-9950.1, U.S. Envirombenta1 Protection Agency, Washington, D.C. .

EPA, 1986b, Test Hethods for Evaluating Solfd Waste, Sy-846, Third Edition, U.S. Environmenta] Protection Agency, Hashington, 0.C.

Harris, S. F., 1990, Groundwater Qualfty Assessfient Plan for the 216-B-3 Pond Systen, WHC-SD-EN-AP-030, Rev. 0, Westinghouse Hanford Company, Richiand, Hashington.

Hartman, M. J., 1991, Groundwater Nonitoring PJan for the 100-D Ponds, WHC-\$D-EN-AP-048, Rev. 0, Westinghouse Hanford Company, Richland, Washingtori.

Jensen, E. J., S. P. Airhart, M. A. Chamess, T. J. Gilmore, D. R. Newconer, and K. R. Oster, 1989, 40 CFR 265 Interin-Status Ground-Water Monitoring Plan for the Single-She 11 Tanks, WHC-SD-EA-AP-012, Westinghouse Hanford Company, Rjchland, Washington

Kasza, 6. L., and \$. M. Goodwin, 1991, Groundwater Honitoring Plan for the 216-A-29 Ditch, UHC-SD-EN-AP-045, Rev. 0, Westinghouse Hanford Company, Richland, Washington.

PNL, 1989, 40 CFR 265 Interio-Status Indicator-Evaluation Groundwater Nonitoring Plan for the 216-8-63 Trench, PNL-6862, Pacific Northwest Laboratory, Richland, Washington.

RCW 248, "Public Water Supplies," Revised Code of Washington, as amended.

Resource Conservation and Recovery Act of 1976, 42 USC 6901, et seq.

Schal]a, R., 1988, Revised Ground-Water Honitoring Coopliance Plan for the 300 Area Process Trenches, PAl -6671 , Pacific Northwest Laboratory, Richiand, Hashington.

Schalidt, J. W., A. R. Johnson, 5. M. McKinney, C. J. Perkins, and C. R. Webb, 1992, Westinghouse Hamford Company Environnental Surveil iance Annua? Report, WHC-EP-0573, Westinghouse Hanford Company, Richland, Washington. 
Sweeney, M. D., 1994, Interin-Status Groundwater Monitoring Plan for the 216-B-3 Pond Systen, WHC-SO-EN-AP-013, Rev. 1, Westinghouse Hanford Company, Richland, Washington.

Thornton, E. C.; 1995, "2101-W Pond," in Annual Report for RCRA Groundwater Honitoring Projects at Hanford Site Facilities for 1994, DOE/RL-94-136, U.S. Department of Energy, Richland, Washington.

WAC 173-160, "Mintmum Standards for Construction and Maintenance of Wel1s," Washington Adginistrative Code, as amended.

WAC 173-200, "Water Quality Standards of the State of Washington, "Washington Administrative Code, as anended.

WAC 173-303, "Dangerous Waste Regulations," Washington Administrative Code, as amended.

WHC-CM-7-8, Environmental Engineering and Geotechnology function Procedures, Volume 4, Section 2.1, Rev. 1, Westinghouse Hanford Company, Rjchland, Washington.

WHC, 1990a, Interio-Status Groundwater Monitoring Plan for the 216-S-10 Pond and Ditch. WHC-SO-EM-AP-018, Rev, 0, Westinghouse Hanford Company, RichTand, Washington.

WHC, 1990b, Interim-Status Groundwater Honitoring Plan for the 216-U-12 Crib, HC-SO-EN-AP-019, Rev, 0, Westinghouse Hanford Company, RichTand, Washington.

WHC, 1991, Interin Status Groundwater Monitoring Plan for the 200 East Area Liquid Effiuent Retention Faci7ity, WHC-SB-EN-AP-024, Rev. I, Westinghouse Hanford Company, Richland, Washington.

WHC, 1992a, Generic Specifications--Groundwater Monitoring Wells, WHC-5-014, Rev. 7, Mestínghouse Hanford Company, Richland, Washington.

WHC, 1992b, Quality Assurance Project Plan for RCRA Groundwater Honitoring Actfyities, HHC-SD-ER-QAPP-001, Rev. 2, Hestinghouse Hanford Company, Richland, Washingtion.

WHC, 1993a, Interim-Status Groundwater Quality Assessment PIan for the 216-U-12 Crib, HHC-SD-EN-AP-108, Rev. O, Westinghouse Hanford Company, Richland, Washington.

WHC, 1993b, Interim Status Groundwater Honitoring P7an for the Nonradioactive Dangerous Waste Landfill, Hanford, Hashington, WHC-SD-EN-AP-026, Rev. 0, West inghouse Hanford Company, Rich] and, Washington.

WHC, 1993c, Radionuclide and Chemical Inventories for the Single-Shell Tanks: Report Prepared for Westinghouse Hanford Company by WASTREN, Inc., WHC-SD-WH-T-I-565, Rev. I, Westinghouse Hanford Company, Rich] and, Washington. 
DOE/RL-94-36-4

This page intentionally left blank. 
DOE/RL-94-36-4

DISTRIBUTIOH.

Nunber of copies

OFFSITE

2

Washington State Department of

Ecologr - Kenneytck Branch

1315 W. 4th

Kennewick, Washington 99336-6018

J. Hallace

1

Hesttinghouse Savannah River Company

P. 0. Box A

Aiken, South Carol ina 29801

R. C. Tuckfield

ONSITE

10

U.S. Department of Energy, Bichland Operations office

R. D. Freeberg

M. J. Furman (4)

J. M: Hennig

R3-81

R. G. HcLeod

$57-55$

R. P. Saget

A5-19

RL Public Reading Room (2)

13

Bechtel_Hanford. Inc.

K. R. Fecht

H6-04

B. H. Ford

H4-85

H. J. Galgoul

H6-01

W. H. Hayward

T7-05

M. C. Hughes

H4-84

L. C. Hulstrom

H6- 05

R. L. Jackson

H4-90

G. L. Kasza

H6-04

A. J. Knepp

H4-85

H. A. Mihalife

X5-53

D. L. Parker

H6-02

R. K. Tranbarger

C. D. Wittreich

H6-04

H6-02 


\section{DISTRIBUTION (cont.)}

Number of copies

ONSIIE

4

Kaiser Entineers, Hanford

L. 5. Angerman

R. N. Brown

L6-61

V. A. Retterer

ᄂ6-79

J. T. Sams

\$2-21

H5-14

Pactfic Morthwest Laboratory

R. W. Bryce

K6-96

B. E. Opitz

D. 1. Stewart

$K 6-79$

Technical files

K6-96

K1-11

Yestinghouse Hanford Company

M. R. Adams

M. D. Alchele

D. 3. Alexander

D. B. Barnett

D. J. Brown

J. A. Caggiano

D. J. Carreli

C. J. Chou

J. J. Dorian

H. H. Edrington

D. 6. Farwick

P. B. Freeman

W. 6. Gardner

C. J. Geier

R. D. Gustavson

V. H. Hamfilton

M. J. Hartman

F. H. Hodges

D. G. Horton

V. G. Johnson

J. C. Johnston

J. W. Lindberg

A. K. HcDowe 11

R. B. Hercer

R. D. Pierce

D. B. Powel1

D. A. Pratt

H6-30

T4-04

H6-06

H6-06

B4-63

H6-06

H6-22

H6-06

H6-20

H6-06

H4-16

H6-06

13-06

R2-36

R1-51

T4-85

H6-06

H6-06

H6-06

H6- 06

H6-05

H6-06

R1-51

H6-06

T3-04

T4-03

S. H. Price

T4-03

H6-23 


\section{DISTRIBUTION (cont.)}

Howber of conies

\section{DNSITE}

Yestinghouse Hanford Company (cont.)

R. W. Reddinger

T4-05

J. S. Schmid

J. A. Serkowski

M. D. Sweeney

R. R. Thampson

E. C. Thornton

D. J. Hatson

B. A. Williams

S. A. Williams (10)

H. T. York

H. -06

H6-06

H6-06

H6-32

H6-06

$\times 3-79$

H6-06

H6-32

Central Fjos

EP.IC (7)

Information Release

H6-32

L8-04

H6-08

Administration

H4-17

0STI (2)

L8-07

Tank Farms Information

Center

R1-20 
DOE/RL-94-36-4 -

Th1s page intentiona11y left blank.

Distr-4 